





1 


\title{
CONFINED ZONE DISPERSION \\ FLUE GAS DESULFURIZATION DEMONSTRATION
}

Quarterly Report No. 8

For the Period

August 17, 1992 to November 16, 1992

\author{
Prepared for: \\ U.S. Department of Energy \\ Pennsylvania Electric Company \\ Pennsylvania Energy Development Authority \\ New York State Electric \& Gas Corporation \\ Rockwell Lime Company
}

\author{
Prepared by: \\ Bechtel Corporation \\ Fifty Beale Street \\ San Francisco, California 94105-1895
}

September 27, 1993

\section{Cooperative Agreement No. DE-FC22-90/ 9190546 \\ Patents. Cleared by Chicago on May 4, 1993 DISCLAIMER}

This report was prepared as an account of work sponsored by an agency of the United States Government. Neither the United States Government nor any agency thereof, nor any of their employees, makes any warranty, express or implied, or assumes any legal liability or responsibility for the accuracy, completeness, or usefulness of any information, apparatus, product, or process disclosed, or represents that its use would not infringe privately owned rights. Reference herein to any specific commercial product, process, or service by trade name, trademark, manufacturer, or otherwise does not necessarily constitute or imply its endorsement, recommendation, or favoring by the United States Government or any agency thereof. The views and opinions of authors expressed herein do not necessarily state or reflect those of the United States Government or any agency thereof. 


\section{CONTENTS}

Section

Page

LEGAL NOTICE vi

1 SUMMARY 1-1

2 INTRODUCTION $2-1$

3 PROJECT STATUS 3-1

3.1 COMPLETION OF DESIGN, PROCUREMENT, AND INSTALLATION (WBS No. 1.3.3) 3-1

3.2 COMPLETION OF FACILITY CONSTRUCTION (WBS No. 1.3.4)

3.3 CZD DEMONSTRATION WITH CONTINUOUS OPERATION (WBS No. 1.3.6)

3.3.1 August 17 to September 16, 1992

3.3.2 September 17 to October 16, 1992

3.3.3 October 17 to November 16, 1992

3.3.4 Percentage of Lime Utilization and the Factors that Could Affect It 3-38

3.3.5 Importance of Heat Balance in the CZD Process 3-49

3.3.6 CZD System Influence on ESP Particulate 3-57 Removal Efficiency

3.3.7 Summary of CZD Operating Data on August 21, $1992 \quad 3-60$

3.3.8 Summary of CZD Operating Data - August 17 to Noyember 16, 1992 3-61

3.3.9 Operational Data - August 17 to November 16, 1992 3-62

3.3.10 Conclusions 3-62

3.4 BASELINE TESTING - FUEL 3-63

4 PLANNED ACTIVITIES 4-1

4.1 PHASE 3 - OPERATION AND DISPOSITION 4-1

4.2 PHASE 3 - PROJECT MANAGEMENT 4-1 


\section{APPENDICES}

Appendix

Page

A Description of Project and Work Breakdown Structure A-1

A.1 Project Description A-1

A.2 Work Breakdown Structure Description A-2

B ' Report Distribution List B-1

C Tables with Summary of Lime Slurry Injection Data August 17 to September 16, 1992

D Tables with Summary of Lime Slurry Injection Data September 17 to October 16, 1992

E Tables with Summary of Lime Slurry Injection Data October 17 to November 16, 1992

F Baseline Testing - Fuel F-1

G Procedures for Calculating $\mathrm{SO}_{2}$ and $\mathrm{NO}_{x}$ Removal and the Percentage of Lime Utilization

$\mathrm{H} \quad$ Tables with Lime Slurry Injection Operating Data August 17 to November 16, 1992

H-1

I Buell ESP Fields Operating Log

I-1 


\section{ILLUSTRATIONS}

Figure

Page

1-1 Major Milestone Status from Pre-Award Activities through November 16, 1992

2-1 Seward Station Arrangement 2-3

3-1 Seward Station Overall Process Flow Diagram 3-2

3-2 Seward CZD System Overview 3-3

3-3 Lime Storage and Slurry Preparation - Continuous Operation 3-4

3-4 Lime Slurry Feed System - Continuous Operation 3-5

3-5 Lime Slurry Injection - Continuous Operation 3-6

3-6 Lime Flow vs $\mathrm{SO}_{2}$ Removal - August 17 to September 16, $1992 \quad 3-12$

3-6A Daily Average Lime Flow vs $\mathrm{SO}_{2}$ Removal - August 17 to September 16, 1992

3-6B Daily Average Lime Slurry Concentration vs $\mathrm{SO}_{2}$ Removal August 17 to September 16, 1992

3-7 Removal vs Injection Rate, Based on August 17 to September 16, 1992 Daily Averages

3-8 Lime Flow vs $\mathrm{SO}_{2}$ Removal - September 17 to October 16, $1992 \quad 3-20$

3-8A Daily Average Lime Flow vs $\mathrm{SO}_{2}$ Removal - September 17 to October 16, 1992

3-8B Daily Average Lime Slurry Concentration vs $\mathrm{SO}_{2}$ Removal September 17 to October 16, 1992

3-9 Boiler Load and Flue Gas Flow - September 17 to October 16, 1992 3-23

3-10 Boiler Load and Flue Gas Flow - October 12 to October 16, $1992 \quad$ 3-25

3-11 Boiler Load and Flue Gas Flow - October 12, 1992

3-12 Boiler Load and Flue Gas Flow - October 13, 1992

3-13 Boiler Load and Flue Gas Flow - October 15, 1992

3-14 Lime Flow vs $\mathrm{SO}_{2}$ Removal - October 17 to November 16, 1992 3-35

3-14A Daily Average Lime Flow vs $\mathrm{SO}_{2}$ Removal - October 17 to

November 16, 1992 


\section{ILLUSTRATIONS (Cont'd)}

Figure

Page

3-14B Daily Average Lime Slurry Concentration vs $\mathrm{SO}_{2}$ Removal October 17 to November 16, 1992

3-15 CZD Performance Summary - October 17 to November 16, 1992

3-16 Percent of $\mathrm{SO}_{2}$ Removal at Constant $34 \mathrm{gpm}$ Injection Rate and Variable Lime Concentration

3-17 $\mathrm{SO}_{2}$ Removal at Different Injection Rates and Percentages of Lime Slurries

3-18 Temperature vs Lime Flow and $\mathrm{SO}_{2}$ Removal - August 17 to November 16, 1992

3-18A Daily Average Lime Flow vs $\mathrm{SO}_{2}$ Removal - August 17 to November 16, 1992

3-18B Daily Average Duct Inlet Temperature vs $\mathrm{SO}_{2}$ Removal August 17 to November 16, 1992

3-19 Lime Flow vs $\mathrm{SO}_{2}$ Removal - August 17 to November 16, 1992

3-19A Daily Average Lime Slurry Concertration vs $\mathrm{SO}_{2}$ Removal August 17 to November 16, 1992

3-20 B, C, D, and E Cross-Section Temperatures vs Water Flow Rate in gpm

3-21 B-Section Temperatures

3-22 C-Section Temperatures 3-52

3-23 D-Section Temperatures 3-53

3-24 E-Section Temperatures 3-54

3-25 Plan View of Duct with Thermocouple Locations 3-55

3-26 Typical Thermocouple Section (24 per Section) 3-56 


\section{TABLES}

Table

Page

3-1 Results of Different Lime Slurry Injection Rates and Lime Slurry Concentrations

3-2 Operations Data Recorded During the Lime Slurry Injection on August 17, 1992, at 5:10 p.m.

3-3 Variation of the Average Percentage of $\mathrm{SO}_{2}$ Removal with Percentage of Lime Slurry Concentration Between August 17 and November 16, 1992

3-4 Elemental LOI Analysis of Ash Samples Removed from Boiler \#15 Electrostatic Precipitator

3-5 Summary of CZD Operating Data

3-6 Results of Coal Analyses During September 1992 


\section{LEGAL NOTICE}

This report was prepared by Bechtel Corporation, pursuant to a Cooperative Agreement partially funded by the U.S. Department of Energy, and neither Bechtel Corporation, nor any of its subcontractors nor the U.S. Department of Energy, nor any person acting on behalf of either:

(A) Makes any warranty or representation, express or implied, with respect to the accuracy, completeness, or usefulness of the information contained in this report, or that the use of any information, apparatus, method, or process disclosed in this report may not infringe privatelyowned rights; or

(B) Assumes any liabilities with respect to the use of, or for damages resulting from the use of, any information, apparatus, method or process disclosed in this report.

Reference herein to any specific commercial product, process, or service by trade name, trademark, manufacturer, or otherwise, does not necessarily constitute or imply its endorsement, recommendation, or favoring by the U.S. Department of Energy. The views and opinions of authors expressed hereini do not necessarily state or reflect those of the U.S. Department of Energy. 


\section{LEGAL NOTICE}

This report was prepared by Bechtel Corporation, pursuant to a Cooperative Agreement partially funded by the U.S. Department of Energy, and neither Bechtel Corporation, nor any of its subcontractors nor the U.S. Department of Energy, nor any person acting on behalf of either:

(A) Makes any warranty or representation, express or implied, with respect to the accuracy, completeness, or usefulness of the information contained in this report, or that the use of any information, apparatus, method, or process disclosed in this report may not infringe privatelyowned rights; or

(B) Assumes any liabilities with respect to the use of, or for damages resulting from the use of, any information, apparatus, method or process disclosed in this report.

Reference herein to any specific commercial product, process, or service by trade name, trademark, manufacturer, or otherwise, does not necessarily constitute or imply its endorsement, recommendation, or favoring by the U.S. Department of Energy. The views and opinions of authors expressed herein do not necessarily state or reflect those of the U.S. Department of Energy. 


\section{Section 1}

\section{SUMMARY}

This is the eighth quarterly report for this project. It covers work performed on Phase 3b from August 17, 1992 through November 16, 1992.

Parametric testing was completed and the six-month continuous demonstration run was initiated in mid-August. Most of the automation of the CZD system has been completed, and the system integrated with the boiler operation of Unit 15.

The continuous demonstration run started on August 17. After about 10 hours of operation, the boiler of Unit 15 blew a tube and was shut down. During the shutdown, new permanent thermocouples were installed on the turning vanes in the duct. These thermocouples monitor temperature and automatically control lime injection. Operations (24 hours/day) resumed on August 20. In the following 10 days, consistent $\mathrm{SO}_{2}$ removal was obtained in the range of $45-54 \%$, and the operation of the automatic lime preparation and feed system was completely satisfactory. Type $\mathrm{S}$ - lime (pressure-hydrated dolomitic lime) was used during this period.

After 10 days of continuous ( 24 hours/day) lime injection operations, the steam pressure at which the duct sootblowers were operating was set too low to prevent deposits from forming on the bottom of the duct. The steam supply pressure to the sootblowers was increased to the maximum; however, it was not possible to remove some of the deposits that had already formed. In a few areas, these deposits were significant, and testing confirmed that further lime injection operations would cause accelerated growth of these deposits. Consequently, lime injection was halted, and it was decided to remove the deposits manually during the next boiler outage. At Penelec's request, the CZD system was kept online but put in the standby mode, and water injected into the duct to assist in opacity control.

To prepare the CZD system for winter operations, final heat tracing and insulation requirements for piping and equipment were completed in October. Penelec operated unit 15 for the entire month of September without a forced outage or maintenance shutdown. As a result, it was not possible to enter the duct to inspect or remove deposits.

Boiler \#15 was taken offline on October 5 due to boiler leaks. Subsequent inspection of the duct during the outage revealed substantial fly ash/lime deposits. Evidence confirmed that a large contributing factor to deposit formation was the previous faulty operation of the sootblowers. The duct was thoroughly cleaned before Boiler \#15 was restarted. As a preventive measure to limit duct deposits, an agreement was made with Penelec to operate the sootblowers more frequently. 
On October 9, the boiler was brought to full load, and lime injection was resumed. The CZD system was returned to full automatic control (load following); however, frequent opacity excursions were observed. Analysis of the Buell ESP field currents for the CZD duct revealed that the first field current was stable, or was slightly increased with the lime injection, but the other three fields showed a dramatic drop in currents. This drop in field currents with lime slurry was the direct opposite of the reaction of the ESP observed so far in the test program. During the past year's testing, it has been observed that the addition of lime increased the field currents and led to a lowering of duct and stack opacity. Further investigation and consultation with Buell Engineers led to the causes of the problem, which were faulty ground connections in the second and third fields. Since repairs required a boiler shutdown, lime slurry density was reduced to about $4 \%$ to permit continued injection. This, coupled with low inlet flue gas temperatures, limited $\mathrm{SO}_{2}$ removal to the range of $20-30 \%$.

On October 26, Boiler $\# 15$ was shut down due to a steam leak in the superheater. An inspection of the duct showed that the increased frequency of the sootblower operations was effective in controlling deposits. Although some buildup was noted on duct walls, floor, and turning vanes, the amount was small and not considered a problem. During the outage, the ESP ground problem was fixed, and the fan room coils were brushed and washed down to permit higher inlet flue gas temperatures. Boiler startup was initiated on October 28.

CZD lime injection was resumed on November 2 following the startup of Boiler \#15 and continued until November 16. Inlet flue gas temperatures to the CZD duct ranged from lows of about $260^{\circ} \mathrm{F}$ to highs of $280^{\circ} \mathrm{F}$, with average temperatures of $275^{\circ} \mathrm{F}$. During this period, the CZD system operated in the load following mode with lime injection rates determined automatically by the temperature at the turning vanes. Due to the low inlet temperatures, lime injection rates varied from $26 \mathrm{gpm}$ to $40 \mathrm{gpm}$, and $\mathrm{SO}_{2}$ removal rates ranged from $18 \%$ to $41 \%$. Lime slurry concentrations varied, in steps, from $5 \%$ to $10 \%$. For reference, the Seward Station CZD installation was designed to achieve $50 \%$ $\mathrm{SO}_{2}$ based on inlet flue gas temperatures of about $300^{\circ} \mathrm{F}$ with accompanying lime injection rates of about $50 \mathrm{gpm}$.

On November 16, lime injection was halted due to a leaking throttle valve in the secondary recirculation line of the lime feed system. The badly worn valve was permanently replaced with a heavy duty spool piece to receive the high pressure drop across the main control valve.

Since the commencement of the continuous demonstration period on August $17,100 \%$ mechanical and process availability of the CZD system has been experienced. Operability has been excellent, and no major equipment or instrumentation failures have occurred. Whenever Boiler \#15 has been in operation, the CZD system has been on-line, injecting lime for $\mathrm{SO}_{2}$ removal or water for stack opacity control. 
Figure 1-1 shows the major milestone status from the pre-award activities through November 16,1992, including the units planned and the units completed.



\section{Flgure 1-1 Major Milestone Status from Pre-Award Actlvities through November 16, 1992}




\section{Section 2}

\section{INTRODUCTION}

The CZD process involves injecting a finely atomized slurry of reactive lime into the flue gas duct work of a coal-fired utility boiler. The principle of the confined zone is to form a wet zone of slurry droplets in the middle of the duct confined in an envelope of hot gas between the wet zone and the duct walls. The lime slurry reacts with part of the $\mathrm{SO}_{2}$ in the gas, and the reaction products dry to form solid particles. A solids collector, typically an electrostatic precipitator (ESP) downstream from the point of injection, captures the reaction products along with the fly ash entrained in the flue gas.

The features that distinguish the CZD process from other similar injection processes are:

- Injection of an alkaline slurry directly into the duct, instead of injection of dry solids into the duct ahead of a fabric filter.

- Use of an ultrafine calcium/magnesium hydroxide, type $S$ pressurehydrated dolomitic lime. This commercial product is made from plentiful, naturally occurring dolomite.

- Low residence time, made possible by the high effective surface area of the Type $\mathrm{S}$ lime.

- Localized dispersion of the reagent. Slurry droplets contact only part of the gas while the droplets are drying, to remove up to 50 percent of the $\mathrm{SO}_{2}$. The process uses dual fluid rather than rotary atomizers.

- Improved electrostatic precipitator performance via gas conditioning from the increased water vapor content, and lower temperatures.

The waste product is composed of magnesium and calcium sulfite and sulfate, with some excess lime. This product mixed with fly ash is self-stabilizing because of the excess lime values, and thus tends to retain heavy metals in insoluble forms within the fly ash.

The demonstration is being conducted at Penelec's Seward Station, Unit No. 15. This boiler is a 147 MWe coal-fired unit, which utilizes Pennsylvania bituminous coal (approximately 1.2 to $2.5 \%$ sulfur). One of the two flue gas ducts leading from the boiler has been retrofitted with the CZD technology. The first existing ESP installed in the station is immediately behind the air preheater. The second ESP, installed about 15 years ago, is about 80 feet away from the first ESP. The station has two more boilers (\#12 and \#14) that burn the same coal and are connected to the common flue gas stack. The arrangement of Seward Station is shown in Figure 2-1. 
The goal of this demonstration is to prove the technical and economic feasibility of the CZD technology on a commercial scale. The process is expected to achieve $50 \% \mathrm{SO}_{2}$ removal at lower capital and O\&M costs than other systems.

The U.S. Department of Energy and Bechtel Corporation are engaged in a cooperative project to demonstrate the CZD Technology. DOE is providing half, or $\$ 5.2$ million, of the project's total $\$ 10.4$ million cost. Others contributing to the project are Pennsylvania Electric Company ( $\$ 3$ million), Bechtel $(\$ 1,340,000)$, the Pennsylvania Energy Development Authority $(\$ 750,000)$, New York Gas and Electric Company $(\$ 100,000)$, and Rockwell Lime Company $(\$ 23,000)$.

To achieve its objectives, the project is divided into the following three phases:

Phase 1: Design and Permitting

Phase 2: Construction and Start-up

Phase 3: Operation and Disposition

Phase 1 activities were completed on January 31,1991. Phase 2 activities were essentially concluded on July 31, 1991, and Phase 3a, Parametric Testing, was initiated on July 1, 1991. This Quarterly Technical Progress Report covers Phase 3b activities from August 17, 1992 through November 16, 1992.

For further information on the structure of this demonstration project, Appendix A provides a project description by phase, a work breakdown structure (WBS) Diagram, and a detailed description of the WBS. 







\section{Section 3}

\section{PROJECT STATUS}

This section of the report provides details of the work performed from August 17, 1992 through November 16, 1992. It also describes the activities carried out as part of the CZD demonstration with continuous operation. Figure 3-1 shows a Seward Station overall process flow diagram.

Figures 3-2 through 3-5 show the Seward CZD system overview, the lime storage and slurry preparation, the lime slurry feed system and the lime slurry injection, respectively, all for the continuous operation.

\subsection{COMPLETION OF DESIGN PROCUREMENT AND INSTALLATION (WBS 1.3.3)}

During the boiler shutdown on August 18, new permanent thermocouples were installed on the turning vanes in the duct. These thermocouples are now monitoring the temperature and automatically controlling the lime injection.

Final heat tracing and insulation requirements for piping and equipment were developed so that installation could be completed on October.

\subsection{COMPLETION OF FACILITY CONSTRUCTION (W.B.S. 1.3.4)}

Facility construction activities have been completed.

\subsection{CZD DEMONSTRATION WITH CONTINUOUS OPERATION (W.B.S. 1.3.6)}

\subsubsection{August 17, 1992 to September 16, 1992}

The starting day for the continuous operation was August 17, 1992. At that time, most of the equipment, instruments, and controls were installed, tested, and debugged.

The retractable soot blowers, the thermocouple lances inside the turning vanes, and the temperature control system for the lime slurry injection rate (based on the lowest temperature at C-cross section) were only in the process of being installed. As a result, during the last two weeks of August 1992, we had difficulties in manually controlling the lime slurry flow rate to the atomizers, based on average flue gas temperature at $C$-cross section. The variations in the boiler load could not be exactly matched manually with the lime slurry injection rate. When the new thermocouple lances on the turning vanes were installed and the temperature controller could regulate the lime slurry injection rate, all the difficulties with following the variation of the boiler and load completely disappeared. 


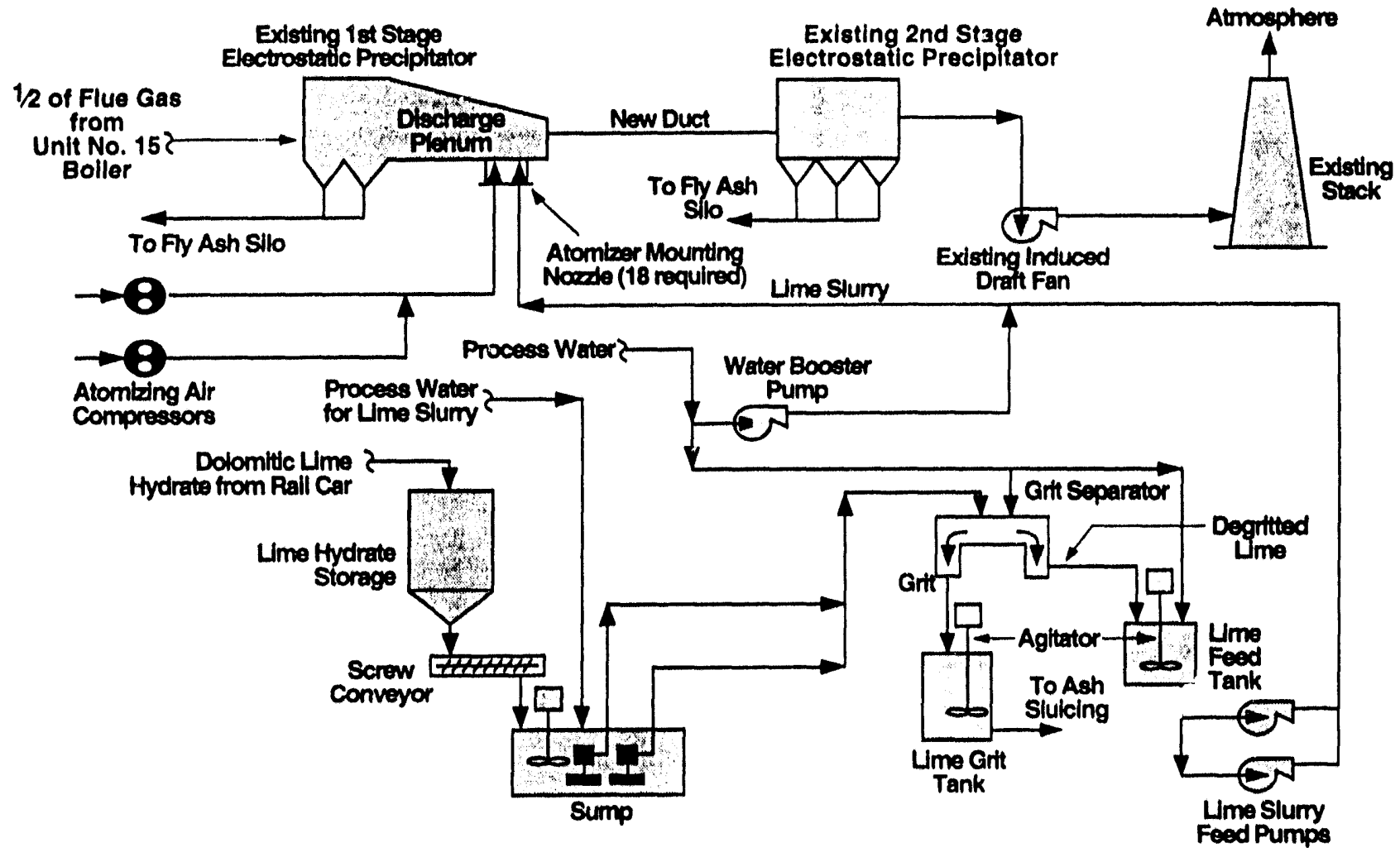

Figure 3-1 Seward Station Overall Process Flow Dlagram 




Figure 3-2 Seward CZD System Overview 




Figure 3-3 Lime Storage and Slurry Preparation - Continuous Operation 




Figure 3-4 Lime Slurry Feed System - Continuous Operation 




Figure 3.5 Lime Slurry Injection - Continuous Operation 


\subsubsection{August 17, 1992}

Continuous operation with the injection of pressure-hydrated dolomitic lime slurry started at 1 p.m. on August 17. Table 3-1 summarizes the results of the operation with different lime slurry injection rates and lime concentrations.

Table 3-1

Results of Different Lime Slurry Injection Rates and Lime Slurry Concentrations

\begin{tabular}{|l|c|c|c|c|}
\hline $\begin{array}{c}\text { Lime Slurny } \\
\text { injection Time }\end{array}$ & $\begin{array}{c}\text { Lime Slurry } \\
\text { Injection Rate } \\
\text { (opm) }\end{array}$ & $\begin{array}{c}\text { Lime } \\
\text { Concentration } \\
(\%)\end{array}$ & $\begin{array}{c}\mathrm{SO}_{2} \text { Removal } \\
(\%)\end{array}$ & $\begin{array}{c}\text { Stack Opacity } \\
(\%)\end{array}$ \\
\hline $2-1 / 2$ hours & $45-55$ & 10.3 & $25-42$ & $9.6-20$ \\
\hline $2-3 / 4$ hours & $54-55$ & 10.7 & $43-46.8$ & $15-20$ \\
\hline $2-1 / 2$ hours & $41-50$ & 13.9 & $38-41.8$ & $11-19.7$ \\
\hline $3 / 4$ hour & 50 & 11.5 & 40 & $13-14$ \\
\hline
\end{tabular}

During the lime slurry injection, the following operations data were recorded every 15 minutes. Table 3-2 shows the data recorded August 17 at 5:10 pm. 
Table 3-2

Operations Data Recorded During the Lime Slurry Injections on August 17 at 5:10 pm

\begin{tabular}{|c|c|}
\hline Operation & Recorded Data \\
\hline Boiler Load & $146.8 \mathrm{MW}$ \\
\hline Stack Opacity & $18.6 \%$ \\
\hline $\mathrm{SO}_{2}$ In & $176 \mathrm{scfm}$ \\
\hline $\mathrm{SO}_{2}$ Out & $92.4 \mathrm{scfm}$ \\
\hline Flue gas temperature in & $308.1^{\circ} \mathrm{F}$ \\
\hline Flue gas temperature out & $195.2^{\circ} \mathrm{F}$ \\
\hline Wt\% Lime Slurry & $14.4 \%$ \\
\hline Lime slurry injection rate & $53.4 \mathrm{gpm}$ \\
\hline Lime slurry level in feed tank & 7.5 feet \\
\hline Compressed air flow rate & $1,154 \mathrm{scfm}$ \\
\hline $\mathrm{SO}_{2}$ In & $922.8 \mathrm{ppm}$ \\
\hline $\mathrm{SO}_{2}$ Out & $392.8 \mathrm{ppm}$ \\
\hline $\mathrm{SO}_{2}$ Removal (Based on dilution of scfm flue gas in and out) & $47.5 \%$ \\
\hline $\mathrm{SO}_{2}$ Reduction (Based on dilution of $\% \mathrm{CO}_{2}$ in and out) & $44.5 \%$ \\
\hline $\mathrm{NO}_{\mathrm{x}}$ In & $351.9 \mathrm{ppm}$ \\
\hline $\mathrm{NO}_{\mathrm{x}}$ Out & $293.0 \mathrm{ppm}$ \\
\hline $\mathrm{NO}_{\mathrm{x}}$ Removal & $-2.37 \%$ \\
\hline $\mathrm{CO}_{2}$ In & $11.6 \%$ \\
\hline $\mathrm{CO}_{2}$ Out & $8.9 \%$ \\
\hline $\mathrm{CO}_{2}$ Dilution & $23.1 \%$ \\
\hline Flue gas in & $190,570 \mathrm{scfm}$ \\
\hline Flue gas out & $234,560 \mathrm{scfm}$ \\
\hline Flue gas dilution & $18.75 \%$ \\
\hline
\end{tabular}

The operation had to be stopped after only 8-1/2 hours of the lime slurry injection because of an emergency shutdown of the boiler.

During this period, the percentage of $\mathrm{SO}_{2}$ removal was usually over $40 \%$ and the percentage of stack opacity under $20 \%$. Increasing the lime slurry concentration 
from $10.3 \%$ to $13.9 \%$ - a $35 \%$ increase -- did not improve the percentage of $\mathrm{SO}_{2}$ removal. (See Appendix $C_{\text {.) }}$

The percent of $\mathrm{SO}_{2}$ removal increased when the lime slurry injection rate increased.

\subsubsection{August 20, 1992}

The dolomitic lime slurry injection into the duct started at 1745 hours and continued until 2345 hours - a total of 6 hours. The cumulative lime slurry injection time was 14.5 hours. The summary of CZD operations for August 20 is as follows:

- Lime injection: $40-50 \mathrm{gpm}$ with a $7.5 \%$ lime concentration

- $\mathrm{SO}_{2}$ removal: $30-41 \%$

- Stack opacity: $11-17 \%$

\subsubsection{August 21, 1992}

Dolomitic lime slurry injection in the duct started at 1445 hours and continued until 2330 hours, a total of 8.75 hours. The cumulative lime injection time was 23.25 hours. The summary of CZD operations on August 21 is as follows:

- Lime injection: $50-55 \mathrm{gpm}$ with a $9 \%$ lime concentration

- $\mathrm{SO}_{2}$ removal: $40-55 \%$

- $\quad$ Stack opacity: $7-9.7 \%$

\subsubsection{August 24, 1992}

The dolomitic lime slurry injection in the duct started at 1400 hours and continued until 2400 hours - a total of 10 hours. The cumulative lime slurry injection time was 33.25 hours. The summary of the CZD operations for August 24 is as follows:

- Lime injection: $40-59 \mathrm{gpm}$ with a $12 \%$ lime concentration

- $\mathrm{SO}_{2}$ removal: $30-50 \%$

- Stack opacity: $11-19.4 \%$ 


\subsubsection{August 25, 1992}

The dolomitic lime slurry injection in the duct started at 2400 hours and continued until 2000 hours - a total of 20 hours. The cumulative lime slurry injection time was 53.25 hours. The summary of CZD operations for August 25 is as follows:

- Lime injection: $48-56 \mathrm{gpm}$ with a $12 \%$ lime concentration

- $\mathrm{SO}_{2}$ removal: $30-44 \%$

- Stack opacity: $10-18 \%$

\subsubsection{August 26, 1992}

The dolomitic lime slurry injection in the duct started at 0245 hours and continued until 2400 hours - a total of 18.75 hours. The cumulative lime slurry injection time was 72 hours. The summary of CZD operations for August 26 is as follows:

- Lime injection: $50-58.8 \mathrm{gpm}$ with a $12 \%$ lime concentration

- $\mathrm{SO}_{2}$ removal: $40-53.5 \%$

- Stack opacity: $6-16 \%$

\subsubsection{August 27, 1992}

The dolomitic lime slurry injection in the duct started at 2400 hours and continued for 18 hours until 2400 hours. The cumulative lime slurry injection was 90 hours. The summary of CZD operations for August 27 is as follows:

- Lime injection: $49-59 \mathrm{gpm}$ with predominantly $12 \%$ lime concentration - $\mathrm{SO}_{2}$ removal: $41-48.8 \%$

- Stack opacity: $6-18 \%$

\subsubsection{August 28, 1992}

The dolomitic lime slurry injection in the duct started at 2400 hours and continued until 2245 hours - a total of 17 hours. The cumulative lime injection time was 107 hours. The summary of CZD operations on August 28 is:

- Lime injection: $40-55.8 \mathrm{gpm}$ with a $12 \%$ lime concentration

- $\quad \mathrm{SO}_{2}$ removal: $34-46.8 \%$

- Stack opacity: Mostly 7-18.8\% 


\subsubsection{September 2, 1992}

The dolomitic lime slurry injection in the duct started at 1530 hours and continued until 2200 hours - a total of 6 hours. The cumulative lime injection time was 113 hours. The summary of CZD operation for September 2 is as follows:

- Lime injection: $33.4-39.6 \mathrm{gpm}$ with a $12 \%$ lime concentration

- $\mathrm{SO}_{2}$ removal: $11-25.4 \%$

- Stack opacity: $8.5-18.7 \%$

\subsubsection{September 3, 1992}

The dolomitic lime slurry injection in the duct started at 0915 hours and continued until 1045 hours - a total of 1.5 hnurs. The cumulative lime injection time was 114.5 hours.

Figure 3-6 shows the variation in the percentage of $\mathrm{SO}_{2}$ removal versus the $\mathrm{gpm}$ of lime slurry flow and percentage of lime slurry concentration, during the period August 17 to September 16, 1992, with the operating data recorded every 15 minutes.

Figure 3-6A shows the daily average lime flow versus $\mathrm{SO}_{2}$ removal for the same period. This cross plot shows that the percentage of $\mathrm{SO}_{2}$ removal is dependent on the lime slurry injection rate, i.e., the higher the lime slurry injection rate, the higher the percentage of $\mathrm{SO}_{2}$ removal. Figure 3-6A also shows that the daily average data varied from less than $20 \% \mathrm{SO}_{2}$ removal when lime flow was about $35 \mathrm{gpm}$, to $47 \% \mathrm{SO}_{2}$ removal with $54 \mathrm{gpm}$ lime flow.

Figure 3-6B shows the daily average lime slurry concentration versus $\mathrm{SO}_{2}$ removal for the same period. This cross plot shows that an increase in daily average lime slurry concentration above $9 \%$ does not increase the percentage of $\mathrm{SO}_{2}$ removal and it decreases the percentage of lime utilization.

For example, on August 17, 1992, the lime slurry concentration varied from $10.3 \%$ to $10.7 \%$ to $13.9 \%$ and then to $11.5 \%$, without any effect on the percentage of $\mathrm{SO}_{2}$ removed, which was mostly dependent on the lime slurry injection rate. (For more details see Appendix C.)

Figure 3-7 shows $\mathrm{SO}_{2}$ removal versus the lime slurry injection rate, expressed in gpm and based on daily averages during the period August 17 to September 16. This graph shows that at a low lime slurry injection rate - for example $36 \mathrm{gpm}$ the percent of $\mathrm{SO}_{2}$ removal was only about $20 \%$. By increasing the injection rate to $50 \mathrm{gpm}, \mathrm{SO}_{2}$ removal increased to $38 \%$. At a $54 \mathrm{gpm}$ injection rate, the $\mathrm{SO}_{2}$ removal rate increased to $45 \%$. By extrapolation, with a $60 \mathrm{gpm}$ injection rate, 


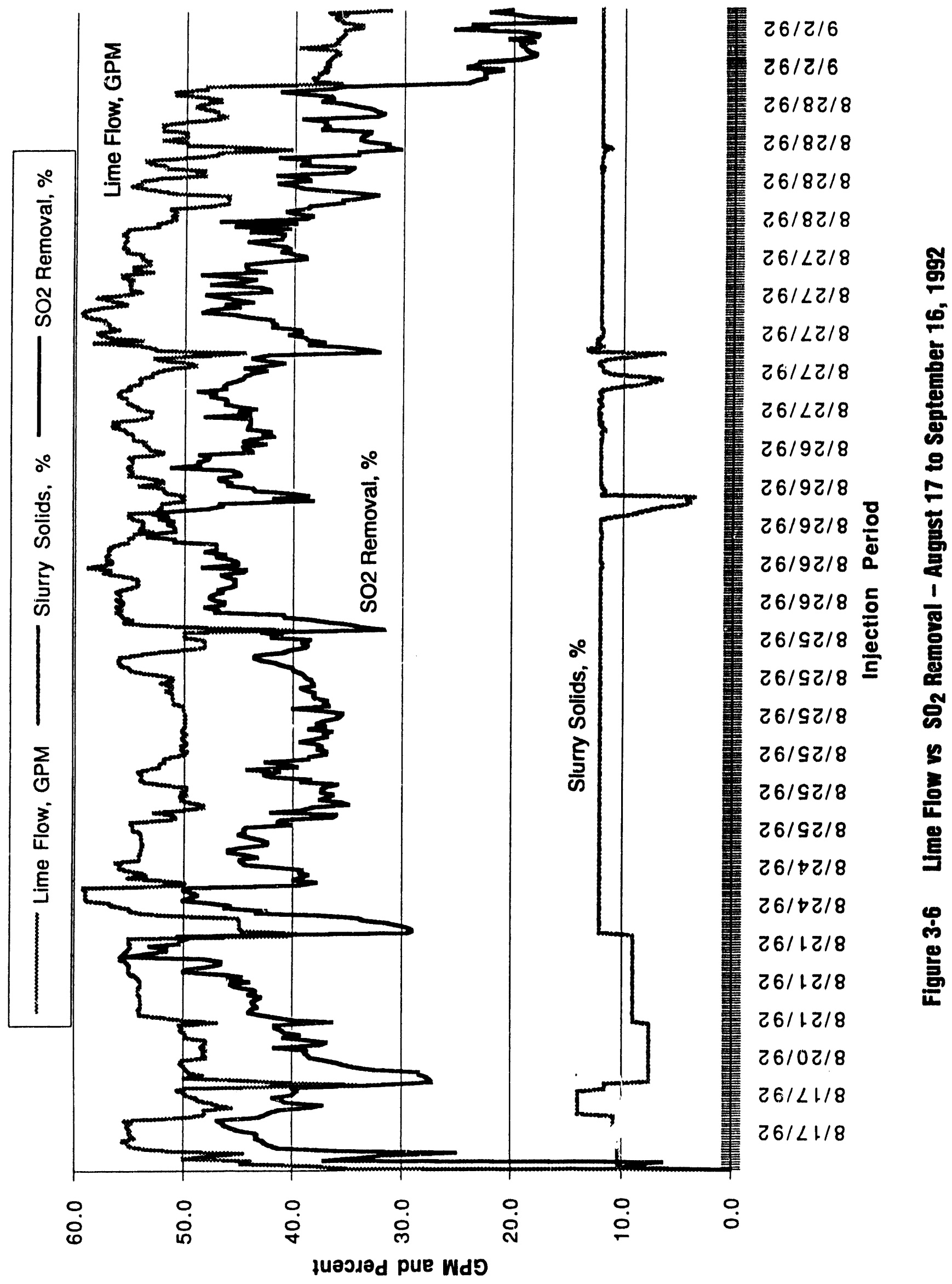


0
0
0



0
0


$\stackrel{2}{\circ}$


₹

오 है

을 은



옹

㑒占


0









the percentage of $\mathrm{SO}_{2}$ removed will reach $55 \%$. As mentioned above, the percentage of $\mathrm{SO}_{2}$ removed represents daily averages and not peak values.

In Figure 3-6, where the values that represent the percentage of $\mathrm{SO}_{2}$ removed were recorded every 15 minutes, a peak value of $55 \% \mathrm{SO}_{2}$ removed at a $55 \mathrm{gpm}$ lime slurry injection rate was recorded on August 21. A peak value of $52 \% \mathrm{SO}_{2}$ removed at a $54 \mathrm{gpm}$ injection rate was recorded on August 26.

The operating data for the period August 17 to September 16, are included in Appendix $C$. These operating data also show the rate of stack opacity every 15 minutes. Almost regularly, the stack opacity percentage was lower than $20 \%$, the limit of the permissible stack opacity percentage.

After 10 days of continuous (24-hour/day) lime injection operations, the steam pressure at which the duct sootblowers were operating was set too low to prevent deposits from forming on the bottom of the duct. The steam supply pressure to the sootblowers was increased to the maximum. However, it was not possible to remove some of the deposits that had already formed. In a few areas, these deposits were significant and testing confirmed that further lime injection operations would cause accelerated growth of these deposits. Consequently, lime injection was halted, and it was decided to remove the deposits manually at the next boiler outage.

For the period September 4 to September 16, 1992, only water was injected in the duct to maintain the percentage opacity at low values.

\subsubsection{September 17 to October 16,1992}

During the startup phase of lime injection automation, there were some maloperations. At the end of August 1992 after 10 days of continuous (24hour/day) operations, it was determined that the steam pressure at which the duct sootblowers were operating was set too low to prevent deposits from forming on the bottom of the duct. A few times when moving from standby mode to ready condition, the flow control valve FCV-10 - being not fully tuned remained fully open and the lime injection rate reached over $80 \mathrm{gpm}$ for 1-2 minutes. At that point the flow control valve closed to limit the lime slurry flow rate to the level to maintain a temperature of about $160^{\circ} \mathrm{F}$ at C-section (at the turning vanes). During these excursions, wet deposits were formed because there was not enough heat in the duct to evaporate the very large amount of lime slurry. Some small quantities of atomized lime slurry reached the ESP, coating the collecting plates and the discharge wires of Fields \#3 and \#4. This lime coating caused some opacity problems.

The steam supply pressure to the sootblowers was increased to the maximum. However, it was not possible to remove some of the deposits that had already formed. In a few areas, the deposits in the duct were significant, and testing 





confirmed that further lime injection operations would cause accelerated growth of these deposits. (See Section 3.3.6 for the chemical analyses of the coating materials.) Consequently, lime injection was halted, and it was decided to remove the deposits manually during the next boiler outage.

\subsubsection{September 29, 1992}

To test the effects on the ESP, operations were switched from water to lime. Dolomitic lime slurry injection in the duct was run at 20-30 gpm, starting at 1015 hours and continuing only until 1145 hours - a total of 1.5 hours. At 1145 hours, the D4 field of the ESP tripped off causing a stack opacity excursion. Operations switched back to water and the D4 field was reset. An increase in the duct deposit heights was also noted.

The summary of the CZD operation on September 29 is as follows:

- Lime slurry injection: $20-30 \mathrm{gpm}$ with a $6.8-7.9 \%$ lime slurry concentration

- $\mathrm{SO}_{2}$ removal: $11.3-20.8 \%$

- Stack opacity: $6.6-7.6 \%$

\subsubsection{September 30,1992}

The dolomitc lime slurry injection in the duct started at 1700 hours and continued until 1815 hours - a total of 1.25 hours. The cumulative lime slurry injection time was 117.25 hours.

The summary of CZD operations on September 30 is as follows:

- Lime slurry injection: $11.1 \mathrm{gpm}$ with a $8 \%$ lime slurry concentration

- $\mathrm{SO}_{2}$ removal: $1.7-3.0 \%$

- Stack opacity: $12.1-13.7 \%$

\subsubsection{October 1, 1992}

The dolomitic lime slurry injection in the duct took only 0.25 hours. The cumulative lime slurry injection time was 117.5 hours.

\subsubsection{October 9, 1992}

The dolomitic lime slurry injection in the duct started at 1515 hours and continued until 1630 hours - a total of 1.25 hours. Cumulative lime slurry injection was 118.75 hours.

The summary of the CZD operation on October 9 is as follows:

- Lime slurry injection: $32-38.2 \mathrm{gpm}$ with a $8.6 \%$ lime slurry concentration 
- $\mathrm{SO}_{2}$ removal: $17.5-19 \%$

- Stack opacity: $8.7-16.7 \%$

\subsubsection{October 12, 1992}

The dolomitic lime slurry injection in the duct started at 1500 hours and operated only 0.75 hours. The cumulative lime slurry injection was 119.5 hours.

The summary of the CZD operation on October 12 is as follows:

- Lime slurry injection: $8.9-14.8 \mathrm{gpm}$ with a $7.8-8.5 \%$ lime slurry concentration

- $\mathrm{SO}_{2}$ removal: $\mathbf{7 . 8 - 8 . 5 \%}$

- Stack opacity: $17.2-18.7 \%$

\subsubsection{October 14,1992}

The dolomitic lime slurry injection in the duct started at 0845 hours and continued until 2100 hours - a total of 12.25 hours. The cumulative lime slurry injection was 131.75 hours.

The summary of the CZD operation on October 14 is as follows:

- Lime slurry injection: $24-36 \%$ with a $1.8-9.1 \%$ lime slurry concentration

- $\mathrm{SO}_{2}$ removal: $7.3-20 \%$

- Stack opacity: $9.4-19.5 \%$

\subsubsection{October 15, 1992}

The dolomitic lime slurry injection in the duct started at 1545 hours and continued until 2400 hours - a total of 8.25 hours. The cumulative lime slurry injection was 140 hours.

The summary of the CZD operation on October 15 is as follows:

- Lime slurry injection: $30 \mathrm{gpm}$ with a $0.2-3 \%$ lime slurry concentration

- $\mathrm{SO}_{2}$ removal: $4.6-22.7 \%$

- Stack opacity: $10.9-21.8 \%$

\subsubsection{October 16,1992}

The dolomitic lime slurry injection in the duct started at midnight and continued until 2130 hours - a total of 14.5 hours. The cumulative lime slurry injection was 154.25 hours.

The summary of the CZD operations on October 16 is as follows:

- Lime slurry injection: 25-35 gpm with a $0.3-3 \%$ lime slurry concentration 
- $\mathrm{SO}_{2}$ removal: $6-15.9 \%$

- Stack opacity: $7.5-37.3 \%$

CZD operations during the period September 17 to October 16 were characterized by lime slurry injections with a very low percentage of solids. This was done to avoid a high percentage of stack opacity until the Buell ESP could be fixed during an emergency boller shutdown.

Operating data for the period September 17 to October 16 are included in Appendix D. These operating data also show the percentage of stack opacity every 15 minutes during the lime slurry injection.

Bechtel attended the U.S. DOE First Annual Clean Coal Technology Conference held in Cleveland, Ohio, on September 22-24, 1992. Jack Abrams presented a paper titled 'Update and Results of Bechtel's Confined Zone Dispersion (CZD) Demonstration at Penrisylvania Electric Company's Seward Station."

Bechtel also attended the DOE Duct Injection Technology Working Group Meeting in Johnstown, PA, on September 30 and October 1, 1992, and conducted a tour of the CZD facilities for the participants.

Figure 3-8 shows, for the period September 17 to October 16, the percentage of $\mathrm{SO}_{2}$ removal versus the lime slurry injection rate in $\mathrm{gpm}$ and the percentage of lime slurry concentration recorded every 15 minutes. For this period, we added two cross plots: Figure 3-8A, daily average lime flow versus $\mathrm{SO}_{2}$ removal, and Figure 3-8B, daily average lime slurry concentration versus $\mathrm{SO}_{2}$ removal.

Figure 3-8A shows that a low daily average lime slurry flow of $11-12 \mathrm{gpm}$ did not remove any $\mathrm{SO}_{2}$ (on September 30 and October 12, 1992). Figure 3-8B shows that with an $8 \%$ daily average lime slurry concentration, the percentage $\mathrm{SO}_{2}$ removal was zero. The reason for this is that, at that time the daily average of lime slurry flow rate was only $11-12 \mathrm{gpm}$, evaporation was too fast and the $120^{\circ} \mathrm{F}$ adiabatic saturation temperature could not be reached.

On the basis of recorded operations data (boiler load, inlet flue gas temperature, lime slurry injection rate, percentage lime slurry concentration, number of atomizers in operation, etc.), we expected a corresponding percentage of $\mathrm{SO}_{2}$ removal and lime utilization. However, the automatically recorded results were disappointing.

We examined in more detail all the recorded data - calling it " $x$-ray data" - to find the cause of the unexpected results. For example, Figure 3-9 shows graphically, every 15 minutes for a period of one month, the boiler load variations and the corresponding flue gas flow rate, in and out, expressed in thousands of scfm. Normally, the inlet flue gas flow rate changes accordingly. 










:








WมSรX 'MOרA MW 'Q707 
This should also be true of the outlet flue gas. Figure 3.9 shows the boiler load during the night decreased from $147 \mathrm{MW}$ to $105 \mathrm{MW}$ and the flue gas flow rate, in and out, changed accordingly - except for the outlet flue gas flow rates marked with circles that show a different pattern. Figures 3-10 through 3-13 show in more detail the variations in the recorded data for the outlet flue gas flowrate. The anomalles are marked with circles. When calculating the percentage of $\mathrm{SO}_{2}$ removed, the flue gas flow rate changes from the inlet to the outlet are thought of as dilution. The resulting $\mathrm{SO}_{2}$ removal rates could be wrong if the inlet or outlet flue gas indicate incorrect values. After correcting the calibration of the outlet flue gas flow meter, we had no more problems.

\subsubsection{October 17, 1992 to November 16, 1992}

CZD operations from October 17 to November 16 are characterized by two distinct periods of continuous lime slurry injection:

ESP Operation Problems. In the first week, there were ESP operation problems (see also Section 3.3.6):

- Suddenly, when we turned lime slurry on with $8 \%$ lime concentration, the percentage opacity increased above $20 \%$. Therefore, we had to switch off the lime slurry to lower the percentage opacity.

- $\quad$ Analysis of the Buell ESP field currents for B-duct revealed that D1 current was stable or slightly increased with lime but that the other three fields exhibited a draniatic drop in field currents. This drop in field currents with lime slurry is the opposite of the reaction of the ESP observed during the CZD program to date. During this past year's testing, we observed that the addition of lime increased field currents and led to a lowering of duct and stack opacity.

- We contacted GE Environmental for consulting services on the Buell ESP.

- We formulated a plan to obtain additional ESP data with lime by using dilute lime slurry concentrations (4\% or less) until stable operation of the ESP was obtained. Therefore, the percentage of $\mathrm{SO}_{2}$ removal was moderately low ( $20 \%$ or lower).

After the boiler's emergency shutdown, problems with ESP operations were partly resolved on October 26 . Faulty grounds to the collecting plates were corrected so the opacity percentages were no longer affected by the percentage of lime slurry concentration. The percentage of lime slurry concentration could then be slowly increased from 4 to $10 \%$ to verify its effects on the percentage of $\mathrm{SO}_{2}$ removed. 







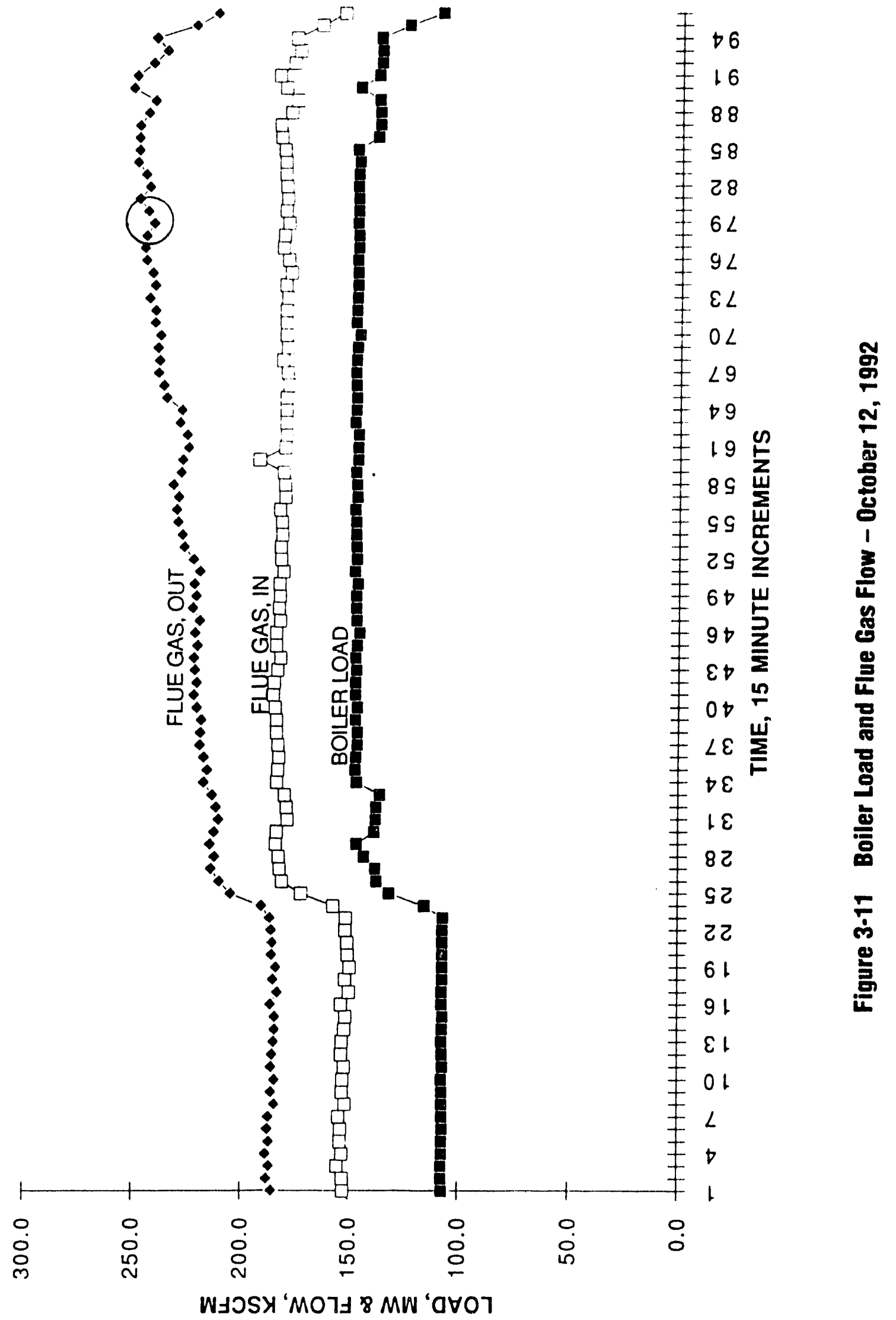












- Low-Temperature Flue Gas. In August, the flue gas temperature in the CZD duct was $300-310^{\circ} \mathrm{F}$, but now the temperature was only $270-275^{\circ} \mathrm{F}$, about $30^{\circ} \mathrm{F}$ lower. The low-temperature flue gas was caused by a large amount of air leakage in the air heater and through cracks in the furnace walls in the superheater area. Low-temperature flue gas in the CZD duct necessitates a substantial reduction in the lime slurry injection rate, resulting in a low percentage of $\mathrm{SO}_{2}$ removal. Until the air heater is improved, we will continue to inject lime slurry at lower rates and therefore have a lower percentage of $\mathrm{SO}_{2}$ removal.

\subsubsection{October 17,1992}

The dolomitic lime slurry injection in the duct started at 0100 hours and continued until 1115 hours - a total of 10.25 hours. The cumulative lime slurry injection time was 164.5 hours.

The summary of the CZD operations on October 17 is as follows:

- Lime slurry injection: $25-35 \mathrm{gpm}$ with a $4 \%$ lime slurry concentration

- $\mathrm{SO}_{2}$ removal: $15-22 \%$

- B-duct temperature: $285-28 \delta^{\prime} \mathrm{F}$

- Stack opacity: 7-18\%

\subsubsection{October 18,1992}

The dolomitic lime slurry injection in the duct started at 1545 hours and continued until 2400 hours - a total of 7.25 hours. The cumulative lime slurry injection time was 172.25 hours.

The summary of the CZD operations on October 18 is as follows:

- Lime slurry injection: $28-35$ gpm with a $4 \%$ lime slurry concentration

- $\mathrm{SO}_{2}$ removal: $14-22 \%$

- B-duct temperature: $273-280^{\circ} \mathrm{F}$

- Stack opacity: $10-18.8 \%$

\subsubsection{October 19, 1992}

The dolomitic lime slurry injection in the duct started at 2400 hours and continued until 2400 hours - a total of 15.25 hours. The cumulative lime slurry injection time was 187.25 hours.

The summary of the CZD operations on October 19 is as follows:

- Lime slurry injection: $30-35 \mathrm{gpm}$ with a $4 \%$ lime slurry concentration

- $\mathrm{SO}_{2}$ removal: $7-11 \%$ 
- B-duct temperature: $280^{\circ} \mathrm{F}$

- Stack opacity: $9-18.5 \%$

\subsubsection{October 20, 1992}

The dolomitic lime slurry injection in the duct started at 0600 hours and continued until 2400 hours - a total of 16.25 hours. The cumulative lime slurry injection time was 204 hours.

The summary of the CZD operations on October 20 is as follows:

- Lime slurry injection: $30-36 \mathrm{gpm}$ with a $4 \%$ lime slurry concentration

- $\mathrm{SO}_{2}$ removal: $10-18 \%$

- B-duct temperature: $270-280^{\circ} \mathrm{F}$

- Stack opacity: $14-20 \%$

\subsubsection{October 21,1992}

The dolomitic lime slurry injection in the duct started at 0630 hours and continued until 2400 hours - a total of 14.5 hours. The cumulative lime slurry injection time was 218.5 hours.

The summary of the CZD operations on October 21 is as follows:

- Lime slurry injection: $30-38 \mathrm{gpm}$ with a $4 \%$ lime slurry concentration

- $\mathrm{SO}_{2}$ removal: $10-16 \%$

- B-dlict temperature: $280-287^{\circ} \mathrm{F}$

- Stack opacity: $12-20.8 \%$

\subsubsection{October 22, 1992}

The dolomitic lime slurry injection in the duct started at 2400 hours and continued until 2400 hours - a total of 22.25 hours. The cumulative lime slurry injection time was 240.75 hours.

The summary of the CZD operations on October 22 is as follows:

- Lime slurry injection: $30-35 \mathrm{gpm}$ with a $4 \%$ lime slurry concentration

- $\mathrm{SO}_{2}$ removal: $10-16 \%$

- B-duct temperature: $276-288^{\circ} \mathrm{F}$

- Stack opacity: $12-21.7 \%$ 


\subsubsection{October 23, 1992}

The dolomitic lime slurry injection in the duct started at 2400 hours and continued until 2400 hours - a total of 19 hours. The cumulative lime slurry injection time was 259.75 hours.

The summary of the CZD operations on October 23 is as follows:

- Lime slurry injection: $30-40 \mathrm{gpm}$ with a $4 \%$ lime slurry concentration

- $\mathrm{SO}_{2}$ removal: $10-18 \%$

- B-duct temperature: $280-290^{\circ} \mathrm{F}$

- Stack opacity: $13-20 \%$

\subsubsection{November 2, 1992}

The dolomitic lime slurry injection in the duct started at 1615 hours and continued until 2400 hours - a total of 7.75 hours. The cumulative lime slurry injection time was 267.5 hours.

The summary of the CZD operations on November 2 is as follows:

- Lime slurry injection: $30-33 \mathrm{gpm}$ with a 5\% lime slurry concentration

- $\mathrm{SO}_{2}$ removal: $12-20 \%$

- B-duct inlet temperature: $280^{\circ} \mathrm{F}$

- Stack opacity: $12-16 \%$

\subsubsection{November 3, 1992}

The dolomitic lime slurry injection in the duct started at 2400 hours and continued until 2400 hours - a total of 24 hours. The cumulative lime slurry injection time was 291.5 hours.

The summary of the CZD operations on November 3 is as follows:

- Lime slurry injection: $28-35 \mathrm{gpm}$ with a $5 \%$ lime slurry concentration

- $\mathrm{SO}_{2}$ removal: $10-20 \%$

- B-duct inlet temperature: $275-280^{\circ} \mathrm{F}$

- Stack opacity: $10-14 \%$

\subsubsection{November 4,1992}

The dolomitic lime slurry injection in the duct started at 2400 hours and continued until 2400 hours - a total of 24 hours. The cumulative lime slurry injection time was 315.5 hours. 
The summary of the CZD operations on November 4 is as follows:

- Lime slurry injection: $27-30 \mathrm{gpm}$ with a $6 \%$ lime slurry concentration

- $\mathrm{SO}_{2}$ removal: $18-23 \%$

- B-duct inlet temperature: $273-282^{\circ} \mathrm{F}$

- Stack opacity: $12-15 \%$

\subsubsection{November 5, 1992}

The dolomitic lime slurry injection in the duct started at 2400 hours and continued until 2400 hours - a total of 24 hours. The cumulative lime slurry injection time was 339.5 hours.

The summary of the CZD operations on November 5 is as follows:

- Lime slurry injection: $30-36 \mathrm{gpm}$ with a $7 \%$ lime slurry concentration

- $\mathrm{SO}_{2}$ removal: $18-25 \%$

- B-duct inlet temperature: $272-280^{\circ} \mathrm{F}$

- Stack opacity: $12-15 \%$

\subsubsection{November 6, 1992}

The dolomitic lime slurry injection in the duct started at 2400 hours and continued until 2400 hours - a total of 23.75 hours. The cumulative lime slurry injection time was 363.25 hours.

The summary of the CZD operations on November 6 is as follows:

- Lime slurry injection: $30-36 \mathrm{gpm}$ with a 8-9\% lime slurry concentration

- $\mathrm{SO}_{2}$ removal: $20-26 \%$

- B-duct inlet temperature: $275-279^{\circ} \mathrm{F}$

- Stack opacity: $10-17 \%$

\subsubsection{November 7, 1992}

The dolomitic lime slurry injection in the duct started at 2400 hours and continued until 2400 hours - a total of 24 hours. The cumulative lime slurry injection time was 387.25 hours.

The summary of the CZD operations on November 7 is as follows:

- Lime slurry injection: $31-34 \mathrm{gpm}$ with a 9\% lime slurry concentration

- $\mathrm{SO}_{2}$ removal: $22-26 \%$

- B-duct inlet temperature: $272-274^{\circ} \mathrm{F}$ 
- Stack opacity: $10-15 \%$

\subsubsection{November 8, 1992}

The dolomitic lime slurry injection in the duct started at 2400 hours and continued until 2400 hours - a total of 24 hours. The cumulative lime slurry injection time was 411.25 hours.

The summary of the CZD operations on November 8 is as follows:

- Lime slurry injection: $33-35 \mathrm{gpm}$ with a $9 \%$ lime slurry concentration

- $\mathrm{SO}_{2}$ removal: $25-29 \%$

- B-duct inlet temperature: $268-276^{\circ} \mathrm{F}$

- Stack opacity: $7-15 \%$

\subsubsection{November 9, 1992}

The dolomitic lime slurry injection in the duct started at 2400 hours and continued until 0915 hours - a total of 9.25 hours. The cumulative lime slurry injection time was 420.5 hours.

The summary of the CZD operations on November 9 is as follows:

- Lime slurry injection: $27-30 \mathrm{gpm}$ with a $9 \%$ lime slurry concentration

- $\mathrm{SO}_{2}$ removal: $30-37 \%$

- B-duct inlet temperature: $267-275^{\circ} \mathrm{F}$

- Stack opacity: $7-14 \%$

It should be noted that from November 3 through November 9 , the CZD continuously operated 24 hours/day, and proved to be a reliable system.

\subsubsection{November 12, 1992}

The dolomitic lime slurry injection in the duct started at 1130 hours and continued until 2245 hours - a total of 11.25 hours. The cumulative lime slurry injection time was 431.75 hours.

The summary of the CZD operations on November 12 is as fol.ows:

- Lime slurry injection: $37-39 \mathrm{gpm}$ with a $10-16 \%$ lime slurry concentration

- $\mathrm{SO}_{2}$ removal: $25-35 \%$

- B-duct inlet temperature: $290^{\circ} \mathrm{F}$

- Stack opacity: $12-17 \%$ 


\subsubsection{November 13, 1992}

The dolomitic lime slurry injection in the duct started at 0845 hours and continued untll 2400 hours - a total of 9.25 hours. The cumulative lime slurry injection time was 441 hours.

The summary of the CZD operations on November 13 is as follows:

- Lime slurry injection: $32-40 \mathrm{gpm}$ with a $10 \%$ lime slurry concentration

- $\mathrm{SO}_{2}$ removal: $16-22 \%$

- B-duct inlet temperature: $277^{\circ} \mathrm{F}$

- Stack opacity: $10 \cdot 14 \%$

\subsubsection{November 14,1992}

The dolomitic lime slurry injection in the duct started at 2400 hours and continued until 2400 hours - a total of 21 hours. The cumulative lime slurry injection time was 462 hours.

The summary of the CZD operations on November 14 is as follows:

- Lime slurry injection: $30-41 \mathrm{gpm}$ with a $10 \%$ lime slurry concentration

- $\mathrm{SO}_{2}$ removal: $20-24 \%$

- B-duct inlet temperature: $273^{\circ} \mathrm{F}$

- Stack opacity: $14-20 \%$

\subsubsection{November 15, 1992}

The dolomitic lime slurry injection in the duct started at 2400 hours and continued until 1900 hours - a total of 16.5 hours. The cumulative lime slurry injection time was 478.5 hours.

The summary of the CZD operations on November 15 is as follows:

- Lime slurry injection: $32-38 \mathrm{gpm}$ with a $10 \%$ lime slurry concentration

- $\mathrm{SO}_{2}$ removal: $20-24 \%$

- B-duct inlet temperature: $273.275^{\circ} \mathrm{F}$

- Stack opacity: $8.15 \%$

The summary data for the period October 17 through November 16, 1992 are included in Appendix E. These summary data show also the stack opacity percentage every 15 minutes during the lime slurry injections.

Figure 3-14 shows the lime slurry flow rate and percentage of lime concentration versus the percentage of $\mathrm{SO}_{2}$ removal recorded every 15 minutes during the period October 17 to November 16, 1992. The graph shows that the lime slurry 


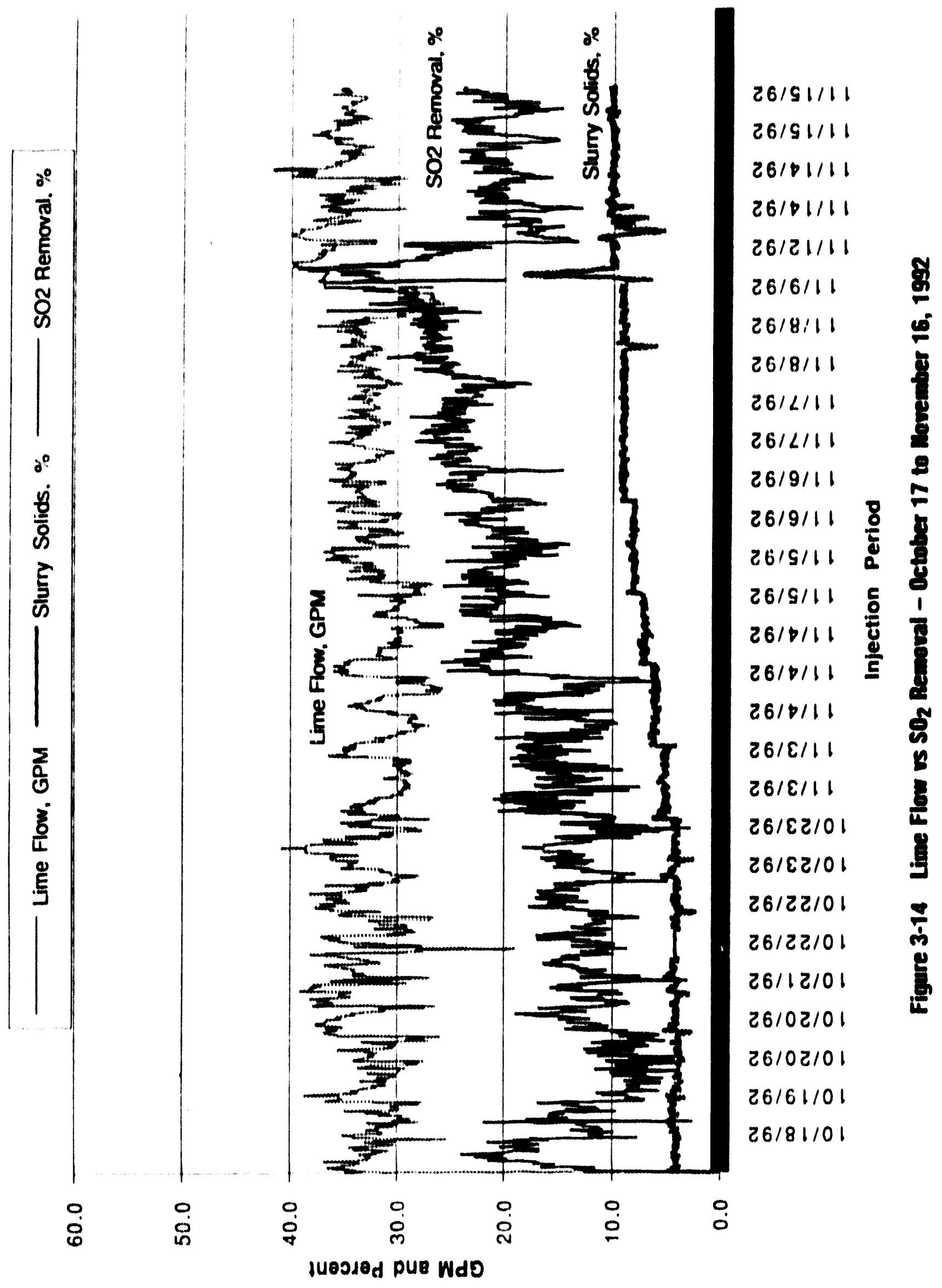


$\infty$









injection rates were maintained at around $35 \mathrm{gpm}$ because of the low flue gas inlet temperature. Figure 3-14A, the cross plot of dally average lime flow versus $\mathrm{SO}_{2}$ removal for the same period, shows that a low lime slurry flow (28-38 $\mathrm{gpm}$ ) limits the percentage of $\mathrm{SO}_{2}$ removal to $20.30 \%$ because the cooling to adiabatic saturation temperature can not be maintained by the low lime slurry flow rate. Figure 3-14B, the cross plot of daily average lime slurry concentration versus $\mathrm{SO}_{2}$ removal for this period, shows that the increase of lime slurry concentration from $6.5-10 \%$ does not improve the percentage of $\mathrm{SO}_{2}$ removal.

Figure 3-15 shows the CZD performance summary for the period October 17 to November 16, 1992 with online availability for 744 hours (100\%). It is remarkable that the automated and integrated CZD systems were online $100 \%$ of the time during this one-month period.

\subsubsection{Percentage of Lime Utilization and the Factors that Could Affect It}

The percentage of lime utilization in the CZD system significantly affects the total cost per ton of $\mathrm{SO}_{2}$ removed. For this reason, we have attempted to determine methods that can improve the percentage of lime utilization. Based on the results of continuous operational data compiled to date, the percentage of lime utilization is directly dependent on two factors:

- The percentage of $\mathrm{SO}_{2}$ removal

- The percentage of lime slurry concentration

For operating conditions at Seward Station, data show that $40-50 \% \mathrm{SO}_{2}$ removal rate and a $6.8 \%$ lime slurry concentration will assure a $40-50 \%$ lime utllization rate. That is, 2 to $2-1 / 2$ moles of $\mathrm{CaO}$ are required for every mole of $\mathrm{SO}_{2}$ removed; or assuming $92 \%$ lime purity, 1.9-2.4 tons of lime are required for every ton of $\mathrm{SO}_{2}$ removed.

The following graphs of operational data recorded between August 17 and November 16, 1992 show the effect of the percentage of lime slurry concentration on the percentage of $\mathrm{SO}_{2}$ removed. Figure 3-16 shows the relationship between the percentage of $\mathrm{SO}_{2}$ removed at a constant $34 \mathrm{gpm}$ injection rate with concentrations of lime slurry varying from 3 to $12 \%$.

The average percentage of $\mathrm{SO}_{2}$ removal varied with the percentage of lime slurry concentration, as shown in Table 3-3. 


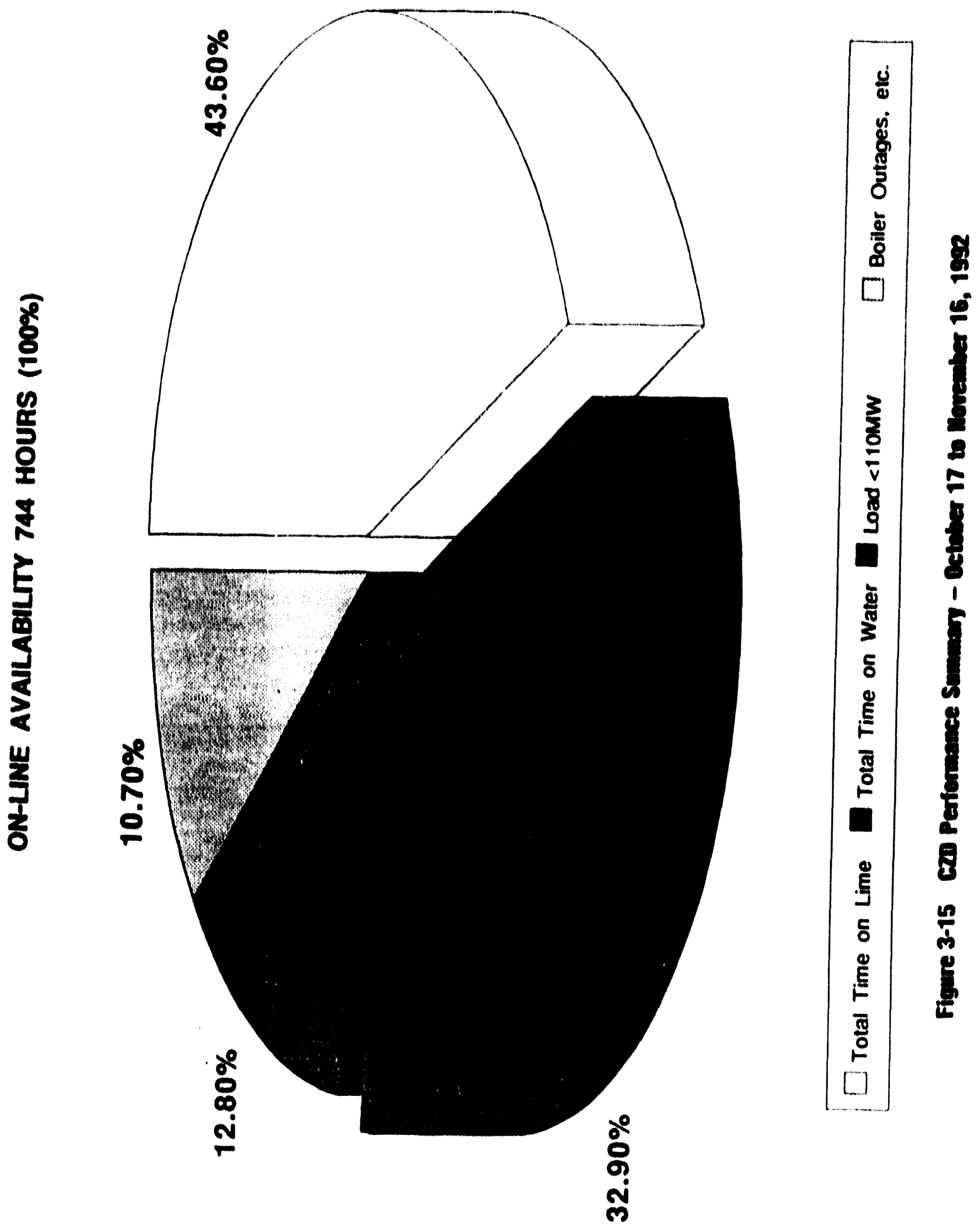




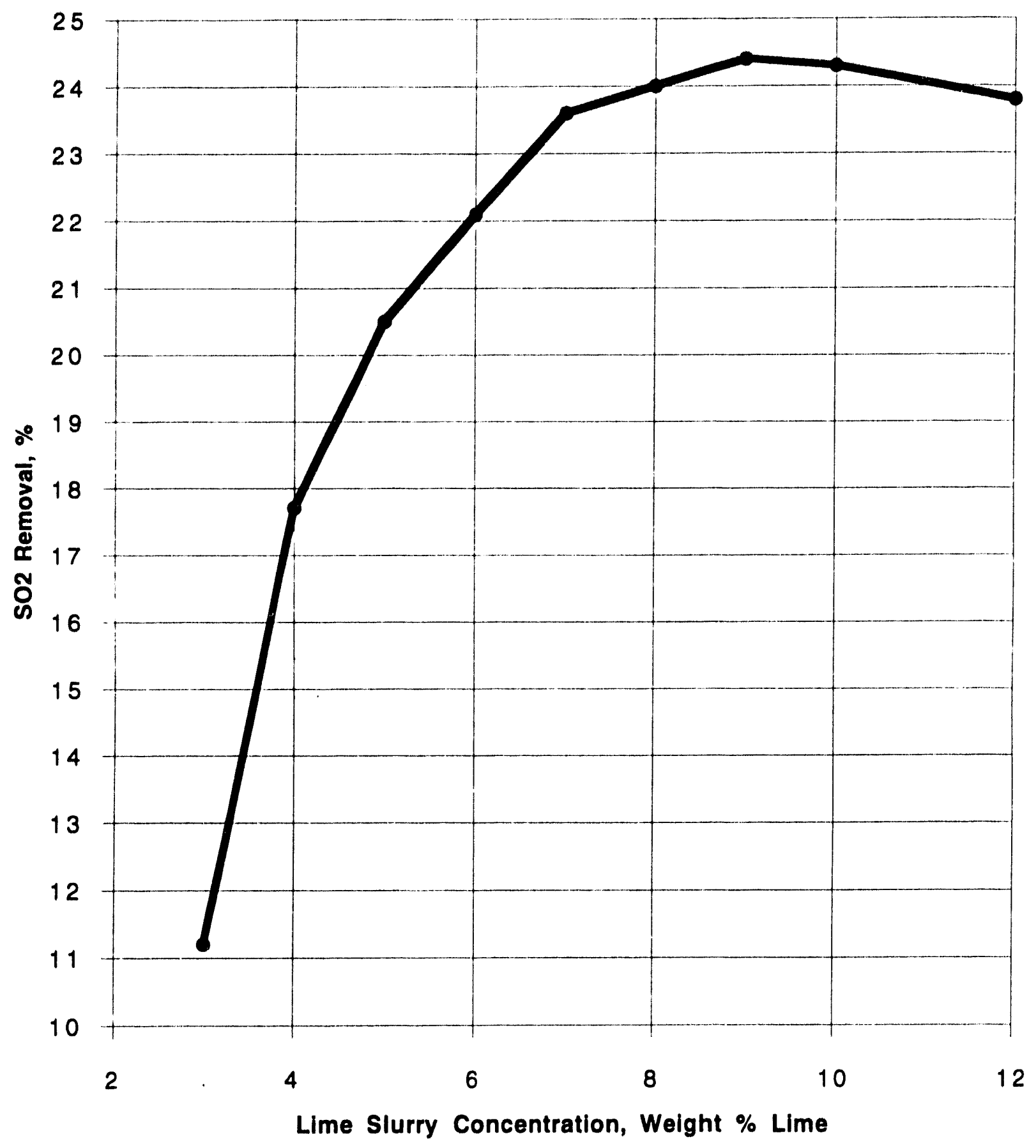

Figure 3-16 Percent oi $\mathrm{SO}_{2}$ Removal at Constant $34 \mathrm{gpm}$ Injection Rate and Variable Lime Concentration

CZD Demo

Page 3-40

Quarterly Report No. 8

93.0131 c.063/WO/st/R4 
Table 3-3

Variation of the Average Percentage of $\mathrm{SO}_{2}$ Removal with Percentage of Lime Slurry Concentration and $34 \mathrm{gpm}$ Lime Slurry Injection Rate, August 17 and November 16, 1992

\begin{tabular}{|c|c|c|c|}
\hline Date & Time & $\begin{array}{c}\text { Lime Slury } \\
\text { Concentration } \\
(\%)\end{array}$ & $\begin{array}{c}\mathrm{SO}_{2} \text { Removal } \\
(\%)\end{array}$ \\
\hline $10-21-92$ & $9: 45$ & 3.0 & 11.2 \\
$10-14-92$ & $15: 45$ & 4.0 & 17.7 \\
$11-2-92$ & $18: 45$ & 5.0 & 20.5 \\
$11-4-92$ & $13: 30$ & 6.0 & 22.1 \\
$11-4-92$ & $15: 15$ & 7.0 & 23.6 \\
$11-5-92$ & $17: 00$ & 8.0 & 24.0 \\
$11-6-92$ & $19: 00$ & 9.0 & 24.4 \\
$11-14-92$ & $20: 45$ & 10.0 & 24.3 \\
$9-2-92$ & $21: 45$ & 12.0 & 23.8 \\
\hline
\end{tabular}

The data in Table 3-3 show that a lime slurry concentration above 7-8\% does not increase the percentage of $\mathrm{SO}_{2}$ removal. An increase in the lime slurry concentration above $7-8 \%$ results in a decrease in the percentage of lime utilization.

Figure 3-17 shows the relationship between the percentage of $\mathrm{SO}_{2}$ removal at different injection rates and the percentage of the lime slurries. This relationship indicates that the percentage of $\mathrm{SO}_{2}$ removed is directly dependent on the lime injection rate. Between the 27 and $37 \mathrm{gpm}$ injection rate, the percentage of $\mathrm{SO}_{2}$ removal is limited to $20-25 \%$ with very slight changes resulting from the percentage of lime concer tration. Between the $45 \mathrm{gpm}$ and 57 gpm lime slurry injection rate, the percentage of $\mathrm{SO}_{2}$ removal increases from $25 \%$ to $50 \%$. Therefore, to obtain a high percentage of $\mathrm{SO}_{2}$ removal, the lime slurry injection rate has to be increased to $52-57 \mathrm{gpm}$.

It is clear that a high lime slurry injection rate is required to achieve a high percentage of $\mathrm{SO}_{2}$ removal. Following are the conditions that limit the injection rate:

- The flue gas flow rate and temperature

- The residence time (in seconds) in the straight duct 


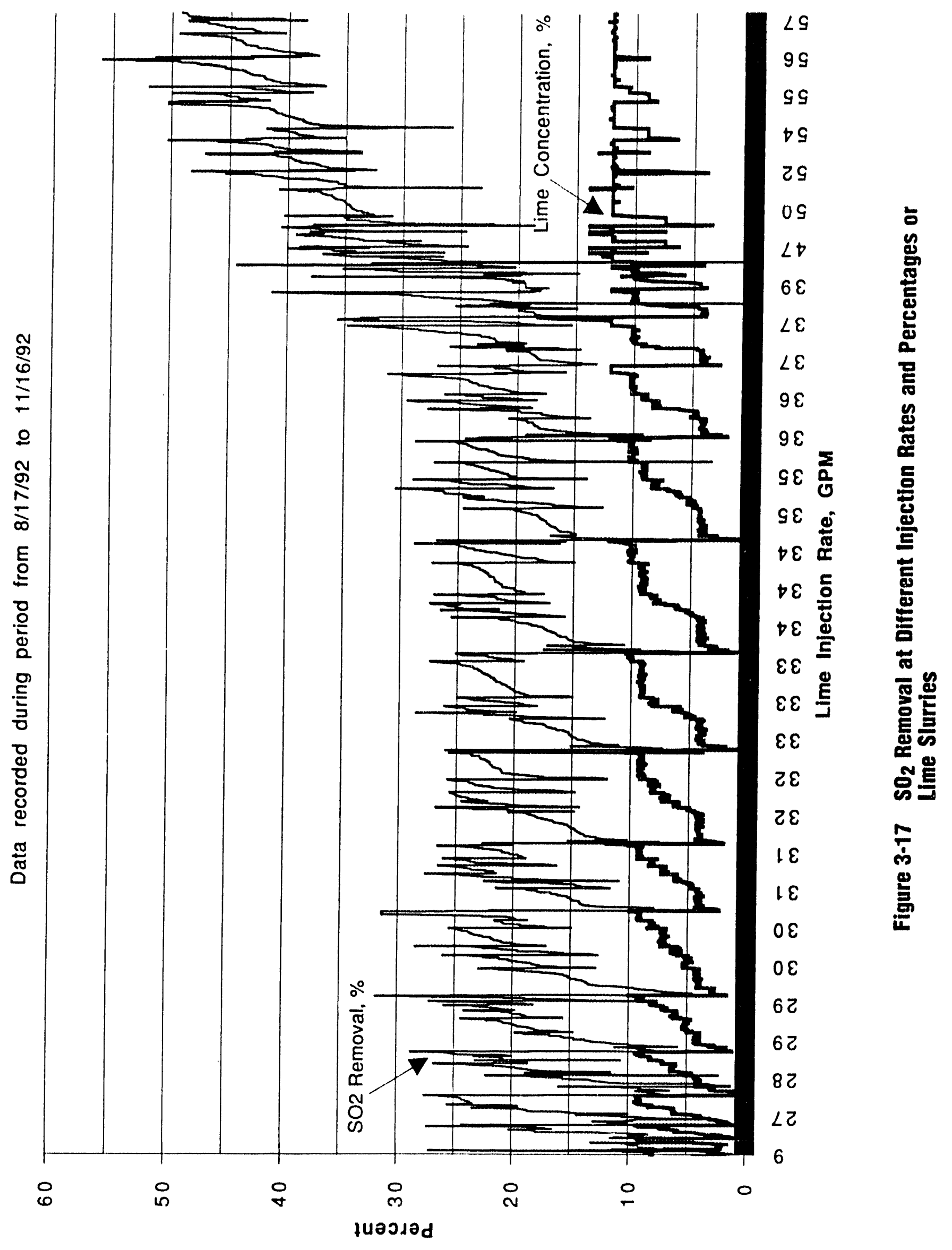


The design of the CZD system at Seward Station is based on the following assumptions:
- An inlet flue gas temperature in the B-duct of $300^{\circ} \mathrm{F}$
- A boiler load of $147 \mathrm{MW}$, equivalent to a flue gas velocity in the duct of 55- 60 fps
- A 120 -foot long straight duct, equivalent to 2 seconds' residence time

During the second half of August, the flue gas temperature was $300-310^{\circ} \mathrm{F}$, the boiler load was 145-147 MW, the residence time in the duct was 2 seconds, and the injection of lime slurry was $52-57 \mathrm{gpm}$ with $\mathrm{SO}_{2}$ removal above $50 \%$.

Figure 3-18 shows the CZD duct temperature versus the lime slurry injection rate and percentage of $\mathrm{SO}_{2}$ removal during the August 17 to November 16, 1992 period. To include the duct inlet temperature in this graph, it has been divided by $5-$ i.e., $300^{\circ} \mathrm{F}$ is represented by $60^{\circ} \mathrm{F}$. The graph shows that during the months of October and November, the inlet flue gas temperature continued to descend from above $300^{\circ} \mathrm{F}$ to $260-280^{\circ} \mathrm{F}$ due to heavy-air leakage in the boiler air heater. This low temperature limited the lime slurry injection rate to $30-40 \mathrm{gpm}$, and resulted in a $15-30 \%$ variation in the percentage of $\mathrm{SO}_{2}$ removal. Figure 3-18A, the cross plot of the daily lime slurry flow versus $\mathrm{SO}_{2}$ removal for this period, shows that:

- With a low lime slurry injection rate of $11-12 \mathrm{gpm}, \mathrm{SO}_{2}$ removal is zero.

- By increasing lime slurry injection to $37 \mathrm{gpm}, \mathrm{SO}_{2}$ removal is $30 \%$.

- By increasing lime slurry injection to $54 \mathrm{gpm}, \mathrm{SO}_{2}$ removal is $47 \%$.

Figure 3-18B, the cross plot of the daily average duct inlet temperature versus percentage $\mathrm{SO}_{2}$ removal for the same period, shows that under the $300^{\circ} \mathrm{F}$ average duct inlet temperature, the percentage $\mathrm{SO}_{2}$ removal is less than $35 \%$ and at $307^{\circ} \mathrm{F}$, the average $\mathrm{SO}_{2}$ removal is over $45 \%$.

Figure 3-19 shows operating data for the same period, but with the lime slurry injection rate $(\mathrm{gpm})$ and the percentage of lime slurry solids versus the percentage of $\mathrm{SO}_{2}$ removal. This graph shows that the percentage of $\mathrm{SO}_{2}$ removal is dependent on the lime slurry injection rate and that the percentage of lime slurry concentration above a specific level does not affect the percentage of $\mathrm{SO}_{2}$ removal.

Figure 3-19A, the cross plot of the daily average lime slurry concentration versus $\mathrm{SO}_{2}$ removal for this period, shows that with $9 \%$ lime slurry concentration by weight, the same percentage $\mathrm{SO}_{2}$ removal can be obtained as with $12 \%$ lime slurry concentration. 


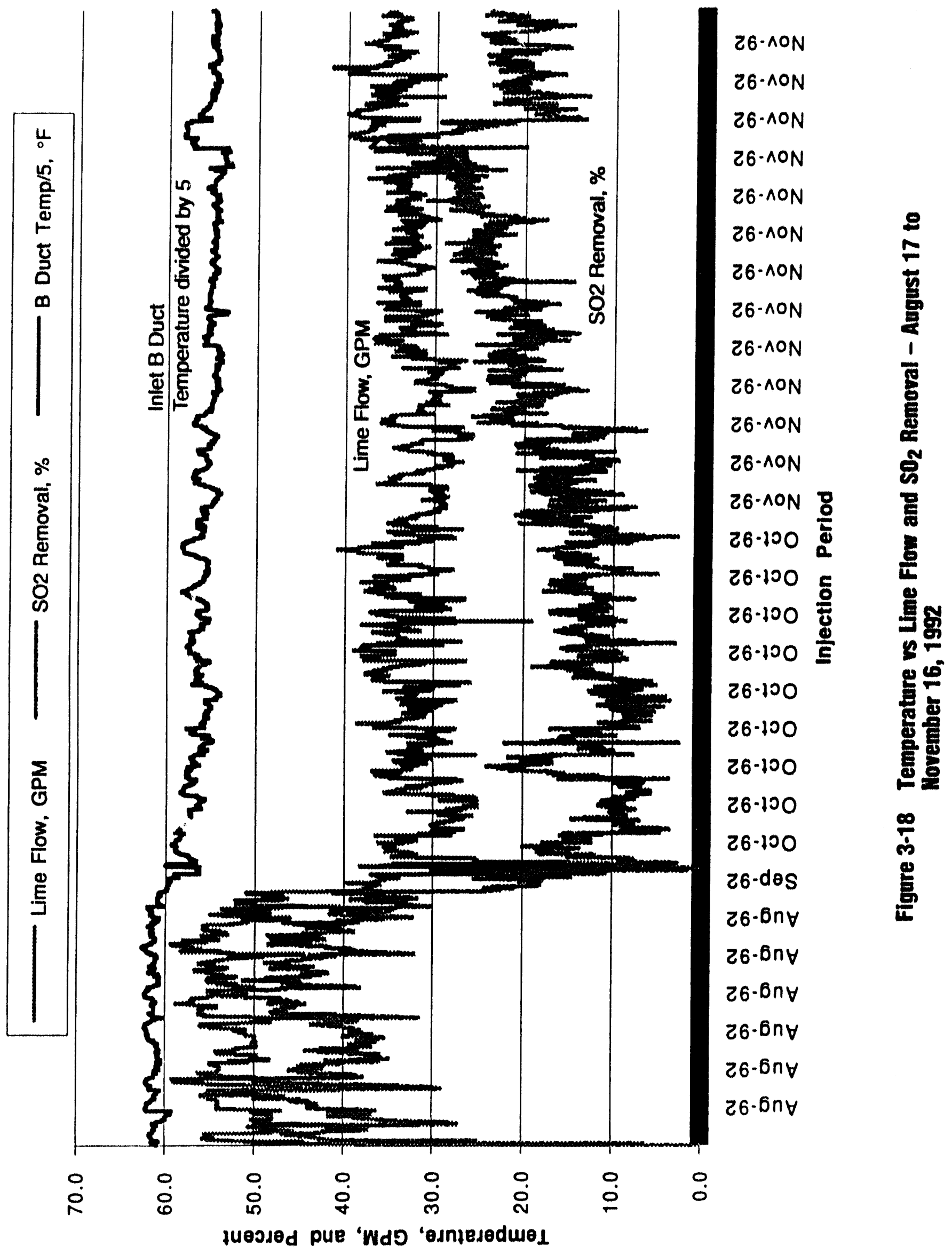









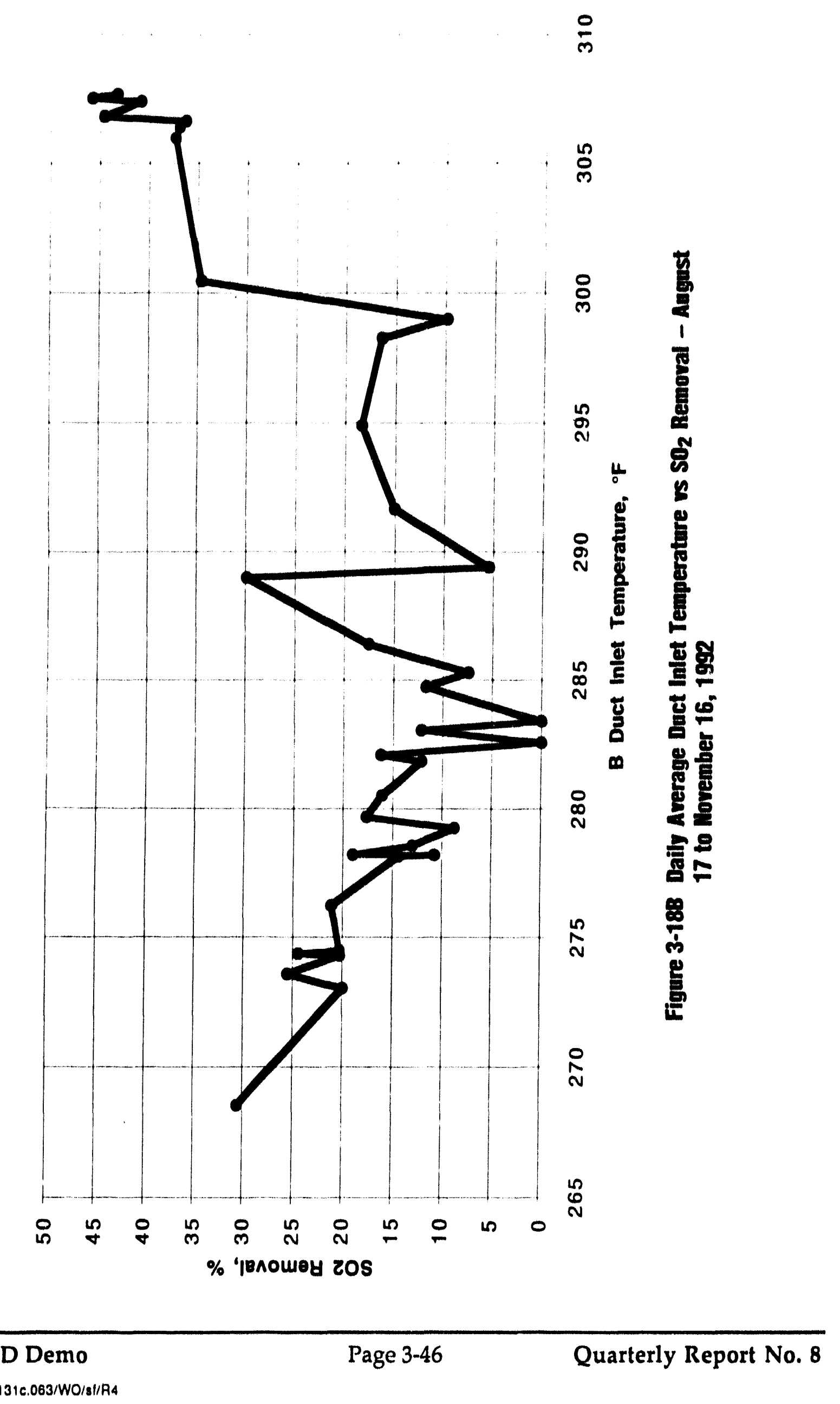




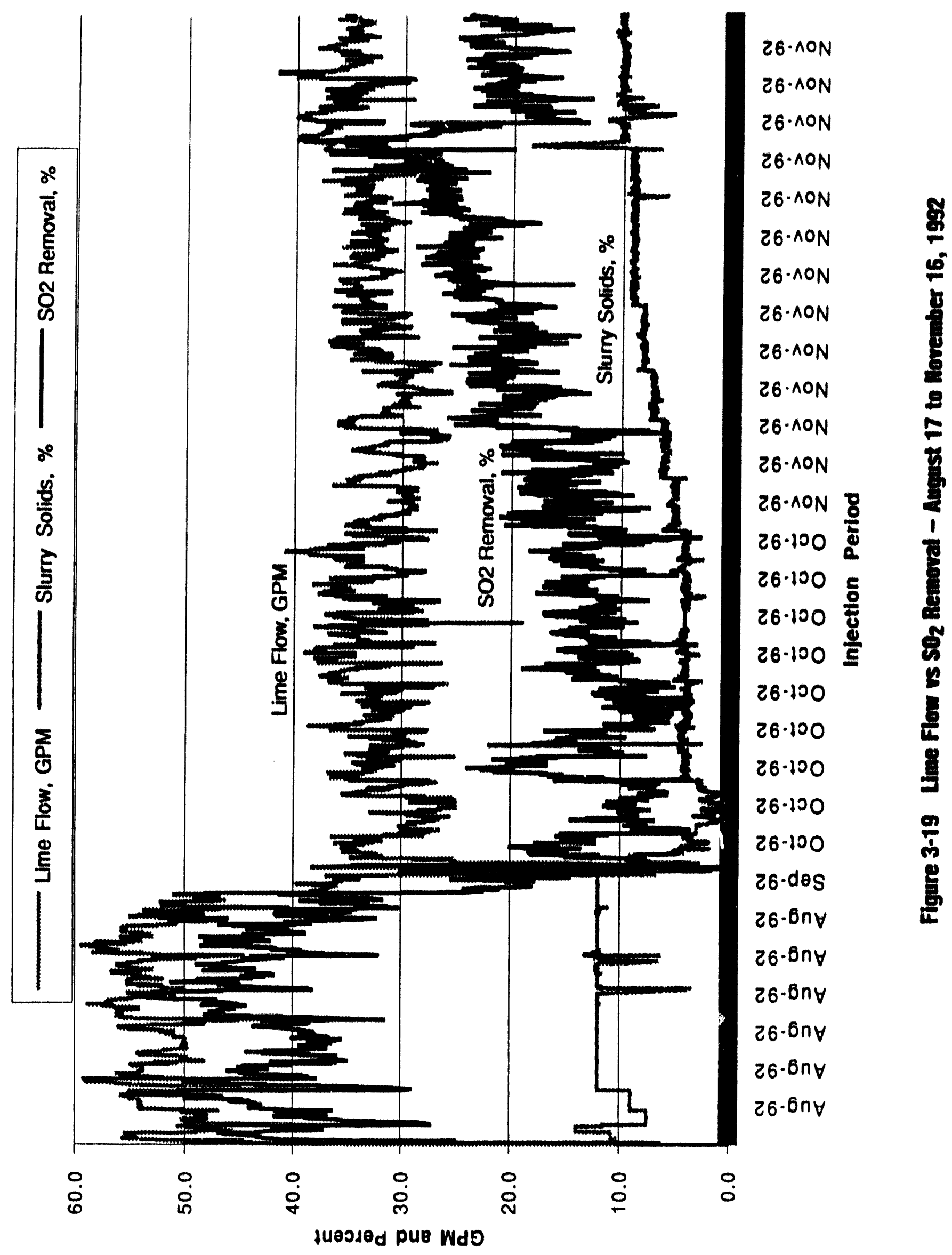









\subsubsection{Importance of Heat Balance in the CZD Process}

For a better understanding of the CZD operation we offer the following explanation.

In the CZD process, the reaction between the $\mathrm{SO}_{2}$ in the hot flue gas and the lime hydroxide slurry injected into the duct takes place in the presence of water. The reaction requires enough water to cool the flue gas at the adiabatic saturation temperature where water and gas mix. At Seward Station, it takes more than 30 gpm of atomized water in the B-duct to cool only a small portion of the flue gas to about $120^{\circ} \mathrm{F}$, the adiabatic saturation temperature. Because the flue gas

temperature in the B-duct is not uniform (hotter on the right side of the duct and top, than on the left side and bottom), to remove about $50 \%$ of the inlet $\mathrm{SO}_{2}$ takes about $52.55 \mathrm{gpm}$ of lime slurry to cool about $65 \%$ of the incoming flue gas to $120^{\circ} \mathrm{F}$ in the first 60 feet of the duct. In the next 60 feet of duct, the hot flue gas along the duct walls will warm the cold gas to about $165^{\circ} \mathrm{F}$. If the inlet flue gas is input at a temperature lower than $300^{\circ} \mathrm{F}$, there will not be enough heat to warm up the cold gas ahead of the turning vanes; in addition, wet deposits of fly ash and reacted products could stick to the turning vanes.

Because of boiler air leakage during the months of October and November 1992 , the flue gas temperature was too low, and the lime slurry injection rate was limited.

Figures 3-20 through 3-24 show the average temperatures and the low temperatures measured in the flue gas duct during different water injection rates and at different distances from the atomizer lances. Figure 3-25 shows the plan view of the B-duct with the thermocouple locations (B, C, D, and E), and Figure 3-26 shows the typical thermocouple section, computed as an average of the $\mathbf{2 4}$ thermocouples in a section.

The B-cross section is about 60 feet from the injection point. With 60 fps gas velocity, it will take the flue gas about one second to reach the B-cross section. If the flue gas in the wet zone is cooled at the adiabatic saturation temperature during this one second, the reaction between the $\mathrm{SO}_{2}$ and lime will be complete and the $\mathrm{SO}_{2}$ removal will be above $50 \%$. If only part of the wet zone is cooled at $120^{\circ} \mathrm{F}$, then the percentage of $\mathrm{SO}_{2}$ removal will be lower.

Figure 3-21 shows that at a $45 \mathrm{gpm}$ water injection rate, only part of the flue gas in the wet zone will reach adiabatic saturation. Experience shows that at a 50-52 gpm lime slurry injection rate, the average temperature at the B-cross section will reach the adiabatic saturation temperature and the percentage of $\mathrm{SO}_{2}$ removal will be $50 \%$. Because of the non-uniform temperature of the in-coming flue gas, the graph shows an average temperature and a lowest temperature at Bsection at different water injection rates. 


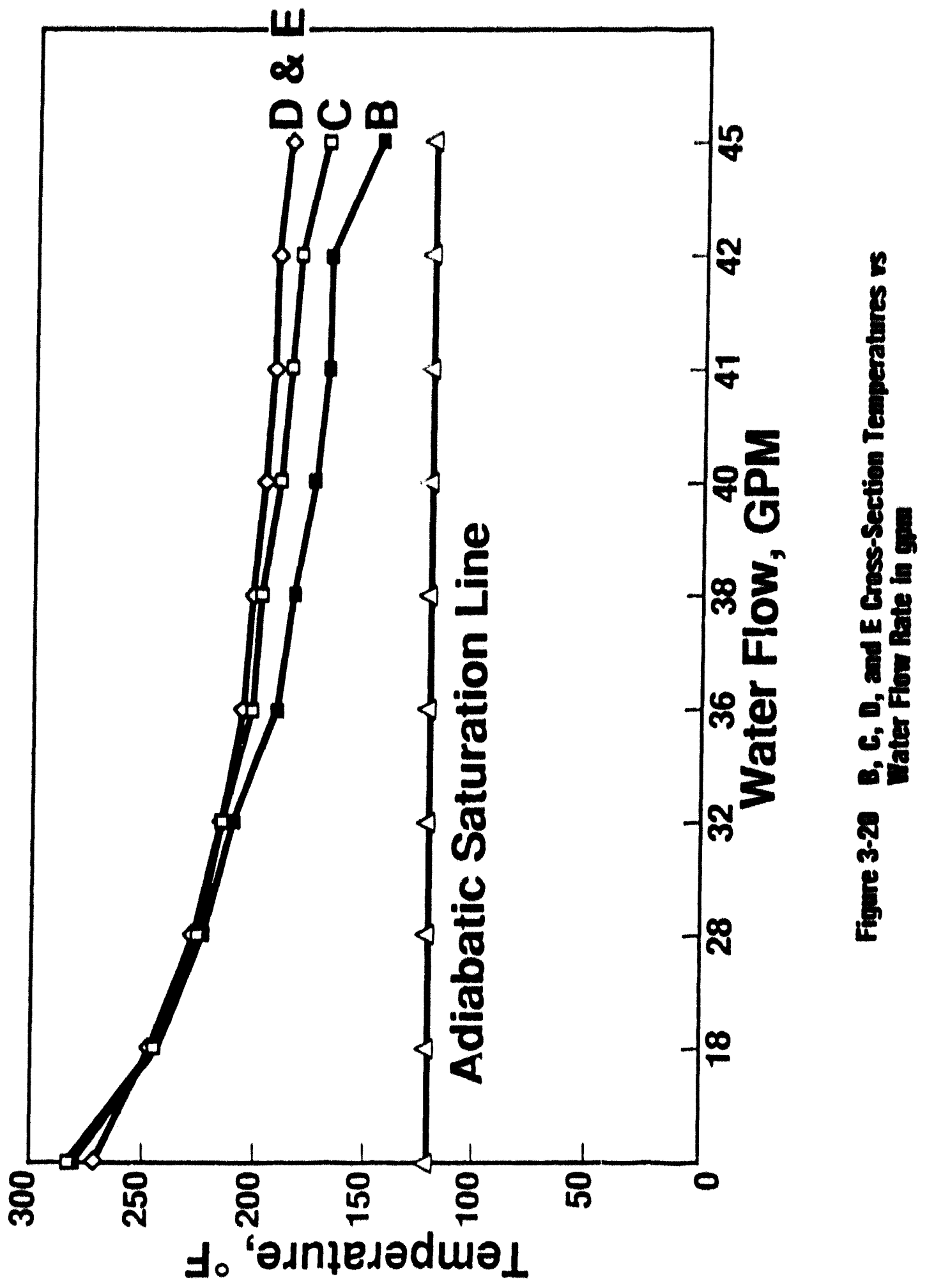














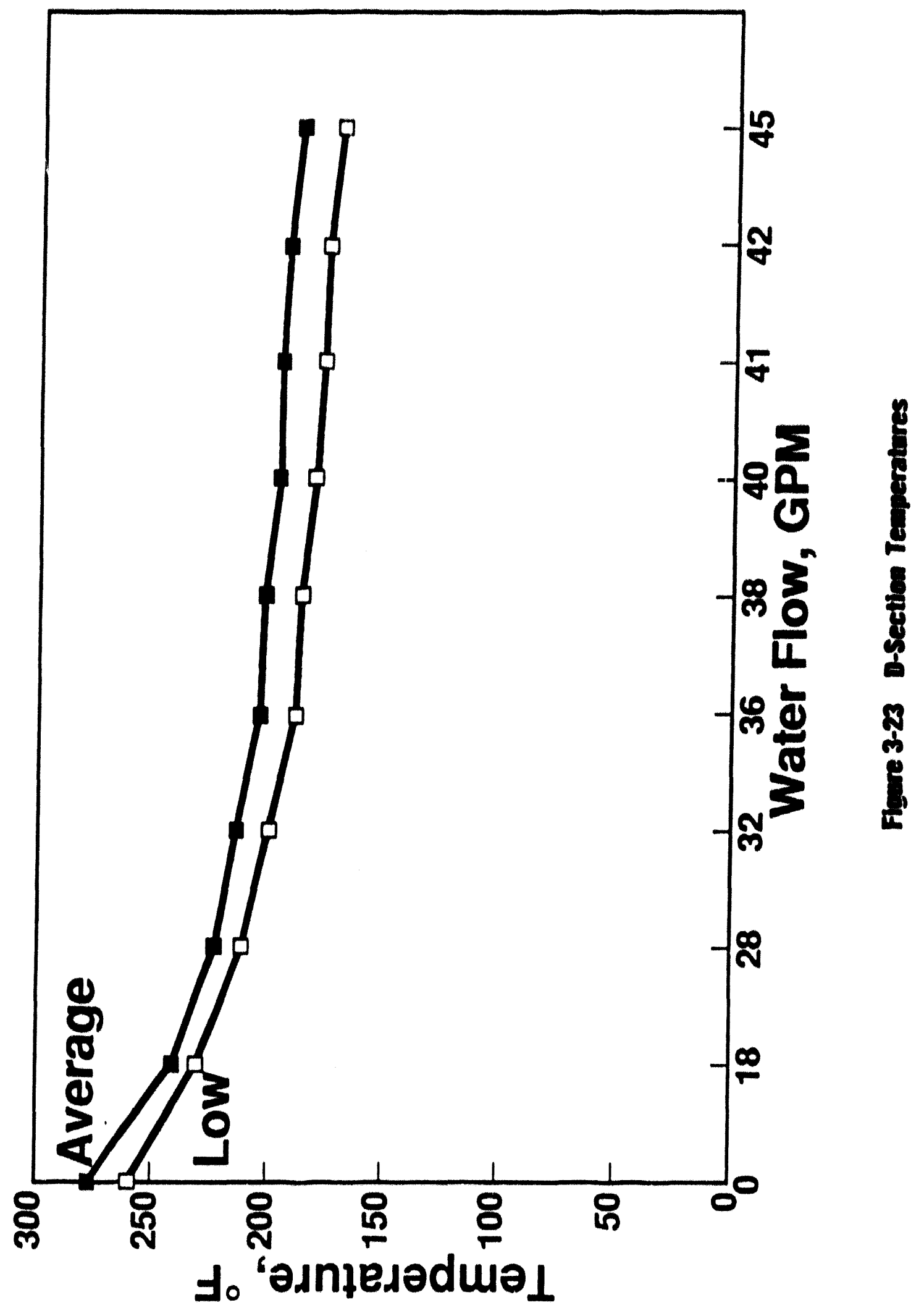




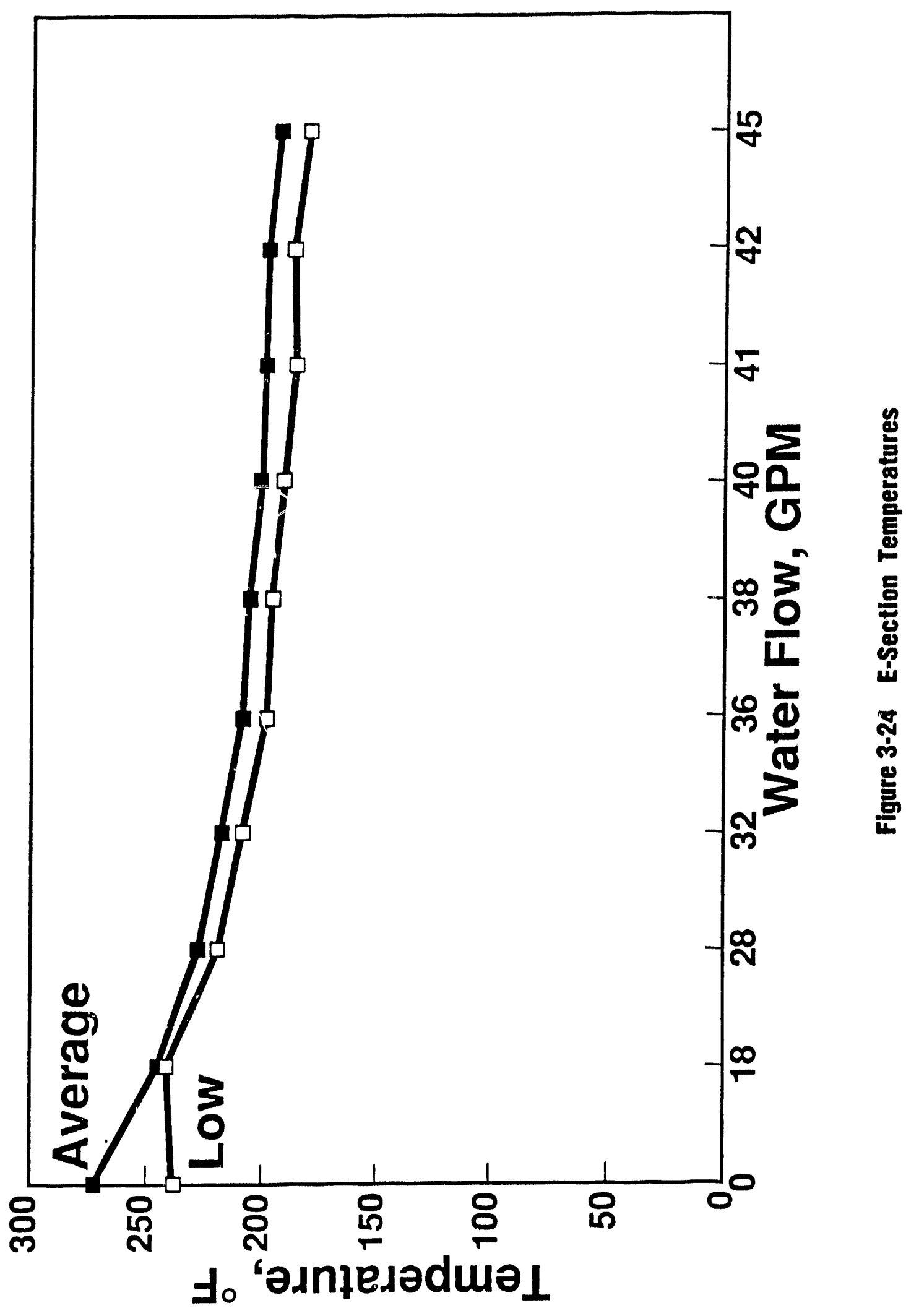




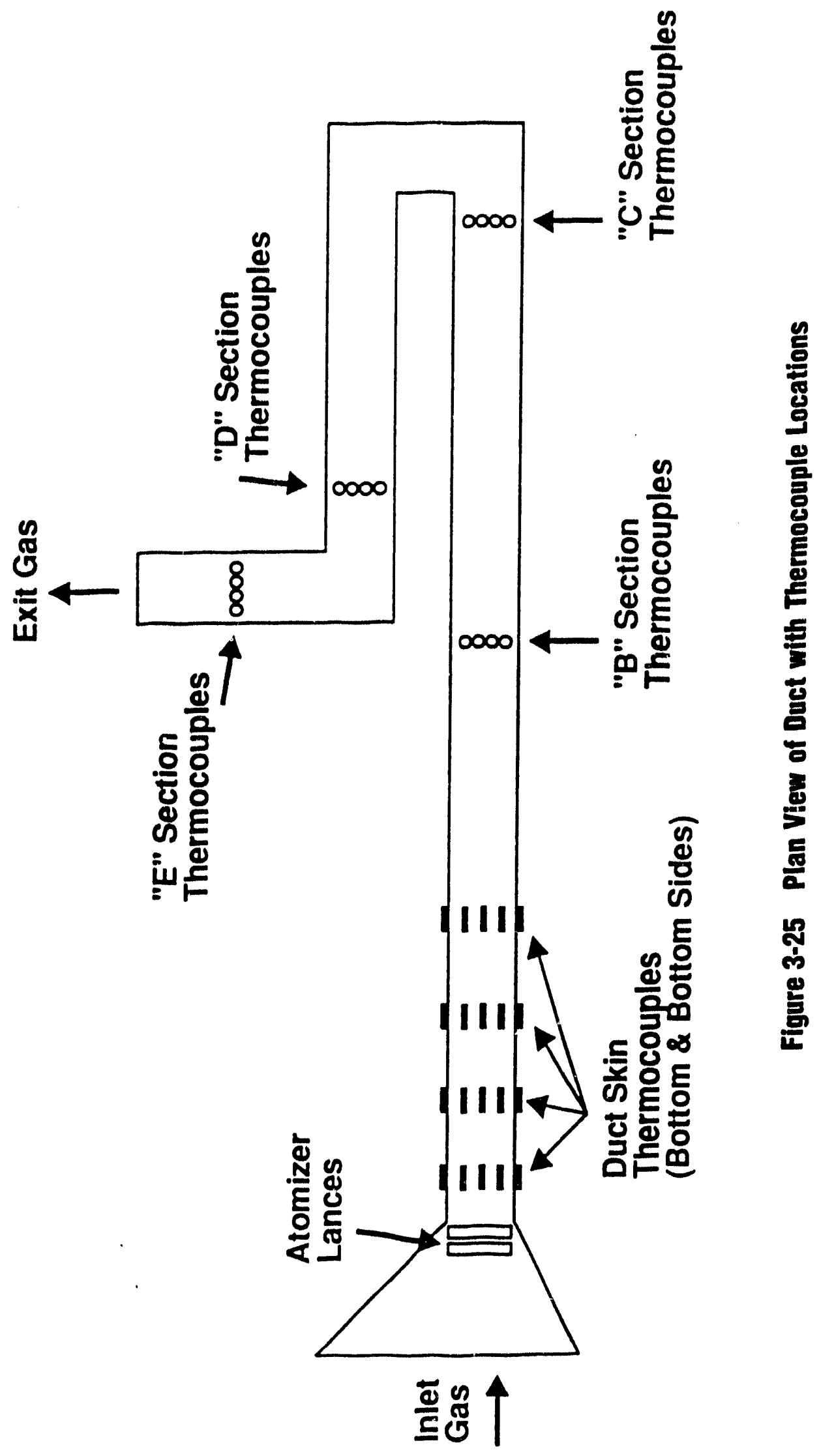




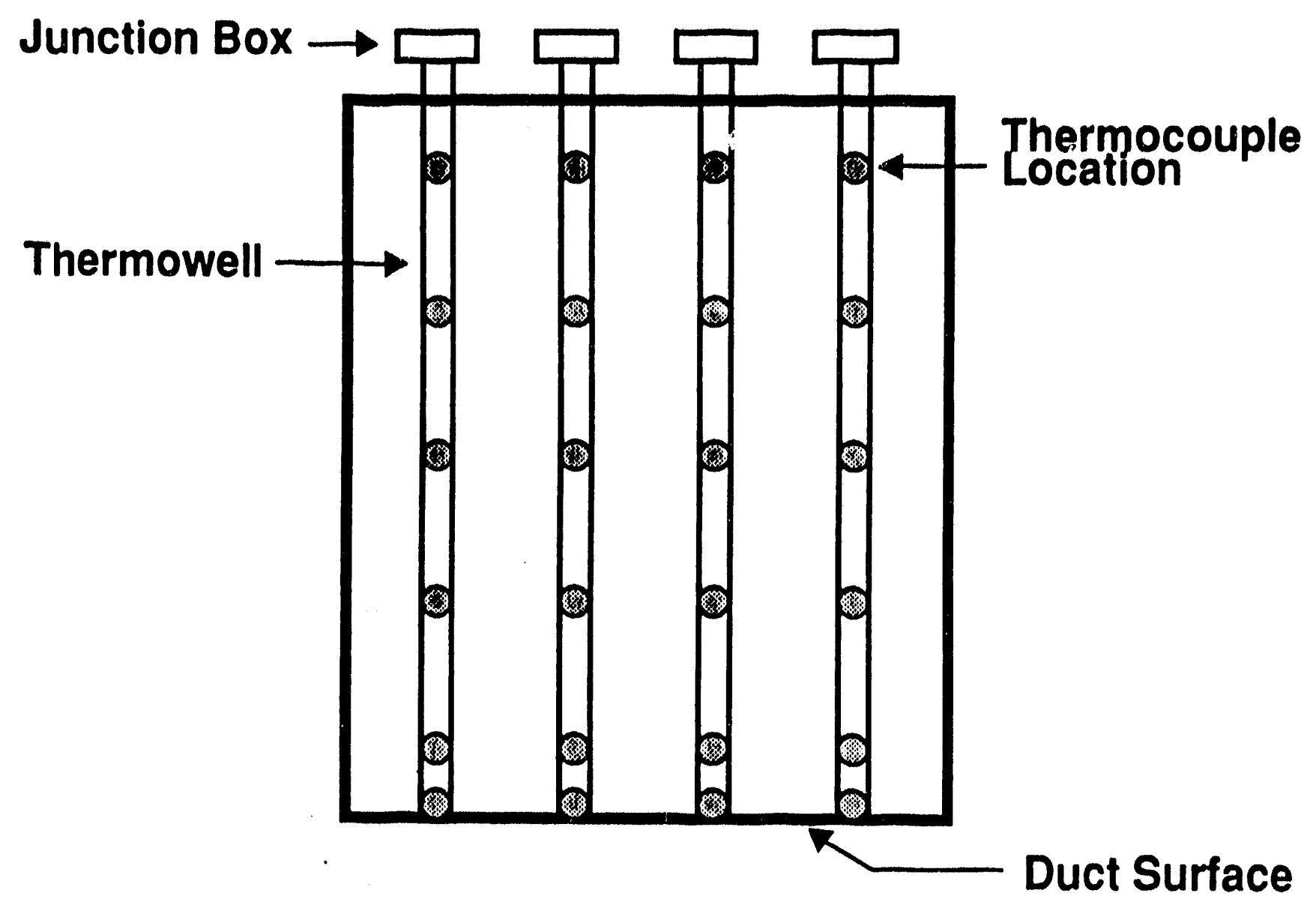

Figure 3-26 Typical Thermocouple Section (24 per Section) 
Figure 3-22 shows that at the C-section, in front of the turning vanes (120 feet from the atomizer lances), the flue gas is warmer and deposition on the turning vanes will not take place.

Figure 3-24 shows that at the E-section, ahead of entrance of the ESP, the flue gas temperature is about $180^{\circ} \mathrm{F}$, about $60^{\circ} \mathrm{F}$ approach to saturation.

\subsubsection{CZD System Influence on ESP Particulate Removal Efficiency}

\subsubsection{Overview}

In general, during CZD parametric testing for short or long durations (48 hours) as well as during continuous operation, the CZD system had a beneficial effect on ESP performance, as measured by lower percentages of stack and B-duct opacities.

During CZD operation in August and November 1992, the Buell ESP worked well and the duct and plant stack opacities were comfortably low. However, in September and October 1992, faced with difficulties with ESP operation, we had to limit the percentage lime slurry concentration to under $4 \%$ to avoid violations on high percentage opacity emissions. In late October 1992, during a boiler emergency shutdown, we corrected faulty grounds to Field \#2 collecting plates and opacity was no longer affected by the percentage of lime slurry concentration. The following discussion describes these events in detail.

\subsubsection{ESP Particulate Removal Efficiency-October 14 to November 16, 1992}

Appendix I includes the Seward Plant Buell ESP fields operating log from October 14 through November 16, 1992. On October 27, 1992, at 1700 hours (see page I-20), with no water or lime slurry injection, the data indicate high percentage stack and duct opacities ( 28.5 and $33 \%$, respectively) and a power consumption $(\mathrm{kW})$ that increases instead of decreases from field to field through the precipitator - because of less particulate in the gas stream. The variation of power input in this unexpected way is symptomatic of problems with the precipitator. After faulty grounds to collecting plates of Field \#2 were corrected, the stack and duct opacities ( 12.4 and $8.7 \%$, respectively) were no longer detrimentally affected by lime slurry injection into the duct and ESP collecting efficiency was improved. (See data collected on November 3 at 1200 hours [page [-30].)

Appendices $C, D$, and $E$ contain tables for the CZD operation for the period August 17 to November 16,1992. The data in these tables include 1-minute average values of the opacity percentage in the plant stack recorded every 15 minutes during lime injection.

In Appendix I, instant values of the duct opacities recorded from October 12 through November 16,1992, are presented. We took samples of coating materials containing ash and CZD solids waste from Unit \#15 discharge wires 
and collecting plates of each of the four Buell ESP fields. The ESP connected with $B$-duct where CZD is operating is named " $D$ " and the one connected with A-duct - without CZD - is named "C." For comparison, eight samples were taken from D.ESP and eight samples from C-ESP. Elements were analyzed semiquantitatively by Energy Dispersive X-ray Spectrometry (EDS). Table 3-4 shows the results of the analyzed samples. The most interesting data are the results for sulfur (S), calcium (Ca), and loss of ignition (LOI as wt\%) which characterize the CZD solids waste. The sulfur concentration of the samples taken from the collecting plates of all fields of " $\mathrm{D}$ " and " $\mathrm{C}$ " ESPs are about the same. However, the sulfur concentration of the samples from the wires from Fields \#2 and \#3 are substantially larger on D-ESP versus C-ESP. The same is true of the increase of calcium concentration on the discharge wires on the Fields \#2 and \#3, and the $w t \%$ of the LOI on the wires in these fields. This means that more CZD reaction products $\left(\mathrm{CaSO}_{4}, 2 \mathrm{H}_{2} \mathrm{O}\right)$ were collected on the discharge wires of Fields $\# 2$ and \#3.

\subsubsection{Summary}

The beneficial influence on CZD on ESP performance is explained as follows:

- Through evaporation of water in the lime slurry injected into the duct, the flue gas is cooled from $300^{\circ} \mathrm{F}$ to about $180^{\circ} \mathrm{F}$. This cooling decreases the gas volume and gas velocity in the ESP, and the longer residence time improves particulate removal efficiency.

- By decreasing the temperature, the particulates' resistivity is lowered, thereby improving performance.

- The increased humidity becomes a conditioning agent for reducing the cake resistivity, improving ESP performance.

- The size of fly ash particles, from the pulverized coal boiler varies from submicron to about 100 microns, with a mean diameter of 10-20 microns. The larger particles are easily removed by the ESP, but the submicron to about 2-micron particles are more difficult to remove due to reintrainment during rapping. The ESP removal efficiency depends on the percentage of very fine particles in the flue gas. If a method to agglomerate the fines into larger particles were available, then ESP performance would come close to 100 percent.

The injected fine atomized lime slurry - made of fine particles of hydrated lime or PHDL with a mean diameter of 5 microns - will intercept and impact the fly ash particles, agglomerating them in larger particles more easily removed by the ESP. 
Table 3-4

Elemental LOI Analysis of Ash Samples Removed from Boiler \#15 Electrostatic Precipitators

\begin{tabular}{|l|c|c|c|c|c|c|c|c|c|}
\hline \multicolumn{1}{|c|}{ Item } & Al & SI & $S$ & $K$ & Ca & Ti & Fo & O & LOI (Wt\%) \\
\hline C-1 Wire & 14.2 & 18.4 & 3.5 & 2.7 & 2.0 & 1.7 & 12.7 & 44.9 & 3.94 \\
C-2 Wire & 15.3 & 20.3 & 1.6 & 2.6 & 1.7 & 1.6 & 12.0 & 44.9 & 5.17 \\
C-3 Wire & 15.5 & 20.6 & 1.6 & 2.7 & 1.8 & 1.4 & 11.3 & 45.1 & 10.70 \\
C-4 Wire & 14.6 & 18.7 & 2.3 & 2.7 & 2.0 & 1.6 & 13.8 & 44.2 & 9.42 \\
C-1 Plate & 15.2 & 20.8 & 1.7 & 2.6 & 1.8 & 1.5 & 11.2 & 45.2 & 6.43 \\
C-2 Plate & 15.5 & 21.6 & 2.0 & 2.4 & 1.6 & 1.2 & 9.8 & 46.0 & 4.47 \\
C-3 Plate & 14.5 & 18.4 & 3.0 & 2.6 & 1.9 & 1.8 & 13.3 & 44.6 & 10.67 \\
C-4 Plate & 15.3 & 19.3 & 2.1 & 2.6 & 1.8 & 1.4 & 12.9 & 44.6 & 6.50 \\
\hline D-1 Wire & 14.0 & 18.7 & 3.6 & 2.6 & 2.4 & 1.6 & 11.9 & 45.2 & 10.16 \\
D-2 Wire & 13.0 & 16.7 & 6.0 & 2.4 & 2.3 & 1.7 & 12.1 & 45.7 & 21.94 \\
D-3 Wire & 14.3 & 18.2 & 3.7 & 2.7 & 2.4 & 1.6 & 11.4 & 45.3 & 16.96 \\
D-4 Wire & 14.6 & 20.1 & 2.2 & 2.5 & 2.0 & 1.6 & 11.8 & 45.1 & 8.45 \\
D-1 Plate & 15.2 & 20.3 & 1.3 & 2.6 & 2.1 & 1.5 & 12.5 & 44.5 & 3.54 \\
D-2 Plate & 14.9 & 19.2 & 2.3 & 2.8 & 2.7 & 1.6 & 11.9 & 44.7 & 4.64 \\
D-3 Plate & 14.2 & 18.1 & 3.0 & 2.8 & 3.0 & 1.6 & 12.9 & 44.3 & 6.26 \\
D-4 Plate & 15.5 & 21.3 & 1.3 & 2.7 & 2.1 & 1.4 & 10.3 & 45.3 & 4.59 \\
\hline
\end{tabular}

Note:

Elements were analyzed semi-quantitatively by Energy Dispersive X-Ray Spectrometry (EDS), The SEM or scanning electron microscope is used to view small specimens or deposits. Electrons rather than light are used to illuminate the sample, allowing the instrument to reach very high levels of magnification. When these electrons impact the sample they generate $X$-rays of varying element-specific wavelengths that can be measured by an energy dispersive spectrometer (EDS) attached to the SEM.

EDS instrument used at GPUN is only sensitive to elements equal to or greater than sodium in atomic number and the sensitivity of the technique generally increases with increasing atomic number. Consequently, common elements such as carbon, oxygen, and nitrogen are not detected by this technique. For example, a sample containing 50\% graphite (crystalline carbon) and 50\% iron oxide would be reported as $100 \%$ iron oxide. Also, EDS is an element-specific method and can not differentiate between various compounds. EDS cannot differentiate between iron filings (elemental iron) and rust (iron oxide). This distinction must be made using other techniques.

Since it is attached to the SEM, the EDS analysis beam can be focused on a very small area, allowing elemental analysis of very small areas or discrete particles. The beam can also be defocused and used to analyze a relatively large area, about a centimeter in diameter. Effective bulk analysis using a defocused beam requires special sample preparation since the electron beam penetrates only a few hundred angstroms into the sample. Consequently, bulk analysis of a sample using a defocused beam requires that the sample be reduced to a fine powder and 
thoroughly mixed before analysis. Generally, unless otherwise specified, the laboratory performs and reports bulk sample analyses.

When semi-quantitative results are desired, a calculation is made based on the relative peak intensities of the various elements requested. The elements requested are assigned an arbitrary value of $100 \%$ based on the assumption that the elements are present as their common oxides. Relative peak intensities are then used to calculate the approximate percentage of each individual element. Although this technique is fairly reliable, it is subject to errors due to undetected elements, matrix interference, and sample preparation. Also, since standards are not used in the analysis, analytical accuracy is not extremely good. A general rule of thumb is to allow a $10-15 \%$ relative error for the elements reported.

If additional accuracy, specific compound identification, or analysis for undetected components is required, the laboratory should be contacted for more information about available techniques for the sample of interest.

\subsubsection{Summary of CZD Operating Data on August 21, 1992}

The following summarizes CZD operating data on August 21, 1992:

- Inlet $\mathrm{SO}_{2}$

- $\mathrm{SO}_{2}$ removed

- Percent $\mathrm{SO}_{2}$ removed

- Hours of lime slurry injection

- Lime slurry injection rate

- Lime slurry concentration

- Lime consumed

- Lime utilization

- Ca-to-sulfur ratio

- Ca-to-sulfur ratio

- CZD solids waste

- Calculated fly ash from B-duct

- Total solids waste
$1738.8 \mathrm{lb} / \mathrm{hr}=237.7 \mathrm{lb}$ moles $/$ day

$796.3 \mathrm{lb} / \mathrm{hr}=108.85 \mathrm{lb}$ moles $/$ day

$45.8 \%$ average per day

8.75 hours

$53.8 \mathrm{gpm}$ average per day

$9 \%$ average per day

$2,553 \mathrm{lb} / \mathrm{hr}=318.3 \mathrm{lb}$ moles $/$ day

$34.3 \% \mathrm{lb}$ average per day

$1.34 \mathrm{lb}$ moles $\mathrm{Ca}$ per $\mathrm{lb}$ mole inlet $\mathrm{SO}_{2}$

$2.92 \mathrm{lb}$ moles $\mathrm{Ca}$ per $\mathrm{lb}$ mole $\mathrm{SO}_{2}$ removed

$32,563 \mathrm{lb} /$ day of which:

$17,886 \mathrm{lb} /$ day reaction products + $14,677 \mathrm{lb} /$ day spent PHDL

$19,032 \mathrm{lb} /$ day

25.8 tons/day of which:

$36.9 \%$ fly ash $+63.1 \%$ CZD solids waste

The CZD operation on August 21, 1992 is characterized by:

- High temperature of inlet flue gas $\left(307.5^{\circ} \mathrm{F}\right)$

- High flow lime slurry injection of $53.8 \mathrm{gpm}$ average per day

- High percent $\mathrm{SO}_{2}$ removed of $45.8 \%$ average per day

- Lime utilization of only $34.3 \%$ average per day, because of higher lime slurry concentration (9\%) than required 
- Calcium-to-sulfur ratio of $2.92 \mathrm{lb}$ moles per $\mathrm{lb}$ mole of $\mathrm{SO}_{2}$ removed, instead of expected $2-2.5 \mathrm{lb}$, due to the higher limr slurry concentration that would be required

- The total solids waste during the lime injection period contains about $37 \%$ fly ash and 63\% CZD solids waste. Without the first ESP that removes $60 \%$ of the fly ash ahead of the B-duct, the total solids waste would contain $60 \%$ fly ash and $40 \%$ CZD solids waste

\subsubsection{Summary of CZD Operating Data - August 17 to November 16, 1992}

The following summarizes CZD operating data from August 17 to November 16, 1992:

- Inlet $\mathrm{SO}_{2}$

- $\quad \mathrm{SO}_{2}$ removed

- Percent $\mathrm{SO}_{2}$ removed

- Hours of lime slurry injection

- Lime slurry injection rate

- Lime slurry concentration

- Lime consumed

- Lime utilization

- Ca-to-sulfur ratio

- Ca-to-sulfur ratio

- CZD solids waste

- Calculated fly ash from B-duct

Total solids waste
$1,493 \mathrm{lb} / \mathrm{hr}=11,156 \mathrm{lb}$ moles $\mathrm{SO}_{2}$ for the period

$322 \mathrm{lb} / \mathrm{hr}=2,406 \mathrm{lb}$ moles $\mathrm{SO}_{2}$ for the period

$21.57 \%$ average for the period

478.25 hours

$35.8 \mathrm{gpm}$ average for the period

$7.8 \%$ average for the period

$697,670 \mathrm{lb}$ lime $=9,938 \mathrm{lb}$ moles for the period

$25.9 \% \mathrm{lb}$ average

$0.89 \mathrm{lb}$ mole $\mathrm{Ca}$ per $\mathrm{lb}$ mole inlet $\mathrm{SO}_{2}$

$4.13 \mathrm{lb}$ mole $\mathrm{Ca}$ per $\mathrm{lb}$ mole $\mathrm{SO}_{2}$ removed

468.8 tons for the period, of which:

210.3 tons reaction products +

258.5 tons spent PHDL

491 tons fly ash

959.8 tons, of which:

$48.84 \%$ CZD solids waste $+51.16 \%$ fly ash

The CZD operation during this period is characterized by:

- Adjustments to the automation for the continuous operation

- Mechanical problems with the Buell ESP (grounding wires broken, etc.) that limited for a while the lime slurry concentration to under $4 \%$

- Low temperature of the inlet flue gas due to air in-leakage in the boiler system ahead of the B-duct. This low temperature flue gas limited the lime slurry injection rate to an average of $35.8 \mathrm{gpm}$. The low injection rate 
caused a reduced percentage of $\mathrm{SO}_{2}$ removal to $21.57 \%$ average, and therefore a low percent lime utilization of $25.9 \%$, and a low calcium-tosulfur ratio of $4.13 \mathrm{lb}$ moles $\mathrm{Ca}$ per $\mathrm{lb}$ mole $\mathrm{SO}_{2}$ removed

- Mechanical problems with the steam soot blowing system that allowed deposits to accumulate in the duct

\subsubsection{Operating Data - August 17 to November 16, 1992}

Operating data for lime slurry injections from August 17 to November 16, 1992 are included in Appendix $\mathrm{H}$.

\subsubsection{Conclusions}

This eighth quarterly report is the first describing the CZD continuous operation and integration with Boiler \#15 operating from the control room with Seward Station operators. Although we were at the beginning of the continuous demonstration period, operability was excellent. Figure 3-15 shows the CZD performance summary for the period October 17 to November 16, 1992. As shown in the figure, when Boiler \#15 was in operation, the CZD system was online injecting lime for $\mathrm{SO}_{2}$ removal or water for stack opacity control.

During the period when the automation of lime injection was in the startup phase, we had some maloperations. A few times moving from standby mode to ready condition, the flow control valve remained fully open and the lime injection rate reached over $80 \mathrm{gpm}$ for 1-2 minutes. The flow control valve had to be closed to limit the lime slurry flow rate to the level that would maintain a temperature of about $160^{\circ} \mathrm{F}$ at the turning vanes. During these excursions, wet deposits were formed because there was not enough heat in the duct to evaporate the very large amount of lime slurry. In addition to the deposits in the duct, some small amount of atomized lime slurry reached the ESP, coating the collecting plates and the discharge wires of ESP Fields \#3 and \#4. This lime coating caused some opacity problems. By fully tuning the flow control valve (FCV-10), the problems disappeared.

Due to large air in-leakage in the boiler and air heater, the duct flue gas inlet temperature started to decrease due to the cold weather. In August, the flue gas temperature at the inlet of the B-duct was about $310^{\circ} \mathrm{F}$, but in November, the temperature was only $275^{\circ} \mathrm{F}$. This lower flue gas temperature limited the lime slurry injection rate and the percent $\mathrm{SO}_{2}$ removal.

Because of the significant effect of low temperature flue gas at the duct inlet on the operating data, we prepared two summaries. Table 3-5 presents a summary of CZD operating data of one day in August (August 21, 1992) (see Section 3.37), and a summary of operating data for the period August 17 to November 16, 1992 (see Section 3.3.8). 
Table 3.5

Summary of CZD Operating Data

\begin{tabular}{|l|l|l|}
\hline \multicolumn{1}{|c|}{ Item } & \multicolumn{1}{|c|}{ Augus1 21, 1992 } & Aug. 17 lo Nov. 18, 1992 \\
\hline Percent $\mathrm{SO}_{2}$ removed & $45.8 \%$ average & $21.57 \%$ average \\
Hours of lime injection & 8.75 hours & 478 hours \\
Lime slurry injection rate & $53.8 \mathrm{gpm}$ average & $35.8 \mathrm{gpm}$ average \\
Lime slurry concentration & $9.0 \%$ & $7.8 \%$ average \\
Lirne utilization & $34.3 \%$ & $25.9 \%$ average \\
Ca-to-sulfur ratio & $2.92 \mathrm{lb}$ moles Ca / lb & $4.13 \mathrm{lb}$ moles Ca / lb \\
& mole $\mathrm{SO}_{2}$ removed & mole SO $\mathrm{SO}_{2}$ removed \\
Total solids waste & $36.9 \%$ fly ash $+63.1 \%$ & $51.16 \%$ fly ach $+48.84 \%$ \\
& CZD solids waste & CZD solids waste \\
\hline
\end{tabular}

The CZD system has a positive influence on ESP particulate removal efficiency, by lowering the percent stack and duct opacity, although the amount of particulate in the flue gas is substantially increased, assuming that the following conditions are observed:

- The ESP normal operating conditions are maintained.

- CZD operation avoids at all times operation that would result in wet lime entering the precipitator (whether planned or upset).

- The existing rapping system is able to remove the fly ash and dried lime and reaction products.

\subsection{BASELINE TESTING - FUEL}

Penelec analyzed samples of the incoming coal for moisture, ash, sulfur, Btu/lb, and volatile matter. The coal samples analyzed are as-received coal, taken ahead of the mills. Table 3-6 shows the results of the coal analyses for September 1992. The full results of these analyses during September, October, and November, 1992 are presented in Appendix F. 
Table 3.6

Results of Coal Analyses During September 1992

SEWARO STATION HEAT RATE OUALIIY SEPTEROER - 1992

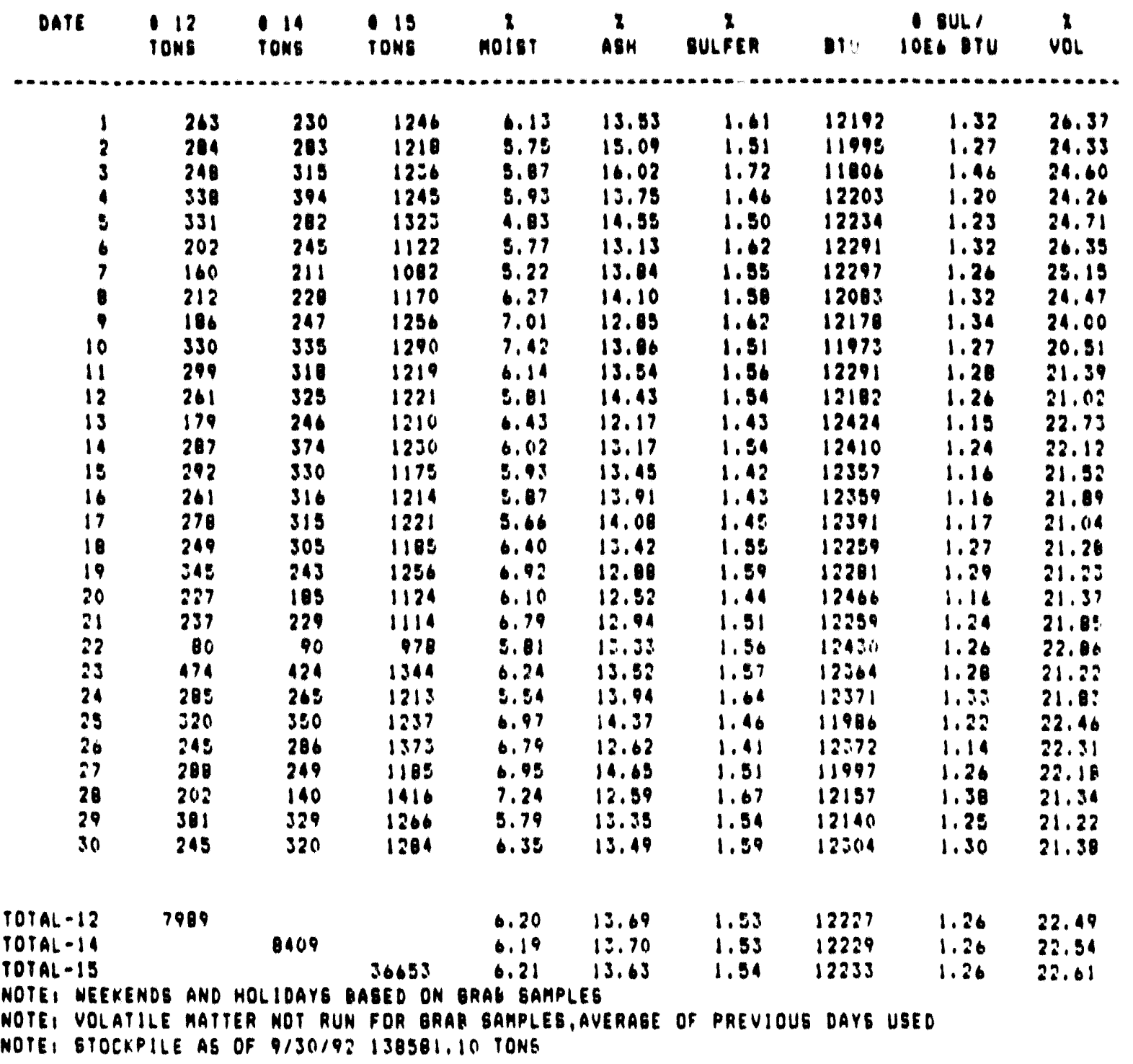




\section{Section 4}

\section{PLANNED ACTIVITIES}

During the next Quarter (November 17, 1992 through February 16, 1993), the following work is planned.

\subsection{PHASE 3 - OPERATION AND DISPOSITION}

We will continue CZD demonstrations with continuous operation for another 3 months, as follows:

- Operate the CZD system under optimized conditions completely integrated with the power plant operation.

- Measure emission control system performance:

- $\mathrm{SO}_{2}$ and $\mathrm{NO}_{x}$ performance tests

- $\quad$ ESP performance tests

- $\quad$ ESP performance improvements, if required

- Chemical analysis of solid wastes

- Continuous data recording and acquisition

- Determine power plant impacts

- Determine effects on CZD continuous operation when higher sulfur coal is burned in boiler \#15

\subsection{PHASE 3 - PROJECT MANAGEMENT}

Bechtel will provide overall management of the work required to complete Phase 3, including:

- Coordinating with Penelec and the project financial assistance groups

- Planning and conducting meetings with the DOE and project team to review the work

- Submitting reports in accordance with the federal assistance reporting checklist 


\section{Appendix A}

\section{Description of Project Work Breakdown Structure}




\section{Appendix A}

\section{DESCRIPTION OF PROJECT AND WORK BREAKDOWN STRUCTURE}

\section{A.1 PROJECT DESCRIPTION}

To accomplish the objectives of this project, the test program contains two distinct periods:

- The first perlod, of six months duration, includes daily factorial runs to test different reagents, atomizers, additives, and slurry concentrations.

- The second period, of one year duration, will demonstrate the performance of the CZD system on a continuous basis under actual power plant operating conditions.

A detalled description of the Work Breakdown Structure for this project, is shown in Figure A-1 on page 4-8. The work is scheduled to be accomplished in three phases as follows:

- Phase 1 work was completed on January 31, 1991. During this phase, Bechtel developed a process design, P\&IDs, and performed detalled engineering for a new extended duct to be used in the CZD demonstration in Unit 15 at Penelec's Seward Station. Bechtel wrote all the specifications for equipment and instruments, and started procurement activities. Interface with the appropriate environmental agencies was accomplished through Penelec, with Bechtel providing assistance as needed.

- Phaje 2 work was substantially completed by July 31, 1991. This phase included equipment acquisition and installation, facility construction, and start-up operations. Bechtel completed procurement of the equipment, instruments, materials, and fabrication of the new long duct. Penelec performed construction and installation of these items in accordance with specifications prepared by Bechtel. At completion of construction, using Penelec operating and maintenance personnel, Bechtel trained the operators, started all major elements of the CZD system, and verified that the installation and control system functioned according to requirements. During this phase, the installation of equipment and instrumentation was limited to that necessary for parametric testing to be accomplished in Phase 3. 
- Phase 3 work will be conducted in two parts. Parametric testing, Phase 3a, was scheduled for the period from June 3, 1991 through November 29, 1991, but the start was delayed until early August. During this period, Bechtel planned to carry out a parametric test program to optimize the performance of the CZD process. The test program is designed to develop operating conditions that will achieve high reliability and low cost operation. Phase 3b was scheduled from December 2, 1991 to July 1, 1993, to complete this demonstration project. The initial program included a period of time from December 2,1991 to July 1992 when no CZD testing was scheduled. With DOE's concurrence, this "no CZD testing" was modified by extending the CZD parametric testing until July 1992 for debugging and training the Penelec operating and maintenance personnel. In this way, the demonstration in July 1992 will be assured.

Based on the results of the parametric tests, Bechtel had completed additional design, procurement, installation and facility construction as necessary to permit a 6-month continuous demonstration. At this time the CZD system is being fully instrumented and integrated with the operation of Penelec's Unit \#15. The goal is to demonstrate the periormance of the CZD process for $\mathrm{SO}_{2}$ removal without significantly affecting either the boiler operation or plant particulate emissions.

Bechtel will supervise the 6-month continuous demonstration, and will carry out various performance tests, data acquisition, and chemical analyses. After the demonstration is completed, the system may remain in operation or the test apparatus may be dismantled and the site restored to its former condition, depending on Penelec's wishes. In either case, the new long straight duct :vill remain in operation.

To promote the dissemination of technical information to industry during the C.ZD demonstration, technical papers will be presented at various conferences. These papers will provide non-proprietary technical and economic data, results, and conclusions. The technical papers will also be made available for publication in appropriate journals. Representatives of the utilities will be invited to visit the demonstration site to learn first hand how the CZD process handles $\mathrm{SO}_{2}$ and $\mathrm{NO}_{x}$ removal from utility flue gas operations.

\section{A.2 WORK BREAKDOWN STRUCTURE DESCRIPTION}

The following tasks will be performed during the three phases of the project to demonstrate the cost effective control of $\mathrm{SO}_{2}$ and $\mathrm{NC}_{x}$ emissions from the preNSPS coal-fired utility boiler No. 15 at Penelec's Seward Station: 


\section{Phase 1: Design and Permitting}

WBS No.1.1.2 Design and Procurement

- Prepare process flow diagrams for each of the two periods, including material balances and stream properties. Prepare technical specifications for process equipment.

- Prepare detailed designs for the fabrication of the duct extension and its associated construction and installation.

- Prepare site-specific detailed designs for installation of the compressed air system, lime feed system, and atomizers with their related headers and distribution piping, including instruments and controls.

- Prepare technical specifications for procurement of required support equipment, and its associated construction and installation.

- Issue bids for equipment with long delivery times.

WBS No.1.1.3 Management and Travel

- Project Management. Provide overall management of the work required to complete this phase, including coordination with Penelec and the groups providing project financial assistance. Bechtel's project management plan includes provisions for meetings with the DOE and formal reviews of project work, as well as provisions for submitting reports in accordance with the Federal Assistance Reporting Checklist, Attachment $C$.

- Travel. Bechtel travel includes project management travel to the Penelec site and to DOE's offices for meetings, and travel by the project engineer and other members of the project team as required to complete the task activities. All travel and subsistence to be in accordance with Government regulations.

\section{Phase 2: Construction and Start-up}

\section{WBS No.1.2.1 Equipment Acquisition and Installation}

- Procure air compressors and electric power supply.

- Procure atomizers.

- Procure instruments and controls.

- Procure lime feed system.

- Procure lime tranfer system.

- Frovide assistance to Penelec for instailation of all process and support equipment, including drawings, specifications, design packages for bidding, and other related design information. 


\section{WBS No. 1.2.2 Facility Construction}

- Approve shop drawings for fabrication of duct extension.

- Provide site engineering support and related assistance to Penelec as required for quality and schedule control.

WBS No. 1.2.3 Start-up (Checkout) and Operator Training for Parametric Testing

- Provide start-up engineer to supervise commissioning of all CZD components and systems.

- Train Penelec's operators to run the CZD system and perform the tasks required to conduct parametric testing.

- Establish procedures for coordination of test operations with Penelec's plant management and operations personnel.

- Finalize all elements of Test Plan.

WBS No.1.2.4 Construction Management

- Provide construction management support to Penelec as requested. Penelec will have prime control and responsibility for all construction activities at Seward Station.

\section{WBS No.1.2.5 Management and Travel}

- Project Management. Provide overall management of the work required to complete this phase, including coordination with Penelec and the groups providing project financial assistance. Plan and conduct meetings with the DOE and the project team for review of project work. Submit reports in accordance with the Federal Assistance Reporting Checklist, Attachment $C$.

- Travel. Bechtel travel includes project management travel to the Penelec site and to DOE's offices for meetings, and travel by the project engineer and other members of the project team as required to complete the task activities. All travel and subsistence to be in accordance with Government regulations.

\section{Phase 3: Operation and Disposition}

\section{WBS No. 1.3.1 Lime Procurement}

- Procure all types of lime and other reagents required to conduct parametric and continuous (one-year) test programs.

\section{WBS No. 1.3.2 CZD Parametric Testing}

- Perform wind tunnel tests on atomizers at the University of California at Davis to $d$. termine performance characteristics. Select atomizers to be used in the parametric test program. 
- Evaluate performance of electrostatic precipitators (ESPs) during parametric tests. Consult with ESP experts, if necessary, to improve performance.

- Evaluate effects of process variables on emission control performance.

- Determine operating conditions for optimum overall performance.

- Evaluate effects of using alternative coals and reagents:

- $\quad$ High and medium sulfur coals

- Alternate reagents including pressure-hydrated dolomitic lime, hydrated calcitic lime, and fresh slaked calcitic lime.

WBS No. 1.3.3 Completion of Design, Procurement and Installation

- Based on the results of the parametric tests, complete the design packages and procure all process equipment needed to completely automate and integrate the operation of the CZD system with boiler No. 15 at Seward Station.

- Procure all instruments and controls needed for continuous operation, monitoring, and data collection.

\section{WBS No. 1.3.4 Completion of Facility Construction}

- Install facilities for lime unloading and storage to support the increased capacity required for continuous operation of the CZD system.

- Insulate piping and provide weather protection and enclosures as required to permit coninuous, all-year operation of the CZD system.

- $\quad$ Provide construction management support to Penelec as requested.

Penelec will have prime control and responsibility for all construction activities at Seward Station.

WBS No. 1.3.5 Start-up (Checkout) and Operator Training for Continuous Operation

- Provide start-up engineer to supervise commissioning of all new CZD components and systems.

- Train Penelec's operators to run the CZD system and perform the tasks required to conduct the one-year continuous demonstration.

- Establish procedures for coordination of data collection and reduction with Penelec's plant management and operations personnel.

WBS No, 1.3.6 CZD Demonstration with Continuous Operaticn

- Operate the CZD system under optimized conditions for one year completely integrated with the power plant operation.

- Measure emission control system performance: 
- $\mathrm{SO}_{2}$ and $\mathrm{NO}_{x}$ performance tests

- $\quad$ ESP performance tests

- ESP performance improvements, if required

- Chemical analysis of solid wastes

- Continuous data recording and acquisition

- Determine power plant impacts.

WBS No. 1.3.7 Duct and Hopper Cleaning

- Perform periodic inspection and cleaning, if necessary, during intervals when the boiler is not operating to determine the long-term extent of duct fouling resulting from CZD system operation.

WBS No. 1.3.8 Data Evaluation and Reporting

- Correlate and evaluate all data collected during the entire CZD test program, including parametric tests as well as continuous operation.

- Summarize key data and test results:

- Inlet and outlet concentration of $\mathrm{SO}_{2}, \mathrm{NO}_{x}$, and oxygen

- Amount of lime used and lime utilization

- Variation in flue gas temperature during lime slurry injection

- Variation in ESP recorded data

- Variation in percent opacity at the stack

- Lime deposition on duct surfaces

- Use technological, economic, environmental, and operating evaluations from the demonstration project to prepare Demonstration Test Reports and a Technology Performance and Economic Evaluation Report.

\section{WBS No. 1.3.9 Disposition}

- Disposition of the CZD system equipment installation:

- The new long straight duct will remain in operation.

- The disposition of all other equipment (continued operation or removal and storage) will be decided by Penel

WBS No. 1.3.10 Management and Travel

- Project Management. Provide overall management of the work required to complete this phase, including coordination with Penelec and the groups providing project financial assistance. Plan and conduct meetings with the DOE and the project team for review of project work. Submit 
reports in accordance with the Federal Assistance Reporting Checklist, Attachment C.

- Travel. Bechtel travel includes project management travel to the Penelec site and to DOE's offices for meetings, and travel by the project engineer and other members of the project team as required to complete the task activities. All travel and subsistence to be in accordance with Government regulations. 


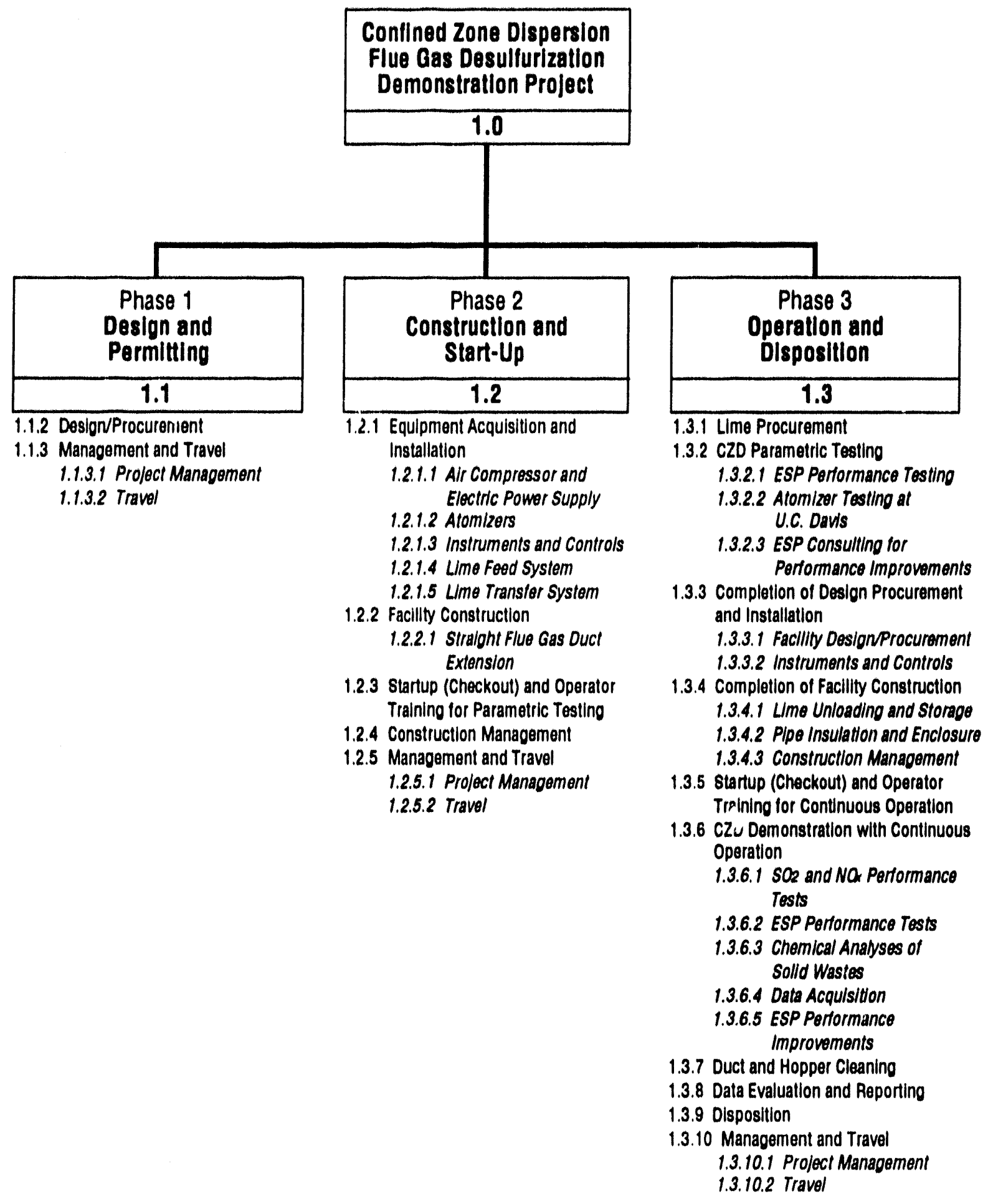

Figure A-1 Work Breakdown Structure (WBS) Dlagram 


\section{Appendix B}

\section{Report Distribution List}




\section{Appendix B}

\section{REPORT DISTRIBUTION LIST}

The number in parentheses () indicates the number of copies submitted.

U.S. Department of Energy - PETC

Mr. Arthur L. Baldwin (2)

PETC Technical Project Manager

Mail Stop 920-L

U.S. Department of Energy/PETC

P.O. Box 10940

Pittsburgh, PA 15236

Mr. Martin J. Byrnes (1)

Contract Specialist

AD-21, Mail Stop 921-165

U.S. Department of Energy/PETC

P.O. Box 10940

Pittsburgh, PA 15236

Dr. C. Lowell Miller (1)

Associate Deputy Assistant

FE-22, 3E-042, Forrestal

U.S. Department of Energy

Washington, D.C. 20585

Mr. Robert Fischer (1)

Office of Patent Counsel

U.S. Department of Energy

9800 South Cass Avenue

Argonne, IL 60439

Office of Technology Transfer (3)

Mail Stop 58-MEZZ

U.S. Department of Energy/PETC

P.O. Box 10940

Pittsburgh, PA 15236

Dr. S. N. Roger Rao (1)

Burns and Roe Technical Group Manager

P.O. Box 18288

Pittsburgh, PA 15236 
Mr. Larry M. Joseph (1)

HQ DOE Program Manager

FE-22, 3E-042, Forrestal

U.S. Department of Energy

Washington, D.C. 20585

Pennsylvania Electric Company

Mr. J. J. Battista (1)

Project Manager

Pennsylvania Electric Company

1001 Broad Street

Johnstown, PA 15907

Mr. D. Kessler (1)

Pennsylvania Electric Company

1001 Broad Street

Johnstown, PA 15907

Mr. J. A. Seebacher (1)

Manager, Fuels Contracts and Planning

Pennsylvania Electric Company

1001 Broad Street

Johnstown, PA 15907

Pennsylvania Energy Development Authority

Mr. Dane C. Bickley (1)

Director

Pennsylvania Energy Development Authority

116 Pine Street, Second Floor

Harrisburg, Pennsylvania 17101-1227

New York State Electric \& Gas Corporation

Mr. John Holler (1)

Manager - Homer City Operations

New York State Electric \& Gas Corporation

Homer City Power Plant

P.O. Box 29

Homer City, Pennsylvania 15748 


\section{Rockwell Lime Company}

Mr. Joseph H. Brisch (1)

Sales Manager

Rockwell Lime Company

4223 Rockwood Road

Manitowoc, Wisconsin 54220-9619 


\title{
Appendix C
}

\author{
Tables With Summary of \\ Lime Slurry Injection Data \\ August 17 to September 16, 1992
}


SUMMARY OF LIME SLURRY INJECTION FROM AUGUST 17, 1992 TO SEPTEMBER 16, 1992

\begin{tabular}{|c|c|c|c|c|c|c|c|c|}
\hline Dalo & Time & Hours of & Cumulative & B Duct Temp & Lime Flow & Slurry Sollds & SO2 Removal & Stack Opacily \\
\hline & & Injection & Hours & ${ }^{\circ} \mathrm{F}$ & GPM & Percent & Percent & Percent \\
\hline $8 / 17 / 92$ & 1300 & 0.25 & 0.25 & 304.3 & 35.0 & 10.3 & 9.8 & 15.1 \\
\hline $8 / 17 / 92$ & 1315 & 0.25 & 0.50 & 304.3 & 38.3 & 10.3 & 8.8 & 18.3 \\
\hline $8 / 17 / 92$ & 1330 & 0.25 & 0.75 & 305.3 & 44.8 & 10.3 & 6.3 & 14.5 \\
\hline $8 / 17 / 92$ & 1345 & 0.25 & 1.00 & 304.8 & 43.8 & 10.3 & 37.0 & 15.0 \\
\hline $8 / 17 / 92$ & 1400 & 0.25 & 1.25 & 306.4 & 50.0 & 10.3 & 34.4 & 16.2 \\
\hline $8 / 17 / 92$ & 1415 & 0.25 & 1.50 & 307.5 & 17.7 & 10.3 & 32.0 & 20.0 \\
\hline $8 / 17 / 92$ & 1430 & 0.25 & 1.75 & 303.2 & 44.5 & 10.3 & 24.9 & 9.6 \\
\hline $8 / 17 / 92$ & 1445 & 0.25 & 2.00 & 303.2 & 51.1 & 10.3 & 26.0 & 11.6 \\
\hline $8 / 17 / 92$ & 1500 & 0.25 & 2.25 & 306.6 & 54.0 & 10.3 & 41.3 & 18.7 \\
\hline $8 / 17 / 92$ & 1515 & 0.25 & 2.50 & 307.0 & 55.1 & 10.3 & 42.0 & 11.2 \\
\hline $8 / 17 / 92$ & 1530 & 0.25 & 2.75 & 307.4 & 55.1 & 10.7 & 42.5 & 11.5 \\
\hline $8 / 17 / 92$ & 1545 & 0.25 & 3.00 & 307.0 & 55.7 & 10.7 & 42.8 & 17.2 \\
\hline $8 / 17 / 92$ & 1600 & 0.25 & 3.25 & 307.4 & 54.5 & 10.7 & 43.4 & 15.9 \\
\hline $8 / 17 / 92$ & 1615 & 0.25 & 3.50 & 307.4 & 54.9 & 10.7 & 43.2 & 16.1 \\
\hline $8 / 17 / 92$ & 1630 & 0.25 & 3.75 & 308.3 & 55.1 & 10.7 & 43.5 & 15.0 \\
\hline $8 / 17 / 92$ & 1645 & 0.25 & 4.00 & 308.1 & 54.9 & 10.7 & 45.0 & 16.0 \\
\hline $8 / 17 / 92$ & 1700 & 0.25 & 4.25 & 308.3 & 55.2 & 10.7 & 45.7 & 15.3 \\
\hline $8 / 17 / 92$ & 1715 & 0.25 & 4.50 & 308.8 & 55.3 & 10.7 & 46.3 & 167 \\
\hline $8 / 17 / 92$ & 1730 & 0.25 & 4.75 & 307.5 & 54.8 & 10.7 & 46.4 & 15.1 \\
\hline $8 / 17 / 92$ & 1745 & 0.25 & 5.00 & 308.1 & 55.5 & 10.7 & 46.8 & 20.6 \\
\hline $8 / 17 / 92$ & 1800 & 0.25 & 5.25 & 308.3 & 49.9 & 10.7 & 43.2 & 18.5 \\
\hline $8 / 17 / 92$ & 1815 & 0.25 & 5.50 & 307.0 & 48.2 & 13.9 & 42.5 & 19.4 \\
\hline $8 / 17 / 92$ & 1830 & 0.25 & 5.75 & 306.2 & 48.0 & 13.9 & 42.1 & 19.7 \\
\hline $8 / 17 / 92$ & 1845 & 0.25 & 6.00 & 306.6 & 48.1 & 13.9 & 41.2 & 11.7 \\
\hline $8 / 17 / 92$ & 1900 & 0.25 & 6.25 & 306.4 & 45.6 & 13.9 & 38.3 & 11.0 \\
\hline $8 / 17 / 92$ & 1915 & 0.25 & 6.50 & 306.8 & 46.7 & 13.9 & 37.2 & 11.3 \\
\hline $8 / 17 / 92$ & 1930 & 0.25 & 6.75 & 305.3 & 47.5 & 13.9 & 40.5 & 10.8 \\
\hline $8 / 17 / 92$ & 1945 & 0.25 & 7.00 & 305.7 & 48.7 & 13.9 & 40.9 & 11.9 \\
\hline $8 / 17 / 92$ & 2000 & 0.25 & 7.25 & 305.9 & 49.0 & 13.9 & 41.8 & 12.4 \\
\hline $8 / 17 / 92$ & 2015 & 0.25 & 7.50 & 306.2 & 49.7 & 13.9 & 40.1 & 12.1 \\
\hline $8 / 17 / 92$ & 2030 & 0.25 & 7.75 & 305.9 & 49.8 & 13.9 & 39.8 & 11.1 \\
\hline $8 / 17 / 92$ & 2045 & 0.25 & 8.00 & 305.9 & 50.6 & 11.5 & 39.5 & 13.6 \\
\hline $8 / 17 / 92$ & 2100 & 0.25 & 8.25 & 306.2 & 49.8 & 11.5 & 40.1 & 13.9 \\
\hline $8 / 17 / 92$ & 2115 & 0.25 & 8.50 & 304.0 & 34.2 & 11.5 & 21.6 & 13.5 \\
\hline Total Hour & & 8.50 & & & & & & \\
\hline $8 / 20 / 92$ & 1745 & 0.25 & 8.75 & 305.1 & 40.1 & 7.5 & 17.2 & 16.8 \\
\hline $8 / 20 / 92$ & 1800 & 0.25 & 9.00 & 306.0 & 44.8 & 7.5 & 21.5 & 15.8 \\
\hline $8 / 20 / 92$ & 1815 & 0.25 & 9.25 & 306.9 & 49.9 & 7.5 & 27.7 & 17.7 \\
\hline $8 / 20 / 92$ & 1830 & 0.25 & 9.50 & 305.1 & 48.2 & 7.5 & 28.4 & 16.1 \\
\hline $8 / 20 / 92$ & 1845 & 0.25 & 9.75 & 304.7 & 49.4 & 7.5 & 28.4 & 15.3 \\
\hline $8 / 20 / 92$ & 1900 & 0.25 & 10.00 & 304.2 & 49.7 & 7.5 & 32.5 & 14.8 \\
\hline $8 / 20 / 92$ & 1915 & 0.25 & 10.25 & 302.4 & 49.9 & 7.5 & 34.0 & 16.7 \\
\hline $8 / 20 / 92$ & 1930 & 0.25 & 10.50 & 301.6 & 50.0 & 7.5 & 35.4 & 14.5 \\
\hline $8 / 20 / 92$ & 1945 & 0.25 & 10.75 & 301.3 & 50.3 & 7.5 & 36.0 & 15.4 \\
\hline $8 / 20 / 92$ & 2000 & 0.25 & 11.00 & 300.3 & 50.1 & 7.5 & 37.1 & 16.1 \\
\hline $8 / 20 / 92$ & 2015 & 0.25 & 11.25 & 299.4 & 47.9 & 7.5 & 33.4 & 14.4 \\
\hline
\end{tabular}


SUMMARY OF LIME SLURAY INJECTION FROM AUGUST 17, 1992 TO SEPTEMBER 16, 1992

\begin{tabular}{|c|c|c|c|c|c|c|c|c|}
\hline Dale & Timo & $\begin{array}{l}\text { Hours of } \\
\text { Injection }\end{array}$ & $\begin{array}{c}\text { Cumulalive } \\
\text { Hours }\end{array}$ & $\begin{array}{c}\text { B Duct Temp: } \\
\text { of }\end{array}$ & $\begin{array}{c}\text { Lime Flow } \\
\text { GPM }\end{array}$ & $\begin{array}{l}\text { Slurry Solids } \\
\text { Percent }\end{array}$ & $\begin{array}{c}\text { SO2 Removal } \\
\text { Percent }\end{array}$ & $\begin{array}{c}\text { Slack Opacily } \\
\text { Percenl }\end{array}$ \\
\hline $8 / 20 / 92$ & 2030 & 0.25 & 11.50 & 299.4 & 48.2 & 7.5 & 33.6 & 16.5 \\
\hline $8 / 20 / 92$ & 2045 & 0.25 & 11.75 & 299.6 & 48.2 & 7.5 & 34.9 & 16.7 \\
\hline $8 / 20 / 92$ & 2100 & 0.25 & 12.00 & 299.9 & 47.9 & 7.5 & 35.6 & 15.0 \\
\hline $8 / 20 / 92$ & 2115 & 0.25 & 12.25 & 299.6 & 48.4 & 7.5 & 41.6 & 14.1 \\
\hline $8 / 20 / 92$ & 2130 & 0.7 .5 & 12.50 & 299.2 & 48.1 & 7.5 & 37.5 & 14.1 \\
\hline $8 / 20 / 92$ & 2145 & 0.25 & 12.75 & 298.8 & 47.9 & 7.5 & 36.8 & 13.5 \\
\hline $8 / 20 / 92$ & 2200 & 0.25 & 13.00 & 297.4 & 48.1 & 7.5 & 38.8 & 13.4 \\
\hline $8 / 20 / 92$ & 2215 & 0.25 & 13.25 & 296.7 & 49.8 & 7.5 & 40.8 & 13.8 \\
\hline $8 / 20 / 92$ & 2230 & 0.25 & 13.50 & 297.4 & 49.8 & 7.5 & 39.3 & 11.6 \\
\hline $8 / 20 / 92$ & 2245 & 0.25 & 13.75 & 296.6 & 49.9 & 7.5 & 40.8 & 14.2 \\
\hline $8 / 20 / 92$ & 2300 & 0.25 & 14.00 & 296.7 & 50.3 & 7.5 & 41.0 & 12.2 \\
\hline $8 / 20 / 92$ & 2315 & 0.25 & 14.25 & 296.7 & 50.1 & 7.5 & 41.6 & 13.0 \\
\hline $8 / 20 / 92$ & 2330 & 0.25 & 14.50 & 296.1 & 50.4 & 7.5 & 36.6 & 12.4 \\
\hline Total Hour & & 6.00 & & & & & & \\
\hline $8 / 21 / 92$ & 1445 & 0.25 & 14.75 & 310.5 & 46.9 & 9.0 & 32.3 & 9.4 \\
\hline $8 / 21 / 92$ & 1500 & 0.25 & 15.00 & 310.3 & 50.1 & 9.0 & 36.2 & 8.6 \\
\hline $8 / 21 / 92$ & 1515 & 0.25 & 15.25 & 311.2 & 52.1 & 9.0 & 38.7 & 9.2 \\
\hline $8 / 21 / 92$ & 1530 & 0.25 & 15.50 & 311.2 & 54.1 & 9.0 & 40.0 & 8.1 \\
\hline $8 / 21 / 92$ & 1545 & 0.25 & 15.75 & 310.7 & 54.0 & 9.0 & 41.8 & 8.7 \\
\hline $8 / 21 / 92$ & 1600 & 0.25 & 16.00 & 310.7 & 54.2 & 9.0 & 42.0 & 9.2 \\
\hline $8 / 21 / 92$ & 1615 & 0.25 & 16.25 & 310.6 & 54.0 & 9.0 & 43.2 & 8.1 \\
\hline $8 / 21 / 92$ & 1630 & 0.25 & 16.50 & 310.3 & 53.9 & 9.0 & 42.7 & 9.0 \\
\hline $8 / 21 / 92$ & 1645 & 0.25 & 16.75 & 309.8 & 54.0 & 9.0 & 42.7 & 7.8 \\
\hline $8 / 21 / 92$ & 1700 & 0.25 & 17.00 & 310.3 & 54.0 & 9.0 & 43.3 & 7.8 \\
\hline $8 / 21 / 92$ & 1715 & 0.25 & 17.25 & 310.4 & 54.0 & 9.0 & 42.8 & 7.8 \\
\hline $8 / 21 / 92$ & 1730 & 0.25 & 17.50 & 310.3 & 54.2 & 9.0 & 44.0 & 8.4 \\
\hline $8 / 21 / 92$ & 1745 & 0.25 & 17.75 & 309.5 & 54.2 & 9.0 & 43.3 & 7.6 \\
\hline $8 / 21 / 92$ & 1800 & 0.25 & 18.00 & 309.6 & 54.2 & 9.0 & 43.8 & 8.4 \\
\hline $8 / 21 / 92$ & 1815 & 0.25 & 18.25 & 308.3 & 54.0 & 9.0 & 45.1 & 9.0 \\
\hline $8 / 21 / 92$ & 1830 & 0.25 & 18.50 & 308.1 & 54.0 & 9.0 & 45.7 & 9.0 \\
\hline $8 / 21 / 92$ & 1845 & 0.25 & 18.75 & 307.9 & 54.0 & 9.0 & 44.0 & 8.4 \\
\hline $8 / 21 / 92$ & 1900 & 0.25 & 19.00 & 307.7 & 54.2 & 9.0 & 45.9 & 7.5 \\
\hline $8 / 21 / 92$ & 1915 & 0.25 & 19.25 & 307.2 & 54.3 & 9.0 & 44.7 & 9.1 \\
\hline $8 / 21 / 92$ & 1930 & 0.25 & 19.50 & 30.5 .3 & 54.8 & 9.0 & 46.4 & 7.7 \\
\hline $8 / 21 / 92$ & 1945 & 0.25 & 19.75 & 305.1 & 55.1 & 9.0 & 50.0 & 7.7 \\
\hline $8 / 21 / 92$ & 2000 & 0.25 & 20.00 & 305.8 & 55.1 & 9.0 & 49.6 & 6.9 \\
\hline $8 / 21 / 92$ & 2015 & 0.25 & 20.25 & 306.0 & 55.0 & 9.0 & 47.5 & 8.1 \\
\hline $8 / 21 / 92$ & 2030 & 0.25 & 20.50 & 305.9 & 55.1 & 9.0 & 46.6 & 7.6 \\
\hline $8 / 21 / 92$ & 2045 & 0.25 & 20.75 & 306.2 & 55.4 & 9.0 & 46.5 & 8.8 \\
\hline $8 / 21 / 92$ & 2100 & 0.25 & 21.00 & 306.1 & 55.9 & 9.0 & 48.2 & 9.7 \\
\hline $8 / 21 / 92$ & 2115 & 0.25 & 21.25 & 305.2 & 55.8 & 9.0 & 55.3 & 7.8 \\
\hline $8 / 21 / 92$ & 2130 & 0.25 & 21.50 & 305.3 & 55.5 & 9.0 & 53.4 & 8.6 \\
\hline $8 / 21 / 92$ & 2145 & 0.25 & 21.75 & 304.4 & 55.2 & 9.0 & 51.7 & 7.6 \\
\hline $8 / 21 / 92$ & 2200 & 0.25 & 22.00 & 304.2 & 55.1 & 9.0 & 51.5 & 7.2 \\
\hline $8 / 21 / 92$ & 2215 & 0.25 & 22.25 & 304.4 & 54.9 & 9.0 & 53.1 & 7.8 \\
\hline $8 / 21 / 92$ & 2230 & 0.25 & 22.50 & 304.4 & 55.1 & 9.0 & 50.4 & 7.4 \\
\hline
\end{tabular}




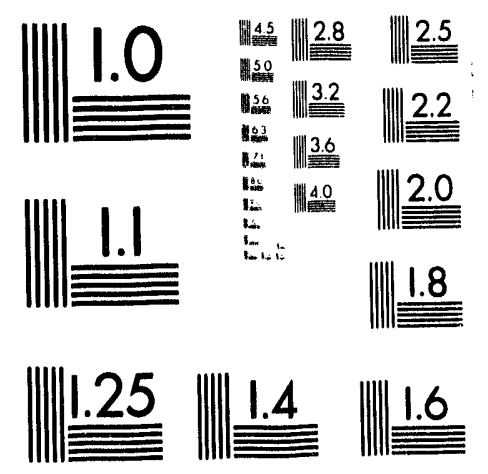



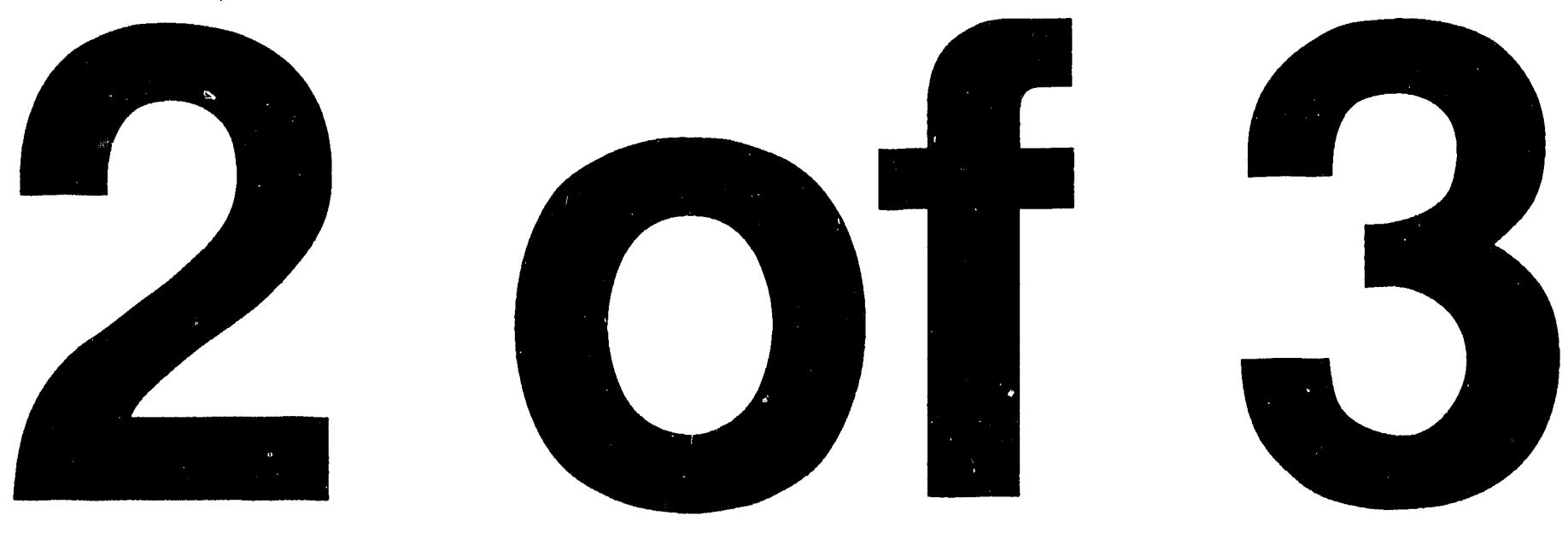
SUMMARY OF LIME SLURRY INJECTION FROM AUGUST 17, 1992 TO SEPTEMBER 16, 1992

\begin{tabular}{|c|c|c|c|c|c|c|c|c|}
\hline Date & Time & Hours of & Cumulative & B Duct Temp & Lime Flow & Slurry Solids & SO2 Removal & Stack Opacity \\
\hline & & Injection & Hours & ${ }^{\circ} \mathrm{F}$ & GPM & Percent & Percent & Percent \\
\hline $8 / 21 / 92$ & 2245 & 0.25 & 22.75 & 304.1 & 55.1 & 9.0 & 50.4 & 8.0 \\
\hline $8 / 21 / 92$ & 2300 & 0.25 & 23.00 & 303.3 & 55.1 & 9.0 & 49.7 & 6.5 \\
\hline $8 / 21 / 92$ & 2315 & 0.25 & 23.25 & 302.6 & 40.6 & 9.0 & 50.5 & 8.0 \\
\hline \multicolumn{2}{|c|}{ Total Hours } & 8.75 & & & & & & \\
\hline & & & & & & & & \\
\hline $8 / 24 / 92$ & 1400 & 0.25 & 23.50 & 308.6 & 39.8 & 12.0 & 24.5 & 15.9 \\
\hline $8 / 24 / 92$ & 1415 & 0.25 & 23.75 & 308.5 & 44.7 & 12.0 & 28.0 & 16.7 \\
\hline $8 / 24 / 92$ & 1430 & 0.25 & 24.00 & 309.1 & 44.9 & 12.0 & 29.4 & 18.0 \\
\hline $8 / 24 / 92$ & 1445 & 0.25 & 24.25 & 308.7 & 44.9 & 12.0 & 30.3 & 19.8 \\
\hline $8 / 24 / 92$ & 1500 & 0.25 & 24.50 & 307.6 & 44.9 & 12.0 & 31.3 & 17.0 \\
\hline $8 / 24 / 92$ & 1515 & 0.25 & 24.75 & 307.6 & 45.0 & 12.0 & 33.5 & 17.6 \\
\hline $8 / 24 / 92$ & 1530 & 0.25 & 25.00 & 307.9 & 45.1 & 12.0 & 34.0 & 18.9 \\
\hline $8 / 24 / 92$ & 1545 & 0.25 & 25.25 & 308.5 & 51.3 & 12.0 & 37.6 & 19.4 \\
\hline $8 / 24 / 92$ & 1600 & 0.25 & 25.50 & 309.9 & 53.9 & 12.0 & 43.4 & 17.1 \\
\hline $8 / 24 / 92$ & 1615 & 0.25 & 25.75 & 309.8 & 55.0 & 12.0 & 42.4 & 18.6 \\
\hline $8 / 24 / 92$ & 1630 & 0.25 & 26.00 & 310.5 & 55.2 & 12.0 & 46.3 & 12.7 \\
\hline $8 / 24 / 92$ & 1645 & 0.25 & 26.25 & 310.9 & 56.5 & 12.0 & 45.8 & 13.4 \\
\hline $8 / 24 / 92$ & 1700 & 0.25 & 26.50 & 311.2 & 56.9 & 12.0 & 47.7 & 13.5 \\
\hline $8 / 24 / 92$ & 1715 & 0.25 & 26.75 & 310.3 & 58.9 & 12.0 & 50.1 & 13.4 \\
\hline $8 / 24 / 92$ & 1730 & 0.25 & 27.00 & 310.8 & 59.0 & 12.0 & 49.4 & 12.3 \\
\hline $8 / 24 / 92$ & 1745 & 0.25 & 27.25 & 310.4 & 59.0 & 12.0 & 48.8 & 11.8 \\
\hline $8 / 24 / 92$ & 1800 & 0.25 & 27.50 & 310.3 & 58.9 & 12.0 & 49.2 & 11.0 \\
\hline $8 / 24 / 92$ & 1815 & 0.25 & 27.75 & 309.8 & 58.9 & 12.0 & 50.0 & 11.5 \\
\hline $8 / 24 / 92$ & 1830 & 0.25 & 28.00 & 310.0 & 59.2 & 12.0 & 49.5 & 11.3 \\
\hline $8 / 24 / 92$ & 1845 & 0.25 & 28.25 & 309.1 & 50.2 & 12.0 & 39.4 & 11.5 \\
\hline $8 / 24 / 92$ & 1900 & 0.25 & 28.50 & 307.4 & 49.9 & 12.0 & 37.8 & 11.8 \\
\hline $8 / 24 / 92$ & 1915 & 0.25 & 28.75 & 306.3 & 52.2 & 12.0 & 39.9 & 13.0 \\
\hline $8 / 24 / 92$ & 1930 & 0.25 & 29.00 & 306.1 & 55.2 & 12.0 & 39.3 & 11.2 \\
\hline $8 / 24 / 92$ & 1945 & 0.25 & 29.25 & 306.3 & 53.5 & 12.0 & 38.6 & 11.0 \\
\hline $8 / 24 / 92$ & 2000 & 0.25 & 29.50 & 306.4 & 54.0 & 12.0 & 39.4 & 11.2 \\
\hline $8 / 24 / 92$ & 2015 & 0.25 & 29.75 & 306.1 & 55.5 & 12.0 & 39.1 & 12.7 \\
\hline $8 / 24 / 92$ & 2030 & 0.25 & 30.00 & 306.3 & 56.0 & 12.0 & 41.3 & 13.0 \\
\hline $8 / 24 / 92$ & 2045 & 0.25 & 30.25 & 304.3 & 55.9 & 12.0 & 44.3 & 12.6 \\
\hline $8 / 24 / 92$ & 2100 & 0.25 & 30.50 & 305.5 & 56.3 & 12.0 & 44.7 & 13.2 \\
\hline $8 / 24 / 92$ & 2115 & 0.25 & 30.75 & 305.4 & 54.1 & 12.0 & 44.9 & 14.8 \\
\hline $8 / 24 / 92$ & 2130 & 0.25 & 31.00 & 306.1 & 54.2 & 12.0 & 43.2 & 12.0 \\
\hline $8 / 24 / 92$ & 2145 & 0.25 & 31.25 & 305.8 & 54.2 & 12.0 & 44.2 & 12.3 \\
\hline $8 / 24 / 92$ & 2200 & 0.25 & 31.50 & 304.3 & 54.0 & 12.0 & 46.0 & 12.4 \\
\hline $8 / 24 / 92$ & 2215 & 0.25 & 31.75 & 302.7 & 54.0 & 12.0 & 46.0 & 12.8 \\
\hline $8 / 24 / 92$ & 2230 & 0.25 & 32.00 & 303.1 & 53.9 & 12.0 & 44.7 & 14.7 \\
\hline $8 / 24 / 92$ & 2245 & 0.25 & 32.25 & 304.8 & 53.8 & 12.0 & 42.5 & 13.8 \\
\hline $8 / 24 / 92$ & 2300 & 0.25 & 32.50 & 303.7 & 53.9 & 12.0 & 42.3 & 14.7 \\
\hline $8 / 24 / 92$ & 2315 & 0.25 & 32.75 & 304.8 & 53.9 & 12.0 & 43.0 & 14.5 \\
\hline $8 / 24 / 92$ & 2330 & 0.25 & 33.00 & 305.1 & 54.0 & 12.0 & 31.3 & 15.6 \\
\hline $8 / 24 / 92$ & 2345 & 0.25 & 33.25 & 304.9 & 54.0 & 12.0 & 30.7 & 13.8 \\
\hline \multicolumn{2}{|c|}{ Total Hours } & 10.00 & & & & & & \\
\hline & & & & & & & & \\
\hline
\end{tabular}


SUMMARY OF LIME SLURRY INJECTION FROM AUGUST 17, 1992 TO SEPTEMBER 16, 1992

\begin{tabular}{|c|c|c|c|c|c|c|c|c|}
\hline Date & Time & Hours of & Cumulative & B Duct Temp & Lime Flow & Slurry Solids & SO2 Removal & Stack Opacity \\
\hline & & Injection & Hours & ${ }^{\circ} \mathrm{F}$ & GPM & Percent & Percent & Percent \\
\hline $8 / 25 / 92$ & 0 & 0.25 & 33.50 & 303.6 & 54.1 & 12.0 & 34.4 & 14.8 \\
\hline $8 / 25 / 92$ & 15 & 0.25 & 33.75 & 303.0 & 54.7 & 12.0 & 38.0 & 14.0 \\
\hline $8 / 25 / 92$ & 30 & 0.25 & 34.00 & 303.4 & 54.8 & 12.0 & 39.3 & 15.3 \\
\hline $8 / 25 / 92$ & 45 & 0.25 & 34.25 & 302.7 & 54.8 & 12.0 & 40.1 & 12.9 \\
\hline $8 / 25 / 92$ & 100 & 0.25 & 34.50 & 301.8 & 55.0 & 12.0 & 41.4 & 11.8 \\
\hline $8 / 25 / 92$ & 115 & 0.25 & 34.75 & 302.6 & 51.5 & 12.0 & 41.0 & 12.7 \\
\hline $8 / 25 / 92$ & 130 & 0.25 & 35.00 & 304.6 & 50.8 & 12.0 & 36.4 & 12.4 \\
\hline $8 / 25 / 92$ & 145 & 0.25 & 35.25 & 302.6 & 51.2 & 12.0 & 36.0 & 16.1 \\
\hline $8 / 25 / 92$ & 200 & 0.25 & 35.50 & 302.2 & 52.8 & 12.0 & 42.0 & 13.4 \\
\hline $8 / 25 / 92$ & 215 & 0.25 & 35.75 & 301.8 & 50.1 & 12.0 & 39.1 & 9.7 \\
\hline $8 / 25 / 92$ & 230 & 0.25 & 36.00 & 301.9 & 48.2 & 12.0 & 38.1 & 10.4 \\
\hline $8 / 25 / 92$ & 245 & 0.25 & 36.25 & 304.1 & 48.3 & 12.0 & 34.9 & 13.7 \\
\hline $8 / 25 / 92$ & 300 & 0.25 & 36.50 & 303.7 & 49.6 & 12.0 & 35.7 & 9.9 \\
\hline $8 / 25 / 92$ & 315 & 0.25 & 36.75 & 303.9 & 50.3 & 12.0 & 37.5 & 11.4 \\
\hline $8 / 25 / 92$ & 330 & 0.25 & 37.00 & 304.1 & 50.4 & 12.0 & 36.7 & 13.1 \\
\hline $8 / 25 / 92$ & 345 & 0.25 & 37.25 & 304.2 & 49.8 & 12.0 & 36.3 & 11.0 \\
\hline $8 / 25 / 92$ & 400 & 0.25 & 37.50 & 304.5 & 50.3 & 12.0 & 37.4 & 14.8 \\
\hline $8 / 25 / 92$ & 415 & 0.25 & 37.75 & 303.4 & 49.8 & 12.0 & 37.4 & 14.0 \\
\hline $8 / 25 / 92$ & 430 & 0.25 & 38.00 & 304.4 & 49.8 & 12.0 & 37.0 & 10.1 \\
\hline $8 / 25 / 92$ & 445 & 0.25 & 38.25 & 304.2 & 52.1 & 12.0 & 35.9 & 10.7 \\
\hline $8 / 25 / 92$ & 500 & 0.25 & 38.50 & 304.5 & 53.7 & 12.0 & 39.3 & 10.6 \\
\hline $8 / 25 / 92$ & 515 & 0.25 & 38.75 & 304.2 & 54.1 & 12.0 & 38.4 & 11.0 \\
\hline $8 / 25 / 92$ & 530 & 0.25 & 39.00 & 304.2 & 53.9 & 12.0 & 42.0 & 10.4 \\
\hline $8 / 25 / 92$ & 545 & 0.25 & 39.25 & 305.0 & 54.0 & 12.0 & 42.7 & 11.4 \\
\hline $8 / 25 / 92$ & 600 & 0.25 & 39.50 & 302.8 & 54.3 & 12.0 & 41.2 & 11.5 \\
\hline $8 / 25 / 92$ & 615 & 0.25 & 39.75 & 303.2 & 53.3 & 12.0 & 44.2 & 15.0 \\
\hline $8 / 25 / 92$ & 630 & 0.25 & 40.00 & 303.9 & 52.3 & 12.0 & 39.6 & 10.1 \\
\hline $8 / 25 / 92$ & 645 & 0.25 & 40.25 & 303.6 & 52.3 & 12.0 & 40.6 & 10.8 \\
\hline $8 / 25 / 92$ & 700 & 0.25 & 40.50 & 304.1 & 51.8 & 12.0 & 42.5 & 9.5 \\
\hline $8 / 25 / 92$ & 715 & 0.25 & 40.75 & 304.1 & 51.9 & 12.0 & 38.5 & 10.4 \\
\hline $8 / 25 / 92$ & 730 & 0.25 & 41.00 & 304.4 & 51.2 & 12.0 & 38.5 & 10.7 \\
\hline $8 / 25 / 92$ & 745 & 0.25 & 41.25 & 304.5 & 50.0 & 12.0 & 37.0 & 10.6 \\
\hline $8 / 25 / 92$ & 800 & 0.25 & 41.50 & 304.9 & 49.7 & 12.0 & 36.9 & 10.9 \\
\hline $8 / 25 / 92$ & 815 & 0.25 & 41.75 & 305.4 & 50.3 & 12.0 & 37.8 & 11.3 \\
\hline $8 / 25 / 92$ & 830 & 0.25 & 42.00 & 304.2 & 50.3 & 12.0 & 37.3 & 11.0 \\
\hline $8 / 25 / 92$ & 845 & 0.25 & 42.25 & 304.4 & 49.8 & 12.0 & 37.8 & 10.4 \\
\hline $8 / 25 / 92$ & 900 & 0.25 & 42.50 & 303.7 & 49.9 & 12.0 & 39.3 & 10.6 \\
\hline $8 / 25 / 92$ & 915 & 0.25 & 42.75 & 304.8 & 50.2 & 12.0 & 37.9 & 10.3 \\
\hline $8 / 25 / 92$ & 930 & 0.25 & 43.00 & 305.6 & 50.0 & 12.0 & 36.8 & 11.0 \\
\hline $8 / 25 / 92$ & 945 & 0.25 & 43.25 & 306.2 & 49.8 & 12.0 & 37.2 & 12.4 \\
\hline $8 / 25 / 92$ & 1000 & 0.25 & 43.50 & 306.4 & 50.2 & 12.0 & 36.4 & 10.5 \\
\hline $8 / 25 / 92$ & 1015 & 0.25 & 43.75 & 307.7 & 50.0 & 12.0 & 38.5 & 12.5 \\
\hline $8 / 25 / 92$ & 1030 & 0.25 & 44.00 & 306.1 & 50.0 & 12.0 & 37.0 & 13.1 \\
\hline $8 / 25 / 92$ & 1045 & 0.25 & 44.25 & 307.9 & 49.9 & 12.0 & 37.8 & 15.2 \\
\hline $8 / 25 / 92$ & 1100 & 0.25 & 44.50 & 309.2 & 50.0 & 12.0 & 37.1 & 11.9 \\
\hline $8 / 25 / 92$ & 1115 & 0.25 & 44.75 & 309.7 & 49.9 & 12.0 & 36.1 & 9.7 \\
\hline $8 / 25 / 92$ & 1130 & 0.25 & 45.00 & 308.8 & 49.9 & 12.0 & 35.7 & 14.6 \\
\hline
\end{tabular}


SUMMARY OF LIME SLURRY INJECTION FROM AUGUST 17, 1992 TO SEPTEMBER 16, 1992

\begin{tabular}{|c|c|c|c|c|c|c|c|c|}
\hline Date & Time & Hours of & Cumulative & B Duct Temp & Lime Flow & Slurry Solids & SO2 Removal & Stack Opacity \\
\hline & & Injection & Hours & ${ }^{\circ} \mathrm{F}$ & GPM & Percent & Percent & Percent \\
\hline $8 / 25 / 92$ & 1145 & 0.25 & 45.25 & 309.8 & 50.0 & 12.0 & 35.4 & 10.7 \\
\hline $8 / 25 / 92$ & 1200 & 0.25 & 45.50 & 309.7 & 50.0 & 12.0 & 37.0 & 9.8 \\
\hline $8 / 25 / 92$ & 1215 & 0.25 & 45.75 & 310.0 & 50.1 & 12.0 & 40.0 & 9.3 \\
\hline $8 / 25 / 92$ & 1230 & 0.25 & 46.00 & 309.5 & 50.1 & 12.0 & 36.6 & 12.2 \\
\hline $8 / 25 / 92$ & 1245 & 0.25 & 46.25 & 309.2 & 51.0 & 12.0 & 37.1 & 11.0 \\
\hline $8 / 25 / 92$ & 1300 & 0.25 & 46.50 & 309.0 & 51.0 & 12.0 & 36.8 & 11.4 \\
\hline $8 / 25 / 92$ & 1315 & 0.25 & 46.75 & 310.2 & 51.1 & 12.0 & 38.0 & 9.8 \\
\hline $8 / 25 / 92$ & 1330 & 0.25 & 47.00 & 310.7 & 51.1 & 12.0 & 38.2 & 14.6 \\
\hline $8 / 25 / 92$ & 1345 & 0.25 & 47.25 & 310.6 & 51.1 & 12.0 & 38.0 & 18.2 \\
\hline $8 / 25 / 92$ & 1400 & 0.25 & 47.50 & 310.2 & 51.7 & 12.0 & 38.7 & 15.3 \\
\hline $8 / 25 / 92$ & 1415 & 0.25 & 47.75 & 309.9 & 51.1 & 12.0 & 39.3 & 21.8 \\
\hline $8 / 25 / 92$ & 1430 & 0.25 & 48.00 & 309.9 & 51.1 & 12.0 & 38.0 & 15.6 \\
\hline $8 / 25 / 92$ & 1445 & 0.25 & 48.25 & 310.6 & 52.2 & 12.0 & 38.3 & 14.5 \\
\hline $8 / 25 / 92$ & 1500 & 0.25 & 48.50 & 310.9 & 51.0 & 12.0 & 38.3 & 13.1 \\
\hline $8 / 25 / 92$ & 1515 & 0.25 & 48.75 & 311.1 & 51.0 & 12.0 & 36.9 & 13.9 \\
\hline $8 / 25 / 92$ & 1530 & 0.25 & 49.00 & 310.5 & 52.1 & 12.0 & 22.5 & 16.0 \\
\hline $8 / 25 / 92$ & 1545 & 0.25 & 49.25 & 311.2 & 53.8 & 12.0 & 24.4 & 15.5 \\
\hline $8 / 25 / 92$ & 1600 & 0.25 & 49.50 & 311.8 & 55.0 & 12.0 & 27.7 & 18.6 \\
\hline $8 / 25 / 92$ & 1615 & 0.25 & 49.75 & 311.2 & 55.1 & 12.0 & 28.1 & 15.1 \\
\hline $8 / 25 / 92$ & 1630 & 0.25 & 50.00 & 311.4 & 55.9 & 12.0 & 28.9 & 16.4 \\
\hline $8 / 25 / 92$ & 1645 & 0.25 & 50.25 & 311.1 & 56.1 & 12.0 & 30.7 & 15.0 \\
\hline $8 / 25 / 92$ & 1700 & 0.25 & 50.50 & 311.3 & 56.1 & 12.0 & 32.9 & 14.9 \\
\hline $8 / 25 / 92$ & 1715 & 0.25 & 50.75 & 311.0 & 56.0 & 12.0 & 33.6 & 14.7 \\
\hline $8 / 25 / 92$ & 1730 & 0.25 & 51.00 & 310.9 & 54.6 & 12.0 & 33.4 & 15.9 \\
\hline $8 / 25 / 92$ & 1745 & 0.25 & 51.25 & 310.0 & 53.1 & 12.0 & 31.0 & 16.6 \\
\hline $8 / 25 / 92$ & 1800 & 0.25 & 51.50 & 309.4 & 50.2 & 12.0 & 30.2 & 14.0 \\
\hline $8 / 25 / 92$ & 1815 & 0.25 & 51.75 & 309.2 & 48.2 & 12.0 & 28.9 & 15.9 \\
\hline $8 / 25 / 92$ & 1830 & 0.25 & 52.00 & 308.0 & 48.1 & 12.0 & 28.6 & 16.7 \\
\hline $8 / 25 / 92$ & 1845 & 0.25 & 52.25 & 307.4 & 48.1 & 12.0 & 29.2 & 15.0 \\
\hline $8 / 25 / 92$ & 1900 & 0.25 & 52.50 & 306.7 & 48.5 & 12.0 & 28.4 & 15.1 \\
\hline $8 / 25 / 92$ & 1915 & 0.25 & 52.75 & 305.5 & 50.0 & 12.0 & 32.0 & 13.0 \\
\hline $8 / 25 / 92$ & 1930 & 0.25 & 53.00 & 305.9 & 50.0 & 12.0 & 31.0 & 13.5 \\
\hline $8 / 25 / 92$ & 1945 & 0.25 & 53.25 & 304.7 & 50.0 & 12.0 & 32.7 & 14.1 \\
\hline \multicolumn{2}{|c|}{ Total Hours } & 20.00 & & & & & & \\
\hline $8 / 26 / 92$ & 245 & 0.25 & 53.50 & 301.3 & 40.1 & 12.0 & 18.5 & 10.7 \\
\hline $8 / 26 / 92$ & 300 & 0.25 & 53.75 & 301.0 & 48.0 & 12.0 & 25.5 & 10.4 \\
\hline $8 / 26 / 92$ & 315 & 0.25 & 54.00 & 306.8 & 53.4 & 12.0 & 31.8 & 8.2 \\
\hline $8 / 26 / 92$ & 330 & 0.25 & 54.25 & 307.0 & 55.6 & 12.0 & 36.6 & 8.1 \\
\hline $8 / 26 / 92$ & 345 & 0.25 & 54.50 & 307.4 & 54.8 & 12.0 & 38.3 & 8.6 \\
\hline $8 / 26 / 92$ & 400 & 0.25 & 54.75 & 306.4 & 55.7 & 12.0 & 40.8 & 12.5 \\
\hline $8 / 26 / 92$ & 415 & 0.25 & 55.00 & 306.6 & 55.9 & 12.0 & 40.9 & 14.8 \\
\hline $8 / 26 / 92$ & 430 & 0.25 & 55.25 & 308.6 & 56.3 & 12.0 & 41.2 & 7.8 \\
\hline $8 / 26 / 92$ & 445 & 0.25 & 55.50 & 309.0 & 55.8 & 12.0 & 40.8 & 13.1 \\
\hline $8 / 26 / 92$ & 500 & 0.25 & 55.75 & 306.7 & 56.3 & 12.0 & 42.1 & 10.2 \\
\hline $8 / 26 / 92$ & 515 & 0.25 & 56.00 & 306.4 & 55.9 & 12.0 & 41.5 & 7.3 \\
\hline $8 / 26 / 92$ & 530 & 0.25 & 56.25 & 306.2 & 55.7 & 12.0 & 43.3 & 8.5 \\
\hline
\end{tabular}


SUMMARY OF LIME SLURRY INJECTION FROM AUGUST 17, 1992 TO SEPTEMBER 16, 1992

\begin{tabular}{|c|c|c|c|c|c|c|c|c|}
\hline Date & Time & Hours of & Cumulative & B Duct Temp & Lime Flow & Slurry Solids & SO2 Removal & Stack Opacity \\
\hline & & Injection & Hours & ${ }^{\circ} \mathrm{F}$ & GPM & Percent & Percent & Percent \\
\hline $8 / 26 / 92$ & 545 & 0.25 & 56.50 & 306.6 & 56.3 & 12.0 & 42.3 & 6.0 \\
\hline $8 / 26 / 92$ & 600 & 0.25 & 56.75 & 306.7 & 55.9 & 12.0 & 43.7 & 8.4 \\
\hline $8 / 26 / 92$ & 615 & 0.25 & 57.00 & 306.5 & 55.9 & 12.0 & 43.3 & 16.8 \\
\hline $8 / 26 / 92$ & 630 & 0.25 & 57.25 & 306.4 & 56.3 & 12.0 & 43.5 & 10.4 \\
\hline $8 / 26 / 92$ & 645 & 0.25 & 57.50 & 306.2 & 55.9 & 12.0 & 42.9 & 11.8 \\
\hline $8 / 26 / 92$ & 700 & 0.25 & 57.75 & 306.0 & 54.8 & 12.0 & 43.8 & 8.8 \\
\hline $8 / 26 / 92$ & 715 & 0.25 & 58.00 & 306.5 & 54.2 & 12.0 & 40.3 & 7.7 \\
\hline $8 / 26 / 92$ & 730 & 0.25 & 58.25 & 306.7 & 54.4 & 12.0 & 40.5 & 8.2 \\
\hline $8 / 26 / 92$ & 745 & 0.25 & 58.50 & 306.9 & 54.2 & 12.0 & 41.3 & 7.3 \\
\hline $8 / 26 / 92$ & 800 & 0.25 & 58.75 & 307.5 & 55.1 & 12.0 & 43.1 & 7.5 \\
\hline $8 / 26 / 92$ & 815 & 0.25 & 59.00 & 307.9 & 56.5 & 12.0 & 42.6 & 7.3 \\
\hline $8 / 26 / 92$ & 830 & 0.25 & 59.25 & 306.6 & 57.4 & 12.0 & 44.8 & 6.9 \\
\hline $8 / 26 / 92$ & 845 & 0.25 & 59.50 & 307.3 & 56.8 & 12.0 & 44.3 & 6.5 \\
\hline $8 / 26 / 92$ & 900 & 0.25 & 59.75 & 306.3 & 58.8 & 11.8 & 48.3 & 6.6 \\
\hline $8 / 26 / 92$ & 915 & 0.25 & 60.00 & 304.9 & 57.1 & 11.9 & 45.0 & 6.3 \\
\hline $8 / 26 / 92$ & 930 & 0.25 & 60.25 & 305.5 & 56.7 & 12.0 & 45.2 & 6.4 \\
\hline $8 / 26 / 92$ & 945 & 025 & 60.50 & 306.2 & 57.1 & 12.0 & 45.8 & 6.5 \\
\hline $8 / 26 / 92$ & 1000 & 0.25 & 60.75 & 306.7 & 57.2 & 12.0 & 47.1 & 6.5 \\
\hline $8 / 26 / 92$ & 1015 & 0.25 & 61.00 & 307.1 & 56.9 & 12.0 & 46.3 & 6.8 \\
\hline $8 / 26 / 92$ & 1030 & 0.25 & 61.25 & 306.4 & 56.9 & 12.0 & 48.1 & 6.5 \\
\hline $8 / 26 / 92$ & 1045 & 0.25 & 61.50 & 306.4 & 57.0 & 12.0 & 47.0 & 7.0 \\
\hline $8 / 26 / 92$ & 1100 & 0.25 & 61.75 & 307.2 & 56.8 & 12.0 & 47.0 & 6.7 \\
\hline $8 / 26 / 92$ & 1115 & 0.25 & 62.00 & 308.3 & 56.0 & 12.0 & 47.2 & 6.1 \\
\hline $8 / 26 / 92$ & 1130 & 0.25 & 62.25 & 309.6 & 55.2 & 12.0 & 51.0 & 6.0 \\
\hline $8 / 26 / 92$ & 1145 & 0.25 & 62.50 & 311.5 & 55.2 & 11.9 & 50.9 & 6.7 \\
\hline $8 / 26 / 92$ & 1200 & 0.25 & 62.75 & 311.2 & 54.7 & 11.9 & 53.5 & 6.0 \\
\hline $8 / 26 / 92$ & 1215 & 0.25 & 63.00 & 311.2 & 54.7 & 11.9 & 53.5 & 6.0 \\
\hline $8 / 26 / 92$ & 1230 & 0.25 & 63.25 & 310.9 & 53.0 & 12.0 & 50.8 & 6.8 \\
\hline $8 / 26 / 92$ & 1245 & 0.25 & 63.50 & 311.9 & 54.2 & 12.0 & 50.9 & 7.0 \\
\hline $8 / 26 / 92$ & 1300 & 0.25 & 63.75 & 312.0 & 53.9 & 12.0 & 51.1 & 7.2 \\
\hline $8 / 26 / 92$ & 1315 & 0.25 & 64.00 & 311.0 & 53.8 & 12.0 & 51.6 & 6.8 \\
\hline $8 / 26 / 92$ & 1330 & 0.25 & 64.25 & 310.7 & 54.0 & 12.0 & 51.0 & 7.0 \\
\hline $8 / 26 / 92$ & 1345 & 0.25 & 64.50 & 310.8 & 54.8 & 11.0 & 52.4 & 6.7 \\
\hline $8 / 26 / 92$ & 1400 & 0.25 & 64.75 & 310.8 & 55.2 & 9.4 & 51.5 & 6.7 \\
\hline $8 / 26 / 92$ & 1415 & 0.25 & 65.00 & 310.5 & 55.2 & 8.4 & 52.9 & 7.5 \\
\hline $8 / 26 / 92$ & 1430 & 0.25 & 65.25 & 309.5 & 52.0 & 7.4 & 51.1 & 6.6 \\
\hline $8 / 26 / 92$ & 1445 & 0.25 & 65.50 & 310.2 & 52.4 & 6.4 & 48.0 & 6.8 \\
\hline $8 / 26 / 92$ & 1500 & 0.25 & 65.75 & 308.7 & 52.1 & 4.3 & 46.5 & 7.8 \\
\hline $8 / 26 / 92$ & 1515 & 0.25 & 66.00 & 309.5 & 52.1 & 3.9 & 46.8 & 7.0 \\
\hline $8 / 26 / 92$ & 1530 & 0.25 & 66.25 & 309.9 & 50.0 & 4.3 & 24.2 & 7.8 \\
\hline $8 / 26 / 92$ & 1545 & 0.25 & 66.50 & 309.9 & 50.3 & 3.5 & 41.8 & 7.3 \\
\hline $8 / 26 / 92$ & 1830 & 0.25 & 66.75 & 306.4 & 50.0 & 11.9 & 38.6 & 9.6 \\
\hline $8 / 26 / 92$ & 1845 & 0.25 & 67.00 & 306.6 & 51.6 & 11.6 & 40.5 & 9.3 \\
\hline $8 / 26 / 92$ & 1900 & 0.25 & 67.25 & 305.6 & 52.0 & 11.7 & 43.6 & 12.2 \\
\hline $8 / 26 / 92$ & 1915 & 0.25 & 67.50 & 305.0 & 52.0 & 11.9 & 43.8 & 9.3 \\
\hline $8 / 26 / 92$ & 1930 & 0.25 & 67.75 & 303.1 & 54.2 & 12.0 & 46.1 & 11.1 \\
\hline $8 / 26 / 92$ & 1945 & 0.25 & 68.00 & 303.1 & 52.0 & 12.0 & 45.7 & 9.9 \\
\hline
\end{tabular}


SUMMARY OF LIME SLURRY INJECTION FROM AUGUST 17, 1992 TO SEPTEMBER 16, 1992

\begin{tabular}{|c|c|c|c|c|c|c|c|c|}
\hline Date & Time & Hours of & Cumulative & B Duct Temp & Lime Flow & Slurry Solids & SO2 Removal & Stack Opacity \\
\hline & & Injection & Hours & ${ }^{\circ} \mathrm{F}$ & GPM & Percent & Percent & Percent \\
\hline $8 / 26 / 92$ & 2000 & 0.25 & 68.25 & 303.0 & 52.2 & 12.0 & 46.7 & 11.0 \\
\hline $8 / 26 / 92$ & 2015 & 0.25 & 68.50 & 302.8 & 53.3 & 12.0 & 46.8 & 9.7 \\
\hline $8 / 26 / 92$ & 2030 & 0.25 & 68.75 & 303.9 & 55.2 & 12.0 & 44.8 & 10.4 \\
\hline $8 / 26 / 92$ & 2045 & 0.25 & 69.00 & 304.4 & 54.8 & 12.0 & 45.1 & 10.1 \\
\hline $8 / 26 / 92$ & 2100 & 0.25 & 69.25 & 304.3 & 54.9 & 12.0 & 45.7 & 11.3 \\
\hline $8 / 26 / 92$ & 2115 & 0.25 & 69.50 & 304.4 & 55.3 & 12.0 & 51.2 & 10.2 \\
\hline $8 / 26 / 92$ & 2130 & 0.25 & 69.75 & 304.1 & 54.9 & 12.0 & 48.6 & 10.5 \\
\hline $8 / 26 / 92$ & 2145 & 0.25 & 70.00 & 304.1 & 54.9 & 12.0 & 48.8 & 10.7 \\
\hline $8 / 26 / 92$ & 2200 & 0.25 & 70.25 & 303.5 & 54.8 & 12.0 & 48.0 & 12.6 \\
\hline $8 / 26 / 92$ & 2215 & 0.25 & 70.50 & 303.8 & 55.2 & 12.0 & 47.7 & 11.0 \\
\hline $8 / 26 / 92$ & 2230 & 0.25 & 70.75 & 302.9 & 52.4 & 12.0 & 48.7 & 11.4 \\
\hline $8 / 26 / 92$ & 2245 & 0.25 & 71.00 & 304.2 & 52.0 & 12.0 & 43.8 & 10.0 \\
\hline $8 / 26 / 92$ & 2300 & 0.25 & 71.25 & 306.1 & 53.2 & 12.0 & 44.1 & 12.7 \\
\hline $8 / 26 / 92$ & 2315 & 0.25 & 71.50 & 304.8 & 53.3 & 12.0 & 44.7 & 11.2 \\
\hline $8 / 26 / 92$ & 2330 & 0.25 & 71.75 & 304.1 & 53.8 & 12.0 & 42.6 & 11.2 \\
\hline $8 / 26 / 92$ & 2345 & 0.25 & 72.00 & 303.6 & 53.6 & 12.0 & 44.6 & 10.9 \\
\hline \multicolumn{2}{|c|}{ Total Hours } & 18.75 & & & & & & \\
\hline $8 / 27 / 92$ & 0 & 0.25 & 72.25 & 304.7 & 54.7 & 11.9 & 44.0 & 11.0 \\
\hline $8 / 27 / 92$ & 15 & 0.25 & 72.50 & 308.4 & 54.8 & 11.9 & 41.8 & 11.0 \\
\hline $8 / 27 / 92$ & 30 & 0.25 & 72.75 & 309.6 & 54.9 & 12.0 & 42.8 & 9.9 \\
\hline $8 / 27 / 92$ & 45 & 0.25 & 73.00 & 309.1 & 55.4 & 11.6 & 42.1 & 14.0 \\
\hline $8 / 27 / 92$ & 100 & 0.25 & 73.25 & 308.7 & 55.7 & 11.9 & 44.3 & 7.7 \\
\hline $8 / 27 / 92$ & 115 & 0.25 & 73.50 & 308.4 & 56.7 & 12.1 & 44.2 & 8.2 \\
\hline $8 / 27 / 92$ & 130 & 0.25 & 73.75 & 307.8 & 56.1 & 11.9 & 43.9 & 11.6 \\
\hline $8 / 27 / 92$ & 145 & 0.25 & 74.00 & 307.7 & 56.6 & 12.2 & 44.1 & 9.3 \\
\hline $8 / 27 / 92$ & 200 & 0.25 & 74.25 & 307.3 & 54.0 & 12.2 & 43.9 & 9.6 \\
\hline $8 / 27 / 92$ & 215 & 0.25 & 74.50 & 305.5 & 53.2 & 12.0 & 48.2 & 9.4 \\
\hline $8 / 27 / 92$ & 230 & 0.25 & 74.75 & 305.4 & 53.1 & 12.2 & 14.0 & 8.9 \\
\hline $8 / 27 / 92$ & 245 & 0.25 & 75.00 & 306.8 & 53.0 & 12.2 & 45.1 & 9.3 \\
\hline $8 / 27 / 92$ & 300 & 0.25 & 75.25 & 309.0 & 54.3 & 12.1 & 43.5 & 8.6 \\
\hline $8 / 27 / 92$ & 315 & 0.25 & 75.50 & 308.2 & 54.5 & 11.9 & 45.3 & 13.4 \\
\hline $8 / 27 / 92$ & 330 & 0.25 & 75.75 & 305.5 & 55.1 & 12.0 & 46.3 & 9.9 \\
\hline $8 / 27 / 92$ & 345 & 0.25 & 76.00 & 306.8 & 55.4 & 12.2 & 46.1 & 10.0 \\
\hline $8 / 27 / 92$ & 400 & 0.25 & 76.25 & 307.4 & 55.4 & 12.0 & 46.4 & 18.2 \\
\hline $8 / 27 / 92$ & 415 & 0.25 & 76.50 & 306.7 & 56.1 & 11.9 & 47.7 & 10.0 \\
\hline $8 / 27 / 92$ & 430 & 0.25 & 76.75 & 305.8 & 56.2 & 12.1 & 47.1 & 7.1 \\
\hline $8 / 27 / 92$ & 445 & 0.25 & 77.00 & 305.6 & 55.9 & 11.8 & 48.8 & 7.8 \\
\hline $8 / 27 / 92$ & 500 & 0.25 & 77.25 & 305.8 & 55.0 & 10.5 & 46.2 & 6.1 \\
\hline $8 / 27 / 92$ & 515 & 0.25 & 77.50 & 306.0 & 55.2 & 8.3 & 46.5 & 5.4 \\
\hline $8 / 27 / 92$ & 530 & 0.25 & 77.75 & 306.0 & 54.1 & 7.2 & 46.1 & 6.0 \\
\hline $8 / 27 / 92$ & 545 & 0.25 & 78.00 & 305.4 & 54.0 & 6.5 & 45.3 & 5.9 \\
\hline $8 / 27 / 92$ & 600 & 0.25 & 78.25 & 305.7 & 54.2 & 8.2 & 44.7 & 5.5 \\
\hline $8 / 27 / 92$ & 615 & 0.25 & 78.50 & 305.9 & 53.0 & 10.8 & 44.5 & 6.0 \\
\hline $8 / 27 / 92$ & 630 & 0.25 & 78.75 & 305.8 & 53.0 & 11.7 & 43.7 & 6.1 \\
\hline $8 / 27 / 92$ & 645 & 0.25 & 79.00 & 305.4 & 52.3 & 11.8 & 42.5 & 5.5 \\
\hline $8 / 27 / 92$ & 700 & 0.25 & 79.25 & 304.4 & 51.0 & 11.8 & 44.5 & 6.0 \\
\hline
\end{tabular}


SUMMARY OF LIME SLURRY INJECTION FROM AUGUST 17, 1992 TO SEPTEMBER 16, 1992

\begin{tabular}{|c|c|c|c|c|c|c|c|c|}
\hline Date & Time & Hours of & Cumulative & B Duct Temp & Lime Flow & Slurry Solids & SO2 Removal & Stack Opacity \\
\hline & & Injection & Hours & ${ }^{\circ} \mathrm{F}$ & GPM & Percent & Percent & Percent \\
\hline $8 / 27 / 92$ & 715 & 0.25 & 79.50 & 304.4 & 49.0 & 12.2 & 42.1 & 6.0 \\
\hline $8 / 27 / 92$ & 730 & 0.25 & 79.75 & 305.3 & 49.4 & 12.1 & 40.8 & 6.8 \\
\hline $8 / 27 / 92$ & 745 & 0.25 & 80.00 & 305.7 & 52.1 & 11.2 & 43.0 & 6.3 \\
\hline $8 / 27 / 92$ & 800 & 0.25 & 80.25 & 306.1 & 52.8 & 8.9 & 43.8 & 6.0 \\
\hline $8 / 27 / 92$ & 830 & 0.25 & 80.50 & 305.5 & 47.7 & 6.3 & 42.3 & 5.5 \\
\hline $8 / 27 / 92$ & 1430 & 0.25 & 80.75 & 308.4 & 44.5 & 12.8 & 25.1 & 10.5 \\
\hline $8 / 27 / 92$ & 1445 & 0.25 & 81.00 & 307.7 & 52.4 & 13.2 & 31.0 & 9.6 \\
\hline $8 / 27 / 92$ & 1500 & 0.25 & 81.25 & 309.6 & 53.0 & 11.8 & 35.2 & 10.8 \\
\hline $8 / 27 / 92$ & 1515 & 0.25 & 81.50 & 309.9 & 54.2 & 12.3 & 38.6 & 9.9 \\
\hline $8 / 27 / 92$ & 1530 & 0.25 & 81.75 & 310.6 & 58.3 & 12.4 & 37.2 & 9.5 \\
\hline $8 / 27 / 92$ & 1545 & 0.25 & 82.00 & 310.9 & 53.9 & 12.2 & 38.8 & 10.3 \\
\hline $8 / 27 / 92$ & 1600 & 0.25 & 82.25 & 311.0 & 56.7 & 12.0 & 39.0 & 13.5 \\
\hline $8 / 27 / 92$ & 1615 & 0.25 & 82.50 & 310.9 & 57.7 & 11.8 & 40.4 & 9.7 \\
\hline $8 / 27 / 92$ & 1630 & 0.25 & 82.75 & 311.6 & 58.0 & 11.8 & 39.2 & 8.6 \\
\hline $8 / 27 / 92$ & 1645 & 0.25 & 83.00 & 312.3 & 56.1 & 12.0 & 39.5 & 7.8 \\
\hline $8 / 27 / 92$ & 1700 & 0.25 & 83.25 & 312.8 & 56.9 & 12.0 & 42.0 & 8.2 \\
\hline $8 / 27 / 92$ & 1715 & 0.25 & 83.50 & 313.3 & 57.0 & 12.0 & 41.5 & 8.7 \\
\hline $8 / 27 / 92$ & 1730 & 0.25 & 83.75 & 312.3 & 57.3 & 12.0 & 42.1 & 9.1 \\
\hline $8 / 27 / 92$ & 1745 & 0.25 & 84.00 & 312.3 & 57.3 & 12.0 & 41.9 & 8.6 \\
\hline $8 / 27 / 92$ & 1800 & 0.25 & 84.25 & 311.2 & 58.7 & 12.0 & 44.2 & 8.0 \\
\hline $8 / 27 / 92$ & 1815 & 0.25 & 84.50 & 309.6 & 59.3 & 12.0 & 45.5 & 9.0 \\
\hline $8 / 27 / 92$ & 1830 & 0.25 & 84.75 & 308.5 & 59.4 & 12.0 & 48.4 & 10.3 \\
\hline $8 / 27 / 92$ & 1845 & 0.25 & 85.00 & 307.7 & 58.8 & 12.0 & 47.6 & 8.7 \\
\hline $8 / 27 / 92$ & 1900 & 0.25 & 85.25 & 308.0 & 57.4 & 12.0 & 45.0 & 9.7 \\
\hline $8 / 27 / 92$ & 1915 & 0.25 & 85.50 & 307.3 & 55.4 & 12.0 & 46.3 & 10.4 \\
\hline $8 / 27 / 92$ & 1930 & 0.25 & 85.75 & 306.6 & 56.0 & 12.0 & 43.8 & 9.7 \\
\hline $8 / 27 / 92$ & 1945 & 0.25 & 86.00 & 305.9 & 57.1 & 12.0 & 45.6 & 9.9 \\
\hline $8 / 27 / 92$ & 2000 & 0.25 & 86.25 & 305.7 & 58.1 & 12.0 & 46.9 & 9.6 \\
\hline $8 / 27 / 92$ & 2015 & 0.25 & 86.50 & 306.2 & 55.6 & 12.0 & 48.1 & 9.2 \\
\hline $8 / 27 / 92$ & 2030 & 0.25 & 86.75 & 305.8 & 54.0 & 12.0 & 42.1 & 10.8 \\
\hline $8 / 27 / 92$ & 2045 & 0.25 & 87.00 & 305.4 & 55.0 & 12.0 & 42.3 & 12.5 \\
\hline $8 / 27 / 92$ & 2100 & 0.25 & 87.25 & 306.0 & 55.1 & 12.0 & 43.2 & 14.0 \\
\hline $8 / 27 / 92$ & 2115 & 0.25 & 87.50 & 306.1 & 55.0 & 12.0 & 47.6 & 12.5 \\
\hline $8 / 27 / 92$ & 2130 & 0.25 & 87.75 & 306.3 & 55.2 & 12.0 & 44.8 & 12.1 \\
\hline $8 / 27 / 92$ & 2145 & 0.25 & 88.00 & 307.6 & 54.8 & 12.0 & 43.5 & 12.4 \\
\hline $8 / 27 / 92$ & 2200 & 0.25 & 88.25 & 308.3 & 55.7 & 12.0 & 45.9 & 12.0 \\
\hline $8 / 27 / 92$ & 2215 & 0.25 & 88.50 & 307.6 & 55.9 & 12.0 & 48.5 & 11.5 \\
\hline $8 / 27 / 92$ & 2230 & 0.25 & 88.75 & 307.8 & 53.0 & 12.0 & 42.7 & 12.4 \\
\hline $8 / 27 / 92$ & 2245 & 0.25 & 89.00 & 306.9 & 54.8 & 12.0 & 44.2 & 12.5 \\
\hline $8 / 27 / 92$ & 2300 & 0.25 & 89.25 & 306.7 & 54.8 & 12.0 & 44.4 & 12.0 \\
\hline $8 / 27 / 92$ & 2315 & 0.25 & 89.50 & 307.8 & 53.2 & 12.0 & 44.5 & 13.6 \\
\hline $8 / 27 / 92$ & 2330 & 0.25 & 89.75 & 308.9 & 53.2 & 12.0 & 41.8 & 11.4 \\
\hline $8 / 27 / 92$ & 2345 & 0.25 & 90.00 & 307.2 & 53.9 & 12.0 & 38.9 & 10.9 \\
\hline \multicolumn{2}{|c|}{ Total Hours } & 18.00 & & & & & & \\
\hline & & & & & & & & \\
\hline$\frac{8 / 28 / 92}{8 / 28 / 92}$ & $\begin{array}{r}0 \\
15\end{array}$ & 0.25 & 90.25 & 307.6 & 54.1 & 12.0 & $\frac{39.0}{40 ?}$ & $\frac{10.1}{70}$ \\
\hline
\end{tabular}


SUMMARY OF LIME SLURRY INJECTION FROM AUGUST 17, 1992 TO SEPTEMBER 16, 1992

\begin{tabular}{|c|c|c|c|c|c|c|c|c|}
\hline Date & Time & Hours of & Cumulative & B Duct Temp & Lime Flow & Slurry Solids & SO2 Removal & Stack Opacity \\
\hline & & Injection & Hours & ${ }^{\circ} \mathrm{F}$ & GPM & Percent & Percent & Percent \\
\hline $8 / 28 / 92$ & 30 & 0.25 & 90.75 & 307.6 & 55.5 & 12.0 & 41.2 & 7.6 \\
\hline $8 / 28 / 92$ & 45 & 0.25 & 91.00 & 306.9 & 55.3 & 12.0 & 40.3 & 6.9 \\
\hline $8 / 28 / 92$ & 100 & 0.25 & 91.25 & 307.8 & 55.8 & 12.0 & 40.8 & 7.8 \\
\hline $8 / 28 / 92$ & 115 & 0.25 & 91.50 & 308.0 & 55.7 & 12.0 & 42.5 & 6.9 \\
\hline $8 / 28 / 92$ & 130 & 0.25 & 91.75 & 308.0 & 55.7 & 12.0 & 44.2 & 10.2 \\
\hline $8 / 28 / 92$ & 145 & 0.25 & 92.00 & 308.0 & 55.4 & 12.0 & 41.2 & 8.5 \\
\hline $8 / 28 / 92$ & 200 & 0.25 & 92.25 & 310.0 & 55.4 & 12.0 & 41.1 & 7.5 \\
\hline $8 / 28 / 92$ & 215 & 0.25 & 92.50 & 309.1 & 55.8 & 12.0 & 40.9 & 14.1 \\
\hline $8 / 28 / 92$ & 230 & 0.25 & 92.75 & 308.7 & 55.5 & 12.0 & 44.0 & 8.9 \\
\hline $8 / 28 / 92$ & 245 & 0.25 & 93.00 & 308.4 & 55.3 & 12.0 & 40.4 & 7.3 \\
\hline $8 / 28 / 92$ & 300 & 0.25 & 93.25 & 307.0 & 52.5 & 12.0 & 45.5 & 6.7 \\
\hline $8 / 28 / 92$ & 315 & 0.25 & 93.50 & 306.5 & 52.6 & 12.0 & 39.9 & 10.1 \\
\hline $8 / 28 / 92$ & 330 & 0.25 & 93.75 & 305.7 & 51.6 & 12.0 & 46.8 & 10.2 \\
\hline $8 / 28 / 92$ & 345 & 0.25 & 94.00 & 307.8 & 50.9 & 12.0 & 39.0 & 16.4 \\
\hline $8 / 28 / 92$ & 400 & 0.25 & 94.25 & 306.7 & 51.3 & 12.0 & 38.3 & 11.9 \\
\hline $8 / 28 / 92$ & 415 & 0.25 & 94.50 & 304.7 & 50.8 & 12.0 & 40.0 & 13.7 \\
\hline $8 / 28 / 92$ & 430 & 0.25 & 94.75 & 304.0 & 51.0 & 12.0 & 40.7 & 11.2 \\
\hline $8 / 28 / 92$ & 445 & 0.25 & 95.00 & 304.7 & 51.4 & 12.0 & 38.8 & 10.1 \\
\hline $8 / 28 / 92$ & 500 & 0.25 & 95.25 & 303.8 & 47.9 & 12.0 & 38.6 & 10.8 \\
\hline $8 / 28 / 92$ & 515 & 0.25 & 95.50 & 304.0 & 46.2 & 12.0 & 35.5 & 11.8 \\
\hline $8 / 28 / 92$ & 530 & 0.25 & 95.75 & 303.4 & 46.1 & 12.0 & 35.6 & 12.2 \\
\hline $8 / 28 / 92$ & 545 & 0.25 & 96.00 & 305.1 & 46.2 & 12.0 & 33.5 & 14.2 \\
\hline $8 / 28 / 92$ & 600 & 0.25 & 96.25 & 306.6 & 46.0 & 12.0 & 32.3 & 14.5 \\
\hline $8 / 28 / 92$ & 615 & 0.25 & 96.50 & 307.3 & 50.9 & 12.0 & 34.2 & 13.6 \\
\hline $8 / 28 / 92$ & 630 & 0.25 & 96.75 & 307.9 & 52.3 & 12.0 & 37.2 & 11.3 \\
\hline $8 / 28 / 92$ & 645 & 0.25 & 97.00 & 307.9 & 53.5 & 12.0 & 39.2 & 11.4 \\
\hline $8 / 28 / 92$ & 700 & 0.25 & 97.25 & 308.9 & 55.0 & 12.0 & 40.1 & 11.2 \\
\hline $8 / 28 / 92$ & 715 & 0.25 & 97.50 & 307.8 & 54.1 & 12.0 & 41.5 & 10.6 \\
\hline $8 / 28 / 92$ & 730 & 0.25 & 97.75 & 308.0 & 54.1 & 12.0 & 38.6 & 11.2 \\
\hline $8 / 28 / 92$ & 745 & 0.25 & 98.00 & 307.5 & 52.2 & 12.0 & 39.1 & 11.8 \\
\hline $8 / 28 / 92$ & 800 & 0.25 & 98.25 & 307.1 & 48.2 & 12.0 & 41.6 & 13.3 \\
\hline $8 / 28 / 92$ & 815 & 0.25 & 98.50 & 308.2 & 49.1 & 12.1 & 35.3 & 14.1 \\
\hline $8 / 28 / 92$ & 830 & 0.25 & 98.75 & 308.7 & 48.3 & 11.8 & 35.0 & 13.8 \\
\hline $8 / 28 / 92$ & 845 & 0.25 & 99.00 & 307.9 & 51.7 & 12.1 & 34.6 & 14.0 \\
\hline $8 / 28 / 92$ & 900 & 0.25 & 99.25 & 308.0 & 52.0 & 12.0 & 39.5 & 13.2 \\
\hline $8 / 28 / 92$ & 915 & 0.25 & 99.50 & 309.4 & 53.2 & 12.0 & 38.7 & 11.6 \\
\hline $8 / 28 / 92$ & 930 & 0.25 & 99.75 & 309.1 & 53.7 & 12.0 & 38.9 & 11.8 \\
\hline $8 / 28 / 92$ & 945 & 0.25 & 100.00 & 309.4 & 50.1 & 11.9 & 41.4 & 13.6 \\
\hline $8 / 28 / 92$ & 1000 & 0.25 & 100.25 & 310.4 & 47.2 & 11.9 & 34.1 & 18.0 \\
\hline $8 / 28 / 92$ & 1015 & 0.25 & 100.50 & 310.4 & 46.8 & 11.8 & 34.2 & 20.8 \\
\hline $8 / 28 / 92$ & 1615 & 0.25 & 100.75 & 301.6 & 40.4 & 11.1 & 24.3 & 14.8 \\
\hline $8 / 28 / 92$ & 1630 & 0.25 & 101.00 & 301.4 & 44.5 & 12.0 & 24.6 & 13.3 \\
\hline $8 / 28 / 92$ & 1645 & 0.25 & 101.25 & 302.2 & 49.9 & 12.0 & 31.3 & 11.5 \\
\hline $8 / 28 / 92$ & 1700 & 0.25 & 101.50 & 303.1 & 49.9 & 12.0 & 33.3 & 12.8 \\
\hline $8 / 28 / 92$ & 1715 & 0.25 & 101.75 & 304.0 & 52.1 & 12.0 & 34.0 & 16.4 \\
\hline $8 / 28 / 92$ & 1730 & 0.25 & 102.00 & 304.7 & 50.1 & 12.0 & 33.8 & 13.8 \\
\hline $8 / 28 / 92$ & 1745 & 0.25 & 102.25 & 304.9 & 49.9 & 12.0 & 33.2 & 13.7 \\
\hline
\end{tabular}


SUMMARY OF LIME SLURRY INJECTION FROM AUGUST 17, 1992 TO SEPTEMBER 16, 1992

\begin{tabular}{|c|c|c|c|c|c|c|c|c|}
\hline Date & Time & Hours of & Cumulative & B Duct Temp & Lime Flow & Slurry Solids & SO2 Removal & Stack Opacity \\
\hline & & Injection & Hours & ${ }^{\circ} \mathrm{F}$ & GPM & Percent & Percent & Percent \\
\hline $8 ! 28 / 92$ & 1800 & 0.25 & 102.50 & 304.5 & 50.1 & 12.0 & 33.0 & 15.4 \\
\hline $8 / 28 / 92$ & 1815 & 0.25 & 102.75 & 303.9 & 52.1 & 12.0 & 37.5 & 15.6 \\
\hline $8 / 28 / 92$ & 1830 & 0.25 & 103.00 & 304.6 & 52.1 & 12.0 & 36.8 & 13.7 \\
\hline $8 / 28 / 92$ & 1845 & 0.25 & 103.25 & 304.9 & 52.2 & 12.0 & 37.3 & 13.9 \\
\hline $8 / 28 / 92$ & 1900 & 0.25 & 103.50 & 304.8 & 49.9 & 12.0 & 37.1 & 15.4 \\
\hline $8 / 28 / 92$ & 1915 & 0.25 & 103.75 & 303.9 & 47.3 & 12.0 & 39.2 & 15.2 \\
\hline $8 / 28 / 92$ & 1930 & 0.25 & 104.00 & 304.7 & 46.3 & 12.0 & 32.5 & 18.8 \\
\hline $8 / 28 / 92$ & 1945 & 0.25 & 104.25 & 304.5 & 47.1 & 12.0 & 31.8 & 15.8 \\
\hline $8 / 28 / 92$ & 2000 & 0.25 & 104.50 & 304.3 & 47.1 & 12.0 & 32.4 & 16.4 \\
\hline $8 / 28 / 92$ & 2015 & 0.25 & 104.75 & 304.0 & 48.1 & 12.0 & 33.1 & 14.9 \\
\hline $8 / 28 / 92$ & 2030 & 0.25 & 105.00 & 303.8 & 49.1 & 12.0 & 34.2 & 14.9 \\
\hline $8 / 28 / 92$ & 2045 & 0.25 & 105.25 & 303.3 & 48.5 & 12.0 & 35.5 & 14.5 \\
\hline $8 / 28 / 92$ & 2100 & 0.25 & 105.50 & 302.6 & 46.8 & 12.0 & 37.5 & 21.1 \\
\hline $8 / 28 / 92$ & 2115 & 0.25 & 105.75 & 302.8 & 47.0 & 12.0 & 36.4 & 13.2 \\
\hline $8 / 28 / 92$ & 2130 & 0.25 & 106.00 & 303.6 & 48.8 & 12.0 & 36.7 & 16.7 \\
\hline $8 / 28 / 92$ & 2145 & 0.25 & 106.25 & 304.2 & 50.9 & 12.0 & 39.4 & 11.0 \\
\hline $8 / 28 / 92$ & 2200 & 0.25 & 106.50 & 303.3 & 51.0 & 12.0 & 41.2 & 11.2 \\
\hline $8 / 28 / 92$ & 2215 & 0.25 & 106.75 & 302.3 & 48.1 & 12.0 & 36.5 & 12.4 \\
\hline $8 / 28 / 92$ & 2230 & 0.25 & 107.00 & 301.4 & 48.1 & 12.0 & 35.5 & 12.0 \\
\hline \multicolumn{2}{|c|}{ Total Hours } & 17.00 & & & & & & \\
\hline & & & & & & & & \\
\hline $9 / 2 / 92$ & 1530 & 0.25 & 107.25 & 299.2 & 35.8 & 12.0 & 14.1 & 8.5 \\
\hline $9 / 2 / 92$ & 1545 & 0.25 & 107.50 & 299.2 & 38.0 & 12.0 & 13.6 & 8.6 \\
\hline $9 / 2 / 92$ & 1600 & 0.25 & 107.75 & 299.9 & 38.4 & 12.0 & 12.5 & 10.4 \\
\hline $9 / 2 / 92$ & 1615 & 0.25 & 108.00 & 299.5 & 37.8 & 12.0 & 12.7 & 12.7 \\
\hline $9 / 2 / 92$ & 1630 & 0.25 & 108.25 & 299.5 & 37.8 & 12.0 & 12.7 & 12.7 \\
\hline $9 / 2 / 92$ & 1715 & 0.25 & 108.50 & 298.7 & 37.0 & 12.0 & 11.0 & 10.0 \\
\hline $9 / 2 / 92$ & 1730 & 0.25 & 108.75 & 299.5 & 37.3 & 12.0 & 14.2 & 10.0 \\
\hline $9 / 2 / 92$ & 1745 & 0.25 & 109.00 & 299.3 & 36.8 & 12.0 & 13.3 & 9.4 \\
\hline $9 / 2 / 92$ & 1800 & 0.25 & 109.25 & 298.9 & 37.1 & 12.0 & 13.1 & 15.0 \\
\hline $9 / 2 / 92$ & 1815 & 0.25 & 109.50 & 298.5 & 36.8 & 12.0 & 14.9 & 12.8 \\
\hline $9 / 2 / 92$ & 1830 & 0.25 & 109.75 & 298.3 & 36.6 & 12.0 & 16.3 & 11.2 \\
\hline $9 / 2 / 92$ & 1845 & 0.25 & 110.00 & 298.5 & 36.2 & 12.0 & 14.0 & 11.9 \\
\hline $9 / 2 / 92$ & 1900 & 0.25 & 110.25 & 298.4 & 35.9 & 12.0 & 14.6 & 14.2 \\
\hline $9 / 2 / 92$ & 1915 & 0.25 & 110.50 & 298.2 & 36.4 & 12.0 & 15.9 & 13.8 \\
\hline $9 / 2 / 92$ & 1930 & 0.25 & 110.75 & 298.2 & 39.6 & 12.0 & 15.9 & 12.3 \\
\hline $9 / 2 / 92$ & 1945 & 0.25 & 111.00 & 298.3 & 36.6 & 12.0 & 19.4 & 13.2 \\
\hline $9 / 2 / 92$ & 2000 & 0.25 & 111.25 & 297.3 & 36.0 & 12.0 & 18.2 & 14.2 \\
\hline $9 / 2 / 92$ & 2015 & 0.25 & 111.50 & 297.3 & 36.2 & 12.0 & 20.4 & 15.8 \\
\hline $9 / 2 / 92$ & 2030 & 0.25 & 111.75 & 298.9 & 35.8 & 12.0 & 17.9 & 17.2 \\
\hline $9 / 2 / 92$ & 2045 & 0.25 & 112.00 & 297.6 & 35.6 & 12.0 & 17.8 & 18.7 \\
\hline $9 / 2 / 92$ & 2100 & 0.25 & 112.25 & 297.1 & 35.4 & 12.0 & 19.3 & 18.0 \\
\hline $9 / 2 / 92$ & 2115 & 0.25 & 112.50 & 298.8 & 35.5 & 12.0 & 25.4 & 17.5 \\
\hline $9 / 2 / 92$ & 2130 & 0.25 & 112.75 & 296.4 & 33.9 & 12.0 & 22.4 & 14.2 \\
\hline $9 / 2 / 92$ & 2145 & 0.25 & 113.00 & 292.7 & 33.8 & 12.0 & 23.8 & 17.5 \\
\hline \multicolumn{2}{|c|}{ Total Hours } & 6.00 & & & & & & \\
\hline
\end{tabular}


SUMMARY OF LIME SLURRY INJECTION FROM AUGUST 17, 1992 TO SEPTEMBER 16, 1992

\begin{tabular}{|c|c|c|c|c|c|c|c|c|}
\hline Date & Time & Hours of & Cumulative & B Duct Temp & Lime Flow & Slurry Solids & SO2 Removal & Stack Opacity \\
\hline & & Injection & Hours & of & GPM & Percent & Percent & Percent \\
\hline $9 / 3 / 92$ & 915 & 0.25 & 113.25 & 294.5 & 35.6 & 12.0 & 14.6 & 14.7 \\
\hline $9 / 3 / 92$ & 930 & 0.25 & 113.50 & 294.5 & 35.9 & 12.0 & 14.6 & 16.4 \\
\hline $9 / 3 / 92$ & 945 & 0.25 & 113.75 & 294.9 & 36.3 & 12.0 & 18.7 & 15.0 \\
\hline $9 / 3 / 92$ & 1000 & 0.25 & 114.00 & 294.9 & 35.2 & 12.0 & 20.0 & 18.1 \\
\hline $9 / 3 / 92$ & 1015 & 0.25 & 114.25 & 295.4 & 36.9 & 12.0 & 22.1 & 16.1 \\
\hline $9 / 3 / 92$ & 1030 & 0.25 & 114.50 & 295.1 & 31.3 & 12.0 & 20.1 & 13.8 \\
\hline Total Hours & 1.50 & & & & & & \\
\hline
\end{tabular}




\title{
Appendix D
}

\author{
Tables With Summary of \\ Lime Slurry Injection Data \\ September 17 to October 16, 1992
}


SUMMARY OF LIME SLURRY INJECTION FROM SEPTEMBER 17, 1992 TO OCTOBER 16, 1992

\begin{tabular}{|c|c|c|c|c|c|c|c|c|}
\hline Date & Time & Hours of & Cumulative & B Duct Temp & Lime Flow & Slurry Solids & SO2 Removal & Stack Opacity \\
\hline & & Injection & Hours & ${ }^{\circ} \mathrm{F}$ & GPM & Percent & Percent & Percent \\
\hline $9 / 29 / 92$ & 1015 & 0.25 & 114.75 & 281.1 & 20.8 & 6.8 & 11.3 & 7.4 \\
\hline $9 / 29 / 92$ & 1030 & 0.25 & 115.00 & 281.5 & 28.4 & 7.5 & 10.7 & 7.9 \\
\hline $9 / 29 / 92$ & 1045 & 0.25 & 115.25 & 282.1 & 30.1 & 7.5 & 18.0 & 6.8 \\
\hline $9 / 29 / 92$ & 1100 & 0.25 & 115.50 & 282.8 & 29.9 & 7.9 & 19.4 & 6.5 \\
\hline $9 / 29 / 92$ & 1115 & 0.25 & 115.75 & 282.4 & 30.0 & 7.9 & 20.8 & 6.6 \\
\hline $9 / 29 / 92$ & 1130 & 0.25 & 116.00 & 282.6 & 30.1 & 7.8 & 20.8 & 7.6 \\
\hline \multicolumn{2}{|l|}{ Total Hours } & 1.50 & & & & & & \\
\hline $9 / 30 / 92$ & 1700 & $00=$ & 11005 & 0040 & 111 & 80 & 47 & 101 \\
\hline $9 / 30 / 92$ & $\mid 1715$ & 0.25 & $\frac{110.20}{116.50}$ & $\frac{281.3}{2019}$ & $\frac{11.1}{11.2}$ & 0.0 & $\frac{1.8}{17}$ & $\frac{12.1}{120}$ \\
\hline $9 / 30 / 92$ & 1730 & 0.25 & 116.75 & $\frac{281.2}{282.7}$ & $\frac{11.2}{109}$ & $\frac{8.0}{80}$ & $\frac{1.8}{17}$ & $\frac{73.2}{12.6}$ \\
\hline $9 / 30 / 92$ & 1745 & 0.25 & 117.00 & $\frac{202.7}{282.9}$ & $\frac{10.0}{11.1}$ & $\frac{0.0}{8.0}$ & 1.0 & 12.9 \\
\hline $9 / 30 / 92$ & 1800 & 0.25 & 117.25 & 283.5 & 11.1 & 8.0 & 3.1 & 13.7 \\
\hline \multicolumn{2}{|l|}{ Total Hours } & 1.25 & & & & & & \\
\hline & & & & & & & & \\
\hline $10 / 1 / 92$ & 1115 & 0.25 & 117.50 & 281.0 & 14.8 & 8.0 & 0.4 & 8.4 \\
\hline \multicolumn{2}{|l|}{ Total Hours } & 0.25 & & & & & & \\
\hline & & & & & & & & \\
\hline $10 / 9 / 92$ & 1515 & 0.25 & 117.75 & 299.1 & 38.2 & 8.6 & 19.0 & 8.7 \\
\hline $10 / 9 / 92$ & 1530 & 0.25 & 118.00 & 298.6 & 35.7 & 8.6 & 18.3 & 10.4 \\
\hline $10 / 9 / 92$ & 1545 & 0.25 & 118.25 & 300.2 & 35.2 & 8.4 & 18.7 & 11.7 \\
\hline $10 / 9 / 92$ & 1600 & 0.25 & 118.50 & 299.1 & 32.0 & 8.8 & 17.5 & 16.7 \\
\hline $10 / 9 / 92$ & 1615 & 0.25 & 118.75 & 298.0 & 34.4 & 8.5 & 17.5 & 14.2 \\
\hline \multicolumn{2}{|c|}{ Total Hours } & 1.25 & & & & & & \\
\hline $10 / 12 / 92$ & 1500 & 0.25 & 119.00 & 283.7 & 8.9 & 7.8 & 4.4 & 17.2 \\
\hline $10 / 12 / 92$ & 1515 & 0.25 & 119.25 & 282.9 & 12.6 & 8.4 & 2.7 & 18.1 \\
\hline $10 / 12 / 92$ & 1530 & 0.25 & 119.50 & 283.6 & 14.8 & 8.5 & 3.8 & 18.7 \\
\hline \multicolumn{2}{|l|}{ Total Hours } & 0.75 & & & & & & \\
\hline & & & & & & & & \\
\hline $10 / 14 / 92$ & 845 & 0.25 & 119.75 & 283.0 & 24.9 & 9.1 & 8.6 & 12.9 \\
\hline $10 / 14 / 92$ & 900 & 0.25 & 120.00 & 283.0 & 25.0 & 9.1 & 7.3 & 12.8 \\
\hline $10 / 14 / 92$ & 915 & 0.25 & 120.25 & 284.5 & 25.0 & 9.1 & 9.2 & 12.0 \\
\hline $10 / 14 / 92$ & 930 & 0.25 & 120.50 & 284.4 & 25.3 & 9.1 & 8.3 & 14.9 \\
\hline $10 / 14 / 92$ & 945 & 0.25 & 120.75 & 285.0 & 25.4 & 9.1 & 8.3 & 14.7 \\
\hline $10 / 14 / 92$ & 1000 & 0.25 & 121.00 & 286.9 & 25.3 & 9.1 & 9.4 & 15.8 \\
\hline $10 / 14 / 92$ & 1015 & 0.25 & 121.25 & 286.7 & 25.4 & 9.1 & 7.7 & 17.1 \\
\hline $10 / 14 / 92$ & 1030 & 0.25 & 121.50 & 286.8 & 28.9 & 9.1 & 8.1 & 15.9 \\
\hline $10 / 14 / 92$ & 1045 & 0.25 & 121.75 & 287.7 & 34.6 & 9.1 & 13.9 & 12.2 \\
\hline $10 / 14 / 92$ & 1100 & 0.25 & 122.00 & 287.7 & 33.0 & 9.1 & 14.9 & 9.4 \\
\hline $10 / 14 / 92$ & 1115 & 0.25 & 122.25 & 287.4 & 32.0 & 9.1 & 12.8 & 10.1 \\
\hline $10 / 14 / 92$ & 1130 & 0.25 & 122.50 & 288.1 & 32.0 & 9.1 & 12.0 & 12.8 \\
\hline $10 / 14 / 92$ & 1145 & 0.25 & 122.75 & 290.0 & 31.8 & 8.5 & 11.9 & 12.9 \\
\hline $10 / 14 / 92$ & 1200 & 0.25 & 123.00 & 291.6 & 32.4 & 8.2 & 15.5 & 14.2 \\
\hline $10 / 14 / 92$ & 1215 & 0.25 & 123.25 & 292.4 & 32.3 & 5.8 & 18.0 & 15.0 \\
\hline $10 / 14 / 92$ & 1230 & 0.25 & 123.50 & 293.3 & 32.7 & 4.3 & 14.7 & 14.9 \\
\hline $10 / 14 / 92$ & 1245 & 0.25 & 123.75 & 294.0 & 32.8 & 4.4 & 16.9 & 13.4 \\
\hline
\end{tabular}


SUMMARY OF LIME SLURRY INJECTION FROM SEPTEMBER 17, 1992 TO OCTOBER 16, 1992

\begin{tabular}{|c|c|c|c|c|c|c|c|c|}
\hline Date & Time & Hours of & Cumulative & B Duct Temp & Lime Flow & Slurry Solids & SO2 Removal & Stack Opacity \\
\hline & & Injection & Hours & ${ }^{\circ} \mathrm{F}$ & GPM & Percent & Percent & Percent \\
\hline $10 / 14 / 92$ & 1300 & 0.25 & 124.00 & 294.2 & 33.9 & 5.0 & 18.3 & 14.1 \\
\hline $10 / 14 / 92$ & 1315 & 0.25 & 124.25 & 294.2 & 35.3 & 5.2 & 18.3 & 12.9 \\
\hline $10 / 14 / 92$ & 1330 & 0.25 & 124.50 & 295.3 & 34.2 & 4.6 & 17.0 & 14.9 \\
\hline $10 / 14 / 92$ & 1345 & 0.25 & 124.75 & 294.9 & 34.0 & 4.8 & 18.1 & 13.0 \\
\hline $10 / 14 / 92$ & 1400 & 0.25 & 125.00 & 294.6 & 35.1 & 4.8 & 16.9 & 16.3 \\
\hline $10 / 14 / 92$ & 1415 & 0.25 & 125.25 & 294.4 & 34.4 & 1.8 & 17.5 & 16.6 \\
\hline $10 / 14 / 92$ & 1430 & 0.25 & 125.50 & 294.9 & 34.2 & 2.7 & 13.7 & 18.1 \\
\hline $10 / 14 / 92$ & 1445 & 0.25 & 125.75 & 294.6 & 35.2 & 4.3 & 18.6 & 16.0 \\
\hline $10 / 14 / 92$ & 1500 & 0.25 & 126.00 & 294.0 & 35.5 & 4.5 & 17.9 & 15.0 \\
\hline $10 / 14 / 92$ & 1515 & 0.25 & 126.25 & 294.6 & 35.0 & 4.2 & 20.0 & 16.3 \\
\hline $10 / 14 / 92$ & 1530 & 0.25 & 126.50 & 294.8 & 34.7 & 4.3 & 19.6 & 15.3 \\
\hline $10 / 14 / 92$ & 1545 & 0.25 & 126.75 & 296.7 & 35.0 & 4.0 & 17.7 & 16.6 \\
\hline $10 / 14 / 92$ & 1600 & 0.25 & 127.00 & 297.0 & 34.4 & 3.3 & 17.2 & 15.8 \\
\hline $10 / 14 / 92$ & 1615 & 0.25 & 127.25 & 295.5 & 34.1 & 3.5 & 15.5 & 14.7 \\
\hline $10 / 14 / 92$ & 1630 & 0.25 & 127.50 & 294.9 & 33.5 & 2.8 & 16.1 & 17.2 \\
\hline $10 / 14 / 92$ & 1645 & 0.25 & 127.75 & 294.7 & 36.1 & 2.0 & 17.7 & 14.4 \\
\hline $10 / 14 / 92$ & 1700 & 0.25 & 128.00 & 294.5 & 32.9 & 2.0 & 12.3 & 15.6 \\
\hline $10 / 14 / 92$ & 1715 & 0.25 & 128.25 & 294.6 & 32.8 & 1.9 & 15.1 & 17.1 \\
\hline $10 / 14 / 92$ & 1730 & 0.25 & 128.50 & 294.6 & 32.4 & 2.9 & 15.3 & 16.8 \\
\hline $10 / 14 / 92$ & 1745 & 0.25 & 128.75 & 294.1 & 31.9 & 3.2 & 13.0 & 16.1 \\
\hline $10 / 14 / 92$ & 1800 & 0.25 & 129.00 & 293.0 & 31.8 & 3.7 & 15.2 & 15.3 \\
\hline $10 / 14 / 92$ & 1815 & 0.25 & 129.25 & 293.2 & 32.7 & 3.8 & 15.3 & 14.5 \\
\hline $10 / 14 / 92$ & 1830 & 0.25 & 129.50 & 292.4 & 34.2 & 3.7 & 14.1 & 16.1 \\
\hline $10 / 14 / 92$ & 1845 & 0.25 & 129.75 & 292.0 & 33.3 & 3.3 & 12.3 & 17.0 \\
\hline $10 / 14 / 92$ & 1900 & 0.25 & 130.00 & 292.3 & 32.9 & 4.2 & 13.4 & 16.2 \\
\hline $10 / 14 / 92$ & 1915 & 0.25 & 130.25 & 292.3 & 32.5 & 3.9 & 15.2 & 16.4 \\
\hline $10 / 14 / 92$ & 1930 & 0.25 & 130.50 & 291.9 & 33.0 & 3.8 & 12.7 & 16.7 \\
\hline $10 / 14 / 92$ & 1945 & 0.25 & 130.75 & 291.7 & 36.4 & 3.7 & 15.7 & 15.0 \\
\hline $10 / 14 / 92$ & 2000 & 0.25 & 131.00 & 291.4 & 28.0 & 3.7 & 13.1 & 19.5 \\
\hline $10 / 14 / 92$ & 2015 & 0.25 & 131.25 & 290.6 & 29.8 & 4.0 & 11.1 & 17.5 \\
\hline $10 / 14 / 92$ & 2030 & 0.25 & 131.50 & 290.8 & 31.5 & 4.0 & 13.9 & 16.0 \\
\hline $10 / 14 / 92$ & 2045 & 0.25 & 131.75 & 289.7 & 29.7 & 4.3 & 15.4 & 17.9 \\
\hline Total Hours & & 12.25 & & & & & & \\
\hline $10 / 15 / 92$ & 1545 & 0.25 & 132.00 & 294.9 & 30.1 & 3.0 & 4.6 & 10.9 \\
\hline $10 / 15 / 92$ & 1600 & 0.25 & 132.25 & 294.5 & 28.9 & 3.0 & 5.8 & 11.8 \\
\hline $10 / 15 / 92$ & 1615 & 0.25 & 132.50 & 292.7 & 27.5 & 3.0 & 9.0 & 12.6 \\
\hline $10 / 15 / 92$ & 1630 & 0.25 & 132.75 & 292.7 & 27.7 & 3.0 & 7.6 & 11.5 \\
\hline $10 / 15 / 92$ & 1645 & 0.25 & 133.00 & 292.6 & 27.7 & 3.0 & 8.9 & 14.3 \\
\hline $10 / 15 / 92$ & 1700 & 0.25 & 133.25 & 292.0 & 26.8 & 3.0 & 4.9 & 15.1 \\
\hline $10 / 15 / 92$ & 1715 & 0.25 & 133.50 & 292.0 & 26.6 & 3.0 & 3.7 & 13.5 \\
\hline $10 / 15 / 92$ & 1730 & 0.25 & 133.75 & 292.2 & 30.0 & 3.0 & 4.5 & 14.7 \\
\hline $10 / 15 / 92$ & 1745 & 0.25 & 134.00 & 291.7 & 29.7 & 3.0 & 5.5 & 12.7 \\
\hline $10 / 15 / 92$ & 1800 & 0.25 & 134.25 & 291.1 & 30.0 & 3.0 & 7.2 & 12.4 \\
\hline $10 / 15 / 92$ & 1815 & 0.25 & 134.50 & 291.3 & 29.7 & 3.0 & 8.5 & 12.6 \\
\hline $10 / 15 / 92$ & 1830 & 0.25 & 134.75 & 290.8 & 29.9 & 3.0 & 9.1 & 12.8 \\
\hline $10 / 15 / 92$ & 1845 & 0.25 & 135.00 & 290.4 & 30.2 & 3.0 & 7.6 & 13.3 \\
\hline
\end{tabular}

Page D-2 
SUMMARY OF LIME SLURRY INJECTION FROM SEPTEMBER 17, 1992 TO OCTOBER 16, 1992

\begin{tabular}{|c|c|c|c|c|c|c|c|c|}
\hline Date & Time & Hours of & Cumulative & B Duct Temp & Lime Flow & Slurry Solids & SO2 Removal & Stack Opacity \\
\hline & & Injection & Hours & ${ }^{\circ} \mathrm{F}$ & GPM & Percent & Percent & Percent \\
\hline $10 / 15 / 92$ & 1900 & 0.25 & 135.25 & 290.0 & 29.6 & 3.0 & 9.7 & 15.1 \\
\hline $10 / 15 / 92$ & 1915 & 0.25 & 135.50 & 289.7 & 29.5 & 2.7 & 7.8 & 16.4 \\
\hline $10 / 15 / 92$ & 1930 & 0.25 & 135.75 & 288.8 & 29.4 & 2.6 & 8.6 & 17.3 \\
\hline $10 / 15 / 92$ & 1945 & 0.25 & 136.00 & 287.3 & 28.7 & 1.8 & 8.5 & 14.5 \\
\hline $10 / 15 / 92$ & 2000 & 0.25 & 136.25 & 289.0 & 29.4 & 1.6 & 7.7 & 16.4 \\
\hline $10 / 15 / 92$ & 2015 & 0.25 & 136.50 & 289.3 & 28.7 & 1.1 & 7.9 & 17.7 \\
\hline $10 / 15 / 92$ & 2030 & 0.25 & 136.75 & 288.7 & 28.0 & 1.2 & 9.2 & 16.9 \\
\hline $10 / 15 / 92$ & 2045 & 0.25 & 137.00 & 287.8 & 27.7 & 0.6 & 7.7 & 15.2 \\
\hline $10 / 15 / 92$ & 2100 & 0.25 & 137.25 & 287.8 & 28.0 & 0.2 & 7.0 & 15.4 \\
\hline $10 / 15 / 92$ & 2115 & 0.25 & 137.50 & 287.5 & 27.2 & 0.3 & 22.7 & 14.4 \\
\hline $10 / 15 / 92$ & 2130 & 0.25 & 137.75 & 286.7 & 26.9 & 0.2 & 14.5 & 15.6 \\
\hline $10 / 15 / 92$ & 2145 & 0.25 & 138.00 & 286.5 & 26.6 & 1.5 & 11.6 & 17.0 \\
\hline $10 / 15 / 92$ & 2200 & 0.25 & 138.25 & 286.7 & 26.6 & 1.7 & 13.0 & 16.3 \\
\hline $10 / 15 / 92$ & 2215 & 0.25 & 138.50 & 286.9 & 27.4 & 1.4 & 10.1 & 17.0 \\
\hline $10 / 15 / 92$ & 2230 & 0.25 & 138.75 & 286.2 & 26.6 & 1.5 & 10.0 & 18.7 \\
\hline $10 / 15 / 92$ & 2245 & 0.25 & 139.00 & 285.6 & 25.6 & 3.3 & 8.4 & 21.8 \\
\hline $10 / 15 / 92$ & 2300 & 0.25 & 139.25 & 286.0 & 28.6 & 3.1 & 8.4 & 18.0 \\
\hline $10 /: 5 / 92$ & 2315 & 0.25 & 139.50 & 286.6 & 29.4 & 2.4 & 8.5 & 15.2 \\
\hline $10 / 15 / 92$ & 2330 & 0.25 & 139.75 & 287.1 & 29.4 & 2.2 & 10.0 & 13.9 \\
\hline $10 / 15 / 92$ & 2345 & 0.25 & 140.00 & 286.9 & 28.9 & 2.0 & 9.3 & 13.8 \\
\hline Total Hours & & 8.25 & & & & & & \\
\hline & & & & & & & & \\
\hline $10 / 16 / 92$ & 0 & 0.25 & 140.25 & 286.2 & 28.5 & 1.4 & 8.9 & 14.9 \\
\hline $10 / 16 / 92$ & 15 & 0.25 & 140.50 & 286.2 & 29.8 & 1.6 & 9.1 & 14.5 \\
\hline $10 / 16 / 92$ & 30 & 0.25 & 140.75 & 285.8 & 32.8 & 1.0 & 10.2 & 10.7 \\
\hline $10 / 16 / 92$ & 45 & 0.25 & 141.00 & 285.9 & 27.6 & 0.6 & 9.3 & 12.6 \\
\hline $10 / 16 / 92$ & 100 & 0.25 & 141.25 & 287.5 & 26.8 & 0.5 & 8.9 & 16.9 \\
\hline $10 / 16 / 92$ & $1: 5$ & 0.25 & 141.50 & 286.3 & 28.4 & 0.6 & 8.0 & 17.2 \\
\hline $10 / 16 / 92$ & 130 & 0.25 & 141.75 & 284.9 & 28.4 & 0.6 & 7.4 & 13.8 \\
\hline $10 / 16 / 92$ & 145 & 0.25 & 142.00 & 280.5 & 26.4 & 2.5 & 8.2 & 10.7 \\
\hline $10 / 16 / 92$ & 200 & 0.25 & 142.25 & 281.5 & 26.5 & 2.3 & 10.2 & 11.0 \\
\hline $10 / 16 / 92$ & 215 & 0.25 & 142.50 & 283.3 & 26.5 & 2.0 & 9.6 & 9.3 \\
\hline $10 / 16 / 92$ & 230 & 0.25 & 142.75 & 282.5 & 25.0 & 1.5 & 10.1 & 9.4 \\
\hline $10 / 16 / 92$ & 245 & 0.25 & 143.00 & 283.5 & 26.6 & 1.2 & 11.1 & 7.6 \\
\hline $10 / 16 / 92$ & 300 & 0.25 & 143.25 & 281.2 & 25.7 & 1.1 & 11.2 & 7.5 \\
\hline $10 / 16 / 92$ & 315 & 0.25 & 143.50 & 281.9 & 25.6 & 0.6 & 11.5 & 9.0 \\
\hline $10 / 16 / 92$ & 330 & 0.25 & 143.75 & 282.4 & 25.2 & 0.3 & 9.0 & 10.4 \\
\hline $10 / 16 / 92$ & 345 & 0.25 & 144.00 & 281.1 & 25.0 & 0.3 & 8.6 & 11.0 \\
\hline $10 / 16 / 92$ & 400 & 0.25 & 144.25 & 281.5 & 25.1 & 0.3 & 9.1 & 13.4 \\
\hline $10 / 16 / 92$ & 415 & 0.25 & 144.50 & 281.5 & 25.0 & 0.2 & 9.8 & 8.6 \\
\hline $10 / 16 / 92$ & 430 & 0.25 & 144.75 & 281.6 & 25.6 & 1.7 & 9.8 & 12.0 \\
\hline $10 / 16 / 92$ & 445 & 0.25 & 145.00 & 280.0 & 25.6 & 2.0 & 8.7 & 10.1 \\
\hline $10 / 16 / 92$ & 500 & 0.25 & 145.25 & 279.6 & 29.2 & 1.6 & 11.2 & 8.9 \\
\hline $10 / 16 / 92$ & 515 & 0.25 & 145.50 & 278.7 & 25.0 & 2.4 & 9.7 & 9.0 \\
\hline $10 / 16 / 92$ & 530 & 0.25 & 145.75 & 279.0 & 25.2 & 2.1 & 9.1 & 10.9 \\
\hline $10 / 16 / 92$ & 545 & 0.25 & 146.00 & 278.3 & 25.1 & 2.2 & 7.5 & 11.4 \\
\hline $10 / 16 / 92$ & 600 & 0.25 & 146.25 & 281.2 & 25.2 & 2.7 & 8.0 & 37.3 \\
\hline
\end{tabular}

Page D-3 B-Duct Opacity >> * = Lower, ${ }^{* *}=$ Higher 
SUMMARY OF LIME SLURRY INJECTION FROM SEPTEMBER 17, 1992 TO OCTOBER 16, 1992

\begin{tabular}{|c|c|c|c|c|c|c|c|c|}
\hline Date & Time & Hours of & Cumulative & B Duct Temp & Lime Flow & Slurry Solids & SO2 Removal & Stack Opacity \\
\hline & & Injection & Hours & ${ }^{\circ} \mathrm{F}$ & GPM & Percent & Percent & Percent \\
\hline $10 / 16 / 92$ & 615 & 0.25 & 146.50 & 282.0 & 25.3 & 2.0 & 7.8 & 17.8 \\
\hline $10 / 16 / 92$ & 630 & 0.25 & 146.75 & 282.3 & 25.6 & 2.1 & 10.0 & 16.9 \\
\hline $10 / 16 / 92$ & 645 & 0.25 & 147.00 & 283.4 & 25.5 & 1.4 & 8.5 & 18.0 \\
\hline $10 / 16 / 92$ & 700 & 0.25 & 147.25 & 282.9 & 29.6 & 1.8 & 8.1 & 12.7 \\
\hline $10 / 16 / 92$ & 715 & 0.25 & 147.50 & 288.4 & 27.9 & 1.3 & 7.1 & 19.2 \\
\hline $10 / 16 / 92$ & 730 & 0.25 & 147.75 & 289.5 & 28.0 & 1.0 & 8.3 & 14.8 \\
\hline $10 / 16 / 92$ & 1500 & 0.25 & 148.00 & 290.7 & 33.0 & 0.8 & 7.4 & 11.2 \\
\hline $10 / 16 / 92$ & 1515 & 0.25 & 148.25 & 290.9 & 35.0 & 0.8 & 5.7 & 10.8 \\
\hline $10 / 16 / 92$ & 1530 & 0.25 & 148.50 & 291.2 & 35.2 & 0.8 & 7.1 & 10.7 \\
\hline $10 / 16 / 92$ & 1545 & 0.25 & 148.75 & 292.3 & 35.4 & 0.8 & 8.3 & 10.2 \\
\hline $10 / 16 / 92$ & 1600 & 0.25 & 149.00 & $291 . \overline{5}$ & 34.4 & 0.8 & 7.0 & 12.0 \\
\hline $10 / 16 / 92$ & 1615 & 0.25 & 149.25 & 291.2 & 34.3 & 1.3 & 5.7 & 12.0 \\
\hline $10 / 16 / 92$ & 1630 & 0.25 & 149.50 & 290.5 & 33.9 & 1.9 & 6.8 & 12.2 \\
\hline $10 / 16 / 92$ & 1645 & 0.25 & 149.75 & 290.8 & 34.1 & 2.2 & 6.0 & 12.3 \\
\hline $10 / 16 / 92$ & 1700 & 0.25 & 150.00 & 290.2 & 33.3 & 2.4 & 7.3 & 11.9 \\
\hline $10 / 16 / 92$ & 1715 & 0.25 & 150.25 & 290.4 & 33.2 & 2.1 & 7.3 & 12.1 \\
\hline $10 / 16 / 92$ & 1730 & 0.25 & 150.50 & 290.1 & 31.7 & 2.7 & 7.5 & 11.8 \\
\hline $10 / 16 / 92$ & 1745 & 0.25 & 150.75 & 289.9 & 31.2 & 2.3 & 6.8 & 13.9 \\
\hline $10 / 16 / 92$ & 1800 & 0.25 & 151.00 & 289.7 & 31.5 & 2.2 & 7.6 & 13.6 \\
\hline $10 / 16 / 92$ & 1815 & 0.25 & 151.25 & 290.0 & 33.0 & 2.4 & 9.2 & 12.6 \\
\hline $10 / 16 / 92$ & 1830 & 0.25 & 151.50 & 288.5 & 30.8 & 2.9 & 8.0 & 13.9 \\
\hline $10 / 16 / 92$ & 1845 & 0.25 & 151.75 & 287.5 & 31.1 & 2.6 & 8.0 & 14.0 \\
\hline $10 / 16 / 92$ & 1900 & 0.25 & 152.00 & 287.1 & 30.5 & 2.5 & 8.7 & 14.8 \\
\hline $10 / 16 / 92$ & 1915 & 0.25 & 152.25 & 286.1 & 30.1 & 2.8 & 6.7 & 16.6 \\
\hline $10 / 16 / 92$ & 1930 & 0.25 & 152.50 & 285.1 & 29.6 & 2.6 & 9.6 & 15.7 \\
\hline $10 / 16 / 92$ & 1945 & 0.25 & 152.75 & 284.3 & 28.8 & 2.8 & 9.8 & 16.3 \\
\hline $10 / 16 / 92$ & 2000 & 0.25 & 153.00 & 284.6 & 28.2 & 2.5 & 6.4 & 15.8 \\
\hline $10 / 16 / 92$ & 2015 & 0.25 & 153.25 & 284.6 & 27.9 & 2.7 & 9.1 & 18.0 \\
\hline $10 / 16 / 92$ & 2030 & 0.25 & 153.50 & 284.0 & 27.2 & 3.0 & 7.7 & 18.5 \\
\hline $10 / 16 / 92$ & 2045 & 0.25 & 153.75 & 283.1 & 26.8 & 2.5 & 9.3 & 18.6 \\
\hline $10 / 16 / 92$ & 2100 & 0.25 & 154.00 & 283.4 & 27.7 & 2.6 & 10.5 & 18.6 \\
\hline $10 / 16 / 92$ & 2115 & 0.25 & 154.25 & 283.6 & 27.5 & 2.6 & 15.9 & 17.9 \\
\hline \multicolumn{2}{|l|}{ Total Hours } & 14.25 & & & & & & \\
\hline
\end{tabular}




\title{
Appendix E
}

\author{
Tables With Summary of \\ Lime Slurry Injection Data \\ October 17 to November 16, 1992
}


SUMMARY OF LIME SLURRY INJECTION FROM OCTOBER 17, 1992 TO NOVEMBER 17, 1992

\begin{tabular}{|c|c|c|c|c|c|c|c|c|}
\hline Date & Time & Hours of & Cumulative & B Duct Temp & Lime Flow & Slurry Solids & SO2 Removal & Stack Opacity \\
\hline & & Injection & Hours & ${ }^{\circ} \mathrm{F}$ & GPM & Percent & Percent & Percent \\
\hline $10 / 17 / 92$ & 100 & 0.25 & 154.50 & 287.1 & 31.4 & 4.4 & 3.7 & 10.7 \\
\hline $10 / 17 / 92$ & 115 & 0.25 & 154.75 & 287.9 & 34.8 & 4.7 & 11.0 & 11.4 \\
\hline $10 / 17 / 92$ & 130 & 0.25 & 155.00 & 286.9 & 34.6 & 4.6 & 11.7 & 12.1 \\
\hline $10 / 17 / 92$ & 145 & 0.25 & 155.25 & 287.3 & 33.9 & 4.2 & 13.4 & 16.6 \\
\hline $10 / 17 / 92$ & 200 & 0.25 & 155.50 & 289.3 & 35.1 & 4.4 & 13.5 & 12.9 \\
\hline $10 / 17 / 92$ & 215 & 0.25 & 155.75 & 288.9 & 36.5 & 4.0 & 11.6 & 5.4 \\
\hline $10 / 17 / 92$ & 230 & 0.25 & 156.00 & 288.6 & 34.9 & 4.1 & 16.5 & 9.5 \\
\hline $10 / 17 / 92$ & 245 & 0.25 & 156.25 & 288.4 & 35.4 & 3.7 & 15.7 & 7.0 \\
\hline $10 / 17 / 92$ & 300 & 0.25 & 156.50 & 287.3 & 34.9 & 4.2 & 16.4 & 9.4 \\
\hline $10 / 17 / 92$ & 315 & 0.25 & 156.75 & 289.3 & 34.1 & 4.0 & 15.3 & 9.7 \\
\hline $10 / 17 / 92$ & 330 & 0.25 & 157.00 & 288.4 & 34.0 & 4.2 & 17.2 & 11.0 \\
\hline $10 / 17 / 92$ & 345 & 0.25 & 157.25 & 288.0 & 33.5 & 4.2 & 16.7 & 9.2 \\
\hline $10 / 17 / 92$ & 400 & 0.25 & 157.50 & 289.2 & 33.6 & 4.1 & 17.0 & 9.1 \\
\hline $10 / 17 / 92$ & 415 & 0.25 & 157.75 & 287.4 & 36.8 & 4.0 & 19.4 & 9.4 \\
\hline $10 / 17 / 92$ & 430 & 0.25 & 158.00 & 287.1 & 33.5 & 3.9 & 21.4 & 8.7 \\
\hline $10 / 17 / 92$ & 445 & 0.25 & 158.25 & 286.4 & 32.7 & 4.0 & 19.7 & 8.5 \\
\hline $10 / 17 / 92$ & 500 & 0.25 & 158.50 & 285.8 & 32.8 & 4.1 & 19.8 & 8.9 \\
\hline $10 / 17 / 92$ & 515 & 0.25 & 158.75 & 287.8 & 32.4 & 4.4 & 21.1 & 6.4 \\
\hline $10 / 17 / 92$ & 530 & 0.25 & 159.00 & 288.3 & 32.9 & 3.7 & 22.7 & 6.9 \\
\hline $10 / 1 \% / 92$ & 545 & 0.25 & 159.25 & 287.9 & 32.4 & 4.0 & 20.5 & 7.2 \\
\hline $10 / 17 / 92$ & 600 & 0.25 & 159.50 & 287.1 & 32.7 & 4.0 & 20.1 & 6.7 \\
\hline $10 / 17 / 92$ & 615 & 0.25 & 159.75 & 285.7 & 33.5 & 4.4 & 24.0 & 7.2 \\
\hline $10 / 17 / 92$ & 630 & 0.25 & 160.00 & 283.7 & 32.1 & 4.3 & 22.1 & 7.0 \\
\hline $10 / 17 / 92$ & 645 & 0.25 & 160.25 & 281.7 & 31.7 & 4.3 & 19.8 & 8.0 \\
\hline $10 / 17 / 92$ & 700 & 0.25 & 160.50 & 284.1 & 32.3 & 4.1 & 19.1 & 7.9 \\
\hline $10 / 17 / 92$ & 715 & 0.25 & 160.75 & 285.5 & 31.5 & 4.2 & 16.8 & 8.7 \\
\hline $10 / 17 / 92$ & 730 & 0.25 & 161.00 & 285.0 & 30.7 & 4.0 & 18.3 & 12.0 \\
\hline $10 / 17 / 92$ & 745 & 0.25 & 161.25 & 283.5 & 34.0 & 4.1 & 16.7 & 8.9 \\
\hline $10 / 17 / 92$ & 800 & 0.25 & 161.50 & 284.4 & 32.8 & 4.0 & 18.9 & 10.3 \\
\hline $10 / 17 / 92$ & 815 & 0.25 & 161.75 & 284.6 & 32.9 & 3.8 & 17.8 & 10.5 \\
\hline $10 / 17 / 92$ & 830 & 0.25 & 162.00 & 285.0 & 34.4 & 4.3 & 20.3 & 10.8 \\
\hline $10 / 17 / 92$ & 845 & 0.25 & 162.25 & 285.9 & 32.1 & 4.1 & 17.9 & 11.0 \\
\hline $10 / 17 / 92$ & 900 & 0.25 & 162.50 & 285.6 & 31.5 & 3.8 & 17.3 & 13.1 \\
\hline $10 / 17 / 92$ & 915 & 0.25 & 162.75 & 286.1 & 30.2 & 3.8 & 16.7 & 12.6 \\
\hline $10 / 17 / 92$ & 930 & 0.25 & 163.00 & 286.1 & 31.1 & 4.2 & 19.0 & 12.5 \\
\hline $10 / 17 / 92$ & 945 & 0.25 & 163.25 & 286.6 & 32.4 & 4.2 & 17.6 & 12.6 \\
\hline $10 / 17 / 92$ & 1000 & 0.25 & 163.50 & 285.3 & 33.1 & 3.9 & 21.5 & 10.4 \\
\hline $10 / 17 / 92$ & 1015 & 0.25 & 163.75 & 285.5 & 30.2 & 4.2 & 17.9 & 34.8 \\
\hline $10 / 17 / 92$ & 1030 & 0.25 & 164.00 & 285.2 & 32.0 & 3.8 & 19.0 & 13.7 \\
\hline $10 / 17 / 92$ & 1045 & 0.25 & 164.25 & 284.3 & 28.5 & 4.2 & 15.9 & 15.4 \\
\hline $10 / 17 / 92$ & 1100 & 0.25 & 164.50 & 284.2 & 25.2 & 4.3 & 17.5 & 17.8 \\
\hline \multicolumn{2}{|l|}{ Total Hours } & 10.25 & & & & & & \\
\hline & & & & & & & & \\
\hline $10 / 18 / 92$ & 1545 & 0.25 & 164.75 & 279.2 & 31.9 & 3.6 & 7.7 & 8.9 \\
\hline $10 / 18 / 92$ & 1600 & 0.25 & 165.00 & 281.2 & 33.8 & 3.9 & 11.0 & 7.0 \\
\hline $10 / 18 / 92$ & 1615 & 0.25 & 165.25 & 282.1 & 34.1 & 4.2 & 11.2 & 18.8 \\
\hline $10 / 18 / 92$ & 1630 & 0.25 & 165.50 & 281.7 & 34.1 & 3.9 & 10.8 & 12.9 \\
\hline
\end{tabular}

Page E-1 B-Duct Opacity>> " $=$ Lower, "* = Higher 
SUMMARY OF LIME SLURRY INJECTION FROM OCTOBER 17, 1992 TO NOVEMBER 17, 1992

\begin{tabular}{|c|c|c|c|c|c|c|c|c|}
\hline Date & Time & Hours of & Cumulative & B Duct Temp & Lime Flow & Slurry Solids & SO2 Removal & Stack Opacity \\
\hline & & Injection & Hours & ${ }^{\circ} \mathrm{F}$ & GPM & Percent & Percent & Percent \\
\hline $10 / 18 / 92$ & 1645 & 0.25 & 165.75 & 281.2 & 35.1 & 3.8 & 12.2 & 10.3 \\
\hline $10 / 18 / 92$ & 1700 & 0.25 & 166.00 & 279.6 & 32.9 & 4.2 & 11.1 & 8.9 \\
\hline $10 / 18 / 92$ & 1715 & 0.25 & 166.25 & 277.7 & 31.6 & 4.1 & 11.1 & 9.6 \\
\hline $10 / 18 / 92$ & 1730 & 0.25 & 166.50 & 277.0 & 31.2 & 4.6 & 9.8 & 9.2 \\
\hline $10 / 18 / 92$ & 1745 & 0.25 & 166.75 & 277.7 & 31.7 & 4.3 & 12.2 & 8.8 \\
\hline $10 / 18 / 92$ & 1800 & 0.25 & 167.00 & 279.7 & 31.8 & 4.1 & 13.7 & 8.5 \\
\hline $10 / 18 / 92$ & 1815 & 0.25 & 167.25 & 281.6 & 32.9 & 4.1 & 10.9 & 8.8 \\
\hline $10 / 18 / 92$ & 1830 & 0.25 & 167.50 & 281.3 & 32.0 & 3.9 & 12.0 & 28.1 \\
\hline $10 / 18 / 92$ & 1845 & 0.25 & 167.75 & 279.6 & 31.7 & 4.2 & $1 \overline{1.1}$ & 13.5 \\
\hline $10 / 18 / 92$ & 1900 & 0.25 & 168.00 & 281.0 & 31.9 & 4.4 & 11.5 & 11.8 \\
\hline $10 / 18 / 92$ & 1915 & 0.25 & 168.25 & 280.5 & 32.0 & 4.3 & 12.7 & 11.9 \\
\hline $10 / 18 / 92$ & 1930 & 0.25 & 168.50 & 279.9 & 30.7 & 4.7 & 12.6 & 14.4 \\
\hline $10 / 18 / 92$ & 1945 & 0.25 & 168.75 & 279.2 & 29.9 & 4.3 & 13.1 & 13.5 \\
\hline $10 / 18 / 92$ & 2000 & 0.25 & 169.00 & 279.0 & 30.3 & 4.4 & 13.3 & 13.4 \\
\hline $10 / 18 / 92$ & 2015 & 0.25 & 169.25 & 278.7 & 32.8 & 4.3 & 12.4 & 12.3 \\
\hline $10 / 18 / 92$ & 2030 & 0.25 & 169.50 & 276.9 & 28.4 & 4.6 & 21.9 & 13.0 \\
\hline $10 / 18 / 92$ & 2115 & 0.25 & 169.75 & 275.6 & 28.0 & 4.9 & 2.6 & 14.1 \\
\hline $10 / 18 / 92$ & 2130 & 0.25 & 170.00 & 274.6 & 29.4 & 4.6 & 16.3 & 13.0 \\
\hline $10 / 18 / 92$ & 2145 & 0.25 & 170.25 & 274.8 & 30.4 & 4.6 & 17.9 & 10.3 \\
\hline $10 / 18 / 92$ & 2200 & 0.25 & 170.50 & 273.9 & 28.7 & 4.1 & 16.5 & 11.5 \\
\hline $10 / 18 / 92$ & 2215 & 0.25 & 170.75 & 274.8 & 29.2 & 4.5 & 14.0 & 13.4 \\
\hline $10 / 18 / 92$ & 2230 & 0.25 & 171.00 & 275.9 & 29.9 & 4.2 & 15.8 & 13.3 \\
\hline $10 / 18 / 92$ & 2245 & 0.25 & 171.25 & 276.8 & 29.7 & 4.4 & 15.4 & 13.1 \\
\hline $10 / 18 / 92$ & 2300 & 0.25 & 171.50 & 276.2 & 29.4 & 3.9 & 15.9 & 12.7 \\
\hline $10 / 18 / 92$ & 2315 & 0.25 & 171.75 & 280.3 & 31.7 & 4.2 & 15.0 & 12.6 \\
\hline $10 / 18 / 92$ & 2330 & 0.25 & 172.00 & 279.0 & 30.7 & 4.0 & 14.4 & 13.5 \\
\hline $10 / 18 / 92$ & 2345 & 0.25 & 172.25 & 278.5 & 30.6 & 4.6 & 15.7 & 14.4 \\
\hline \multicolumn{2}{|l|}{ Total Hours } & 7.75 & & & & & & \\
\hline & & & & & & & & \\
\hline $10 / 19 / 92$ & 0 & 0.25 & 172.50 & 276.0 & 32.9 & 4.4 & 15.0 & 13.4 \\
\hline $10 / 19 / 92$ & 15 & 0.25 & 172.75 & 276.7 & 31.4 & 4.2 & 9.6 & 12.0 \\
\hline $10 / 19 / 92$ & 30 & 0.25 & 173.00 & 279.0 & 34.8 & 4.7 & 12.8 & 13.6 \\
\hline $10 / 19 / 92$ & 45 & 0.25 & 173.25 & 283.6 & 29.7 & 4.6 & 14.2 & 10.5 \\
\hline $10 / 19 / 92$ & 100 & 0.25 & 173.50 & 285.6 & 30.5 & 4.3 & 11.2 & 9.1 \\
\hline $10 / 19 / 92$ & 115 & 0.25 & 173.75 & 285.5 & 29.7 & 4.5 & 11.7 & 9.1 \\
\hline $10 / 19 / 92$ & 130 & 0.25 & 174.00 & 286.6 & 30.2 & 4.3 & 11.5 & 10.5 \\
\hline $10 / 19 / 92$ & 145 & 0.25 & 174.25 & 285.6 & 29.8 & 4.4 & 13.0 & 14.4 \\
\hline $10 / 19 / 92$ & 200 & 0.25 & 174.50 & 285.2 & 30.3 & 4.2 & 13.7 & 10.3 \\
\hline $10 / 19 / 92$ & 215 & 0.25 & 174.75 & 284.8 & 30.7 & 4.4 & 14.6 & 13.2 \\
\hline $10 / 19 / 92$ & 230 & 0.25 & 175.00 & 283.6 & 28.1 & 4.5 & 13.1 & 11.4 \\
\hline $10 / 19 / 92$ & 245 & 0.25 & 175.25 & 284.9 & 30.0 & 4.1 & 13.1 & 13.1 \\
\hline $10 / 19 / 92$ & 300 & 0.25 & 175.50 & 285.8 & 27.9 & 4.3 & 16.8 & 11.8 \\
\hline $10 / 19 / 92$ & 315 & 0.25 & 175.75 & 288.9 & 27.6 & 4.4 & 11.8 & 14.6 \\
\hline $10 / 19 / 92$ & 1200 & 0.25 & 176.00 & 278.9 & 36.6 & 4.7 & 7.2 & 13.9 \\
\hline $10 / 19 / 92$ & 1215 & 0.25 & 176.25 & 279.4 & 36.0 & 4.5 & 7.0 & 14.0 \\
\hline $10 / 19 / 92$ & 1230 & 0.25 & 176.50 & 279.8 & 35.6 & 3.9 & 8.7 & 17.1 \\
\hline $10 / 19 / 92$ & 1245 & 0.25 & 176.75 & 279.4 & 35.0 & 3.3 & 7.5 & 13.9 \\
\hline
\end{tabular}

Page E-2 B-Duct Opacity>> " = Lower, "* = Higher 
SUMMARY OF LIME SLURRY INJECTION FROM OCTOBER 17, 1992 TO NOVEMBER 17, 1992

\begin{tabular}{|c|c|c|c|c|c|c|c|c|}
\hline Date & Time & Hours of & Cumulative & B Duct Temp & Limo Flow & Slurry Solids & SO2 Removal & Stack Opacity \\
\hline & & Injection & Hours & ${ }^{\circ} \mathrm{F}$ & GPM & Percent & Percent & Percent \\
\hline $10 / 19 / 92$ & 1300 & 0.25 & 177.00 & 279.4 & 35.3 & 3.3 & 8.7 & 14.9 \\
\hline $10 / 19 / 92$ & 1315 & 0.25 & 177.25 & 280.1 & 35.4 & 4.9 & 8.7 & 18.2 \\
\hline $10 / 19 / 92$ & 1330 & 0.25 & 177.50 & 280.0 & 35.3 & 4.9 & 8.5 & 13.4 \\
\hline $10 / 19 / 92$ & 1345 & 0.25 & 177.75 & 281.0 & 38.6 & 4.2 & 10.9 & 18.5 \\
\hline $10 / 19 / 92$ & 1400 & 0.25 & 178.00 & 279.6 & 37.8 & 4.5 & 9.0 & 16.8 \\
\hline $10 / 19 / 92$ & 1415 & 0.25 & 178.25 & 279.4 & 34.8 & 4.2 & 11.4 & 13.8 \\
\hline $10 / 19 / 92$ & 1430 & 0.25 & 178.50 & 278.3 & 33.7 & 4.5 & 8.8 & 13.9 \\
\hline $10 / 19 / 92$ & 1445 & 0.25 & 178.75 & 279.9 & 34.0 & 4.0 & 8.8 & 17.5 \\
\hline $10 / 19 / 92$ & 1500 & 0.25 & 179.00 & 279.4 & 34.3 & 3.7 & 5.6 & 14.3 \\
\hline $10 / 19 / 92$ & 1515 & 0.25 & 179.25 & 278.8 & 33.5 & 3.8 & 8.6 & 25 \\
\hline $10 / 19 / 92$ & 1530 & 0.25 & 179.50 & 279.1 & 33.5 & 4.3 & 8.4 & 14.2 \\
\hline $10 / 19 / 92$ & 1545 & 0.25 & 179.75 & 279.2 & 32.9 & 4.3 & 8.0 & 16.1 \\
\hline $10 / 19 / 92$ & 1600 & 0.25 & 180.00 & 277.5 & 32.4 & 4.1 & 8.2 & 16.5 \\
\hline $10 / 19 / 92$ & 1615 & 0.25 & 180.25 & 276.7 & 31.9 & 3.8 & 7.4 & 12.0 \\
\hline $10 / 19 / 92$ & 1630 & 0.25 & 180.50 & 279.1 & 32.3 & 4.1 & 8.7 & 10.8 \\
\hline $10 / 19 / 92$ & 1645 & 0.25 & 180.75 & 277.8 & 31.0 & 4.1 & 7.7 & 9.9 \\
\hline $10 / 19 / 92$ & 1700 & 0.25 & 181.00 & 278.0 & 31.7 & 3.9 & 7.6 & 8.7 \\
\hline $10 / 19 / 92$ & 1715 & 0.25 & 181.25 & 278.7 & 31.4 & 3.6 & 5.7 & 12.6 \\
\hline $10 / 19 / 92$ & 1730 & 0.25 & 181.50 & 278.7 & 31.2 & 3.8 & 5.5 & 12.2 \\
\hline $10 / 19 / 92$ & 1745 & 0.25 & 181.75 & 278.9 & 31.0 & 3.8 & 8.4 & 10.3 \\
\hline $10 / 19 / 92$ & 1800 & 0.25 & 182.00 & 278.0 & 33.4 & 3.5 & 8.8 & 11.2 \\
\hline $10 / 19 / 92$ & 1815 & 0.25 & 182.25 & 277.7 & 30.8 & 3.7 & 7.4 & 11.1 \\
\hline $10 / 19 / 92$ & 1830 & 0.25 & 182.50 & 276.9 & 31.3 & 3.5 & 6.8 & 15.1 \\
\hline $10 / 19 / 92$ & 1845 & 0.25 & 182.75 & 277.6 & 31.8 & 4.2 & 5.3 & 16.4 \\
\hline $10 / 19 / 92$ & 1900 & 0.25 & 183.00 & 278.1 & 31.9 & 4.3 & 7.9 & 15.1 \\
\hline $10 / 19 / 92$ & 1915 & 0.25 & 183.25 & 276.3 & 30.7 & 3.8 & 7.0 & 15.9 \\
\hline $10 / 19 / 92$ & 1930 & 0.25 & 183.50 & 276.2 & 30.1 & 3.9 & 9.8 & 14.6 \\
\hline $10 / 19 / 92$ & 1945 & 0.25 & 183.75 & 276.3 & 30.1 & 3.7 & 6.8 & 16.0 \\
\hline $10 / 19 / 92$ & 2000 & 0.25 & 184.00 & 276.2 & 30.6 & 4.0 & 7.4 & 15.1 \\
\hline $10 / 19 / 92$ & 2015 & 0.25 & 184.25 & 276.3 & 32.7 & 3.9 & 10.1 & 14.5 \\
\hline $10 / 19 / 92$ & 2030 & 0.25 & 184.50 & 274.8 & 29.9 & 4.1 & 10.1 & 15.3 \\
\hline $10 / 19 / 92$ & 2045 & 0.25 & 184.75 & 275.0 & 29.9 & 3.8 & 5.8 & 12.8 \\
\hline $10 / 19 / 92$ & 2100 & 0.25 & 185.00 & 275.2 & 29.8 & 3.8 & 4.3 & 12.4 \\
\hline $10 / 19 / 92$ & 2115 & 0.25 & 185.25 & 275.9 & 29.6 & 3.9 & 6.8 & 10.8 \\
\hline \begin{tabular}{|l|}
$0 / 19 / 92$ \\
\end{tabular} & 2130 & 0.25 & 185.50 & 276.5 & 29.8 & 3.9 & 11.6 & 10.5 \\
\hline $10 / 19 / 92$ & 2145 & 0.25 & 185.75 & 276.3 & 29.1 & 3.8 & 8.6 & 11.6 \\
\hline $10 / 19 / 92$ & 2200 & 0.25 & 186.00 & 276.5 & 33.6 & 3.9 & 6.7 & 9.6 \\
\hline $10 / 19 / 92$ & 2215 & 0.25 & 186.25 & 277.2 & 32.3 & 3.7 & 8.0 & 9.6 \\
\hline $10 / 19 / 92$ & 2230 & 0.25 & 186.50 & 278.5 & 32.4 & 3.7 & 9.7 & 10.5 \\
\hline $10 / 19 / 92$ & 2245 & 0.25 & 186.75 & 277.9 & 29.0 & 3.8 & 6.3 & 15.2 \\
\hline $10 / 19 / 92$ & 2300 & 0.25 & 187.00 & 277.4 & 28.7 & 4.0 & 7.4 & 12.3 \\
\hline $10 / 19 / 92$ & 2315 & 0.25 & 187.25 & 277.6 & 27.9 & 3.8 & 3.6 & 14.4 \\
\hline $10 / 19 / 92$ & 2330 & 0.25 & 187.50 & 278.7 & 29.3 & 3.8 & 9.8 & 11.6 \\
\hline $10 / 19 / 92$ & 2345 & 0.25 & 187.75 & 276.8 & 27.5 & 3.8 & 10.6 & 15.8 \\
\hline Total Hours & & 15.50 & & & & & & \\
\hline $10 / 20 / 92$ & 600 & 0.25 & 188.00 & 272.0 & 33.6 & 3.4 & 8.0 & 9.7 \\
\hline
\end{tabular}

Page E-3 B-Duct Opacity>> " = Lower, "* Higher 
SUMMARY OF LIME SLURRY INJECTION FROM OCTOBER 17, 1992 TO NOVEMBER 17, 1992

\begin{tabular}{|c|c|c|c|c|c|c|c|c|}
\hline Date & Time & Hours of & Cumulative & B Duct Temp & Lime Flow & Slurry Solids & SO2 Removal & Stack Opacity \\
\hline & & Injection & Hours & ${ }^{\circ} \mathrm{F}$ & GPM & Percent & Percent & Percent \\
\hline $10 / 20 / 92$ & 615 & 0.25 & 188.25 & 272.3 & 34.1 & 3.4 & 4.3 & 13.5 \\
\hline $10 / 20 / 92$ & 630 & 0.25 & 188.50 & 273.2 & 33.3 & 3.7 & 8.2 & 12.0 \\
\hline $10 / 20 / 92$ & 645 & 0.25 & 188.75 & 271.8 & 33.1 & 3.8 & 4.6 & 14.5 \\
\hline $10 / 20 / 92$ & 700 & 0.25 & 189.00 & 272.0 & 32.8 & 3.7 & 8.4 & 14.3 \\
\hline $10 / 20 / 92$ & 715 & 0.25 & 189.25 & 272.0 & 32.7 & 3.8 & 4.1 & 13.7 \\
\hline $10 / 20 / 92$ & 730 & 0.25 & 189.50 & 270.5 & 32.1 & 3.7 & 11.3 & 15.2 \\
\hline $10 / 20 / 92$ & 745 & 0.25 & 189.75 & 269.6 & 32.7 & 3.6 & 7.7 & 15.7 \\
\hline $10 / 20 / 92$ & 800 & 0.25 & 190.00 & 271.2 & 32.5 & 4.1 & 10.5 & 15.9 \\
\hline $10 / 20 / 92$ & 815 & 0.25 & 190.25 & 269.6 & 32.5 & 4.0 & 9.7 & 15.3 \\
\hline $10 / 20 / 92$ & 830 & 0.25 & 190.50 & 270.5 & 31.8 & 3.8 & 12.4 & 15.3 \\
\hline $10 / 20 / 92$ & 845 & 0.25 & 190.75 & 270.3 & 33.1 & 3.6 & 8.3 & 14.8 \\
\hline $10 / 20 / 92$ & 900 & 0.25 & 191.00 & 271.6 & 33.2 & 4.1 & 8.0 & 15.7 \\
\hline $10 / 20 / 92$ & 915 & 0.25 & 191.25 & 271.6 & 35.5 & 4.2 & 7.9 & 17.3 \\
\hline $10 / 20 / 92$ & 930 & 0.25 & 191.50 & 272.0 & 33.9 & 3.8 & 12.6 & 17.1 \\
\hline $10 / 20 / 92$ & 945 & 0.25 & 191.75 & 272.5 & 31.1 & 3.8 & 10.2 & 16.4 \\
\hline $10 / 20 / 92$ & 1000 & 0.25 & 192.00 & 272.3 & 30.4 & 3.9 & 8.0 & 15.7 \\
\hline $10 / 20 / 92$ & 1015 & 0.25 & 192.25 & 273.4 & 30.7 & 3.7 & 6.7 & 17.6 \\
\hline $10 / 20 / 92$ & 1030 & 0.25 & 192.50 & 273.9 & 30.7 & 3.8 & 10.8 & 16.3 \\
\hline $10 / 20 / 92$ & 1045 & 0.25 & 192.75 & 274.5 & 30.0 & 3.9 & 8.3 & 15.4 \\
\hline $10 / 20 / 92$ & 1100 & 0.25 & 193.00 & 275.5 & 30.2 & 3.8 & 7.0 & 18.5 \\
\hline $10 / 20 / 92$ & 1115 & 0.25 & 193.25 & 276.9 & 32.4 & 4.5 & 11.9 & 16.2 \\
\hline $10 / 20 / 92$ & 1130 & 0.25 & 193.50 & 278.4 & 33.3 & 4.0 & 10.3 & 15.8 \\
\hline $10 / 20 / 92$ & 1145 & 0.25 & 193.75 & 278.3 & 31.3 & 3.9 & 10.6 & 15.7 \\
\hline $10 / 20 / 92$ & 1200 & 0.25 & 194.00 & 277.7 & 27.1 & 3.7 & 5.1 & 19.4 \\
\hline $10 / 20 / 92$ & 1215 & 0.25 & 194.25 & 283.6 & 28.7 & 4.1 & 6.6 & 17.8 \\
\hline $10 / 20 / 92$ & 1230 & 0.25 & 194.50 & 285.6 & 29.0 & 3.9 & 8.5 & 20.0 \\
\hline $10 / 20 / 92$ & 1245 & 0.25 & 194.75 & 282.3 & 27.7 & 3.9 & 8.0 & 19.9 \\
\hline $10 / 20 / 92$ & 1300 & 0.25 & 195.00 & 279.9 & 27.5 & 3.9 & 6.2 & 17.6 \\
\hline $10 / 20 / 92$ & 1315 & 0.25 & 195.25 & 280.6 & 25.9 & 4.0 & 9.3 & 19.6 \\
\hline $10 / 20 / 92$ & 1515 & 0.25 & 195.50 & 282.0 & 35.5 & 3.7 & 7.5 & 14.4 \\
\hline $10 / 20 / 92$ & 1530 & 0.25 & 195.75 & 282.4 & 35.5 & 3.3 & 5.7 & 16.3 \\
\hline $10 / 20 / 92$ & 1545 & 0.25 & 196.00 & 282.4 & 36.1 & 3.3 & 6.7 & 14.6 \\
\hline $10 / 20 / 92$ & 1600 & 0.25 & 196.25 & 283.9 & 36.6 & 2.7 & 9.4 & 16.5 \\
\hline $10 / 20 / 92$ & 1615 & 0.25 & 196.50 & 283.3 & 36.8 & 3.4 & 10.1 & 14.8 \\
\hline $10 / 20 / 92$ & 1630 & 0.25 & 196.75 & 282.5 & 36.3 & 5.2 & 8.6 & 14.2 \\
\hline $10 / 20 / 9^{\prime} c$ & 1645 & 0.25 & 197.00 & 281.6 & 35.7 & 5.1 & 10.8 & 20.2 \\
\hline $10 / 20 / 92$ & 1700 & 0.25 & 197.25 & 281.4 & 35.5 & 4.3 & 14.3 & 16.3 \\
\hline $10 / 20 / 92$ & 1715 & 0.25 & 197.50 & 282.0 & 35.6 & 4.5 & 10.5 & 18.5 \\
\hline $10 / 20 / 92$ & 1730 & 0.25 & 197.75 & 281.1 & 36.7 & 4.2 & 13.6 & 16.8 \\
\hline $10 / 20 / 92$ & 1745 & 0.25 & 198.00 & 280.3 & 35.0 & 4.1 & 12.3 & 15.3 \\
\hline $10 / 20 / 92$ & 1800 & 0.25 & 198.25 & 279.8 & 35.8 & 4.5 & 10.3 & 15.8 \\
\hline $10 / 20 / 92$ & 1815 & 0.25 & 198.50 & 281.8 & 36.7 & 4.7 & 12.1 & 14.9 \\
\hline $10 / 20 / 92$ & 1830 & 0.25 & 198.75 & 284.0 & 37.6 & 3.9 & 11.0 & 14.2 \\
\hline $10 / 20 / 92$ & 1845 & 0.25 & 199.00 & 283.3 & 37.4 & 4.3 & 11.9 & 15.1 \\
\hline $10 / 20 / 92$ & 1900 & 0.25 & 199.25 & 282.2 & 36.8 & 4.3 & 14.1 & 14.4 \\
\hline $10 / 20 / 92$ & 1915 & 0.25 & 199.50 & 283.3 & 36.6 & 4.3 & 13.6 & 14.0 \\
\hline $10 / 20 / 92$ & 1930 & 0.25 & 199.75 & 283.6 & 36.5 & 4.0 & 14.2 & 14.5 \\
\hline
\end{tabular}

Page E-4 B-Duct Opacity>> " = Lower, " = Higher 
SUMMARY OF LIME SLURRY INJECTION FROM OCTOBER 17, 1992 TO NOVEMBER 17, 1992

\begin{tabular}{|c|c|c|c|c|c|c|c|c|}
\hline Date & Time & Hours of & Cumulalive & B Duct Temp & Lime Flow & Slurry Solids & SO2 Removal & Stack Opacily \\
\hline & & Injection & Hours & ${ }^{\circ} \mathrm{F}$ & GPM & Percent & Percent & Percent \\
\hline $10 / 20 / 92$ & 1945 & 0.25 & 200.00 & 284.0 & 36.7 & 4.4 & 14.0 & 15.0 \\
\hline $10 / 20 / 92$ & 2000 & 0.25 & 200.25 & 283.7 & 36.3 & 4.3 & 14.9 & 15.9 \\
\hline $10 / 20 / 92$ & 2015 & 0.25 & 200.50 & 283.1 & 35.2 & 4.3 & 12.1 & 16.6 \\
\hline $10 / 20 / 92$ & 2030 & 0.25 & 200.75 & 283.3 & 35.3 & 4.3 & 14.0 & 13.3 \\
\hline $10 / 20 / 92$ & 2045 & 0.25 & 201.00 & 282.9 & 35.6 & 3.9 & 14.6 & 15.6 \\
\hline $10 / 20 / 92$ & 2100 & 0.25 & 201.25 & 282.3 & 34.9 & 4.2 & 15.4 & 13.5 \\
\hline $10 / 20 / 92$ & 2115 & 0.25 & 201.50 & 282.0 & 34.8 & 4.2 & 17.2 & 13.6 \\
\hline $10 / 20 / 92$ & 2130 & 0.25 & 201.75 & 281.4 & 34.0 & 3.9 & 18.9 & 14.0 \\
\hline $10 / 20 / 92$ & 2145 & 0.25 & 202.00 & 281.4 & 34.2 & 4.1 & $1 \% .1$ & 14.5 \\
\hline $10 / 20 / 92$ & 2200 & 0.25 & 202.25 & 281.3 & 33.3 & 4.3 & 14.4 & 12.9 \\
\hline $10 / 20 / 92$ & 2215 & 0.25 & 202.50 & 280.1 & 32.3 & 3.9 & 12.4 & 13.1 \\
\hline $10 / 20 / 92$ & 2230 & 0.25 & 202.75 & 278.8 & 31.7 & 4.2 & 14.7 & 11.7 \\
\hline $10 / 20 / 92$ & 2245 & 0.25 & 203.00 & 278.6 & 31.1 & 4.2 & 14.5 & 15.1 \\
\hline $10 / 20 / 92$ & 2300 & 0.25 & 203.25 & 276.9 & 31.6 & 4.3 & 13.3 & 14.7 \\
\hline $10 / 20 / 92$ & 2315 & 0.25 & 203.50 & 277.8 & 31.7 & 4.0 & 13.7 & 14.3 \\
\hline $10 / 20 / 92$ & 2330 & 0.25 & 203.75 & 275.9 & 27.6 & 4.1 & 16.8 & 15.4 \\
\hline $10 / 20 / 92$ & 2345 & 0.25 & 204.00 & 276.5 & 28.1 & 4.0 & 12.0 & 25.6 \\
\hline Total Hours & & 16.25 & & & & & & \\
\hline $10 / 21 / 92$ & $630^{\circ}$ & 0.25 & 204.25 & 276.7 & 26.4 & 4.1 & 9.9 & 10.5 \\
\hline $10 / 21 / 92$ & 645 & 0.25 & 204.50 & 283.1 & 37.8 & 4.5 & 9.3 & 12.7 \\
\hline $10 / 21 / 92$ & 700 & 0.25 & 204.75 & 283.1 & 37.4 & 4.6 & 10.6 & 9.5 \\
\hline $10 / 21 / 92$ & 715 & 0.25 & 205.00 & 282.2 & 36.7 & 4.1 & 8.4 & 10.5 \\
\hline $10 / 21 / 92$ & 730 & 0.25 & 205.25 & 281.1 & 36.0 & 4.2 & 10.4 & 12.6 \\
\hline $10 / 21 / 92$ & 745 & 0.25 & 205.50 & 281.8 & 36.0 & 3.9 & 8.6 & 12.2 \\
\hline $10 / 21 / 92$ & 800 & 0.25 & 205.75 & 284.2 & 37.0 & 3.9 & 10.7 & 12.1 \\
\hline $10 / 21 / 92$ & 815 & 0.25 & 206.00 & 283.8 & $37 . \overline{5}$ & 4.1 & 13.3 & 13.3 \\
\hline $10 / 21 / 92$ & 830 & 0.25 & 206.25 & 282.3 & 36.4 & 3.8 & 10.7 & 12.5 \\
\hline $10 / 21 / 92$ & 845 & 0.25 & 206.50 & 282.0 & 38.1 & 3.8 & 13.8 & 12.1 \\
\hline $10 / 21 / 92$ & 900 & 0.25 & 206.75 & 282.0 & 35.4 & 4.1 & 11.2 & 13.0 \\
\hline $10 / 21 / 92$ & 915 & 0.25 & 207.00 & 282.0 & 34.3 & 3.9 & 10.9 & 12.7 \\
\hline $10 / 21 / 92$ & 930 & 0.25 & 207.25 & 284.3 & 35.1 & 4.1 & 11.3 & 13.7 \\
\hline $10 / 21 / 92$ & 945 & 0.25 & 207.50 & 285.5 & 35.2 & 3.0 & 11.2 & 14.2 \\
\hline $10 / 21 / 92$ & 1000 & 0.25 & 207.75 & 287.3 & 35.4 & 2.9 & 9.0 & 13.9 \\
\hline $10 / 21 / 92$ & 1015 & 0.25 & 208.00 & 287.3 & 35.2 & 3.7 & 10.2 & 14.8 \\
\hline $10 / 21 / 92$ & 1030 & 0.25 & 208.25 & 286.7 & 34.6 & 4.5 & 10.4 & 16.8 \\
\hline $10 / 21 / 92$ & 1045 & 0.25 & 208.50 & 286.9 & 34.3 & 4.5 & 9.4 & 15.7 \\
\hline $10 / 21 / 92$ & 1100 & 0.25 & 208.75 & 286.1 & 39.0 & 4.9 & 13.3 & 14.1 \\
\hline $10 / 21 / 92$ & 1115 & 0.25 & 209.00 & 286.8 & 38.2 & 4.6 & 15.1 & 14.1 \\
\hline $10 / 21 / 92$ & 1130 & 0.25 & 209.25 & 287.1 & 37.9 & 4.3 & 13.9 & 14.3 \\
\hline $10 / 21 / 92$ & 1145 & 0.25 & 209.50 & 287.3 & 38.0 & 4.3 & 12.8 & 14.8 \\
\hline $10 / 21 / 92$ & 1200 & 0.25 & 209.75 & 287.4 & 38.1 & 4.6 & 15.7 & 12.7 \\
\hline $10 / 21 / 92$ & 1215 & 0.25 & 210.00 & 287.6 & 37.8 & 4.0 & 14.5 & 12.0 \\
\hline $10 / 21 / 92$ & 1230 & 0.25 & 210.25 & 289.0 & 37.1 & 4.1 & 15.0 & 12.8 \\
\hline $10 / 21 / 92$ & 1245 & 0.25 & 210.50 & 288.8 & 37.8 & 4.0 & 12.5 & 12.7 \\
\hline $10 / 21 / 92$ & 1300 & 0.25 & 210.75 & 288.1 & 37.9 & 3.9 & 13.2 & 13.9 \\
\hline $10 / 21 / 92$ & 1315 & 0.25 & 211.00 & 287.6 & 29.0 & 4.0 & 11.4 & 14.0 \\
\hline
\end{tabular}

Page E-5 B.Duct Opacitys> " = Lower, " = Higher 
SUMMARY OF LIME SLURRY INJECTION FROM OCTOBER 17, 1992 TO NOVEMBER 17, 1992

\begin{tabular}{|c|c|c|c|c|c|c|c|c|}
\hline Date & Time & Hours of & Cumulative & B Duat Temp & Lime Flow & Slurry Solids & SO2 Removal & Stack Opacity \\
\hline & & Injection & Hours & ${ }^{\circ} \mathrm{F}$ & GPM & Percent & Percent & Percent \\
\hline $10 / 21 / 92$ & 1330 & 0.25 & 211.25 & 286.9 & 31.1 & 4.2 & 9.5 & 14.7 \\
\hline $10 / 21 / 92$ & 1345 & 0.25 & 211.50 & 285.7 & 30.6 & 4.1 & 7.1 & 15.3 \\
\hline $10 / 21 / 92$ & 1615 & 0.25 & 211.75 & 286.2 & 36.2 & 3.8 & 3.1 & 12.8 \\
\hline $10 / 21 / 92$ & 1630 & 0.25 & 212.00 & 286.4 & 35.0 & 4.3 & 6.6 & 11.7 \\
\hline $10 / 21 / 92$ & 1645 & 0.25 & 212.25 & 285.5 & 30.6 & 4.2 & 9.8 & 16.5 \\
\hline $10 / 21 / 92$ & 1700 & 0.25 & 212.50 & 284.7 & 27.0 & 4.4 & 9.0 & 17.2 \\
\hline $10 / 21 / 92$ & 1800 & 0.25 & 212.75 & 284.3 & 27.8 & 5.2 & 12.9 & 19.0 \\
\hline $10 / 21 / 92$ & 1815 & 0.25 & 213.00 & 285.1 & 33.0 & 4.9 & 7.4 & 13.7 \\
\hline $10 / 21 / 92$ & 1830 & 0.25 & 213.25 & 286.9 & 34.1 & 4.4 & 10.6 & 14.5 \\
\hline $10 / 21 / 92$ & 1845 & 0.25 & 213.50 & 286.0 & 33.4 & 4.4 & 11.0 & 15.9 \\
\hline $10 / 21 / 92$ & 1900 & 0.25 & 213.75 & 285.8 & 33.1 & 4.8 & 12.1 & 14.7 \\
\hline $10 / 21 / 92$ & 1915 & 0.25 & 214.00 & 286.7 & 34.1 & 4.4 & 12.1 & 15.4 \\
\hline $10 / 21 / 92$ & 1930 & 0.25 & 214.25 & 285.9 & 34.0 & 4.4 & 13.0 & 16.2 \\
\hline $10 / 21 / 92$ & 1945 & 0.25 & 214.50 & 286.1 & 33.9 & 4.2 & 12.8 & 13.9 \\
\hline $10 / 21 / 92$ & 2000 & 0.25 & 214.75 & 285.3 & 34.1 & 4.3 & 10.5 & 13.5 \\
\hline $10 / 21 / 92$ & 2015 & 0.25 & 215.00 & 285.0 & 34.0 & 4.3 & 13.3 & 13.9 \\
\hline $10 / 21 / 92$ & 2030 & 0.25 & 215.25 & 285.3 & 34.0 & 4.3 & 12.3 & 13.8 \\
\hline $10 / 21 / 92$ & 2045 & 0.25 & 215.50 & 286.2 & 34.7 & 4.1 & 13.7 & 15.8 \\
\hline $10 / 21 / 92$ & 2100 & 0.25 & 215.75 & 286.1 & 35.2 & 4.6 & 13.1 & 13.8 \\
\hline $10 / 21 / 92$ & 2115 & 0.25 & 216.00 & 285.7 & 35.8 & 4.5 & 15.1 & 13.1 \\
\hline $10 / 21 / 92$ & 2130 & 0.25 & 216.25 & 286.1 & 36.1 & 4.6 & 13.5 & 15.2 \\
\hline $10 / 21 / 92$ & 2145 & 0.25 & 216.50 & 287.3 & 36.8 & 4.4 & 15.7 & 13.2 \\
\hline $10 / 21 / 92$ & 2200 & 0.25 & 216.75 & 283.0 & 34.3 & 4.3 & 16.3 & 15.6 \\
\hline $10 / 21 / 92$ & 2215 & 0.25 & 217.00 & 281.9 & 31.5 & 4.3 & 16.4 & 13.4 \\
\hline $10 / 21 / 92$ & 2230 & 0.25 & 217.25 & 282.4 & 31.9 & 4.1 & 14.6 & 14.6 \\
\hline $10 / 21 / 92$ & 2245 & 0.25 & 217.50 & 281.4 & 32.8 & 4.0 & 15.4 & 13.5 \\
\hline $10 / 21 / 92$ & 2300 & 0.25 & 217.75 & 279.8 & 32.7 & 4.5 & 15.6 & 20.8 \\
\hline $10 / 21 / 92$ & 2315 & 0.25 & 218.00 & 280.3 & 33.5 & 3.9 & 13.0 & 12.7 \\
\hline $10 / 21 / 92$ & 2330 & 0.25 & 218.25 & 279.4 & 33.6 & 4.2 & 14.7 & 12.3 \\
\hline $10 / 21 / 92$ & 2345 & 0.25 & 218.50 & 279.3 & 34.4 & 4.2 & 14.6 & 17.2 \\
\hline \multicolumn{2}{|l|}{ Total Hours } & 14.50 & & & & & & \\
\hline $10 / 22 / 92$ & 0 & 0.25 & 218.75 & 279.3 & 34.6 & 4.2 & 14.6 & 12.8 \\
\hline $10 / 22 / 92$ & 15 & 0.25 & 219.00 & 280.0 & 35.4 & 4.2 & 14.1 & 14.1 \\
\hline $10 / 22 / 92$ & 30 & 0.25 & 219.25 & 280.3 & 35.8 & 4.2 & 12.3 & 14.1 \\
\hline $10 / 22 / 92$ & 45 & 0.25 & 219.50 & 281.0 & 37.4 & 4.2 & 9.9 & 14.7 \\
\hline $10 / 22 / 92$ & 100 & 0.25 & 219.75 & 280.8 & 38.1 & 4.2 & 11.2 & 15.5 \\
\hline $10 / 22 / 92$ & 115 & 0.25 & 220.00 & 279.5 & 35.2 & 4.2 & 10.4 & 12.2 \\
\hline $10 / 22 / 92$ & 130 & 0.25 & 220.25 & 278.9 & 29.1 & 4.2 & 12.9 & 10.9 \\
\hline $10 / 22 / 92$ & 145 & 0.25 & 220.50 & 276.6 & 27.6 & 4.2 & 11.3 & 12.2 \\
\hline $10 / 22 / 92$ & 200 & 0.25 & 220.75 & 276.8 & 28.1 & 4.2 & 11.5 & 12.1 \\
\hline $10 / 22 / 92$ & 215 & 0.25 & 221.00 & 276.7 & 27.9 & 4.2 & 10.9 & 12.0 \\
\hline $10 / 22 / 92$ & 230 & 0.25 & 221.25 & 277.8 & 19.0 & 4.2 & 8.6 & $\because 31.7$ \\
\hline $10 / 22 / 92$ & 245 & 0.25 & 221.50 & 276.5 & 28.0 & 4.2 & 11.3 & 9.6 \\
\hline $10 / 22 / 92$ & 300 & 0.25 & 221.75 & 277.4 & 28.1 & 4.2 & 11.2 & 10.1 \\
\hline $10 / 22 / 92$ & 315 & 0.25 & 222.00 & 277.4 & 34.0 & 4.2 & 13.7 & 30.8 \\
\hline $10 / 22 / 92$ & 330 & 0.25 & 222.25 & 276.2 & 27.7 & 4.2 & 11.5 & 12.0 \\
\hline
\end{tabular}

Page E-6 B.Duct Opacity>> ". Lower, ". " Hlgher 
SUMMARY OF LIME SLURRY INJECTION FROM OCTOBER 17, 1992 TO NOVEMBER 17, 1992

\begin{tabular}{|c|c|c|c|c|c|c|c|c|}
\hline Date & Time & Hours of & Cumulative & B Ducl Temp & Lime Flow & Slurry Solids & SO2 Removal & Stack Opacily \\
\hline & & Injection & Hours & ${ }^{\circ} \mathrm{F}$ & GPM & Percent & Porcent & Percent \\
\hline $10 / 22 / 92$ & 345 & 0.25 & 222.50 & 280.0 & 31.0 & 4.2 & 8.7 & 14.4 \\
\hline $10 / 22 / 92$ & 400 & 0.25 & 222.75 & 281.7 & 31.2 & 4.2 & 10.3 & 17.6 \\
\hline $10 / 22 / 92$ & 415 & 0.25 & 223.00 & 281.5 & 34.3 & 4.2 & 11.5 & 14.4 \\
\hline $10 / 22 / 92$ & 430 & 0.25 & 223.25 & 281.7 & 34.6 & 4.2 & 11.1 & 13.1 \\
\hline $10 / 22 / 92$ & 445 & 0.25 & 223.50 & 281.3 & 35.3 & 4.2 & 13.8 & 31.2 \\
\hline $10 / 22 / 92$ & 500 & 0.25 & 223.75 & 282.6 & 35.5 & 4.2 & 12.1 & 13.1 \\
\hline $10 / 22 / 92$ & 515 & 0.25 & 224.00 & 283.5 & 36.4 & 4.2 & 15.0 & 13.0 \\
\hline $10 / 22 / 92$ & 530 & 0.25 & 224.25 & 283.1 & 36.0 & 4.2 & 16.9 & 11.9 \\
\hline $10 / 22 / 92$ & 545 & 0.25 & 224.50 & 282.8 & 35.2 & 4.2 & 14.0 & 13.0 \\
\hline $10 / 22 / 92$ & 600 & 0.25 & 224.75 & 282.8 & 36.9 & 4.2 & 13.5 & 13.3 \\
\hline $10 / 22 / 92$ & 615 & 0.25 & 225.00 & 282.8 & 37.1 & 4.2 & 15.8 & 14.3 \\
\hline $10 / 22 / 92$ & 630 & 0.25 & 225.25 & 282.8 & 35.9 & 4.2 & 17.0 & 15.7 \\
\hline $10 / 22 / 92$ & 645 & 0.25 & 225.50 & 280.6 & 29.1 & 4.2 & 10.6 & 16.5 \\
\hline $10 / 22 / 92$ & 700 & 0.25 & 225.75 & 278.2 & 30.9 & 4.2 & 16.2 & 11.0 \\
\hline $10 / 22 / 92$ & 715 & 0.25 & 226.00 & 277.8 & 30.2 & 4.2 & 12.6 & 15.9 \\
\hline $10 / 22 / 92$ & 730 & 0.25 & 226.25 & 277.3 & 28.2 & 4.2 & 12.1 & 15.1 \\
\hline $10 / 22 / 92$ & 745 & 0.25 & 226.50 & 276.9 & 28.9 & 4.2 & 12.0 & 15.0 \\
\hline $10 / 22 / 92$ & 800 & 0.25 & 226.75 & 278.4 & 28.5 & 4.0 & 11.1 & 16.4 \\
\hline $10 / 22 / 92$ & 815 & 0.25 & 227.00 & 277.6 & 29.1 & 3.8 & 10.2 & 16.6 \\
\hline $10 / 22 / 92$ & 830 & 0.25 & 227.25 & 278.3 & 32.1 & 4.0 & 12.5 & 13.5 \\
\hline $10 / 22 / 92$ & 845 & 0.25 & 227.50 & 276.9 & 30.3 & 4.1 & 13.7 & 13.7 \\
\hline $10 / 22 / 92$ & 900 & 0.25 & 227.75 & 277.7 & 29.0 & 4.4 & 12.5 & 12.7 \\
\hline $10 / 22 / 92$ & 915 & 0.25 & 228.00 & 277.7 & 29.0 & 4.4 & 12.5 & 12.7 \\
\hline $10 / 22 / 92$ & 930 & 0.25 & 228.25 & 277.7 & 29.0 & 4.4 & 12.5 & 12.7 \\
\hline $10 / 22 / 92$ & 945 & 0.25 & 228.50 & 277.9 & 30.3 & 3.9 & 11.9 & 13.7 \\
\hline $10 / 22 / 92$ & 1000 & 0.25 & 228.75 & 278.5 & 31.7 & 4.1 & 11.3 & 15.9 \\
\hline $10 / 22 / 92$ & 1015 & 0.25 & 229.00 & 279.4 & 31.7 & 3.8 & 10.6 & 10.9 \\
\hline $10 / 22 / 92$ & 1045 & 0.25 & 229.25 & 279.1 & 31.7 & 4.2 & 11.0 & 10.5 \\
\hline $10 / 22 / 92$ & 1100 & 0.25 & 229.50 & 279.8 & 32.2 & 3.8 & 11.1 & 9.9 \\
\hline $10 / 22 / 92$ & 1115 & 0.25 & 229.75 & 280.2 & 32.7 & 4.1 & 11.5 & 7.1 \\
\hline $10 / 22 / 92$ & 1130 & 0.25 & 230.00 & 281.3 & 27.0 & 4.0 & 11.9 & 7.9 \\
\hline $10 / 22 / 92$ & 1145 & 0.25 & 230.25 & 281.3 & 27.1 & 4.5 & 10.2 & 11.1 \\
\hline $10 / 22 / 92$ & 1200 & 0.25 & 230.50 & 282.6 & 26.6 & 4.1 & 7.6 & 11.0 \\
\hline $10 / 22 / 92$ & 1215 & 0.25 & 230.75 & 283.1 & 26.7 & 4.2 & 8.2 & 11.1 \\
\hline $10 / 22 / 92$ & 1230 & 0.25 & 231.00 & 284.6 & 31.1 & 4.0 & 9.1 & 19.3 \\
\hline $10 / 22 / 92$ & 1415 & 0.25 & 231.25 & 286.6 & 34.8 & 3.8 & 12.2 & 14.8 \\
\hline $10 / 22 / 92$ & 1430 & 0.25 & 231.50 & 287.0 & 35.4 & 3.7 & 11.2 & 15.2 \\
\hline $10 / 22 / 92$ & 1445 & 0.25 & 231.75 & 287.4 & 35.0 & 2.8 & 11.8 & 12.0 \\
\hline $10 / 22 / 92$ & 1500 & 0.25 & 232.00 & 288.0 & 34.2 & 2.9 & 10.4 & 13.7 \\
\hline $10 / 22 / 92$ & 1515 & 0.25 & 232.25 & 288.0 & 34.4 & 2.4 & 10.3 & 13.4 \\
\hline $10 / 22 / 92$ & 1530 & 0.25 & 232.50 & 289.1 & 31.5 & 4.1 & 12.1 & 14.0 \\
\hline $10 / 22 / 92$ & 1545 & 0.25 & 232.75 & 288.9 & 36.2 & 4.1 & 12.4 & 13.6 \\
\hline $10 / 22 / 92$ & 1600 & 0.25 & 233.00 & 288.8 & 35.9 & 4.0 & 14.3 & 12.4 \\
\hline $10 / 22 / 92$ & 1615 & 0.25 & 233.25 & 289.7 & 36.3 & 4.2 & 14.5 & 15.3 \\
\hline $10 / 22 / 92$ & 1630 & 0.25 & 233.50 & 292.0 & 37.0 & 3.6 & 13.6 & 14.2 \\
\hline $10 / 22 / 92$ & 1645 & 0.25 & 233.75 & 291.3 & 37.7 & 4.1 & 17.7 & 11.2 \\
\hline $10 / 22 / 92$ & 1700 & 0.25 & 234.00 & 289.4 & 35.5 & 4.1 & 16.6 & 11.1 \\
\hline
\end{tabular}

Page E-7 B-Duct Opacitys> " - Lower, "- - Higher 
SUMMARY OF LIME SLURRY INJECTION FROM OCTOBER 17, 1992 TO NOVEMBER 17, 1992

\begin{tabular}{|c|c|c|c|c|c|c|c|c|}
\hline Date & Time & Hours of & Cumulative & B Duct Temp & Lime Flow & Slurry Solids & SO2 Removal & Slack Opacity \\
\hline & & Injection & Hours & ${ }^{\circ} \mathrm{F}$ & GPM & Percent & Percent & Percent \\
\hline $10 / 22 / 92$ & 1715 & 0.25 & 234.25 & 289.1 & 36.9 & 3.8 & 14.4 & 12.6 \\
\hline $10 / 22 / 92$ & 1730 & 0.25 & 234.50 & 288.3 & 36.7 & 4.2 & 14.8 & 15.2 \\
\hline $10 / 22 / 92$ & 1745 & 0.25 & 234.75 & 287.7 & 37.2 & 4.4 & 15.9 & 14.3 \\
\hline $10 / 22 / 92$ & 1800 & 0.25 & 235.00 & 286.4 & 34.4 & 4.0 & 15.4 & 14.9 \\
\hline $10 / 22 / 92$ & 1815 & 0.25 & 235.25 & 286.2 & 35.4 & 4.2 & 12.3 & 11.2 \\
\hline $10 / 22 / 92$ & 1830 & 0.25 & 235.50 & 285.6 & 35.8 & 3.9 & 15.8 & 11.8 \\
\hline $10 / 22 / 92$ & 1845 & 0.25 & 235.75 & 284.9 & 35.9 & 4.5 & 14.8 & 12.3 \\
\hline $10 / 22 / 92$ & 1900 & 0.25 & 236.00 & 284.4 & 34.7 & 4.1 & 14.6 & 15.8 \\
\hline $10 / 22 / 92$ & 1915 & 0.25 & 236.25 & 285.0 & 36.6 & 4.2 & $16 . \overline{6}$ & 11.8 \\
\hline $10 / 22 / 92$ & 1930 & 0.25 & 236.50 & 284.1 & 35.9 & 3.8 & 17.1 & 14.1 \\
\hline $10 / 22 / 92$ & 1945 & 0.25 & 236.75 & 283.1 & 35.2 & 3.8 & 13.2 & 12.7 \\
\hline $10 / 22 / 92$ & 2000 & 0.25 & 237.00 & 282.1 & 35.1 & 4.4 & 14.2 & 14.4 \\
\hline $10 / 22 / 92$ & 2015 & 0.25 & 237.25 & 281.8 & 35.1 & 4.0 & 15.3 & 10.3 \\
\hline $10 / 22 / 92$ & 2030 & 0.25 & 237.50 & 281.6 & 35.2 & 4.2 & 15.8 & 13.8 \\
\hline $10 / 22 / 92$ & 2045 & 0.25 & 237.75 & 281.8 & 38.2 & 4.0 & 16.7 & 14.7 \\
\hline $10 / 22 / 92$ & 2100 & 0.25 & 238.00 & 279.8 & 35.6 & 4.2 & 16.1 & 13.4 \\
\hline $10 / 22 / 92$ & 2115 & 0.25 & 238.25 & 279.8 & 35.2 & 4.1 & 16.3 & 12.3 \\
\hline $10 / 22 / 92$ & 2130 & 0.25 & 238.50 & 280.0 & 33.9 & 4.2 & 16.9 & 17.5 \\
\hline $10 / 22 / 92$ & 2145 & 0.25 & 238.75 & 279.4 & 32.8 & 4.0 & 13.1 & 11.6 \\
\hline $10 / 22 / 92$ & 2200 & 0.25 & 239.00 & 279.1 & 33.6 & 3.7 & 15.2 & 21.7 \\
\hline $10 / 22 / 92$ & 2215 & 0.25 & 239.25 & 280.0 & 33.2 & 4.2 & 14.0 & 13.2 \\
\hline $10 / 22 / 92$ & 2230 & 0.25 & 239.50 & 279.9 & 33.9 & 4.3 & 14.6 & 11.8 \\
\hline $10 / 22 / 92$ & 2245 & 0.25 & 239.75 & 279.3 & 32.7 & 4.1 & 13.7 & 10.6 \\
\hline $10 / 22 / 92$ & 2300 & 0.25 & 240.00 & 279.3 & 32.7 & 4.3 & 13.7 & 13.2 \\
\hline $10 / 22 / 92$ & 2315 & 0.25 & 240.25 & 279.3 & 36.7 & 4.2 & 12.5 & 13.8 \\
\hline $10 / 22 / 92$ & 2330 & 0.25 & 240.50 & 277.9 & 31.3 & 4.3 & 15.4 & 11.0 \\
\hline $10 / 22 / 92$ & 2345 & 0.25 & 240.75 & 277.6 & 32.7 & 3.8 & 11.7 & 14.4 \\
\hline Total Hours & & 22.25 & & & & & & \\
\hline $10 / 23 / 92$ & 0 & 0.25 & 241.00 & 277.7 & 31.6 & 4.1 & 13.3 & 12.5 \\
\hline $10 / 23 / 92$ & 15 & 0.25 & 241.25 & 278.6 & 29.6 & 4.2 & 4.9 & 11.8 \\
\hline $10 / 23 / 92$ & 30 & 0.25 & 241.50 & 278.7 & 30.3 & 3.9 & 0.1 & 20.0 \\
\hline $10 / 23 / 92$ & 45 & 0.25 & 241.75 & 276.9 & 30.2 & 4.2 & 9.2 & 12.3 \\
\hline $10 / 23 / 92$ & 100 & 0.25 & 242.00 & 278.9 & 30.4 & 4.1 & 10.1 & 15.7 \\
\hline $10 / 23 / 92$ & 115 & 0.25 & 242.25 & 278.7 & 29.6 & 4.1 & 8.8 & 11.7 \\
\hline $10 / 23 / 92$ & 130 & 0.25 & 242.50 & 277.3 & 29.1 & 4.4 & 9.0 & 15.6 \\
\hline $10 / 23 / 92$ & 600 & 0.25 & 242.75 & 276.8 & 28.4 & 5.5 & 9.4 & 14.6 \\
\hline $10 / 23 / 92$ & 615 & 0.25 & 243.00 & 276.6 & 27.9 & 5.1 & 9.0 & 15.6 \\
\hline $10 / 23 / 92$ & 630 & 0.25 & 243.25 & 276.6 & 30.3 & 4.8 & 8.9 & 13.8 \\
\hline $10 / 23 / 92$ & 645 & 0.25 & 243.50 & 277.4 & 30.5 & 4.8 & 7.9 & 13.5 \\
\hline $10 / 23 / 92$ & 700 & 0.25 & 243.75 & 277.6 & 30.5 & 4.7 & 11.0 & 14.2 \\
\hline $10 / 23 / 92$ & 715 & 0.25 & 244.00 & 277.6 & 30.6 & 4.6 & 10.1 & 14.6 \\
\hline $10 / 23 / 92$ & 730 & 0.25 & 244.25 & 277.6 & 31.1 & 4.3 & 12.8 & 16.5 \\
\hline $10 / 23 / 92$ & 745 & 0.25 & 244.50 & 278.0 & 31.6 & 4.7 & 11.8 & 12.8 \\
\hline $10 / 23 / 92$ & 800 & 0.25 & 244.75 & 278.2 & 31.4 & 4.5 & 12.2 & 14.9 \\
\hline $10 / 23 / 92$ & 815 & 0.25 & 245.00 & 278.2 & 31.4 & 4.5 & 12.2 & 14.9 \\
\hline $10 / 23 / 92$ & 830 & 0.25 & 245.25 & 277.9 & 35.2 & 4.2 & 13.7 & 12.4 \\
\hline
\end{tabular}

Page E-8 B-Duct Opacltys> " Lower, "- Hlgher 
SUMMARY OF LIME SLURRY INJECTION FROM OCTOBER 17, 1992 TO NOVEMBER 17, 1992

\begin{tabular}{|c|c|c|c|c|c|c|c|c|}
\hline Date & Time & Hours of & Cumulative & B Duct Temp & Lime Flow & Slurry Solids & SO2 Removal & Stack Opacily \\
\hline & & Injection & Hours & ${ }^{\circ} \mathrm{F}$ & GPM & Percent & Percent & Percent \\
\hline $10 / 23 / 92$ & 845 & 0.25 & 245.50 & 277.4 & 34.7 & 4.2 & 15.4 & 13.1 \\
\hline $10 / 23 / 92$ & 900 & 0.25 & 245.75 & 276.9 & 34.5 & 4.2 & 15.1 & 17.3 \\
\hline $10 / 23 / 92$ & 915 & 0.25 & 246.00 & 277.3 & 34.1 & 4.1 & 13.8 & 12.1 \\
\hline $10 / 23 / 92$ & 930 & 0.25 & 246.25 & 276.7 & 34.1 & 3.8 & 14.3 & 16.4 \\
\hline $10 / 23 / 92$ & 945 & 0.25 & 246.50 & 277.8 & 34.4 & 4.0 & 12.6 & 14.3 \\
\hline $10 / 23 / 92$ & 1000 & 0.25 & 246.75 & 278.0 & 34.5 & 4.1 & 15.1 & 13.3 \\
\hline $10 / 23 / 92$ & 1015 & 0.25 & 247.00 & 278.3 & 34.6 & 3.9 & 12.2 & 14.2 \\
\hline $10 / 23 / 92$ & 1030 & 0.25 & 247.25 & 279.0 & 33.8 & 3.7 & 15.1 & 12.3 \\
\hline $10 / 23 / 92$ & 1045 & 0.25 & 247.50 & 278.9 & 33.6 & 3.0 & 11.7 & 13.5 \\
\hline $10 / 23 / 92$ & 1100 & 0.25 & 247.75 & 280.0 & 35.8 & 2.6 & 16.2 & 14.1 \\
\hline $10 / 23 / 92$ & 1115 & 0.25 & 248.00 & 281.5 & 35.9 & 4.8 & 14.3 & 16.1 \\
\hline $10 / 23 / 92$ & 1130 & 0.25 & 248.25 & 282.8 & 36.2 & 4.2 & 12.9 & 14.2 \\
\hline $10 / 23 / 92$ & 1145 & 0.25 & 248.50 & 283.7 & 36.3 & 4.6 & 16.5 & 17.8 \\
\hline $10 / 23 / 92$ & 1200 & 0.25 & 248.75 & 284.1 & 36.2 & 4.0 & 14.4 & 13.1 \\
\hline $10 / 23 / 92$ & 1215 & 0.25 & 249.00 & 284.5 & 34.9 & 4.4 & 12.9 & 14.5 \\
\hline $10 / 23 / 92$ & 1230 & 0.25 & 249.25 & 285.2 & 33.6 & 4.1 & 12.5 & 14.1 \\
\hline $10 / 23 / 92$ & 1245 & 0.25 & 249.50 & 286.4 & 35.6 & 4.3 & 14.9 & 12.5 \\
\hline $10 / 23 / 92$ & 1300 & 0.25 & 249.75 & 288.2 & 36.4 & 3.8 & 14.9 & 14.0 \\
\hline $10 / 23 / 92$ & 1315 & 0.25 & 250.00 & 289.4 & 38.1 & 4.3 & 13.8 & 14.6 \\
\hline $10 / 23 / 92$ & 1330 & 0.25 & 250.25 & 289.9 & 38.6 & 3.9 & 16.0 & 16.8 \\
\hline $10 / 23 / 92$ & 1345 & 0.25 & 250.50 & 290.7 & 38.5 & 4.4 & 16.3 & 13.0 \\
\hline $10 / 23 / 92$ & 1400 & 0.25 & 250.75 & 290.7 & 38.5 & 4.4 & 16.3 & 13.0 \\
\hline $10 / 23 / 92$ & 1415 & 0.25 & 251.00 & 290.7 & 38.5 & 4.4 & 16.3 & 13.0 \\
\hline $10 / 23 / 92$ & 1430 & 0.25 & 251.25 & 290.7 & 38.5 & 4.4 & 16.3 & 13.0 \\
\hline $10 / 23 / 92$ & 1445 & 0.25 & 251.50 & 291.2 & 40.8 & 4.1 & 18.3 & 13.8 \\
\hline $10 / 23 / 92$ & 1500 & 0.25 & 251.75 & 290.7 & 38.5 & 4.4 & 16.3 & 13.0 \\
\hline $10 / 23 / 92$ & 1515 & 0.25 & 252.00 & 290.7 & 38.5 & 4.4 & 16.3 & 13.0 \\
\hline $10 / 23 / 92$ & 1530 & 0.25 & 252.25 & 290.7 & 38.5 & 4.4 & 16.3 & 13.0 \\
\hline $10 / 23 / 92$ & 1545 & 0.25 & 252.50 & 292.0 & 37.8 & 3.9 & 14.3 & 15.5 \\
\hline $10 / 23 / 92$ & 1600 & 0.25 & 252.75 & 291.7 & 33.6 & 4.3 & 13.5 & 16.5 \\
\hline $10 / 23 / 92$ & 1615 & 0.25 & 253.00 & 291.4 & 34.1 & 4.4 & 12.7 & 13.0 \\
\hline $10 / 23 / 92$ & 1630 & 0.25 & 253.25 & 291.0 & 36.2 & 4.4 & 11.9 & 11.8 \\
\hline $10 / 23 / 92$ & 1645 & 0.25 & 253.50 & 291.1 & 36.8 & 4.4 & 12.8 & 12.1 \\
\hline $10 / 23 / 92$ & 1700 & 0.25 & 253.75 & 291.8 & 36.9 & 4.4 & 12.1 & 13.0 \\
\hline $10 / 23 / 92$ & 1715 & 0.25 & 254.00 & 291.0 & 36.9 & 4.1 & 14.7 & 13.3 \\
\hline $10 / 23 / 92$ & 1730 & 0.25 & 254.25 & 290.0 & 31.6 & 4.3 & 8.1 & 15.1 \\
\hline $10 / 23 / 92$ & 1745 & 0.25 & 254.50 & 288.9 & 33.0 & 4.3 & 15.3 & 13.6 \\
\hline $10 / 23 / 92$ & 1800 & 0.25 & 254.75 & 290.0 & 34.8 & 4.2 & 12.7 & 12.6 \\
\hline $10 / 23 / 92$ & 1815 & 0.25 & 255.00 & 287.7 & 30.5 & 4.0 & 11.0 & 14.9 \\
\hline $10 / 23 / 92$ & 1830 & 0.25 & 255.25 & 287.1 & 30.8 & 3.8 & 9.0 & 17.2 \\
\hline $10 / 23 / 82$ & 1845 & 0.25 & 255.50 & 285.5 & 30.3 & 3.9 & 7.4 & 13.5 \\
\hline $10 / 23 / 92$ & 1900 & 0.25 & 255.75 & 286.0 & 31.7 & 4.0 & 9.6 & 15.1 \\
\hline $10 / 23 / 92$ & 1915 & 0.25 & 256.00 & 285.0 & 30.9 & 4.0 & 11.6 & 15.2 \\
\hline $10 / 23 / 92$ & 1830 & 0.25 & 256.25 & 285.0 & 30.4 & 4.1 & 5.7 & 16.6 \\
\hline $10 / 23 / 92$ & 1945 & 0.25 & 256.50 & 282.5 & 27.7 & 3.9 & 5.8 & 13.0 \\
\hline $10 / 23 / 92$ & 2000 & 0.25 & 256.75 & 281.4 & 27.9 & 4.2 & 5.2 & 15.2 \\
\hline $10 / 23 / 92$ & 2015 & 0.25 & 257.00 & 279.8 & 29.0 & 4.4 & 2.8 & 16.8 \\
\hline
\end{tabular}

Page E-9 B.Duct Opacity>> " - Lower, " - Higher 
SUMMARY OF LIME SLURAY INJECTION FROM OCTOBER 17, 1992 TO NOVEMBER 17, 1992

\begin{tabular}{|c|c|c|c|c|c|c|c|c|}
\hline Date & Time & Hours of & Cumulative & B Duct Temp & Lime Flow & Slurry Solids & SO2 Removal & Stack Opacily \\
\hline & & Injection & Hours & ${ }^{\circ} \mathrm{F}$ & GPM & Percent & Percent & Percent \\
\hline $10 / 23 / 92$ & 2030 & 0.25 & 257.25 & 280.7 & 28.1 & 4.1 & 12.1 & 14.7 \\
\hline $10 / 23 / 92$ & 2045 & 0.25 & 257.50 & 281.6 & 30.5 & 4.4 & 8.9 & 15.4 \\
\hline $10 / 23 / 92$ & 2100 & 0.25 & 257.75 & 281.0 & 32.5 & 4.1 & 6.7 & 14.4 \\
\hline $10 / 23 / 92$ & 2115 & 0.25 & 258.00 & 280.9 & 34.5 & 3.9 & 9.5 & 12.8 \\
\hline $10 / 23 / 92$ & 2130 & 0.25 & 258.25 & 279.9 & 32.8 & 3.9 & 14.5 & 14.2 \\
\hline $10 / 23 / 92$ & 2145 & 0.25 & 258.50 & 280.0 & 33.8 & 4.2 & 14.8 & 14.8 \\
\hline $10 / 23 / 92$ & 2200 & 0.25 & 258.75 & 279.9 & 32.4 & 4.5 & 14.6 & 14.6 \\
\hline $10 / 23 / 92$ & 2215 & 0.25 & 259.00 & 279.9 & 35.2 & 4.2 & 11.0 & 14.7 \\
\hline $10 / 23 / 92$ & 2230 & 0.25 & 259.25 & 279.6 & 32.6 & 3.7 & 9.8 & 13.9 \\
\hline $10 / 23 / 92$ & 2245 & 0.25 & 259.50 & 279.4 & 32.5 & 4.1 & 10.6 & 15.1 \\
\hline $10 / 23 / 92$ & 2300 & 0.25 & 259.75 & 279.0 & 32.4 & 4.2 & 10.9 & 16.8 \\
\hline \multicolumn{2}{|l|}{ Total Hours } & 19.00 & & & & & & \\
\hline $11 / 2 / 92$ & 1615 & 0.25 & 260.00 & 280.1 & 26.9 & 6.2 & 10.4 & 12.3 \\
\hline $11 / 2 / 92$ & 1630 & 0.25 & 260.25 & 281.5 & 31.3 & 6.1 & 9.7 & 11.8 \\
\hline $11 / 2 / 92$ & 1645 & 0.25 & 260.50 & 281.7 & 31.7 & 5.9 & 10.5 & 11.7 \\
\hline $11 / 2 / 92$ & 1700 & 0.25 & 260.75 & 283.4 & 30.8 & 5.1 & 12.6 & 12.9 \\
\hline $11 / 2 / 92$ & 1715 & 0.25 & 261.00 & 280.6 & 29.7 & 5.7 & 10.2 & 12.6 \\
\hline $11 / 2 / 92$ & 1730 & 0.25 & 261.25 & 278.8 & 30.7 & 5.8 & 11.9 & 11.7 \\
\hline $11 / 2 / 92$ & 1745 & 0.25 & 261.50 & 282.0 & 33.3 & 5.4 & 14.8 & 11.7 \\
\hline $11,2 / 92$ & 1800 & 0.25 & 261.75 & 282.9 & 34.6 & 5.4 & 12.4 & 12.0 \\
\hline $11 / 2 / 92$ & 1815 & 0.25 & 262.00 & 280.2 & 34.2 & 4.8 & 18.1 & 14.6 \\
\hline $11 / 2 / 92$ & 1830 & 0.25 & 262.25 & 282.1 & 33.0 & 5.7 & 14.8 & 13.2 \\
\hline $11 / 2 / 92$ & 1845 & 0.25 & 262.50 & 283.7 & 35.2 & 5.3 & 20.5 & 13.1 \\
\hline $11 / 2 / 92$ & 1900 & 0.25 & 262.75 & 282.7 & 33.2 & 5.1 & 16.8 & 13.3 \\
\hline $11 / 2 / 92$ & 1915 & 0.25 & 263.00 & 282.1 & 33.9 & 5.4 & 18.6 & 12.4 \\
\hline $11 / 2 / 92$ & 1930 & 0.25 & 263.25 & 283.5 & 33.3 & 4.7 & 13.7 & 17.6 \\
\hline $11 / 2 / 92$ & 1945 & 0.25 & 263.50 & 281.3 & 33.3 & 4.9 & 14.9 & 14.8 \\
\hline $11 / 2 / 92$ & 2000 & 0.25 & 263.75 & 282.2 & 34.4 & 5.2 & 17.8 & 12.9 \\
\hline $11 / 2 / 92$ & 2015 & 0.25 & 264.00 & 283.5 & 34.1 & 5.4 & 13.5 & 14.0 \\
\hline $11 / 2 / 92$ & 2030 & 0.25 & 264.25 & 281.4 & 33.2 & 4.8 & 14.5 & 13.8 \\
\hline $11 / 2 / 92$ & 2045 & 0.25 & 264.50 & 278.1 & 34.1 & 5.2 & 18.3 & 14.5 \\
\hline $11 / 2 / 92$ & 2100 & 0.25 & 264.75 & 278.5 & 32.0 & 5.1 & 19.0 & 13.2 \\
\hline $11 / 2 / 92$ & 2115 & 0.25 & 265.00 & 279.9 & 32.8 & 4.9 & 12.5 & 14.8 \\
\hline $11 / 2 / 92$ & 2130 & 0.25 & 265.25 & 279.2 & 32.6 & 5.2 & 20.2 & 15.8 \\
\hline $11 / 2 / 92$ & 2145 & 0.25 & 265.50 & 278.5 & 32.6 & 5.3 & 18.6 & 14.0 \\
\hline $11 / 2 / 92$ & 2200 & 0.25 & 265.75 & 279.0 & 31.9 & 5.1 & 16.1 & 14.1 \\
\hline $11 / 2 / 92$ & 2215 & 0.25 & 266.00 & 278.5 & 31.7 & 4.9 & 20.9 & 14.2 \\
\hline $11 / 2 / 92$ & 2230 & 0.25 & 266.25 & 280.1 & 32.1 & 5.1 & 15.0 & 13.0 \\
\hline $11 / 2 / 92$ & 2245 & 0.25 & 266.50 & 280.3 & 31.8 & 4.9 & 19.0 & 164 \\
\hline $11 / 2 / 92$ & 2300 & 0.25 & 266.75 & 277.9 & 30.4 & 5.0 & 18.9 & 15.7 \\
\hline $11 / 2 / 92$ & 2315 & 0.25 & 267.00 & 277.7 & 30.6 & 4.8 & 17.3 & 13.4 \\
\hline $11 / 2 / 92$ & 2330 & 0.25 & 267.25 & 278.8 & 30.4 & 4.9 & 20.3 & 13.0 \\
\hline $11 / 2 / 92$ & 2345 & 0.25 & 267.50 & 279.9 & 29.4 & 4.9 & 11.8 & 14.8 \\
\hline \multicolumn{2}{|l|}{ Total Hours } & 7.75 & & & & & & \\
\hline $11 / 3 / 92$ & 0 & 0.25 & 267.75 & 278.1 & 29.6 & 5.3 & 19.9 & 12.6 \\
\hline
\end{tabular}

Page E-10 B.Duct Opacitys> ' = Lower, ". - Hlgher 
SUMMARY OF LIME SLURRY INJECTION FROM OCTOBER 17, 1992 TO NOVEMBER 17, 1992

\begin{tabular}{|c|c|c|c|c|c|c|c|c|}
\hline Date & Time & Hours of & Cumulative & B Duct Temp & Lime Flow & Slurry Solids & SO2 Removal & Stack Opacity \\
\hline & & Injection & Hours & ${ }^{\circ} \mathrm{F}$ & GPM & Percent & Percent & Percent \\
\hline $11 / 3 / 92$ & 15 & 0.25 & 268.00 & 280.0 & 30.0 & 5.1 & 16.7 & 10.5 \\
\hline $11 / 3 / 92$ & 30 & 0.25 & 268.25 & 281.0 & 29.7 & 5.0 & 12.0 & 10.0 \\
\hline $11 / 3 / 92$ & 45 & 0.25 & 268.50 & 278.7 & 29.5 & 5.4 & 8.5 & 10.1 \\
\hline $11 / 3 / 92$ & 100 & 0.25 & 268.75 & 279.1 & 29.4 & 5.1 & 13.1 & 9.4 \\
\hline $11 / 3 / 92$ & 115 & 0.25 & 269.00 & 280.2 & 29.5 & 5.4 & 10.1 & 10.1 \\
\hline $11 / 3 / 92$ & 130 & 0.25 & 269.25 & 281.0 & 28.7 & 4.8 & 11.6 & 10.9 \\
\hline $11 / 3 / 92$ & 145 & 0.25 & 269.50 & 279.1 & 29.4 & 4.9 & 7.5 & 11.7 \\
\hline $11 / 3 / 92$ & 200 & 0.25 & 269.75 & 276.4 & 28.8 & 4.8 & 16.5 & 10.1 \\
\hline $11 / 3 / 92$ & 215 & 0.25 & 270.00 & 275.3 & 29.3 & 5.3 & 16.7 & 10.4 \\
\hline $11 / 3 / 92$ & 230 & 0.25 & 270.25 & 275.4 & 29.0 & 5.1 & 17.1 & 11.0 \\
\hline $11 / 3 / 92$ & 245 & 0.25 & 270.50 & 275.6 & 28.7 & 5.2 & 13.6 & 10.2 \\
\hline $11 / 3 / 92$ & 300 & 0.25 & 270.75 & 274.6 & 29.3 & 5.2 & 15.5 & 11.6 \\
\hline $11 / 3 / 92$ & 315 & 0.25 & 271.00 & 274.5 & 29.4 & 5.8 & 15.0 & 14.0 \\
\hline $11 / 3 / 92$ & 330 & 0.25 & 271.25 & 274.2 & 29.0 & 5.4 & 13.1 & 13.2 \\
\hline $11 / 3 / 92$ & 345 & 0.25 & 271.50 & 274.8 & 29.1 & 5.4 & 12.0 & 11.8 \\
\hline $11 / 3 / 92$ & 400 & 0.25 & 271.75 & 273.5 & 29.4 & 5.5 & 12.2 & 12.1 \\
\hline $11 / 3 / 92$ & 415 & 0.25 & 272.00 & 272.9 & 29.9 & 5.6 & 10.9 & 24.1 \\
\hline $11 / 3 / 92$ & 430 & 0.25 & 272.25 & 274.0 & 29.9 & 5.6 & 16.0 & 11.4 \\
\hline $11 / 3 / 92$ & 445 & 0.25 & 272.50 & 272.8 & 31.3 & 5.4 & 17.0 & 9.4 \\
\hline $11 / 3 / 92$ & 500 & 0.25 & 272.75 & 273.6 & 30.0 & 5.4 & 12.7 & 9.0 \\
\hline $11 / 3 / 92$ & 515 & 0.25 & 273.00 & 273.6 & 29.9 & 5.0 & 14.6 & 9.4 \\
\hline $11 / 3 / 92$ & 530 & 0.25 & 273.25 & 272.1 & 29.0 & 5.1 & 13.9 & 9.2 \\
\hline $11 / 3 / 92$ & 545 & 0.25 & 273.50 & 271.5 & 29.3 & 4.9 & 13.8 & 9.9 \\
\hline $11 / 3 / 92$ & 600 & 0.25 & 273.75 & 272.2 & 28.5 & 5.2 & 14.4 & 9.9 \\
\hline $11 / 3 / 92$ & 615 & 0.25 & 274.00 & 272.2 & 29.2 & 5.1 & 15.9 & 9.8 \\
\hline $11 / 3 / 92$ & 630 & 0.25 & 274.25 & 271.9 & 29.8 & 5.0 & 16.1 & 10.7 \\
\hline $11 / 3 / 92$ & 645 & 0.25 & 274.50 & 271.7 & 29.0 & 5.0 & 13.8 & 12.0 \\
\hline $11 / 3 / 92$ & 700 & 0.25 & 274.75 & 272.2 & 29.4 & 5.0 & 9.0 & 12.6 \\
\hline $11 / 3 / 92$ & 715 & 0.25 & 275.00 & 270.7 & 29.7 & 5.0 & 17.6 & 14.0 \\
\hline $11 / 3 / 92$ & 730 & 0.25 & 275.25 & 269.8 & 30.0 & 5.3 & 11.7 & 13.7 \\
\hline $11 / 3 / 92$ & 745 & 0.25 & 275.50 & 272.5 & 29.8 & 5.2 & 18.0 & 11.3 \\
\hline $11 / 3 / 92$ & 800 & 0.25 & 275.75 & 272.5 & 29.8 & 5.1 & 10.3 & 11.0 \\
\hline $11 / 3 / 92$ & 815 & 0.25 & 276.00 & 272.9 & 29.2 & 4.9 & 19.2 & 59.0 \\
\hline $11 / 3 / 92$ & 830 & 0.25 & 276.25 & 271.3 & 30.4 & 5.0 & 11.5 & 12.2 \\
\hline $11 / 3 / 92$ & 845 & 0.25 & 276.50 & 271.8 & 30.5 & 5.4 & 17.5 & 12.0 \\
\hline $11 / 3 / 92$ & 900 & 0.25 & 276.75 & 272.5 & 29.9 & 5.0 & 14.2 & 12.0 \\
\hline $11 / 3 / 92$ & 915 & 0.25 & 277.00 & 274.9 & 28.7 & 5.0 & 16.0 & 11.6 \\
\hline $11 / 3 / 92$ & 930 & 0.25 & 277.25 & 274.7 & 29.3 & 5.0 & 15.0 & 10.3 \\
\hline $11 / 3 / 92$ & 945 & 0.25 & 277.50 & 275.4 & 28.6 & 5.2 & 18.5 & 11.0 \\
\hline $11 / 3 / 92$ & 1000 & 0.25 & 277.75 & 276.9 & 30.4 & 5.2 & 17.2 & 10.4 \\
\hline $11 / 3 / 92$ & 1015 & 0.25 & 278.00 & 276.6 & 28.7 & 4.9 & 17.0 & 11.8 \\
\hline $11 / 3 / 92$ & 1030 & 0.25 & 278.25 & 279.4 & 29.8 & 5.2 & 19.4 & 11.7 \\
\hline $11 / 3 / 92$ & 1045 & 0.25 & 278.50 & 279.1 & 30.4 & 5.5 & 15.2 & 11.4 \\
\hline $11 / 3 / 92$ & 1100 & 0.25 & 278.75 & 280.2 & 30.4 & 5.5 & 18.3 & 10.4 \\
\hline $11 / 3 / 92$ & 1115 & 0.25 & 279.00 & 281.3 & 35.5 & 5.4 & 18.7 & 12.4 \\
\hline $11 / 3 / 92$ & 1130 & 0.25 & 279.25 & 282.8 & 36.4 & 4.9 & 15.4 & 10.5 \\
\hline $11 / 3 / 92$ & 1145 & 0.25 & 279.50 & 283.3 & 34.7 & 5.4 & 14.1 & 11.5 \\
\hline
\end{tabular}

Page E-11 B-Duct Opacity >> " = Lower, "* = Higher 
SUMMARY OF LIME SLURRY INJECTION FROM OCTOBER 17, 1992 TO NOVEMBER 17, 1992

\begin{tabular}{|c|c|c|c|c|c|c|c|c|}
\hline Date & Time & Hours of & Cumulative & B Duct Temp & Lime Flow & Slurry Solids & SO2 Removal & Stack Opacity \\
\hline & & Injection & Hours & ${ }^{\circ} \mathrm{F}$ & GPM & Percent & Percent & Percent \\
\hline $11 / 3 / 92$ & 1200 & 0.25 & 279.75 & 285.2 & 34.9 & 5.1 & 15.2 & 12.4 \\
\hline $11 / 3 / 92$ & 1215 & 0.25 & 280.00 & 285.0 & 34.7 & 5.3 & 15.5 & 11.1 \\
\hline $11 / 3 / 92$ & 1230 & 0.25 & 280.25 & 284.7 & 34.9 & 5.0 & 17.9 & 10.6 \\
\hline $11 / 3 / 92$ & 1245 & 0.25 & 280.50 & 285.0 & 34.4 & 4.8 & 12.1 & 10.8 \\
\hline $11 / 3 / 92$ & 1300 & 0.25 & 280.75 & 283.2 & 35.0 & 5.1 & 18.3 & 12.1 \\
\hline $11 / 3 / 92$ & 1315 & 0.25 & 281.00 & 282.0 & 34.6 & 5.1 & 18.1 & 11.0 \\
\hline $11 / 3 / 92$ & 1330 & 0.25 & 281.25 & 283.4 & 33.9 & 5.2 & 14.7 & 13.0 \\
\hline $11 / 3 / 92$ & 1345 & 0.25 & 281.50 & 286.1 & 35.1 & 5.1 & 18.8 & 11.9 \\
\hline $11 / 3 / 92$ & 1400 & 0.25 & 281.75 & 285.8 & 34.5 & 4.2 & 14.7 & 12.3 \\
\hline $11 / 3 / 92$ & 1415 & 0.25 & 282.00 & 284.8 & 33.8 & 5.9 & 18.6 & 11.3 \\
\hline $11 / 3 / 92$ & 1430 & 0.25 & 282.25 & 285.8 & 34.4 & 6.5 & 18.2 & 12.0 \\
\hline $11 / 3 / 92$ & 1445 & 0.25 & 282.50 & 285.0 & 34.1 & 6.1 & 17.2 & 13.2 \\
\hline $11 / 3 / 92$ & 1500 & 0.25 & 282.75 & 285.2 & 34.1 & 6.3 & 16.5 & 11.3 \\
\hline $11 / 3 / 92$ & 1515 & 0.25 & 283.00 & 285.4 & 33.6 & 6.0 & 17.2 & 12.5 \\
\hline $11 / 3 / 92$ & 1530 & 0.25 & 283.25 & 284.1 & 32.4 & 6.3 & 13.4 & 12.8 \\
\hline $11 / 3 / 92$ & 1545 & 0.25 & 283.50 & 283.6 & 31.4 & 6.1 & 12.8 & 12.6 \\
\hline $11 / 3 / 92$ & 1600 & 0.25 & 283.75 & 285.2 & 31.2 & 6.0 & 14.9 & 12.4 \\
\hline $11 / 3 / 92$ & 1615 & 0.25 & 284.00 & 282.8 & 31.6 & 6.1 & 18.1 & 13.8 \\
\hline $11 / 3 / 92$ & 1630 & 0.25 & 284.25 & 282.5 & 31.6 & 6.1 & 10.9 & 12.5 \\
\hline $11 / 3 / 92$ & 1645 & 0.25 & 284.50 & 284.5 & 32.1 & 6.6 & 20.8 & 12.5 \\
\hline $11 / 3 / 92$ & 1700 & 0.25 & 284.75 & 280.9 & 30.6 & 6.2 & 13.8 & 11.9 \\
\hline $11 / 3 / 92$ & 1715 & 0.25 & 285.00 & 280.7 & 30.8 & 6.1 & 12.4 & 12.1 \\
\hline $11 / 3 / 92$ & 1730 & 0.25 & 285.25 & 280.4 & 30.1 & 6.6 & 12.3 & 12.5 \\
\hline $11 / 3 / 92$ & 1745 & 0.25 & 285.50 & 278.9 & 29.9 & 5.9 & 18.1 & 11.7 \\
\hline $11 / 3 / 92$ & 1800 & 0.25 & 285.75 & 278.1 & 29.2 & 6.3 & 11.0 & 11.9 \\
\hline $11 / 3 / 92$ & 1815 & 0.25 & 286.00 & 278.0 & 29.6 & 5.8 & 12.8 & 12.3 \\
\hline $11 / 3 / 92$ & 1830 & 0.25 & 286.25 & 278.2 & 29.7 & 5.8 & 11.8 & 12.1 \\
\hline $11 / 3 / 92$ & 1845 & 0.25 & 286.50 & 277.4 & 28.1 & 6.3 & 13.9 & 13.5 \\
\hline $11 / 3 / 92$ & 1900 & 0.25 & 286.75 & 277.8 & 28.4 & 6.4 & 10.4 & 12.7 \\
\hline $11 / 3 / 92$ & 1915 & 0.25 & 287.00 & 276.8 & 28.2 & 6.3 & 11.2 & 12.2 \\
\hline $11 / 3 / 92$ & 1930 & 0.25 & 287.25 & 277.2 & 28.3 & 6.2 & 11.2 & 12.8 \\
\hline $11 / 3 / 92$ & 1945 & 0.25 & 287.50 & 277.1 & 28.2 & 6.4 & 10.3 & 12.0 \\
\hline $11 / 3 / 92$ & 2000 & 0.25 & 287.75 & 276.2 & 28.0 & 6.4 & 12.1 & 12.6 \\
\hline $11 / 3 / 92$ & 2015 & 0.25 & 288.00 & 276.9 & 28.6 & 6.4 & 12.6 & 13.1 \\
\hline $11 / 3 / 92$ & 2030 & 0.25 & 288.25 & 277.1 & 26.9 & 6.0 & 10.8 & 14.1 \\
\hline $11 / 3 / 92$ & 2045 & 0.25 & 288.50 & 276.8 & 28.2 & 6.1 & 9.5 & 15.0 \\
\hline $11 / 3 / 92$ & 2100 & 0.25 & 288.75 & 277.8 & 28.6 & 6.2 & 9.8 & 14.0 \\
\hline $11 / 3 / 92$ & 2115 & 0.25 & 289.00 & 278.5 & 29.0 & 5.8 & 10.9 & 12.0 \\
\hline $11 / 3 / 92$ & 2130 & 0.25 & 289.25 & 278.0 & 28.9 & 6.1 & 9.9 & 12.3 \\
\hline $11 / 3 / 92$ & 2145 & 0.25 & 289.50 & 278.0 & 28.6 & 5.9 & 13.4 & 14.3 \\
\hline $11 / 3 / 92$ & 2200 & 0.25 & 289.75 & 278.6 & 28.8 & 5.8 & 12.6 & 12.7 \\
\hline $11 / 3 / 92$ & 2215 & 0.25 & 290.00 & 278.1 & 28.9 & 5.9 & 18.1 & 13.1 \\
\hline $11 / 3 / 92$ & 2230 & 0.25 & 290.25 & 279.0 & 28.0 & 6.3 & 17.5 & 14.3 \\
\hline $11 / 3 / 92$ & 2245 & 0.25 & 290.50 & 278.1 & 28.6 & 5.9 & 13.0 & 13.2 \\
\hline $11 / 3 / 92$ & 2300 & 0.25 & 290.75 & 278.5 & 28.6 & 5.8 & 14.2 & 12.6 \\
\hline $11 / 3 / 92$ & 2315 & 0.25 & 291.00 & 278.0 & 28.2 & 5.9 & 13.3 & 15.5 \\
\hline $11 / 3 / 92$ & 2330 & 0.25 & 291.25 & 279.5 & 28.2 & 6.1 & 12.2 & 12.2 \\
\hline
\end{tabular}

Page E-12 B-Duct Opacity $>>$ " = Lower, "* = Higher 
SUMMARY OF LIME SLURRY INJECTION FROM OCTOBER 17, 1992 TO NOVEMBER 17, 1992

\begin{tabular}{|c|c|c|c|c|c|c|c|c|}
\hline Date & Time & Hours of & Cumulative & B Duct Temp & Lime Flow & Slurry Solids & SO2 Removal & Stack Opacity \\
\hline & & Injection & Hours & ${ }^{\circ} \mathrm{F}$ & GPM & Percent & \begin{tabular}{|l|} 
Percent \\
\end{tabular} & Percent \\
\hline $11 / 3 / 92$ & 2345 & 0.25 & 291.50 & 281.7 & 30.2 & 5.8 & 15.9 & 12.3 \\
\hline \multicolumn{2}{|c|}{ Total Hours } & 24.00 & & & & & & \\
\hline & & & & & & & & \\
\hline $11 / 4 / 92$ & 0 & 0.25 & 291.75 & 282.8 & 30.8 & 6.0 & 20.8 & 13.8 \\
\hline $11 / 4 / 92$ & 15 & 0.25 & 292.00 & 280.6 & 30.1 & 6.1 & 13.6 & 15.0 \\
\hline $11 / 4 / 92$ & 30 & 0.25 & 292.25 & 282.5 & 30.6 & 5.6 & 10.0 & 11.5 \\
\hline $11 / 4 / 92$ & 45 & 0.25 & 292.50 & 280.3 & 31.4 & 5.9 & 15.5 & 11.6 \\
\hline $11 / 4 / 92$ & 100 & 0.25 & 292.75 & 280.7 & 33.1 & 5.9 & 15.3 & 10.7 \\
\hline $11 / 4 / 92$ & 115 & 0.25 & 293.00 & 281.6 & 32.9 & 6.1 & 18.9 & 10.9 \\
\hline $11 / 4 / 92$ & 130 & 0.25 & 293.25 & 283.3 & 33.4 & 5.6 & 18.4 & 10.8 \\
\hline $11 / 4 / 92$ & 145 & 0.25 & 293.50 & 279.7 & 33.9 & 6.3 & 16.5 & 10.6 \\
\hline $11 / 4 / 92$ & 200 & 0.25 & 293.75 & 276.8 & 33.1 & 6.1 & 18.6 & 10.8 \\
\hline $11 / 4 / 92$ & 215 & 0.25 & 294.00 & 277.2 & 34.6 & 6.0 & 18.0 & 12.8 \\
\hline $11 / 4 / 92$ & 230 & 0.25 & 294.25 & 276.1 & 33.4 & 6.0 & 20.8 & 11.4 \\
\hline $11 / 4 / 92$ & 245 & 0.25 & 294.50 & 277.1 & 34.0 & 6.3 & 20.3 & 11.0 \\
\hline $11 / 4 / 92$ & 300 & 0.25 & 294.75 & 277.1 & 33.5 & 6.2 & 18.5 & 10.0 \\
\hline $11 / 4 / 92$ & 315 & 0.25 & 295.00 & 276.1 & 32.6 & 6.0 & 19.8 & 10.7 \\
\hline $11 / 4 / 92$ & 330 & 0.25 & 295.25 & 276.6 & 32.8 & 6.4 & 17.8 & 13.6 \\
\hline $11 / 4 / 92$ & 345 & 0.25 & 295.50 & 277.7 & 32.5 & 5.8 & 21.0 & 12.3 \\
\hline $11 / 4 / 92$ & 400 & 0.25 & 295.75 & 275.7 & 32.1 & 6.2 & 17.3 & 12.2 \\
\hline $11 / 4 / 92$ & 415 & 0.25 & 296.00 & 274.6 & 31.4 & 5.9 & 18.3 & 14.1 \\
\hline $11 / 4 / 92$ & 430 & 0.25 & 296.25 & 275.8 & 31.9 & 6.0 & 17.2 & 11.5 \\
\hline $11 / 4 / 92$ & 445 & 0.25 & 296.50 & 276.1 & 31.6 & 5.8 & 20.0 & 10.9 \\
\hline $11 / 4 / 92$ & 500 & 0.25 & 296.75 & 276.1 & 31.5 & 6.3 & 16.5 & 9.7 \\
\hline $11 / 4 / 92$ & 515 & 0.25 & 297.00 & 273.7 & 31.3 & 6.2 & 12.7 & 10.0 \\
\hline $11 / 4 / 92$ & 530 & 0.25 & 297.25 & 274.9 & 31.3 & 6.1 & 17.9 & 9.6 \\
\hline $11 / 4 / 92$ & 545 & 0.25 & 297.50 & 274.3 & 27.5 & 5.9 & 19.9 & 9.3 \\
\hline $11 / 4 / 92$ & 600 & 0.25 & 297.75 & 273.2 & 26.5 & 6.1 & 11.9 & 9.3 \\
\hline $11 / 4 / 92$ & 615 & 0.25 & 298.00 & 273.9 & 26.1 & 6.5 & 13.3 & 12.2 \\
\hline $11 / 4 / 92$ & 630 & 0.25 & 298.25 & 273.6 & 25.9 & 5.7 & 12.3 & 13.0 \\
\hline $11 / 4 / 92$ & 645 & 0.25 & 298.50 & 273.2 & 25.9 & 6.0 & 11.2 & 12.9 \\
\hline $11 / 4 / 92$ & 700 & 0.25 & 298.75 & 272.9 & 27.4 & 6.2 & 13.3 & 13.2 \\
\hline $11 / 4 / 92$ & 715 & 0.25 & 299.00 & 271.9 & 26.4 & 6.2 & 14.6 & 15.4 \\
\hline $11 / 4 / 92$ & 730 & 0.25 & 299.25 & 273.5 & 25.7 & 5.9 & 12.4 & 14.4 \\
\hline $11 / 4 / 92$ & 745 & 0.25 & 299.50 & 273.3 & 25.7 & 5.7 & 13.6 & 14.6 \\
\hline $11 / 4 / 92$ & 800 & 0.25 & 299.75 & 274.5 & 26.8 & 5.9 & 10.7 & 14.7 \\
\hline $11 / 4 / 92$ & 815 & 0.25 & 300.00 & 275.3 & 26.6 & 6.1 & 14.5 & 14.0 \\
\hline $11 / 4 / 92$ & 830 & 0.25 & 300.25 & 276.6 & 27.0 & 6.3 & 11.9 & 14.9 \\
\hline $11 / 4 / 92$ & 845 & 0.25 & 300.50 & 276.9 & 27.6 & 6.2 & 12.7 & 14.0 \\
\hline $11 / 4 / 92$ & 900 & 0.25 & 300.75 & 277.1 & 27.7 & 5.7 & 12.3 & 13.9 \\
\hline $11 / 4 / 92$ & 915 & 0.25 & 301.00 & 277.8 & 30.1 & 6.0 & 6.4 & 12.5 \\
\hline $11 / 4 / 92$ & 930 & 0.25 & 301.25 & 278.9 & 30.2 & 6.2 & 7.6 & 12.5 \\
\hline $11 / 4 / 92$ & 945 & 0.25 & 301.50 & 278.4 & 28.6 & 6.2 & 8.3 & 14.6 \\
\hline $11 / 4 / 92$ & 1000 & 0.25 & 301.75 & 278.9 & 27.3 & 6.2 & 9.0 & 14.1 \\
\hline $11 / 4 / 92$ & 1015 & 0.25 & 302.00 & 279.5 & 29.6 & 5.9 & 7.6 & 14.6 \\
\hline $11 / 4 / 92$ & 1030 & 0.25 & 302.25 & 280.8 & 28.2 & 6.2 & 15.7 & 14.2 \\
\hline $11 / 4 / 92$ & 1045 & 0.25 & 302.50 & 279.2 & 26.9 & 6.1 & 15.4 & 13.8 \\
\hline
\end{tabular}

Page E-13 B-Duct Opacity >> " = Lower, "* = Higher 
SUMMARY OF LIME SLURRY INJECTION FROM OCTOBER 17, 1992 TO NOVEMBER 17, 1992

\begin{tabular}{|c|c|c|c|c|c|c|c|c|}
\hline Date & Time & Hours of & Cumulative & B Ducl Temp & Lime Flow & Slurry Solids & SO2 Removal & Stack Opacily \\
\hline & & Injection & Hours & ${ }^{\circ} \mathrm{F}$ & GPM & Percent & Percent & Percent \\
\hline $11 / 4 / 92$ & 1100 & 0.25 & 302.75 & 279.3 & 29.6 & 6.4 & 14.0 & 14.4 \\
\hline $11 / 4 / 92$ & 1115 & 0.25 & 303.00 & 281.6 & 33.3 & 6.4 & 15.8 & 13.9 \\
\hline $11 / 4 / 92$ & 1130 & 0.25 & 303.25 & 282.0 & 34.1 & 5.9 & 16.1 & 12.6 \\
\hline $11 / 4 / 92$ & 1145 & 0.25 & 303.50 & 285.0 & 35.3 & 6.1 & 21.4 & 15.7 \\
\hline $11 / 4 / 92$ & 1200 & 0.25 & 303.75 & 284.7 & 35.1 & 6.3 & 19.2 & 14.8 \\
\hline $11 / 4 / 92$ & 1215 & 0.25 & 304.00 & 285.4 & 35.0 & 6.1 & 19.1 & 13.5 \\
\hline $11 / 4 / 92$ & 1230 & 0.25 & 304.25 & 285.9 & 34.5 & 6.0 & 18.6 & 14.0 \\
\hline $11 / 4 / 92$ & 1245 & 0.25 & 304.50 & 285.5 & 36.0 & 6.2 & 25.2 & 14.9 \\
\hline $11 / 4 / 92$ & 1300 & 0.25 & 304.75 & 284.0 & 34.9 & 5.8 & 21.3 & 16.6 \\
\hline $11 / 4 / 92$ & $10: 5$ & 0.25 & 305.00 & 284.1 & 34.6 & 6.4 & 21.3 & 39.3 \\
\hline $11 / 4 / 92$ & 1330 & 0.25 & 305.25 & 284.9 & 35.2 & 5.9 & 22.1 & 8.7 \\
\hline $11 / 4 / 92$ & 1345 & 0.25 & 305.50 & 284.6 & 35.5 & 6.1 & 21.6 & 64.5 \\
\hline $11 / 4 / 92$ & 1400 & 0.25 & 305.75 & 284.4 & 34.9 & 6.1 & 21.4 & 12.1 \\
\hline $11 / 4 / 92$ & 1415 & 0.25 & 306.00 & 285.0 & 35.9 & 6.2 & 22.3 & 15.5 \\
\hline $11 / 4 / 92$ & 1430 & 0.25 & 306.25 & 282.8 & 35.0 & 6.2 & 23.8 & 13.9 \\
\hline $11 / 4 / 92$ & 1445 & 0.25 & 306.50 & 283.2 & 34.9 & 7.5 & 22.6 & 13.6 \\
\hline $11 / 4 / 92$ & 1500 & 0.25 & 306.75 & 284.1 & 34.6 & 7.1 & 22.5 & 12.4 \\
\hline $11 / 4 / 92$ & 1515 & 0.25 & 307.00 & 284.2 & 34.4 & 7.2 & 23.6 & 14.8 \\
\hline $11 / 4 / 92$ & 1530 & 0.25 & 307.25 & 284.1 & 35.0 & 7.2 & 25.8 & 12.7 \\
\hline $11 / 4 / 92$ & 1545 & 0.25 & 307.50 & 283.3 & 34.5 & 7.0 & 21.3 & 14.4 \\
\hline $11 / 4 / 92$ & 1600 & 0.25 & 307.75 & 281.2 & 34.6 & 6.9 & 24.5 & 13.7 \\
\hline $11 / 4 / 92$ & 1615 & 0.25 & 308.00 & 279.9 & 33.9 & 7.3 & 22.8 & 12.9 \\
\hline $11 / 4 / 92$ & 1630 & 0.25 & 308.25 & 279.9 & 30.9 & 7.0 & 21.8 & 13.8 \\
\hline $11 / 4 / 92$ & 1645 & 0.25 & 308.50 & 278.8 & 30.1 & 7.0 & 17.5 & 13.9 \\
\hline $11 / 4 / 92$ & 1700 & 0.25 & 308.75 & 279.6 & 31.1 & 7.0 & 19.4 & 12.8 \\
\hline $11 / 4 / 92$ & 1715 & 0.25 & 309.00 & 278.9 & 31.4 & 7.1 & 22.3 & 17.2 \\
\hline $11 / 4 / 92$ & 1730 & 0.25 & 309.25 & 278.9 & 32.0 & 7.4 & 21.7 & 18.4 \\
\hline $11 / 4 / 92$ & 1745 & 0.25 & 309.50 & 277.7 & 32.0 & 7.1 & 22.2 & 16.4 \\
\hline $11 / 4 / 92$ & 1800 & 0.25 & 309.75 & 275.5 & 31.6 & 6.9 & 20.0 & 15.7 \\
\hline $11 / 4 / 92$ & 1815 & 0.25 & 310.00 & 276.4 & 31.8 & 6.7 & 21.2 & 14.6 \\
\hline $11 / 4 / 92$ & 1830 & 0.25 & 310.25 & 277.2 & 31.8 & 6.9 & 20.7 & 14.0 \\
\hline $11 / 4 / 92$ & 1845 & 0.25 & 310.50 & 277.4 & 31.3 & 7.3 & 23.7 & 15.3 \\
\hline $11 / 4 / 92$ & 1900 & 0.25 & 310.75 & 276.5 & 31.8 & 6.8 & 20.2 & 14.1 \\
\hline $11 / 4 / 92$ & 1915 & 0.25 & 311.00 & 275.7 & 31.5 & 6.9 & 21.4 & 13.8 \\
\hline $11 / 4 / 92$ & 1930 & 0.25 & 311.25 & 275.2 & 31.5 & 7.0 & 19.6 & 14.3 \\
\hline $11 / 4 / 92$ & 1945 & 0.25 & 311.50 & 275.7 & 31.7 & 7.5 & 18.2 & 15.7 \\
\hline $11 / 4 / 92$ & 2000 & 0.25 & 311.75 & 276.1 & 31.8 & 7.0 & 19.8 & 14.0 \\
\hline $11 / 4 / 92$ & 2015 & 0.25 & 312.00 & 275.9 & 28.5 & 7.6 & 20.6 & 16.1 \\
\hline $11 / 4 / 92$ & 2030 & 0.25 & 312.25 & 275.9 & 30.6 & 7.3 & 18.0 & 15.4 \\
\hline $11 / 4 / 92$ & 2045 & 0.25 & 312.50 & 275.2 & 29.8 & 7.3 & 19.3 & 13.8 \\
\hline $11 / 4 / 92$ & 2100 & 0.25 & 312.75 & 275.1 & 30.1 & 6.9 & 19.0 & 15.5 \\
\hline $11 / 4 / 92$ & 2115 & 0.25 & 313.00 & 275.1 & 29.9 & 7.2 & 19.6 & 15.6 \\
\hline $11 / 4 / 92$ & 2130 & 0.25 & 313.25 & 275.5 & 30.9 & 7.6 & 23.0 & 14.9 \\
\hline $11 / 4 / 92$ & 2145 & 0.25 & 313.50 & 274.9 & 29.4 & 7.3 & 19.6 & 15.3 \\
\hline $11 / 4 / 92$ & 2200 & 0.25 & 313.75 & 274.5 & 30.2 & 6.9 & 20.7 & 16.9 \\
\hline $11 / 4 / 92$ & 2215 & 0.25 & 314.00 & 275.4 & 30.3 & 7.1 & 17.7 & 15.0 \\
\hline $11 / 4 / 92$ & 2230 & 0.25 & 314.25 & 274.3 & 30.3 & 7.1 & 18.5 & 10.6 \\
\hline
\end{tabular}


SUMMARY OF LIME SLURRY INJECTION FROM OCTOBER 17, 1992 TO NOVEMBER 17, 1992

\begin{tabular}{|c|c|c|c|c|c|c|c|c|}
\hline Date & Time & Hours of & Cumulative & B Duct Temp & Lime Flow & Slurry Solids & SO2 Removal & Stack Opacity \\
\hline & & Injection & Hours & ${ }^{\circ} \mathrm{F}$ & GPM & Percent & Percent & Percent \\
\hline $11 / 4 / 92$ & 2245 & 0.25 & 314.50 & 274.0 & 29.5 & 7.2 & 19.1 & 10.9 \\
\hline $11 / 4 / 92$ & 2300 & 0.25 & 314.75 & 274.0 & 29.9 & 6.4 & 18.6 & 11.4 \\
\hline $11 / 4 / 92$ & 2315 & 0.25 & 315.00 & 274.7 & 29.9 & 7.3 & 19.0 & 11.4 \\
\hline $11 / 4 / 92$ & 2330 & 0.25 & 315.25 & 273.5 & 29.9 & 6.9 & 16.9 & 11.8 \\
\hline $11 / 4 / 92$ & 2345 & 0.25 & 315.50 & 273.8 & 29.9 & 6.6 & 19.9 & 12.8 \\
\hline \multicolumn{2}{|l|}{ Total Hours } & 24.00 & & & & & & \\
\hline & & & & & & & & \\
\hline $11 / 5 / 92$ & 0 & 0.25 & 315.75 & 273.7 & 29.9 & 7.0 & 20.8 & 10.7 \\
\hline $11 / 5 / 92$ & 15 & 0.25 & 316.00 & 274.3 & 30.1 & 7.0 & 19.2 & 54.1 \\
\hline $11 / 5 / 92$ & 30 & 0.25 & 316.25 & 272.6 & 29.3 & 6.7 & 16.2 & 10.9 \\
\hline $11 / 5 / 92$ & 45 & 0.25 & 316.50 & 274.1 & 29.5 & 6.8 & 20.8 & 10.4 \\
\hline $11 / 5 / 92$ & 100 & 0.25 & 316.75 & 273.6 & 29.9 & 7.1 & 16.1 & 10.2 \\
\hline $11 / 5 / 92$ & 115 & 0.25 & 317.00 & 274.2 & 29.5 & 7.2 & 17.1 & 11.2 \\
\hline $11 / 5 / 92$ & 130 & 0.25 & 317.25 & 275.6 & 29.5 & 7.1 & 15.1 & 10.3 \\
\hline $11 / 5 / 92$ & 145 & 0.25 & 317.50 & 275.1 & 30.6 & 7.0 & 19.0 & 9.7 \\
\hline $11 / 5 / 92$ & 200 & 0.25 & 317.75 & 272.5 & 26.0 & 7.3 & 13.0 & 11.3 \\
\hline $11 / 5 / 92$ & 215 & 0.25 & 318.00 & 271.6 & 25.6 & 7.2 & 14.1 & 12.2 \\
\hline $11 / 5 / 92$ & 230 & 0.25 & 318.25 & 272.1 & 27.9 & 7.1 & 13.9 & 10.1 \\
\hline $11 / 5 / 92$ & 245 & 0.25 & 318.50 & 270.3 & 27.1 & 6.7 & 16.6 & 9.4 \\
\hline $11 / 5 / 92$ & 300 & 0.25 & 318.75 & 272.6 & 25.6 & 7.1 & 14.3 & 15.0 \\
\hline $11 / 5 / 92$ & 315 & 0.25 & 319.00 & 272.6 & 27.8 & 7.0 & 16.4 & 15.1 \\
\hline $11 / 5 / 92$ & 330 & 0.25 & 319.25 & 272.6 & 28.4 & 7.0 & 16.2 & 13.7 \\
\hline $11 / 5 / 92$ & 345 & 0.25 & 319.50 & 273.0 & 28.4 & 7.1 & 21.3 & 12.4 \\
\hline $11 / 5 / 92$ & 400 & 0.25 & 319.75 & 273.2 & 28.1 & 7.2 & 15.7 & 12.8 \\
\hline $11 / 5 / 92$ & 415 & 0.25 & 320.00 & 272.1 & 28.7 & 6.9 & 16.3 & 12.3 \\
\hline $11 / 5 / 92$ & 430 & 0.25 & 320.25 & 271.8 & 29.1 & 6.8 & 15.6 & 13.1 \\
\hline $11 / 5 / 92$ & 445 & 0.25 & 320.50 & 271.3 & 29.2 & 7.2 & 16.8 & 12.4 \\
\hline $11 / 5 / 92$ & 500 & 0.25 & 320.75 & 272.3 & 30.7 & 7.3 & 17.0 & 12.1 \\
\hline $11 / 5 / 92$ & 515 & 0.25 & 321.00 & 272.8 & 31.1 & 7.3 & 21.8 & 12.5 \\
\hline $11 / 5 / 92$ & 530 & 0.25 & 321.25 & 272.1 & 32.3 & 7.3 & 23.9 & 15.8 \\
\hline $11 / 5 / 92$ & 545 & 0.25 & 321.50 & 273.0 & 32.2 & 7.2 & 21.3 & 12.5 \\
\hline $11 / 5 / 92$ & 600 & 0.25 & 321.75 & 273.5 & 30.9 & 7.1 & 21.1 & 12.0 \\
\hline $11 / 5 / 92$ & 615 & 0.25 & 322.00 & 271.2 & 31.1 & 7.3 & 23.4 & 10.8 \\
\hline $11 / 5 / 92$ & 630 & 0.25 & 322.25 & 271.8 & 32.0 & 7.2 & 21.8 & 9.8 \\
\hline $11 / 5 / 92$ & 645 & 0.25 & 322.50 & 273.5 & 31.2 & 7.3 & 24.1 & 12.2 \\
\hline $11 / 5 / 92$ & 700 & 0.25 & 322.75 & 272.2 & 30.5 & 6.9 & 22.2 & 11.2 \\
\hline $11 / 5 / 92$ & 715 & 0.25 & 323.00 & 271.6 & 30.1 & 7.2 & 20.7 & 11.3 \\
\hline $11 / 5 / 92$ & 730 & 0.25 & 323.25 & 272.7 & 29.7 & 6.9 & 21.2 & 11.0 \\
\hline $11 / 5 / 92$ & 745 & 0.25 & 323.50 & 273.4 & 30.4 & 7.3 & 20.6 & 11.5 \\
\hline $11 / 5 / 92$ & 800 & 0.25 & 323.75 & 273.8 & 30.3 & 6.9 & 21.9 & 12.4 \\
\hline $11 / 5 / 92$ & 815 & 0.25 & 324.00 & 273.0 & 30.0 & 6.9 & 24.1 & 12.0 \\
\hline $11 / 5 / 92$ & 830 & 0.25 & 324.25 & 274.7 & 29.9 & 7.2 & 20.5 & 8.2 \\
\hline $11 / 5 / 92$ & 845 & 0.25 & 324.50 & 275.0 & 29.9 & 7.4 & 20.8 & 8.4 \\
\hline $11 / 5 / 92$ & 900 & 0.25 & 324.75 & 274.2 & 29.6 & 6.9 & 20.2 & 8.8 \\
\hline $11 / 5 / 92$ & 915 & 0.25 & 325.00 & 273.7 & 29.4 & 7.2 & 19.9 & 9.5 \\
\hline $11 / 5 / 92$ & 930 & 0.25 & 325.25 & 273.3 & 29.2 & 7.0 & 20.4 & 11.3 \\
\hline $11 / 5 / 92$ & 945 & 0.25 & 325.50 & 273.2 & 29.3 & 7.1 & 20.7 & 12.0 \\
\hline
\end{tabular}

Page E-15 B-Duct Opacity>> " = Lower, "* = Higher 
SUMMARY OF LIME SLURRY INJECTION FROM OCTOBER 17, 1992 TO NOVEMBER 17, 1992

\begin{tabular}{|c|c|c|c|c|c|c|c|c|}
\hline Date & Time & Hours of & Cumulative & B Ducl Temp & Lime Flow & Slurry Solids & SO2 Removal & Stack Opacliy \\
\hline & & Injection & Hours & of & GPM & Percent & Percent & Percent \\
\hline $11 / 5 / 92$ & 1000 & 0.25 & 325.75 & 274.2 & 30.7 & 7.2 & 21.2 & 9.8 \\
\hline $11 / 5 / 92$ & 1015 & 0.25 & 326.00 & 273.0 & 29.5 & 7.1 & 20.9 & 9.8 \\
\hline $11 / 5 / 92$ & 1030 & 0.25 & 326.25 & 273.3 & 30.0 & 7.3 & 21.5 & 9.3 \\
\hline $11 / 5 / 92$ & 1045 & 0.25 & 326.50 & 273.4 & 29.8 & 7.1 & 23.9 & 9.9 \\
\hline $11 / 5 / 92$ & 1100 & 0.25 & 326.75 & 272.9 & 30.4 & 7.3 & 15.9 & 11.4 \\
\hline $11 / 5 / 92$ & 1115 & 0.25 & 327.00 & 273.3 & 28.7 & 7.1 & 18.5 & 13.3 \\
\hline $11 / 5 / 92$ & 1130 & 0.25 & 327.25 & 271.9 & 33.7 & 7.6 & 20.5 & 10.6 \\
\hline $11 / 5 / 92$ & 1145 & 0.25 & 327.50 & 271.9 & 27.4 & 7.9 & 18.2 & 12.0 \\
\hline $11 / 5 / 92$ & 1200 & 0.25 & 327.75 & 272.0 & 27.8 & 8.6 & 18.5 & 11.3 \\
\hline $11 / 5 / 92$ & 1215 & 0.25 & 328.00 & 271.5 & 28.5 & 8.1 & 20.6 & 11.6 \\
\hline $11 / 5 / 92$ & 1230 & 0.25 & 328.25 & 273.6 & 27.9 & 8.2 & 19.6 & 11.8 \\
\hline $11 / 5 / 92$ & 1245 & 0.25 & 328.50 & 272.2 & 27.9 & 8.2 & 21.8 & 12.5 \\
\hline $11 / 5 / 92$ & 1300 & 0.25 & 328.75 & 271.4 & 28.4 & 8.0 & 20.1 & 12.1 \\
\hline $11 / 5 / 92$ & 1315 & 0.25 & 329.00 & 273.6 & 28.3 & 8.0 & 19.9 & 14.8 \\
\hline $11 / 5 / 92$ & 1330 & 0.25 & 329.25 & 271.6 & 27.9 & 8.1 & 20.8 & 14.2 \\
\hline $11 / 5 / 92$ & 1345 & 0.25 & 329.50 & 271.4 & 28.3 & 8.2 & 20.2 & 12.6 \\
\hline $11 / 5 / 92$ & 1400 & 0.25 & 329.75 & 271.5 & 29.6 & 8.0 & 19.9 & 12.9 \\
\hline $11 / 5 / 92$ & 1415 & 0.25 & 330.00 & 269.4 & 27.3 & 8.0 & 25.7 & 14.8 \\
\hline $11 / 5 / 92$ & 1430 & 0.25 & 330.25 & 271.3 & 28.6 & 8.1 & 20.7 & 13.9 \\
\hline $11 / 5 / 92$ & 1445 & 0.25 & 330.50 & 271.1 & 26.6 & 8.0 & 17.8 & 15.2 \\
\hline $11 / 5 / 92$ & 1500 & 0.25 & 330.75 & 269.3 & 27.1 & 8.2 & 18.2 & 15.1 \\
\hline $11 / 5 / 92$ & 1515 & 0.25 & 331.00 & 270.2 & 29.0 & 7.7 & 23.4 & 12.3 \\
\hline $11 / 5 / 92$ & 1530 & 0.25 & 331.25 & 270.9 & 31.5 & 8.2 & 18.6 & 14.4 \\
\hline $11 / 5 / 92$ & 1545 & 0.25 & 331.50 & 271.1 & 32.6 & 7.7 & 20.8 & 12.1 \\
\hline $11 / 5 / 92$ & 1600 & 0.25 & 331.75 & 273.2 & 31.6 & 8.1 & 19.9 & 11.4 \\
\hline $11 / 5 / 92$ & 1615 & 0.25 & 332.00 & 271.8 & 32.0 & 7.7 & 23.9 & 11.6 \\
\hline $11 / 5 / 92$ & 1630 & 0.25 & 332.25 & 271.8 & 34.8 & 7.9 & 21.4 & 11.6 \\
\hline $11 / 5 / 92$ & 1645 & 0.25 & 332.50 & 270.7 & 31.1 & 8.0 & 20.0 & 10.8 \\
\hline $11 / 5 / 92$ & 1700 & 0.25 & 332.75 & 271.8 & 33.3 & 8.1 & 24.0 & 9.7 \\
\hline $11 / 5 / 92$ & 1715 & 0.25 & 333.00 & 269.8 & 31.3 & 8.0 & 24.0 & 14.2 \\
\hline $11 / 5 / 92$ & 1730 & 0.25 & 333.25 & 272.3 & 31.8 & 7.9 & 22.3 & 15.5 \\
\hline $11 / 5 / 92$ & 1745 & 0.25 & 333.50 & 269.2 & 31.0 & 8.1 & 22.0 & 12.5 \\
\hline $11 / 5 / 92$ & 1800 & 0.25 & 333.75 & 269.8 & 31.3 & 7.9 & 21.1 & 14.6 \\
\hline $11 / 5 / 92$ & 1815 & 0.25 & 334.00 & 270.3 & 31.8 & 8.2 & 21.9 & 15.7 \\
\hline $11 / 5 / 92$ & 1830 & 0.25 & 334.25 & 271.2 & 31.5 & 7.9 & 23.5 & 15.4 \\
\hline $11 / 5 / 92$ & 1845 & 0.25 & 334.50 & 271.1 & 34.5 & 8.1 & 22.9 & 13.7 \\
\hline $11 / 5 / 92$ & 1900 & 0.25 & 334.75 & 271.1 & 31.1 & 8.2 & 22.0 & 14.9 \\
\hline $11 / 5 / 92$ & 1915 & 0.25 & 335.00 & 271.3 & 31.9 & 8.2 & 22.0 & 13.4 \\
\hline $11 / 5 / 92$ & 1930 & 0.25 & 335.25 & 272.8 & 33.2 & 8.2 & 21.6 & 34.0 \\
\hline $11 / 5 / 92$ & 1945 & 0.25 & 335.50 & 271.4 & 32.5 & 7.9 & 19.4 & 13.4 \\
\hline $11 / 5 / 92$ & 2000 & 0.25 & 335.75 & 270.7 & 34.1 & 8.1 & 18.7 & 16.0 \\
\hline $11 / 5 / 92$ & 2015 & 0.25 & 336.00 & 272.2 & 32.0 & 7.9 & 19.9 & 15.0 \\
\hline $11 / 5 / 92$ & 2030 & 0.25 & 336.25 & 272.4 & 32.7 & 7.8 & 19.9 & 13.1 \\
\hline $11 / 5 / 92$ & 2045 & 0.25 & 336.50 & 272.6 & 33.2 & 8.2 & 19.9 & 14.6 \\
\hline $11 / 5 / 92$ & 2100 & 0.25 & 336.75 & 271.7 & 32.3 & 8.1 & 19.8 & 13.0 \\
\hline $11 / 5 / 92$ & 2115 & 0.25 & 337.00 & 274.2 & 33.4 & 7.8 & 18.7 & 14.3 \\
\hline $11 / 5 / 92$ & 2130 & 0.25 & 337.25 & 280.0 & 35.2 & 7.9 & 25.4 & 13.7 \\
\hline
\end{tabular}

Page E-16 B-Duct Opacity>> " = Lower, "* = Higher 
SUMMARY OF LIME SLURRY INJECTION FROM OCTOBER 17, 1992 TO NOVEMBER 17, 1992

\begin{tabular}{|c|c|c|c|c|c|c|c|c|}
\hline Date & Time & Hours of & Cumulative & B Ducl Temp & Lime Flow & Slurry Solids & SO2 Removal & Stack Opacity \\
\hline & & Injection & Hours & ${ }^{\circ} \mathrm{F}$ & GPM & Percent & Percent & Percent \\
\hline $11 / 5 / 92$ & 2145 & 0.25 & 337.50 & 280.4 & 34.6 & 8.4 & 21.4 & 15.6 \\
\hline $11 / 5 / 92$ & 2200 & 0.25 & 337.75 & 277.9 & 34.4 & 8.4 & 18.0 & 14.1 \\
\hline $11 / 5 / 92$ & 2215 & 0.25 & 338.00 & 278.3 & 36.7 & 8.0 & 20.8 & 13.2 \\
\hline $11 / 5 / 92$ & 2230 & 0.25 & 338.25 & 279.1 & 35.6 & 8.1 & 18.9 & 13.4 \\
\hline $11 / 5 / 92$ & 2245 & 0.25 & 338.50 & 279.0 & 36.1 & 8.4 & 20.9 & 14.3 \\
\hline $11 / 5 / 92$ & 2300 & 0.25 & 338.75 & 279.1 & 34.7 & 7.8 & 21.1 & 14.6 \\
\hline $11 / 5 / 92$ & 2315 & 0.25 & 339.00 & 278.0 & 33.7 & 8.0 & 15.0 & 15.2 \\
\hline $11 / 5 / 92$ & 2330 & 0.25 & 339.25 & 279.2 & 35.7 & 7.8 & 18.2 & 14.6 \\
\hline $11 / 5 / 92$ & 2345 & 0.25 & 339.50 & 279.7 & 36.0 & 7.9 & 20.5 & 13.7 \\
\hline \multicolumn{2}{|l|}{ Total Hours } & 24.00 & & & & & & \\
\hline $11 / 6 / 92$ & 0 & 0.25 & 339.75 & 279.0 & 36.0 & 8.1 & 21.3 & 12.9 \\
\hline $11 / 6 / 92$ & 15 & 0.25 & 340.00 & 277.9 & 36.3 & 8.2 & 16.6 & 14.7 \\
\hline $11 / 6 / 92$ & 30 & 0.25 & 340.25 & 279.8 & 36.9 & 8.1 & 19.0 & 13.5 \\
\hline $11 / 6 / 92$ & 45 & 0.25 & 340.50 & 279.2 & 36.3 & 8.0 & 16.6 & 14.3 \\
\hline $11 / 6 / 92$ & 100 & 0.25 & 340.75 & 280.6 & 35.9 & 8.0 & 18.7 & 11.6 \\
\hline $11 / 6 / 92$ & 115 & 0.25 & 341.00 & 279.2 & 35.2 & 8.3 & 19.4 & 9.4 \\
\hline $11 / 6 / 92$ & 130 & 0.25 & 341.25 & 277.0 & 32.4 & 8.1 & 15.1 & 10.9 \\
\hline $11 / 6 / 92$ & 145 & 0.25 & 341.50 & 277.9 & 36.2 & 8.1 & 23.0 & 9.2 \\
\hline $11 / 6 / 92$ & 215 & 0.25 & 341.75 & 278.1 & 28.7 & 8.6 & 16.5 & 14.8 \\
\hline $11 / 6 / 92$ & 230 & 0.25 & 342.00 & 275.7 & 29.9 & 8.4 & 14.0 & 14.5 \\
\hline $11 / 6 / 92$ & 245 & 0.25 & 342.25 & 276.6 & 30.2 & 8.2 & 15.0 & 13.4 \\
\hline $11 / 6 / 92$ & 300 & 0.25 & 342.50 & 278.0 & 31.6 & 8.7 & 17.1 & 9.8 \\
\hline $11 / 6 / 92$ & 315 & 0.25 & 342.75 & 277.7 & 32.5 & 8.4 & 18.8 & 9.7 \\
\hline $11 / 6 / 92$ & 330 & 0.25 & 343.00 & 277.5 & 33.1 & 8.0 & 18.6 & 9.3 \\
\hline $11 / 6 / 92$ & 345 & 0.25 & 343.25 & 277.0 & 32.2 & 8.0 & 19.9 & 11.2 \\
\hline $11 / 6 / 92$ & 400 & 0.25 & 343.50 & 278.1 & 33.7 & 8.1 & 16.9 & 11.9 \\
\hline $11 / 6 / 92$ & 415 & 0.25 & 343.75 & 278.2 & 33.8 & 8.0 & 18.6 & 10.0 \\
\hline $11 / 6 / 92$ & 430 & 0.25 & 344.00 & 277.0 & 32.8 & 8.1 & 19.8 & 10.4 \\
\hline $11 / 6 / 92$ & 445 & 0.25 & 344.25 & 275.9 & 32.9 & 8.1 & 21.7 & 8.7 \\
\hline $11 / 6 / 92$ & 500 & 0.25 & 344.50 & 276.0 & 34.0 & 8.4 & 20.4 & 8.9 \\
\hline $11 / 6 / 92$ & 515 & 0.25 & 344.75 & 276.7 & 32.5 & 8.2 & 20.9 & 8.1 \\
\hline $11 / 6 / 92$ & 530 & 0.25 & 345.00 & 276.4 & 31.6 & 7.9 & 21.2 & 8.4 \\
\hline $11 / 6 / 92$ & 545 & 0.25 & 345.25 & 276.9 & 32.8 & 8.2 & 19.8 & 9.3 \\
\hline $11 / 6 / 92$ & 600 & 0.25 & 345.50 & 277.9 & 29.8 & 8.0 & 17.8 & 11.8 \\
\hline $11 / 6 / 92$ & 615 & 0.25 & 345.75 & 275.5 & 29.4 & 8.0 & 19.3 & 16.7 \\
\hline $11 / 6 / 92$ & 630 & 0.25 & 346.00 & 274.9 & 29.3 & 8.0 & 19.1 & 13.1 \\
\hline $11 / 6 / 92$ & 645 & 0.25 & 346.25 & 274.4 & 30.4 & 8.2 & 20.5 & 12.3 \\
\hline $11 / 6 / 92$ & 700 & 0.25 & 346.50 & 274.7 & 32.7 & 7.7 & 21.2 & 13.3 \\
\hline $11 / 6 / 92$ & 715 & 0.25 & 346.75 & 276.7 & 30.8 & 8.3 & 20.4 & 12.8 \\
\hline $11 / 6 / 92$ & 730 & 0.25 & 347.00 & 276.0 & 30.5 & 8.3 & 18.2 & 13.6 \\
\hline $11 / 6 / 92$ & 745 & 0.25 & 347.25 & 276.1 & 31.4 & 7.9 & 21.1 & 12.9 \\
\hline $11 / 6 / 92$ & 800 & 0.25 & 347.50 & 277.0 & 35.6 & 8.0 & 21.3 & 11.5 \\
\hline $11 / 6 / 92$ & 815 & 0.25 & 347.75 & 277.3 & 33.9 & 7.9 & 22.3 & 10.0 \\
\hline $11 / 6 / 92$ & 830 & 0.25 & 348.00 & 277.4 & 33.9 & 8.3 & 22.8 & 10.5 \\
\hline $11 / 6 / 92$ & 845 & 0.25 & 348.25 & 277.1 & 34.4 & 8.0 & 23.7 & 10.5 \\
\hline $11 / 6 / 92$ & 900 & 0.25 & 348.50 & 277.5 & 33.8 & 7.9 & 22.8 & 11.7 \\
\hline
\end{tabular}

Page E-17 B-Duct Opacity >> " = Lower, "* = Higher 
SUMMARY OF LIME SLURRY INJECTION FROM OCTOBER 17, 1992 TO NOVEMBER 17, 1992

\begin{tabular}{|c|c|c|c|c|c|c|c|c|}
\hline Date & Time & Hours of & Cumulative & B Ducl Temp & Lime Flow & Slurry Solids & SO2 Removal & Stack Opaclly \\
\hline & & Injection & Hours & ${ }^{\circ} \mathrm{F}$ & GPM & Percent & Percent & Percent \\
\hline $11 / 6 / 92$ & 915 & 0.25 & 348.75 & 278.4 & 34.0 & 7.9 & 22.4 & 11.1 \\
\hline $11 / 6 / 92$ & 930 & 0.25 & 349.00 & 277.2 & 34.6 & 8.3 & 23.6 & 12.1 \\
\hline $11 / 6 / 92$ & 945 & 0.25 & 349.25 & 277.6 & 35.6 & 8.1 & 24.2 & 10.8 \\
\hline $11 / 6 / 92$ & 1000 & 0.25 & 349.50 & 277.2 & 30.9 & 8.0 & 23.3 & 10.8 \\
\hline $11 / 6 / 82$ & 1015 & 0.25 & 349.75 & 274.3 & 31.5 & 8.3 & 19.7 & 10.5 \\
\hline $11 / 6 / 92$ & 1030 & 0.25 & 350.00 & 269.7 & 30.9 & 8.1 & 23.0 & 10.6 \\
\hline $11 / 6 / 92$ & 1045 & 0.25 & 350.25 & 267.3 & 30.4 & 8.0 & 23.0 & 10.2 \\
\hline $11 / 6 / 92$ & 1100 & 0.25 & 350.50 & 267.2 & 29.8 & 8.2 & 22.3 & 10.7 \\
\hline $11 / 6 / 92$ & 1115 & 0.25 & 350.75 & 267.5 & 29.9 & 8.0 & 23.5 & 10.2 \\
\hline $11 / 6 / 92$ & 1130 & 0.25 & 351.00 & 267.2 & 29.7 & 8.1 & 22.3 & 11.6 \\
\hline $11 / 6 / 92$ & 1145 & 0.25 & 351.25 & 266.2 & 31.4 & 7.9 & 25.5 & 10.7 \\
\hline $11 / 6 / 92$ & 1200 & 0.25 & 351.50 & 268.4 & 29.4 & 8.1 & 21.5 & 11.3 \\
\hline $11 / 6 / 92$ & 1215 & 0.25 & 351.75 & 273.0 & 32.3 & 7.9 & 20.9 & 10.4 \\
\hline $11 / 6 / 92$ & 1230 & 0.25 & 352.00 & 273.8 & 34.2 & 7.9 & 21.1 & 10.6 \\
\hline $11 / 6 / 92$ & 1245 & 0.25 & 352.25 & 275.9 & 34.2 & 8.0 & 18.3 & 11.1 \\
\hline $11 / 6 / 92$ & 1300 & 0.25 & 352.50 & 278.9 & 35.7 & 8.1 & 22.3 & 11.7 \\
\hline $11 / 6 / 92$ & 1315 & 0.25 & 352.75 & 279.2 & 35.3 & 8.1 & 22.0 & 11.6 \\
\hline $11 / 6 / 92$ & 1330 & 0.25 & 353.00 & 279.7 & 35.4 & 7.9 & 21.7 & 11.2 \\
\hline $11 / 6 / 92$ & 1345 & 0.25 & 353.25 & 278.3 & 35.0 & 8.0 & 19.3 & 11.5 \\
\hline $11 / 6 / 92$ & 1400 & 0.25 & 353.50 & 277.2 & 34.8 & 8.0 & 20.6 & 11.7 \\
\hline $11 / 6 / 92$ & 1415 & 0.25 & 353.75 & 276.8 & 34.6 & 8.0 & 17.9 & 12.0 \\
\hline $11 / 6 / 92$ & 1430 & 0.25 & 354.00 & 277.0 & 31.7 & 8.1 & 17.7 & 12.5 \\
\hline $11 / 6 / 92$ & 1445 & 0.25 & 354.25 & 277.6 & 32.3 & 8.0 & 16.2 & 13.1 \\
\hline $11 / 6 / 92$ & 1500 & 0.25 & 354.50 & 277.8 & 32.4 & 8.2 & 17.3 & 13.7 \\
\hline $11 / 6 / 92$ & 1515 & 0.25 & 354.75 & 277.0 & 33.0 & 7.9 & 17.7 & 13.3 \\
\hline $11 / 6 / 92$ & 1530 & 0.25 & 355.00 & 277.1 & 36.5 & 9.2 & 21.2 & 10.9 \\
\hline $11 / 6 / 92$ & 1545 & 0.25 & 355.25 & 276.4 & 31.7 & 9.0 & 17.6 & 13.3 \\
\hline $11 / 6 / 92$ & 1600 & 0.25 & 355.50 & 276.9 & 32.7 & 9.1 & 18.8 & 13.3 \\
\hline $11 / 6 / 92$ & 1615 & 0.25 & 355.75 & 276.2 & 32.8 & 8.9 & 20.6 & 13.1 \\
\hline $11 / 6 / 92$ & 1630 & 0.25 & 356.00 & 277.3 & 32.9 & 8.8 & 21.3 & 13.7 \\
\hline $11 / 6 / 92$ & 1645 & 0.25 & 356.25 & 277.1 & 33.5 & 8.9 & 21.1 & 13.4 \\
\hline $11 / 6 / 92$ & 1700 & 0.25 & 356.50 & 276.9 & 33.8 & 9.0 & 21.5 & 14.0 \\
\hline $1, \overline{6 / 92}$ & 1715 & 0.25 & 356.75 & 277.3 & 33.0 & 9.0 & 21.3 & 13.5 \\
\hline $11 / 6 / 92$ & 1730 & 0.25 & 357.00 & 277.5 & 33.3 & 9.0 & 21.0 & 14.4 \\
\hline $11 / 6 / 92$ & 1745 & 0.25 & 357.25 & 276.8 & 33.3 & 9.0 & 21.2 & 12.3 \\
\hline $11 / 6 / 92$ & 1800 & 0.25 & 357.50 & 277.1 & 33.4 & 9.1 & 21.7 & 13.8 \\
\hline $11 / 6 / 92$ & 1815 & 0.25 & 357.75 & 277.2 & 33.4 & 9.0 & 22.2 & 13.9 \\
\hline $11 / 6 / 92$ & 1830 & 0.25 & 358.00 & 276.9 & 34.0 & 8.8 & 24.8 & 12.8 \\
\hline $11 / 6 / 92$ & 1845 & 0.25 & 358.25 & 276.9 & 33.6 & 9.3 & 22.7 & 13.1 \\
\hline $11 / 6 / 92$ & 1900 & 0.25 & 358.50 & 277.7 & 33.9 & 8.9 & 24.4 & 14.9 \\
\hline $11 / 6 / 92$ & 1915 & 0.25 & 358.75 & 277.0 & 33.3 & 9.0 & 24.3 & 16.3 \\
\hline $11 / 6 / 92$ & 1930 & 0.25 & 359.00 & 276.6 & 33.3 & 8.9 & 25.4 & 16.2 \\
\hline $11 / 6 / 92$ & 1945 & 0.25 & 359.25 & 277.2 & 33.3 & 9.2 & 24.4 & 14.5 \\
\hline $11 / 6 / 92$ & 2000 & 0.25 & 359.50 & 276.1 & 32.7 & 9.2 & 23.8 & 14.1 \\
\hline $11 / 6 / 92$ & 2015 & 0.25 & 359.75 & 276.0 & 35.2 & 9.1 & 23.0 & 14.2 \\
\hline $11 / 6 / 92$ & 2030 & 0.25 & 360.00 & 276.4 & 35.2 & 9.1 & 26.4 & 15.6 \\
\hline $11 / 6 / 92$ & 2045 & 0.25 & 360.25 & 276.9 & 31.7 & 9.3 & 25.2 & 17.0 \\
\hline
\end{tabular}

Page E-18 B.Duct Opacitys> " = Lower, "* Higher 
SUMMARY OF LIME SLURRY INJECTION FROM OCTOBER 17, 1992 TO NOVEMBER 17, 1992

\begin{tabular}{|c|c|c|c|c|c|c|c|c|}
\hline Date & Time & Hours of & Cumulative & B Ducl Temp & Lime Flow & Slurry Solids & SO2 Removal & Slack Opacily \\
\hline & & Injection & Hours & ${ }^{\circ} \mathrm{F}$ & GPM & Percent & Percent & Percent \\
\hline $11 / 6 / 92$ & 2100 & 0.25 & 360.50 & 276.5 & 31.2 & 9.1 & 24.6 & 15.1 \\
\hline $11 / 6 / 92$ & 2115 & 0.25 & 360.75 & 276.6 & 31.1 & 8.9 & 25.3 & 17.1 \\
\hline $11 / 6 / 92$ & 2130 & 0.25 & 361.00 & 276.1 & 31.1 & 8.9 & 25.3 & 17.2 \\
\hline $11 / 6 / 92$ & 2145 & 0.25 & 361.25 & 276.4 & 36.1 & 9.1 & 23.0 & 16.6 \\
\hline $11 / 6 / 92$ & 2200 & 0.25 & 361.50 & 276.6 & 36.4 & 9.2 & 22.7 & 13.8 \\
\hline $11 / 6 / 92$ & 2215 & 0.25 & 361.75 & 276.4 & 35.1 & 9.0 & 23.2 & 12.9 \\
\hline $11 / 6 / 92$ & 2230 & 0.25 & 362.00 & 274.9 & 34.7 & 9.4 & 23.5 & 12.8 \\
\hline $11 / 6 / 92$ & 2245 & 0.25 & 362.26 & 275.5 & 34.5 & 9.1 & 22.8 & 12.8 \\
\hline $11 / 6 / 92$ & 2300 & 0.25 & 362.50 & 275.8 & 34.0 & 8.7 & 23.0 & 13.8 \\
\hline $11 / 6 / 92$ & 2315 & 0.25 & 362.75 & 274.9 & 34.4 & 8.8 & 22.0 & 12.4 \\
\hline $11 / 6 / 92$ & 2330 & 0.25 & 363.00 & 275.1 & 34.6 & 8.9 & 25.1 & 11.8 \\
\hline $11 / 6 / 92$ & 2345 & 0.25 & 363.25 & 275.5 & 34.1 & 9.3 & 24.8 & 10.7 \\
\hline Total Hours & & 23.75 & & & & & & \\
\hline $11 / 7 / 92$ & 0 & 0.25 & 363.50 & 274.7 & 33.9 & 9.0 & 23.4 & 10.5 \\
\hline $11 / 7 / 92$ & 15 & 0.25 & 363.75 & 274.9 & 34.3 & 8.8 & 14.6 & 10.7 \\
\hline $11 / 7 / 92$ & 30 & 0.25 & 364.00 & 275.6 & 34.4 & 9.0 & 20.3 & 14.7 \\
\hline $11 / 7 / 92$ & 45 & 0.25 & 364.25 & 277.4 & 34.5 & 9.2 & 19.9 & 10.8 \\
\hline $11 / 7 / 92$ & 100 & 0.25 & 364.50 & 277.0 & 34.6 & 8.8 & 21.5 & 10.2 \\
\hline $11 / 7 / 92$ & 115 & 0.25 & 364.75 & 276.7 & 35.6 & 9.3 & 21.4 & 10.3 \\
\hline $11 / 7 / 92$ & 130 & 0.25 & 365.00 & 277.2 & 35.1 & 8.9 & 21.7 & 11.3 \\
\hline $11 / 7 / 92$ & 145 & 0.25 & 365.25 & 275.1 & 34.5 & 9.2 & 23.2 & 8.3 \\
\hline $11 / 7 / 92$ & 200 & 0.25 & 365.50 & 273.5 & 34.5 & 9.0 & 25.5 & 8.2 \\
\hline $11 / 7 / 92$ & 215 & 0.25 & 365.75 & 274.4 & 34.2 & 9.1 & 24.3 & 10.5 \\
\hline $11 / 7 / 92$ & 230 & 0.25 & 366.00 & 275.3 & 34.5 & 8.9 & 24.7 & 10.2 \\
\hline $11 / 7 / 92$ & 245 & 0.25 & 366.25 & 275.1 & 35.9 & 9.2 & 25.9 & 9.6 \\
\hline $11 / 7 / 92$ & 300 & 0.25 & 366.50 & 274.1 & 34.2 & 8.8 & 25.5 & 9.7 \\
\hline $11 / 7 / 92$ & 315 & 0.25 & 366.75 & 275.4 & 33.0 & 9.2 & 24.0 & 8.8 \\
\hline $11 / 7 / 92$ & 330 & 0.25 & 367.00 & 275.3 & 32.0 & 8.6 & 23.1 & 10.6 \\
\hline $11 / 7 / 92$ & 345 & 0.25 & 367.25 & 274.0 & 33.0 & 9.2 & 23.9 & 9.8 \\
\hline $11 / 7 / 92$ & 400 & 0.25 & 367.50 & 273.1 & 31.7 & 9.2 & 23.4 & 10.2 \\
\hline $11 / 7 / 92$ & 415 & 0.25 & 367.75 & 272.0 & 31.7 & 9.1 & 27.1 & 10.0 \\
\hline $1117 / 92$ & 430 & 0.25 & 368.00 & 272.4 & 31.7 & 9.0 & 26.2 & 9.1 \\
\hline $11 / 7 / 92$ & 445 & 0.25 & 368.25 & 274.2 & 31.8 & 8.9 & 25.0 & 9.6 \\
\hline $11 / 7 / 92$ & 500 & 0.25 & 368.50 & 273.9 & 32.1 & 9.0 & 24.7 & 9.9 \\
\hline $11 / 7 / 92$ & 515 & 0.25 & 368.75 & 271.9 & 31.3 & 9.4 & 26.3 & 9.2 \\
\hline $11 / 7 / 92$ & 530 & 0.25 & 369.00 & 271.6 & 32.1 & 9.3 & 23.6 & 9.1 \\
\hline $11 / 7 / 92$ & 545 & 0.25 & 369.25 & 272.0 & 31.1 & 9.3 & 23.6 & 10.5 \\
\hline $11 / 7 / 92$ & 600 & 0.25 & 369.50 & 273.1 & 30.9 & 8.9 & 25.3 & 9.4 \\
\hline $11 / 7 / 92$ & 615 & 0.25 & 369.75 & 273.1 & 30.3 & 9.0 & 23.8 & 11.4 \\
\hline $11 / 7 / 92$ & 630 & 0.25 & 370.00 & 273.3 & 30.7 & 9.0 & 22.6 & 10.8 \\
\hline $11 / 7 / 92$ & 645 & 0.25 & 370.25 & 274.2 & 31.2 & 9.0 & 23.3 & 12.1 \\
\hline $1117 / 92$ & 700 & 0.25 & 370.50 & 274.5 & 31.2 & 8.8 & 23.5 & 12.8 \\
\hline $11 / 7 / 92$ & 715 & 0.25 & 370.75 & 274.6 & 32.1 & 8.9 & 22.1 & 10.7 \\
\hline $11 / 7 / 92$ & 730 & 0.25 & 371.00 & 275.4 & 33.1 & 9.2 & 23.6 & 9.8 \\
\hline $11 / 7 / 92$ & 745 & 0.25 & 371.25 & 275.3 & 33.1 & 9.1 & 25.5 & 10.8 \\
\hline $11 / 7 / 92$ & 800 & 0.25 & 371.50 & 274.9 & 33.1 & 9.0 & 25.4 & 9.1 \\
\hline
\end{tabular}

Page E-19 B-Duct Opacity>> " = Lower, "* = Hlgher 
SUMMARY OF LIME SLURRY INJECTION FROM OCTOBER 17, 1992 TO NOVEMBER 17, 1992

\begin{tabular}{|c|c|c|c|c|c|c|c|c|}
\hline Date & Time & Hours of & Cumulative & B Ducl Temp & Lime Flow & Slurry Solids & SO2 Removal & Stack Opacily \\
\hline & & Injection & Hours & of & GPM & Percent & Percent & Percent \\
\hline $11 / 7 / 92$ & 815 & 0.25 & 371.75 & 276.6 & 33.7 & 9.0 & 22.8 & 9.8 \\
\hline $11 / 7 / 92$ & 830 & 0.25 & 372.00 & 276.1 & 34.3 & 9.2 & 25.1 & 11.8 \\
\hline $11 / 7 / 92$ & 845 & 0.25 & 372.25 & 276.8 & 34.1 & 8.2 & 25.6 & 12.3 \\
\hline $11 / 7 / 92$ & 800 & 0.25 & 372.50 & 276.8 & 35.1 & 9.1 & 25.7 & 12.7 \\
\hline $11 / 7 / 82$ & 915 & 0.25 & 372.75 & 275.8 & 36.5 & 9.0 & 28.4 & 17.2 \\
\hline $11 / 7 / 92$ & 930 & 0.25 & 373.00 & 275.3 & 34.6 & 9.2 & 27.9 & 12.9 \\
\hline $11 / 7 / 92$ & 945 & 0.25 & 373.25 & 275.5 & 34.2 & 9.4 & 27.2 & 13.3 \\
\hline $11 / 7 / 92$ & 1000 & 0.25 & 373.50 & 274.0 & 34.4 & 9.1 & 23.8 & 11.2 \\
\hline $11 / 7 / 92$ & 1015 & 0.25 & 373.75 & 274.4 & 34.1 & 9.3 & 26.1 & 12.1 \\
\hline $11 / 7 / 92$ & 1030 & 0.25 & 374.00 & 274.4 & 33.4 & 8.8 & 26.5 & 12.1 \\
\hline $11 / 7 / 92$ & 1045 & 0.25 & 374.25 & 273.9 & 33.8 & 8.0 & 26.7 & 12.6 \\
\hline $11 / 7 / 92$ & 1100 & 0.25 & 374.50 & 273.3 & 33.2 & 8.9 & 26.7 & 13.4 \\
\hline $11 / 7 / 92$ & 1115 & 0.25 & 374.75 & 272.2 & 33.5 & 9.0 & 26.7 & 11.6 \\
\hline $11 / 7 / 82$ & 1130 & 0.25 & 375.00 & 274.0 & 32.4 & 8.8 & 25.7 & 11.1 \\
\hline $11 / 7 / 92$ & 1145 & 0.25 & 375.25 & 273.7 & 34.0 & 9.0 & 25.2 & 11.7 \\
\hline $11 / 7 / 92$ & 1200 & 0.25 & 375.50 & 273.8 & 32.6 & 8.9 & 28.0 & 14.5 \\
\hline $11 / 7 / 92$ & 1215 & 0.25 & 375.75 & 274.1 & 34.9 & 9.2 & 23.2 & 12.3 \\
\hline $11 / 7 / 92$ & 1230 & 0.25 & 376.00 & 274.2 & 32.8 & 9.2 & 27.5 & 12.2 \\
\hline $11 / 7 / 92$ & 1245 & 0.25 & 376.25 & 274.9 & 32.5 & 8.0 & 27.3 & 12.7 \\
\hline $11 / 7 / 92$ & 1300 & 0.25 & 376.50 & 273.8 & 31.9 & 9.1 & 24.3 & 13.3 \\
\hline $11 / 7 / 92$ & 1315 & 0.25 & 376.75 & 275.6 & 33.5 & 9.0 & 26.1 & 13.4 \\
\hline $11 / 7 / 92$ & 1330 & 0.25 & 377.00 & 276.4 & 34.4 & 8.8 & 23.4 & 14.1 \\
\hline $11 / 7 / 92$ & 1345 & 0.25 & 377.25 & 276.5 & 31.8 & 9.2 & 23.8 & 13.8 \\
\hline $11 / 7 / 92$ & 1400 & 0.25 & 377.50 & 275.3 & 34.7 & 8.9 & 24.9 & 12.6 \\
\hline $11 / 7 / 92$ & 1415 & 0.25 & 377.75 & 274.8 & 32.7 & 9.1 & 26.9 & 14.5 \\
\hline $11 / 7 / 92$ & 1430 & 0.25 & 378.00 & 274.7 & 31.3 & 9.0 & 24.7 & 13.6 \\
\hline $11 / 7 / 92$ & 1445 & 0.25 & 378.25 & 274.5 & 31.2 & 9.2 & 24.1 & 15.1 \\
\hline $11 / 7 / 92$ & 1500 & 0.25 & 378.50 & 273.6 & 30.8 & 9.3 & 23.2 & 13.7 \\
\hline $11 / 7 / 92$ & 1515 & 0.25 & 378.75 & 273.3 & 30.7 & 8.9 & 25.0 & 14.2 \\
\hline $11 / 7 / 92$ & 1530 & 0.25 & 379.00 & 273.4 & 32.1 & 9.0 & 22.8 & 13.9 \\
\hline $11 / 7 / 92$ & 1545 & 0.25 & 379.25 & 273.4 & 32.1 & 9.0 & 25.5 & 13.5 \\
\hline $11 / 7 / 92$ & 1600 & 0.25 & 379.50 & 274.6 & 32.6 & 9.2 & 24.2 & 13.9 \\
\hline $11 / 7 / 92$ & 1615 & 0.25 & 379.75 & 274.6 & 31.7 & 9.2 & 25.6 & 14.2 \\
\hline $11 / 7 / 92$ & 1630 & 0.25 & 380.00 & 274.1 & 33.1 & 8.9 & 26.3 & 14.0 \\
\hline $11 / 7 / 92$ & 1645 & 0.25 & 380.25 & 273.6 & 35.6 & 8.8 & 28.9 & 12.0 \\
\hline $11 / 7 / 92$ & 1700 & 0.25 & 380.50 & 273.4 & 34.6 & 8.8 & 24.7 & 12.4 \\
\hline $11 / 7 / 92$ & 1715 & 0.25 & 380.75 & 274.0 & 32.0 & 9.1 & 24.6 & 13.9 \\
\hline $11 / 7 / 92$ & 1730 & 0.25 & 381.00 & 275.9 & 33.1 & 8.9 & 23.7 & 15.3 \\
\hline $11 / 7 / 92$ & 1745 & 0.25 & 381.25 & 274.7 & 32.9 & 8.8 & 24.0 & 14.3 \\
\hline $11 / 7 / 92$ & 1800 & 0.25 & 381.50 & 273.8 & 33.7 & 9.0 & 26.3 & 14.4 \\
\hline $11 / 7 / 92$ & 1815 & 0.25 & 381.75 & 273.8 & 33.5 & 8.9 & 25.1 & 14.0 \\
\hline $11 / 7 / 92$ & 1830 & 0.25 & 382.00 & 274.7 & 34.2 & 9.1 & 25.5 & 13.2 \\
\hline $11 / 7 / 92$ & 1845 & 0.25 & 382.25 & 273.8 & 32.3 & 9.2 & 23.7 & 12.7 \\
\hline $11 / 7 / 92$ & $10 \cap 0$ & 0.25 & 382.50 & 274.2 & 34.9 & 8.8 & 25.5 & 13.2 \\
\hline $11 / 7 / 92$ & 1915 & 0.25 & 382.75 & 273.5 & 32.3 & 8.7 & 25.9 & 14.1 \\
\hline $11 / 7 / 92$ & 1930 & 0.25 & 383.00 & 273.6 & 31.6 & 8.9 & 23.7 & 13.7 \\
\hline $11 / 7 / 92$ & 1945 & 0.25 & 383.25 & 272.8 & 31.6 & 8.9 & 23.6 & 14.7 \\
\hline
\end{tabular}

Page E-20 B.Duct Opacity >> " = Lower, " = Higher 
SUMMARY OF LIME SLURRY INJECTION FROM OCTOBER 17, 1992 TO NOVEMBER 17, 1992

\begin{tabular}{|c|c|c|c|c|c|c|c|c|}
\hline Dalo & Time & Hours of & Cumulative & B Duct Temp. & Lime Flow & Slurry Solids & SO2 Removal & Slack Opacily \\
\hline & & Injection & Hours & ${ }^{\circ} \mathrm{F}$ & GPM & Percent & Percent & Percent \\
\hline $11 / 7 / 92$ & 2000 & 0.25 & 383.50 & 272.6 & 32.0 & 8.8 & 24.0 & 14.9 \\
\hline $11 / 7 / 92$ & 2015 & 0.25 & 383.75 & 274.2 & 32.3 & 9.1 & 21.1 & 14.1 \\
\hline $11 / 7 / 92$ & 2030 & 0.25 & 384.00 & 273.3 & 32.2 & 9.2 & 25.8 & 12.9 \\
\hline $11 / 7 / 92$ & 2045 & 0.25 & 384.25 & 273.5 & 32.9 & 9.1 & 22.8 & 14.0 \\
\hline $11 / 7 / 92$ & 2100 & 0.25 & 384.50 & 274.2 & 32.8 & 8.8 & 22.8 & 14.2 \\
\hline $11 / 7 / 92$ & 2115 & 0.25 & 384.75 & 274.6 & 33.4 & 9.0 & 24.4 & 14.5 \\
\hline $11 / 7 / 92$ & 2130 & 0.25 & 385.00 & 274.2 & 32.8 & 9.0 & 25.6 & 13.3 \\
\hline $11 / 7 / 92$ & 2145 & 0.25 & 385.25 & 273.8 & 32.6 & 9.0 & 24.8 & 15.0 \\
\hline $11 / 7 / 92$ & 2200 & 0.25 & 385.50 & 272.7 & 32.3 & 9.4 & 25.8 & 12.7 \\
\hline $11 / 7 / 92$ & 2215 & 0.25 & 385.75 & 273.3 & 32.6 & 8.0 & 23.1 & 13.2 \\
\hline $11 / 7 / 92$ & 2230 & 0.25 & 386.00 & 274.2 & 34.8 & 8.8 & 23.1 & 13.9 \\
\hline $11 / 7 / 92$ & 2245 & 0.25 & 386.25 & 274.7 & 33.1 & 9.1 & 22.1 & 13.2 \\
\hline $11 / 7 / 92$ & 2300 & 0.25 & 386.50 & 274.2 & 34.2 & 9.1 & 22.1 & 13.3 \\
\hline $11 / 7 / 92$ & 2315 & 0.25 & 386.75 & 275.0 & 33.8 & 9.0 & 22.8 & 14.4 \\
\hline $11 / 7 / 82$ & 2330 & 0.25 & 387.00 & 274.1 & 31.8 & 9.0 & 22.7 & 14.7 \\
\hline $11 / 7 / 92$ & 2345 & 0.25 & 387.25 & 274.3 & 32.0 & 9.2 & 22.6 & 13.1 \\
\hline \multicolumn{2}{|c|}{ Tolal Hours } & 24.00 & & & & & & \\
\hline $11 / 8 / 92$ & 0 & 0.25 & 387.50 & 274.0 & 31.4 & 8.8 & 24.5 & 13.3 \\
\hline $11 / 8 / 92$ & 15 & 0.25 & 387.75 & 273.7 & 33.1 & 8.8 & 22.5 & 13.0 \\
\hline $11 / 8 / 92$ & 30 & 0.25 & 388.00 & 274.2 & 33.0 & 9.1 & 24.6 & 14.8 \\
\hline $11 / 8 / 92$ & 45 & 0.25 & 388.25 & 275.2 & 33.5 & 9.0 & 21.8 & 13.6 \\
\hline $11 / 8 / 92$ & 100 & 0.25 & 388.50 & 275.0 & 32.8 & 9.2 & 23.8 & 14.3 \\
\hline $11 / 8 / 92$ & 115 & 0.25 & 388.75 & 275.5 & 32.6 & 9.1 & 23.3 & 15.0 \\
\hline $11 / 8 / 92$ & 130 & 0.25 & 389.00 & 274.3 & 31.9 & 8.8 & 19.1 & 10.7 \\
\hline $11 / 8 / 92$ & 145 & 0.25 & 389.25 & 273.4 & 31.1 & 9.2 & 19.3 & 10.7 \\
\hline $11 / 8 / 92$ & 200 & 0.25 & 389.50 & 270.9 & 31.3 & 8.9 & 24.0 & 10.6 \\
\hline $11 / 8 / 92$ & 215 & 0.25 & 389.75 & 270.6 & 33.9 & 9.2 & 17.6 & 9.1 \\
\hline $11 / 8 / 92$ & 230 & 0.25 & 390.00 & 273.4 & 30.2 & 8.9 & 18.3 & 10.0 \\
\hline $11 / 8 / 92$ & 245 & 0.25 & 390.25 & 273.2 & 29.5 & 8.9 & 20.2 & 11.8 \\
\hline $11 / 8 / 92$ & 300 & 0.25 & 390.50 & 272.7 & 30.1 & 9.2 & 20.2 & 11.7 \\
\hline $11 / 8 / 92$ & 315 & 0.25 & 390.75 & 273.1 & 31.2 & 9.2 & 21.1 & 9.5 \\
\hline $11 / 8 / 92$ & 330 & 0.25 & 391.00 & 273.9 & 30.7 & 8.8 & 21.8 & 9.7 \\
\hline $11 / 8 / 92$ & 345 & 0.25 & 391.25 & 272.4 & 30.9 & 9.3 & 20.9 & 12.3 \\
\hline $11 / 8 / 92$ & 400 & 0.25 & 391.50 & 272.0 & 31.0 & 9.3 & 21.0 & 9.8 \\
\hline $11 / 8 / 92$ & 415 & 0.25 & 391.75 & 272.7 & 31.8 & 9.1 & 24.9 & 9.3 \\
\hline $11 / 8 / 92$ & 430 & 0.25 & 392.00 & 273.8 & 31.4 & 8.9 & 22.3 & 10.3 \\
\hline $11 / 8 / 92$ & 445 & 0.25 & 392.25 & 273.2 & 34.2 & 8.8 & 24.6 & 8.6 \\
\hline $11 / 8 / 92$ & 500 & 0.25 & 392.50 & 273.5 & 31.7 & 9.3 & 23.6 & 10.0 \\
\hline $11 / 8 / 92$ & 515 & 0.25 & 392.75 & 273.7 & 33.3 & 8.9 & 24.4 & 8.8 \\
\hline $11 / 8 / 92$ & 530 & 0.25 & 393.00 & 273.8 & 33.1 & 9.1 & 25.4 & 9.5 \\
\hline $11 / 8 / 92$ & 545 & 0.25 & 393.25 & 273.5 & 32.3 & 8.8 & 25.6 & 8.4 \\
\hline $11 / 8 / 92$ & 600 & 0.25 & 393.50 & 272.5 & 32.2 & 9.1 & 25.9 & 8.2 \\
\hline $11 / 8 / 92$ & 615 & 0.25 & 393.75 & 273.5 & 32.2 & 9.3 & 24.8 & 9.1 \\
\hline $11 / 8 / 92$ & 630 & 0.25 & 394.00 & 271.8 & 33.2 & 9.2 & 27.7 & 7.3 \\
\hline $11 / 8 / 92$ & 645 & 0.25 & 394.25 & 271.0 & 35.7 & 9.0 & 26.9 & 6.5 \\
\hline $11 / 8 / 92$ & 700 & 0.25 & 394.50 & 273.0 & 32.1 & 8.8 & 28.5 & 7.1 \\
\hline
\end{tabular}

Page E-21 B.Duct Opacity >> " - Lower, "* = Higher 
SUMMARY OF LIME SLURRY INJECTION FROM OCTOBER 17, 1992 TO NOVEMBER 17, 1992

\begin{tabular}{|c|c|c|c|c|c|c|c|c|}
\hline Dale & Time & $\begin{array}{l}\text { Hours of } \\
\text { Injection }\end{array}$ & $\begin{array}{l}\text { Cumulalive } \\
\text { Hours }\end{array}$ & $\begin{array}{c}\text { B Ducl Tomp } \\
{ }^{\circ} \mathrm{F}\end{array}$ & $\begin{array}{c}\text { Lime Flow } \\
\text { GPM }\end{array}$ & $\begin{array}{c}\text { Slurry Solids } \\
\text { Percent }\end{array}$ & $\begin{array}{c}\text { SO2 Removal } \\
\text { Porcent }\end{array}$ & $\begin{array}{c}\text { Slack Opacily } \\
\text { Percent }\end{array}$ \\
\hline $11 / 8 / 82$ & 715 & 0.25 & 394.75 & 272.3 & 31.6 & 9.0 & 25.6 & 8.3 \\
\hline $11 / 8 / 92$ & 730 & 0.25 & 395.00 & 272.9 & 32.4 & 9.0 & 24.8 & 8.3 \\
\hline $11 / 8 / 92$ & 745 & 0.25 & 395.25 & 274.2 & 32.8 & 8.8 & 26.0 & 8.8 \\
\hline $11 / 8 / 82$ & 800 & 0.25 & 395.50 & 274.7 & 32.9 & 0.0 & 25.6 & 8.7 \\
\hline $11 / 8 / 82$ & 815 & 0.25 & 395.75 & 273.4 & 35.5 & 9.2 & 24.2 & 9.3 \\
\hline $11 / 8 / 92$ & 830 & 0.25 & 396.00 & 273.8 & 33.3 & 8.8 & 25.4 & 8.4 \\
\hline $11 / 8 / 82$ & 845 & 0.25 & 396.25 & 275.2 & 34.6 & 0.1 & 24.6 & 10.0 \\
\hline $11 / 8 / 82$ & 900 & 0.25 & 396.50 & 275.9 & 34.7 & 0.1 & 27.8 & 0.3 \\
\hline $11 / 8 / 92$ & 915 & 0.25 & 396.75 & 276.0 & 34.6 & 8.0 & 26.7 & 9.0 \\
\hline $11 / 8 / 82$ & 830 & 0.25 & 397.00 & 275.1 & 34.0 & 8.9 & 27.3 & 9.0 \\
\hline $11 / 8 / 92$ & 945 & 0.25 & 397.25 & 274.5 & 34.5 & 8.8 & 25.7 & 8.9 \\
\hline $11 / 8 / 92$ & 1000 & 0.25 & 397.50 & 273.6 & 33.1 & 8.9 & 28.3 & 0.0 \\
\hline $11 / 8 / 92$ & 1015 & 0.25 & 397.75 & 272.6 & 33.4 & 9.3 & 26.5 & 8.3 \\
\hline $11 / 8 / 92$ & 1030 & 0.25 & 398.00 & 272.3 & 33.5 & 8.9 & 25.2 & 8.2 \\
\hline $11 / 8 / 92$ & 1045 & 0.25 & 398.25 & 271.0 & 33.2 & 9.0 & 31.0 & 7.8 \\
\hline $11 / 8 / 92$ & 1100 & 0.25 & 398.50 & 271.3 & 34.5 & 8.8 & 27.5 & 0.0 \\
\hline $11 / 8 / 82$ & 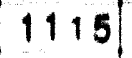 & 0.25 & 388.75 & 271.8 & 33.3 & 9.0 & 28.5 & 8.9 \\
\hline $11 / 8 / 92$ & 1130 & 0.25 & 399.00 & 271.5 & 31.8 & 8.1 & 28.3 & 9.8 \\
\hline $11 / 8 / 82$ & 1145 & 0.25 & 399.25 & 273.3 & 33.5 & 9.2 & 28.3 & 0.5 \\
\hline $11 / 8 / 92$ & 1200 & 0.25 & 399.50 & 273.3 & 32.8 & 9.2 & 26.8 & 8.3 \\
\hline $11 / 8 / 92$ & 1215 & 0.25 & 399.75 & 274.0 & 32.4 & 8.8 & 25.9 & 8.6 \\
\hline $11 / 8 / 92$ & 1230 & 0.25 & 400.00 & 273.6 & 33.0 & 8.7 & 24.8 & 8.0 \\
\hline $11 / 8 / 92$ & 1245 & 0.25 & 400.25 & 272.6 & 32.1 & 8.1 & 26.9 & 7.8 \\
\hline $11 / 8 / 92$ & 1300 & 0.25 & 400.50 & 271.8 & 32.1 & 7.3 & 25.3 & 7.5 \\
\hline $11 / 8 / 92$ & 1315 & 0.25 & 400.75 & 272.1 & 31.2 & 6.7 & 27.6 & 8.0 \\
\hline $11 / 8 / 92$ & 1330 & 0.25 & 401.00 & 273.0 & 32.4 & 6.0 & 24.9 & 9.8 \\
\hline $11 / 8 / 92$ & 1345 & 0.25 & 401.25 & 274.5 & 31.7 & 7.5 & 25.2 & 8.3 \\
\hline $11 / 8 / 92$ & 1400 & 0.25 & 401.50 & 275.9 & 32.4 & 8.4 & 26.6 & 8.4 \\
\hline $11 / 8 / 92$ & 1415 & 0.25 & 401.75 & 275.9 & 35.5 & 8.8 & 27.7 & 10.3 \\
\hline $11 / 8 / 92$ & 1430 & 0.2 .5 & 402.00 & 276.8 & 34.8 & 9.6 & 26.7 & 9.0 \\
\hline $11 / 8 / 92$ & 1445 & 0.25 & 402.25 & 276.1 & 34.2 & 9.2 & 26.8 & 8.8 \\
\hline $11 / 8 / 92$ & 1500 & 0.25 & 402.50 & 275.0 & 33.2 & 9.2 & 26.6 & 7.1 \\
\hline $11 / 8 / 92$ & 1515 & 0.25 & 402.75 & 274.4 & 33.5 & 9.1 & 27.3 & 8.5 \\
\hline $11 / 8 / 92$ & 1530 & 0.25 & 403.00 & 276.0 & 33.7 & 8.7 & 26.6 & 6.8 \\
\hline $11 / 8 / 92$ & 1545 & 0.25 & 403.25 & 277.2 & 34.4 & 9.3 & 26.0 & 6.3 \\
\hline $11 / 8 / 92$ & 1600 & 0.25 & 403.50 & 278.7 & 34.2 & 8.8 & 26.0 & 6.5 \\
\hline $11 / 8 / 92$ & 1615 & 0.25 & 403.75 & 278.8 & 34.4 & 8.7 & 25.0 & 8.0 \\
\hline $11 / 8 / 92$ & 1630 & 0.25 & 404.00 & 278.4 & 33.7 & 9.2 & 28.0 & 9.1 \\
\hline $11 / 8 / 92$ & 1645 & 0.25 & 404.25 & 276.4 & 33.1 & 9.0 & 25.9 & 7.6 \\
\hline $11 / 8 / 92$ & 1700 & 0.25 & 404.50 & 276.5 & 33.2 & 9.1 & 27.3 & 7.9 \\
\hline $11 / 8 / 92$ & 1715 & 0.25 & 404.75 & 275.2 & 33.0 & 9.1 & 26.3 & 7.3 \\
\hline $11 / 8 / 92$ & 1730 & 0.25 & 405.00 & 275.1 & 33.6 & 9.4 & 26.1 & 7.6 \\
\hline $11 / 8 / 92$ & 1745 & 0.25 & 405.25 & 274.6 & 34.1 & 8.8 & 26.7 & 7.3 \\
\hline $11 / 8 / 92$ & 1800 & 0.25 & 405.50 & 274.4 & 33.6 & 9.0 & 27.7 & 8.8 \\
\hline $11 / 8 / 92$ & 1815 & 0.25 & 405.75 & 274.8 & 34.5 & 8.8 & 26.9 & 8.2 \\
\hline $11 / 8 / 92$ & 1830 & 0.25 & 406.00 & 274.8 & 32.9 & 9.0 & 26.3 & 7.3 \\
\hline $11 / 8 / 92$ & 1845 & 0.25 & 406.25 & 274.7 & 35.8 & 9.0 & 27.5 & 6.8 \\
\hline
\end{tabular}

Page E-22 B.Duct Opacity>> " = Lower, " - Higher 
SUMMARY OF LIME SLUARY INJECTION FROM OCTOBER 17, 1992 TO NOVEMBER 17, 1992

\begin{tabular}{|c|c|c|c|c|c|c|c|c|}
\hline Dale & Time & $\begin{array}{l}\text { Hours of } \\
\text { Injection }\end{array}$ & $\begin{array}{c}\text { Cumulalive } \\
\text { Hours }\end{array}$ & $\begin{array}{c}\text { B Ducl Tomp } \\
\text { of }\end{array}$ & $\begin{array}{c}\text { Lime Flow } \\
\text { QPM }\end{array}$ & $\begin{array}{c}\text { Slurry Solids } \\
\text { Percent }\end{array}$ & $\begin{array}{c}\text { SO2 Removal } \\
\text { Percent }\end{array}$ & $\begin{array}{c}\text { Slack Opacily } \\
\text { Percent }\end{array}$ \\
\hline $11 / 8 / 92$ & 1900 & 0.26 & 406.50 & 274.5 & 33.9 & 8.7 & 25.4 & 7.1 \\
\hline $11 / 8 / 92$ & 1915 & 0.25 & 406.75 & 274.5 & 33.5 & 9.1 & 26.0 & 7.4 \\
\hline $11 / 8 / 92$ & 1930 & 0.25 & 407.00 & 274.3 & 34.2 & 8.8 & 26.7 & 7.9 \\
\hline $11 / 8 / 92$ & 1845 & 0.26 & 407.25 & 273.8 & 33.8 & 8.6 & 28.2 & 8.0 \\
\hline $11 / 8 / 92$ & 2000 & 0.25 & 407.50 & 273.0 & 34.0 & 8.9 & 25.3 & 7.7 \\
\hline $11 / 8 / 92$ & 2015 & 0.25 & 407.75 & 273.0 & 37.6 & 8.9 & 27.6 & 7.7 \\
\hline $11 / 8 / 82$ & 2030 & 0.25 & 408.00 & 273.2 & 34.2 & 8.9 & 28.9 & 7.8 \\
\hline $11 / 8 / 82$ & 2045 & 0.25 & 408.26 & 272.5 & 32.8 & 8.4 & 26.8 & 7.7 \\
\hline $11 / 8 / 92$ & 2100 & 0.25 & 408.50 & 271.2 & 33.3 & 8.0 & 27.1 & 6.8 \\
\hline $11 / 8 / 82$ & 2118 & 0.25 & 408.75 & 271.0 & 32.1 & 8.3 & 24.3 & 7.4 \\
\hline $11 / 8 / 92$ & 2130 & 0.25 & 409.00 & 271.4 & 35.4 & 9.1 & 28.1 & 6.8 \\
\hline $11 / 8 / 92$ & 2145 & 0.25 & 409.25 & 271.0 & 34.1 & 8.8 & 28.0 & 7.4 \\
\hline $11 / 8 / 82$ & 2200 & 0.25 & 409.50 & 271.1 & 32.9 & 9.0 & 28.6 & 7.8 \\
\hline $11 / 8 / 92$ & 2215 & 0.25 & 409.75 & 270.8 & 32.8 & 9.1 & 26.0 & 8.1 \\
\hline $11 / 8 / 82$ & 2230 & 0.25 & 410.00 & 269.4 & 33.3 & 9.1 & 27.9 & 8.5 \\
\hline $11 / 8 / 92$ & 2245 & 0.25 & 410.25 & 270.1 & 29.1 & 9.3 & 27.1 & 8.5 \\
\hline $11 / 8 / 82$ & 2300 & 0.25 & 410.50 & 269.6 & 29.8 & 9.2 & 26.3 & 0.5 \\
\hline $11 / 8 / 92$ & 2315 & 0.25 & 410.75 & 268.8 & 30.1 & 9.0 & 27.1 & 8.7 \\
\hline $11 / 8 / 92$ & 2330 & 0.26 & 411.00 & 271.8 & 30.1 & 9.3 & 27.8 & 7.7 \\
\hline $11 / 8 / 92$ & 2345 & 0.25 & 411.25 & 274.8 & 28.9 & 8.8 & 22.3 & 7.1 \\
\hline Tolal Hours & & 24.00 & & & & & & \\
\hline $11 / 9 / 92$ & 0 & 0.25 & 411.50 & 275.2 & 28.6 & 8.8 & 24.6 & 8.0 \\
\hline $11 / 9 / 92$ & 15 & 0.25 & 411.75 & 273.2 & 28.1 & 8.9 & 28.3 & 7.1 \\
\hline $11 / 9 / 92$ & 30 & 0.25 & 412.00 & 275.4 & 29.5 & 8.9 & 27.8 & 7.8 \\
\hline $11 / 9 / 92$ & 45 & 0.25 & 412.25 & 277.8 & 29.4 & 9.0 & 36.7 & 8.4 \\
\hline $11 / 9 / 92$ & 100 & 0.25 & 412.50 & 269.9 & 28.3 & 8.9 & 24.4 & 6.9 \\
\hline $11 / 9 / 92$ & 115 & 0.25 & 412.75 & 266.1 & 26.9 & 9.2 & 29.1 & 7.5 \\
\hline $11 / 9 / 92$ & 130 & 0.25 & 413.00 & 264.4 & 26.9 & 9.0 & 28.7 & 7.3 \\
\hline $11 / 9 / 92$ & 145 & 0.25 & 413.25 & 265.3 & 27.1 & 9.4 & 26.7 & 7.7 \\
\hline $11 / 9 / 92$ & 200 & 0.25 & 413.50 & 265.8 & 26.6 & 9.0 & 27.3 & 7.9 \\
\hline $11 / 9 / 92$ & 215 & 0.25 & 413.75 & 265.7 & 27.1 & 8.8 & 27.5 & 6.0 \\
\hline $11 / 9 / 92$ & 230 & 0.25 & 414.00 & 265.5 & 25.7 & 9.0 & 31.9 & 7.2 \\
\hline $11 / 9 / 92$ & 245 & 0.25 & 414.25 & 266.7 & 27.5 & 8.9 & 32.8 & 6.3 \\
\hline $11 / 9 / 92$ & 300 & 0.25 & 414.50 & 268.8 & 26.2 & 9.3 & 30.4 & 8.3 \\
\hline $11 / 9 / 92$ & 315 & 0.25 & 414.75 & 268.8 & 26.2 & 9.0 & 27.6 & 7.2 \\
\hline $11 / 9 / 92$ & 330 & 0.25 & 415.00 & 267.4 & 26.6 & 9.2 & 27.7 & 7.1 \\
\hline $11 / 9 / 92$ & 345 & 0.25 & 415.25 & 268.1 & 26.9 & 9.3 & 28.3 & 8.5 \\
\hline $11 / 9 / 92$ & 400 & 0.25 & 415.50 & 268.5 & 27.2 & 9.1 & 28.5 & 7.7 \\
\hline $11 / 9 / 92$ & 415 & 0.25 & 415.75 & 267.8 & 26.6 & 9.2 & 30.0 & 7.1 \\
\hline $11 / 9 / 92$ & 430 & 0.25 & 410.00 & 268.3 & 26.6 & 8.9 & 28.2 & 7.6 \\
\hline $11 / 9 / 92$ & 445 & 0.25 & 416.25 & 268.7 & 26.8 & 9.1 & 29.1 & 7.1 \\
\hline $11 / 9 / 92$ & 500 & 0.25 & 416.50 & 268.8 & 27.0 & 8.9 & 29.4 & 7.0 \\
\hline $11 / 9 / 92$ & 515 & 0.25 & 416.75 & 269.6 & 26.8 & 9.0 & 28.3 & 7.4 \\
\hline $11 / 9 / 92$ & 530 & 0.25 & 417.00 & 268.4 & 26.7 & 8.9 & 28.1 & 10.9 \\
\hline $11 / 9 / 92$ & 545 & 0.25 & 417.25 & 268.8 & 28.0 & 9.2 & 29.5 & 11.6 \\
\hline $11 / 9 / 92$ & 600 & 0.25 & 417.50 & 268.5 & 28.0 & 9.4 & 30.0 & 10.7 \\
\hline
\end{tabular}

Page E-23 B-Duct Opacity >> " Lower, " = Higher 
SUMMARY OF LIME SLUARY INJECTION FROM OCTOBER 17, 1992 TO NOVEMBER 17, 1992

\begin{tabular}{|c|c|c|c|c|c|c|c|c|}
\hline Date & Time & Houre of & Cumulalive & B Duat Temp & Lime Flow & Shury Solids & S02 Removal & Stack Opactiy \\
\hline & & Injection & Hours & of & GPM & Percent & Percent & Porcent \\
\hline $11 / 0 / 02$ & 615 & 0.28 & 417.78 & 260.0 & 28.4 & 8.8 & 29.1 & 10.8 \\
\hline $11 / 9102$ & 630 & 0.25 & $\$ 18.00$ & 268.7 & 28.1 & 8.9 & 31.0 & 9.5 \\
\hline $11 / 9 / 02$ & 648 & 0.28 & 418.25 & 268.0 & 28.8 & 0.3 & 33.3 & 10.2 \\
\hline $11 / 0102$ & 700 & 0.26 & 418.50 & 268.0 & 28.8 & 8.9 & 30.6 & 11.1 \\
\hline $11 / 9192$ & 715 & 0.25 & 418.75 & 271.0 & 29.8 & 8.0 & 32.5 & 10.7 \\
\hline $11 / 9 / 92$ & 730 & 0.25 & 418.00 & 266.0 & 26.7 & 9.3 & 32.2 & 14.2 \\
\hline $11 / 9 / 92$ & 746 & 0.25 & 410.26 & 267.1 & 30.3 & 8.9 & 36.0 & 0.8 \\
\hline $11 / 9 / 02$ & 800 & 0.26 & $\mathbf{4 1 9 . 5 0}$ & 267.1 & 30.3 & 8.9 & 36.9 & 9.8 \\
\hline $11 / 8182$ & 815 & 0.28 & 419.75 & 267.1 & 30.3 & 8.9 & 36.9 & 9.8 \\
\hline $11 / 8 / 82$ & 830 & 0.26 & 420.00 & 267.1 & 30.3 & 8.9 & 36.8 & 9.8 \\
\hline $11 / 9102$ & 845 & 0.25 & 420.26 & 267.1 & 30.3 & 8.9 & 36.0 & 9.8 \\
\hline $11 / 9 / 92$ & 900 & 0.28 & 420.50 & 267.1 & 30.3 & 8.9 & 36.8 & 9.8 \\
\hline Total Hour: & & 9.25 & & 268.4 & 28.3 & 9.0 & 31.6 & 9.1 \\
\hline $11 / 12 / 92$ & 1130 & 0.25 & 420.76 & 286.7 & 37.1 & 6.6 & 20.0 & 16.8 \\
\hline $11 / 12 / 92$ & 1145 & 0.25 & 421.00 & 286.2 & 37.3 & 8.2 & 34.2 & 15.1 \\
\hline $11 / 12 / 82$ & 1200 & 0.25 & 421.26 & 284.8 & 37.6 & 9.4 & 31.7 & 16.7 \\
\hline $11 / 12 / 92$ & 1218 & 0.26 & 421.50 & 286.5 & 36.8 & 10.3 & 31.5 & 16.8 \\
\hline $11 / 12 / 92$ & 1230 & 0.25 & 421.75 & 286.5 & 36.9 & 12.8 & 31.8 & 17.2 \\
\hline $11 / 12 / 92$ & 1245 & 0.25 & 422.00 & 287.1 & 37.2 & 16.4 & 32.7 & 16.3 \\
\hline $11 / 12 / 92$ & 1300 & 0.25 & 422.25 & 286.7 & 37.0 & 18.3 & 32.3 & 18.0 \\
\hline $11 / 12 / 92$ & 1315 & 0.25 & 422.50 & 287.5 & 37.0 & 18.0 & 33.2 & 15.5 \\
\hline $11 / 12 / 92$ & 1330 & 0.25 & 422.75 & 287.7 & 36.9 & 17.7 & 32.8 & 18.2 \\
\hline $11 / 12 / 92$ & 1345 & 0.25 & 423.00 & 288.2 & 36.8 & 16.5 & 35.4 & 16.1 \\
\hline $11 / 12 / 92$ & 1400 & 0.25 & 423.25 & 285.9 & 36.8 & 14.6 & 35.1 & 24.1 \\
\hline $11 / 12 / 92$ & 1415 & 0.25 & 423.50 & 287.0 & 36.7 & 12.9 & 31.8 & 17.1 \\
\hline $11 / 12 / 92$ & 1430 & 0.25 & 423.75 & 286.4 & 36.5 & 12.1 & 32.7 & 18.6 \\
\hline $11 / 12 / 92$ & 1445 & 0.25 & 424.00 & 287.0 & 36.8 & 11.2 & 33.5 & 14.1 \\
\hline $11 / 12 / 92$ & 1600 & 0.25 & 424.25 & 285.7 & 38.5 & 10.1 & 35.6 & 14.3 \\
\hline $11 / 12 / 92$ & 1515 & 0.25 & 424.50 & 285.1 & 37.5 & 9.7 & 35.7 & 15.4 \\
\hline $11 / 12 / 92$ & 1530 & 0.25 & 424.75 & 284.7 & 37.7 & 10.0 & 39.3 & 14.2 \\
\hline $11 / 12 / 92$ & 1545 & 0.25 & 425.00 & 289.2 & 38.2 & 10.1 & 36.2 & 17.9 \\
\hline $11 / 12 / 92$ & 1600 & 0.25 & 425.25 & 291.8 & 39.9 & 10.2 & 29.8 & 15.8 \\
\hline $11 / 12 / 92$ & 1615 & 0.25 & 425.50 & 291.8 & 39.8 & 9.9 & 31.7 & 17.3 \\
\hline $11 / 12 / 92$ & 1630 & 0.25 & 426.75 & 291.9 & 39.5 & 9.7 & 35.4 & 14.2 \\
\hline $11 / 12 / 92$ & 1645 & 0.25 & 426.00 & 291.9 & 39.5 & 10.3 & 30.8 & 12.2 \\
\hline $11 / 12 / 82$ & 1700 & 0.25 & 426.25 & 291.8 & 39.8 & 9.7 & 31.0 & 12.4 \\
\hline $11 / 12 / 92$ & 1715 & 0.25 & 426.50 & 291.1 & 39.1 & 10.1 & 29.6 & 12.8 \\
\hline $11 / 12 / 92$ & 1730 & 0.25 & 426.75 & 290.5 & 39.5 & 10.2 & 29.5 & 12.8 \\
\hline $11 / 12 / 92$ & 1745 & 0.25 & 427.00 & 290.5 & 38.1 & 10.0 & 27.2 & 13.5 \\
\hline $11 / 12 / 92$ & 1800 & 0.25 & 427.25 & 290.2 & 38.1 & 9.8 & 28.1 & 13.7 \\
\hline $11 / 12 / 92$ & 1815 & 0.25 & 427.50 & 290.1 & 37.7 & 10.2 & 28.3 & 14.0 \\
\hline $11 / 12 / 92$ & 1830 & 0.25 & 427.75 & 290.3 & 37.5 & 9.9 & 26.8 & 14.5 \\
\hline $11 / 12 / 92$ & 1845 & 0.25 & 428.00 & 290.7 & 37.7 & 10.1 & 27.4 & 14.7 \\
\hline $11 / 12 / 92$ & 1900 & 0.25 & 428.25 & 290.2 & 37.1 & 10.1 & 27.9 & 15.6 \\
\hline $11 / 12 / 92$ & 1915 & 0.25 & 428.50 & 290.0 & 36.9 & 10.0 & 25.7 & 14.8 \\
\hline $11 / 12 / 92$ & 1930 & 0.25 & 428.75 & 290.2 & 36.9 & 10.1 & 26.4 & 15.3 \\
\hline
\end{tabular}

Page E-24 B-Duct Opacity>> " = Lower, " - Hlgher 
SUMMARY OF LIME SLURRY INJECTION FROM OCTOBER 17, 1992 TO NOVEMBER 17, 1992

\begin{tabular}{|c|c|c|c|c|c|c|c|c|}
\hline Date & Time & Hours of & Cumulative & B Duct Temp & Lime Flow & Slurry Solids & SO2 Removal & Stack Opacity \\
\hline & & Injection & Hours & ${ }^{\circ} \mathrm{F}$ & GPM & Percent & Percent & Percent \\
\hline $11 / 12 / 92$ & 1945 & 0.25 & 429.00 & 290.3 & 36.8 & 10.1 & 25.0 & 14.8 \\
\hline $11 / 12 / 92$ & 2000 & 0.25 & 429.25 & 291.3 & 37.5 & 10.1 & 25.9 & 16.0 \\
\hline $11 / 12 / 92$ & 2015 & 0.25 & 429.50 & 291.3 & 37.0 & 10.1 & 24.3 & 15.8 \\
\hline $11 / 12 / 92$ & 2030 & 0.25 & 429.75 & 291.4 & 36.8 & 10.2 & 23.2 & 17.0 \\
\hline $11 / 12 / 92$ & 2045 & 0.25 & 430.00 & 290.7 & 37.2 & 9.6 & 27.6 & 18.1 \\
\hline $11 / 12 / 92$ & 2100 & 0.25 & 430.25 & 290.6 & 35.9 & 10.3 & 26.5 & 14.0 \\
\hline $11 / 12 / 92$ & 2115 & 0.25 & 430.50 & 291.2 & 36.3 & 10.1 & 21.3 & 16.7 \\
\hline $11 / 12 / 92$ & 2130 & 0.25 & 430.75 & 291.2 & 36.0 & 10.3 & 22.6 & 15.0 \\
\hline $11 / 12 / 92$ & 2145 & 0.25 & 431.00 & 290.1 & 36.0 & 10.1 & 29.2 & 15.1 \\
\hline $11 / 12 / 92$ & 2200 & 0.25 & 431.25 & 288.1 & 35.9 & 9.7 & 29.5 & 15.5 \\
\hline $11 / 12 / 92$ & 2215 & 0.25 & 431.50 & 287.7 & 36.0 & 9.8 & 29.4 & 16.4 \\
\hline $11 / 12 / 92$ & 2230 & 0.25 & 431.75 & 288.6 & 37.3 & 10.1 & 28.8 & 19.6 \\
\hline \multicolumn{2}{|c|}{ Total Hours } & 11.25 & & & & & & \\
\hline $11 / 13 / 92$ & 845 & 0.25 & 432.00 & 277.0 & 32.1 & 10.2 & 13.3 & 12.4 \\
\hline $11 / 13 / 92$ & 900 & 0.25 & 432.25 & 277.3 & 32.4 & 10.4 & 14.2 & 8.0 \\
\hline $11 / 13 / 92$ & 915 & 0.25 & 432.50 & 277.3 & 32.1 & 10.7 & 14.2 & 8.8 \\
\hline $11 / 13 / 92$ & 930 & 0.25 & 432.75 & 277.2 & 32.0 & 10.8 & 14.1 & 9.1 \\
\hline $11 / 13 / 92$ & 1500 & 0.25 & 433.00 & 277.4 & 35.2 & 11.4 & 14.4 & 9.7 \\
\hline $11 / 13 / 92$ & 1515 & 0.25 & 433.25 & 281.8 & 38.3 & 11.0 & 15.9 & 10.3 \\
\hline $11 / 13 / 92$ & 1530 & 0.25 & 433.50 & 283.2 & 38.7 & 11.2 & 16.6 & 9.2 \\
\hline $11 / 13 / 92$ & 1545 & 0.25 & 433.75 & 284.1 & 38.9 & 10.0 & 16.6 & 9.0 \\
\hline $11 / 13 / 92$ & 1600 & 0.25 & 434.00 & 284.1 & 39.3 & 9.1 & 18.2 & 10.6 \\
\hline $11 / 13 / 92$ & 1615 & 0.25 & 434.25 & 283.5 & 39.6 & 8.0 & 17.7 & 10.1 \\
\hline $11 / 13 / 92$ & 1630 & 0.25 & 434.50 & 283.2 & 39.8 & 7.0 & 19.0 & 10.3 \\
\hline $11 / 13 / 92$ & 1645 & 0.25 & 434.75 & 282.6 & 40.0 & 6.1 & 19.0 & 10.4 \\
\hline $11 / 13 / 92$ & 1700 & 0.25 & 435.00 & 281.7 & 39.7 & 5.8 & 17.0 & 10.8 \\
\hline $11 / 13 / 92$ & 1715 & 0.25 & 435.25 & 281.6 & 39.1 & 5.4 & 17.7 & 11.5 \\
\hline $11 / 13 / 92$ & 1730 & 0.25 & 435.50 & 282.0 & 39.1 & 6.6 & 18.0 & 12.9 \\
\hline $11 / 13 / 92$ & 1745 & 0.25 & 435.75 & 282.0 & 38.8 & 8.5 & 16.4 & 13.4 \\
\hline $11 / 13 / 92$ & 1800 & 0.25 & 436.00 & 281.5 & 39.2 & 8.9 & 16.7 & 12.9 \\
\hline $11 / 13 / 92$ & 1815 & 0.25 & 436.25 & 281.8 & 39.0 & 9.5 & 16.4 & 12.1 \\
\hline $11 / 13 / 92$ & 1830 & 0.25 & 436.50 & 280.6 & 38.0 & 9.7 & 14.6 & 12.7 \\
\hline $11 / 13 / 92$ & 1845 & 0.25 & 436.75 & 279.7 & 37.5 & 9.7 & 18.0 & 12.8 \\
\hline $11 / 13 / 92$ & 1900 & 0.25 & 437.00 & 279.4 & 37.7 & 9.1 & 20.3 & 14.5 \\
\hline $11 / 13 / 92$ & 1915 & 0.25 & 437.25 & 279.9 & 36.9 & 8.2 & 17.9 & 13.4 \\
\hline $11 / 13 / 92$ & 1930 & 0.25 & 437.50 & 279.7 & 36.9 & 9.4 & 17.7 & 13.1 \\
\hline $11 / 13 / 92$ & 1945 & 0.25 & 437.75 & 278.6 & 36.7 & 9.8 & 18.5 & 13.3 \\
\hline $11 / 13 / 92$ & 2000 & 0.25 & 438.00 & 278.2 & 35.6 & 10.2 & 18.5 & 14.4 \\
\hline $11 / 13 / 92$ & 2015 & 0.25 & 438.25 & 278.1 & 35.3 & 9.1 & 16.7 & 12.8 \\
\hline $11 / 13 / 92$ & 2030 & 0.25 & 438.50 & 278.1 & 34.5 & 8.4 & 18.7 & 14.0 \\
\hline $11 / 13 / 92$ & 2130 & 0.25 & 438.75 & 278.6 & 33.5 & 7.6 & 16.8 & 14.6 \\
\hline $11 / 13 / 92$ & 2145 & 0.25 & 439.00 & 277.3 & 38.0 & 7.0 & 23.6 & 12.3 \\
\hline $11 / 13 / 92$ & 2200 & 0.25 & 439.25 & 277.3 & 37.6 & 7.6 & 23.7 & 11.6 \\
\hline $11 / 13 / 92$ & 2215 & 0.25 & 439.50 & 277.6 & 36.5 & 9.4 & 22.3 & 13.6 \\
\hline $11 / 13 / 92$ & 2230 & 0.25 & 439.75 & 277.5 & 36.2 & 10.2 & 19.6 & 13.0 \\
\hline $11 / 13 / 92$ & 2245 & 0.25 & 440.00 & 277.4 & 35.5 & 10.6 & 20.6 & 12.9 \\
\hline
\end{tabular}

Page E-25 B-Duct Opacity >> " = Lower, "* = Higher 
SUMMARY OF LIME SLURRY INJECTION FROM OCTOBER 17, 1992 TO NOVEMBER 17, 1992

\begin{tabular}{|c|c|c|c|c|c|c|c|c|}
\hline Date & Time & Hours of & Cumulative & B Duct Temp & Lime Flow & Slurry Solids & SO2 Removal & Stack Opacity \\
\hline & & Injection & Hours & ${ }^{\circ} \mathrm{F}$ & GPM & Percent & Percent & Percent \\
\hline $11 / 13 / 92$ & 2300 & 0.25 & 440.25 & 277.3 & 35.2 & 10.6 & 20.4 & 12.6 \\
\hline $111 / 13 / 92$ & 2315 & 0.25 & 440.50 & 275.9 & 34.6 & 10.5 & 20.4 & 12.5 \\
\hline $11 / 13 / 92$ & 2330 & 0.25 & 440.75 & 275.4 & 34.9 & 10.2 & 22.5 & 11.3 \\
\hline $11 / 13 / 92$ & 2345 & 0.25 & 441.00 & 275.0 & 34.4 & 10.2 & 22.6 & 12.3 \\
\hline \multicolumn{2}{|l|}{ Total Hours } & 9.25 & & & & & & \\
\hline & & & & & & & & \\
\hline $11 / 14 / 92$ & 0 & 0.25 & 441.25 & 275.0 & 34.6 & 10.4 & 16.3 & 11.4 \\
\hline $11 / 14 / 92$ & 15 & 0.25 & 441.50 & 276.3 & 35.5 & 10.2 & 16.7 & 12.0 \\
\hline $11 / 114 / 92$ & 30 & 0.25 & 441.75 & 277.0 & 36.7 & 9.9 & 15.7 & 11.2 \\
\hline $11 / 14 / 92$ & 45 & 0.25 & 442.00 & 276.6 & 29.2 & 9.2 & 12.9 & 11.6 \\
\hline $11 / 14 / 92$ & 100 & 0.25 & 442.25 & 274.7 & 36.3 & 8.4 & 14.8 & 10.0 \\
\hline $11 / 14 / 92$ & 115 & 0.25 & 442.50 & 274.7 & 35.5 & 9.0 & 16.0 & 9.9 \\
\hline $11 / 14 / 92$ & 130 & 0.25 & 442.75 & 275.4 & 35.4 & 10.4 & 16.9 & 9.7 \\
\hline $11 / 14 / 92$ & 145 & 0.25 & 443.00 & 275.3 & 37.3 & 10.3 & 18.4 & 16.4 \\
\hline $11 / 14 / 92$ & 200 & 0.25 & 443.25 & 277.4 & 36.7 & 9.9 & 17.4 & 11.5 \\
\hline $\mid 11 / 14 / 92$ & 215 & 0.25 & 443.50 & 276.4 & 36.0 & 10.3 & 21.3 & 9.7 \\
\hline $11 / 14 / 92$ & 230 & 0.25 & 443.75 & 274.4 & 35.4 & 10.5 & 22.6 & 11.6 \\
\hline $11 / 14 / 92$ & 245 & 0.25 & 444.00 & 273.9 & 34.2 & 10.7 & 18.5 & 9.7 \\
\hline $11 / 14 / 92$ & 300 & 0.25 & 444.25 & 274.3 & 36.1 & 10.2 & 22.3 & 9.8 \\
\hline $11 / 14 / 92$ & 315 & 0.25 & 444.50 & 276.9 & 36.2 & 10.2 & 21.5 & 8.5 \\
\hline $11 / 14 / 92$ & 330 & 0.25 & 444.75 & 274.5 & 36.6 & 10.1 & 22.0 & 9.0 \\
\hline $11 / 14 / 92$ & 345 & 0.25 & 445.00 & 273.3 & 33.6 & 10.1 & 21.4 & 9.4 \\
\hline $11 / 14 / 92$ & 400 & 0.25 & 445.25 & 273.2 & 36.9 & 10.2 & 19.8 & 8.7 \\
\hline $11 / 14 / 92$ & 415 & 0.25 & 445.50 & 274.3 & 35.7 & 10.3 & 20.6 & 9.0 \\
\hline $11 / 14 / 92$ & 430 & 0.25 & 445.75 & 275.2 & 35.3 & 10.1 & 23.0 & 8.5 \\
\hline $11 / 14 / 92$ & 445 & 0.25 & 446.00 & 275.0 & 35.1 & 9.6 & 20.0 & 8.7 \\
\hline $11 / 14 / 92$ & 500 & 0.25 & 446.25 & 272.6 & 35.7 & 10.1 & 21.5 & 10.4 \\
\hline $11 / 14 / 92$ & 515 & 0.25 & 446.50 & 272.6 & 37.4 & 9.9 & 20.3 & 8.2 \\
\hline $11 / 14 / 92$ & 530 & 0.25 & 446.75 & 272.7 & 33.0 & 10.2 & 21.5 & 8.8 \\
\hline $11 / 14 / 92$ & 545 & 0.25 & 447.00 & 274.3 & 33.8 & 9.9 & 23.7 & 8.1 \\
\hline $11 / 14 / 92$ & 600 & 0.25 & 447.25 & 275.5 & 33.4 & 10.0 & 21.4 & 8.6 \\
\hline $11 / 14 / 92$ & 615 & 0.25 & 447.50 & 273.9 & 33.8 & 9.8 & 21.9 & 10.2 \\
\hline $11 / 14 / 92$ & 630 & 0.25 & 447.75 & 273.5 & 34.9 & 10.4 & 19.6 & 11.0 \\
\hline $11 / 14 / 92$ & 645 & 0.25 & 448.00 & 272.2 & 33.9 & 10.3 & 20.6 & 11.3 \\
\hline $11 / 14 / 92$ & 700 & 0.25 & 448.25 & 271.9 & 32.1 & 9.9 & 22.6 & 9.7 \\
\hline $11 / 14 / 92$ & 715 & 0.25 & 448.50 & 271.3 & 33.2 & 10.1 & 21.0 & 9.9 \\
\hline $11 / 14 / 92$ & 730 & 0.25 & 448.75 & 272.1 & 34.6 & 10.2 & 21.6 & 10.3 \\
\hline $11 / 14 / 92$ & 745 & 0.25 & 449.00 & 271.6 & 33.1 & 10.0 & 20.5 & 9.9 \\
\hline $11 / 14 / 92$ & 800 & 0.25 & 449.25 & 272.6 & 31.8 & 9.9 & 19.7 & 10.4 \\
\hline $11 / 14 / 92$ & 815 & 0.25 & 449.50 & 273.9 & 29.7 & 9.9 & 21.2 & 15.2 \\
\hline $11 / 14 / 92$ & 830 & 0.25 & 449.75 & 273.4 & 30.8 & 10.3 & 18.2 & 17.4 \\
\hline $11 / 14 / 92$ & 845 & 0.25 & 450.00 & 274.4 & 31.6 & 10.0 & 19.4 & 17.8 \\
\hline $11 / 14 / 92$ & 900 & 0.25 & 450.25 & 274.1 & 30.8 & 10.1 & 19.0 & 16.6 \\
\hline $11 / 14 / 92$ & 915 & 0.25 & 450.50 & 272.6 & 33.3 & 9.9 & 20.6 & 13.9 \\
\hline $11 / 14 / 92$ & 930 & 0.25 & 450.75 & 272.9 & 30.7 & 10.1 & 19.4 & 14.8 \\
\hline $11 / 14 / 92$ & 945 & 0.25 & 451.00 & 273.3 & 29.1 & 10.0 & 17.4 & 17.3 \\
\hline $11 / 14 / 92$ & 1000 & 0.25 & 451.25 & 273.1 & 29.9 & 10.0 & 15.7 & 20.0 \\
\hline
\end{tabular}

Page E-26 B-Duct Opacity >> " = Lower, "* = Higher 
SUMMARY OF LIME SLURRY INJECTION FROM OCTOBER 17, 1992 TO NOVEMBER 17, 1992

\begin{tabular}{|c|c|c|c|c|c|c|c|c|}
\hline Date & Time & Hours of & Cumulative & B Duct Temp & Lime Flow & Slurry Solids & SO2 Removal & Stack Opacity \\
\hline & & Injection & Hours & ${ }^{\circ} \mathrm{F}$ & GPM & Percent & Percent & Percent \\
\hline $11 / 14 / 92$ & 1300 & 0.25 & 451.50 & 274.5 & 36.3 & 9.6 & 16.9 & 14.9 \\
\hline $11 / 14 / 92$ & 1315 & 0.25 & 451.75 & 274.1 & 37.9 & 10.5 & 18.6 & 15.4 \\
\hline $11 / 14 / 92$ & 1330 & 0.25 & 452.00 & 274.0 & 40.0 & 10.0 & 17.5 & 9.3 \\
\hline $11 / 14 / 92$ & 1345 & 0.25 & 452.25 & 276.5 & 38.3 & 9.9 & 19.5 & 8.0 \\
\hline $11 / 14 / 92$ & 1400 & 0.25 & 452.50 & 276.8 & 37.9 & 9.9 & 19.0 & 7.2 \\
\hline $11 / 14 / 92$ & 1415 & 0.25 & 452.75 & 276.5 & 39.4 & 9.9 & 23.0 & 7.3 \\
\hline $11 / 14 / 92$ & 1430 & 0.25 & 453.00 & 275.8 & 38.4 & 10.2 & 19.9 & 8.5 \\
\hline $11 / 14 / 92$ & 1445 & 0.25 & 453.25 & 275.4 & 37.9 & 10.0 & 20.7 & 8.2 \\
\hline $11 / 14 / 92$ & 1500 & 0.25 & 453.50 & 276.2 & 37.7 & 10.3 & 19.5 & 7.3 \\
\hline $11 / 14 / 92$ & 1515 & 0.25 & 453.75 & 276.7 & 38.7 & 10.1 & 21.7 & 7.8 \\
\hline $11 / 14 / 92$ & 1530 & 0.25 & 454.00 & 275.5 & 41.7 & 10.1 & 22.2 & 7.4 \\
\hline $11 / 14 / 92$ & 1545 & 0.25 & 454.25 & 275.5 & 41.7 & 10.1 & 22.2 & 7.4 \\
\hline $11 / 14 / 92$ & 1600 & 0.25 & 454.50 & 278.6 & 36.8 & 9.8 & 20.6 & 9.1 \\
\hline $11 / 14 / 92$ & 1615 & 0.25 & 454.75 & 278.6 & 36.8 & 9.8 & 20.6 & 9.1 \\
\hline $11 / 14 / 92$ & 1645 & 0.25 & 455.00 & 275.6 & 36.2 & 9.9 & 20.1 & 8.0 \\
\hline $11 / 14 / 92$ & 1700 & 0.25 & 455.25 & 276.3 & 35.5 & 9.9 & 19.4 & 10.3 \\
\hline $11 / 14 / 92$ & 1715 & 0.25 & 455.50 & 276.5 & 35.3 & 9.9 & 21.5 & 13.5 \\
\hline $11 / 14 / 92$ & 1730 & 0.25 & 455.75 & 273.5 & 35.4 & 10.3 & 24.2 & 15.3 \\
\hline $11 / 14 / 92$ & 1745 & 0.25 & 456.00 & 273.4 & 36.0 & 10.0 & 22.7 & 12.9 \\
\hline $11 / 14 / 92$ & 1800 & 0.25 & 456.25 & 274.8 & 35.8 & 10.0 & 19.7 & 15.3 \\
\hline $11 / 14 / 92$ & 1815 & 0.25 & 456.50 & 274.8 & 35.1 & 9.9 & 21.4 & 13.7 \\
\hline $11 / 14 / 92$ & 1830 & 0.25 & 456.75 & 273.5 & 34.6 & 10.0 & 20.1 & 15.2 \\
\hline $11 / 14 / 92$ & 1845 & 0.25 & 457.00 & 272.8 & 34.6 & 9.7 & 23.1 & 14.7 \\
\hline $11 / 14 / 92$ & 1900 & 0.25 & 457.25 & 272.5 & 34.7 & 9.7 & 23.4 & 14.8 \\
\hline $11 / 14 / 92$ & 1915 & 0.25 & 457.50 & 271.8 & 33.9 & 10.2 & 22.4 & 14.5 \\
\hline $11 / 14 / 92$ & 1930 & 0.25 & 457.75 & 272.7 & 34.3 & 10.2 & 21.9 & 14.5 \\
\hline $11 / 14 / 92$ & 1945 & 0.25 & 458.00 & 272.7 & 34.7 & 10.0 & 23.2 & 14.2 \\
\hline $11 / 14 / 92$ & 2000 & 0.25 & 458.25 & 273.5 & 34.3 & 9.8 & 22.4 & 14.2 \\
\hline $11 / 14 / 92$ & 2015 & 0.25 & 458.50 & 272.1 & 33.2 & 10.2 & 22.1 & 14.9 \\
\hline $11 / 14 / 92$ & 2030 & 0.25 & 458.75 & 272.9 & 34.5 & 10.2 & 22.8 & 13.4 \\
\hline $11 / 14 / 92$ & 2045 & 0.25 & 459.00 & 273.3 & 35.5 & 10.0 & 24.3 & 14.5 \\
\hline $11 / 14 / 92$ & 2100 & 0.25 & 459.25 & 273.5 & 33.5 & 9.9 & 24.2 & 15.9 \\
\hline $11 / 14 / 92$ & 2115 & 0.25 & 459.50 & 272.9 & 35.2 & 10.0 & 21.7 & 14.8 \\
\hline $11 / 14 / 92$ & 2130 & 0.25 & 459.75 & 273.5 & 34.0 & 9.6 & 20.2 & 14.6 \\
\hline $11 / 14 / 92$ & 2145 & 0.25 & 460.00 & 273.8 & 33.6 & 9.6 & 18.6 & 14.6 \\
\hline $11 / 14 / 92$ & 2200 & 0.25 & 460.25 & 273.4 & 33.5 & 10.0 & 20.0 & 16.0 \\
\hline $11 / 14 / 92$ & 2215 & 0.25 & 460.50 & 272.6 & 32.9 & 9.8 & 21.5 & 14.3 \\
\hline $11 / 14 / 92$ & 2230 & 0.25 & 460.75 & 272.7 & 32.3 & 10.1 & 20.1 & 14.5 \\
\hline $11 / 14 / 92$ & 2245 & 0.25 & 461.00 & 274.3 & 34.0 & 9.7 & 22.8 & 13.9 \\
\hline $11 / 14 / 92$ & 2300 & 0.25 & 461.25 & 273.8 & 34.2 & 9.9 & 22.7 & 16.6 \\
\hline $11 / 14 / 92$ & 2315 & 0.25 & 461.50 & 274.6 & 34.2 & 10.1 & 18.1 & 13.4 \\
\hline $11 / 14 / 92$ & 2330 & 0.25 & 461.75 & 275.2 & 35.9 & 10.0 & 17.9 & 12.3 \\
\hline $11 / 14 / 92$ & 2345 & 0.25 & 462.00 & 274.4 & 35.7 & 10.2 & 16.4 & 9.6 \\
\hline \multicolumn{2}{|l|}{ Total Hours } & 21.00 & & & & & & \\
\hline & & & & & & & & \\
\hline $11 / 15 / 92$ & 0 & 0.25 & 462.25 & 274.3 & 35.3 & 9.8 & 15.0 & 9.3 \\
\hline $11 / 15 / 92$ & 15 & 0.25 & 462.50 & 275.0 & 36.0 & 9.8 & 16.4 & 9.2 \\
\hline
\end{tabular}

Page E-27 B-Duct Opacity >> * = Lower, ** = Higher 
SUMMARY OF LIME SLURRY INJECTION FROM OCTOBER 17, 1992 TO NOVEMBER 17, 1992

\begin{tabular}{|c|c|c|c|c|c|c|c|c|}
\hline Date & Time & Hours of & Cumulative & B Duct Temp & Lime Flow & Slurry Solids & SO2 Removal & Stack Opacily \\
\hline & & Injection & Hours & of & GPM & Percent & Percent & Percent \\
\hline $11 / 15 / 92$ & 30 & 0.25 & 462.75 & 276.5 & 36.0 & 10.1 & 15.3 & 7.6 \\
\hline $11 / 15 / 92$ & 45 & 0.25 & 463.00 & 274.9 & 35.2 & 10.1 & 16.7 & 7.6 \\
\hline $11 / 15 / 92$ & 100 & 0.25 & 463.25 & 273.8 & 34.3 & 9.8 & 17.2 & 7.2 \\
\hline $11 / 15 / 92$ & 115 & 0.25 & 463.50 & 273.9 & 36.6 & 10.1 & 17.9 & 8.4 \\
\hline $11 / 15 / 92$ & 130 & 0.25 & 463.75 & 273.2 & 36.7 & 10.2 & 19.7 & 9.6 \\
\hline $11 / 15 / 92$ & 145 & 0.25 & 464.00 & 275.5 & 36.3 & 9.9 & 20.5 & 8.5 \\
\hline $11 / 15 / 92$ & 200 & 0.25 & 464.25 & 277.0 & 36.9 & 9.9 & 20.5 & 7.3 \\
\hline $11 / 15 / 92$ & 215 & 0.25 & 464.50 & 274.3 & 38.1 & 9.7 & 23.6 & 8.2 \\
\hline $11 / 15 / 92$ & 230 & 0.25 & 464.75 & 274.3 & 36.6 & 10.2 & 21.5 & 7.5 \\
\hline $11 / 15 / 92$ & 245 & 0.25 & 465.00 & 274.6 & 36.8 & 10.1 & 21.2 & 8.5 \\
\hline $11 / 15 / 92$ & 300 & 0.25 & 465.25 & 276.5 & 37.3 & 9.7 & 24.1 & 7.1 \\
\hline $11 / 15 / 92$ & 315 & 0.25 & 465.50 & 277.3 & 36.6 & 9.6 & 21.3 & 8.2 \\
\hline $11 / 15 / 92$ & 330 & 0.25 & 465.75 & 274.1 & 36.5 & 10.0 & 22.9 & 9.0 \\
\hline $11 / 15 / 92$ & 345 & 0.25 & 466.00 & 273.8 & 35.5 & 9.8 & 23.2 & 8.8 \\
\hline $11 / 15 / 92$ & 400 & 0.25 & 466.25 & 273.6 & 35.6 & 10.3 & 24.0 & 10.3 \\
\hline $11 / 15 / 92$ & 415 & 0.25 & 466.50 & 275.5 & 35.7 & 10.3 & 21.0 & 9.9 \\
\hline $11 / 15 / 92$ & 430 & 0.25 & 466.75 & 275.2 & 35.7 & 10.2 & 23.4 & 9.9 \\
\hline $11 / 15 / 92$ & 445 & 0.25 & 467.00 & 276.5 & 35.8 & 10.0 & 23.1 & 8.2 \\
\hline $11 / 15 / 92$ & 500 & 0.25 & 467.25 & 277.2 & 35.8 & 10.3 & 24.1 & 8.2 \\
\hline $11 / 15 / 92$ & 515 & 0.25 & 467.50 & 274.2 & 34.5 & 10.2 & 23.4 & 8.7 \\
\hline $11 / 15 / 92$ & 530 & 0.25 & 467.75 & 273.7 & 35.8 & 10.3 & 24.6 & 8.9 \\
\hline $11 / 15 / 92$ & 545 & 0.25 & 468.00 & 273.8 & 33.5 & 10.4 & 22.4 & 8.6 \\
\hline $11 / 15 / 92$ & 600 & 0.25 & 468.25 & 273.5 & 34.5 & 10.3 & 23.5 & 8.3 \\
\hline $11 / 15 / 92$ & 615 & 0.25 & 468.50 & 273.3 & 34.4 & 10.2 & 25.0 & 8.0 \\
\hline $11 / 15 / 92$ & 630 & 0.25 & 468.75 & 273.6 & 36.1 & 10.6 & 22.1 & 9.0 \\
\hline $11 / 15 / 92$ & 645 & 0.25 & 469.00 & 272.8 & 33.8 & 10.1 & 23.1 & 8.9 \\
\hline $11 / 15 / 92$ & 730 & 0.25 & 469.25 & 274.1 & 32.4 & 10.4 & 21.5 & 11.2 \\
\hline $11 / 15 / 92$ & 745 & 0.25 & 469.50 & 273.7 & 33.2 & 9.8 & 20.8 & 11.7 \\
\hline $11 / 15 / 92$ & 800 & 0.25 & 469.75 & 274.8 & 34.3 & 10.0 & 19.1 & 9.0 \\
\hline $11 / 15 / 92$ & 815 & 0.25 & 470.00 & 274.2 & 33.1 & 10.5 & 18.9 & 8.6 \\
\hline $11 / 15 / 92$ & 830 & 0.25 & 470.25 & 274.8 & 34.4 & 9.9 & 19.2 & 10.8 \\
\hline $11 / 15 / 92$ & 845 & 0.25 & 470.50 & 275.3 & 34.4 & 10.2 & 18.3 & 11.5 \\
\hline $11 / 15 / 92$ & 1100 & 0.25 & 470.75 & 274.9 & 35.1 & 9.6 & 17.6 & 8.1 \\
\hline $11 / 15 / 92$ & 1115 & 0.25 & 471.00 & 275.0 & 35.0 & 10.0 & 17.5 & 7.2 \\
\hline $11 / 15 / 92$ & 1130 & 0.25 & 471.25 & 272.9 & 34.4 & 10.7 & 18.9 & 7.2 \\
\hline $11 / 15 / 92$ & 1145 & 0.25 & 471.50 & 273.1 & 34.0 & 10.6 & 16.2 & 8.3 \\
\hline $11 / 15 / 92$ & 1200 & 0.25 & 471.75 & 273.2 & 34.0 & 10.3 & 18.2 & 8.2 \\
\hline $11 / 15 / 92$ & 1215 & 0.25 & 472.00 & 273.3 & 34.3 & 10.1 & 14.8 & 7.2 \\
\hline $11 / 15 / 92$ & 1230 & 0.25 & 472.25 & 272.2 & 34.9 & 10.1 & 21.2 & 7.6 \\
\hline $11 / 15 / 92$ & 1245 & 0.25 & 472.50 & 274.2 & 35.0 & 9.9 & 19.7 & 7.5 \\
\hline $11 / 15 / 92$ & 1300 & 0.25 & 472.75 & 275.3 & 35.4 & 10.0 & 17.0 & 7.8 \\
\hline $11 / 15 / 92$ & 1315 & 0.25 & 473.00 & 274.6 & 34.9 & 10.2 & 18.8 & 8.4 \\
\hline $11 / 15 / 92$ & 1330 & 0.25 & 473.25 & 273.1 & 34.4 & 10.1 & 19.2 & 8.4 \\
\hline $11 / 15 / 92$ & 1345 & 0.25 & 473.50 & 273.3 & 34.4 & 10.1 & 17.7 & 8.7 \\
\hline $11 / 15 / 92$ & 1400 & 0.25 & 473.75 & 273.3 & 34.0 & 10.1 & 20.1 & 7.4 \\
\hline $11 / 15 / 92$ & 1415 & 0.25 & 474.00 & 274.7 & 34.3 & 10.0 & 16.8 & 8.7 \\
\hline $11 / 15 / 92$ & 1430 & 0.25 & 474.25 & 274.7 & 34.5 & 10.1 & 23.1 & 7.8 \\
\hline
\end{tabular}

Page E-28 B-Duct Opacity >> " = Lower, "* = Higher 
SIJMMARY OF LIME SLURRY INJECTION FROM OCTOBER 17, 1992 TO NOVEMBER 17, 1992

\begin{tabular}{|c|c|c|c|c|c|c|c|c|}
\hline Dale & Time & Hours of & Cumulative & B Duct Temp & Lime Flow & Slurry Solids & SO2 Removal & Stack Opacity \\
\hline & & Injection & Hours & ${ }^{2}$ & GPM & Percent & Percent & Percent \\
\hline $11 / 15 / 92$ & 1445 & 0.25 & 474.50 & 274.2 & 33.7 & 9.9 & 20.0 & 8.9 \\
\hline $11 / 15 / 92$ & 1500 & 0.25 & 474.75 & 274.7 & 33.5 & 10.3 & 19.8 & 11.6 \\
\hline $11 / 15 / 92$ & 1515 & 0.25 & 475.00 & 273.6 & 33.4 & 10.0 & 20.3 & 8.4 \\
\hline $11 / 15 / 92$ & 1530 & 0.25 & 475.25 & 274.2 & 33.1 & 10.0 & 19.9 & 7.9 \\
\hline $11 / 15 / 92$ & 1545 & 0.25 & 475.50 & 274.4 & 32.9 & 10.1 & 20.8 & 8.2 \\
\hline $11 / 15 / 92$ & 1600 & 0.25 & 475.75 & 275.2 & 32.9 & 10.3 & 22.3 & 9.9 \\
\hline $11 / 15 / 92$ & 1615 & 0.25 & 476.00 & 274.3 & 33.4 & 10.0 & 19.8 & 11.3 \\
\hline $11 / 15 / 92$ & 1630 & 0.25 & 476.25 & 273.6 & 34.1 & 9.9 & 19.9 & 8.4 \\
\hline $11 / 15 / 92$ & 1645 & 0.25 & 476.50 & 273.8 & 34.9 & 10.0 & 20.5 & 9.3 \\
\hline $11 / 15 / 92$ & 1700 & 0.25 & 476.75 & 274.0 & 36.2 & 10.3 & 24.6 & 12.6 \\
\hline $11 / 15 / 92$ & 1715 & 0.25 & 477.00 & 275.1 & 34.7 & 10.1 & 21.7 & 12.2 \\
\hline $11 / 15 / 92$ & 1730 & 0.25 & 477.25 & 274.8 & 34.9 & 9.9 & 22.4 & 15.5 \\
\hline $11 / 15 / 92$ & 1745 & 0.25 & 477.50 & 274.4 & 34.2 & 10.2 & 22.6 & 13.0 \\
\hline $11 / 15 / 92$ & 1800 & 0.25 & 477.75 & 274.6 & 34.9 & 10.1 & 22.8 & 16.0 \\
\hline $11 / 15 / 92$ & 1815 & 0.25 & 478.00 & 275.5 & 35.2 & 10.4 & 24.0 & 12.0 \\
\hline $11 / 15 / 92$ & 1830 & 0.25 & 478.25 & 277.1 & 35.5 & 10.0 & 23.8 & 12.0 \\
\hline $11 / 15 / 92$ & 1845 & 0.25 & 478.50 & 275.5 & 34.7 & 9.9 & 23.8 & 13.7 \\
\hline Total Hours & 16.50 & & & & & & \\
\hline
\end{tabular}

Page E-29 B-Duct Opacity >> = Lower, "* = Higher 


\section{Appendix F}

\section{Baseline Testing - Fuel}


Appendix F

Baseline Testing - Fuel

Penelec conducted sampling of the incoming coal. The coal is analyzed for moisture, ash, sulfur, Btu/lb, and volatile matter. The results of these analyses during September, October, and November 1992 are attached. 


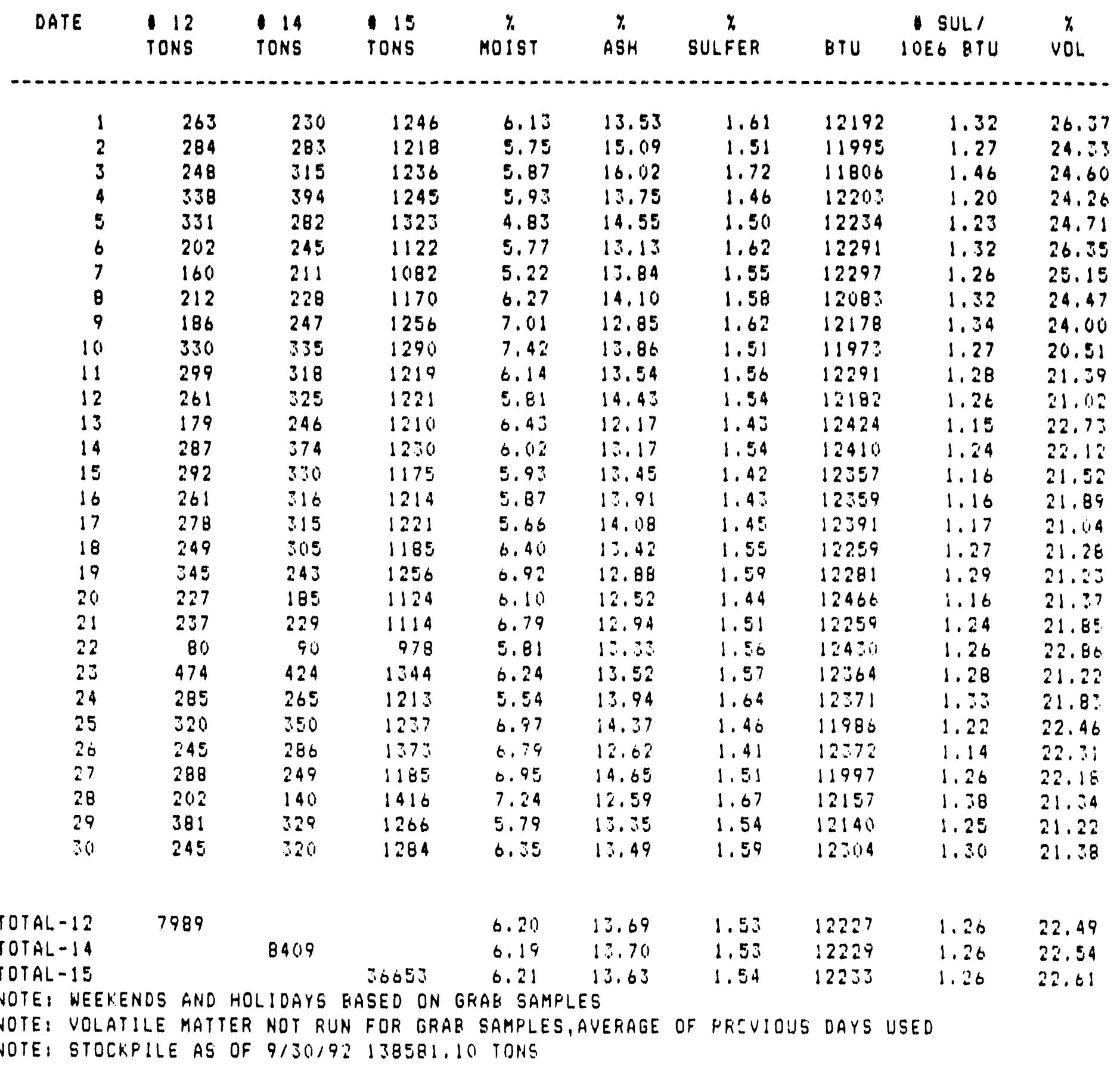


SEMARO STAIION HEAT RATE OUALITY OCTOBER - 1992

\begin{tabular}{|c|c|c|c|c|c|c|c|c|}
\hline DATE & $\begin{array}{l}112 \\
\text { rous }\end{array}$ & $\begin{array}{l}114 \\
\text { rons }\end{array}$ & $\begin{array}{l}118 \\
10018\end{array}$ & ${ }_{1}^{2}$ & $\underset{A B K}{2}$ & $\begin{array}{c}8 \\
\text { SULFER }\end{array}$ & BIV & $\begin{array}{c}18421 \\
10 E 6 \text { BrV }\end{array}$ \\
\hline
\end{tabular}

\begin{tabular}{|c|c|c|c|c|c|c|c|c|c|}
\hline 1 & 427 & 126 & 1145 & 6.45 & 13.34 & 1.63 & 12309 & 1.33 & 21.90 \\
\hline 2 & 265 & 329 & 1257 & 6.23 & 13.83 & 1.40 & 12274 & 1.15 & 22.05 \\
\hline 3 & 364 & 319 & 1430 & 6.32 & 12.81 & 1.61 & 12440 & 1.29 & 22.01 \\
\hline 1 & 258 & 313 & 1118 & 5.85 & 11.71 & 1.38 & 12612 & 1.09 & 21.59 \\
\hline 3 & 316 & 260 & 784 & 6.34 & 13.09 & 1.60 & 12321 & 1.31 & 21.93 \\
\hline 6 & 205 & 315 & 0 & 5.54 & 14.58 & 1.60 & 12219 & 1.31 & 21.59 \\
\hline 1 & 278 & 308 & 0 & 3.41 & 14.16 & 1.57 & 12354 & 1.28 & 21.50 \\
\hline 8 & 314 & 288 & 385 & 5.33 & 12.45 & 1.54 & 12614 & 1.22 & 22.57 \\
\hline 9 & 127 & 189 & 1109 & 6.33 & 13.58 & 1.61 & 12322 & 1.31 & 20.49 \\
\hline 10 & 287 & 283 & 1195 & 5.58 & 13.24 & 1.53 & 12470 & 1.23 & 20.19 \\
\hline 11 & 243 & 190 & 1165 & 6.09 & 12.75 & 1.61 & 12519 & 1.29 & 22.12 \\
\hline 12 & 239 & 291 & 1217 & 6.38 & 13.38 & 1.69 & 12324 & 1.38 & 21.51 \\
\hline 13 & 293 & 360 & 1195 & 6.76 & 13.32 & 1.66 & 12270 & 1.36 & 21.12 \\
\hline 14 & 259 & 287 & 1216 & 7.08 & 13.37 & 1.57 & 12196 & 1.29 & 20.49 \\
\hline 15 & 215 & 333 & 1237 & 6.30 & 13.66 & 1.19 & 12296 & 1.22 & 21.76 \\
\hline 16 & 265 & 276 & 1221 & 3.91 & 14.12 & 1.59 & 12260 & 1.30 & 21.73 \\
\hline 17 & 247 & 296 & 1215 & 5.79 & 15.00 & 1.64 & 12134 & 1.35 & 21.75 \\
\hline 18 & 303 & 194 & 1216 & 6.24 & 13.70 & 1.47 & 12264 & 1.20 & 20.96 \\
\hline 19 & 220 & 321 & 1260 & 5.81 & 15.13 & 1.53 & 12151 & 1.26 & 21.24 \\
\hline 20 & $30 B$ & 304 & 1313 & 3.03 & 13.96 & 1.53 & 12411 & 1.24 & 22.87 \\
\hline 21 & 40 & 303 & 1296 & 3.30 & 14.31 & 1.61 & 12389 & 1.30 & 22.48 \\
\hline 22 & 0 & 261 & 1370 & 3.86 & 13.71 & 1.62 & 12292 & 1.32 & 22.01 \\
\hline 23 & 0 & 357 & 1338 & 3.41 & 15.22 & 1.63 & 12132 & 1.35 & 21.70 \\
\hline 24 & 138 & 239 & 1264 & 5.55 & 13.44 & 1.44 & 12481 & 1.15 & 21.76 \\
\hline 25 & 300 & 277 & 1316 & 5.54 & 14.64 & 1.62 & 12256 & 1.32 & 22.17 \\
\hline 26 & 258 & 187 & 605 & 5.65 & 14.59 & 1.48 & 12209 & 1.21 & 22.63 \\
\hline 27 & 285 & 149 & 0 & 3.24 & 14.24 & 1.56 & 12293 & 1.27 & 22.37 \\
\hline 28 & 298 & 26 & 383 & 5.28 & 14.04 & 1.54 & 12339 & 1.25 & 22.37 \\
\hline 29 & 186 & 159 & 1068 & 5.40 & 15.53 & 1.38 & 12124 & 1.31 & 20.94 \\
\hline 30 & 204 & 184 & 1200 & 5.77 & 13.05 & 1.53 & 12441 & 1.23 & 20.66 \\
\hline 31 & 328 & 344 &.$^{1284}$ & 5.35 & 12.06 & 1.63 & 12619 & 1.29 & 20.66 \\
\hline$L-12$ & 7470 & & & 5.88 & 13.62 & 1.56 & 12347 & 1.27 & 21.69 \\
\hline TOTAL-14 & & 8364 & & 5.88 & 13.71 & 1.57 & 12335 & 1.27 & 21.67 \\
\hline TOTAL-15 & & & 31802 & 5.91 & 13.70 & 1.57 & 12334 & 1.27 & 21.61 \\
\hline
\end{tabular}

NOTE, MEEKENDS AND HOLJDAYS BASED ON GRAB SAKPLES

NOTE, VOLATILE MATTER NOT RUN FOR GRAB SAYPLES, AVERAGE OF PREVIOUS DAYS USEO

NOTE, STOCKPILE AS OF 10/31/92 161319,88 TOWS 
TU: EUECHIEL UTr

... MAR-01-'93 MON 10:48 ID:BECHTEL DEMO SEWD PL FAX NO:814-446-6956 HO31 PO2

SEMAKL GTAI:UN HEII RATE QUALITY NJVEMBEH - IYY?



HDTE, WHE! ENE AND RULIDAYS BABED OH GHAE SATFLEB

NCTE, VOLATILE MATTE NOT RUN FOK BHAB SAMPLEB, AVERAGE GF PREULOUS DAYS USEO






\section{Appendix G}

\section{Procedures for Calculating $\mathrm{SO}_{2}$ and $\mathrm{NO}_{x}$ Removal and the Percentage of Lime Utilization}




\section{ESTIMATES OF $\mathrm{SO}_{2}$ AND NOX REMOVAL}

Bases: Measured Quandivies are:

- $\mathrm{SO}_{2}, \mathrm{NOx}$ and $\mathrm{O}_{2}$ concentrations in injet and outlet flue gas

- Flue gas flow at the discharge from the test duce

- Inlet and ouclet gas temperarures

- Barometric pressure

- Flue gas pressure

- Lime usage

- Lime concentration

- Atomizing air usage

- Dilucion water usage

Essimared Quandities are:

- Fue gas nows in and out

- $\mathrm{SO}_{2}$ and NOx removal

- Lime urilizacion

- Solid waste requiring disposal

\section{Flue Gas Flon Measurements}

The flue gas flow meter is located at the end of the eas duct, just upseream of the second stage Buell ESP. When noching is being injected into the lue gas, it indicates the instantaneous gas flow in SCFM on a mass flow basis. During the injection of water or of a lime slurry, this meter will be used to indicale the blow of treated flue gas withour correction for the effects of changed gas properties on the sccuricy of these readings.

Figure 1 contains the planar arrangement of the test duct with the locations of the lue gas flow meter, the lime atomizers and the upstream and downsoream $\mathrm{SO}_{2}$, NOx and $\mathrm{O}_{2}$ analyzers in dicated on it.

Figure 2 contains the flue gas flow dingram showing the location of ESP, abomizers and instrumentation required for the determination of $\mathrm{SO}_{2}$ removal and lime ualization.

Figures 3 and 4 contain the laboratory correlation of lime slurry concentrations with their specific gravicies at $40^{\circ} \mathrm{F}, 60^{\circ} \mathrm{F}$ and $72^{\circ} \mathrm{F}$. 
Figure 1. Test Duct Arrangement



prepres 1

TLST DUCP ARRANGEMENT

SHOW:NA ReLATIUT LoCATIONS

of rawe oas flow metrer,

LIMO SLURRY ATOMILEAS ANO

WNSTREAT AND DOWNSTREAMT

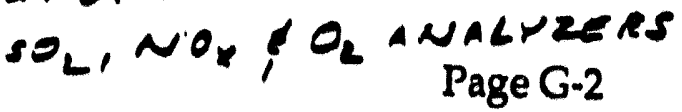


Figure 2. Seward CZD Systern, Test Duct, Flue Ges Flow Diagrum

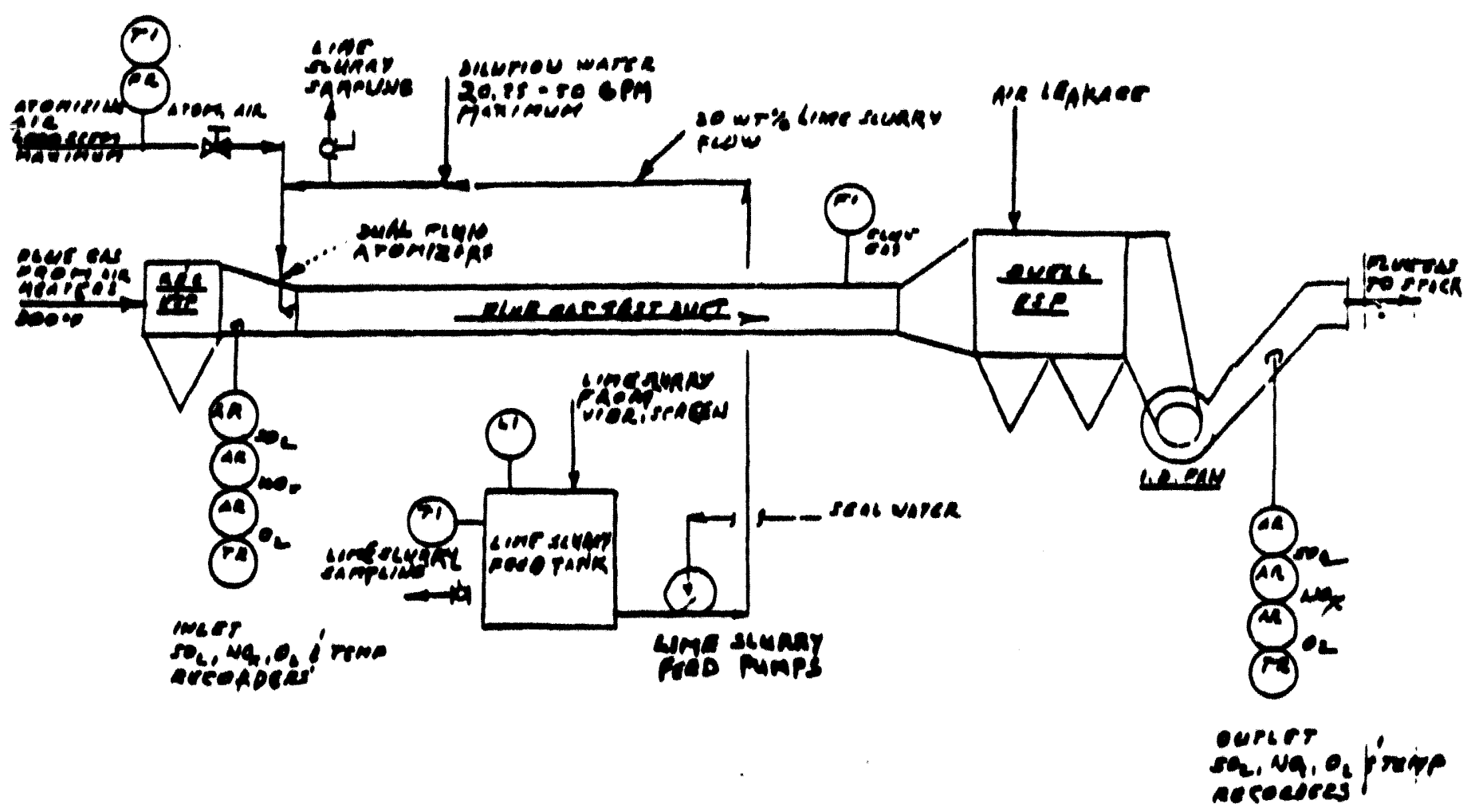




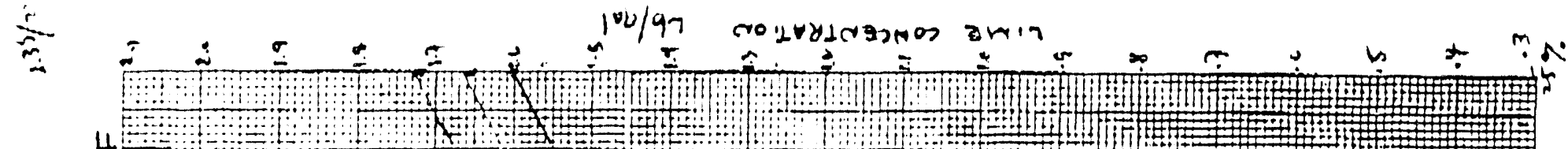

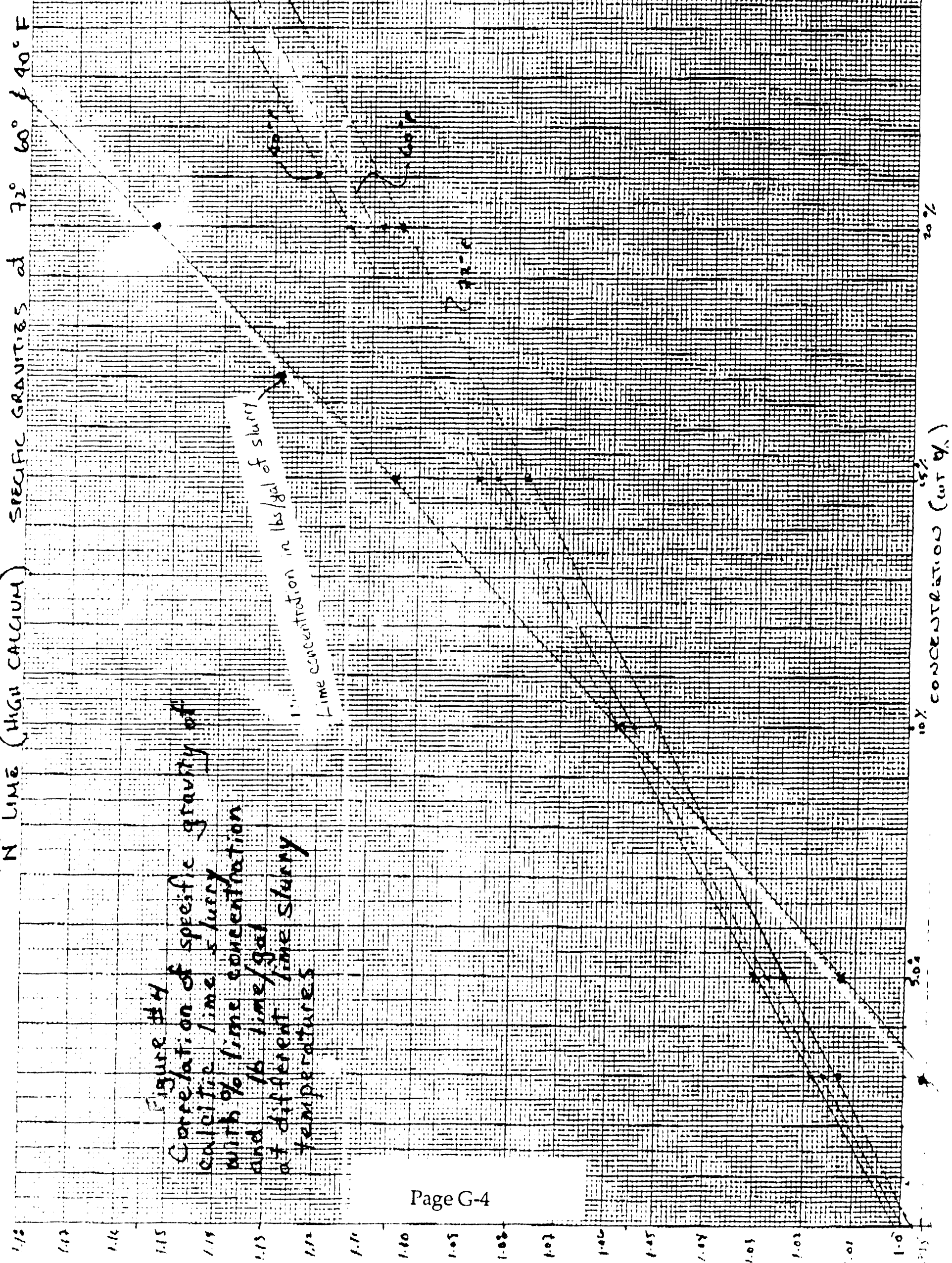




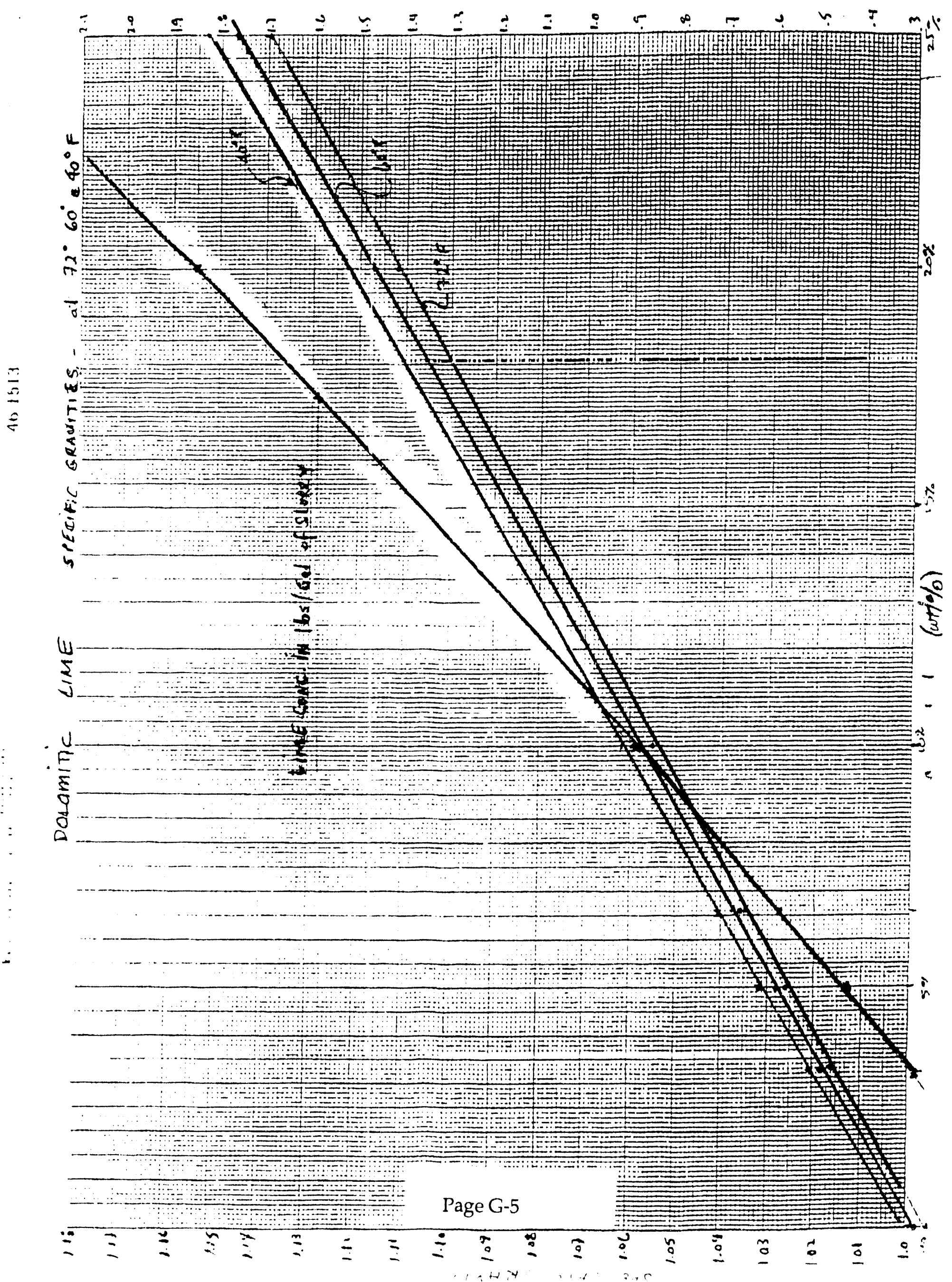




\section{CZD TESTING AT SEWARD - ESTIMATING PROCEDURE}

In order to un : :rstand the operation and performance of CZD system we must know:

- The inlet and outlet flue gas flows and their compositions

- The atomizing air usage

- Lime slurry and dilution water injection rates

- Lime slurry concentration

The inlet and outet flue gas flows are estimated from the flue gas flow meter reading located downstream of the atomizing nozzles at the inlet to the second stage ESP, from the atomizing air and water usage and from inlet and outlet flue gas analysis ( $\mathrm{SO}_{2}, \mathrm{NOx}$ and $\mathrm{O}_{2}$ ).

Atomizing air usage is indicated by a flow indicator. Water usage is estimated from measured lime slurry usage and its water content and from the dilution water usage.

Plant air leakage is based on the oxygen mass balance.

In all, there is a set of 5 linear equation to be solved to estimate the actual $\mathrm{SO}_{2}$ and $\mathrm{NO}$ removal.

Lime usage and utilization are based on the measured volumes and specific gravities of the lime slurries injected into the flue gas stream and the above estimates of the associated $\mathrm{SO}_{2}$ and NOx removals. 
MATHEMATICAL PROCEDURE NOMENCLATURE

\section{Descriotion}

Inlet flue gas flow

Outet glue gas flow

Flue gas flow meter reading

Atomizing air flow meter reading

Water content of atomized lime slury

(This is estimated from the flow of lime slurry and dilution water and expressed as the flow of water vapor in SCFM)

$\mathrm{SO}_{2}$ absorbed from flue gas

NOx absorbed frim flue gas

Air leakage

Oxygen concentration in inlet flue gas

Oxygen concentration in outlet flue gas

$\mathrm{SO}_{2}$ concentration in inlet flue gas

$\mathrm{SO}_{2}$ concentration in oudet flue gas

NOx concentration in inlet flue gas

NOx concentration in outlet flue gas
Symbol Units

F.G. IN SCFM

F.G. OUT SCFM

F.G. SCFM
A.A.
SCFM
W.C.
SCFM

$\mathrm{XSO}_{2}$

SCFM

YNOX

SCFM

$Z_{d}$

SCFM

$O X_{\mathbb{N}}$

Vol \%

OXOUT Vol\%

$\mathrm{SO}_{2} \mathbb{N} \quad \frac{\mathrm{ppm}}{10^{6}}(\mathrm{Vol})$

$\mathrm{SO}_{2}$ OUT $\quad \frac{\mathrm{ppm}}{106}(\mathrm{Vol})$

NOX $\mathbb{I N} \quad \frac{\mathrm{ppm}}{106}(\mathrm{Vol})$

NOx out $\quad \frac{\mathrm{ppm}}{106}(\mathrm{Vol})$ 


\section{MASS BALANCE EQUATIONS}

1. Inlet Flue Gas ${ }^{\circ}$ = Flue gas flow meter reading (FG) less atomizing air meter reading $\left(F G_{\mathbb{N}}\right)$ (AA) less water content of lime slurry used (WC) plus $\mathrm{SO}_{2}$ absorbed ( $\mathrm{XSO}_{\mathrm{SO}}$ ) plus NOx absorbed ( $\mathrm{Y}_{\mathrm{NO}}$ ).

$$
\left(F G_{\mathbb{N}}\right)=(\mathrm{FG}) \cdot(\mathrm{AA}) \cdot(\mathrm{WC})+\left(\mathrm{X}_{\mathrm{SO}}\right)+\left(\mathrm{Y}_{\mathrm{NO}}\right)
$$

2. Outlet Flue $G_{a s}=$ Flue gas flowmeter reading $(F G)$ plus air leakage $\left(Z_{A L}\right)$

(FG Discharged

from $\mathbb{D}$ fan)

$$
(F G \text { Out })=(F G)+\left(Z_{A L}\right)
$$

3. Oxygen Balance

Inlet Flue Gas $(\mathrm{FGIN}) \times \frac{\text { Measured } \mathrm{O}_{2} \text { Conc. }(\mathrm{OX} \mathrm{NN})}{100}+$ Atom. Air $\times 0.21+\mathrm{Z}_{\mathrm{AL}} \times 0.21$

$$
\begin{aligned}
& =\text { Outlet Flue gas }\left(F G_{O U T}\right) \times \frac{\text { Measured } O_{2} \text { Conc. }\left(\mathrm{OX}_{\mathrm{OUT}}\right)}{100} \\
& \left(\mathrm{FG}_{\mathrm{LN}}\right) \times \frac{\left(\mathrm{OX} \mathrm{XNN}_{\mathrm{N}}\right)}{100}+(\mathrm{AA}) \times 0.21+\left(\mathrm{Z}_{\mathrm{AL}}\right) \times 0.21=\left(\mathrm{FGOUT}_{\mathrm{OUT}}\right) \times \frac{(\mathrm{OX} \mathrm{OUTT})}{100}
\end{aligned}
$$

4. $\mathrm{SO}_{2}$ Balance

$\mathrm{SO}_{2}$ absorbed $\left(\mathrm{XSO}_{2}\right)=$ Inlet Flue $\mathrm{Gas}_{2}\left(\mathrm{FG}_{\mathrm{NN}}\right) \times$ measured $\mathrm{SO}_{2}$ concentrate $\left(\mathrm{SO}_{2} \mathrm{ppm}\right)-$ Outlet Flue Gas (FGOUT) $x$ measured $\mathrm{SO}_{2}$ concentrate $\left(\mathrm{SO}_{2} \mathrm{ppm}\right)$

$$
(\mathrm{XSO} 2)=(\mathrm{FGIN}) \times \frac{(\mathrm{SO} 2 \mathrm{~N})}{10^{6}} \cdot(\mathrm{FGOUT}) \times \frac{(\mathrm{SO} 2 \text { OUT })}{10^{6}}
$$

5. NOx Balance

NOx absorbed $\left(Y_{\text {NOJ }}=\right.$ inlet flue gas $\left(F G_{N}\right) x$ measured NOx concentrate $\frac{\text { NOx }}{106}$ - outlet flue gas (FGOUT) $x$ measured NOx concentrate $\frac{N O x}{10^{6}}$

$Y_{N O_{2}}=F G_{\mathbb{N}} \times\left(\frac{N O_{\times N N}}{10^{6}}\right) \cdot\left(F G_{\text {and }} \times\left(\frac{N O_{x \text { art }}}{10^{6}}\right)\right.$

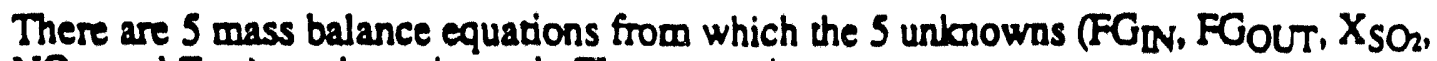
$N O_{x}$, and $Z_{A L}$ can be estimated. These equarions are:

(1) $\quad\left(F G_{\mathbb{N}}\right)=(F G) \cdot(A A) \cdot(W C)+\left(X_{S O 2}\right)+\left(Y_{N O X}\right)$

(2) $\quad($ FGOUT $)=(F G)+\left(Z_{A D}\right.$

(3) $\quad\left(F_{\mathbb{N N}}\right) \times \frac{\left(O X_{\mathbb{N}}\right)}{100}+A A \times 0.21+Z_{A L} \times 0.21=\left(F_{O U T T}\right) \times \frac{\left(O X_{O U T}\right)}{100}$ 
(4) $\left(\mathrm{XSO}_{2}\right)=\left(\mathrm{FG}_{\mathrm{IN}}\right) \times \frac{\left(\mathrm{SO}_{2 \mathrm{IN}}\right)}{10^{6}} \cdot(\mathrm{FG}$ OUT $) \times \frac{\left(\mathrm{SO}_{2} \mathrm{OUT}\right)}{10^{6}}$

(5) $\quad\left(\mathrm{NO}_{\mathrm{X}}\right)=\left(\mathrm{FG}_{\mathrm{IN}} \times \frac{\left(\mathrm{NO}_{\times} \mathrm{IN}\right)}{10^{6}} \cdot\left(\mathrm{FG}_{\mathrm{OUT}}\right) \times \frac{\left(\mathrm{NO}_{\mathrm{O}} \mathrm{OUT}\right)}{10^{6}}\right.$

To eliminate $Z_{A L}$ (air leakage) multiply equation (2) by 0.21 and add it to equation (3)

$$
\begin{aligned}
& 0.21 \text { (FGOUT) }=0.21(\mathrm{FG})+0.21\left(\mathrm{Z}_{\mathrm{AL}}\right)
\end{aligned}
$$

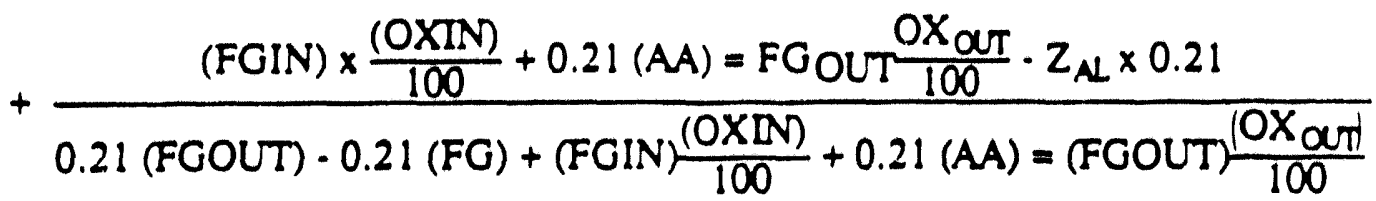

$$
\begin{aligned}
& \left(F_{\text {OUT }}\right)\left[0.21 \cdot \frac{\left(\mathrm{OX}_{\mathrm{OUT}}\right)}{100}\right]=-0.21 \mathrm{AA}+0.21(\mathrm{FG}) \cdot\left(\mathrm{FG}_{\mathrm{NN}}\right) \frac{\left(\mathrm{OX}_{\mathrm{NN}}\right)}{100}
\end{aligned}
$$

Substituting $X_{S O 2}$ and $Y_{N O X}$ in Equation (1) by their values from equations (4) and (5) we will get:

$$
\begin{aligned}
& F G_{I N}=(F G) \cdot(A A) \cdot(W C)+\left(F G_{I N} \times \frac{S_{2} I N}{10^{6}} \cdot F G_{O U T} \times \frac{S_{2} \text { OUT }}{10^{6}}\right. \\
& +\left(F _ { I N } \text { INO } x \text { IN - FGOUTI } \left(\text { FO }_{x}\right.\right. \text { OUT) } \\
& F G_{I N}\left(1 \cdot \frac{S_{2} \text { IN }}{10^{6}}\right) \cdot \frac{N O_{x} \text { IN }}{10^{6}}=F G \cdot A A \cdot W C \cdot \frac{S O_{2} \text { OUT }}{10^{6}}+\frac{N_{x} \text { OUT }}{10^{6}} \\
& F G_{I N} \times \frac{O X_{I N}}{10^{6}}=0.21 A A+0.21 F G \cdot F G_{O U T}\left|0.21 \cdot \frac{O X_{I N}}{10_{6}}\right|
\end{aligned}
$$

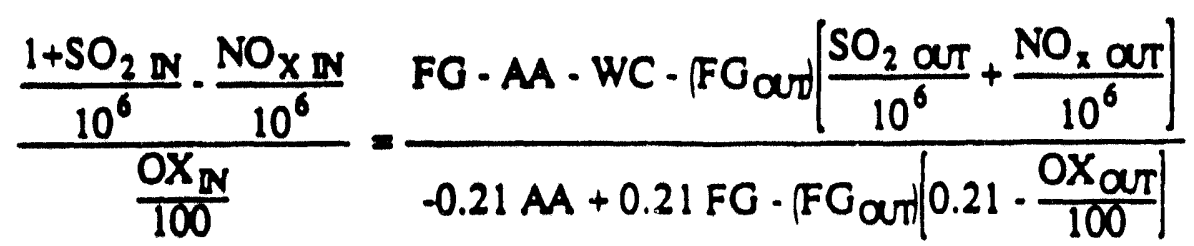

$$
\begin{aligned}
& =\frac{F G \cdot A A \cdot W C \cdot F G_{\alpha u r} \mid\left[\frac{S_{2} \alpha r}{10^{6}}+\frac{N_{x} \alpha r}{10^{6}}\right)}{\left.-0.21(A A+F G) \cdot F G_{\alpha r} \mid 0.21 \cdot \frac{O X_{\alpha r}}{100}\right)}
\end{aligned}
$$


$\frac{1 \cdot \frac{S_{2} \mathbb{N}}{10^{6}}+\frac{N O_{X \mathbb{N}}}{10^{6}}}{\frac{O X_{\mathbb{N}}}{100}}\left(0.21(A A+F G)=\frac{1 \cdot \frac{S_{2} \mathbb{N}}{10^{6}} \cdot \frac{N_{\times} \mathbb{N}}{10^{6}}}{\frac{O X_{\mathbb{N}}}{100}} F G_{\alpha r}\left(0.21 \cdot \frac{O X_{Q T}}{100}\right)\right.$

$+F G \cdot A A \cdot W C \cdot F G_{\alpha T}\left[\frac{S_{2} a r}{10^{6}}+\frac{\mathrm{NO}_{2} \alpha \pi}{10^{6}}\right)$

$\mathrm{FG}_{\mathrm{ar}}\left[\frac{1+\frac{\mathrm{SO}_{2 \mathrm{NV}}}{10^{6}}+\frac{\mathrm{NO}_{\times \mathrm{N}}}{10^{6}}}{\frac{\mathrm{OX} \mathrm{NV}}{100}}\left(0.21 \cdot \frac{\mathrm{OX} \mathrm{QT}}{100}\right) \cdot \frac{\mathrm{SO}_{2} \mathrm{ar}}{10^{6}} \cdot \frac{\mathrm{NO}_{\times} \mathrm{ar}}{10^{6}}\right]$

$=\left[\frac{1-\frac{\mathrm{SO}_{2} \mathbb{N}}{10^{6}} \cdot \frac{\mathrm{NO}_{\times} \mathrm{N}}{10^{6}}}{\frac{O X_{\mathbb{N}}}{100}} \times 10.21(A A+F G)\right] \cdot F G+A A+W C$

$=F G\left[0.21 \frac{1 \cdot \frac{\mathrm{SO}_{2 \mathrm{~N}}}{10^{6}} \cdot \frac{\mathrm{NO}_{\mathrm{XN}}}{10^{6}}}{\frac{O X_{\mathrm{N}}}{100}} \cdot 1\right] \cdot 0.21 \frac{1 \cdot \frac{\mathrm{SO}_{2 \mathrm{~N}}}{10^{6}} \cdot \frac{\mathrm{NO}_{\times} \mathrm{N}}{10^{6}}}{\frac{O \mathrm{X}_{\mathrm{N}}}{100}} \mathrm{AA}+\mathrm{AA}+\mathrm{WC}$

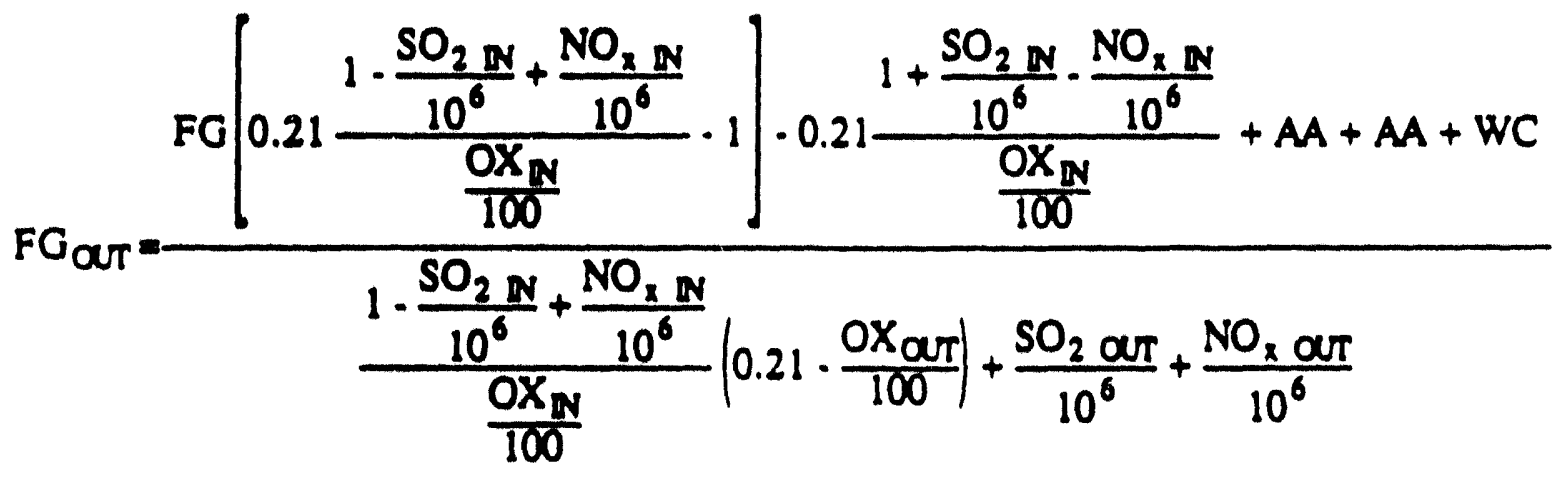


$F G_{\mathbb{N}}=\frac{F G \cdot A A \cdot W C \cdot F G_{\alpha T}\left(\frac{S O_{2} \alpha T}{10^{6}}+\frac{N O_{x} \alpha T}{10^{6}}\right)}{1 \cdot \frac{S O_{2} \mathbb{N}}{10^{6}} \cdot \frac{N O_{x} \mathbb{N}}{10^{6}}}$

$Z_{\mathcal{A L}}=F G_{\text {OT }} \cdot F G$

$X_{\mathrm{SO}_{2}}=\mathrm{FG}_{\mathbb{N}} \times \frac{\mathrm{SO}_{2 \mathrm{~N}}}{10^{6}} \cdot \mathrm{FG}_{\mathrm{OUT}} \times \frac{\mathrm{SO}_{2} \text { ar }}{10^{6}}$

$X_{N_{1}}=F G_{\mathbb{N}} \times \frac{N O_{\times} N}{10^{6}} \cdot F G_{O T} \times \frac{N O_{x} \text { OT }}{10^{6}}$

$\% \mathrm{SO}_{2}$ REMOVAL $=\frac{\mathrm{X}_{\mathrm{SO}_{2}}}{F_{\mathrm{NN}} \times \mathrm{SO}_{2 \mathrm{~N}}} \times 100$

\% NO REMOVAL $=\frac{Y_{N_{1}}}{F G_{N} \times N_{2 N}} \times 100$

Flue Gas Humidity $=\frac{\mathrm{Lb}_{\mathrm{H}} \mathrm{O} \mathrm{V} \mathrm{p}}{\mathrm{b} \text { of Dry Air Equivalent of Flue Gas }}=W$

Relative Humidity $=\frac{P}{P_{1}} \times 100$

where:

$W=$ Flue gas hurnidity

$P=$ Water vapor partial pressure in flue gas

$P_{s}=$ Saturated steam pressure at flue gas temperanure

$P=\frac{\text { Relative Humidity } \times P_{2}}{100}$

$W=\frac{18.016 p}{18.967(P \cdot p)}$ 


\section{DETERMINING LIME USAGE}

\section{Basis:}

There is no data available for correlating the specific gravity of dolomitic hydrate slurries with the is hydrate content. Local laboratory will measure specific gravidies of dolomitic lime sluries and their hydrate contenes at $40^{\circ} \mathrm{F}, 60^{\circ} \mathrm{F}$ and $80^{\circ} \mathrm{F}$ and prepare a chart showing the dependence of specific gravity on hydrate concentration at the above temperanures.

During the test runs the actual dolomitic lime usage will be determined by:

- measuring the specific gravity and temperanure of the lime slurry in the feed tank

- using the laboratory correlation of the specific gravity with the hydrate concentration to determine the hydrate content of this slurry

- dipping the lime feed tank to determine the volume of the lime slurty and, hence, the amount of lime hydrate used

\section{DETERMINATION OF SPECIFIC GRAVITY OF THE DOLOMITIC LME SLURRY IN THE LIME FEED TANK}

This tank handles up to 20 WT\% of lime slurries which are 100 viscous for determining their specific gravity with a simple hydrometer. However, a hydrometer could be used to determine specific gravity of $20 \%$ lime slurry after threefold dilution with water.

\section{Method}

Take $100 \mathrm{ml}$ sample of concentrated slurty, weigh it and dilute it with $200 \mathrm{ml}$ of water $10 \mathrm{a}$ tocal volume of $300 \mathrm{~mL}$. Measure its specific gravity and then calculate the spectific gravity of original sample as follows: 


\section{Calculations:}

Suppose that $100 \mathrm{ml}$ of original sample weight equals $112.5 \mathrm{gm}$ and that after mixing it wich 200 $\mathrm{ml}$ of water it has specific gravity equais 1.04 at $80^{\circ} \mathrm{F}$.

This specific gravity (from the graph of concentrated vs specific gravity) corresponds to the lime concentrate of $7.3 \%$.

$\therefore \frac{\text { Lime content of original sample }}{\text { WT of } 100 \mathrm{ml} \text { of original sample }+200} \times 100=7.3$

Lime content of original sample $=0.073$ (weight of original sample +200 )

Concentration of lime in original sample: =

$$
\begin{aligned}
& =\frac{0.073 \text { (Wt. of original sample }+200)}{\text { W. of original sample }} \times 100 \\
& =\frac{0.073(112.5+200)}{112.5} \times 100=20.28 W_{t .9}
\end{aligned}
$$

Lime concentrated below $10 W_{L}$ \% can be measured using hydrometer to determine their specific gravity and then a char of specific gravity vs. concentrate. To determine slurry concentrate. the lime feed pumps will recirculate 100 GPM of 20 Wis lime slurry of which about 20 GPM will be fed to the atomizers and about 80 GPM renumed to the feed ank. The two lime slurry feed pumps will use up $101.1 / 2$ GPM of seal water, 80\% of which will enter the lime feed ank resulting in progressive dilution of its content. In order to account for it the concentrzte of lime slurty in the feed cank should be checked at least twice for each lest. The first check should be when lime injection stars and the second check when it stops. 


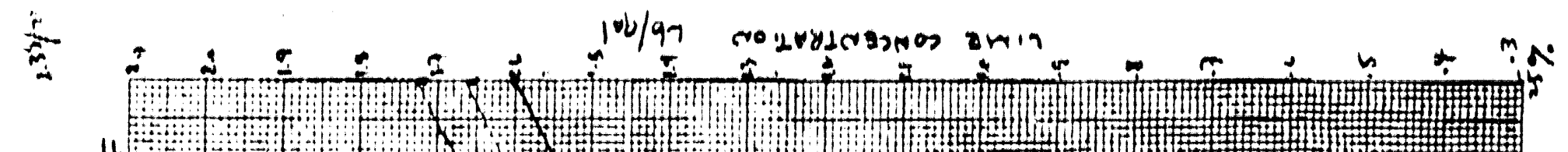

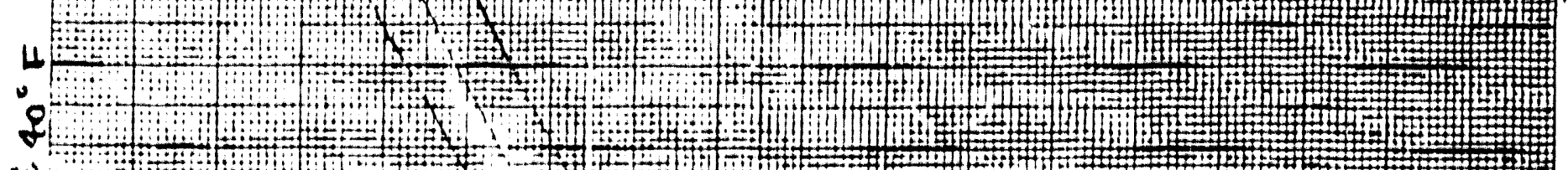



$3-t+5$

r

3

r.

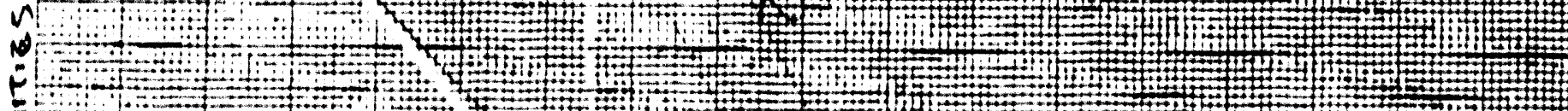





3.

J

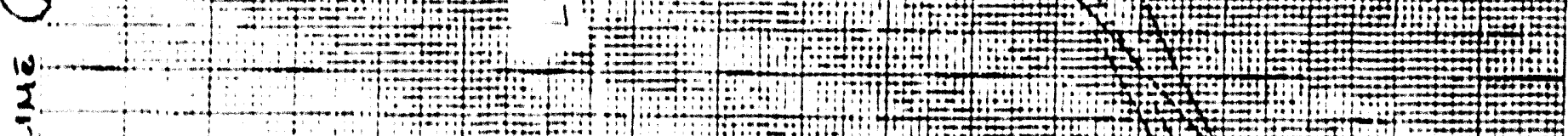

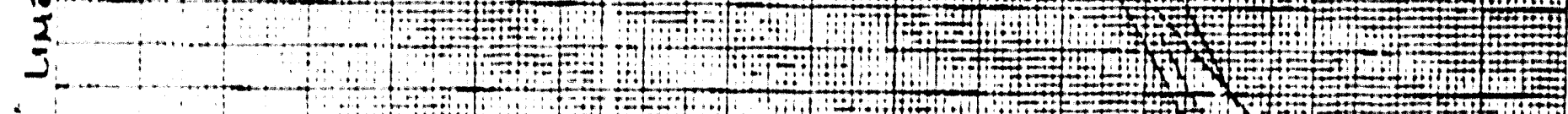

$\mathbf{z}$

6.กए।

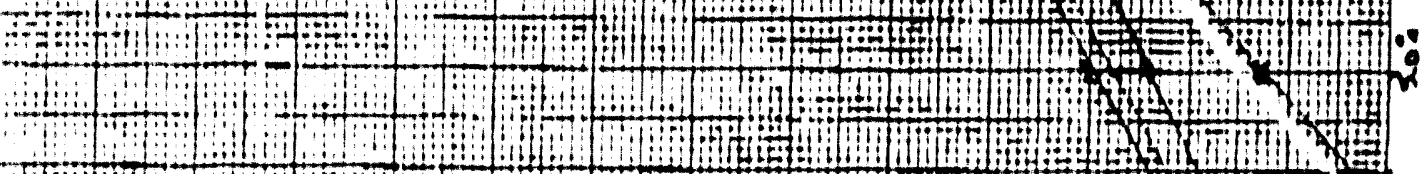
650 Tom 400 of 150 14

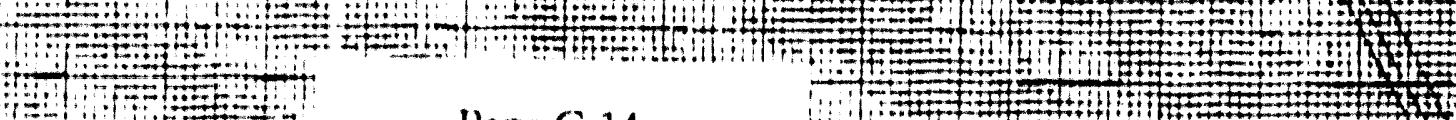
Jage $C_{1}-14$

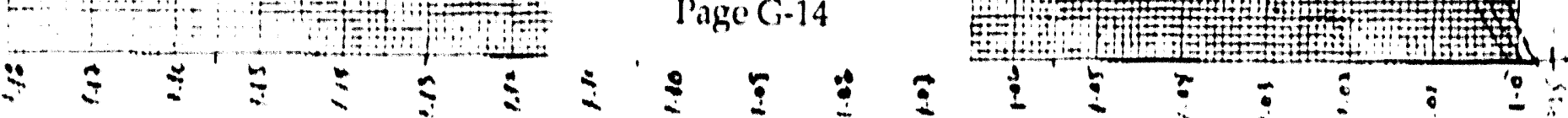




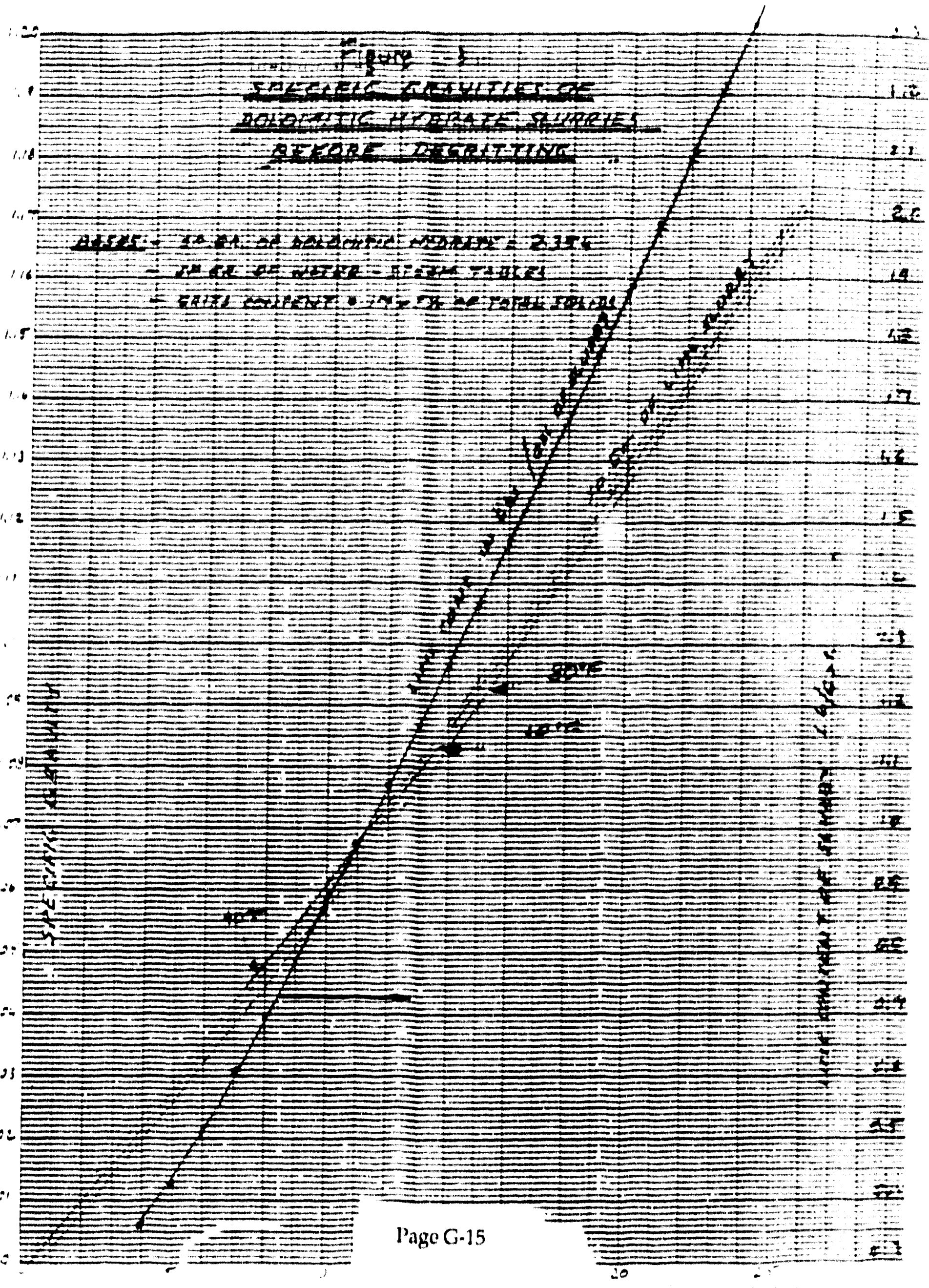


Lime Usage = (Content of lime feed lank at the star of test run in gallon) $\times 8.328 \times$ (its specific eravity) $x$ (corresponding slumry concentrate) - content of lime feed unk at the end of test run in gallon) $\times 8.326 \times$ (FLS specific gravity) $\times$ (corresponding slumy concentrate)

Example: Esomate of lime content of the lime slurry in the lime feed tank at the stan of test:

Tank diameter $=12^{\prime} \cdot 0^{\prime \prime}$

Slurry Depth $=10^{\prime} \cdot 0^{\prime \prime}$ (by tank dipping)

$\therefore$ Volume of slurry $=\frac{12^{2} \pi}{4} \times 10=1,130^{4} \mathrm{cu} . \mathrm{ft}$.

- 8.459.4 GAL.

Specific gravity of slury $=1.125$ (as 60\%, measured)

Comesponding lime concentrice $=19.5 \mathrm{~m} \%$ (from the chan relating specific eravity lo concentrate)

$\therefore$ Lime content of the feed unk at the star of lest $=8,455.4 \times 8.328$ $\times 1.125 \times 0.195=19,447.6 \mathrm{lbs}$.

Estimute of lime content of the lime slurry in the lime feed unk at the end of tess:

Slumy Depth = 5' 'of" (by ank dipping)

$\therefore$ Volume of Slurry $=\frac{13^{2} \pi}{4} \times 5.7792 \times 7.48=4.961 .4 \mathrm{GL}$.

Specific gravity of slumy $=1.12$

Corresponding lime concentrute $=18.9$ WT \&

$\therefore$ Lime content of the feed unk at the end of wast

- 4,961.4 ×8.328 × $1.12 \times 0.189=8,614.3 \mathrm{lbs}$.

Hence lime used $=19,447.6 \cdot 8,614.3=6,833 \mathrm{lbs}$.

Avernge concentration of lime slurry used

$=\frac{6,833}{8,455.4-4,886.5+307.5}$

Esdimuted volume of seal water used by P.102A and P. $102 B$ during the test.

- 1.7627 lbe of lime hydrue/gallon of slurty corresponding to slurry concentrate of 18.9 WT\% trom the chare 


$$
\begin{aligned}
& \text { Lime utilization }=\frac{\text { Mols } \mathrm{SO}_{2} \mathrm{ABS}=\frac{1}{2} \text { Mols } \mathrm{NO}_{2} \mathrm{ABS}}{\text { Mols of lime used }} \\
& \text { Mols } \mathrm{SO}_{2} \mathrm{ABS}=\frac{\mathrm{x}_{\mathrm{OO}_{1}}}{379.3} \\
& \text { Mols NO } A B S=\frac{Y_{N O}}{379.5} \\
& \text { Mols of lime used } \\
& =\frac{\text { WT of lime hydrice } \times 0.385}{38}+\frac{W T \text { of lime hydnce } \times 0.57}{74} \\
& \text { - WT of lime hydrte used }(0.0066+0.0077) \\
& \text { - WT of lime hydnie used } \times 0.0143
\end{aligned}
$$




\section{TEST DATA}

$10 / 10 / 91$

Flue gas flow meter reading $\quad=260,000$ SCFM $=F G=C$ Atomizing air flow meter reading $=1,350 \mathrm{SCFM}=\mathrm{AA}=\mathrm{D}$ Water content of lime slurry $\quad=5,180$ SCFM $=W C=F$

$$
\begin{aligned}
\text { Concentrate } & =3.4 \mathrm{WT} \% \\
\text { Specific Gravity } & =1.018 \mathrm{at} 60^{\circ} \mathrm{F} \\
\text { Flow } & =30 \mathrm{gpm}
\end{aligned}
$$$$
W C=30 \times 8.328 \times 1.018 \times 0.966=245.7 \mathrm{lb} / \mathrm{mid}=5,180 \mathrm{SCFM}
$$

Inlet $\mathrm{SO}_{2}$ concentate in ppmv $=905.81 \mathrm{ppmv}=\mathrm{SO}_{2} \mathbb{N}=\mathrm{F}$

Outlet $\mathrm{SO}_{2}$ concentrate in ppinv $=703.87$

Injet $\mathrm{NO}_{\mathrm{x}}$ concentrate in ppmv $=383.25$

Outlet $\mathrm{NO}_{x}$ concentrate in ppmv $=313.87$

Inlet $O_{2}$ concentrate in Vol. \% $=6.16$

Oudet $O_{2}$ concentrate in Vol. \% $=7.41$

$$
\begin{aligned}
& =\mathrm{SO}_{2} \text { OUT }=\mathrm{O} \\
& =N \mathrm{NO}_{\times} \mathbb{N}=\mathrm{H} \\
& =\mathrm{NO}_{\mathrm{OUTT}}=\mathrm{I} \\
& =O_{2} \mathbb{N}=J \\
& =\mathrm{O}_{2} \text { OUT }=\mathrm{K}
\end{aligned}
$$



$$
=\frac{260,000\left|.21 \frac{1.0012891}{0.0616}-1\right|+1,350\left|1+0.21 \frac{1.0012891}{0.0616}\right|+5,180}{\frac{1.0012891}{0.0616}(0.21-0.0741)+0.00070387+0.000313851}
$$

$$
=\frac{627,506.2+3,258.2+5,180}{2.2090+0.001017 .74}=\frac{629,428}{2.2100}=284,809 \mathrm{SCFM}
$$

$$
\begin{aligned}
& \text { Inlet } F G=F G_{\mathbb{N}}=\frac{F G \cdot A A \cdot W C \cdot F G_{\alpha r}\left(S_{2} a r+N_{x} a r t\right)}{1+S_{2 N}-N_{2 N}} \\
& =\frac{260,000 \cdot 1,350 \cdot 5,180 \cdot 284,809(0.00070367+0.00031387)}{1 \cdot 0.00090581 \cdot 0.00038325} \\
& =\frac{253,470-289.9}{0.9987109} \\
& =253,506 \text { SCFM }
\end{aligned}
$$


Air Leakage $=Z_{A L}=F G_{O U T}-F G$

$=284,809-260,000$

$=24,809$ SCFM

$\mathrm{SO}_{2}$ Removed $=\mathrm{FG}_{\mathbb{N}} \times \mathrm{SO}_{2} \mathbb{N} \cdot \mathrm{FGOUT} \times \mathrm{SO}_{2} \mathrm{OUT}$

$$
\begin{aligned}
& =253,506 \times 0.00090581 \cdot 284,809 \times 0.00070387 \\
& =229.63 \cdot 200.47 \\
& X_{\mathrm{SO}_{2}}=2916 \mathrm{SCFM}
\end{aligned}
$$

$\mathrm{NO}_{3}$ Removed $=\mathrm{FG}_{\mathbb{N N}} \times \mathrm{NO}_{\times \mathbb{N}} \cdot \mathrm{FGOUT} \times \mathrm{NO}_{\times}$OUT

$$
=253,506 \times 0.00038325 \cdot 284,809 \times 0.00031387
$$

$$
Y_{\text {NOX }}=97.16 \cdot 89.39+7.76 \text { SCFM }
$$

$\% \mathrm{SO}_{2}$ Removal $=\frac{\mathrm{X}_{\mathrm{SO}_{2}}}{\mathrm{FG}_{\mathrm{NN}} \times \mathrm{SO}_{2 \mathrm{~N}}} 100=\frac{25: 6}{229.56} \times 100=12.7 \%$

$\% \mathrm{NO}_{x}$ Removal $=\frac{\mathrm{Y}_{\mathrm{NO}_{1}}}{F G_{\mathbb{N}} \times \mathrm{NO}_{\times \mathbb{N}}} 100=\frac{7.76}{97.13} \times 100=8.00 \%$

Estimate of lime usage:

$30 \mathrm{gpm}$ of 3.4 WT\% lime slurry

$$
\text { SP GT at } 60^{\circ} \mathrm{F}=1.018
$$$$
\mathrm{Ca}(\mathrm{OH})_{2} \text { Used }=30 \times 8.328 \times 1.018 \times 0.034
$$

$=8.6475 \mathrm{lb} /$ minute

Assume $95.5 \%$ purity

$$
=\frac{8.6475 \times 0.955}{74}
$$

$=0.1116 \mathrm{lb} \mathrm{Mol} /$ minute

$\mathrm{SO}_{2}$ Removed $=\frac{29.16}{379}=0.079 \mathrm{Lb} \mathrm{Molmminute}$

NO $_{x}$ Removed $=\frac{7.76}{379}=0.0204 \mathrm{Lb}$ Mol/minute

Lime Utilization $=\frac{0.0769 \times \frac{0.0204}{2}}{0.1116} \times 100=78.07 \%$ 


\section{TEST DATA}

$10 / 11 / 91$

Flue gas Flow Meter Reading $=268,000$ SCFM

Atomizing Air Meter Reading $=1,350$ SCFM

Water Content of Lime Slurry $=5,127$ SCFM

Concentrate $=5.5 \mathrm{WT} \%$

$\mathrm{Sp} \mathrm{Gr}=1.03$ at $60^{\circ} \mathrm{F}$

Flow $\quad=30 \mathrm{gpm}$

W.C. $=30 \times 8.328 \times 1.03 \times 0.945=243.18 \mathrm{Lb} / \mathrm{Min}$

$=5,127$ SCFM

$$
\begin{aligned}
& \text { Inlet } \mathrm{SO}_{2} \text { Concentrate }=868.56 \mathrm{ppm} \\
& \text { Outlet SO2 } 2 \text { Concentrate }=660.49 \mathrm{ppm} \\
& \text { Inlet } \mathrm{NO}_{x} \text { Concentrate }=400.23 \mathrm{ppm} \\
& \text { Outlet NO } \mathrm{x}_{x} \text { Concentrate }=328.05 \mathrm{ppm} \\
& \text { Inlet } \mathrm{O}_{2} \text { Concentrate }=6.32 \% \\
& \text { Oudet } \mathrm{O}_{2} \text { Concentrate }=7.38 \% \\
& F G_{\text {OT }}=\frac{\left.268,000 \times\left[\frac{1.0012688}{0.0623} \cdot 1\right]+1,350 \mid 1 \cdot .21 \times \frac{1.0012688}{0.0623}\right]+5,127}{\frac{1.0012688}{0.0623}(.21-0.0738)+0.00066049+0.000328 .05} \\
& =\frac{636,517.0-3,206.3+5,127}{2,1890+0.0009885}=\frac{638,437.7}{2.1900} \\
& =291,524 \text { SCFM } \\
& F G_{\mathbb{N}}=\frac{\mathrm{FG}-\mathrm{AA}-\mathrm{WC}+\mathrm{FG}_{\mathrm{arr}}\left(\mathrm{SO}_{2} \mathrm{ar}+\mathrm{NO}_{2} \text { arr }\right)}{1+\mathrm{SO}_{2 \mathrm{~N}}+\mathrm{NO}_{2 \mathrm{~N}}} \\
& =\frac{268,000 \cdot 1,350 \cdot 5,127+295,690.7(0.00066049+0.00032805)}{1+0.00086856+0.00040023} \\
& =\frac{261,523+292.3}{0.9987312} \\
& =261,562 \text { SCFM }
\end{aligned}
$$


Air Leakage $=Z_{A L}=F G_{O U T} \cdot F G$

$=241,524 \cdot 268,000$

$=23,524$ SCFM

$\mathrm{SO}_{2}$ Removed $\quad=\mathrm{FG}_{\mathbb{N}} \times \mathrm{SO}_{2} \mathbb{N} \cdot \mathrm{FGOUT} \times \mathrm{SO}_{2} \mathrm{OUT}$

$=261,562 \times 0.00086856 \cdot 291,524 \times 0.0006604$

$=227.18 \cdot 192.55$

$=34.63$ SCFM

$\mathrm{NO}_{\mathrm{x}}$ Removed $\quad=261,562 \times 0.00040023 \cdot 291,524 \times 0.00032805$

$=104.68 \cdot 95.63$

$=9.05$ SCFM

\% $\mathrm{SO}_{2}$ REMOVAL $=\frac{34.63}{227.18} 100=15.24 \%$

\% NO REMOVAL $=\frac{9.05}{104.68} 100=8.65 \%$

Estimate of Lime Usage

$30 \mathrm{gpm}$ of 5.5 WT\% lime slurry

$\mathrm{Sp} \mathrm{Gr}=1.03$

$30 \times 8.328 \times 1.03 \times 0.55=14.15 \mathrm{Lb} / \mathrm{Hr}$

$=13.5 \mathrm{Lb}$ of pure $\mathrm{Ca}\left(\mathrm{OH}_{2}\right)$

$=0.1827 \mathrm{Lb}$ Mol/Minute

$\mathrm{SO}_{2}$ Removed $=\frac{34.63}{379.5}=0.0913 \mathrm{Lb}$ MolMinute

NO Removed $=\frac{9.05}{379.5}=0.0238 \mathrm{Lb}$ MolMinute

Lime Utilization $=\frac{0.0913+\frac{0.0238}{2}}{0.1827} 100$

$=56.50 \%$ 
FG

$=252,000$ SCFM

AA

$=1,350$ SCFM

Water Content of Lime Slurry $=5,054$ SCFM

$$
\begin{aligned}
\text { Concentrate } & =8 \% \\
\text { Sp Gr } & =1.043 \mathrm{~F} \\
\text { Fow } & =30 \mathrm{gpm} \\
\text { W.C. } & =30 \times 8.328 \times 1.03 \times 0.92=239.74 \mathrm{Lb} / \mathrm{Min} \\
& =5,054 \text { SCFM }
\end{aligned}
$$

Inlet $\mathrm{SO}_{2}$ Concentrate $=891.1 \mathrm{ppm}$

Outlet $\mathrm{SO}_{2}$ Concentrate $=665.9 \mathrm{ppm}$

Inlet $\mathrm{NO}_{\mathrm{x}}$ Concentrate $=423.9 \mathrm{ppm}$

Oudet $\mathrm{NO}_{\mathrm{x}}$ Concentrate $=326.6 \mathrm{ppm}$

Inlet $\mathrm{O}_{2}$ Concentrate $=6.2 \%$

Outlet $\mathrm{O}_{2}$ Concentrate $=7.52 \%$

$F G_{\alpha T}=\frac{252.000\left|.21 \times \frac{1.001315}{0.062} \cdot 1\right|+1,350\left|1 \cdot .21 \times \frac{1.001315}{0.062}\right|+5,000}{\frac{1.001315}{0.062}(0.21 \cdot 0.0738)+0.000659+0.0003266}$

$=\frac{602,670.8-3,228.6+5,054}{2,1771+0.0009925}=\frac{604,496.2}{2.1781}$

$=277,534.7$ SCFM

$F G_{\mathbb{N}}=\frac{252,000 \cdot 1,350 \cdot 5,054+277,534.7(0.0006659+0.0003266)}{1+0.000891 .1 \cdot 0.000423 .9}$

$$
=\frac{245,320}{0.9987}=245,643 \text { SCFM }
$$

Air Leakage $=Z_{A L}=$ FGoUT $\cdot F G$ 


$$
\begin{aligned}
& =277,534 \cdot 252,000 \\
& =25, \$ 31 \text { SCFM }
\end{aligned}
$$

$\mathrm{SO}_{2}$ Removed $\quad=\mathrm{FGN}_{\mathrm{N}} \times \mathrm{SO}_{2} \mathrm{~N} \cdot \mathrm{FGOUT} \times \mathrm{SO}_{2} \mathrm{OUT}$

$=245,643 \times 891.1 \cdot 277,535 \times 665.9$

$=218.89 \cdot 184.81$

$=34.08$ SCFM

NO Removed $=245,643 \times 423.9-277,535 \times 326.6$

$=104.13 \cdot 10.64$

$=13.49$ SCFM

$\% \mathrm{SO}_{2}$ REMOVAL $=\frac{34.08}{218.89} 100=15.57 \%$

\% NO $\times$ REMOVAL $=\frac{13.49}{104.13} 100=12.95 \%$

Estimate of Lime Usage

$30 \mathrm{gpm}$ of 8 WT\% lime slurry

$$
\begin{aligned}
& \text { Sp } G t=1.043 \\
& \text { Purity }=95.5 \%
\end{aligned}
$$

$30 \times 8.328 \times 1.043 \times 0.08 \times 0.955=19.9 \mathrm{Lb} / \mathrm{Min}=0.269 \mathrm{Lb}$ Mol/Minute

$\mathrm{SO}_{2}$ Removed $=\frac{34.08}{379.5}=0.0898$

NO Recoved $=\frac{13.49}{379.5}=0.0355$

Lime Udilization $=\frac{0.0898+\frac{0.0355}{2}}{0.259}=40 \%$ 


\title{
Appendix $\mathrm{H}$
}

\author{
Tables With Lime Slurry \\ Injection Operating Data \\ August 17 to November 16, 1992
}


CZD LIME INJECTION DATA. AUQUST 17, 10 NOVEMBEA 16.1002

\begin{tabular}{|c|c|c|c|c|c|c|c|c|c|c|c|c|c|c|c|c|c|c|}
\hline Dalo & Timo & $\begin{array}{l}\text { Boiler } \\
\text { Load } \\
\text { NW }\end{array}$ & \begin{tabular}{|l|} 
Duct \\
Inlol \\
Tomp of
\end{tabular} & $\begin{array}{l}\text { Avg "C" } \\
\text { Section } \\
\text { Tomp "F }\end{array}$ & $\begin{array}{l}\text { Ducl } \\
\text { Oullet } \\
\text { Temp of }\end{array}$ & $\begin{array}{l}\text { Flue Oas } \\
\text { Flow In } \\
\text { KSCFM }\end{array}$ & $\begin{array}{l}\text { Flue Gas } \\
\text { Flow Ous } \\
\text { KSCFM }\end{array}$ & $\begin{array}{l}502 \\
\ln 101 \\
\mathrm{ppm}\end{array}$ & $\begin{array}{c}802 \\
\text { Outloit } \\
\mathrm{ppm}\end{array}$ & $\begin{array}{l}\mathrm{NOx} \\
\text { Inloi } \\
\mathrm{ppm}\end{array}$ & $\begin{array}{c}\text { NOx } \\
\text { Outlol } \\
\text { ppm }\end{array}$ & $\begin{array}{l}\text { Lime } \\
\text { Flow } \\
\text { opm }\end{array}$ & $\begin{array}{c}\text { Lime } \\
\text { Conc } \\
\%\end{array}$ & $\begin{array}{c}502 \\
\text { Pamoval } \\
\%\end{array}$ & $\begin{array}{c}\text { NOn } \\
\text { Hemovai } \\
\%\end{array}$ & $\begin{array}{c}802 \\
\ln 101 \\
\text { Lo/He }\end{array}$ & $\begin{array}{c}802 \\
\text { Hemoved } \\
\text { Lb/H }\end{array}$ & $\begin{array}{c}\text { Limo } \\
\text { Uthlization } \\
\%\end{array}$ \\
\hline Aup. 17 & 1300 & 1452 & 304.3 & N/A & 221.4 & 189.7 & 2282 & 806 & $6 B O$ & 346 & 298 & 350 & 10.3 & 0.8 & .98 & 1741.8 & 169.0 & 9.8 \\
\hline Aug.17 & 1315 & 144.6 & 304.3 & N/A & 220.4 & 100.2 & 227.1 & 807 & 693 & 323 & 290 & $38 ?$ & 10.3 & 8.8 & .10 .5 & 17485 & 154.3 & BI \\
\hline Aug.17 & 1330 & 145.4 & 305.3 & N/A & 209.3 & 1002 & 230.3 & 883 & 691 & 352 & 297 & 44.8 & 10.3 & 63 & .20 & 1710.8 & 1080 & 48 \\
\hline 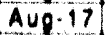 & 1345 & 146.3 & 304.8 & $N / A$ & 211.6 & 189.3 & 228.4 & 883 & 459 & 318 & 302 & 43.8 & 10.3 & 370 & .14 .0 & 16946 & 027.4 & 288 \\
\hline Aup.17 & 1400 & 140.5 & 306.4 & $N / A$ & 203.5 & 189.8 & 232.2 & 908 & 487 & 351 & 286 & 50.0 & 103 & 344 & .30 & 17457 & 600.8 & 24.1 \\
\hline Aug.17 & 1415 & 134.4 & 307.5 & $N / A$ & 204.8 & 76.6 & 22.1 & 860 & 487 & 340 & 286 & 17.7 & 103 & 320 & .4 .5 & 667.5 & 213.4 & 24.2 \\
\hline Aup.17 & 1430 & 140.0 & 303.2 & N/A & 2091 & 183.8 & 228.6 & $87 \mathrm{~A}$ & 528 & 358 & 296 & 445 & 103 & 24.8 & .2 .2 & 1627.0 & 1061 & 18.3 \\
\hline Aug.17 & 1445 & 140.0 & 303.2 & $N / A$ & 1094 & 189.5 & 232.2 & 674 & 528 & 358 & 296 & $51 !$ & 103 & 260 & .0 .7 & 1677.6 & 436.6 & 17.2 \\
\hline Aup.17 & 1500 & 146.1 & 306.6 & $N / A$ & 186.8 & 181.0 & 232.7 & 801 & 434 & 370 & 313 & 54.0 & 10.3 & 41.3 & 20 & 17444 & 720.8 & 268 \\
\hline Aug.17 & 1515 & 1468 & 307.0 & N/A & 194.9 & 190.7 & 232.8 & 900 & 432 & 364 & 308 & 55.1 & 103 & 420 & .33 & 17560 & 736.6 & 26.0 \\
\hline Aug. 17 & 1530 & 146.8 & 307.4 & N/A & 104.4 & 100.4 & 231.0 & 002 & 427 & 357 & 303 & 35.1 & 107 & 42.5 & 2.7 & 1730.7 & 730.6 & 25.8 \\
\hline Aug.17 & 1545 & 147.2 & 307.0 & $N / A$ & 1941 & 101.2 & 2348 & 910 & 424 & 354 & 308 & 557 & 10.7 & 42.8 & .7 .3 & 1763.5 & 754.6 & 26.1 \\
\hline Aug. 17 & 1600 & 1468 & 307.4 & $N / A$ & 195.0 & 190.0 & 235.6 & 909 & 415 & 352 & 322 & 54.5 & 107 & 43.4 & .13 .4 & 17505 & 759.1 & 268 \\
\hline Aug - 17 & 1615 & 146.9 & 307.4 & N/A & 194.7 & 180.3 & 234.7 & 900 & 413 & 320 & 293 & 54.0 & 10.7 & 432 & 132 & 1726.8 & 1451 & 26.2 \\
\hline Aup. 17 & 1630 & 146.6 & 308.3 & $N / A$ & 184.7 & 1801 & 235.6 & 906 & 413 & 348 & 293 & 55.1 & 107 & 43.5 & .3 .8 & 17456 & 7500 & 26.6 \\
\hline Aup. 17 & 1645 & 145.8 & 3081 & $N / A$ & 195.2 & 182.5 & 234.9 & 803 & 407 & 355 & 335 & 540 & 10.7 & 45.0 & .153 & 1760.8 & 792.6 & 27.8 \\
\hline Aug. 17 & 1700 & 146.8 & 308.3 & $N / A$ & 184.8 & $180 . ?$ & 233.3 & 800 & 403 & 350 & 297 & 55.2 & 10.7 & 45.7 & .3 .6 & 17550 & 8028 & 24.1 \\
\hline A $\cup 9.17$ & 1715 & 1471 & 308.8 & $N / A$ & 105.2 & 190.4 & 232.5 & 896 & 384 & 321 & 283 & 553 & 107 & 46.3 & .114 & 17263 & 798.7 & 27.8 \\
\hline Aug : 17 & 1730 & 147.3 & 307.5 & $N / A$ & 194.8 & 189.3 & 232.5 & 896 & 301 & 352 & 295 & 54.8 & 10.7 & 46.4 & 28 & 1718.1 & 707.5 & 28.1 \\
\hline Aup.17 & 1745 & 146.8 & 3081 & N/A & 185.3 & 180.4 & 233.8 & 806 & 392 & 355 & 336 & 55.5 & 10.7 & 46.8 & .16 .1 & 1748.4 & 818.1 & B. 5 \\
\hline Aug. 17 & 1800 & 146.3 & 308.3 & N/A & 189.4 & 180.6 & 2330 & 903 & 420 & 356 & 298 & 490 & 107 & 432 & .23 & 1744.4 & 763.4 & 29.1 \\
\hline A $\cup 0.17$ & 1815 & 1465 & 3070 & $N / A$ & 2023 & 1807 & 2317 & 687 & 424 & 326 & 298 & 482 & 13.8 & 425 & .11 .1 & 17328 & 736.7 & 22.3 \\
\hline A $\cup 0.17$ & 1830 & 1460 & 3062 & N/A & 203.3 & 181.3 & 2294 & 906 & 438 & 356 & 298 & 480 & 139 & 421 & 0.1 & 1755.7 & 738.3 & 22.4 \\
\hline Aug.17 & 1845 & 146.5 & 306.6 & $N / A$ & 2040 & 1928 & 232.2 & 910 & 444 & 354 & 335 & 481 & 13.9 & 412 & .13 .8 & 1777.2 & 732.2 & 22.2 \\
\hline Aup.17 & 1000 & 146.8 & 306.4 & N/A & 2058 & 1818 & 230.2 & 880 & 462 & 352 & 286 & 456 & 139 & 38.3 & .07 & 17476 & 689.6 & 21.4 \\
\hline Aug. 17 & 1815 & 1462 & 3068 & $N / A$ & 205.9 & 1818 & 2307 & 825 & 467 & 323 & 286 & 46.7 & 138 & 37.2 & 0.8 & 17380 & 646.8 & 20.2 \\
\hline Aup. 17 & 1930 & 146.4 & 305.3 & $N / A$ & 2051 & 181.7 & 231.8 & 816 & 450 & 352 & 296 & 47.5 & 138 & 40.5 & -1.7 & 17787 & 7210 & 22.1 \\
\hline Aug. 17 & 1945 & 146.7 & 305.7 & $N / A$ & 2044 & 192.8 & 230.8 & 908 & 448 & 348 & 332 & 48.7 & 138 & 40.9 & .138 & 1774.2 & 726.2 & 17 \\
\hline$A \cup 0.17$ & 2000 & 146.8 & 305.8 & $N / A$ & 202.8 & 181.3 & 228.0 & 908 & 444 & 354 & 300 & 49.0 & 13.8 & 41.8 & .0 .8 & 1760.5 & 735.6 & 1.9 \\
\hline Aug. 17 & 2015 & 147.0 & 306.2 & $N / A$ & 2014 & 192.8 & 232.5 & 894 & 444 & 325 & 296 & 497 & 13.8 & 40.1 & 97 & 17 & 6 & 5 \\
\hline Aug. 17 & 2030 & 1468 & 305.8 & $N / A$ & 200.8 & 1827 & 232.4 & 915 & 457 & 358 & 298 & 498 & 13.8 & 39.8 & a & 17 & 7 & 20.8 \\
\hline Aug.17 & 2045 & 1471 & 305.8 & $N / A$ & 2006 & 1834 & 232.0 & 917 & 463 & 356 & 334 & 50.6 & 11.5 & 39.5 & .126 & 17965 & 7088 & 250 \\
\hline Aug.17 & 2100 & 1471 & 306.2 & $N / A$ & 2000 & 1932 & 2320 & 917 & 458 & 665 & 208 & 49.8 & 11.5 & 40.1 & 46.3 & 17846 & & 8 \\
\hline Aug .17 & 2115 & 146.8 & 304.0 & N/A & 2144 & 191,8 & 2300 & 833 & 610 & 734 & 263 & 34.2 & 11.5 & 21.6 & 57.0 & 18 & 2 & 204 \\
\hline AVE & PAGES & 1456 & 306.4 & $N / A$ & 2021 & 187.5 & 2277 & 9021 & 468.4 & 368.1 & 303.2 & 48.6 & 116 & 370 & .3 .3 & 1714.8 & 6363 & 228 \\
\hline Aug.20 & 1745 & 1420 & 305.1 & $N / A$ & 212.0 & 100.8 & 2281 & 868 & 601 & 360 & 337 & 401 & 75 & 17.2 & .11 .6 & 16788 & 288.8 & 20.2 \\
\hline Aug. 20 & 1800 & 1415 & 306.0 & N/A & 200.9 & 1810 & 226.2 & 856 & 568 & 358 & 100 & 448 & 75 & 21.5 & 0.6 & 16563 & 5.7 & 22.3 \\
\hline Aug. 20 & 1815 & 1443 & 306.8 & $N / A$ & 2021 & 182.1 & 2281 & 876 & 534 & 330 & 301 & 49.9 & 7.5 & 277 & 83 & 17058 & 472.8 & 26.6 \\
\hline Aug. 20 & 1830 & 1432 & 305.1 & N/A & 201.6 & 182.6 & 228.9 & 881 & 528 & 350 & 298 & 48.2 & 7.5 & 28.4 & 0.8 & 1718.0 & 487.7 & 284 \\
\hline Aug. 20 & 1845 & 1452 & 304.7 & N/A & 200.3 & 192.8 & 230.9 & 877 & 524 & 360 & 338 & 494 & 7.5 & 28.4 & 12.3 & 17130 & 486.8 & 276 \\
\hline Aug $20^{\circ}$ & 1800 & 1448 & 304.2 & $N / A$ & 1994 & 1933 & 2324 & 874 & 491 & 362 & 303 & 40.7 & 7.5 & 5 & .0 .6 & 17 & 1 & 4 \\
\hline Aug.20 & 1915 & 144.6 & 3024 & $N / A$ & 1984 & 181.3 & 2286 & 860 & 475 & 333 & 303 & 48.8 & 7.5 & 34.0 & .8 .4 & 16667 & 7.2 & $\theta$ \\
\hline A $\cup 0.20$ & 1930 & 1443 & 3016 & $N / A$ & 1880 & 192.9 & 2297 & 864 & 468 & 363 & 304 & 50.0 & 7.5 & 35.4 & 0.2 & 16884 & 597.5 & 3.5 \\
\hline Aup.20 & 1845 & 144.8 & 301.3 & $N / A$ & 196.5 & 192.2 & 2281 & 857 & 463 & 361 & 338 & 50.3 & 7.5 & 360 & .108 & 1669.7 & 600.3 & 13.5 \\
\hline Aup.20 & 2000 & 143.8 & 300.3 & $N / A$ & 194.6 & 192.3 & 230.0 & 857 & 451 & 364 & 303 & 50.1 & 7.5 & 7 & 0.6 & 0.5 & 2 & .7 \\
\hline$A \cup g \cdot 20$ & 2015 & 1439 & 2994 & $N / A$ & 187.1 & 1934 & 230.3 & 847 & 474 & 333 & 303 & 479 & 7.5 & 4 & 8.0 & 0.5 & 0 & 24 \\
\hline$A \cup 0.20$ & 2030 & 1443 & 299.4 & $N / A$ & 197.8 & 182.6 & 2310 & 858 & 475 & 369 & 302 & 48.2 & 7.5 & 33.6 & 1.8 & 1674.1 & 5631 & 2.8 \\
\hline$A \cup 0.20$ & 2045 & 1435 & 299.6 & $N / A$ & 198.3 & 194.3 & 231.9 & 864 & 471 & 365 & 341 & 48.2 & 75 & 349 & .11 .5 & 17007 & 5836 & 4.5 \\
\hline Aug. 20 & 2100 & 144.0 & 298.8 & N/A & 1982 & 193.7 & 230.5 & 864 & 468 & 365 & 304 & 47.9 & 7.5 & 56 & 00 & 5.5 & 2 & \\
\hline A $\cup 0.20$ & 2115 & 1440 & 289.6 & N/A & 1990 & 184.1 & 231.8 & 864 & 422 & 365 & 254 & $\triangle B A$ & 7.5 & 416 & 11 & 0 & .1 & 1.0 \\
\hline Aup.20 & 2130 & 143.7 & 2992 & $N / A$ & 198.6 & 194.3 & 234.0 & 864 & 448 & 365 & 271 & 481 & 7.5 & 37.5 & 10.7 & 1700.7 & 636.1 & 17.2 \\
\hline 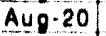 & 2145 & 1443 & 298.8 & $N / A$ & 1882 & 1936 & 2348 & 864 & 450 & 365 & 278 & 47.8 & 7.5 & 8 & 7.4 & 1684.6 & 6 & .5 \\
\hline Aug.20 & 2200 & 143.6 & 2874 & $N / A$ & $196 ?$ & 1934 & 231.2 & 864 & 442 & 365 & 279 & 48.1 & 75 & 38.8 & 7 & 2.8 & 657.0 & 3.3 \\
\hline $\bar{A} \cup_{0}-20$ & 2215 & 1430 & 296.7 & N/A & 183.8 & 191.3 & 2335 & 904 & 438 & 365 & 280 & 49.8 & 7.5 & 40.8 & 6.5 & 1751.8 & 713.9 & 0.2 \\
\hline Aug. 20 & 2230 & 143.8 & 297.4 & $N / A$ & 1927 & 1894 & 2316 & 888 & 441 & 365 & 288 & 49.8 & 75 & 393 & 3.5 & 17048 & 6695 & 37.7 \\
\hline$A \cup Q \cdot 20$ & 2245 & 144.4 & 296.6 & $N / A$ & 182.2 & 192.3 & 233.9 & 898 & 437 & 365 & 282 & 49. & 75 & 40.8 & 6.0 & 17492 & 7131 & 40.1 \\
\hline Aug. 20 & 2300 & 143.7 & 296.7 & $N / A$ & 192.4 & 192.1 & 232.9 & 898 & 437 & 365 & 282 & 503 & 75 & 410 & 6.3 & 17474 & & 38.8 \\
\hline A U : 20 & 2315 & 1438 & 2867 & $N / A$ & 192.4 & 1914 & 228.6 & 898 & 437 & 365 & 282 & 50.1 & 75 & 41.6 & 7.3 & 1741.1 & 724.0 & 0.5 \\
\hline 40.20 & 2330 & 140.8 & 296.1 & $N / A$ & 1914 & 1894 & 231.8 & 898 & 465 & 305 & 282 & 50.4 & 75 & 36.6 & .13 .2 & 1722.9 & 629.8 & 35.1 \\
\hline$A \overrightarrow{V E}$ & RAGES & 1438 & 300.5 & N/A & 1980 & 182.4 & 230.8 & 872.7 & 475.5 & 357.2 & 2979 & 48.6 & 7.5 & 34.6 & 0.3 & 17007 & 588.1 & 33.8 \\
\hline Aug.21 & 1445 & 145.2 & 310.5 & $N / A$ & 2056 & 186.9 & 229.7 & 041 & 510 & 456 & 290 & 46.9 & 80 & 32.3 & 21.7 & 6 & 575.8 & 28.4 \\
\hline $4 \cup 0.21$ & 1500 & 145.3 & 310.3 & N/A & 2056 & 1855 & 230.1 & 940 & 484 & 448 & 290 & 501 & 9.0 & 36.2 & 201 & 17673 & 639.8 & 29.6 \\
\hline 0.21 & 1515 & 144.5 & 3112 & IA & 2010 & 187.7 & 2326 & 921 & 455 & 440 & 291 & 52.1 & 90 & 387 & 18.1 & 1751.1 & 6784 & 30.2 \\
\hline Aug-21 & 1530 & 145.6 & & & 99.7 & 189.8 & 2330 & 921 & 450 & 436 & 289 & 541 & 80 & 400 & 188 & 17707 & 709.1 & 30.4 \\
\hline$A \cup 0.21$ & 1545 & 145.5 & 3107 & $N / A$ & 198.9 & $180 ?$ & 2334 & & 431 & 430 & $n \Omega^{2}$ & 540 & 9.0 & 41.8 & 107 & & & 31.4 \\
\hline 18.21 & 1600 & 1454 & 310.7 & $N / A$ & 198.6 & 180.2 & 235.3 & 811 & 425 & 423 & 290 & 54.2 & 8.0 & 42.0 & 14.7 & 17 & 734.2 & 31.4 \\
\hline 0.21 & 1615 & 145.5 & & & 188.6 & 191,7 & 2330 & 91 & 427 & 419 & 294 & 540 & 9.0 & 43.2 & 14.7 & 1772.8 & 7654 & 32.8 \\
\hline$A \cup 0.21$ & 1630 & 145.0 & 310.3 & $N / A$ & 197.8 & 188.7 & 233.3 & 9 & 424 & 413 & 000 & 53.8 & 90 & 427 & 12.5 & 1747.1 & 745.3 & 32.0 \\
\hline Aug.21 & 1645 & 145.3 & 3098 & N/A & 198.4 & 188.4 & 234.9 & 921 & 423 & 409 & 289 & 54.0 & 9.0 & 42.7 & 11.8 & 1757.7 & 750.2 & 32.2 \\
\hline 0.21 & 1700 & 145.4 & 310.3 & $N / A$ & 197.8 & 190.2 & 234.5 & 920 & 423 & 408 & 284 & 54.0 & 90 & 43.3 & 14.2 & 17725 & 768.0 & 33.0 \\
\hline 0.21 & 1715 & 143.7 & 310.4 & A & 197.8 & 189.1 & 2325 & 911 & 423 & 413 & 283 & 540 & 9.0 & 42.9 & 15.8 & 1745.0 & 7478 & 32.1 \\
\hline Aug-21 & 1730 & 141.9 & 0.3 & & 1980 & 189.8 & 233.4 & 901 & 410 & 395 & 277 & 54.2 & 90 & 440 & 13.6 & 1732.3 & 762.0 & 32.6 \\
\hline Aug. 21 & 1745 & 140.5 & 309.5 & N/A & 197.5 & 190.0 & 233.5 & 883 & 407 & 384 & 275 & 54.2 & 8.0 & 433 & 12.0 & 1700.6 & 737.0 & 31.5 \\
\hline Aug.21 & 1800 & 140.3 & 309.6 & $\mathrm{~N} / \mathrm{A}$ & 198.0 & 191.1 & 235.1 & 880 & 402 & 383 & 270 & 54.2 & 90 & 438 & 13.2 & 17037 & 745.4 & 31.9 \\
\hline
\end{tabular}


C2D LIME INJECTKON DATA AUQUST 17, TO NOVEMBEA 16, 1992

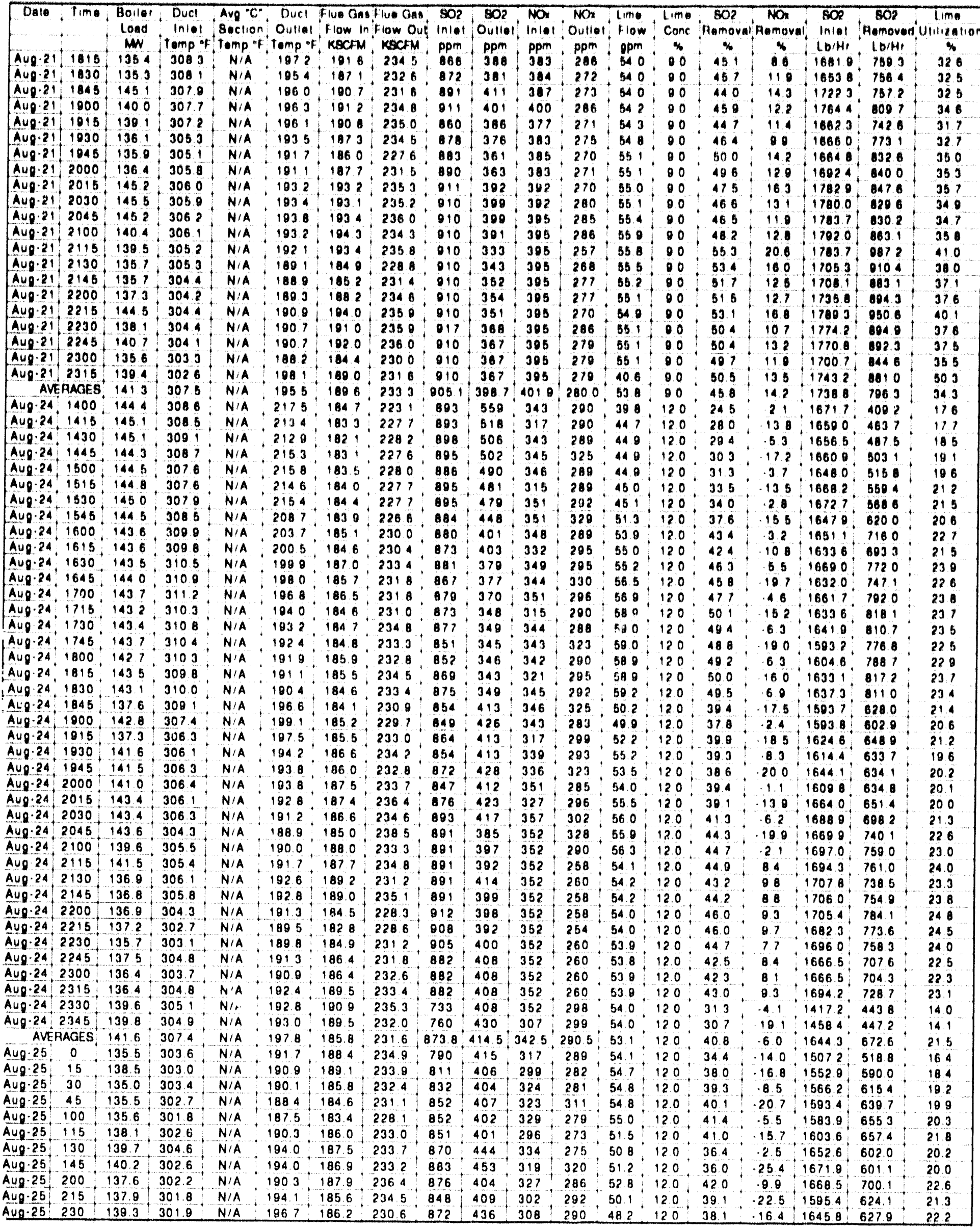


C20 LIME NJECIION DAIA. AUGUST 17, TO NOVEMAKA 16, 190 ?

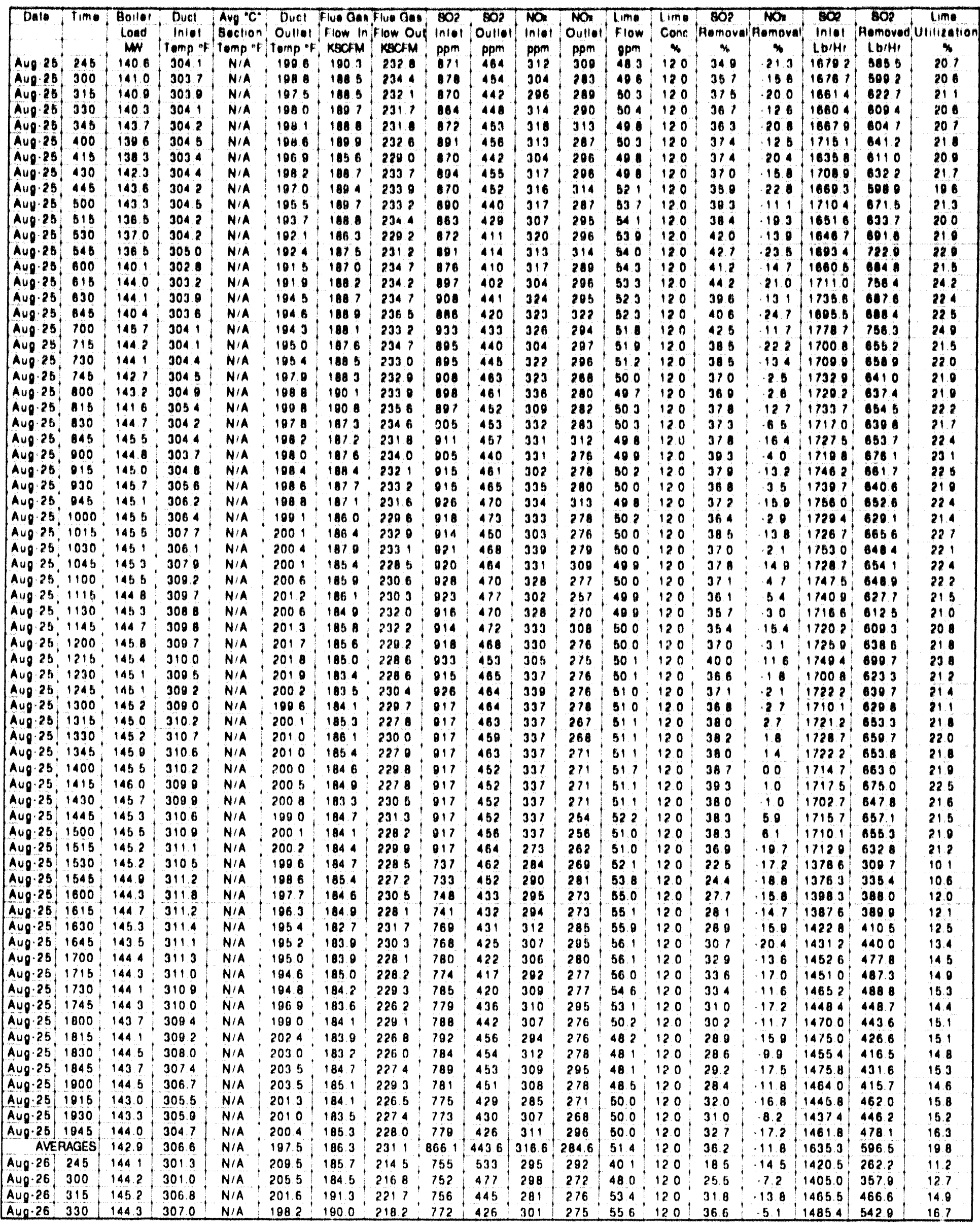


C20 LIME NJEC TION DATA. AUQUST 17, TO NOVEMBE 1 16, 1008

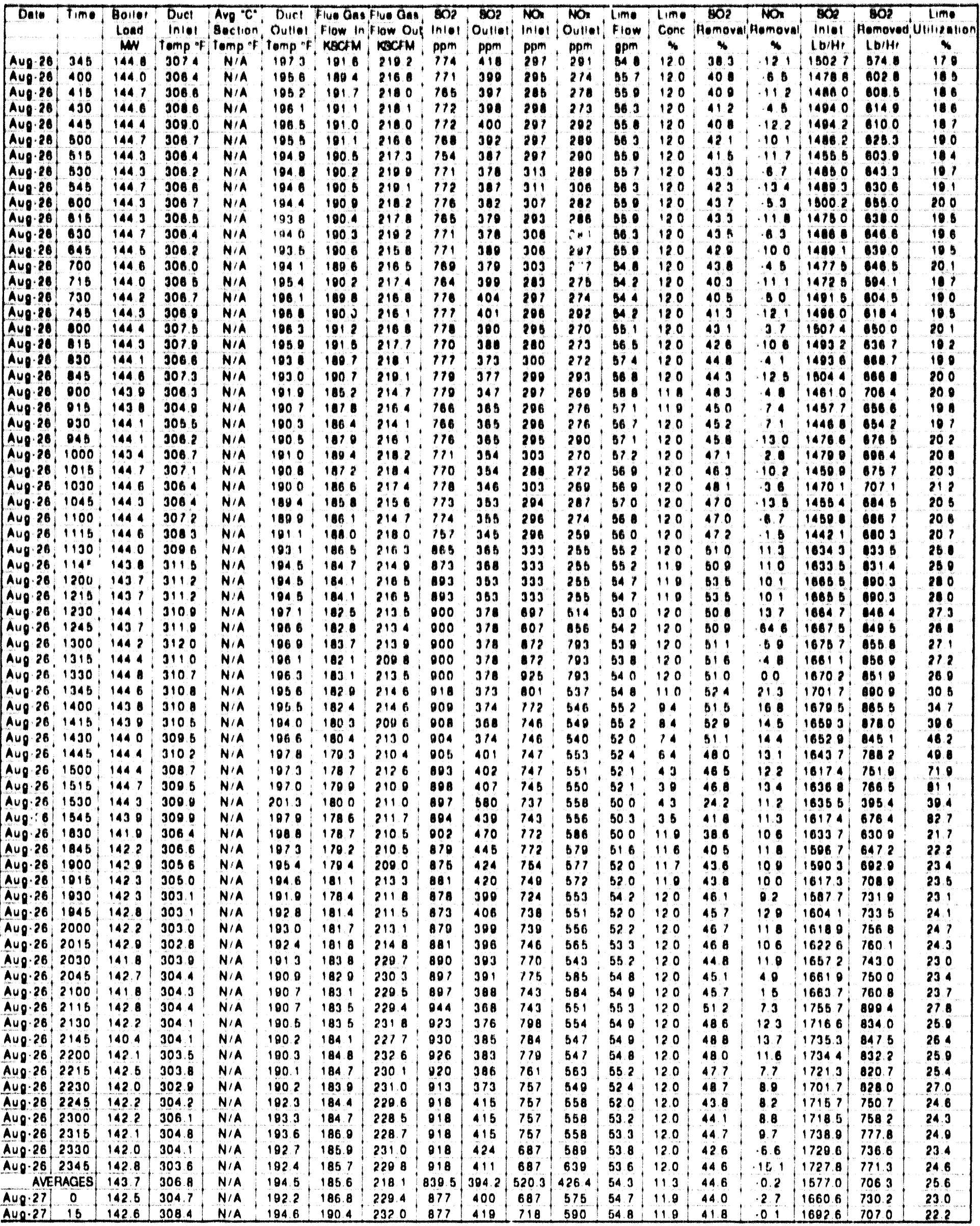




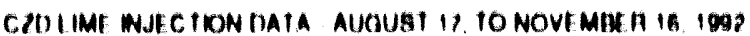

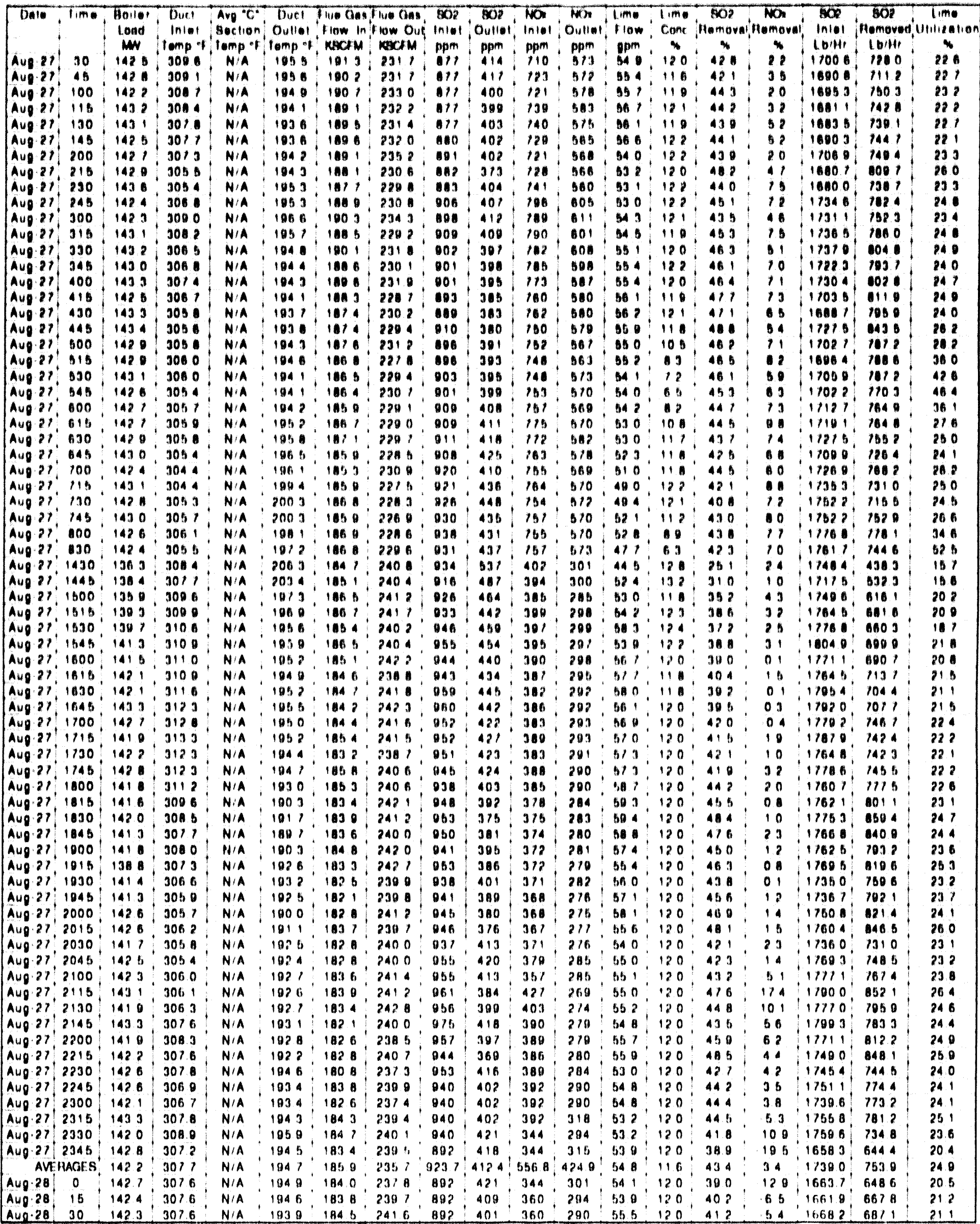


C2OLIME NJICION DATA AUGUST 17, 10 NOVEMAKA 16. 199?

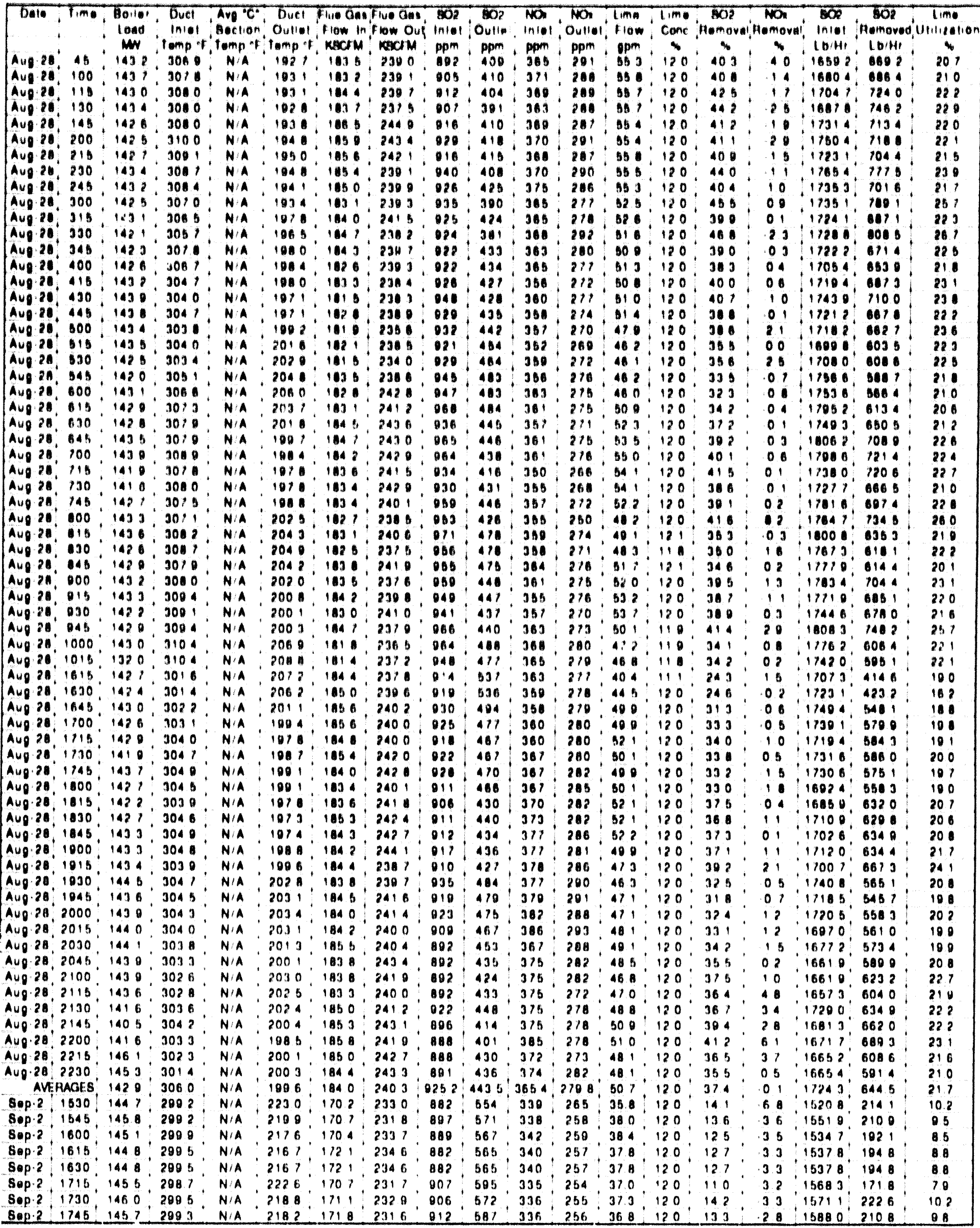




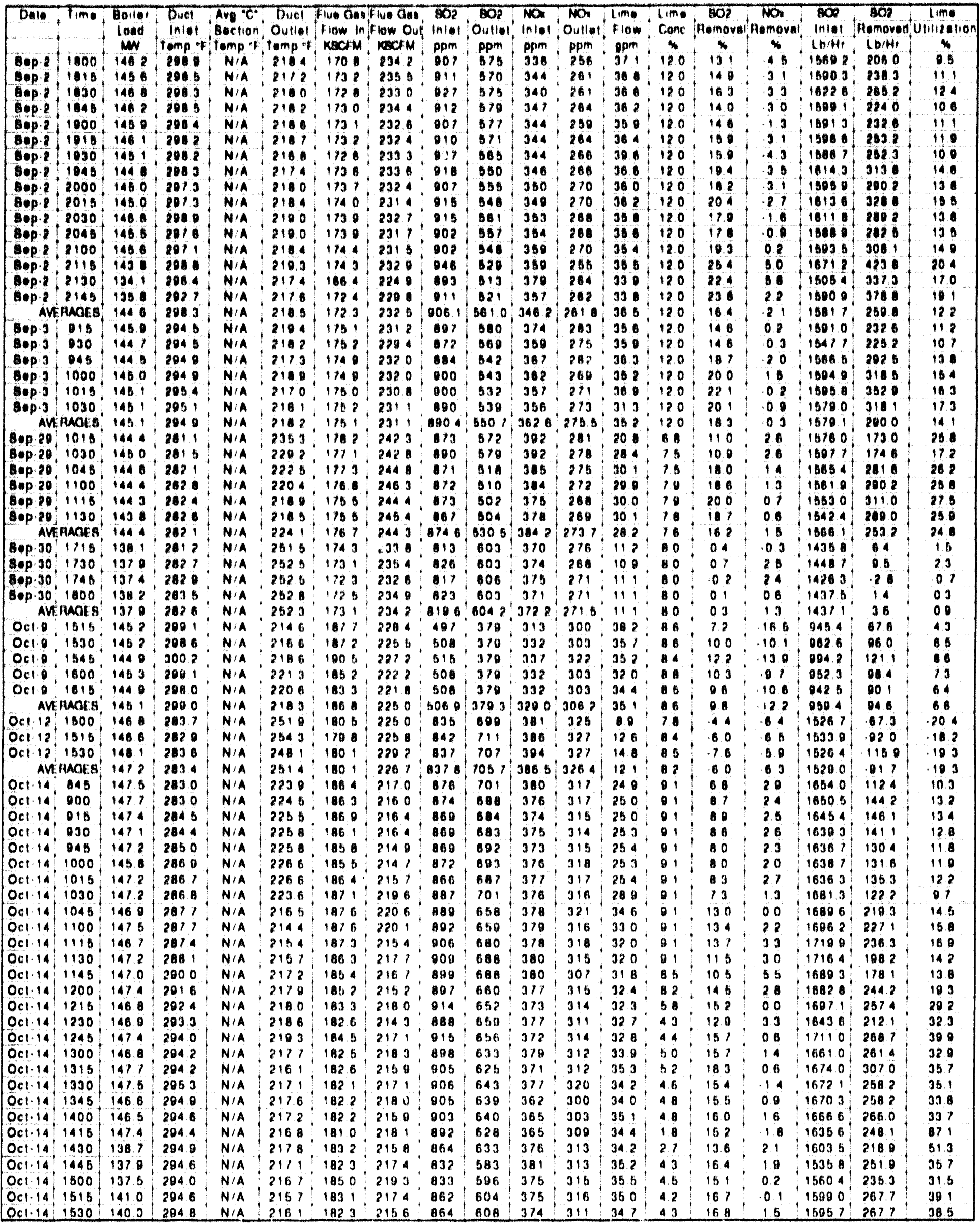


CZD LIME INJECTION DATA. AUGUST 17, TO NOVEMBER 16, 1992

\begin{tabular}{|c|c|c|c|c|c|c|c|c|c|c|c|c|c|c|c|c|c|c|}
\hline Dale & Time & Boiler & Duct & Avg "C" & Duct & Flue Gas & Flue Ge? & $\mathrm{SO2}$ & $\mathrm{SO} 2$ & NOx & NOx & $\operatorname{Lim} \theta$ & $\operatorname{Lim} \theta$ & 502 & NOx & 502 & 502 & $\operatorname{Lim} \theta$ \\
\hline & & Load & Inlet & Section & Outlet & Flow in & Flow Out & $\ln \mid \theta t$ & Outlet & Inlol & Outlet & Flow & Conc & Removal & Removal & $\ln |\theta|$ & Removed & Utilizatio \\
\hline & & MN & Tomp of & Temp of & Temp of & KSCFM & KSCFM & $\mathrm{ppm}$ & $\mathrm{ppm}$ & $\mathrm{ppm}$ & $\mathrm{ppm}$ & $\mathrm{gpm}$ & $\%$ & $\%$ & $\%$ & $\mathrm{Lb} / \mathrm{Hr}$ & Lb/Hr & $\%$ \\
\hline Oct-14 & 1545 & 141.4 & 296.7 & $N / A$ & 217.2 & 1814 & 217.8 & 878 & 621 & 371 & 309 & 35.0 & 40 & 15. & .0 .1 & 1616.3 & 244.4 & 37.5 \\
\hline Oct.14 & 1600 & 141.0 & 297.0 & $N / A$ & 218.0 & 180.9 & 214.4 & 882 & 621 & 373 & 310 & 34.4 & 3.3 & 16.6 & 1.6 & 1616.4 & 267.6 & 50.8 \\
\hline Oct.14 & 1615 & 145.8 & 295.5 & $N / A$ & 218.2 & 179.0 & 213.7 & 886 & 639 & 382 & 307 & 34.1 & 3.5 & 14.0 & 3.8 & 1607.6 & 224.5 & 40.5 \\
\hline Oct.14 & 1630 & 145.8 & 294.9 & $N / A$ & 217.5 & 179.2 & 213.3 & 888 & 642 & 380 & 313 & 33.5 & 2.8 & 13.9 & 1.8 & 1612.7 & 224.6 & 51.8 \\
\hline Oct.14 & 1645 & 146.4 & 294.7 & $N / A$ & 215.6 & 178.7 & 213.8 & 897 & 636 & 376 & 306 & 36.1 & 2.0 & 15.1 & 2.6 & 1623.7 & 245.4 & 73.8 \\
\hline Oct.14 & 1700 & 145.9 & 294.5 & N/A & 218.4 & 180.0 & 212.1 & 890 & 667 & 375 & 315 & 32.8 & 2.0 & 11.7 & 1.0 & 1623.0 & 180.0 & 62.7 \\
\hline Oct-14 & 1715 & 142.6 & 294.6 & $N / A$ & 219.0 & 181.6 & 211.8 & 875 & 646 & 373 & 310 & 32.8 & 1.9 & 14.0 & 2.9 & 1610.7 & 224.8 & 78.4 \\
\hline Oct-14 & 1730 & 143.0 & 294.6 & $N / A$ & 219.2 & 179.9 & 207.3 & 867 & 645 & 370 & 310 & 32.4 & 2.9 & 14.3 & 3.7 & 1581.0 & 226.7 & 52.1 \\
\hline Oct.14 & 1745 & 144.8 & 294.1 & N/A & 219.3 & 178.9 & 2044 & 859 & 657 & 375 & 305 & 31.8 & 3.2 & 12.6 & 7.1 & 1556.8 & 196.8 & 41.6 \\
\hline Oct-14 & 1800 & 144.3 & 293.0 & $N / A$ & 218.1 & 179.2 & 206.8 & 887 & 630 & 369 & 306 & 31.8 & 3.7 & 18.1 & 4.5 & 1610.3 & 290.7 & 53.1 \\
\hline Oct.14 & 1815 & 140.7 & 293.2 & $N / A$ & 218.3 & 180.7 & 203.9 & 852 & 633 & 372 & 312 & 32.7 & 3.8 & 16.2 & 5.4 & 1560.6 & 252.3 & 43.6 \\
\hline $\mathrm{OCl}-14$ & 1830 & 142.4 & 292.4 & $N / A$ & 219.0 & 181.1 & $2 C 2.4$ & 855 & 637 & 378 & 309 & 34.2 & 3.7 & 16.7 & 8.8 & 1568.6 & 261.7 & 44.5 \\
\hline Oct-14 & 1845 & 146.0 & 292.0 & N/A & 217.6 & 180.3 & 198.3 & 883 & 674 & 378 & 306 & 33.3 & 3.3 & 16.0 & 11.0 & 1613.8 & 259.0 & 50.8 \\
\hline Oct.14 & 1900 & 147.3 & 292.3 & N/A & 217.4 & 180.7 & 195.8 & 910 & 680 & 366 & 307 & 32.9 & 4.2 & 19.0 & 8.8 & 1665.7 & 316.1 & 48.1 \\
\hline Oct.14 & 1915 & 147.2 & 292.3 & N/A & 217.4 & 181.5 & 190.1 & 913 & 661 & 367 & 306 & 32.5 & 3.8 & 24.1 & 12.7 & 1678.6 & 404.9 & 68.6 \\
\hline Oct.14 & 1930 & 147.5 & 291.9 & N/A & 216.7 & 1796 & 188.4 & 893 & 671 & 362 & 308 & 33.0 & 3.8 & 21.1 & 10.7 & 1624.8 & 343.4 & 58.9 \\
\hline sct-14 & 1945 & 147.1 & 291.7 & N/A & 214.2 & 180.7 & 185.8 & 883 & 660 & 363 & 306 & 36.4 & 3.7 & 23.1 & 13.1 & 1616.4 & 373.7 & 59.7 \\
\hline Oct.14 & 2000 & 147.8 & 291.4 & N/A & 220.0 & 180.2 & 183.5 & 889 & 036 & 365 & 310 & 280 & 3.7 & 29.4 & 13.6 & 1623.8 & 347.9 & 72.2 \\
\hline Oct-14 & 2015 & 146.7 & 290.6 & $N / A$ & 221.2 & 180.7 & 181.6 & 899 & 702 & 363 & 309 & 29.8 & 4.0 & 21.5 & 14.5 & 1646.5 & 354.5 & 63.8 \\
\hline Oct-14 & 2030 & 147.4 & 290.8 & $N / A$ & 218.8 & 180.2 & 180.4 & $88 \hat{\varepsilon}$ & 667 & 366 & 307 & 31.5 & 4.0 & 24.3 & 16.1 & 1611.1 & 391.4 & 66.7 \\
\hline Oct-14 & 2045 & 137.7 & 289.7 & N/A & 218.8 & 183.5 & 182.9 & 865 & 632 & 376 & 317 & 29.7 & 4.3 & 27.1 & 15.9 & 1608.0 & 436.3 & 73.2 \\
\hline AVE & RAGES & 145.3 & 291.7 & $N / A$ & 218.8 & 132.8 & 209.9 & 883.5 & 654.5 & 373.4 & 311.7 & 32.0 & 5.3 & 15.0 & 4.2 & 1636.3 & 245.3 & 39.6 \\
\hline Oct. 15 & 1545 & 137.1 & 294.9 & N/A & 219.0 & 177.6 & 206.7 & 774 & 663 & 358 & 336 & 30.1 & 3.0 & 0. & .9 .3 & 1392.0 & 4.0 & 0.8 \\
\hline Oct. 15 & 1600 & 134.9 & 294.5 & $N / A$ & 221.8 & 177.8 & 213.1 & 778 & 652 & 353 & 325 & 28.9 & 3.0 & -0.3 & .10 .2 & 1403.5 & .3 .8 & -0.9 \\
\hline Oct. 15 & 1615 & 137.5 & 292.7 & N/A & 223.4 & 175.3 & 208.1 & 809 & 660 & 353 & 32 & 27.5 & 3.0 & 3.0 & .8 .7 & 1436.0 & 43.8 & 11.5 \\
\hline $0 \mathrm{cl} \cdot 15$ & 1630 & 136.6 & 292.7 & $N / A$ & 224.6 & 176.1 & 211.3 & 821 & 668 & 376 & 32 & 27.7 & 3 & 2.3 & .5 .1 & 1464.0 & 34.3 & 8.8 \\
\hline Oct.15 & 1645 & 139.8 & 292.6 & N/A & 224.5 & 175.6 & 206.9 & 822 & 660 & 376 & 321 & 27.7 & 3.0 & 5.4 & .0 .6 & 1461.6 & 78.4 & 20.4 \\
\hline $0 c t-15$ & 1700 & 137.7 & 292.0 & N/A & 225.3 & 175.2 & 207.3 & 837 & 689 & 385 & 328 & 26.8 & 3.0 & 2.6 & -0.7 & 1485.8 & 39.1 & 10.5 \\
\hline Oct-15 & 1715 & 144.1 & $25 ? .0$ & $N / 6$ & 225.4 & 173.8 & 206.2 & 837 & 684 & 387 & 319 & 26.6 & 3. & 2.9 & 2.1 & 14 & 43.4 & 11.7 \\
\hline Oct. 15 & 1730 & 140.5 & 292. & $N / A$ & 223.5 & 175.8 & 209.3 & 828 & 705 & 38 & 337 & 30.0 & 3. & -1.4 & .5 .1 & 1473.9 & -20.5 & .4 .9 \\
\hline Oct-15 & 1745 & 145.6 & 291.7 & N/A & 221.5 & 175.6 & 2079 & 833 & 683 & 385 & 324 & 28.7 & 3.0 & 2.9 & 0.2 & 1481.2 & 42.9 & 10.4 \\
\hline Oct.15 & 1800 & 144.8 & 291.1 & N/A & 220.3 & 176.9 & 209.2 & 839 & 690 & 3 & 324 & 30.0 & 3. & 2.8 & -1.1 & 1503.8 & 41.7 & 10.0 \\
\hline Oct.15 & 1815 & 143.6 & 291.3 & A & 220.4 & 176.9 & 208.1 & 850 & 675 & 388 & 331 & 29.7 & 3. & 6.6 & .0 .5 & 15 & 4 & 24 \\
\hline Oct.15 & 1830 & 145.1 & 280.8 & N/A & 220.2 & 177.7 & 212.7 & $80 i$ & 675 & 389 & 324 & 29.8 & 3.0 & 2.8 & 0.3 & 1496.2 & 41.9 & 0.1 \\
\hline Oct.15 & 1845 & 145.3 & 290.4 & N/A & 219.6 & 177.1 & 209.8 & 857 & 687 & 383 & 32 & 30.2 & 3.0 & 5.0 & 0.5 & 1537.6 & 76.6 & 18.3 \\
\hline Oct.15 & 1800 & 145.3 & 290.0 & N/A & 219.8 & 176.8 & 212.3 & 859 & 675 & 38 & 32 & 29.6 & 3 & 5.7 & -1.8 & 15 & 88 & 4 \\
\hline Oct.15 & 1915 & 145.4 & 289.7 & $N / A$ & 2196 & 178.0 & 211.5 & 862 & 683 & 385 & 320 & 29.5 & 2.7 & 5.8 & 1.1 & 1554.4 & 90.2 & 5 \\
\hline Oct. 15 & 1930 & 145.4 & 288.8 & N/A & 219.6 & 178.3 & 212.1 & 849 & 667 & 383 & 324 & 29.4 & 2.6 & 6.5 & .0 .7 & 1533.6 & 99.5 & 8.2 \\
\hline Oct-15 & 1945 & 144.8 & 287.3 & $N / A$ & 219.3 & 176.4 & 211.8 & 866 & 681 & 380 & 324 & 28.7 & 1.8 & 5.6 & .2 .3 & 1548.5 & 5 & 6.4 \\
\hline Oct.15 & 2000 & 145.3 & 289.0 & N/A & 220.0 & 179.3 & 215.2 & 853 & 670 & 391 & 33 & 29.4 & 1. & 59 & -1.4 & .3 & 80 & 1.6 \\
\hline Oct.15 & 2015 & 144.7 & 289.3 & $N / A$ & 220.9 & 179.4 & 212.5 & 854 & 677 & 380 & 319 & 28.7 & 1.1 & 6.1 & 0.6 & 1553.0 & 94.8 & 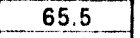 \\
\hline $1 \cdot 15$ & 2030 & 140.8 & 288.7 & N/A & 221.7 & 180.0 & 210.5 & 831 & 686 & 389 & 321 & 28.0 & 1.2 & 4.0 & 3.6 & 1524.7 & 60.9 & 9.6 \\
\hline Oct-15 & 2045 & 139.7 & 287.8 & $N / A$ & 222.1 & 180.0 & 21 & 804 & 64 & 36 & 307 & 27.7 & 0.6 & 4. & 0 & 14 & 9 & 90.8 \\
\hline Oct-15 & 2100 & 139.8 & 287.8 & $N / A$ & 222.6 & 180.3 & 213.7 & 804 & 644 & 367 & 30 & 28.0 & 0. & $\pi$ & 1. & & & 288.8 \\
\hline Oct.15 & 2115 & 140.3 & 287.5 & $N / A$ & 222.3 & 180.0 & 209.4 & 873 & 624 & 367 & 303 & 27.2 & 0.3 & 16.9 & 4.0 & 1592.9 & 269.2 & 5 \\
\hline 1.15 & 2130 & 140.0 & 286.7 & N/A & 223.1 & 180.7 & 213.2 & 839 & 636 & 381 & 30 & 26.8 & 0.2 & 10.5 & 5.7 & 1536.1 & 161.6 & 659.1 \\
\hline 1.15 & 2145 & 139.9 & 286.5 & N/A & 223.2 & 180.7 & 21 & 824 & 64 & 37 & 30 & 26.6 & 1. & 8. & 1.2 & 15 & 130.9 & 71.5 \\
\hline Oct.15 & 2200 & 139.6 & 286.7 & $N / A$ & 224.0 & 181.5 & 214.0 & 823 & 643 & 374 & 305 & 26.6 & 1.7 & 7 & 3.7 & & 8 & 57.2 \\
\hline Oct.15 & 2215 & 140.0 & 286.9 & $N / A$ & 223.3 & 181.7 & 214.8 & 818 & 648 & 371 & 310 & 274 & 1.4 & 6.4 & 1.3 & 1506.7 & 96.8 & 55.0 \\
\hline-15 & 2230 & 140.3 & 286.2 & N/A & 224.0 & 181.0 & 2126 & 814 & 638 & 37 & 31 & 26.6 & 1. & 7.9 & 1.9 & 1.9 & 118.0 & 64.4 \\
\hline Oct.15 & 2245 & 140.2 & 285.6 & N/A & 224.5 & 181.8 & 212.8 & 813 & 64 & 37 & 31 & 25.6 & 9 & 6.6 & 2. & & 98.7 & 5.2 \\
\hline Oct.15 & 2300 & 139.7 & 286.0 & N/A & 221.4 & 182.5 & 213.4 & 813 & 64 & 373 & 310 & 28.6 & 3.1 & 6. & 3.0 & & & 5 \\
\hline Oct. 15 & 2315 & 139.6 & 286.6 & $N / A$ & 219.7 & 182.7 & 212.6 & 813 & 648 & 373 & 310 & 29.4 & 2.4 & 7.3 & 3.4 & 1504.9 & 109.6 & 33.6 \\
\hline Oct-15 & 2330 & 140.1 & 287.1 & N/A & 219.4 & 182.9 & 214.3 & 813 & 638 & 373 & 312 & 29.4 & 2.2 & 8.1 & 2.2 & 1506.6 & .8 & 40.8 \\
\hline Oct. 15 & 2345 & 139.9 & 286.9 & $\mathrm{~N} / \mathrm{A}$ & 219.3 & 182.3 & $2: 1.3$ & 813 & 642 & 319 & 317 & 28.9 & 2.0 & 84 & -15.2 & 1501.7 & 126.6 & 47.6 \\
\hline \multicolumn{2}{|c|}{ AVERAGES } & 141.3 & 289.4 & N/A & 2218 & 178.7 & 211.2 & 829.0 & 662.9 & 374.8 & 318.7 & 28.4 & 2.2 & 54 & 0.6 & 1.0 & 82.6 & 77.9 \\
\hline $3 \mathrm{cl} \cdot 16$ & 0 & 139.9 & 286.2 & N/A & 220.3 & 182.6 & 213.7 & 748 & 645 & 333 & 316 & 28.5 & 1.4 & .0 .9 & -11.1 & 1384.1 & -12.1 & -6.6 \\
\hline $\mathrm{cl}-16$ & 15 & 139.7 & 286.2 & $N / A$ & 219.0 & 182.8 & 213.3 & 766 & 645 & 33 & 31 & 29.8 & 1.6 & 1.7 & -114 & 9 & 24.3 & 11.1 \\
\hline oct-16 & 30 & 139.7 & 285.8 & N/A & 214.6 & 182.8 & 215.3 & 774 & 644 & 34 & 31 & 32.8 & 1.0 & 2. & .8 .0 & & 28.2 & 18.8 \\
\hline $0 \mathrm{c1.16}$ & 45 & 140.2 & 285.9 & $N / A$ & 218.4 & 181.8 & 212.5 & 774 & 63 & 34 & 33 & 27.6 & 0.6 & 4.9 & -12.3 & 5.9 & 60 & \\
\hline & 100 & 139.7 & 287.5 & $N / A$ & 222.5 & 183.1 & 210.5 & 774 & 639 & 352 & 321 & 26.8 & 0.5 & 5.1 & -4.8 & 0.1 & 6 & 118.7 \\
\hline Oct.16 & 115 & 139.6 & 286.3 & $N / A$ & 221.5 & 184.0 & 212.4 & 783 & 653 & 339 & 321 & 28.4 & 0.6 & 3.6 & -9.6 & 14590 & 53.1 & 68.2 \\
\hline Oct. 16 & 130 & 128.0 & 284.9 & $N / A$ & 9 & 177.8 & 205.2 & 760 & 629 & 356 & 321 & 28.4 & 0.6 & 4.4 & -4.1 & 1369.3 & & 78.3 \\
\hline Oct-16 & 145 & 113.8 & 280.5 & N/A & 213.9 & 167.0 & 194.0 & 764 & 605 & 363 & 310 & 26.4 & 2.5 & 80 & 0.9 & 1292.9 & 102.9 & \\
\hline 1.16 & 200 & 105.6 & 281.5 & N/A & 212.8 & 162.1 & 188.7 & 738 & 583 & 350 & 299 & 26.5 & 2.3 & 8.1 & 0.4 & 2.4 & 98.0 & 34.9 \\
\hline 16 & 215 & 4.6 & 33.3 & IA & 213.0 & 162.4 & 189.0 & 734 & 577 & 361 & 306 & 26.5 & 20 & 8.6 & 1.4 & 1208.1 & 104.1 & 42.7 \\
\hline Oct. 16 & 230 & 105.0 & 282.5 & IA & 212.6 & 158.0 & 182.7 & 746 & 583 & 364 & 311 & $25 . C$ & 1.5 & 9.6 & 1.1 & 1193.6 & 114.6 & 66.6 \\
\hline Oct 16 & 245 & 104.7 & 283.5 & N/A & 212.3 & 159.4 & 187.2 & 746 & 577 & 362 & 300 & $26 . t$ & 1.2 & 9.2 & 24 & & 110.6 & 75 \\
\hline 16 & 300 & 104.8 & 281.2 & N/A & 211.1 & 158.9 & 184.8 & 750 & 572 & 364 & 303 & 25.7 & 1.1 & 11 & 3.3 & 1207.6 & 136.6 & 105.5 \\
\hline cat.16 & 315 & 104.7 & 281.9 & $0-$ & 211.5 & 161.3 & 186.6 & 749 & 571 & 364 & 303 & 25.6 & 0.6 & $11 . y$ & 3.6 & 1224.2 & 145.6 & 207.6 \\
\hline Oct-16 & 330 & 105.1 & 282.4 & N/A & & 160.7 & 184.7 & 738 & 578 & 360 & 306 & 25.2 & 0.3 & 10.0 & 2.3 & 1201.1 & 120.4 & 349.3 \\
\hline Oct-16 & 345 & 105.2 & 281.1 & $N / A$ & 212.3 & 159.9 & 183.7 & 734 & 583 & 363 & 30 & 25.0 & a & 8.7 & 3.0 & 8.7 & 103.7 & 303.4 \\
\hline Oct.16 & 400 & 104.7 & 281.5 & N/A & 212.9 & 159.7 & 184.3 & 738 & 584 & 366 & 308 & 25.1 & 0.3 & 8.7 & 2 & 11936 & 103.3 & 30 \\
\hline Dct-16 & 415 & 105.2 & 281.5 & N/A & 213.0 & 159.6 & 184.6 & 738 & 579 & 362 & 303 & 25.0 & 0.2 & 9.3 & 3.4 & 1193.7 & 110.8 & 486.2 \\
\hline
\end{tabular}


CZD LIME INJECTION DATA - AUGUST 17, TO NOVEMBER 16, 1892

\begin{tabular}{|c|c|c|c|c|c|c|c|c|c|c|c|c|c|c|c|c|c|c|}
\hline Dale & $\operatorname{Tim} \theta$ & Boiler & Duct & Avg "C" & Ducl & Flue Gas & Flue Gas & SO2 & $\mathrm{SO} 2$ & Nax & NOx & $\operatorname{Lim} \theta$ & $\operatorname{Lim} \theta$ & SO2 & NOx & 502 & 502 & $\operatorname{Lim} \theta$ \\
\hline & & Load & $\ln |\theta|$ & Soction & Outlol & Flow in & Flow Out & $\operatorname{ln|\theta |}$ & Oullet & Inlet & Outlat & Flow & Conc & Removalif & emoval & $\ln \mid \theta t$ & Pemoved & tilization \\
\hline & & MN & Temp of & Temp of & Tomp of & KSCFM & KSCFM & $\mathrm{ppm}$ & $\mathrm{ppm}$ & $\mathrm{ppm}$ & $\mathrm{ppm}$ & $\mathrm{gpm}$ & $\%$ & $\%$ & $\%$ & $\mathrm{Lb} / \mathrm{HI}$ & LbIHI & $\%$ \\
\hline $0 \mathrm{cl.16}$ & 430 & 105.2 & 281.6 & $N / A$ & 211.3 & 161.1 & 188.7 & 737 & 571 & 365 & 300 & 25.6 & 1.7 & 9.3 & 4.0 & 1202.5 & 11117 & 559 \\
\hline $0 \mathrm{ct} \cdot 16$ & 445 & 104.8 & 280.0 & N/A & 211.3 & 162.2 & 185.8 & 742 & 576 & 373 & 304 & 25.6 & 2.0 & 11.1 & 6.6 & 1218.8 & 135.5 & 57.5 \\
\hline $0 \mathrm{ct.16}$ & 500 & 105.1 & 278.6 & $N / A$ & 208.3 & 161.9 & 187.5 & 746 & 556 & 373 & 303 & 29.2 & 1.6 & 13.7 & 5.8 & 1223.9 & 167.7 & 78.2 \\
\hline $0 c t \cdot 16$ & 515 & 105.0 & 278.7 & $N / A$ & 210.4 & 160.8 & 183.6 & 742 & $5 \div 0$ & 373 & 303 & 25.0 & 2.4 & 12.4 & 7.2 & 1208.2 & 148.8 & 54.1 \\
\hline Oct.16 & 530 & 111.6 & 279.0 & $N / A$ & 212.2 & 167.2 & 190.6 & 751 & 575 & 371 & 306 & 252 & 2.1 & 12.7 & 5.9 & 1271.6 & 162.1 & 66.5 \\
\hline $0<1.16$ & 545 & 121.7 & 278.3 & $N / A$ & 214.0 & 172.2 & 195.5 & 769 & 609 & 373 & 310 & 25.1 & 2.2 & 10.0 & 5.7 & 1341.0 & 133.9 & 52.6 \\
\hline Oct.16 & 600 & 129.1 & 281.2 & $N / A$ & 217.8 & 178.1 & 204.4 & 777 & 620 & 372 & 309 & 25.2 & 2.7 & 8.4 & 4.7 & 1402.3 & 117.4 & 37.3 \\
\hline $0 c t-16$ & 615 & 124.7 & 282.0 & N/A & 216.8 & 171.9 & 196.8 & 795 & 623 & $369^{-}$ & 310 & 25.3 & 2.0 & 10.3 & 3.9 & 1384.6 & 142.6 & 61.2 \\
\hline Oat.16 & 630 & 125.3 & 282.3 & $N / A$ & 216.1 & 174.1 & 198.0 & 788 & 608 & 372 & 300 & 25.6 & 2.1 & 12.2 & 5.8 & 1389.5 & 170.0 & 68.6 \\
\hline 0 ct.16 & 645 & 124.9 & 283.4 & $N / A$ & 217.8 & 173.1 & 199.5 & 785 & 622 & 377 & 312 & 25.5 & 1.4 & 8.6 & 4.5 & 1376.1 & 118.0 & 72.0 \\
\hline $0 \mathrm{ct} \cdot 16$ & 700 & 127.3 & 282.8 & $N / A$ & 213.2 & 172.7 & 188.1 & 773 & 616 & 375 & 311 & 28.6 & 1.8 & 8.5 & 4.9 & 1352.8 & 115.6 & 47.2 \\
\hline $0 c 1 \cdot 16$ & 715 & 130.1 & 288.4 & $N / A$ & 218.9 & 182.8 & 209.9 & 792 & 634 & 377 & 313 & 27.9 & 1.3 & 8.1 & 4.5 & 1467.1 & 118.2 & 71.7 \\
\hline $0 c t-16$ & 730 & 130.0 & 289.5 & $N / A$ & 220.3 & 180.5 & 212.2 & 800 & 631 & 377 & 313 & 280 & 1.0 & 7.2 & 2.2 & 1462.1 & 105.8 & 82.6 \\
\hline Oct.16 & 1500 & 145.5 & 290.7 & $N / A$ & 213.7 & 174.3 & 209.2 & 878 & 707 & 379 & 317 & 33.0 & 0.8 & 3.4 & .0 .3 & 1551.2 & 53.3 & 44.2 \\
\hline Oct.16 & 1515 & 145.6 & 290.9 & $N / A$ & 210.4 & 176.5 & 212.1 & 857 & 696 & 379 & 314 & 35.0 & 0.6 & 2.4 & 0.3 & 1532.4 & 373 & 29.2 \\
\hline Oct.16 & 1530 & 146.0 & 291.2 & N/A & 209.8 & 176.7 & 211.0 & 860 & 680 & 379 & 315 & 35.2 & 0.11 & 5.5 & 0.6 & 1539.5 & 85.1 & 66.1 \\
\hline Oct.16 & 1545 & 144.1 & 292.3 & $N / A$ & 209.9 & 178.6 & 210.7 & 850 & 668 & 376 & 307 & 35.4 & 0.8 & 7.3 & 3.8 & 1538.9 & 112.2 & 86.6 \\
\hline $0 \mathrm{ct} \cdot 16$ & 1600 & 145.1 & 291.5 & N/A & 210.4 & 178.1 & 209.9 & 844 & 667 & 371 & 319 & 34.4 & 0.8 & 6.8 & -1.3 & 1522.1 & 103.0 & 8 \\
\hline Oct.16 & 1615 & 146.5 & 291.2 & N/A & 209.9 & 175.5 & 206.8 & 850 & 683 & 395 & 319 & 34.3 & 1.3 & 5.3 & 4.9 & 1512.1 & 80.4 & 39.3 \\
\hline Oct.16 & 1630 & 145.9 & 290.5 & N/A & 210.4 & 175.9 & 208.8 & 867 & 695 & 392 & 331 & 33.8 & 1.8 & 4.8 & 0.2 & 1545.0 & 75.5 & 5.5 \\
\hline Oct.16 & 1645 & 145.6 & 280.8 & N/A & 210.8 & 176.1 & 21119 & 860 & 688 & 388 & 320 & 34.1 & 2.2 & 3.7 & 0.9 & 1534.3 & 56.5 & 16.3 \\
\hline $0 \mathrm{ct.16}$ & 1700 & 145.7 & 290.2 & N/A & 211.1 & 175.3 & 208.4 & 865 & 690 & 386 & 315 & 33.3 & 2.4 & 5.2 & 3.1 & 1537.1 & 80.6 & 21.8 \\
\hline Oct.16 & 1715 & 145.5 & 2904 & N/A & 211.6 & 175.2 & 207.5 & 870 & 700 & 385 & 316 & 33.2 & 2.1 & 4.7 & 2.8 & 1545.1 & 73.0 & 227 \\
\hline Oct.16 & 1730 & 145.7 & 290.1 & $N / A$ & 212.9 & 175.2 & 205.3 & 868 & 689 & 382 & 320 & 31.7 & 2.7 & 70 & 2.0 & 1541.5 & 107.7 & 27.2 \\
\hline Oct.16 & 1745 & 145.5 & 289.9 & $N / A$ & 214.2 & 174.6 & 208.2 & 865 & 685 & 379 & 313 & 31.2 & 2.3 & 5.5 & 1.5 & 1530.0 & 84.4 & 5.5 \\
\hline Dct.16 & 1800 & 145.7 & 289.7 & $N / A$ & 214.4 & 173.5 & 207.2 & 855 & 678 & 377 & 311 & 31.5 & 2.2 & 5.3 & 1.4 & 1502.8 & 78.8 & 5.0 \\
\hline Oct.16 & 1815 & 145.7 & 290.0 & N/A & 213.7 & 173.5 & 210.4 & 873 & 674 & 373 & 311 & 33.0 & 2.4 & 6.3 & .0 .8 & 1534.5 & 97.0 & 6.5 \\
\hline Oct.16 & 1830 & 145.5 & 288.5 & $N / A$ & 214.2 & 174.6 & 208.8 & 865 & 677 & 375 & 308 & 30.8 & 2.8 & 6.4 & 2.0 & 15300 & 88.2 & 8 \\
\hline $0 \mathrm{ct.16}$ & 1845 & 145.3 & 287.5 & N/A & 214.6 & 175.7 & 210.3 & 855 & 676 & 381 & 310 & 31.1 & 2.6 & 5.4 & 2.5 & 1522.8 & 82.8 & 2 \\
\hline Oct.16 & 1900 & 145.4 & 287.1 & N/A & 215.2 & 177.0 & 212.9 & 856 & 671 & 375 & 310 & 30.5 & 2.5 & 5.7 & 0.7 & 1534.9 & 88.0 & 5.0 \\
\hline Oct.16 & 1915 & 145.3 & 286.1 & N/A & 215.7 & 177.3 & 211.1 & 855 & 677 & 378 & 314 & 30.1 & 2.8 & 5.7 & 1.5 & 1536.6 & 88.0 & 2.6 \\
\hline Oct. 16 & 1930 & 145.7 & 285.1 & N/A & 215.9 & 177.7 & 209.5 & 866 & 666 & 380 & 31 & 29.6 & 2.6 & 9.3 & 2.9 & 1559.0 & 145.8 & 1.0 \\
\hline $0 \mathrm{ct} \cdot 16$ & 1945 & 145.7 & 284.3 & N/A & 216.3 & 176.7 & 213.1 & 861 & 666 & 381 & 316 & 28.8 & 28 & 6.7 & 0.0 & 15413 & $10 \therefore .7$ & 27.5 \\
\hline Oct.16 & 2000 & 145.5 & 284.6 & $N / A$ & 217.6 & 179.2 & 210.3 & 858 & 675 & 384 & 318 & 28.2 & 2.5 & 7.6 & 2.7 & 1557.6 & 118.7 & 36.5 \\
\hline Oct.16 & 2015 & 1450 & 284.6 & N/A & 218.6 & 178.8 & 209.4 & 861 & 679 & 385 & 32 & 27.9 & 2.7 & 7.7 & 2.8 & 1560.5 & 120.3 & 4.6 \\
\hline Oct.16 & 2030 & 145.9 & 284.0 & $N / A$ & 219.2 & 177.6 & 209.4 & 844 & 666 & 377 & 314 & 27.2 & 3.0 & 6.8 & 1.8 & 1517.8 & 10 & 7.6 \\
\hline Oct.16 & 2045 & 145.5 & 283.1 & $N / A$ & 219.4 & 178.0 & 210.7 & 867 & 674 & 384 & 319 & 26.8 & 2.5 & 8.0 & 1.7 & 1564.3 & 124.8 & 0.4 \\
\hline Oct-16 & 2100 & 145.9 & 283.4 & $N / A$ & 218.6 & 176.6 & 2085 & 867 & 673 & 375 & 31 & 27.7 & 2.6 & 8.4 & .0 .4 & 1552.0 & 130.7 & 39.3 \\
\hline \multirow{2}{*}{\multicolumn{2}{|c|}{ VERAGES }} & 144.7 & 283.6 & $N / A$ & 219.0 & 178.8 & 212.0 & 867 & 655 & 375 & 3 & 27.5 & 2.6 & 10.5 & -0.2 & 1571.4 & 164.9 & 9.9 \\
\hline & & 130.9 & 285.3 & $N / A$ & 214.5 & 172.8 & 2024 & 805.9 & 637.6 & 370.6 & 312.4 & 288 & 1.7 & 7.3 & 1.2 & 1413.4 & 101.6 & 72.3 \\
\hline Oct.17 & 100 & 1052 & 287.1 & N/A & 208.7 & 157.4 & 182.7 & 769 & 638 & 364 & 315 & 31.4 & 4.4 & 3.7 & .0 .4 & 1226.5 & 45.0 & 7.0 \\
\hline Oct.17 & 115 & 1050 & 287.9 & $N / A$ & 201.1 & 158.5 & 185.1 & 775 & 5 & 3 & & 34.8 & 4.7 & 11.0 & 2.2 & 1244.7 & 137.5 & 18.0 \\
\hline Oct.17 & 130 & 105.0 & 286.9 & $N / A$ & 198.2 & 156.2 & 183.1 & 777 & 58 & 373 & 31 & 34.6 & 4.6 & 11.7 & 2.3 & 1229.1 & 14 & 19.4 \\
\hline $0 \mathrm{ct} \cdot 17$ & 145 & 105.1 & 287.3 & NIA & 1984 & 157.0 & 183.6 & 778 & 57 & 378 & 311 & 33.9 & 4.2 & 13.4 & 3.6 & 1237.7 & 165.3 & 24.8 \\
\hline 1.17 & 200 & 104.9 & 289.3 & N/A & 197.7 & 156.9 & 186.6 & 770 & 56 & 37 & 304 & 35.1 & 4.4 & 13.5 & 3.5 & 1224.2 & 165.5 & 23. \\
\hline Oct.17 & 215 & 105.2 & 288.9 & $N / A$ & 196.8 & 156.0 & 187.2 & 782 & 576 & 377 & 308 & 36.5 & 4.0 & 196 & 1.8 & 1235.4 & 142.9 & 21.0 \\
\hline Oct-17 & 230 & 105.0 & 288.6 & $N / A$ & 196.6 & 158.2 & 184.8 & 779 & 557 & 376 & 310 & 34.9 & 4.1 & 16.5 & 3.7 & 1248.8 & 20 & 9 \\
\hline oct.17 & 245 & 105.0 & 288.4 & $N / A$ & 197.9 & 158.0 & 186.5 & 780 & 557 & 375 & 310 & 35.4 & 3.7 & 15.7 & 2.6 & 1248.0 & 195.5 & 32.1 \\
\hline Oct. 17 & 300 & 105.0 & 287.3 & $N / A$ & 197.7 & 156.6 & 187.7 & 783 & 54 & 37 & 307 & 34.9 & 4.2 & 16.4 & 2.7 & 1242.5 & 203.2 & 29.7 \\
\hline Oct-17 & 315 & 105.0 & 289.3 & $N / A$ & 198.9 & 159.2 & 186.1 & 769 & 55 & 373 & 306 & 34.1 & 4.0 & 15.3 & 4.1 & 12 & 189.4 & 29.8 \\
\hline Oct 17 & 330 & 104.9 & 288.4 & $N / A$ & 199.1 & 159.2 & 183.1 & 771 & 555 & 376 & 312 & 34.0 & 4.2 & 17.2 & 4.4 & 12 & 214.3 & 2 \\
\hline-17 & 345 & 105.0 & 288.0 & $N / A$ & 1990 & 157.3 & 183.8 & 778 & 555 & 377 & 310 & 33.5 & 4.2 & 16.7 & 3.9 & 1239.3 & 206.7 & 31.5 \\
\hline $0 \mathrm{Cl} \cdot 17$ & 400 & 104.8 & 289.2 & N/A & 200.3 & 159.9 & 184.3 & 768 & 553 & 375 & 310 & 33.6 & 4.1 & 17.0 & 4.9 & 1243.6 & 21 & 33.0 \\
\hline $0 \mathrm{Cl} \cdot 17$ & 415 & 105.2 & 287.4 & N/A & 198.9 & 159.2 & 184.1 & 778 & 542 & 377 & 308 & 36.8 & 4.0 & 19.4 & 5.6 & 1254.3 & 243.3 & 35.5 \\
\hline Oct.17 & 430 & 104.8 & 287.1 & $N / A$ & 197.9 & 159.6 & 182.7 & 781 & 536 & 376 & 308 & 33.5 & 3.9 & 21.4 & 6.2 & 1263.1 & 270.9 & $a$ \\
\hline Oct.17 & 445 & 104.6 & 286.4 & $N / A$ & 199.7 & 158.7 & 183.2 & 785 & 546 & 375 & 306 & 32.7 & 4.0 & 19.7 & 5.7 & 1.7 & 24 & 40.8 \\
\hline $0 \mathrm{cl.17}$ & 500 & 104.8 & 285.8 & $N / A$ & 199.4 & 159.4 & 182.1 & 790 & 555 & 376 & 307 & 32.8 & 4.1 & 19.8 & 6.8 & 1276.0 & 253.0 & 40.4 \\
\hline $2 c t \cdot 17$ & 515 & 104.9 & 287.8 & IA & 200.8 & 158.9 & 181.5 & 791 & 547 & 377 & 306 & 32.4 & 4.4 & 21.1 & 7.3 & $\mid 1273.6$ & & $A 0$ \\
\hline $2 \mathrm{ct} \cdot 17$ & 530 & 104.6 & 288.3 & $N / A$ & 2010 & 158.4 & 181.0 & 807 & 546 & 379 & 303 & 32.9 & 3.7 & 22.7 & 8.9 & 1295.2 & 293.9 & 51.9 \\
\hline act-17 & 545 & 104.8 & 287.9 & N/A & 201.8 & 158.2 & 181.8 & 806 & 558 & 376 & 307 & 32.4 & 40 & 20.5 & 6.1 & 1291.9 & 265.0 & 43.9 \\
\hline Jt-17 & 600 & 104.9 & 287.1 & N/A & 202.3 & 157.0 & 182.5 & 799 & 549 & 374 & 305 & 32.7 & 4.0 & 20.1 & 5.2 & 1270.2 & 255.0 & 41.9 \\
\hline $3 \mathrm{ct}-17$ & 615 & 105.4 & 285.7 & $N / A$ & 199.6 & 159.5 & 181.3 & 792 & $530^{-}$ & 372 & 302 & 33.5 & 4.4 & 24.0 & 7.7 & & 306.5 & 44.6 \\
\hline $3 \mathrm{ct}-17$ & 630 & 114.1 & 283.7 & $N / A$ & 201,6 & 162.9 & 185.1 & 800 & 549 & 376 & 305 & 32.1 & 4.3 & 22.1 & 7.6 & 1320.4 & 291.7 & 45.3 \\
\hline $3 \mathrm{ct} \cdot 17$ & 645 & 4.0 & 281.7 & $N / A$ & 204. & 1734 & 1912 & 801 & 583 & 376 & 311 & $3: 7$ & 4.3 & 158 & 8.7 & $140^{\circ}$ & 278.0 & 43.7 \\
\hline$x t-17$ & 700 & 3.7 & 284.1 & $\mathrm{~N} / \mathrm{A}$ & 206.8 & 179.3 & 199.6 & 831 & 603 & 381 & 312 & 32.3 & 4.1 & 19.1 & 8.8 & 1508.7 & 288.5 & 46.7 \\
\hline$x+17$ & 715 & 135.9 & 285.5 & $N / A$ & 209.4 & 181.7 & 203.0 & 819 & 610 & 376 & 314 & 31.5 & 4.2 & 16.8 & 6.7 & 1506.8 & 252.4 & 40.9 \\
\hline$x t-17$ & 730 & 134.0 & 285.0 & $N / A$ & 210.9 & 179.4 & 200.3 & 8.12 & 616 & 381 & 315 & 30.7 & 40 & 18.3 & 7.8 & 1529.7 & 279.8 & 48.9 \\
\hline $\mathrm{ct}-17$ & 745 & 138.1 & 283.5 & N/A & 2087 & 180.4 & 204.0 & 834 & 615 & 380 & 317 & 34.0 & 4.1 & 16.7 & 5.7 & 1524.4 & 254.3 & 39.1 \\
\hline st. 17 & 800 & 141.2 & 284.4 & $N / A$ & 0.7 & 182.5 & 202.1 & 862 & 631 & 20 & 321 & 32.8 & 4.0 & 18.9 & 7.6 & 1583.0 & 301.3 & 49.3 \\
\hline $3 \mathrm{ct} \cdot 17$ & 815 & 137.2 & 284.6 & N/A & 210.2 & 1815 & 203.1 & 845 & 621 & 382 & 318 & 32.9 & 38 & 17.8 & 7.0 & $15 \leqslant 3.2$ & 276.3 & 47.5 \\
\hline Ct- 17 & 830 & 143.1 & 285.0 & N/A & 211.0 & 182.9 & 2024 & 861 & 620 & 386 & 317 & 34.4 & 4.3 & 20.3 & 9.1 & 15363 & 324.0 & 47.0 \\
\hline ct. 17 & 845 & 145.3 & 285.9 & N/A & 213.1 & 186.6 & 204.9 & 858 & 642 & 381 & 322 & 32.1 & 4.1 & 17.9 & 7.4 & 1622.9 & 290.5 & 47.4 \\
\hline ct.1? & 900 & $1 \overline{45.5}$ & 285.6 & N/A & 213 & 185.3 & 202.9 & 861 & 650 & 387 & 324 & 31.5 & 3.8 & 17.3 & 8.2 & 1617.2 & 280.4 & 50.4 \\
\hline $\mathrm{ct}-17$ & 915 & 139.9 & 286.1 & $N / 4$ & 213.6 & 180.9 & 202.9 & 853 & 634 & 386 & 319 & 30.2 & 3.8 & 16.7 & 7.2 & 1564.2 & 261.2 & 48.9 \\
\hline
\end{tabular}


CZD LIME INJECTION DATA - AUGUST 17, TO NOVEMBER 16, 1992

\begin{tabular}{|c|c|c|c|c|c|c|c|c|c|c|c|c|c|c|c|c|c|c|}
\hline Dale & Time & Boiler & Dual & Avg "C" & Dual & Flue Gas & Flue Gas & 502 & SC? & NOX & NOx & $\lim \theta$ & Lime & 502 & NOx & $\mathrm{SO} 2$ & 502 & $\operatorname{Lime}$ \\
\hline & & Load & Inlel & Section & Outlet & Flow in & Flow Ou & Inlo| & Outlel & Inle| & Outlet & Flow & Conc & Pemoval & Gemov & $\ln |\theta|$ & Removed & tilization \\
\hline & & $M N$ & Temp of & Temp of & Temp ${ }^{\prime} F$ & KSCFM & KSCFM & $\mathrm{ppm}$ & $\mathrm{ppm}$ & $\mathrm{ppm}$ & $\mathrm{ppm}$ & $\mathrm{gpm}$ & $\%$ & $\%$ & $\%$ & $L b / H e$ & Lb/HI & $\%$ \\
\hline $0,1.17$ & 830 & 139.2 & 286.1 & $N / A$ & 2142 & 179.7 & 2001 & 860 & 625 & 392 & 323 & 31.1 & 4.2 & 190 & 8.2 & 1565.6 & 297.7 & 48.8 \\
\hline $0,1.17$ & 945 & 140.8 & 286.6 & N/A & 213.6 & 183.2 & 204.1 & 839 & 620 & 388 & 325 & 32.4 & 4.2 & 17.6 & 6.7 & 1557.3 & 274.4 & 43.2 \\
\hline Oct.17 & 1000 & 139.6 & 285.3 & $N / A$ & 212.2 & 182.5 & 189.8 & 858 & 615 & 381 & 323 & 33.1 & 3.9 & 21.5 & 9.5 & 1586.3 & 341.5 & 56.8 \\
\hline Oct.17 & 1015 & 138.7 & 285.5 & $N / A$ & 2138 & 180.7 & 2012 & 845 & 623 & 389 & 324 & 30.2 & 4.2 & 17.8 & 7.2 & 1547.8 & 277.0 & 46.8 \\
\hline Oct.17 & 1030 & 139.3 & 285.2 & $N / A$ & 213.8 & 181.1 & 200.7 & 864 & 632 & 398 & 328 & 32.0 & 3.8 & 19.0 & 8.6 & 1586.1 & 301.3 & 53.3 \\
\hline Oct.17 & 1045 & 135.0 & 284.3 & $N / A$ & 214.8 & 176.4 & 1857 & 837 & 635 & 398 & 332 & 28.5 & 4.2 & 15.8 & 7.6 & 14959 & 237.3 & 42.5 \\
\hline $0 \mathrm{ct.17}$ & 1100 & 133.2 & 284.2 & N/A & 2180 & 174.4 & 181.6 & 847 & 636 & 398 & 319 & 25.2 & 4.3 & 17.5 & 12.0 & 1496.3 & 262.0 & 51.8 \\
\hline \multicolumn{2}{|c|}{ AVERAGES } & 119.7 & 286.4 & N/A & 205.0 & 168.1 & 1912 & 810.3 & 587.1 & 380.0 & 313.3 & 32.8 & 4.1 & 17.5 & 6.1 & 1384.1 & 243.8 & 39.4 \\
\hline Oct-18 & 1545 & 141.5 & 279.2 & $N / A$ & 206.8 & 176.3 & 212.2 & 836 & 641 & 382 & 310 & 31.9 & 3.6 & 77 & 2.2 & 1492.4 & 114.7 & 21.5 \\
\hline Oct.18 & 1600 & 141.5 & 281.2 & N/A & 205.8 & 181.8 & 219.0 & 832 & 614 & 389 & 321 & 33.8 & 3.8 & 11.0 & 0.6 & 1532.5 & 168.2 & 27.6 \\
\hline 001.18 & 1615 & 145.1 & 282.1 & $N / A$ & 205.7 & 183.6 & 217.6 & 838 & 628 & 398 & 324 & 34.1 & 4.2 & 11.2 & 3.6 & 1558.8 & 174.5 & 26.1 \\
\hline Oct.18 & 1630 & 146.3 & 281.7 & N/A & 205.8 & 182.8 & 220.3 & 834 & 617 & 394 & 331 & 34.1 & 3.9 & 10.8 & -1.1 & 1545.5 & 167.4 & 27.1 \\
\hline Oct.18 & 1645 & 140.4 & 281.2 & N/A & 205.3 & 181.7 & 220.9 & 828 & 597 & 400 & 332 & 35.1 & 3.8 & 12.2 & -0.8 & 1523.4 & 186.3 & 30.0 \\
\hline $0 c t-18$ & 1700 & 139.6 & 279.6 & $N / A$ & 203.8 & 177.5 & 215.1 & 816 & 599 & 388 & 318 & 32.8 & 4.2 & 11.1 & 0.6 & 1467.5 & 162.3 & 25.2 \\
\hline Oct.18 & 1715 & 134.7 & 277.7 & N/A & 203.3 & 173.6 & 210.5 & 805 & 590 & 383 & 315 & 31.6 & 4. & 11.1 & 0.2 & 1415.9 & 156.7 & 26.0 \\
\hline Oct.18 & 1730 & 136.8 & 277.0 & N/A & 203.9 & 174.5 & 213.1 & 813 & 600 & 384 & 311 & 31.2 & 4. & 9.8 & 1.0 & 1437.4 & 141.1 & 21.0 \\
\hline Oct.18 & 1745 & 138.7 & 277.7 & $N / A$ & 204.2 & 177.8 & 217.7 & 804 & 577 & 382 & 310 & 317 & 4. & 12.2 & 0.5 & 1447.5 & 175.9 & 27.7 \\
\hline $0 \mathrm{cl} \cdot 18$ & 1800 & 140.8 & 279.7 & N/A & 206.4 & 182.5 & 221. & 818 & 582 & 380 & 323 & 31.8 & 4 & 13.7 & -2.8 & 1512.5 & 207.9 & 34.2 \\
\hline $0 \mathrm{cl}-18$ & 1815 & 139.6 & 281.6 & N/A & 207.3 & 184.7 & 221.0 & 805 & 589 & 387 & 318 & 32.9 & 4. & 10.8 & 1.6 & 1505.5 & 163.4 & 26.0 \\
\hline $0 c t-18$ & 1830 & 140.0 & 2813 & $N / A$ & 207.8 & 1810 & 2995 & 798 & 579 & 380 & 316 & 32.0 & 38 & 12.0 & .0 .8 & 1462.5 & 176.0 & 0.3 \\
\hline Oct.18 & 1845 & 145.1 & 279.6 & $\mathrm{~N} / \mathrm{A}$ & 207.7 & 180.8 & 2199 & 805 & 588 & 388 & 324 & 31.7 & 4.2 & 11.1 & -1.6 & 1474.6 & 163.7 & 26.4 \\
\hline Oct.18 & 1900 & 145.5 & 281.0 & $N / A$ & 208.5 & 182.0 & 2196 & 815 & 598 & 405 & 332 & 31.9 & 4.4 & 11.5 & 1.3 & 1502.9 & 172.6 & 26.3 \\
\hline $\mathrm{Oct} \cdot 18$ & 1915 & 145.5 & 280.5 & $N / A$ & 208.8 & 1830 & 220.3 & 816 & 592 & 389 & 320 & 32.0 & 4.8 & 12.7 & 0.8 & 1513.0 & 191.8 & 2.8 \\
\hline Oct.18 & 1930 & 145.8 & 279.9 & N/A & 209.4 & 182.5 & 219.3 & 805 & 585 & 388 & 322 & 30.7 & 4. & 12.6 & 0.4 & 1487.6 & 188.0 & 27.9 \\
\hline Oct.18 & 1945 & 145.4 & 279.2 & $N / A$ & 210.5 & 182.1 & 218.8 & 819 & 592 & 385 & 315 & 29.9 & 4. & 13.1 & 1.6 & 1511.1 & 197.8 & 33.0 \\
\hline $0 c t \cdot 18$ & 2000 & 145.4 & 279.0 & N/A & 210.4 & 181.4 & 216.6 & 816 & 592 & 383 & 316 & 30.3 & 4 & 13.3 & 1.5 & 1488.8 & 189.8 & 2.1 \\
\hline $0 \mathrm{ct} \cdot 18$ & 2015 & 140.6 & 278.7 & $N / A$ & 2090 & 181.0 & 220.9 & 831 & 597 & 382 & 313 & 32.8 & 4.3 & 12.4 & 0.1 & 1525.6 & 189.6 & 28.8 \\
\hline Oct.18 & 2030 & 137.8 & 276.9 & $N / A$ & 208.3 & 172.3 & 2036 & 81 & 540 & 379 & $30 \mathrm{~J}$ & 28.4 & 4.6 & 21.9 & 4.1 & 1425.4 & 312.5 & 51.2 \\
\hline Oct.18 & 2115 & 140.1 & 275.6 & N/A & 207.6 & 174.8 & 213.7 & 824 & 657 & 418 & 300 & 28.0 & 4.8 & 2.6 & 12.4 & 1459.4 & 37.7 & 5.9 \\
\hline Oct-18 & 2130 & 134.9 & 274.6 & N/A & 205.6 & 172.8 & 2100 & 853 & 588 & 395 & 308 & 28.4 & 46 & 16.3 & 5.3 & 1494.1 & 243.3 & 38.5 \\
\hline Oct.18 & 2145 & 1344 & 274.8 & N/A & 2049 & 173.1 & 210.5 & 845 & 571 & 391 & 309 & 304 & 4.6 & 17.9 & 3. & 14818 & 265.1 & 40.6 \\
\hline Oct-18 & 2200 & 1346 & 273.9 & $N / A$ & 2039 & 172.8 & 209.5 & 846 & 582 & 386 & 308 & 28.7 & 4.1 & 16.5 & 3 & 1480.8 & & \\
\hline 001.18 & 2215 & 1350 & 274.8 & $N / A$ & 205.2 & 175.4 & 208.9 & 838 & 604 & 390 & 312 & 29.2 & 4.5 & 140 & 4.7 & 1488.4 & 209.1 & 34.1 \\
\hline Oct.18 & 2230 & 134.9 & 275.9 & $N$ & 205.4 & 175.7 & 210.9 & 83 & 583 & 387 & 314 & 29.9 & 4.2 & 158 & 2. & 1478.4 & 232.9 & 39.8 \\
\hline Oct.18 & 2245 & 140.0 & 276.8 & $N / A$ & 206.7 & 179.3 & 216.0 & 846 & 594 & 386 & 315 & 29.7 & 44 & 154 & 1. & 1537.6 & 8 & 38.8 \\
\hline $0 c 1.18$ & 2300 & 134.2 & 276.2 & NIA & 206.2 & 174.4 & 208.9 & 846 & 594 & 386 & 315 & 29.4 & 39 & 15.9 & 2.5 & 1495.6 & 7.5 & 44.5 \\
\hline $0 c 1.18$ & 2315 & 144.1 & 280.3 & $N / A$ & 208.1 & 183.8 & 222.5 & 846 & 594 & 386 & 315 & 31.7 & 4.2 & 150 & 1. & 1576.2 & 6.2 & 38.1 \\
\hline Oct.18 & 2330 & 136.0 & 279.0 & $N / A$ & 206.4 & 174.9 & 213.2 & 846 & 594 & 386 & 320 & 30.7 & 4.0 & 14.4 & .0 .9 & 1489.8 & 9 & 37.8 \\
\hline $0 c 1.18$ & 2345 & 1300 & 278.5 & N/A & 206.3 & 175.7 & 210.8 & 846 & 594 & 329 & 323 & 30.6 & 46 & 15.7 & -17.9 & 15067 & 237.2 & 36.1 \\
\hline$A V E$ & RAGES & 140.0 & 278.6 & $N / A$ & 206.6 & 178.6 & 2155 & 826.3 & 5956 & 386.7 & 316.7 & 31.2 & 4.3 & 130 & 10 & 14948 & & 31.5 \\
\hline Oct.19 & 0 & 134.8 & 276.0 & N/A & 2020 & 173.7 & 210.3 & 840 & 594 & 340 & 318 & 32.9 & 4.4 & 150 & -13.3 & 1489.6 & 223.1 & 0 \\
\hline Oct.19 & 15 & 134.1 & 276.7 & N/A & 203.2 & 174.5 & 208.0 & 784 & 594 & 339 & 320 & 31.4 & 4.2 & 9.6 & .12 .6 & 1385.4 & 132.8 & 21.6 \\
\hline Oct.19 & 30 & 122.6 & 279.0 & N/A & 202.7 & 165.8 & 20 & 80 & 5 & 357 & 319 & 34.8 & 4.7 & 12.8 & -9.5 & & & \\
\hline $0 c t-19$ & 45 & 108.3 & 283.6 & N/A & 203.6 & 160.8 & 197.0 & 798 & 559 & 370 & 312 & 29.7 & 4.6 & 14.2 & -3.3 & 13001 & 184.4 & $\therefore$ \\
\hline Oct-19 & 100 & 105.2 & 2856 & N/A & 203.9 & 159.4 & 197.8 & 752 & 538 & 362 & 31 & 30.5 & 4.3 & 11.2 & -6.5 & 1214.7 & 136.5 & 22.3 \\
\hline Oct-19 & 115 & 105.1 & 285.5 & N/A & 204.7 & 159.6 & 19 & 7 & & 364 & & 29.7 & 4. & & -5.3 & & & \\
\hline Oct.19 & 130 & 105.1 & 286.6 & N/A & 205.0 & 159.7 & 196.4 & 756 & 544 & 369 & 31 & 30.2 & 4.3 & 1.5 & -34 & 12 & 9 & 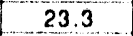 \\
\hline Ocl-19 & 145 & 105.2 & 2856 & $N / A$ & 205.2 & 1596 & 197.0 & 75 & 535 & 377 & 30 & 29.8 & 4.4 & 13.0 & .1 .4 & 1225.9 & 158.8 & 26.0 \\
\hline Oct.19 & 200 & 104.9 & 285.2 & N/A & 2048 & 157.9 & 18 & 7 & 537 & 380 & 3 & 30.3 & 4. & 7 & -3.7 & 12 & .5 & 2 \\
\hline Oet.19 & 215 & 104.8 & 284.8 & N/A & 2042 & 1563 & 197.2 & 77 & 522 & 372 & 31 & 30.7 & 4.4 & 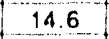 & 5.6 & 1219.7 & 5 & 28.2 \\
\hline Oct.19 & 230 & 105.2 & 283.6 & $N / A$ & 205.7 & 156.8 & 194.3 & 776 & 544 & 381 & 30 & 281 & 4.5 & 13.1 & .0 .1 & 12330 & 161.8 & 27.4 \\
\hline 9 & 245 & 105.0 & 284.9 & A & 20 & 158.3 & 1970 & 774 & 541 & 3 & 30 & 30.0 & 41 & & -1.8 & & & 38 \\
\hline Oct.19 & 300 & 105.0 & 2858 & $N / A$ & 2067 & 156.6 & 196.2 & 77 & 511 & 3 & 30 & 27.9 & 4.3 & 16.8 & 1.9 & & & 37 \\
\hline Oct.19 & 315 & 105.0 & 288.9 & N/A & 211.1 & 158.1 & 193.5 & 781 & 563 & 381 & 314 & 27.6 & 4.4 & 11.8 & -1.0 & 1251.2 & 147.4 & 26.0 \\
\hline 1.18 & 1200 & 145.3 & 278.9 & $N / A$ & 2018 & 181.9 & 228.6 & 841 & 620 & 39 & 334 & 36.6 & 4.7 & 72 & -5.7 & & & \\
\hline Oct.18 & 1215 & 145.2 & 279.4 & $N / A$ & 201.1 & 1837 & 229.1 & 8 & 62 & 40 & 330 & 36.0 & 4.5 & 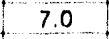 & 3. & & & \\
\hline Oct-19 & 1230 & 145.7 & 279.8 & IA & 2013 & 180.5 & 224.1 & 83 & 613 & 398 & 331 & 35.6 & 3.9 & 8.7 & .33 & 1524.3 & 132.7 & 205 \\
\hline 1.19 & 1245 & 145.9 & 279.4 & 1 & 201.8 & 181.8 & 229.6 & 8 & 61 & 397 & 334 & 35 & 3 & 7 & 6.1 & 1544.5 & 4 & 21.7 \\
\hline Oc1.19 & 1300 & 145.7 & 279.4 & $N / A$ & 201.7 & 180.3 & 226.6 & 8 & 6 & 40 & 332 & 353 & 33 & & 4.2 & 1543.5 & & 210 \\
\hline 1.19 & 1315 & 145.5 & 280.1 & N/A & 201.6 & 179.9 & 225.1 & 84 & 613 & 39 & 330 & 35.4 & 4.9 & 87 & .3 .7 & 1532.9 & 8 & 5 \\
\hline 1.19 & 1330 & 145.2 & & $N / A$ & 2020 & 181.8 & & 8: & 609 & 40 & 334 & 35,3 & 4.9 & 8.5 & .5 .0 & 1544.5 & & 16.2 \\
\hline Oc1.19 & 1345 & 145.4 & 281.0 & $N / A$ & 2016 & 182.8 & 232.9 & 8 & 593 & 40 & 331 & 38.6 & 4.2 & 10.9 & .4 .6 & 1570.4 & 171.3 & 22.7 \\
\hline 1.19 & 1400 & 145.5 & 279.6 & $N / A$ & 2004 & 183.4 & 2330 & 833 & 597 & 399 & 331 & 37.8 & 4.5 & 90 & -5.6 & 15479 & 38.8 & 975 \\
\hline .19 & 1415 & 145.3 & 279.4 & N/A & 201.9 & 183.6 & 2322 & 843 & 590 & 40 & 333 & 34.8 & 4.2 & 11.4 & .46 & 15672 & 179.4 & 26.3 \\
\hline 19 & 1430 & 145.4 & 278.3 & & 202.8 & & 2000 & & & & 33 & 20 & 15 & & -6.7 & & & \\
\hline 1.19 & 1445 & 145.5 & 279.9 & N/A & 204.4 & 183.7 & 2310 & 839 & 608 & 403 & 333 & 34.0 & 4.0 & 8 & .3 .7 & 1561.6 & 137.9 & 21.8 \\
\hline & 1500 & 146.1 & 279.4 & & 203.8 & 1806 & 228.5 & 833 & 621 & 399 & 329 & 34.3 & 3.7 & 5 & .4 .5 & 15233 & & 14.6 \\
\hline 1.19 & 1515 & 145.3 & 278.8 & & 2035 & 1800 & 2298 & & & & 325 & 37 & & & -6.4 & & 134.7 & 2.8 \\
\hline 1.19 & 1530 & 145.3 & 279.1 & $N / A$ & 203.8 & 180.2 & 227.1 & 856 & 622 & 387 & 324 & 33.5 & 4.3 & 8.4 & .5 .5 & 1562.7 & 130.6 & 19.4 \\
\hline-19 & 1545 & 145.7 & 279.2 & $N / A$ & 204.2 & 1792 & 225.2 & 853 & 625 & 385 & 316 & 32.9 & 4.3 & 8.0 & -3.4 & 1549.5 & 123.6 & 18.7 \\
\hline-19 & 1600 & 0.0 & & & 204.6 & & & & & & 321 & 0 & 4 & 8.2 & -7.1 & 1541.6 & 126.5 & 20.4 \\
\hline$c t \cdot 19$ & 1615 & 145.7 & 276.7 & $N / A$ & 205.5 & 1824 & 229.8 & 828 & 608 & 381 & 324 & 31.9 &  & 1 & -7.2 & 1530.2 & 113.6 & 20.2 \\
\hline & 1630 & 145.1 & 279.1 & IV/A & 206.9 & 184.1 & 230.4 & 843 & 615 & 391 & 328 & 32.3 & 4.1 & 8.7 & .5 .1 & 1571.5 & 136.1 & 22.1 \\
\hline ct.19 & 1645 & 145.5 & 277.8 & $\mathrm{~N} / \mathrm{A}$ & 206.5 & 180.1 & 229.6 & 841 & 609 & 385 & 326 & 31.0 & 4.1 & 7.7 & -7.8 & 1534.6 & 118.4 & 20.0 \\
\hline
\end{tabular}




\begin{tabular}{|c|c|c|c|c|c|c|c|c|c|c|c|c|c|c|c|c|c|c|}
\hline Dato & Timo & Bollor & Duct & $A v g$ "C" & Ducl & Flue Gas & Flue Gas & $\mathrm{SO} 2$ & 502 & NOX & Ox & $\operatorname{Lim} \theta$ & $\operatorname{Lim} \theta$ & $\mathrm{SO} 2$ & NOx & 502 & 502 & Lime \\
\hline & & Lood & Inlet & Section & Oullal & Flow in & Flow Out & $\ln |\theta|$ & Oullet & $\ln \mid \theta t$ & Outlet. & Flow & Conc & Removal & Removal & $\ln |0|$ & Pemoved & Utilizatio \\
\hline & & MN & Temp of & Temp of & Tomp of & KSCFM & KSCFM & ppm & $\mathrm{ppm}$ & $\mathrm{ppm}$ & ppm & $\mathrm{gpm}$ & $\%$ & $\%$ & $\%$ & $\mathrm{Lb} / \mathrm{HI}$ & $\mathrm{Lb} / \mathrm{HI}$ & $\%$ \\
\hline Oct.19 & 1700 & 145.6 & 278.0 & N/A & 2064 & 179.6 & 230.3 & 845 & 609 & 389 & 323 & 31.7 & 3.8 & 7.6 & -6.5 & 15384 & 1164 & 202 \\
\hline Oct-19 & 1715 & 145.6 & 278.7 & $N / A$ & 207.3 & 181.4 & 233.4 & 829 & 607 & 386 & 321 & 31.4 & 3.6 & 5.7 & -6.8 & 15227 & 86.4 & 16.4 \\
\hline $0 c 1 \cdot 19$ & 1730 & 145.8 & 278.7 & $N / A$ & 207.1 & 178.9 & 227.8 & 836 & 620 & 384 & 318 & $31.2]$ & 3.8 & 5.5 & .5 .5 & 15153 & 83.8 & 15.2 \\
\hline Oct-19 & 1745 & 145.5 & 278.8 & N/A & 207.9 & 180.5 & 228.5 & 852 & 613 & 380 & 321 & 31.0 & 38 & B. 4 & .7 .4 & 15580 & 131.6 & 24.0 \\
\hline Oct-19 & 1800 & 145.0 & 278.0 & N/A & 206.0 & 180.1 & 231.8 & 852 & 604 & 380 & 317 & 33.4 & 3.5 & 8.8 & .7 .2 & 15554 & 137.1 & 25.3 \\
\hline Oct-19 & 1815 & 145.7 & 277.7 & $N / A$ & 207.7 & 182.9 & 233.6 & 832 & 603 & 379 & 317 & 30.8 & 3.7 & 7.4 & 6.7 & 1541.8 & 113.7 & 21.5 \\
\hline 0 ct-19 & 1830 & 145.2 & 276.8 & $N / A$ & 207.2 & 182.3 & 230.5 & 836 & 616 & 380 & 14 & 31.3 & 3.6 & 6.8 & .4 .6 & 1544.1 & 105.8 & 20.2 \\
\hline Oct.19 & 1845 & 145.5 & 277.6 & N/A & 207.8 & 183.1 & 232.6 & 826 & 615 & 378 & 318 & 31.8 & 4.2 & 5.3 & 6.6 & 1531.4 & 811 & 130 \\
\hline Oct-18 & 1900 & 145.7 & 278.1 & N/A & 207.8 & 185.2 & 234.7 & 826 & 600 & 380 & 318 & 31.8 & 4.3 & 7.9 & .6 .5 & 15490 & 122.5 & 19.1 \\
\hline $001-19$ & 1915 & 145.5 & 276.3 & NIA & 207.6 & 183.1 & 231.5 & 843 & 618 & 381 & 319 & 30.7 & 38 & 7.0 & .56 & 15630 & 1101 & 20.3 \\
\hline Oct.18 & 1930 & 145.5 & 276.2 & N/A & 208.5 & 183.8 & 230.4 & 846 & 608 & 381 & 319 & 30.1 & 3.9 & 98 & 51 & 15753 & 155.0 & 28.4 \\
\hline Oct.18 & 1945 & 145.4 & 276.3 & $N / A$ & 208.8 & 183.4 & 232.6 & 826 & 607 & 381 & 318 & 30.1 & 3.7 & 68 & .62 & 1534.9 & 104.6 & 20.2 \\
\hline Oct.18 & 2000 & 145.8 & 276.2 & N/A & 208.3 & 182.6 & 232.3 & 830 & 604 & 378 & 318 & 30.6 & 4.0 & 74 & .7 .1 & 1535.2 & 112.9 & 18.8 \\
\hline Oct-19 & 2015 & 145.3 & 276.3 & N/A & 206.7 & 185.1 & 235.6 & 829 & 585 & 376 & 319 & 32.7 & 3.8 & 10.1 & 8.0 & $1554 ?$ & 157.3 & 26.5 \\
\hline Oct.19 & 2030 & 145.2 & 274.8 & $N / A$ & 206.9 & 183.3 & 231,3 & 835 & 595 & 378 & 318 & 29.9 & 4.1 & 10.1 & .6 .1 & 15507 & 1566 & 27.4 \\
\hline Oct.19 & 2045 & 145.9 & 275.0 & $N / A$ & 207.5 & 182.0 & 234.0 & 832 & 609 & 380 & 317 & 29.8 & 3.8 & 58 & .7 .2 & 15342 & 89.4 & 16.8 \\
\hline Oct.19] & 2100 & 145.5 & 275.2 & N/A & 2085 & 182.3 & 233.5 & 832 & 622 & 381 & 316 & 298 & 3.8 & 4.3 & .64 & 1536.7 & 5.5 & 12.4 \\
\hline $0 c 1-18$ & 2115 & 145.4 & 275.8 & N/A & 2086 & 181.2 & 234.0 & 832 & 600 & 422 & 302 & 296 & 3.8 & 6.8 & 74 & 1527.5 & 104.0 & 8.4 \\
\hline Oct.19 & 2130 & 145.7 & 276.5 & $N / A$ & 209.9 & 183.2 & 233.6 & 868 & 602 & 393 & 311 & 29.8 & 3.8 & 11.6 & .0 .7 & 1611.0 & 186.4 & 34.5 \\
\hline Oct.19 & 2145 & 144.8 & 276.3 & $N / A$ & 2090 & 179.6 & 2295 & 864 & 618 & 388 & 315 & 28.1 & 3.8 & 8.6 & .3 .8 & 1572.0 & 135.2 & 6.3 \\
\hline Oct.19 & 2200 & 145.5 & 276.5 & $N / A$ & 207.6 & 181.7 & 234.6 & 851 & 615 & 386 & 315 & 33.6 & 3.9 & 6.7 & 5.5 & 1566.5 & 104.8 & 7.2 \\
\hline Oct-19 & 2215 & 145.5 & 277.2 & N/A & 207.1 & 182.8 & 237.1 & 855 & 606 & 386 & 312 & 32.3 & 3.7 & 8.0 & 50 & 15834 & 126.6 & 2.8 \\
\hline Oct.18 & 2230 & 145.2 & 278.5 & $N / A$ & 2080 & 184.2 & 236.3 & 852 & 600 & 389 & 316 & 324 & 3.7 & 9.7 & .4 .2 & 1589.8 & 153.6 & 7.6 \\
\hline Oct-19 & 2245 & 147.3 & 277.8 & $N / A$ & 211.3 & 183.7 & 235.8 & 869 & 634 & 388 & 320 & 29.0 & 3.8 & 6.3 & .58 & 1617.2 & 101.8 & 9.9 \\
\hline Oct.19 & 2300 & 147.5 & 277.4 & N/A & 212.9 & 185.3 & 235.0 & 869 & 634 & 388 & 320 & 28.7 & 4.0 & 7.4 & .46 & 1631.3 & 121.0 & 2.6 \\
\hline Oct-19 & 2315 & 146.0 & 277.6 & $N / A$ & 213.6 & 183.5 & 234.2 & 869 & 656 & 388 & 340 & 27.9 & 3.8 & 3.6 & .120 & 1615.5 & 58.1 & 1.8 \\
\hline Oct.19 & 2330 & 146.1 & 278.7 & $N / A$ & 214.3 & 188.9 & 238.8 & 869 & 620 & 388 & 315 & 29.3 & 3.8 & 9.8 & .2 .7 & 16 & & 31.4 \\
\hline Oct-19 & 2345 & 146.7 & 276.8 & N/A & 215.0 & 187.9 & 239.9 & 735 & 637 & 322 & 324 & 27.5 & 3.8 & .10 .6 & .28 .4 & 1389.7 & .148 .2 & .30 .5 \\
\hline \multicolumn{2}{|c|}{ AVERAGES } & 137.7 & 279.2 & $N / A$ & 206.1 & 177.6 & 224.2 & 826.9 & 598.0 & 383.0 & 320.2 & 31.8 & 4.1 & 8.8 & -5.6 & 1490.4 & 128.3 & 21.3 \\
\hline Ocl.20 & 600 & 146.0 & 272.0 & N/A & 200.6 & 180.9 & 232.4 & 865 & 668 & 371 & 313 & 33 & 3.4 & 0.8 & -8.5 & 1586.2 & 12.5 & 2.4 \\
\hline Oct.20 & 615 & 147.1 & 272.3 & $N / A$ & 198.8 & 184.0 & 234.8 & 840 & 630 & 376 & 311 & 34.1 & 3.4 & 4.3 & -5.6 & 0.1 & 8 & 12.4 \\
\hline Oct.20 & 630 & 1442 & 273.2 & N/A & 198.7 & 178.8 & 226.2 & 857 & 622 & 373 & 308 & 33.3 & 3.7 & 8.2 & .4 .3 & 1553.3 & 128.0 & 3 \\
\hline Oct.20 & 645 & 147.1 & 271.8 & $N / A$ & 1990 & 182.3 & 232.3 & 842 & 630 & 376 & 316 & 331 & 3.8 & 4.6 & -6.8 & 1554.3 & 72.0 & 12.3 \\
\hline Oct.20 & 700 & 144.7 & 272.0 & N/A & 198.8 & 180.7 & 229.6 & 853 & 615 & 377 & 310 & 32.8 & 3. & 8.4 & -4.6 & 1561.5 & & 23.2 \\
\hline Oct.20 & 715 & 142.4 & 272.0 & N/A & 198.5 & 178.7 & 230.5 & 836 & 621 & 378 & 310 & 32.7 & 3.8 & 4.1 & .5 .8 & 15 & & 10.7 \\
\hline $0 c 1-20$ & 730 & 136.7 & 270.5 & $N / A$ & 195.9 & 1714 & 214.6 & 862 & 610 & 368 & 311 & 32.1 & 3.7 & 11.3 & .5 .9 & 1496.8 & 6 & 30.7 \\
\hline Oct.20 & 745 & 138.1 & 269.6 & $N / A$ & 196.9 & 178.6 & 2253 & 829 & 606 & 384 & 327 & 32.7 & 3.6 & 7.7 & .7 .4 & 1499.2 & 15.0 & 210 \\
\hline Oct.20 & 800 & 137.3 & 271.2 & $N / A$ & 196.8 & 177.5 & 225.1 & 845 & 597 & 398 & 319 & 32.5 & 4.1 & 10.5 & -1.5 & 1520.4 & 15 & 25.6 \\
\hline Oct.20 & 815 & 142.2 & 269.6 & N/A & 197.1 & 181.5 & 231.5 & 842 & 596 & 387 & 317 & 32.5 & 40 & 9.7 & .4 .4 & 15484 & 149.5 & 24.7 \\
\hline 1.20 & 830 & 142.8 & 270.5 & N/A & 198.1 & 181.1 & 227.4 & 856 & 597 & 389 & 322 & 31.8 & 3.8 & 12.4 & .4 .0 & 1570.5 & 104.1 & 34.5 \\
\hline Oct.20 & 845 & 147.2 & 270.3 & $N / A$ & 198.7 & 182.4 & 230.4 & 847 & 615 & 390 & 31 & 3 & 36 & 8.3 & -3.2 & 1566.0 & 128.5 & 23.4 \\
\hline Oct-20 & 900 & 147.9 & 271.6 & $N / A$ & 200.7 & 183.2 & 230.8 & 849 & 620 & 390 & 320 & 33.2 & 4.1 & 8.0 & -3.5 & 5.7 & 1 & 19.9 \\
\hline Oct.20 & 915 & 146.9 & 271.6 & N/A & 199.6 & 183.0 & 233.0 & 852 & 616 & 386 & 321 & 35.5 & 4.2 & 7.8 & -5.9 & 1578.5 & 125.6 & 18.1 \\
\hline Oct-20 & 830 & 146.4 & 272.0 & N/A & 199.8 & 183.7 & 227.6 & 866 & 611 & 387 & 318 & 33.9 & 3.8 & 12.6 & -1.9 & 1611.6 & 202.9 & 33.9 \\
\hline Oal.20 & 945 & 146.6 & 272.5 & $N / A$ & 202.4 & 183.2 & 229.8 & 852 & 610 & 381 & 31 & 31. & 38 & 10.2 & .2 .7 & 15 & 161.0 & 29.3 \\
\hline Oct.20 & 1000 & 107.0 & 272.3 & $N / A$ & 202.2 & 180.9 & 226.8 & 859 & 631 & 377 & 316 & 30.4 & 3.9 & 8. & -5.1 & 5.2 & 6 & 22.8 \\
\hline Oct.20 & 1015 & 146.3 & 273.4 & $N / A$ & 203.9 & 182.4 & 228.9 & 830 & 617 & 378 & 309 & 30.7 & 3.7 & 6.7 & -2.6 & 1533.9 & 102.0 & 19.3 \\
\hline $0 c t .20$ & 1030 & 146.8 & 273.9 & $N / A$ & 205.2 & 184.0 & 228.1 & 841 & 605 & 37 & 307 & 30.7 & 3.8 & 10.8 & -0.8 & 79 & 169.9 & 31.3 \\
\hline Ocl.20 & 1045 & 146.3 & 274.5 & $N / A$ & 2060 & 184.1 & 226.1 & 839 & 626 & 37 & 312 & 30 & 39 & 83 & -2.1 & 50 & 0 & 3.8 \\
\hline Oct.20 & 1100 & 146.8 & 275.5 & $N / A$ & 206.9 & 182.1 & 223.3 & 849 & 644 & 379 & 316 & 30.2 & 3.8 & 7.0 & 2.3 & 15 & .7 & 0.5 \\
\hline 1.20 & 1115 & 146.6 & 276.9 & $N / A$ & 204.6 & 183.4 & 228.0 & 863 & $6+1$ & 381 & 313 & 32.4 & 4.5 & 11.9 & -2.0 & 1603.4 & 191.1 & 8.1 \\
\hline Oct-20 & 1130 & 147.2 & 278.4 & N/A & 204.8 & 182.6 & 226.5 & 850 & 615 & 38 & 317 & 33 & 4.0 & 10.3 & -1.7 & 1572.4 & 3 & 26.0 \\
\hline Oct-20 & 1145 & 143.4 & 278.3 & N/A & 204.9 & 179.4 & 223.0 & 850 & 6 & 385 & 316 & 31.3 & 3.9 & 10.6 & -1.9 & 1545.7 & 2 & 28.7 \\
\hline Oct.20 & 1200 & 143.1 & 277.7 & $N / A$ & 2116 & 180.3 & 222.4 & 855 & 658 & 384 & 320 & 27.1 & 3.7 & 5.1 & 2.8 & 2.6 & 4 & 17.2 \\
\hline 1.20 & 1215 & 139.7 & 283.6 & $N / A$ & 214.7 & 184.0 & 222.2 & 843 & 652 & 380 & 325 & 28.7 & 4.1 & 6 & -3.1 & 1570.7 & 103.2 & 18.8 \\
\hline Oct.20 & 1230 & 146.9 & 285.6 & $N / A$ & 217.4 & 188.3 & 228.8 & 852 & 641 & 385 & 322 & 29.0 & 3.9 & 8.5 & .1 .6 & 1625.3 & 138.6 & 26.3 \\
\hline Oct. 20 & 1245 & 1464 & 282.3 & N/A & 216.6 & 182.8 & 222.3 & 854 & 646 & 383 & 317 & 27.7 & 3.9 & 8.0 & -0.8 & 1581.5 & 127.0 & 25.3 \\
\hline 1.20 & 1300 & 146.6 & 279.9 & $N / A$ & 216.9 & 182.0 & 223.6 & 844 & 644 & 379 & 314 & 27.5 & 3.9 & 62 & -1.8 & & & 9.2 \\
\hline 20 & 1315 & 147.3 & 280.6 & $N / A$ & 217.6 & 181.0 & 221.8 & 855 & 633 & 383 & 318 & 25.9 & 4.0 & 9.3 & -1.7 & 1567.8 & 145.7 & 30.2 \\
\hline 1.20 & 1530 & 147.6 & 282.4 & $N / A$ & 206.2 & 182.0 & 2236 & 845 & 649 & 392 & 327 & 35.5 & 3.3 & 5.7 & -2.3 & 1558.0 & 88.1 & 16.2 \\
\hline Oct.20 & 1545 & 148.7 & 2824 & $N / A$ & 205.2 & 1857 & 230.1 & 838 & 631 & 392 & 329 & 36.1 & 3.3 & 6.7 & -4.0 & 1576.7 & 106.1 & 19.2 \\
\hline$c t \cdot 20$ & 1600 & 145.6 & 2839 & $N / A$ & 204.4 & 184.0 & 225.5 & 819 & 605 & 380 & 312 & 36.6 & 2.7 & 9.4 & 0.5 & & 143.8 & 1.5 \\
\hline .20 & 1615 & 146.8 & 283.3 & N/A & 2036 & 183.5 & 227.2 & 845 & 613 & 388 & 321 & 36.8 & 3.4 & 10.1 & -2.6 & 15 & 8 & 274 \\
\hline 20 & 1630 & 147.5 & 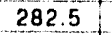 & & 202.7 & 182.5 & 225.8 & 824 & 609 & 390 & 327 & 36.3 & 52 & 8.6 & -3.9 & 1523.6 & 130.8 & 14.8 \\
\hline Oct.20 & 1645 & 147.1 & 281.6 & $N / A$ & 2024 & 179.9 & 222.6 & & 616 & 390 & 322 & 35.7 & 5.1 & 10.8 & -2.1 & & 169.0 & 19.8 \\
\hline 1.20 & 1700 & 145.9 & 281.4 & $N^{\prime} A$ & 202.2 & 180.9 & 220.6 & 860 & 604 & 389 & 338 & 35.5 & 4.3 & 14.3 & -5.9 & 1577.0 & 226.1 & 31.8 \\
\hline 20 & 1715 & 1390 & 282.0 & N/A & 201.8 & 178.6 & 222.2 & 849 & 611 & 404 & 331 & 35.6 & 4.5 & 10.5 & 1.9 & 1537.0 & 161.7 & 21.6 \\
\hline Oct-20 & 1730 & 138.0 & 281.1 & $N A$ & 1988 & 1754 & 18.1 & & 579 & 386 & 316 & 36.7 & 4.2 & 136 & -1.8 & 1480.4 & 200.9 & 28.0 \\
\hline Oct-20 & 1745 & 138.7 & 280.3 & N/A & 200.0 & 177.6 & 218.3 & & 592 & 391 & 330 & 35.0 & 4.1 & 123 & -3.9 & & 184.2 & 27.5 \\
\hline c 1.20 & 1800 & 142.6 & 279.8 & $N / A$ & 199.7 & 176.8 & 2200 & 825 & 594 & 388 & 324 & 35.8 & 4.5 & 10.3 & .4 .0 & 1477.8 & 152.9 & 20.3 \\
\hline 1.20 & 15 & 147.6 & 31.8 & IA & 2009 & 83.1 & 226.7 & 847 & 601 & 392 & 329 & 36.7 & 4.7 & 12.1 & .4 .0 & 1571.9 & 190.5 & 23.6 \\
\hline Oct.20 & 1830 & 146.6 & & & 2016 & 184.0 & 227.9 & 839 & 602 & 388 & 325 & 37.6 & 39 & 11.0 & -3.8 & 1563.2 & 172.2 & 25.2 \\
\hline Oct-20 & 1845 & 146.8 & 283.3 & N/A & 201.1 & 182.8 & 226.1 & 836 & 595 & 389 & 322 & 37.4 & 4.3 & 11.9 & -2.6 & 1548.4 & 184.4 & 24.6 \\
\hline $0 \mathrm{Cl} \cdot 20$ & 1900 & 147.2 & 282.2 & $N / A$ & 201.3 & 182.8 & 225.8 & 851 & 591 & 385 & 320 & 36.8 & 4.3 & 14.1 & -2.5 & 1576.0 & 223.0 & 30.2 \\
\hline
\end{tabular}


CZD LIME INJECTION DATA - AUGUST 17, TO NOVEMBEA 16, 1982

\begin{tabular}{|c|c|c|c|c|c|c|c|c|c|c|c|c|c|c|c|c|c|c|}
\hline Dalo & Time & Boller & Duct & Avg " $C^{\prime \prime}$ & Duct & Flue Ges & Flue $\mathrm{Gay}$ & $80 ?$ & 802 & NOX & NOX & Lime & $\lim \theta$ & 502 & NOx & 802 & 502 & Lime \\
\hline & & $\frac{\text { Load }}{M W}$ & Inlel & $\begin{array}{l}\text { Section } \\
\text { Tomp of }\end{array}$ & $\begin{array}{l}\text { Oullot } \\
\text { Temp of }\end{array}$ & $\begin{array}{l}\text { Flow in } \\
\text { KSCFM }\end{array}$ & $\begin{array}{l}\text { Flow Out } \\
\text { KSCFM }\end{array}$ & $\begin{array}{c}\text { Inlol } \\
\text { ppm }\end{array}$ & $\begin{array}{c}\text { Oullol } \\
\text { ppm }\end{array}$ & inla| & Oullal & $\begin{array}{l}\text { Flow } \\
\text { opm }\end{array}$ & $\begin{array}{c}\text { Conc } \\
\%\end{array}$ & $\begin{array}{c}\text { Gemoval } \\
\%\end{array}$ & Hemoval & $\mid \begin{array}{l}\text { Inlol } \\
\text { lo/Hl }\end{array}$ & $\begin{array}{c}\text { Removed } \\
\mathrm{Lb} / \mathrm{Hr}\end{array}$ & Utilization \\
\hline Oct. 20 & 1015 & 147.1 & 283.3 & N/A & 201.4 & 1806 & 223.9 & 845 & 589 & 384 & 317 & 36.6 & 4.3 & 136 & 2.5 & 15468 & 211.0 & 28.7 \\
\hline Oet.20 & 1930 & 147.0 & 283.6 & $N / A$ & 2018 & 179.5 & 220.3 & 851 & 595 & 384 & 318 & 36.5 & 4.0 & 14.2 & 9.8 & 1548.4 & 2107 & 32.3 \\
\hline $0 c 1.20$ & 1945 & 147.0 & 2840 & $N / A$ & 202.2 & 182.1 & 225.5 & 839 & 583 & 382 & 315 & 36.7 & 44 & 14.0 & 2.2 & 15480 & 216.4 & 28.7 \\
\hline Oct.20 & 2000 & 146.3 & 2837 & $N / A$ & 202.0 & 180.9 & 222.0 & 840 & 582 & 384 & 320 & 36.3 & 4.3 & 14.9 & 2.3 & 15396 & 228.6 & 31.5 \\
\hline $0 c 1.20$ & 2015 & 1470 & 283.1 & $N / A$ & 203.7 & 181.8 & 227.5 & 839 & $580^{\circ}$ & 387 & 318 & 35.2 & 43 & 12.1 & -3.0 & 1545.4 & 186.8 & 26.5 \\
\hline Oc1.20 & 2030 & 146.7 & 283.3 & $N / A$ & 204.0 & 180.5 & 223.5 & 853 & 582 & 389 & 322 & 35.3 & 43 & 14.0 & -24 & 15588 & 2183 & 30.8 \\
\hline Oct.20 & 2045 & 146.8 & 282.8 & $N / A$ & 2046 & 1833 & 224.6 & 824 & 574 & 383 & 315 & 35.6 & 30 & 14.6 & .0 .8 & 15303 & 223.4 & 34.6 \\
\hline Oct.20 & 2100 & 146.5 & 282.3 & N/A & 205.4 & 185.3 & 2255 & 824 & 573 & 383 & 315 & 34.9 & 42 & 154 & 0.1 & 15470 & 237.8 & 34.8 \\
\hline $0 \mathrm{ct} \cdot 20$ & 2115 & 146.1 & 282.0 & N/A & 205.2 & 184.2 & 224.3 & 824 & 560 & 383 & 307 & 34.8 & 42 & 17.2 & 24 & 1537.8 & 265.2 & 38.9 \\
\hline Oct.20 & 2130 & 147.2 & 281.4 & $N / A$ & 205.8 & 184.4 & 225.7 & 858 & 568 & 396 & 313 & 340 & 3.9 & 18.9 & 3.5 & 16038 & 303.7 & 49.2 \\
\hline Oct.20 & 2145 & 146.4 & 281.4 & $N / A$ & 2058 & 183.8 & 224.7 & 847 & 575 & 390 & 314 & 34.2 & 41 & 17.1 & 1.7 & 1578.0 & 270.1 & 41.3 \\
\hline Oct.20 & 2200 & 145.8 & 281.3 & $N / A$ & 207.1 & 185.7 & 228.5 & 837 & 582 & 385 & 314 & 33.3 & 4.3 & 14.4 & 0.2 & 15748 & 226.5 & 33.9 \\
\hline Oct.20 & 2215 & 143.5 & 280.1 & N/A & 207.2 & 1837 & 224.2 & 821 & 589 & 380 & 313 & 32.3 & 3.8 & 124 & 04 & 15281 & 189.2 & 123 \\
\hline Qct.20 & 2230 & 138.4 & 278.8 & $N / A$ & 205.7 & 177.2 & 218.1 & 837 & 580 & 378 & 312 & 34.7 & 4.2 & 14.7 & -1.2 & 1501.8 & 220.4 & 35.5 \\
\hline Oct.20 & 2245 & 137.4 & 278.6 & N/A & 205.2 & 174.7 & 213.6 & 830 & 580 & 386 & 311 & 31.1 & 4.2 & 14.5 & 1.5 & 1468.3 & 212.2 & 34.8 \\
\hline $0 c 1.20$ & 2300 & 137.5 & 276.8 & N/A & 204.3 & 1778 & 220.2 & 830 & 580 & 386 & 311 & 316 & 4.3 & 13.3 & 0.2 & 14943 & 189.4 & 31.5 \\
\hline 0 c1.20 & 2315 & 138.5 & 277.8 & $N / A$ & 205.1 & 179.7 & 216.9 & 830 & 593 & 386 & 327 & 31.7 & 4.0 & 13.7 & .24 & 1510.3 & 206.2 & 4.8 \\
\hline Oct.20 & 2330 & 137.4 & 275.8 & N/A & 207.6 & 176.5 & 213.5 & 830 & 571 & 386 & 313 & 27.6 & 4.1 & 168 & 1.7 & 1483.4 & 248.3 & 7.3 \\
\hline Oct.20 & 2345 & 136.8 & 276.5 & $\mathrm{~N} / \mathrm{A}$ & 209.0 & 175.6 & 217.2 & 830 & 590 & 386 & 320 & 281 & 4.0 & 12.0 & .2 .7 & 14758 & 176.6 & 3.7 \\
\hline$A V E$ & RAGES & 144.5 & 278.2 & N/A & 2037 & 181.3 & 224.8 & 843.1 & 606.9 & 3844 & 3170 & 33.2 & 4.0 & 10.7 & 26 & 15400 & 165.7 & 6.6 \\
\hline Oct.21 & 0 & 137.8 & 276.7 & $N / A$ & 212.0 & 174.4 & 2128 & 830 & 612 & 386 & 317 & 26.4 & 4.1 & 8.9 & 0.5 & 1465.7 & 1454 & 88 \\
\hline Oc1.21 & 645 & 146.5 & 283.1 & $N / A$ & 203.8 & 185.4 & 2263 & $82 ?$ & 614 & 368 & 17 & 37.8 & 45 & 9.3 & 52 & 15525 & 0 & 8.2 \\
\hline 001.21 & 700 & 146.9 & 283.1 & $N / A$ & 2034 & 1849 & 2254 & 831 & 609 & 375 & 307 & 374 & 4.6 & 106 & 0.1 & 15568 & 165.1 & .5 \\
\hline Oct.21 & 715 & 147.3 & 282.2 & NIA & 203.6 & 184.8 & 225.3 & 831 & 624 & 381 & 315 & 36.7 & 4.1 & B.4 & .0 .9 & 15558 & 1306 & 86 \\
\hline Oct.21 & 730 & 146.2 & 281.1 & $N / A$ & 203.4 & 182.5 & 2253 & 834 & 605 & 372 & 12 & 360 & 4.2 & 10.4 & 37 & 1542.1 & 160.2 & 2.7 \\
\hline Oct.21 & 745 & 147.1 & 281.8 & $N / A$ & 204.1 & 182.9 & 225.2 & 823 & 611 & 367 & 308 & 36.0 & 3.8 & 8.6 & .3 .4 & 1525.1 & 131.5 & 01 \\
\hline oct.21 & 800 & 146.6 & 284.2 & N/A & 205.3 & 186.3 & 228.3 & 824 & 600 & 371 & 310 & 370 & 3.9 & 10.7 & 24 & 15544 & 167.0 & 18 \\
\hline $0 c 1.21$ & 815 & 146.5 & 283.8 & $N / P$ & 204.8 & 187.7 & 227.3 & 818 & 585 & 370 & 313 & 37.5 & 41 & 13.3 & 2.6 & 15547 & 206.5 & 3.8 \\
\hline $0 c 1.21$ & 830 & 146.7 & 282.3 & $N / A$ & 205.2 & 184.7 & 228.4 & 819 & 591 & 37 & 313 & 36.4 & 3.8 & 10.7 & 4.2 & 17 & 3 & 5 \\
\hline Oc1.21 & 845 & 146.1 & 282.0 & $\mathrm{~N} / \mathrm{A}$ & 203.5 & 185.6 & 227.3 & 816 & 574 & 374 & 314 & 381 & 3.8 & 13.8 & .2 .6 & 1534.5 & 211.0 & 5 \\
\hline Oc1-21 & 900 & 146.5 & 282.0 & $N / A$ & 2052 & 185.9 & 231.1 & 820 & 585 & 37 & 11 & 35.4 & 41 & 11.2 & .3 .6 & 15435 & 8 & 6 \\
\hline Oct.21 & 915 & 147.2 & 282.0 & $N / A$ & 2062 & 1848 & 2256 & B15 & 595 & 370 & 10 & 343 & 3.8 & 109 & .22 & 1526.8 & 167.0 & 8 \\
\hline Ocl.21 & 930 & 147.1 & 284.3 & $N / A$ & 207.1 & 184.7 & 226.2 & 836 & 605 & 372 & 313 & 35.1 & 41 & 11.3 & 32 & 35 & 177.2 & 6.4 \\
\hline Oct.21, & 945 & 147.2 & 285.5 & N/A & 2077 & 1844 & 224.3 & 833 & 608 & 371 & 311 & 35.2 & 30 & 112 & .1 .8 & 15554 & 1730 & 5.6 \\
\hline Oc1.21 & 1000 & 147.1 & 287.3 & $N / A$ & 208.7 & 183.2 & 228.0 & $84 !$ & 614 & 371 & 312 & 35.4 & 2.9 & 9.0 & 4.7 & 15601 & 140.8 & 29.6 \\
\hline Oct.21 & 1015 & 146.9 & 287.3 & $N / A$ & 2085 & 183.1 & 227.1 & 844 & 611 & 371 & 311 & 35.2 & 3.7 & 102 & 4.0 & 1564.8 & 2 & 3 \\
\hline Oct.21 & 1030 & 146.9 & 286.7 & $N / A$ & 2091 & 1829 & 225.1 & 854 & 622 & 37 & 309 & 346 & 4.5 & 10.4 & 2.4 & 1583.3 & .8 & 7 \\
\hline Oct.21 & 1045 & 146.8 & 286.8 & $N / A$ & 209.7 & 183.4 & 2251 & 844 & 622 & 370 & 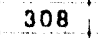 & 34.3 & 4.5 & 9.4 & 2.2 & 15674 & 147.9 & 0.5 \\
\hline Oct.21. & 1100 & 147.3 & 286.4 & $N / A$ & 204.5 & 183.4 & 228.0 & 848 & 591 & 371 & 308 & 390 & 4.8 & 13.3 & .3 .3 & 1576.5 & 0.3 & 3.5 \\
\hline Oct.21 & 1115 & 147.0 & 2868 & NIA & 2045 & 183.7 & 227.6 & 857 & 587 & 374 & $n$ & 382 & 46 & 15.1 & .23 & 4.8 & 3 & \\
\hline Oct.21 & 1130 & 147.2 & 287.1 & $N / A$ & 205.1 & 1844 & 226.8 & 851 & 595 & 70 & 308 & 37. & 43 & 13.9 & .2 .5 & 1589.8 & 221,6 & 1 \\
\hline Oct.21 & 1145 & 147.1 & 287.3 & $N / A$ & 205.2 & 184.0 & 227.0 & 848 & 599 & 37 & 311 & 38.0 & 4.3 & 12.8 & .2 .8 & 15817 & 203.1 & 6 \\
\hline $0<1.21$ & 1200 & 146.6 & 2874 & $N / A$ & 2054 & 1842 & 229.2 & 859 & 582 & 3 & 3 & 38.1 & 4.6 & 15.7 & .34 & 30 & 7 & \\
\hline Oc1.21 & 1215 & 145.8 & 287.6 & N/A & 205.2 & 182.9 & 227.4 & 864 & 594 & 3 & $3 c$ & 37.8 & 4.0 & 14.5 & .2 .7 & 8 & 4 & $\theta$ \\
\hline Oc1.21 & 1230 & 146.4 & 2890 & $N / A$ & 206.7 & 183.8 & 228.2 & 869 & 585 & 375 & 312 & $3 \div 1$ & 4.1 & 150 & -3.3 & 16181 & 242.6 & 34.2 \\
\hline Oct.21 & 1245 & 147.2 & 288.8 & $N / A$ & 206.4 & 1843 & 227.2 & 845 & 600 & 373 & 31 & 37.8 & 40 & 12.5 & 3.3 & 1577.5 & 196.6 & 9 \\
\hline 1.21 & 1300 & 1457 & 288.1 & $N$ & 2054 & 183.3 & 2274 & Q48 & 594 & 378 & 6 & 37.9 & 39 & 132 & 3.8 & 15 & 3 & \\
\hline Oct.21 & 1315 & 142.5 & 287.6 & $N / A$ & 2121 & 1838 & 2300 & 8. & 600 & 389 & 320 & 290 & 40 & 11.4 & -2.8 & 0 & 8 & 3 \\
\hline Oct.21 & 1330 & 140.4 & 286.9 & $N / A$ & 2148 & 182.4 & 221.9 & 850 & 632 & 391 & 322 & 31.1 & 42 & 9.5 & 0.2 & 0.7 & 149.3 & 24.5 \\
\hline Oct-21 & 1345 & 142.2 & 285.7 & $N / A$ & 217.6 & 1834 & 2223 & 847 & 649 & 383 & 321 & 30.6 & 41 & 7.1 & .1 .6 & 15746 & 1122 & 19.2 \\
\hline Oct.21 & 1615 & 144.6 & 286.2 & A & 2116 & 183.4 & 22 & 849 & 668 & 374 & 318 & 36.2 & 38 & 31 & 46 & 15 & & $\pi$ \\
\hline Oct.21 & 1630 & 143.0 & 2864 & N/A & 210.9 & 182.8 & 2244 & 849 & 646 & 385 & 319 & 0 & 4.3 & 6 & -1.8 & 15 & & 8 \\
\hline Ocl.21 & 1645 & 141.8 & 285.5 & $N / A$ & 211.6 & 177.4 & 216.8 & 862 & 636 & 381 & 318 & 30.6 & 42 & 9.8 & -2.1 & 1550.1 & 5 & 6 \\
\hline 1.21 & 1700 & 141.7 & 284.7 & N/A & 215.7 & 175.5 & 214.9 & 864 & 642 & 387 & 322 & 27.0 & 44 & 9.0 & -2.0 & 15361 & 137.6 & 24.8 \\
\hline Ocl.21 & 1800 & 138.8 & 284.3 & N/A & 217.8 & & 21 & 8 & 738 & 388 & 325 & 8 & 52 & .2 .9 & .0 .8 & $2.2 !$ & & .6 .6 \\
\hline Oct.21 & 1815 & 140.3 & 285.1 & & 213.4 & 180.0 & 221.0 & 866 & 653 & 391 & 324 & 33.0 & 49 & 74 & -1.8 & 01 & 5 & 5 \\
\hline Oct.21 & 1830 & 146.7 & 286.9 & $N / A$ & 212.8 & 184.0 & 225.4 & 900 & 657 & 395 & 331 & 34.1 & 44 & 106 & -2.6 & 16784 & 8.7 & 25.5 \\
\hline 1.21 & 1215 & 146.4 & 286.0 & N/A & 212.9 & 185.2 & 227.2 & 865 & 628 & 375 & 316 & 334 & 44 & 110 & .34 & 16239 & 178.5 & 260 \\
\hline Oct.21 & 1900 & 146.8 & 285.8 & $A$ & 2134 & 185.2 & 224.3 & 868 & 630 & 379 & 17 & 33.1 & 8 & 121 & -14 & & 7.3 & \\
\hline Oct.21 & 1915 & 146.4 & 2867 & $N / A$ & $2124^{\circ}$ & 1849 & 2243 & 853 & 618 & 383 & 317 & 34.1 & & 12.1 & .0 .7 & 1598.8 & 33.4 & 276 \\
\hline Oct.21 & 1930 & 146.6 & 2859 & $N / A$ & $212 !$ & 185.6 & 226.4 & 872 & 622 & 386 & 320 & 34.0 & 44 & 130 & .9 .2 & 16405 & 214.0 & 30.6 \\
\hline $0 \mathrm{cl}-21$ & 1945 & 146.2 & 286.1 & A & 2116 & 185.4 & 223.0 & 864 & 626 & 380 & 317 & 33.9 & 4.2 & 12.8 & 0.3 & 16226 & 07.5 & 313 \\
\hline $0 \mathrm{cl}-21$ & 2000 & 147.5 & 2853 & A & 210.9 & 183.8 & 2240 & 858 & 630 & 385 & 317 & 341 & 4.3 & 10.5 & 0.5 & 1597.6 & 8.3 & 24.6 \\
\hline Oct.21 & 2015 & 146.5 & 2850 & $N / A$ & 210.3 & 182.5 & 2206 & 883 & 634 & 380 & 316 & 34.0 & 4.3 & 13.3 & .0 .5 & 16335 & 16.9 & 31.8 \\
\hline Oct.21 & 2030 & 147.3 & 285.3 & $\mathrm{~N} / \mathrm{A}$ & 210.2 & 1820 & 2229 & 886 & 634 & 381 & 318 & 340 & 43 & 12.3 & -21 & 16336 & 201.1 & 29.5 \\
\hline & 45 & 16.8 & 286.2 & & 2101 & 1822 & 2232 & 894 & 630 & 382 & 319 & 34.7 & 4.1 & 137 & 21 & 16509 & 226.7 & 34.2 \\
\hline Oct-21] & 2100 & 146.8 & 286.1 & & 209.5 & & & & 642 & 384 & 318 & 352 & 46 & 131 & .02 & 18 & 8.4 & 289 \\
\hline Oct.21 & 2115 & 146.7 & 285.7 & N/A & 2081 & 183.7 & 223.8 & 894 & 623 & 384 & 312 & 35.8 & 45 & 15.1 & 1.0 & 4.5 & 252.1 & 33.5 \\
\hline Oct.21 & 2130 & 146.6 & 286.1 & $N / A$ & 208.3 & 1856 & 227.2 & 894 & 632 & 384 & 315 & 361 & 46 & 13.5 & .0 .4 & 1681.7 & 227.3 & 29.3 \\
\hline Oc1.21 & 2145 & $14: 0$ & 287.3 & & 208.0 & 1830 & 220.5 & 894 & 625 & 384 & 316 & 36.8 & 44 & 15.7 & 0.8 & 1658.2 & 261.0 & 34.5 \\
\hline Oct.21 & 2200 & 138.3 & 283.0 & & 2062 , & & & 900 & 609 & 374 & 316 & 34.3 & 4.3 & 16.3 & .4 .5 & 1643.7 & 267.4 & 38.9 \\
\hline Oct.21 & 2215 & 140.8 & 281.9 & $N / A$ & 2086 & 182.4 & 2189 & 898 & 626 & 386 & 315 & & 4.3 & 16.4 & 2.1 & 011 & 71.9 & \\
\hline Oct.21 & 2230 & 138.5 & $28 \div 4$ & $N / A$ & 2094 & 180.1 & 2174 & 904 & 640 & 390 & 324 & 319 & 4.1 & 14.6 & 0.3 & 1650.1 & 240.9 & 39.5 \\
\hline Oct.21 & 2245 & 137.5 & 281.4 & $N / A$ & 2072 & 178.1 & 2163 & 889 & 620 & 380 & 309 & 32.8 & 4.0 & 15.4 & 1.3 & 1604.9 & 246.4 & 40.3 \\
\hline
\end{tabular}


C2D IIME INJECTION DATA - AUQUST 17, TO NOVEMBEA 16, 1992

\begin{tabular}{|c|c|c|c|c|c|c|c|c|c|c|c|c|c|c|c|c|c|c|}
\hline Dalo & Time & $\begin{array}{l}\text { Boiler } \\
\text { Load } \\
\text { MN }\end{array}$ & \begin{tabular}{|c|} 
Duct \\
Inlol \\
Tomp of
\end{tabular} & $\begin{array}{l}\text { Avg "C" } \\
\text { Soction } \\
\text { Temp of }\end{array}$ & $\begin{array}{c}\text { Duct } \\
\text { Outlat } \\
\text { Temp of }\end{array}$ & $\begin{array}{l}\text { Flue Oas } \\
\text { Flow in } \\
\text { KSCFM }\end{array}$ & $\begin{array}{l}\text { Flue Oas } \\
\text { Flow Out } \\
\text { KSCFM }\end{array}$ & $\begin{array}{c}502 \\
\ln \mid 01 \\
\mathrm{ppm}\end{array}$ & \begin{tabular}{c|}
$\mathrm{SO} 2$ \\
Oullol \\
$\mathrm{ppm}$
\end{tabular} & $\begin{array}{c}\text { NOx } \\
\text { Inlol } \\
\text { ppm }\end{array}$ & \begin{tabular}{|c|} 
NOx \\
Outlet \\
ppm
\end{tabular} & $\begin{array}{l}\text { Limo } \\
\text { Flow } \\
\text { gpm }\end{array}$ & $\begin{array}{l}\lim \theta \\
\text { Conc } \\
\%\end{array}$ & $\begin{array}{c}502 \\
\text { Hemoval } \\
\%\end{array}$ & $\begin{array}{c}\mathrm{NOx} \\
\text { pemoval } \\
\%\end{array}$ & $\begin{array}{c}\mathrm{SO} \\
\ln \mid \mathrm{Ol} \\
\mathrm{Lb} / \mathrm{HI}\end{array}$ & \begin{tabular}{c|}
$\mathrm{SO} 2$ \\
Hemoved \\
$\mathrm{Lb} / \mathrm{Hf}$
\end{tabular} & \begin{tabular}{|c|}
$\operatorname{Lime}$ \\
Utilization \\
\end{tabular} \\
\hline Oct.21 & 2300 & 137.5 & 278.8 & $N / A$ & 206.2 & 178.2 & 215.8 & 889 & 620 & 380 & 309 & 32.7 & 4.5 & 156 & 1.5 & 1605.8 & 248.8 & 36.3 \\
\hline Oct.21 & 2315 & 138.1 & 280.3 & $N / A$ & 2060 & 1780 & 218.9 & 889 & 636 & 380 & 309 & 33.5 & 38 & 130 & 1.1 & $1621+$ & 21110 & 34.7 \\
\hline Oc1.21 & 2330 & 136.0 & 278.4 & N/A & 2048 & 178.2 & 2140 & 888 & 632 & 380 & 318 & 33.6 & 42 & 14.7 & .0 .3 & 16058 & 235.8 & 35.8 \\
\hline Oc1.21 & 2345 & 141.0 & 278.3 & $N / A$ & 204.8 & 181.8 & 222.3 & 889 & 621 & 380 & 326 & 34.4 & 4.2 & 14.6 & 46 & 1639.2 & 239.6 & 35.6 \\
\hline AVE & PAGES & 144.6 & $284 . ?$ & $N / A$ & 208.4 & 182.9 & 223.8 & 858.5 & 618.4 & 378.4 & 3150 & 346 & 4.2 & 11.7 & .1 .8 & 1580.6 & 186.2 & 27.5 \\
\hline Oct.22 & 0 & 138.1 & 279.3 & N/A & 2034 & 180.4 & 221.6 & 889 & 618 & 380 & 323 & 34.6 & 18 & 14.6 & .4 .3 & 16256 & 237.2 & 35.0 \\
\hline Oct.22 & 15 & 146.3 & 2800 & N/A & 203.9 & 186.8 & 228.5 & 888 & 625 & 380 & 324 & 35.4 & 4.2 & 14.1 & .4 .1 & 1684.2 & 237.5 & 34.3 \\
\hline $0<1.22$ & 30 & 145.1 & 280.3 & N/A & 204.1 & 186.6 & 226.0 & 864 & 623 & 360 & 328 & 358 & 4.2 & 12.3 & .10 .7 & 1634.2 & 201.1 & 28.7 \\
\hline Oct.22 & 45 & 144.1 & 2810 & W/A & 202.2 & 184.1 & 224.4 & 862 & 637 & 362 & 318 & 37.4 & 42 & 9.8 & .7 .1 & 1607.7 & 158.7 & 21.7 \\
\hline Oct.22 & 100 & 144.7 & 2800 & N/A & 200.7 & 182.1 & 2230 & 862 & 625 & 368 & 322 & 381 & 42 & 11.2 & .7 .1 & 15902 & 178.3 & 23.8 \\
\hline Oct.22 & 115 & 144.3 & 279.5 & $N / A$ & 200.7 & 179.3 & 219.1 & 862 & 632 & 374 & 315 & 35.2 & 4.2 & 10.4 & 2.8 & 1565.8 & 162.1 & 23.5 \\
\hline $0 c 1.22$ & 130 & 142.2 & 278.9 & N/A & 2048 & 180.9 & 2197 & 878 & 629 & 361 & 327 & 29.1 & 4.2 & 12.8 & .9 .8 & 1600.1 & 208.3 & 36.5 \\
\hline 001.22 & 145 & 138.5 & 276.6 & $N / A$ & 206.5 & 181.1 & 2192 & 877 & 756 & 376 & 320 & 27.6 & 4.2 & .43 & .3 .2 & 1609.9 & 60.3 & -12.8 \\
\hline Oct.22 & 200 & 140.5 & 276.8 & $N / A$ & 207.3 & 178.1 & 216.5 & 886 & 765 & 376 & 321 & 28.1 & 4.2 & .50 & 3.6 & 1598.8 & .79 .7 & .14 .5 \\
\hline $0 \mathrm{c1.22}$ & 215 & 146.1 & $276 . ?$ & $N / A$ & 200.4 & 184.5 & 220.9 & 881 & 750 & 377 & 317 & 278 & 4.2 & 18 & 0.6 & 16654 & -32.5 & 50 \\
\hline Ocl.22 & 230 & 141.2 & 277.8 & $N / A$ & 220.9 & 178.3 & 2101 & 808 & 783 & 371 & 317 & 190 & 4.2 & 1.6 & 0.7 & 1640.0 & -26.0 & .70 \\
\hline Ocl.22 & 245 & 140.8 & 276.5 & $N / A$ & 206.7 & 178.3 & 216.6 & 804 & 767 & 378 & 318 & 28.0 & 4.2 & -3.1 & -1.8 & 1632.7 & .50 .7 & -9.3 \\
\hline 001.22 & 330 & 141.8 & 276.2 & $N / A$ & 2086 & 178.4 & 215.8 & 806 & 760 & 384 & 324 & 27.7 & 4.2 & .1 .5 & -2.3 & 1638.2 & .23 .8 & -4.4 \\
\hline Oct.22 & 345 & 145.0 & 280.0 & $N / A$ & 210.4 & 188.3 & 224.1 & 803 & 685 & 381 & 325 & 31.0 & 4.2 & 8.7 & .1 .3 & 1723.4 & 167.3 & 27.6 \\
\hline Oct.22 & 400 & 141.9 & 281.7 & $N / A$ & 209.9 & 182.5 & 217.2 & 807 & 683 & 392 & 325 & 31.2 & 4.2 & 10.3 & 1.3 & 16767 & 173.0 & 28.3 \\
\hline Oct.22 & 415 & 138.3 & 281.5 & $N / A$ & 2062 & 178.4 & 213.6 & 803 & 668 & 392 & 322 & 343 & 4.2 & 11.5 & 1.5 & 1632.7 & 187.5 & 27.8 \\
\hline $0 c 1.22$ & 430 & 140.4 & 281.7 & $N / A$ & 204.6 & 180.6 & 220.8 & 907 & 660 & 392 & 327 & 34.6 & $4:$ & 111 & 1.9 & 16602 & 184.1 & 7.2 \\
\hline Oct.22 & 445 & 139.3 & 2813 & $N / A$ & 204.1 & 183.6 & 221.0 & 898 & 643 & 381 & 332 & 35.3 & 4.2 & 138 & 2.2 & 16701 & 2297 & 33.2 \\
\hline Oct.22 & 500 & 140.6 & 2826 & $N / A$ & 203.4 & 1814 & 221.5 & 808 & 654 & 401 & 325 & 35.5 & 4.2 & 12.1 & 0.8 & 1668.5 & 201.2 & 28.9 \\
\hline $0 \mathrm{cl} .22$ & 515 & 1430 & 283.5 & $N / A$ & 204.1 & 1858 & 223.8 & 808 & 641 & 389 & 322 & 36.4 & 4.2 & 15.0 & 0.3 & 1708.9 & 255.8 & 35.8 \\
\hline $0 c 1.22$ & 530 & 138.8 & 283.1 & $N / A$ & 202.0 & 181.4 & 2182 & 908 & 627 & 396 & 322 & 36.0 & 4.2 & 16.8 & 2.0 & 1668.5 & 282.5 & 0.1 \\
\hline Oct.22 & 545 & 143.3 & 2828 & N/A & 2027 & 1828 & 221.1 & 908 & 645 & 396 & 327 & 35.2 & 4.2 & 14.0 & 0.2 & 1681.4 & 2358 & 342 \\
\hline Oct.22 & 600 & 141.0 & 282.8 & $N / A$ & 2027 & 181.7 & 220.8 & 908 & 646 & 400 & 329 & 36.9 & 4.2 & 13.5 & 0.0 & $\mid 1671.2$ & 225.8 & 313 \\
\hline Oct.22 & 615 & 144.2 & 2828 & $\mathrm{~N} / \mathrm{A}$ & 203.2 & 186.8 & 225.1 & 908 & 634 & 387 & 327 & 37.1 & 4.2 & 15.8 & 2.1 & 17181 & 1.5 & 4 \\
\hline Oci.22 & 630 & 143.7 & 282.8 & $N / A$ & 2027 & 184.5 & 220.9 & 908 & 629 & 381 & 320 & 35.8 & 4.2 & 17.0 & 0.6 & 16970 & 288.5 & 41.0 \\
\hline Oct.22 & 645 & 144.3 & 280.6 & $N / A$ & 209.8 & 185.3 & 2210 & 889 & 667 & 378 & 317 & 291 & 4.2 & 10.6 & 0.0 & $1668 \mathrm{~B}$ & 176.7 & 310 \\
\hline Oct.22 & 700 & 1438 & 278.2 & $N / A$ & 207.6 & 183.2 & 2204 & 898 & 625 & 377 & 316 & 30.8 & 4.2 & 16.2 & 0.9 & 16674 & 270.8 & 44.8 \\
\hline Oct.22 & 716 & 1470 & 277.8 & N/A & 2087 & 183.7 & 222.0 & 885 & 640 & 378 & 313 & 30.2 & 4.2 & 12.6 & 0.2 & 1647.0 & 207.8 & 35.2 \\
\hline Oct.22 & 730 & 147.2 & 277.3 & $N / A$ & 207.8 & 1820 & 222.1 & 803 & 643 & 379 & 310 & 28.2 & 4.2 & 12.1 & 0.1 & 1646.5 & 200.0 & 36.2 \\
\hline Ocl.22 & 745 & 147.1 & 276.8 & $N / A$ & 211.6 & 183.1 & 220.8 & 891 & 650 & 378 & 310 & 28.9 & 4.2 & 12.0 & 1.6 & 1653.7 & 198.8 & 35.1 \\
\hline $0<1.22$ & 800 & 146.9 & 278.4 & N/A & 2121 & 180.5 & 2168 & 878 & 650 & 375 & 308 & 285 & 40 & 11.1 & 10 & 1606.4 & 177.8 & 33.5 \\
\hline Oc1.22 & 815 & 146.5 & 277.6 & $N / A$ & 2114 & 1829 & 2216 & 875 & 648 & 381 & 319 & 29.1 & 38 & 10.2 & 1.5 & 16213 & 165.7 & 32.2 \\
\hline Oct.22 & 830 & 1465 & 278.3 & $N / A$ & 210.1 & 180.8 & 218.7 & 888 & 640 & 383 & 317 & 32.1 & 40 & 12.5 & .0 .5 & 1629.2 & 2038 & 31.1 \\
\hline Oc1.22 & B4 5 & 146.8 & 276.8 & $N / A$ & 210.9 & 1838 & 217.8 & 888 & 646 & 383 & 318 & 30.3 & 41 & 13.7 & 1.7 & 16535 & 227.3 & 39.3 \\
\hline Oct.22 & 800 & 146.0 & 277.7 & $N / A$ & 211.6 & 184.1 & 219.5 & 891 & 654 & 388 & 319 & 28.0 & 4.4 & 12.5 & 18 & 1661.8 & 207.8 & 34.8 \\
\hline Oct.22 & 815 & 1460 & 277.7 & $N / A$ & 211.6 & 1841 & 219.5 & 891 & 654 & 388 & 319 & 29.0 & 4.4 & 12.5 & 1.8 & 16618 & 778 & 14.8 \\
\hline Oct.22 & 930 & 146.0 & 277.7 & $N / A$ & 211.6 & 1841 & 219.5 & 891 & 654 & 388 & 318 & 29.0 & 4.4 & 12.5 & 1.9 & 1661.8 & 207.8 & 34.8 \\
\hline 001.22 & 845 & 146.5 & 277.8 & $N / A$ & 2102 & 184.7 & 2213 & 891 & 655 & 397 & 327 & 30.3 & 3.0 & 11.9 & 1.2 & 1667.2 & 1890 & 2 \\
\hline Oct.22 & 1000 & 1464 & 278.5 & $N / A$ & 208.7 & 1838 & 222.8 & 891 & 652 & 396 & 330 & 31.7 & 4.1 & 11.3 & -1.0 & 1659.1 & 187.7 & 0 \\
\hline Oct.22 & 1015 & 146.8 & 279.4 & $N / A$ & 2091 & 186.7 & 2246 & 891 & 662 & 409 & 335 & 31.7 & 3.8 & 10.6 & 1.3 & 1685.3 & 178.1 & 31.8 \\
\hline Oc1.22 & 1045 & 146.4 & 279.1 & N/A & 208.6 & 185.1 & 220.3 & 891 & 666 & 411 & 341 & 31.7 & 4.2 & 11.0 & 1.4 & 1670.8 & 183.6 & 29.6 \\
\hline Oct.22 & 1100 & 146.5 & 279.8 & A & 207.4 & 183.3 & 219.7 & 891 & 661 & 408 & 338 & 32.2 & 3.8 & 11.1 & 06 & 1654.6 & 183.6 & 32.3 \\
\hline Oct.22 & 1115 & 146.0 & 280.2 & $/ A$ & 206.7 & 182.5 & 221.5 & 891 & 650 & 393 & 323 & 32 & 4.1 & 11.5 & 0.4 & 1647.3 & 0 & \\
\hline Oct.22 & 1130 & 146.5 & 281.3 & N/A & 2142 & 183.9 & 217.1 & 904 & 675 & 389 & 320 & 27.0 & 4.0 & 119 & $2 \theta$ & 1684.0 & 199.7 & 8.7 \\
\hline 001.22 & 1145 & 146.6 & 281.3 & A & 217.5 & 183.6 & 221.2 & 804 & 674 & 392 & 318 & 27 & 4.5 & 10.2 & 2.0 & 1681.3 & 171.2 & 0.1 \\
\hline Oct.22 & 1200 & 146.8 & 282.6 & A & 219.1 & 181.6 & 1 & 004 & 686 & 388 & 323 & 26 & 4.1 & 7.6 & 0.0 & 1663.0 & 6 & 7 \\
\hline Oc1.22 & 1215 & 146.2 & 283.1 & A. & 2203 & 182.7 & $218 . ?$ & 904 & 693 & 387 & 319 & 267 & 42 & 8.2 & 1.4 & 30 & 8 & 2 \\
\hline $0 \times 1.22$ & 1230 & 147.2 & 284.6 & N/A & 216.7 & 183.3 & 217.3 & 904 & 693 & 387 & 356 & 31.1 & 4.0 & 0.1 & .8 .8 & 1678.5 & 152.1 & 6.3 \\
\hline Oct.22 & 1415 & 146.6 & 286.6 & $N / A$ & 212.8 & 181.6 & 219.8 & 854 & 619 & 372 & 312 & 34.8 & 3.8 & 12.2 & -1.6 & 1571.1 & 191.7 & 31.2 \\
\hline Oct.22 & 1430 & 146.6 & 287.0 & $N / A$ & 210.9 & 181.5 & 220.5 & 840 & 61 & 372 & 313 & 35.4 & 3.7 & 11.2 & -2.2 & 1543.8 & 172.3 & 8.3 \\
\hline Oct.22 & 1445 & 146.7 & 287.4 & N/A & 210.1 & 182.1 & 218.9 & 848 & 622 & 373 & 309 & 35.0 & 2.8 & 11.8 & 0.6 & 1564.4 & 1851 & 0.8 \\
\hline Oct.22 & 1500 & 146.8 & 288.0 & N/A & 211.1 & 1810 & 219.5 & 835 & 617 & 372 & 306 & 34.2 & 2.8 & 10.4 & 0.0 & 1531.3 & 159.3 & 34.7 \\
\hline Oct.22 & 1515 & 146.7 & 288.0 & & 211.8 & 1807 & 219.5 & 833 & 615 & 369 & 308 & 34.4 & 2.4 & 10.3 & -1.4 & 1525.1 & 1576 & 41.4 \\
\hline Oct.22 & 1530 & 146.5 & 289.1 & $N / A$ & 211.4 & 181.2 & 221.9 & 843 & 605 & 368 & 306 & 31.5 & 4.1 & 12.1 & -20 & 1547.7 & 187.7 & 31.2 \\
\hline Oct.22 & 1545 & 146.4 & 288.9 & $N / A$ & 2107 & 1814 & 2206 & 833 & 600 & 369 & 306 & 36. & $4 !$ & 12.4 & 0.8 & 1531.0 & 180.2 & 7.5 \\
\hline Oct.22 & 1600 & 146.5 & 288.8 & $N / A$ & 210.2 & 1807 & 220.8 & 848 & 595 & 367 & 306 & 35.9 & 40 & 143 & .1 .9 & 1553.3 & 222.4 & 13.3 \\
\hline Oct.22 & 1615 & 146.8 & 19.7 & & 210.9 & 180.9 & 220.5 & 832 & 583 & 374 & 307 & 36.3 & 4.2 & 14.5 & 0.2 & 1524.8 & 221.6 & 31.2 \\
\hline Oc1.22 & 1630 & 146.9 & 292.0 & $N / A$ & 2100 & 1824 & 222.7 & 822 & 109 & 368 & 308 & 37.0 & 3.6 & 13.6 & .2 .3 & 15182 & 206.3 & 3.3 \\
\hline Oct-22 & 1645 & 146.3 & 2813 & $N / A$ & 209.3 & 181.0 & 217.6 & 842 & 577 & 371 & 308 & 37.7 & 4. & 17.7 & 0.1 & 1544.1 & & 37.9 \\
\hline 1.22 & 1700 & 146.5 & 289.4 & & 208.8 & 180.2 & 217.0 & 835 & 578 & 374 & 307 & 35.5 & 41 & 16.6 & 1.2 & 1524.5 & 253.7 & 37.4 \\
\hline Oct-22 & 1715 & 142.8 & & & 208.8 & 181.0 & & 821 & 7 & 373 & 306 & 36.9 & 3.8 & 14.4 & 0.9 & 15056 & 216.1 & 33.1 \\
\hline Jct-22 & 1730 & 147.0 & 288.3 & NIA & 208.1 & 181.7 & 2211 & 831 & 582 & 376 & 305 & 36.7 & 4.2 & 14.8 & 1.1 & 15298 & 226.3 & 31.5 \\
\hline Sct.22 & $174^{5}$ & 146.4 & 287.7 & $N / A$ & 207.9 & 181.0 & 2214 & 844 & 580 & 374 & 310 & 37.2 & 4.4 & 15.9 & .1 .3 & 1548.5 & 246.6 & 32.3 \\
\hline $5 c 1.22$ & 1800 & 146.9 & & & 208.3 & 181.6 & 220.7 & 834 & 580 & 376 & 308 & 34.4 & 4.0 & 15.4 & 0.5 & 1534.5 & 236.7 & 36.9 \\
\hline $5 \mathrm{ct} .22$ & 1815 & 146.5 & 286.2 & N/A & 2090 & 1810 & & 826 & 589 & 368 & 305 & 35.4 & 4.2 & 12.3 & -1.9 & 1514.8 & 186.1 & 26.8 \\
\hline $\mathrm{ct}-22$ & 1830 & 146.4 & 285.6 & N/A & 207.6 & 182.0 & 222.0 & 839 & 579 & 372 & 303 & 35.8 & 3.8 & 15.8 & 0.5 & 1547.1 & 244.8 & 37.7 \\
\hline$c 1.2 ?$ & 1845 & 146.7 & 284.8 & N/A & 207.2 & 183.6 & 224.4 & 833 & 580 & 372 & 305 & 35.9 & 4.5 & 14.8 & .0 .2 & 1549.6 & 2300 & 30.5 \\
\hline ct.-22 & 1900 & 146.8 & 284.4 & N/A & 2078 & 183.4 & 221.2 . & 830 & 587 & 377 & 312 & 34.7 & 4.1 & 14.6 & 0.1 & 1541.4 & 224.9 & 33.9 \\
\hline ct.22 & 1915 & 147.1 & 285.0 & N/A & 207.0 & 1831 & 2180 & 839 & 588 & 381 & 314 & 36.6 & 42 & 160 & 1.9 & 1556.5 & 257.9 & 360 \\
\hline
\end{tabular}


CZD LIME INJECTION DATA - AUGUST 17, TO NOVEMBER 16, 1992

\begin{tabular}{|c|c|c|c|c|c|c|c|c|c|c|c|c|c|c|c|c|c|c|}
\hline Dato & Time & $\begin{array}{l}\text { Bollat } \\
\text { Load } \\
\text { MN }\end{array}$ & \begin{tabular}{|c|} 
Duct \\
Inlel \\
Teinp of
\end{tabular} & $\begin{array}{l}\text { Avg "C" } \\
\text { Section } \\
\text { Temp "F }\end{array}$ & $\begin{array}{l}\text { Ducl } \\
\text { Oullal } \\
\text { Tomp of }\end{array}$ & $\begin{array}{l}\text { Flue Gas } \\
\text { Flow in } \\
\text { KSCFM }\end{array}$ & $\begin{array}{l}\text { Flue Gas } \\
\text { Flow Out } \\
\text { KSCFM }\end{array}$ & $\begin{array}{c}502 \\
\ln |\theta| \\
\mathrm{ppm}\end{array}$ & \begin{tabular}{|c|}
$50 ?$ \\
Oullet \\
ppm
\end{tabular} & $\begin{array}{l}\mathrm{NOx} \\
\ln \mid \mathrm{el} \\
\mathrm{ppm}\end{array}$ & $\begin{array}{c}\mathrm{NOx} \\
\text { Ouflei } \\
\mathrm{ppm}\end{array}$ & $\begin{array}{l}\text { Lime } \\
\text { Flow } \\
\text { opm }\end{array}$ & $\begin{array}{c}\operatorname{lime}_{0} \\
\text { Conc } \\
\%\end{array}$ & $\begin{array}{c}\text { So? } \\
\text { Hemoval } \\
\%\end{array}$ & $\begin{array}{c}\mathrm{NOx} \\
\text { Hemoval } \\
\%\end{array}$ & $\begin{array}{c}\mathrm{SO} 2 \\
\mathrm{Inlol} \\
\mathrm{Lb} / \mathrm{HI}\end{array}$ & \begin{tabular}{c|}
$\mathrm{SO} 2$ \\
Ramaved \\
Lb/Ht
\end{tabular} & $\begin{array}{c}\text { Lime } \\
\text { Uilization } \\
\%\end{array}$ \\
\hline $0 c 1.22$ & 1930 & 1464 & 284.1 & NIA & 2066 & 184.1 & 2223 & 849 & 583 & 383 & $30 B$ & 35.8 & 3.8 & 17.1 & 2.8 & 1584.4 & 270.4 & 42.6 \\
\hline $0 c 1.22$ & 1945 & 147.1 & 2831 & N/A & 2066 & 1833 & 222.5 & 842 & 602 & 380 & $3: 4$ & 35.2 & 3.9 & 13.2 & 0.2 & 1563.7 & 205.7 & 32.2 \\
\hline Ocl.22 & 2000 & 146.6 & 282.1 & $N / A$ & 206.9 & 183.7 & 222.5 & 847 & 600 & 381 & 315 & 35.1 & 44 & 14.2 & .0 .3 & 1577.2 & 223.7 & 31.0 \\
\hline $0,1.22$ & 2015 & 146.5 & 281.8 & N/A & 2066 & 183.8 & 222.0 & 843 & 581 & 380 & 317 & 35.1 & 40 & 15.3 & 0.7 & 15699 & 239.6 & 36.7 \\
\hline $0 c 1.22$ & 2030 & 146.8 & 2816 & $N / A$ & 205.9 & 183.6 & 222.5 & 833 & 579 & 385 & 315 & 35.2 & 42 & 15.8 & 10 & 1549.6 & 244.3 & 35.4 \\
\hline 001.22 & 2045 & 1448 & 2818 & N/A & 2047 & 186.0 & 225.8 & 848 & 582 & 377 & 310 & 38.2 & 4.0 & 16.7 & 0.1 & 15979 & 266.6 & 37.6 \\
\hline 001.22 & 2100 & 146.7 & 279.8 & $N / A$ & 205.2 & 184.6 & 225.8 & 848 & 582 & 371 & 307 & 35.6 & 4.2 & 161 & .1 .4 & 15858 & 254.6 & 36.5 \\
\hline Oct.22 & 2115 & 1466 & 279.8 & $N / A$ & 204.8 & 184.0 & 224.2 & 848 & 582 & 410 & 309 & 352 & 4.1 & 163 & 81 & 1580.7 & 257.8 & 38.3 \\
\hline Oct.22 & 2130 & 146.1 & 2800 & $N / A$ & 206.2 & 183.8 & 223.1 & 868 & 595 & 387 & 304 & 33.8 & 4.2 & 16.9 & 4.7 & 16190 & 273.2 & 41.2 \\
\hline $0 c 1.22$ & 2145 & 146.7 & 279.4 & N/A & 205.3 & 179.2 & 218.1 & 873 & 621 & 384 & 309 & 32.8 & 40 & 131 & 1.4 & 15858 & 207.4 & 34.0 \\
\hline $0,1.22$ & 2200 & 146.7 & 279.1 & N/A & 204.6 & 178.0 & 218.0 & 880 & 610 & 383 & 308 & 33.6 & 37 & 15.2 & 1.3 & 1586.7 & 243.4 & 42.1 \\
\hline $0 c 1.22$ & 2215 & 146.4 & 280.0 & NIA & 2053 & 180.5 & 220.2 & 875 & 617 & 382 & 309 & 33.2 & 4.2 & 14.0 & 1.1 & 1600.9 & 223.5 & 34.4 \\
\hline Oct.22 & 2230 & 146.8 & 279.8 & N/A & 205.7 & $181 . ?$ & 222.7 & 874 & 609 & 381 & 313 & 33.8 & 4.3 & 14.6 & .0 .7 & 1608.8 & $234 . \theta$ & 34.5 \\
\hline Oct.22 & 2245 & 146.4 & 278.3 & $N / A$ & 2062 & 180.7 & 2201 & 869 & 615 & 379 & 311 & 32.7 & 4.1 & 13.7 & 0.1 & 1590.8 & 218.5 & 350 \\
\hline Oct.22 & 2300 & 146.4 & 279.3 & $N / A$ & 2068 & 182.9 & 222.8 & 869 & 615 & 379 & 311 & 32.7 & 43 & 13.7 & 0.1 & 1610.2 & 221.0 & 33.7 \\
\hline $0 c 1.22$ & 2315 & 147.8 & 278.3 & N/A & 205.6 & 182.8 & 221.5 & 869 & 627 & 378 & 311 & 36.7 & 42 & 12.5 & 0.6 & 1609.3 & 201.3 & 280 \\
\hline 001.22 & 2330 & 146.4 & 277.8 & N/A & 207.5 & 181.5 & 220.1 & 869 & 606 & 378 & 310 & 31.3 & 4.3 & 15.4 & 0.8 & 1597.9 & 245.6 & 38.1 \\
\hline 001.22 & 2345 & 146.4 & 277.6 & N/A & 200.6 & 181.6 & 2220 & $86 \theta$ & 628 & 323 & 322 & 32.7 & 3.8 & 11.7 & -21.7 & 1598.7 & 186.4 & 32.3 \\
\hline$A \sqrt{E}$ & RAGES & 145.2 & 2818 & N/A & 2081 & 182.5 & 220.8 & 872.6 & 634.8 & 380.6 & 316.8 & 33.0 & 4.1 & 12.1 & .0 .8 & 1613.3 & 193.9 & 30.6 \\
\hline Oct.23 & 0 & 146.6 & 277.7 & N/A & 208.2 & 180.1 & 218.6 & 869 & 620 & 337 & 318 & 31.6 & 4.1 & 13.3 & 14.6 & 1585.5 & 2114 & 350 \\
\hline $0 \mathrm{cl} \cdot 23$ & 15 & 146.2 & 2786 & $N / A$ & 2103 & 180.3 & 2188 & 815 & 640 & 334 & 318 & 28.6 & 4.2 & 49 & -15.6 & 14920 & 737 & 12.7 \\
\hline $0 \mathrm{Cl}-23$ & 30 & 146.6 & 278.7 & $N / A$ & 210.9 & 1819 & 2200 & 828 & 622 & 357 & 320 & 30.3 & 38 & 9.1 & .84 & 15251 & 138.8 & 252 \\
\hline Oct.23 & 45 & 1470 & 276.9 & $N / A$ & 2105 & iके 1 & 2189 & 818 & 619 & 360 & 346 & $3 c .2$ & 4.2 & 9.2 & .15 .5 & 15111 & 1384 & 23.4 \\
\hline Oct.23 & 100 & 146.6 & 2789 & $N / A$ & 211.1 & 1834 & 2220 & 839 & 623 & $36 !$ & 325 & 30.4 & 41 & 101 & -9.2 & 15581 & 157.0 & 27.0 \\
\hline Oct.23 & 115 & 146.8 & 2787 & $N / A$ & 212.1 & 183.2 & 220.8 & 814 & 615 & 369 & 316 & 29.6 & 4.1 & 8.8 & 3.2 & 15100 & 133.3 & 23.6 \\
\hline $0 \mathrm{ct} .23$ & 130 & 1468 & 277.3 & $N / A$ & 2197 & 182.3 & 222.2 & 828 & 618 & 377 & 317 & 291 & 44 & 9.0 & 2.5 & 15294 & 1383 & 3.1 \\
\hline Oct.23 & 600 & 1461 & 2768 & $N / A$ & 2138 & 185.1 & 221.3 & 861 & 652 & 372 & 311 & 284 & 5.5 & 9.4 & 0.1 & 1614.5 & 152.0 & 20.7 \\
\hline Ocl.23 & 615 & 146.6 & 276.6 & $N / A$ & 213.9 & 183.8 & 2176 & 853 & 655 & 372 & 314 & 27.8 & 5.1 & 9.0 & .0 .2 & 15883 & 14.36 & 1.5 \\
\hline 0.1 .23 & 630 & 147.0 & 276.6 & N/A & 2111 & 184.0 & 219.8 & 841 & 641 & 375 & 312 & 30.3 & 4.8 & 8.8 & 0.6 & 1566.9 & 138.8 & 0.6 \\
\hline Oct.23 & 645 & 146.8 & 2774 & N/A & 210.5 & 184.4 & 2224 & 836 & 638 & 376 & 314 & 305 & 48 & 7.9 & .0 .8 & 1561.9 & 1236 & 8.0 \\
\hline $\mathrm{Oct}-23$ & 700 & 146.4 & 277.6 & $N / A$ & 2104 & 1847 & 221.1 & 848 & 639 & 378 & 316 & 30.5 & 4.7 & 110 & 0.2 & 15895 & 175.3 & 6.2 \\
\hline Oct.23 & 715 & 146.1 & 277.6 & $N / A$ & 2103 & 1857 & 2219 & 843 & 634 & 384 & 322 & 30.6 & 46 & 101 & -0.3 & 15861 & 1600 & 4.3 \\
\hline Oct.23 & 730 & 146.4 & 277.6 & $N / A$ & 2100 & 186.2 & 221.0 & 860 & 632 & 382 & 322 & 311 & 4.3 & 12.8 & 2.4 & 1623.2 & 208.5 & 3.4 \\
\hline Oc1.23 & 745 & 144.9 & 278.0 & $N / A$ & 2093 & 1859 & 2206 & 831 & 617 & 370 & 318 & 31.6 & 4.7 & 11.8 & -1.8 & 1564.0 & 184.8 & 6.6 \\
\hline Oc1.23 & 800 & 146.2 & 2782 & $N / A$ & 209.7 & 187.2 & 2230 & 827 & 609 & 383 & 322 & 31.4 & 4.5 & 12.2 & .0 .1 & 15686 & 181.7 & 90 \\
\hline Oct.23] & 815 & 146.2 & 278.2 & $N / A$ & 2097 & 187.2 & 2230 & 827 & 609 & 383 & 322 & 31.4 & 4.5 & 12.2 & .0 .1 & 1568.6 & 191.7 & 9.0 \\
\hline Oct.23 & 830 & 146.5 & 277.9 & NIA & 2043 & 1843 & 222.9 & 833 & 594 & 379 & 316 & 35.2 & 42 & 13.7 & .0 .7 & 1554.5 & 2133 & 9 \\
\hline Oct.23 & 845 & 146.8 & 277.4 & N/A & 204.5 & 1851 & 221.2 & 841 & 595 & 378 & 314 & 34.7 & $4 ?$ & 15.4 & 0.8 & 15772 & 242.8 & 57 \\
\hline $\mathrm{Oct} \cdot 23$ & 800 & 146.4 & 276.9 & $N / A$ & 202.7 & 1832 & 2222 & 840 & 587 & 376 & 306 & 34.5 & 42 & 151 & 1.3 & 15583 & 2358 & 4.9 \\
\hline $0,1.23$ & 915 & 145.9 & 277.3 & $N / A$ & 2033 & 185.1 & 2213 & 832 & 600 & 376 & 315 & 34.1 & 4.1 & 13.8 & .0 .2 & 15603 & 215.3 & 30 \\
\hline $0<1.23$ & 830 & 146.5 & 276.7 & $N / A$ & 2026 & 1826 & 221.7 & 850 & 600 & 373 & 310 & 34.1 & 3.8 & 14.3 & -1.0 & 15724 & 2249 & 3 \\
\hline Oc1.23 & 845 & 146.7 & 2778 & $N / A$ & 2026 & 182.8 & 224.0 & 841 & 600 & 376 & 311 & 344 & 0 & 126 & .14 & 1558.5 & 195.8 & 6 \\
\hline Oct.23 & 1000 & 146.6 & 278.0 & N/A & 2028 & 182.8 & 221.1 & 842 & 591 & 372 & 310 & 34.5 & 4.1 & 151 & .0 .8 & 1550.4 & 235.7 & 5.8 \\
\hline Oct.23 & 1015 & 146.7 & 278.3 & $N / A$ & 2032 & 182.0 & 223.9 & 836 & 596 & 374 & 309 & 34.6 & 3.8 & 12.2 & $\cdot 1.9$ & 1541.6 & 188.6 & 0 \\
\hline Oct.23 & 1030 & 1464 & 2790 & N/A & 2046 & 1830 & 224.9 & 845 & 584 & 374 & 309 & 33.8 & 37 & 15.1 & .1 .4 & 1567.5 & 237.0 & 40.8 \\
\hline $0 c 1.23$ & 1045 & 146.8 & 278.8 & N/A & 2051 & 182.3 & 221.4 & 841 & 611 & 374 & 311 & 336 & 3.0 & 11.7 & .1 .2 & 1552.4 & 1821 & .0 \\
\hline Oc1.23 & 1100 & 146.7 & 2800 & N/A & 203.4 & 183.5 & 222.6 & 851 & 588 & 371 & 312 & 35.8 & 2.6 & 16.2 & 2.0 & 1582.9 & 2558 & 9.5 \\
\hline Oct.23 & 1115 & 146.8 & 281.5 & N/A & 2036 & 1836 & $\because 22.3$ & 844 & 587 & 375 & 313 & 35.9 & 4.8 & 14.3 & 0.9 & 1570.8 & 225.2 & 27.9 \\
\hline Oct.23 & 1130 & 143.1 & 282.8 & $N / A$ & 203.7 & 183.1 & 223.7 & 834 & 595 & 370 & 310 & 362 & 4.2 & 12.9 & 2.5 & 15472 & 198.9 & 28.1 \\
\hline Oct.23 & 1145 & 145.1 & 283.7 & N/A & 203.4 & 180.3 & 217.1 & 837 & 580 & 362 & 307 & 36.3 & 4.6 & 16.5 & 2.1 & 1528.1 & 252.5 & 4 \\
\hline Oc1.23 & 1200 & 145.3 & 284.1 & N/A & 2042 & 182.6 & 224.0 & 834 & 582 & 373 & 316 & 36.2 & 40 & 14.4 & .4 .1 & 1543.0 & 222.3 & 33.0 \\
\hline Oct.23 & 1215 & 145.3 & 284.5 & N/A & 2052 & 181.5 & 24 & 834 & 592 & 368 & 308 & 34.9 & 4.4 & 12.9 & -26 & 1532.8 & 197.9 & 27.6 \\
\hline Oct.23 & 1230 & 145.7 & 285.2 & N/A & 2086 & 179.8 & 21.3 & 842 & 598 & 367 & 307 & 33.6 & 4.1 & 125 & .2 .9 & 0 & 192.4 & 30.0 \\
\hline Oct.23 & 1245 & 145.7 & 286.4 & N/A & 207.1 & 181.3 & 217.6 & 842 & 586 & 367 & 306 & 35.6 & 4.3 & 149 & .0 .1 & 15458 & 230.8 & 32.3 \\
\hline Oc1.23 & 1300 & 145.9 & 288.2 & N/A & 206.9 & 180.2 & 2194 & 846 & 582 & 365 & 310 & 364 & 3.8 & 14.9 & .3 .3 & 1545.3 & 2286 & 357 \\
\hline Oc1.23 & 1315 & 145.4 & 289.4 & A & 206.4 & 178.8 & 1.5 & 843 & 587 & 365 & 307 & 381 & 4.3 & 138 & 43 & 1527.2 & 210.0 & 27.5 \\
\hline 041.23 & 1330 & 145.7 & 289.8 & $N / A$ & 204.8 & 178.4 & 162 & 845 & 581 & 6 & 19 & 38.6 & 39 & 160 & 3.5 & 15281 & 243.8 & 34.8 \\
\hline Oct.23 & 1345 & 145.8 & 290.7 & N/A & 205.3 & 1778 & 2160 & 842 & 582 & 367 & $30 ?$ & 385 & 44 & 16.3 & .11 & 1515.9 & 247.2 & 31.3 \\
\hline Oct.23 & 1400 & 1458 & 280.7 & & 205.3 & 177.8 & 2150 & 842 & 582 & 367 & 307 & 38.5 & 44 & 16.3 & -1.1 & 15159 & 247.2 & 31.3 \\
\hline Oct.23 & 1415 & 145.8 & 280.7 & & 205.3 & 177.8 & & 842 & 582 & 367 & 307 & 38.5 & 44 & 16.3 & .1 .1 & 1515.9 & 247.2 & 31.3 \\
\hline Oct.23 & 1430 & 145.8 & 290.7 & $N / A$ & 2053 & 177.8 & 50 & 842 & 582 & 367 & 307 & 38.5 & 4.4 & 16.3 & .1 .1 & 1515.9 & 247.2 & 31.3 \\
\hline Oct-23 & 1445 & 145.5 & 2912 & $N / A$ & 2055 & 176.7 & 2187 & 850 & 561 & 359 & 303 & 40.8 & 4.1 & 18.3 & .44 & 15216 & 278.5 & 35.7 \\
\hline Oc1.23 & 1500 & 145.8 & 290.7 & N/A & 2053 & 177.8 & 2150 & 842 & 582 & 367 & 307 & 38.5 & 4.4 & 16.3 & .1 .1 & 15159 & 2472 & 31.3 \\
\hline $0 c 1.23$ & 1515 & 145.8 & 0.7 & & 205.3 & 1778 & 215.0 & 842 & 582 & 367 & 307 & 38.5 & 44 & 16.3 & -1.1 & 15159 & 247.2 & 31.3 \\
\hline Oct.23 & 1530 & 145.8 & 290.7 & $N / A$ & 205.3 & 1778 & 2150 & 842 & 582 & 367 & 307 & 385 & 4.4 & 163 & .1 .1 & 1515.9 & 247.2 & 313 \\
\hline Oc1.23 & 1545 & 145.3 & 292.0 & N/A & 208.3 & 174.8 & 216.2 & 824 & 571 & 360 & 303 & 37.8 & 39 & 14.3 & .38 & 1ren 2 & 208.7 & 30.4 \\
\hline & 1600 & 145.9 & 291.7 & & 211.4 & 174.2 & 214.2 & 830 & 583 & 361 & 301 & 33.6 & 43 & 135 & .2 .4 & 1464.1 & 197.9 & 29.4 \\
\hline Oct.23 & 1615 & 146.0 & 1.4 & & 211.6 & 174.7 & & 812 & $57 ?$ & 332 & 304 & 34.1 & 44 & 12.7 & -12.5 & 1437.3 & 183.2 & 26.2 \\
\hline Oct.23 & 1630 & 147.0 & 281.0 & $N / A$ & 210.5 & 1745 & 214.3 & 815 & 584 & 358 & 300 & 36.2 & 4.4 & 11.9 & 2.8 & 1440.9 & 172.0 & 23.1 \\
\hline Oc1.23 & 1645 & 146.8 & 291.1 & N/A & 209.4 & 175.3 & 215.8 & 800 & 567 & 360 & 306 & 36.8 & 4.4 & 128 & -4.9 & 1420.9 & 181.7 & 240 \\
\hline Oct.23 & 1700 & 147.1 & 281.8 & N/A & 209.3 & 1749 & 216.5 & 803 & 570 & 360 & 304 & 36.9 & 44 & 12.1 & .4 .3 & 14221 & 171.8 & 22.7 \\
\hline Oct.23 & 1715 & 146.5 & & & 208.2 & 173.8 & 216.5 & 806 & 552 & 364 & 306 & 36.9 & 4.1 & 147 & -4.5 & 14184 & 2087 & 296 \\
\hline Oct.23 & 1730 & 146.8 & 2900 & N/A & 2155 & 176.0 & 214.7 & 802 & 604 & 362 & 308 & 31.6 & 43 & 8. 1 & .38 & 1429.3 & 115.6 & 182 \\
\hline $0 \mathrm{cl} \cdot 23$ & 1745 & 146.4 & 288.9 & $N / A$ & 213.3 & 175.6 & 2133 & 806 & 562 & 355 & 338 & 330 & 43 & 153 & .15 .5 & 14331 & 218.6 & 330 \\
\hline
\end{tabular}


CZD LIME INJECTION DATA - AUGUST 17, TO NOVEMBER 16, 1992

\begin{tabular}{|c|c|c|c|c|c|c|c|c|c|c|c|c|c|c|c|c|c|c|}
\hline Dale & Time & $\begin{array}{l}\text { Boller } \\
\text { Load }\end{array}$ & $\begin{array}{l}\text { Duct } \\
\text { Inlo| }\end{array}$ & $\begin{array}{l}\text { Avg "C" } \\
\text { Section }\end{array}$ & $\begin{array}{l}\text { Duct } \\
\text { Outlet }\end{array}$ & $\begin{array}{l}\text { Flue Gas } \\
\text { Flow In }\end{array}$ & $\begin{array}{l}\text { Flue Gas } \\
\text { Flow Out }\end{array}$ & $\begin{array}{l}50 ? \\
\text { Inle! }\end{array}$ & \begin{tabular}{|l|}
$\mathrm{SO} 2$ \\
Sutlol
\end{tabular} & $\begin{array}{l}\text { Nax } \\
\text { Inloi }\end{array}$ & $\begin{array}{c}\text { NOx } \\
\text { Outlel }\end{array}$ & $\begin{array}{l}\text { Lime } \\
\text { Flow }\end{array}$ & $\begin{array}{l}\text { Lime } \\
\text { Conc }\end{array}$ & $\begin{array}{c}802 \\
\text { hemoval }\end{array}$ & $\begin{array}{c}\text { NOx } \\
\text { Hemoval }\end{array}$ & $\begin{array}{l}802 \\
\ln |\theta|\end{array}$ & \begin{tabular}{|c|}
802 \\
Hemoved
\end{tabular} & Lime \\
\hline & & $M N$ & Tomp of & Temp of & $\begin{array}{l}\text { Oullot } \\
\text { Temp of }\end{array}$ & KSCFM & KSCFM & ppm & $\begin{array}{l}\text { Ouflel } \\
\mathrm{ppm}\end{array}$ & $\begin{array}{l}\ln 101 \\
\text { ppm }\end{array}$ & $\frac{\text { Outlel }}{\mathrm{ppm}}$ & $\mathrm{gpm}$ & $\%$ & $\%$ & $\%$ & I b/Ht & $\mathrm{Lb} / \mathrm{Hi}$ & $\frac{120}{\%}$ \\
\hline $0 \mathrm{c1.23}$ & 1800 & 146.3 & 2800 & N/A & 213.8 & 177.3 & 217.3 & 810 & 577 & 362 & 316 & 34.8 & 4.2 & 12.7 & 7.0 & 1454.2 & 184.8 & 27.1 \\
\hline $0 c 1.23$ & 1815 & 1467 & 287.7 & $N / A$ & 216.3 & 175.8 & 215.5 & 804 & 584 & 364 & 307 & 30.5 & 4.0 & 110 & .3 .4 & 1432.1 & 157.2 & 27.7 \\
\hline $0 c t-23$ & 1830 & 146.4 & 287.1 & N/A & 2160 & 176.4 & 214.6 & 786 & 588 & 363 & 300 & 30.8 & 3.8 & 9.0 & .3 .3 & 14050 & 126.7 & 23.3 \\
\hline $0 c t .23$ & 1845 & 146.6 & 285.5 & N/A & 216.5 & 176.3 & 216.2 & 783 & 501 & 371 & 318 & 30.3 & 38 & 7.4 & 5.2 & 1397.9 & 103.5 & 18.8 \\
\hline $0 \mathrm{cl} \cdot 23$ & 1800 & 146.8 & 286.0 & N/A & 215.7 & 177.8 & 213.9 & 780 & 593 & 372 & 316 & 31.7 & 4.0 & 8.6 & 2.3 & 1422.3 & 136.3 & 23.1 \\
\hline $0 c 1.23$ & 1015 & 146.4 & 285.0 & N/A & 2141 & 177.0 & 2168 & 787 & 568 & 371 & 317 & 30.8 & 40 & 11.6 & 4.8 & 1410.6 & 163.0 & 28.3 \\
\hline $0 \mathrm{ct} \cdot 23$ & 1830 & 146.3 & 2850 & $N / A$ & 216.9 & 179.0 & 218.8 & 786 & 606 & 363 & 323 & 30.4 & 4.1 & 5.7 & .0 .0 & 1424.8 & 81.6 & 14.0 \\
\hline $0 c t .23$ & 1045 & 146.1 & 282.5 & $N / A$ & 2164 & 1768 & 2174 & 785 & 602 & 364 & 310 & 27.7 & 3.8 & 5.8 & .7 .8 & 1407.2 & 81.4 & 16.2 \\
\hline $0 \mathrm{cl} \cdot 23$ & 2000 & 146.8 & 281.4 & $N / A$ & 2182 & 177.6 & 2176 & 788 & 608 & 372 & 319 & 27.0 & 4.2 & 5.2 & 60 & 1417.2 & 73.7 & 13.5 \\
\hline Oct-23 & 2015 & 146.4 & 278.8 & N/A & 2179 & 1763 & 219.4 & 784 & 612 & 360 & 313 & 29.0 & 44 & 2.8 & - B. 5 & 1399.7 & 39.5 & 6.6 \\
\hline $0 \mathrm{ct}-23$ & 2030 & 147.1 & 280.7 & N/A & 2139 & 178.5 & 217.3 & 786 & 567 & 359 & 307 & 28.1 & 41 & 12.1 & .4 .1 & 1420.8 & 172.5 & 32.1 \\
\hline $0 \mathrm{ct} \cdot 23$ & 2045 & 146.3 & 281.6 & N/A & 2140 & 179.5 & 214.7 & 788 & 600 & 360 & 336 & 30.5 & 4.4 & 8.8 & .11 .7 & 14324 & 127.4 & 20.3 \\
\hline $0 c 1.23$ & 2100 & 146.2 & 281.0 & $N / A$ & 211.8 & 178.7 & 218.3 & 788 & 601 & 368 & 316 & 32.5 & 4.1 & 67 & .5 .1 & 14260 & 858 & 15.4 \\
\hline Oct.23 & 2115 & 146.0 & 280.8 & N/A & 211.3 & 178.9 & 216.3 & 788 & $58 \theta$ & 368 & 308 & 34.5 & 3.8 & 9.5 & .1 .2 & 1427.6 & 135.8 & 21.7 \\
\hline $0 \mathrm{Cl} \cdot 23$ & 2130 & 146.1 & 279.8 & N/A & 211,3 & 179.7 & 218.6 & 837 & 585 & 380 & 303 & 32.8 & 3.8 & 14.5 & 2.5 & 15230 & 220.5 & 37.0 \\
\hline $0<1.23$ & 2145 & 146.1 & 2800 & $N / A$ & 209.6 & 178.8 & 219.6 & 831 & 580 & 376 & 307 & 33.8 & 4.2 & 148 & 0.2 & 15138 & 223.5 & 33.8 \\
\hline $0 \mathrm{Cl} \cdot 23$ & 2200 & 147.3 & 279.8 & $N / A$ & 209.0 & 178.5 & 215.8 & 832 & 590 & 375 & 307 & 32.4 & 4.5 & 14.6 & 1.6 & 1512.2 & 221.3 & 32.5 \\
\hline 001.23 & 2215 & 146.4 & 279.8 & $N / A$ & 210.8 & 180.2 & 220.4 & 837 & 608 & 377 & 312 & 35.2 & 4.2 & 11.0 & -1.5 & 1627.3 & 168.7 & 24.5 \\
\hline Oct.23 & 2230 & 146.7 & 270.6 & $N / A$ & 210.4 & 178.7 & 2196 & 834 & 615 & 381 & 314 & 32.6 & 3.7 & 8.8 & .0 .6 & 1597.6 & 1483 & 26.4 \\
\hline Oct.23 & 2245 & 146.4 & 279.4 & $N / A$ & 208.6 & 179.1 & 218.2 & 834 & 611 & 384 & 318 & 32.5 & 4.1 & 10.6 & .1 .2 & 15125 & 160.8 & 250 \\
\hline Oct.23 & 2300 & 146.8 & 279.0 & $N / A$ & 208.0 & 180.2 & 218.8 & 834 & 611 & 384 & 310 & 32.4 & 4.2 & 10.8 & .0 .8 & 15218 & 165.8 & 261 \\
\hline AVE & AAGES & 146.3 & 283.1 & $N / A$ & 2092 & 180.2 & 218.9 & 826.4 & 5977 & 368.3 & 312.8 & 33.5 & 4.2 & 12.1 & .3 .3 & 15001 & 183.5 & 27.8 \\
\hline Nov. 2 & 1615 & 1220 & 280.1 & $N / A$ & 214.6 & 150.1 & 174.7 & 795 & 612 & 373 & 324 & 26.9 & 62 & 10.4 & .1 .1 & 12000 & 125.9 & 160 \\
\hline Nov. 2 & 1630 & 121.2 & 281.5 & $N / A$ & 2076 & 152.6 & 178.3 & 780 & 599 & 365 & 327 & 31.3 & 6.1 & 9.7 & 5.2 & 12054 & 116.5 & 12.9 \\
\hline Nov. 2 & 1730 & 1216 & 278.8 & $N / A$ & 199.6 & 154.6 & 184.6 & 783 & 578 & 384 & 318 & 30.7 & 5.8 & 11.9 & 1.0 & 1225.8 & 145.7 & 17.4 \\
\hline Nov.? & 1745 & 121.7 & 282.0 & N/A & 189.4 & 156.6 & 186.4 & 785 & 562 & 380 & 322 & 333 & 54 & 148 & .0 .8 & 1245.7 & 184.3 & 21.8 \\
\hline Nov. 2 & 1800 & 121.2 & 282.8 & $N / A$ & 1977 & 154.4 & 185.4 & 776 & 567 & 381 & 316 & 34.6 & 5.4 & 12.4 & 02 & 1214.1 & 150.0 & 17.1 \\
\hline Nov. 2 & 1815 & 123.1 & 280.2 & $N / A$ & 1980 & 158.1 & 189.3 & 761 & 521 & 378 & 317 & 34.2 & 4.8 & 181 & 0.1 & 1219.2 & 220.7 & 28.7 \\
\hline Nov. 2 & 1830 & 1215 & 282.1 & N/A & 1997 & 157.8 & 1868 & 756 & 545 & 381 & 317 & 33.0 & 5.7 & 14.8 & 1.5 & 1209.6 & 179.1 & 20.2 \\
\hline Nov.2 & 1845 & 121.7 & 283.7 & $N / A$ & 198.8 & 157.2 & 1858 & 789 & 530 & 376 & 321 & 35.2 & 5.3 & 20.5 & .1 .0 & 12560 & 257.5 & 29.4 \\
\hline Nov. 2 & 1800 & 122.5 & 282.7 & NIA & 1990 & 154.8 & 183.5 & 784 & 551 & 377 & 315 & 332 & 5.1 & 168 & 1.2 & 1230.6 & 2062 & 26.0 \\
\hline Nov. 2 & 1915 & 122.1 & 282.1 & $\mathrm{~N} / \mathrm{A}$ & 1980 & 158.1 & 187.4 & 772 & 530 & 381 & 323 & 339 & 5.4 & 18.6 & 0.5 & 12368 & 230.3 & 26.8 \\
\hline Nov. 2 & 1830 & 1213 & 283.5 & $N / A$ & 199.1 & 152.9 & 183.1 & 789 & 560 & 362 & 314 & 33.3 & 47 & 137 & .3 .8 & 1221.7 & 167.0 & 22.8 \\
\hline Nov. 2 & 1945 & 123.3 & 281.3 & $\mathrm{~N} / \mathrm{A}$ & 1991 & 155.5 & 182.0 & 732 & 533 & 367 & 313 & 33.3 & 48 & 14.9 & 0.3 & 11536 & 171.7 & 22.5 \\
\hline Nov. 2 & 2000 & 122.2 & 282.2 & N/A & 189.5 & 160.8 & 190.7 & 753 & 522 & 363 & 333 & 344 & 52 & 17.8 & .8 .8 & 1227.7 & 218.9 & 26.1 \\
\hline Nov. 2 & 2015 & 119.8 & 283.5 & N/A & 198.8 & 158.2 & 188.3 & 734 & 534 & 378 & 306 & 341 & 54 & 13.5 & 4.1 & 1176.0 & 158.2 & 18.3 \\
\hline Nov. 2 & 2030 & 120.6 & 281.4 & $N / A$ & 187.4 & 151.5 & 180.8 & 760 & 544 & 354 & 319 & 332 & 48 & 14.5 & .78 & 1166.0 & 168.4 & 22.7 \\
\hline Nov. 2 & 2045 & 1213 & 2781 & N/A & 186.0 & 152.8 & 183.1 & 760 & 518 & 356 & 325 & 34.1 & 52 & 18.3 & $\cdot 8.5$ & 11760 & 215.8 & 259 \\
\hline Nov. 2 & 2100 & 121.6 & 278.5 & N/A & 196.9 & 151.0 & 180.1 & 760 & 516 & 398 & 397 & 320 & 51 & 18.0 & 51 & 11621 & 221.3 & 28.9 \\
\hline Nov- 2 & 2115 & 120.2 & 279.9 & $N / A$ & 187.5 & 153.8 & 185.6 & 760 & 551 & 389 & 308 & 32.8 & 4.9 & 12.5 & 4.2 & 1183.7 & 148.5 & 19.7 \\
\hline Nov. 2 & 2130 & 120.8 & 279.2 & $N / A$ & 1967 & 149.9 & 180.6 & 817 & 541 & 374 & 315 & 32.6 & 5.2 & 20.2 & -1.4 & 1240.8 & 250.9 & 16 \\
\hline Nov-2 & 2145 & 120.8 & 278.5 & $N / A$ & 196.2 & 150.5 & 180.4 & 788 & 535 & 376 & 306 & 32.6 & 5.3 & 18.6 & 2.5 & 1201.0 & 223.1 & 7.5 \\
\hline Nov. 2 & 2200 & 119.8 & 279.0 & $N / A$ & 186.9 & 1510 & 180.8 & 777 & 544 & 370 & 306 & 31.9 & 5.1 & 16.1 & 0.8 & 11881 & 1811 & 5.1 \\
\hline Nav.2 & 2215 & 120.1 & 278.5 & $N / A$ & 196.8 & 147.8 & 177.3 & 808 & 533 & 364 & 315 & 317 & 4.9 & 20.8 & .39 & 1210.0 & 253.4 & 4.8 \\
\hline Nov- 2 & 2230 & 120.6 & 280.1 & $N / A$ & 197.6 & 150.3 & 181.0 & 770 & 543 & 372 & 315 & 321 & 51 & 15.0 & 2.1 & 11720 & 76.2 & 0 \\
\hline Nov. 2 & 2245 & 1197 & 280.3 & $N / A$ & 198.1 & 154.7 & 186.0 & 778 & 524 & 367 & 314 & 31.8 & 4.9 & 19.0 & 2.8 & 1210.6 & 231.8 & 1.8 \\
\hline Nov. 2 & 2300 & 120.5 & $277 . \theta$ & $N / A$ & 188.3 & 150.1 & 1807 & 778 & 524 & 367 & 314 & 30.4 & 5.0 & 18.8 & .2 .8 & 11833 & 2238 & 1.4 \\
\hline Nov-2 & 2315 & 120.7 & 271.7 & N/A & 199.0 & 151.8 & 182.7 & 778 & 535 & 367 & 326 & 30.6 & 4.8 & 17.3 & .6 .7 & 1196.7 & 207.3 & 0.2 \\
\hline Nov. 2 & 2330 & 120.3 & 278.8 & NA & 200.4 & 156.8 & 187.7 & 778 & 518 & 367 & 312 & 30.4 & 4.8 & 20.3 & .1 .7 & 1236.2 & 250.8 & 6.0 \\
\hline Nov. 2 & 2345 & 119.0 & 279.8 & $N / A$ & 1994 & 1459 & 174.7 & 778 & 573 & 306 & 300 & 29.4 & 49 & 11.8 & .17 .5 & 1150.2 & 136.0 & 20.2 \\
\hline \multicolumn{2}{|c|}{ AVERAGES } & 121.1 & 2805 & N/A & 199.2 & 153.6 & 183.2 & 774.1 & 544.6 & 370.6 & 316.4 & 32.4 & 5.2 & 16.1 & 2.0 & 1204.2 & 194.0 & 4.8 \\
\hline Nov. 3 & 0 & 120.7 & 278.1 & $N / A$ & 201.5 & 154.6 & 181.6 & 778 & 531 & 317 & 321 & 29.6 & 5.3 & 19.9 & .180 & 1218.8 & 242.7 & 3.0 \\
\hline Nov-3 & 15 & 120.1 & 280.0 & $N / A$ & 201.8 & 156.1 & 183.7 & 778 & 551 & 347 & 316 & 30.0 & 5.1 & 16.7 & .7 .0 & 0.6 & 206.0 & 8.7 \\
\hline Nov. 3 & 30 & 118.9 & 281.0 & N/A & 201.3 & 150.2 & 1799 & 772 & 568 & 334 & 321 & 29.7 & 5.0 & 12.0 & .15 .3 & 1175.0 & 140.6 & 0.2 \\
\hline Nov. 3 & 45 & 119.4 & 278.7 & $N / A$ & 201.6 & 152.7 & 179.1 & 709 & 553 & 355 & 314 & 29.5 & 54 & 8.5 & 37 & 10965 & 93.0 & 2.4 \\
\hline Nov. 3 & 100 & 121.1 & 279.1 & $N / A$ & 2028 & 155.6 & 182.3 & 700 & 520 & 332 & 330 & 294 & 5.1 & 13.1 & .16 .6 & 1103.8 & 1442 & 0.5 \\
\hline Nov. 3 & 115 & 119.8 & 280.2 & $N / A$ & 204.6 & 1600 & 1847 & 701 & 546 & 361 & 31 & 29.5 & 5.4 & 10.1 & 0.5 & 6.7 & 115.0 & 5.4 \\
\hline Nov.3 & 130 & 118.1 & 281.0 & N/A & 205.0 & 1561 & 185.8 & 736 & 546 & 342 & 312 & 28.7 & 4.8 & 11.6 & .8 .4 & 11641 & 135.4 & 1.0 \\
\hline Nov-3 & 145 & 119.1 & 279.1 & $N / A$ & 204.0 & 153.4 & 1800 & 717 & 565 & 343 & 308 & 29.4 & 4.9 & 7.5 & 5.3 & 1113.9 & 837 & 12.4 \\
\hline Nov. 3 & 200 & 120.7 & 276.4 & $N / A$ & 2023 & 150.9 & 1764 & 745 & 533 & 315 & 326 & 28.8 & 48 & 16.5 & .21 .0 & 1139.2 & 187.5 & 8.0 \\
\hline Nov-3 & 215 & 120.7 & 275.3 & & 201.6 & 156.3 & 179.7 & 720 & 522 & 349 & 320 & 29.3 & 53 & 16.7 & -5.5 & 1139.7 & 180.2 & 6.1 \\
\hline Nov-3 & 230 & 118.4 & 275.4 & N/A & 2030 & 162.4 & 1880 & 741 & 531 & 326 & 334 & 29.0 & 51 & 17.1 & .18 .4 & 1219.6 & 209.1 & 0.2 \\
\hline Nov. 3 & 245 & 120.2 & 2756 & $N / A$ & 202.5 & 1580 & 1831 & 742 & 554 & 331 & 310 & 287 & 52 & 136 & -8.2 & 11881 & 161.3 & 3.1 \\
\hline Nove 3 & 300 & 120.3 & 2746 & $N / A$ & 201.9 & 160.2 & 182.4 & 713 & 530 & 315 & 315 & 29.3 & 5.2. & 15.5 & .13 .9 & 11576 & 178.9 & 5.0 \\
\hline Nov. 3 & 315 & 120.0 & 274.5 & Ni & 200.3 & $158 . \mathrm{a}$ & 182.5 & 329 & 534 & 37 & 309 & 29.4 & 58 & 15.0 & .66 & 1160.4 & 173.8 & 1.7 \\
\hline Nov-3 & 330 & 115.0 & 274.2 & N/A & 1994 & 154.4 & 1795 & 728 & 545 & 329 & 332 & 290 & 5.4 & 131 & .174 & 11400 & 149.7 & 0.4 \\
\hline Nov.3 & 345 & 1153 & 274.8 & $N / A$ & 199.6 & 154.6 & 1789 & 722 & 546 & 335 & 318 & 29.1 & 5.4 & 12.0 & 10.5 & 11313 & 136.0 & 18.4 \\
\hline Nov-3 & 400 & 114.6 & 273.5 & & 1990 & 157.3 & 180.9 & 696 & 531 & 32 & 318 & 29.4 & 5.5 & 122 & 12.9 & 1108.8 & 135.5 & 178 \\
\hline Nov-3 & 415 & 114.8 & 272.8 & N/A & 198.5 & 159.6 & 182.4 & 701 & 547 & 342 & 312 & 29.9 & 56 & 10.9 & 4.5 & 11339 & 123.9 & 15.7 \\
\hline Nov. 3 & 430 & 114.4 & 274.0 & $N / A$ & 188.4 & 156.8 & 181.4 & 759 & 551 & 328 & 330 & 29.9 & 5.6 & 160 & -16.4 & 12052 & 1934 & 246 \\
\hline Nov.3 & 445 & 116.9 & 272.8 & $\mathrm{~N} / \mathrm{A}$ & 196.5 & 157.1 & 179.7 & 715 & 519 & 340 & 316 & 313 & 5.4 & 17.0 & 6.5 & 1138.4 & 193.3 & 24.4 \\
\hline 2v. 3 & 500 & 116.8 & 2736 & $N / A$ & 198.3 & 161.1 & 183.2 & 703 & 540 & 321 & 313 & 300 & 54 & 127 & .11 .1 & 1147.0 & 145.6 & 19.1 \\
\hline Nov. 3 & 515 & 116.8 & 273.6 & $N / A$ & 197.7 & 158.8 & 181.6 & 727 & 543 & 340 & 317 & 29.9 & 5.0 & 14.6 & .6 .4 & 11700 & 170.8 & 24.4 \\
\hline Vov-3 & 530 & 117.2 & 272.1 & $N / A$ & 197.5 & 1557 & 1794 & 728 & 544 & 341 & 339 & 29.0 & 5.1 & 13.9 & .148 & 11480 & 160.1 & 23.1 \\
\hline
\end{tabular}


CZD LIME INJECTION DATA AUGUST 17, TO NOVEMBEA 16, 1982

\begin{tabular}{|c|c|c|c|c|c|c|c|c|c|c|c|c|c|c|c|c|c|c|}
\hline Dalo & fime & $\begin{array}{l}\text { Boilor } \\
\text { Load } \\
\text { MW }\end{array}$ & $\begin{array}{l}\text { Duct } \\
\text { Inlot } \\
\text { Tomp of }\end{array}$ & $\begin{array}{l}\text { Avg "C" } \\
\text { Section } \\
\text { Temp of }\end{array}$ & $\begin{array}{l}\text { Duct } \\
\text { Outlet } \\
\text { Temp of }\end{array}$ & $\begin{array}{l}\text { Flue Gas } \\
\text { Flow in } \\
\text { KSCFM }\end{array}$ & $\begin{array}{l}\text { Flue Oas } \\
\text { Flow Out } \\
\text { KSCFM }\end{array}$ & $\begin{array}{c}502 \\
\operatorname{lnl}|0| \\
\mathrm{ppm}\end{array}$ & $\begin{array}{c}\mathrm{SO} 2 \\
\text { Outlet } \\
\mathrm{ppm}\end{array}$ & $\begin{array}{l}\text { NOK } \\
\text { Inlol } \\
\text { ppm }\end{array}$ & $\begin{array}{c}\text { NOx } \\
\text { Outlet } \\
\text { ppm }\end{array}$ & $\begin{array}{l}\operatorname{Lim} \theta \\
\text { Flow } \\
\text { opm }\end{array}$ & $\begin{array}{c}\text { Lime } \\
\text { Conc } \\
\%\end{array}$ & $\begin{array}{c}502 \\
\text { Ramoval } \\
\%\end{array}$ & $\begin{array}{c}\text { Mox } \\
\text { Hemoval } \\
\%\end{array}$ & $\begin{array}{l}\mathrm{SO} 2 \\
\ln |\mathrm{l}| \\
\mathrm{lb} / \mathrm{H}\end{array}$ & $\begin{array}{c}802 \\
\text { Pemoved } \\
\mathrm{L} / \mathrm{HI}\end{array}$ & $\begin{array}{c}\text { Lime } \\
\text { Ulilizalio } \\
\%\end{array}$ \\
\hline Nov. 3 & 545 & 117.2 & 271.5 & $N / A$ & 197.6 & 159.2 & 1817 & 708 & 536 & 328 & 317 & 28.3 & 4.8 & 13.8 & 10.2 & 11438 & 1581 & 23.5 \\
\hline Nov. 3 & 600 & 116.8 & 272.2 & $N / A$ & 1982 & 1590 & 182.2 & 711 & 532 & 318 & 316 & 285 & 5.2 & 14.4 & .13 .7 & 1145.7 & 1646 & 237 \\
\hline Nov 3 & 615 & 117.2 & 272.2 & $N / A$ & 1973 & 1570 & 1810 & 741 & 541 & 330 & 318 & 20.2 & 51 & 159 & .8 .2 & 1170.0 & 187.8 & 26.8 \\
\hline Nov. 3 & 630 & 117.1 & 271.9 & N/A & 196.7 & 159.7 & 181.2 & 730 & 540 & 340 & 340 & 20.8 & 5.0 & 16.1 & .13 .6 & 11807 & 1002 & 27.3 \\
\hline Nov. 3 & 645 & 116.6 & 271.7 & N/A & 106.8 & 155.5 & 178.8 & 729 & 544 & 339 & 319 & 290 & 5.0 & 138 & .8 .8 & 1148. & 1588 & 234 \\
\hline Nov. 3 & 700 & 116.8 & 272.2 & N/A & 197.3 & 157.2 & 183.0 & 714 & 558 & 320 & 316 & 29.4 & 5.0 & 9.0 & .14 .7 & 1137.5 & 102.8 & 140 \\
\hline Nov. 3 & 715 & 1176 & 270.7 & $N / A$ & 1952 & 153.8 & 1774 & 751 & 537 & 339 & 310 & 29.7 & 50 & 176 & .8 .5 & 11704 & 206.1 & 29.6 \\
\hline Nov-3 & 730 & 117.2 & 269.8 & NIA & 194.3 & 1554 & 179.1 & 713 & 546 & 335 & 338 & 30.0 & 5.3 & 11.7 & .16 .2 & 1122.1 & 131.4 & 17.6 \\
\hline Nov. 3 & 745 & 116.1 & 272.5 & $N / A$ & 195.2 & 158.8 & 1826 & 741 & 529 & 339 & 318 & 29.8 & 5.2 & 180 & .0 .0 & 1192.6 & 214.6 & 29.5 \\
\hline Nov. 3 & 800 & 116.5 & 272.5 & $N / A$ & 195.8 & 157.3 & 180.7 & 717 & 560 & 324 & 317 & $29 . \dot{B}$ & 5.1 & 10.3 & .123 & 1143.0 & 117.8 & 165 \\
\hline Nov.3 & 815 & 116.5 & 272.8 & $N / A$ & 1965 & 155.4 & 178.0 & 752 & 531 & 350 & 317 & 29.2 & 4.0 & 19.2 & .3 .8 & 11834 & 2266 & 33.8 \\
\hline Nov. 3 & 830 & 118.3 & 271.3 & N/A & 1952 & 156.8 & 180.7 & 702 & 539 & 348 & 319 & 30.4 & 5.0 & 11.5 & .5 .1 & 1116.5 & 128.7 & 181 \\
\hline Nov. 3 & 845 & 116.2 & 271.8 & N/A & 1956 & 160.2 & 182.7 & 719 & 520 & 346 & 317 & 30.5 & 5.4 & 17.5 & .4 .3 & 1166.5 & 2047 & 26.5 \\
\hline Nov -3 & 900 & 117.3 & 272.5 & N/A & 197.6 & 158.0 & 1808 & 731 & 548 & 327 & 317 & 29.8 & 5.0 & $14 . ?$ & .11 .2 & 1170.5 & 166.1 & 3.7 \\
\hline Nov. 3 & 815 & 117.2 & 274.8 & $N / A$ & 1987 & 154.3 & 177.7 & 748 & 547 & 353 & 322 & 28.7 & 50 & 160 & B.1 & 11711 & 187.2 & 27.8 \\
\hline Nov-3 & 930 & 117.3 & 274.7 & N/A & 189.4 & 161.7 & 185.2 & 708 & 526 & 350 & 320 & 29.3 & 50 & 15.0 & .4 .6 & 1160.3 & & 5.4 \\
\hline Nov. 3 & 945 & 117.2 & 2754 & N/A & 199.8 & 158.8 & 1819 & 740 & 530 & 349 & 328 & 28.6 & 5.2 & 185 & 6.8 & 1100.2 & 2222 & 31.9 \\
\hline Nov 3 & 1000 & 116.6 & 276.8 & N/A & 197.6 & 1552 & 179.6 & 745 & 533 & 330 & 307 & 30.4 & 52 & 17.2 & .7 .6 & 1170.8 & & 7.1 \\
\hline Nov. 3 & 1015 & 117.1 & 276.6 & N/A & 1984 & 157.3 & 1784 & 732 & 533 & 351 & 320 & 28.7 & 4.8 & 17.0 & .4 .1 & 1166.0 & 198.1 & 30.1 \\
\hline Nov 3 & 1030 & 117.2 & 279.4 & $N / A$ & 2012 & 160.5 & 181.0 & 742 & 528 & 352 & 310 & 29.8 & 5.2 & 18.4 & .26 & 1206.1 & 233.8 & 2 \\
\hline Nov. 3 & 1045 & 116.5 & 279.1 & $N / A$ & 1996 & 158.9 & 184.7 & 732 & 534 & 346 & 313 & 30.4 & 5.5 & 15.2 & 51 & 11780 & 178.6 & 22.8 \\
\hline Nov 3 & 1100 & 116.6 & 2802 & N/A & 198.2 & 152.7 & 1773 & 758 & 534 & 358 & 315 & 304 & 55 & 18.3 & 18 & & & 73 \\
\hline Nov. 3 & 1115 & 116.8 & 281.3 & $N / A$ & 1936 & 153.8 & 1809 & 761 & 526 & 350 & 318 & 35.5 & 5.4 & 18.7 & .71 & 11 & 1.7 & 4.6 \\
\hline Nov. 3 & 1130 & 117.6 & 282.8 & $N / A$ & 1934 & 156.3 & 183.4 & 729 & 526 & 353 & 318 & 364 & 4.9 & 154 & .5 .7 & 11540 & 177.3 & 21.2 \\
\hline Nov-3 & 1145 & 116.8 & 283.3 & $N / A$ & 1934 & 154.2 & 179.2 & 742 & 548 & 357 & 324 & 34.7 & 54 & 14.1 & 5.4 & 1159.6 & 163.7 & 6 \\
\hline Nov. 3 & 1200 & 116.6 & 285.2 & $N / A$ & 1943 & 153.4 & 179.4 & 750 & 544 & 361 & 311 & 34.9 & 51 & 152 & 0.8 & 1165.1 & & 2 \\
\hline Nov-3 & 1215 & 116.5 & 2850 & N/A & 1951 & 154.9 & 182.3 & 753 & 541 & 351 & 321 & 347 & 53 & 15.5 & 7.5 & 11 & 6 & 3 \\
\hline Nov. 3 & 1230 & 115.9 & 284.7 & N/A & 195.8 & 157.0 & 1848 & 760 & 530 & 343 & 322 & 348 & 50 & 17.8 & .10 .3 & 12083 & .7 & 5 \\
\hline Nov 3 & 1245 & 116.0 & 2850 & N/A & 195.9 & 153.2 & $182 \mathrm{~B}$ & 744 & 549 & 347 & 302 & 34.4 & 4.8 & 12.1 & .3 .8 & 11552 & 138.3 & 30 \\
\hline Nov- 3 & 1300 & 116.1 & 283.2 & $N / A$ & 1956 & 156.6 & 1831 & 742 & 519 & 332 & 321 & 350 & 5.1 & 18.3 & .13 .1 & 11776 & 2155 & 5.8 \\
\hline Nov-3 & 1315 & 116.5 & 2820 & $N / A$ & 1845 & 1554 & 183.2 & 733 & 510 & 352 & 310 & 34.6 & 5.1 & 181 & 6 & 4 & & 2 \\
\hline Nov-3 & 1330 & 115.0 & 2834 & N/A & 1945 & 148.7 & 175.3 & 741 & 536 & 355 & 11 & 33.8 & 5.2 & 14.7 & .3 .3 & 1116.0 & 1639 & 10.8 \\
\hline Nov. 3 & 1345 & 112.2 & 286.1 & $N / A$ & 1953 & 152.0 & 1805 & 745 & 500 & 350 & 321 & 35.1 & 5.1 & 18.8 & 8.8 & 11467 & 2157 & 25.7 \\
\hline Nov. 3 & 1400 & 112.3 & 285.8 & $N / A$ & 195.9 & 149.3 & 178.2 & 743 & 531 & 323 & 310 & 34.5 & 4.2 & 14.7 & -14.4 & 4.2 & 5 & 4.5 \\
\hline Nov. 3 & 1415 & 112.8 & 284.8 & N/A & 195.8 & 1468 & 1760 & 751 & 507 & 342 & 315 & 33.8 & 5.8 & 18.6 & .11 .1 & 1116.4 & 207.5 & 22.1 \\
\hline Nov 3 & 1430 & 1125 & 285.8 & $N / A$ & 186.4 & 1504 & 180.8 & 727 & 495 & 347 & 318 & 344 & 6.5 & 18.2 & .10 .3 & 11074 & 201.3 & 9.1 \\
\hline Nov. 3 & 1445 & 111.7 & 2850 & N/A & 195.6 & 147.7 & 178.5 & 725 & 497 & 349 & 319 & 34.1 & 6.1 & 172 & .10 .7 & 10853 & 1871 & 8.1 \\
\hline Nov. 3 & 1500 & 112.4 & 285.2 & $N / A$ & 196.0 & 147.2 & 1768 & 720 & 501 & 360 & 323 & 34.1 & 6.3 & 165 & .7 .7 & 1073.4 & 6 & 7.4 \\
\hline Nov. 3 & 1515 & 112.7 & 285.4 & $N / A$ & 197.6 & 1452 & 178.0 & 744 & 502 & 351 & 322 & 336 & 60 & 172 & .124 & 1094.1 & 1.6 & 8 \\
\hline Nov. 3 & 1530 & 1128 & 2841 & $N / A$ & 1880 & 1474 & 179.4 & 724 & 515 & 351 & 321 & 32.4 & 63 & 134 & .1112 & $\mid 1081.6$ & 145.3 & 5.1 \\
\hline Nov 3 & 1545 & 112.6 & 283.6 & $N / A$ & 2002 & 147.8 & 179.8 & 718 & 515 & 363 & 328 & 31.4 & 6.1 & 128 & .9 .9 & 1076.2 & 138.3 & 15.3 \\
\hline Nov. 3 & 1600 & 111.2 & 285.2 & $N / A$ & 200.9 & 144.7 & 174.3 & 713 & 504 & 32 & 312 & 31.2 & 60 & 110 & .150 & 10 & 5 & 17.6 \\
\hline Nov-3 & 1615 & 116.6 & 282.8 & $N / A$ & 2008 & 144.6 & 174.8 & 742 & 503 & 352 & 325 & 31.6 & 6.1 & 18.1 & .114 & 1087.4 & 187.2 & 1.7 \\
\hline Nov-3 & 1630 & 1182 & 2825 & N/A & 2021 & 151.5 & 182.9 & 701 & 518 & 361 & 321 & 31.6 & 6.1 & 10.8 & .7 .5 & 1076.3 & 117.2 & 12.9 \\
\hline Nov-3 & 1645 & 117.2 & 2845 & $N / A$ & 202.8 & 1534 & 184.7 & 760 & 500 & 353 & 331 & 32.1 & 6.6 & 20.8 & .131 & 1180.6 & .7 & 24.5 \\
\hline Nov. 3 & 1700 & 1187 & 280.8 & N/A & 2022 & 148.2 & 178.4 & 726 & 520 & 32 & 319 & 3 & 6.2 & 13.8 & .17 .3 & 0.4 & 3 & 168 \\
\hline Nov-3 & 1715 & 117.8 & 280.7 & $\cdots$ & 2035 & 1528 & 1836 & 710 & 518 & 354 & 315 & 30.8 & 6.1 & 12.4 & 6.7 & 10987 & 9 & 3 \\
\hline Nov 3 & 1730 & 118.8 & 2804 & N/A & 203.7 & 152.9 & 182.2 & 125 & 534 & 347 & 318 & 30 : & 6.6 & 12.3 & .8 .2 & 1123.5 & 138.6 & 4.8 \\
\hline Nov. 3 & 1745 & 117.8 & 278.9 & N/A & 2037 & 151.5 & 180.6 & 746 & 513 & 3 & 330 & 29.9 & 58 & 18 & .112 & 11453 & 20 & 25.0 \\
\hline Nov. 3 & 1800 & 118.7 & 278.1 & N/A & 2042 & 151.2 & 179.5 & 715 & 536 & 336 & 322 & 28.2 & 63 & 11.0 & -1 & 1.8 & & 3.8 \\
\hline Nov-3 & 1815 & 110.6 & 278.0 & $N / A$ & 203.9 & 1513 & 1818 & 735 & 533 & 351 & 323 & 296 & 5.8 & 128 & .10 .6 & 1126.3 & 144.5 & 7.9 \\
\hline Nov. 3 & 1830 & 1199 & 278.2 & $N / A$ & 2042 & 152.5 & 182.1 & 725 & 535 & 35 & 327 & 29.7 & 5.8 & 11.8 & .9 .4 & 11 & 132.7 & 16.4 \\
\hline Nov. 3 & 1845 & 117.8 & 277.4 & $N / A$ & 204.7 & 151.5 & 179.7 & 730 & 530 & 3 & 328 & 28.1 & 6.3 & 139 & 9. & 11 & 155.7 & 18.6 \\
\hline Nov. 3 & 1900 & 1179 & 277.8 & N/A & 204.9 & 151.9 & 181.5 & 712 & 534 & 327 & 317 & 284 & 6.4 & 10 & .15 .8 & 10 & 114.3 & 3 \\
\hline Nov 3 & 1915 & 118.8 & 276.8 & N/A & 205.1 & 1510 & 179.7 & 730 & 545 & 345 & 313 & $28 . ?$ & 6.3 & 11.2 & .7 .8 & 1117.1 & 124.9 & 4.9 \\
\hline Nov. 3 & 1930 & 117.9 & 277.2 & N/A & $205 ?$ & 151.3 & 180.6 & 731 & 544 & 353 & 326 & 28.3 & 6.2 & 11.2 & .102 & 1120.9 & 1255 & 152 \\
\hline Nov.3 & 1945 & 117.3 & 77.1 & $N / A$ & 2056 & $150 \mathrm{~B}$ & 1804 & 736 & 552 & 354 & 334 & 28.2 & 64 & 103 & .12 .7 & 1124.1 & 116.0 & 13.6 \\
\hline Nov. 3 & 2000 & 118.6 & 276.2 & $N / A$ & 205.3 & 150.8 & 179.9 & 738 & 544 & 326 & 320 & 28.0 & 64 & 12.1 & -16.8 & & 136.3 & 16.1 \\
\hline Nov. 3 & 2015 & 1180 & 276.9 & A & 2053 & 150.3 & 178.3 & 752 & 554 & 357 & $32 \theta$ & 28.6 & 6.4 & 12.6 & .8 .9 & 11453 & 144.5 & 16.7 \\
\hline Nov.3 & 2030 & 119.1 & 277.1 & & 2058 & 150.6 & 180.8 & 762 & 566 & 356 & 328 & 269 & 60 & 108 & .111 & 11621 & 125.3 & 16.5 \\
\hline Nov. 3 & 2045 & 1194 & 276.8 & N/A & 205.0 & 151.1 & (8) 6 & 755 & 568 & 356 & 327 & 28.2 & 6.1 & 9.5 & .10 .4 & 1155.9 & 1100 & 13.6 \\
\hline Nov -3 & 2100 & 118.8 & 277.8 & N/A & 205.5 & 151.6 & 181.7 & 755 & 568 & 356 & 322 & 8.6 & 6.2 & 98 & .8 .4 & 1159.7 & 1131 & 3.5 \\
\hline Nov-3 & 2115 & 120.1 & 278.5 & N/A & 2044 & 1512 & 1800 & 755 & 565 & 386 & 311 & 290 & 58 & 10.9 & 4.0 & 11 & 126.2 & 15.9 \\
\hline Nov 3 & & 0 & 278.0 & & 204.2 & 151.1 & 179.2 & 755 & 574 & 382 & 308 & 28.9 & 6.1 & 9.8 & 4.2 & 1155.9 & 114.6 & 13.8 \\
\hline Nov.3 & 2145 & 119.7 & 278.0 & & 204.1 & 150.8 & 4707 & 75 & 553 & 377 & 316 & 28.6 & 59 & 13.4 & 1.1 & 11536 & 154.7 & 19.5 \\
\hline Nov 3 & 2200 & 120.2 & 278.6 & N/A & 204.3 & 151.2 & 179.8 & 782 & 575 & 373 & 316 & 380 & 5.8 & 12.6 & .0 .7 & 1197.4 & & 18.2 \\
\hline ov. 3 & 2215 & 121.0 & 278.1 & $1 / A$ & 204.5 & 151.5 & 178.3 & 777 & 541 & 371 & 324 & 28.9 & 59 & 18.1 & 2.7 & & 216.4 & 27.0 \\
\hline Nov. 3 & 2230 & & & & 2052 & 151.8 & 1812 & 792 & 548 & 384 & 322 & 280 & 6.3 & 17.5 & 03 & 1218.3 & 213.1 & 25.6 \\
\hline Nov. 3 & 2245 & 121.3 & 2781 & N/A & 204.7 & 148.7 & 178.2 & 780 & 566 & 374 & 316 & 28.6 & 5.9 & 13.0 & -1.1 & 1174.6 & 152.6 & 18.2 \\
\hline Nov-3 & 2300 & 117.7 & 278.5 & N/A & 205.2 & 1527 & 1804 & 780 & 566 & 374 & 6 & 28.6 & 5.8 & 14.2 & 0.3 & 1206.2 & 171.6 & 22.0 \\
\hline Nov-3 & 2315 & 118.9 & 2780 & N/A & 2050 & 149.8 & 178.9 & 780 & 566 & 374 & 321 & 282 & 5.9 & 13.3 & 2.3 & 1183.2 & 157.3 & 20.1 \\
\hline 3 & 2330 & 116.7 & 273.5 & N/A & 2050 & 145.8 & 175.5 & 780 & 569 & 374 & 313 & 28.2 & 61 & 12.2 & .0 .6 & 11524 & 140.7 & 17.3 \\
\hline av-3 & 2345 & 116.0 & 281.7 & N/A & 2044 & 1494 & 177.5 & 780 & 552 & 374 & 317 & 30.2 & 5.8 & 15.9 & .0 .7 & 1180.1 & 187.4 & 22.7 \\
\hline$A V E$ & RAGES & 117.3 & 78.1 & N/A & 2002 & 1538 & 1806 & 737.3 & 537.0 & 3465 & 3195 & 304 & 55 & 14.4 & .8 .5 & 1148.4 & 1659 & 21.2 \\
\hline
\end{tabular}


C2D LIME INJECTION DATA AUGUST 17, TO NOVEMBEA 16, 1992

\begin{tabular}{|c|c|c|c|c|c|c|c|c|c|c|c|c|c|c|c|c|c|c|}
\hline Dale & Time & $\begin{array}{l}\text { Boile! } \\
\text { Load } \\
\text { MN }\end{array}$ & \begin{tabular}{|c|} 
Ducl \\
Inlel \\
Tomp of
\end{tabular} & $\begin{array}{l}\text { Avg "C" } \\
\text { Section } \\
\text { temp of }\end{array}$ & $\begin{array}{l}\text { Ducl } \\
\text { Oullel } \\
\text { Temp of }\end{array}$ & \begin{tabular}{|l|} 
Flue Oas \\
Flow in \\
KSOCFM
\end{tabular} & $\begin{array}{l}\text { Flue Gas } \\
\text { Flow Out } \\
\text { KSCFM }\end{array}$ & $\begin{array}{c}\mathrm{BO} 2 \\
\ln 1 \mathrm{i} \\
\mathrm{ppm}\end{array}$ & \begin{tabular}{|c|}
802 \\
Oullat \\
ppm
\end{tabular} & $\begin{array}{l}\text { Nor } \\
\text { Inlei } \\
\text { ppm }\end{array}$ & $\begin{array}{c}\text { NOn } \\
\text { Outlat } \\
\text { ppm }\end{array}$ & $\begin{array}{l}\operatorname{lima} \\
\text { Flow } \\
\text { opm }\end{array}$ & $\begin{array}{l}\text { Lime } \\
\text { Cons } \\
\%\end{array}$ & $\begin{array}{c}50 ? \\
\text { Removat } \\
\%\end{array}$ & $\begin{array}{c}\text { Nor } \\
\text { Hemoval } \\
x\end{array}$ & $\begin{array}{c}80 ? \\
\text { Inlel } \\
\text { lo/tif }\end{array}$ & $\begin{array}{c}802 \\
\text { Hemoved } \\
\mathrm{lo} / \mathrm{HI}\end{array}$ & $\begin{array}{c}\text { lime } \\
\text { Utilizatic } \\
*\end{array}$ \\
\hline Nov-4 & 0 & 114.5 & 282.8 & NiA & 2041 & 152.0 & $180 \mathrm{~B}$ & 280 & 522 & 332 & 321 & & 60 & 208 & $14 ?$ & $1207 ?$ & 2513 & 200 \\
\hline Nov- 4 & 15 & 1164 & 280.6 & $N / A$ & 2025 & 1407 & 1776 & 736 & 536 & 337 & 308 & 301 & 61 & 13.6 & 5 & 11109 & 1523 & 176 \\
\hline Nor. 4 & 30 & 118.7 & 62.5 & $N / A$ & 203.5 & 1510 & 1788 & 708 & 542 & 340 & 324 & 30.6 & 56 & 100 & 0.2 & 10900 & 1065 & 13.5 \\
\hline Nov.4 & 45 & 1165 & 80.3 & $\bar{N} / A$ & 2015 & 1535 & 1821 & 124 & 516 & 355 & 335 & 314 & 58 & 155 & 111 & 11255 & 1742 & 200 \\
\hline Nov 4 & 100 & 1166 & 2807 & $N / A$ & 2001 & 1540 & 1820 & 126 & 521 & 338 & 323 & 331 & 50 & 153 & 12.7 & 11331 & 1731 & 188 \\
\hline Nov. 4 & 115 & 1178 & 11.6 & N/A & 1886 & 1520 & 1785 & 734 & 507 & 340 & 317 & 320 & 61 & 189 & 0.7 & 11366 & 2153 & 228 \\
\hline Nov. 4 & 130 & 117.2 & 03.3 & $N / A$ & 188.5 & 154.8 & 1823 & 743 & 515 & 347 & 325 & 33.4 & 66 & 184 & 10.1 & 11649 & 2114 & 244 \\
\hline Nov. 4 & 145 & 117.4 & 270.7 & $N / A$ & 1866 & 1544 & 1826 & 122 & 510 & 358 & 334 & 33.9 & 6.3 & 165 & 10.3 & 1120.8 & 1861 & 185 \\
\hline Nov. 4 & 200 & 1163 & 2.76 .8 & N/A & 1052 & 1551 & 182.6 & 728 & 504 & 339 & 325 & 331 & 6. 1 & 18.6 & .12 .7 & 1144.3 & 212.6 & 223 \\
\hline Nov. 4 & 215 & 116.5 & 2772 & $N / A$ & 1832 & 15 & 180.5 & 728 & 614 & 352 & 321 & 34.6 & 60 & 180 & 5.9 & 1147.2 & 2070 & 21.2 \\
\hline Nov. 4 & 230 & 1165 & 276.1 & $N / A$ & 1920 & 153.8 & 1810 & 742 & 500 & 353 & 332 & 334 & 60 & 20.8 & .108 & 11566 & 240.4 & 5.6 \\
\hline Nov- 4 & 245 & 1157 & 277.1 & NIA & 1930 & 1567 & 1860 & 744 & 489 & 358 & 330 & 34.0 & 63 & 20.3 & 0.4 & 11008 & $240 ?$ & 23.8 \\
\hline Nov. 4 & 300 & 115. & 277.1 & $N / A$ & 192.7 & 1560 & 1845 & 733 & 505 & 332 & 321 & 33.5 & 6.2 & 18.5 & .141 & 11581 & 213.0 & 8 \\
\hline Nov-4 & 315 & 116.0 & 276.1 & $N / A$ & 193.1 & 1552 & 1817 & 744 & 610 & 357 & 320 & 32.6 & 6.0 & 18.8 & .40 & 1170.2 & 231.2 & 5.1 \\
\hline Nov-4 & 330 & 112.8 & 276.6 & $N / A$ & $192 \mathrm{~B}$ & 1542 & 180.5 & 736 & 517 & 352 & 327 & 328 & 64 & 17.8 & .89 & 1140.4 & 2047 & 10.6 \\
\hline Nov. 4 & 346 & 111.3 & 277.7 & $N / A$ & 1032 & 1550 & 1833 & 765 & 511 & 352 & 328 & 325 & 58 & 210 & 10.1 & 12016 & 252.3 & 8.4 \\
\hline Nov. 4 & 400 & 1130 & $275 ?$ & $N / A$ & 1934 & & 170.7 & 723 & 500 & 332 & $32 !$ & 321 & 6.2 & 17.3 & 138 & 11204 & 1034 & 006 \\
\hline Nov 4 & 415 & 1120 & 16 & $N / A$ & 1831 & 151.6 & 1785 & 740 & 517 & 354 & 310 & 31.4 & 50 & 183 & & 1140.8 & 210.3 & 24.1 \\
\hline Nov. 4 & 430 & 113.5 & 275.8 & $N / A$ & 1937 & 152.2 & 180.5 & 147 & 521 & 361 & 330 & 31.8 & 6.0 & 17.2 & . 3 & 11513 & 108.3 & 220 \\
\hline Nov-4 & 445 & 112.8 & 2761 & N/A & 193.0 & 150.3 & 179.8 & 172 & 516 & 348 & 334 & & 5.8 & 200 & .14 .2 & 11758 & 235.6 & 273 \\
\hline Nov. 4 & 500 & 1132 & 276.1 & $N / A$ & 1943 & 1513 & 170.6 & 734 & 516 & 336 & 328 & & 6.3 & 16.5 & .16 .0 & $\mid 11244$ & 185.3 & 108 \\
\hline Nov. 4 & 515 & 114.8 & 2737 & N/A & 194.5 & & 182.3 & 128 & 537 & 364 & 314 & & $6:$ & 12.7 & & 11355 & & 58 \\
\hline Nov. 4 & 530 & 115.6 & 274.8 & $N / A$ & 1847 & 1507 & 1785 & 167 & 532 & 360 & 332 & & 6.1 & 17.8 & $\theta$ & 11713 & 210.0 & 33 \\
\hline Nov 4 & 545 & 1148 & 2743 & $N / A$ & 1958 & 152.0 & 1836 & 751 & 498 & 361 & आ? & 27.5 & 59 & 188 & 6.2 & 11560 & 2303 & o 1 \\
\hline Nov. 4 & 600 & 1157 & 273.2 & $N / A$ & 200.8 & 152.2 & 1803 & 140 & 551 & 331 & 315 & 26. & 61 & 110 & 12.8 & 11414 & 1358 & 78 \\
\hline Nov. 4 & 615 & 117.3 & 273.9 & $N / A$ & 2026 & 150.4 & 176.4 & 176 & 574 & 353 & 317 & 26 & 6.5 & 13.3 & .64 & 11810 & 1568 & 9.6 \\
\hline Nov. 4 & 630 & 1152 & 2736 & $N / A$ & 203.4 & 150.7 & 177.7 & 770 & 57 & 364 & 33 & & 57 & 123 & .7 .7 & 17759 & 145.1 & 0.8 \\
\hline Nov.4 & 645 & 1162 & 273.2 & $N / A$ & 203.6 & 148.2 & 1762 & 757 & 569 & 35 & 328 & 258 & 60 & 112 & 100 & 11437 & 127.0 & 5 \\
\hline Nov 4 & 700 & 1152 & 272.8 & $N / A$ & 2020 & 15 & 17 & 751 & 549 & 3 & 20 & 274 & 6.2 & 13.3 & .137 & 46 & 8 & 1 \\
\hline Noved & 715 & 1161 & 271.8 & $N / A$ & 203.1 & 1522 & 1800 & 755 & 546 & 361 & 320 & 26.4 & 6.2 & 14.6 & .4 .6 & 11643 & 169.4 & 1.9 \\
\hline Nov. 4 & 730 & 116.2 & 273.5 & $N / A$ & 2035 & 1506 & 177.7 & 757 & 562 & 360 & 14 & $25 ?$ & 58 & 124 & .3 & 11545 & 143.5 & 0.1 \\
\hline Nov. 4 & 745 & 1171 & 2733 & $N / A$ & 204.3 & 1508 & 175.8 & 756 & 560 & $36 \theta$ & 331 & & 5.7 & 136 & .4 .8 & 1154.5 & 157.3 & 20 \\
\hline Nov. 4 & 800 & 1165 & 274.5 & $N / A$ & 2042 & 152.0 & 17 & 738 & 564 & 8 & 18 & 2 & 50 & 10.7 & $\therefore 103$ & 84 & 1221 & 64 \\
\hline Nov. 4 & 815 & 1165 & 275.3 & Nil & 2046 & 150.5 & 176.5 & 155 & 551 & 359 & 315 & 26.6 & 61 & 145 & 2. & 11514 & 167.0 & 10 \\
\hline Nov.4 & 830 & 1161 & & NiA & 205.0 & 152.2 & 179.6 & 738 & 551 & 354 & 325 & 27 & 6.3 & 11.9 & 8.2 & 11384 & 135.7 & 6.9 \\
\hline Nov. 4 & 845 & $115 ?$ & 27 & $N / A$ & 204.8 & 151.8 & 17 & 746 & 556 & 240 & 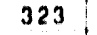 & 2 & 6.2 & 127 & 93 & 7.5 & 7 & 10 \\
\hline Nov. 4 & 900 & 1141 & 277.1 & $N / A$ & 2044 & 152.7 & 17 & 714 & 538 & 35 & n & 27.7 & 57 & 12.3 & 10.3 & 11042 & 4 & 18.2 \\
\hline Nov. 4 & 915 & 117.6 & 2778 & $N / A$ & 203.2 & 152.7 & 183.0 & 714 & 558 & 36 & 307 & 3 & 6.0 & 64 & .06 & 11042 & 70.5 & 8.3 \\
\hline Nov. 4 & 83) & 118.8 & 2789 & $N / A$ & 203.5 & 154.6 & 182.8 & 714 & 558 & 366 & 307 & 2 & 6.2 & 76 & 0.8 & 11177.8 & 85.4 & 9.7 \\
\hline Nov 4 & 94L & 1181 & 2784 & $N / A$ & 205.0 & 153.3 & 178.8 & 714 & 558 & 366 & 307. & 28.6 & 6.2 & 8.3 & 1.5 & 11085 & 924 & 110 \\
\hline Nov. 4 & 1000 & 1164 & 278.9 & $N / A$ & 205.6 & 1547 & 1802 & 71 & & 36 & n & 2 & 6.2 & 8.0 & 2. & 8.7 & 100.8 & 26 \\
\hline Nov. 4 & 1015 & 1176 & 278.5 & $N / A$ & 203.7 & 1523 & 180.1 & 71 & 558 & 36 & 307 & 29.6 & 5.0 & 7.6 & 0.8 & 11013 & 840 & 10.2 \\
\hline Nov. 4 & 1030 & 117.6 & 280.8 & N/A & 2057 & 1540 & 181.0 & 114 & 51 & 30 & 3 & 28.2 & 6.2 & 15.7 & 3.1 & 6 & 174.5 & 12 \\
\hline Nov 4 & 1045 & 1174 & 278.2 & $N / A$ & 205.5 & 1523 & 179.9 & 714 & 51 & 36 & 900 & 26 & 61 & 154 & 0.3 & & 169.7 & 1.8 \\
\hline Nov. 4 & 1900 & 117.8 & 278.3 & $N / A$ & 203.7 & 151.4 & 178.4 & $72:$ & 52 & 36 & 3 & 29.6 & 6.4 & 14.0 & 1.2 & 11 & 155.8 & 74 \\
\hline Nov. 4 & 1115 & 117.0 & 281.6 & $N / A$ & 1978 & 1523 & 179.8 & 718 & 518 & 368 & 306 & 33.3 & 64 & 158 & 1.3 & 11082 & 1753 & 74 \\
\hline Nov. 4 & 1130 & 117.5 & 282.0 & N/A & 198.3 & 152.1 & 182.8 & 738 & 51 & 36 & & 34.1 & 59 & 16.1 & 3.6 & 8.4 & 8 & 9.4 \\
\hline Nov. 4 & 1145 & 1174 & 285.0 & $N / A$ & 1957 & 152.6 & 183.3 & 74 & 48 & 37 & & & 61 & 4 & -1.3 & 0.6 & & 42 \\
\hline Nov 4 & 1200 & 1187 & 284.7 & $N / A$ & 1856 & 15 & 17 & 75 & 5 & 3 & 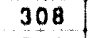 & 35. & 0.3 & 19.2 & 1.5 & 4.6 & 0 & 11 \\
\hline Nov. 4 & 1215 & 118.1 & 285.4 & $N / A$ & 195.8 & 151.7 & 181,1 & 75 & 50 & 37 & 31 & 350 & 6.1 & 19.1 & 0.8 & 1152.1 & 219.8 & 1.8 \\
\hline Nor. 4 & 1230 & 118.4 & 285.8 & $N / A$ & 1958 & 150.2 & 181.8 & 768 & 51 & 37 & 30 & 34. & 60 & 186 & .0 .8 & 8. 9 & & $?$ \\
\hline Nov-4 & 1245 & 1166 & 285.5 & A & 4 & 2 & & 762 & 4 & & 21 & & & $=9$ & .1 & & & 8 \\
\hline Nov. 4 & 1300 & 118.6 & 284.0 & $N / A$ & 195.8 & 1.1 & 17 & 75 & 4 & 37 & 306 & 34. & 5.8 & 3 & 0. & 11 & 8 & 25.5 \\
\hline Nov. 4 & 1315 & 118.4 & 284.1 & $N / A$ & 1947 & 147.7 & 178.5 & 770 & 499 & 371 & 308 & 34.6 & 6.4 & 21.3 & 0.8 & 11517 & 244.8 & 34 \\
\hline Nov. 4 & 1330 & 117.4 & 284.9 & $N / A$ & 1952 & 148.6 & 178.7 & 769 & 481 & 373 & 312 & 35.2 & 58 & 2.1 & 0.5 & 11572 & 256.3 & 6.2 \\
\hline Nov.4 & 1345 & 118.1 & 284.6 & & 195.5 & 149.7 & 181.8 & 75 & $A B$ & 37 & 3 & & 6.1 & 21 & 2. & 1138.5 & 245.8 & 241 \\
\hline Nov. 4 & 1400 & 1181 & 284.4 & 4 & 195.5 & 18 & & 762 & 4 & 374 & 310 & 3 & 6.1 & A7 4 & 834 & 7.7 & 6 & 0.7 \\
\hline Nov. 4 & 1415 & 118.2 & 2850 & $N / A$ & 196.1 & 218.0 & 154.8 & 760 & 47 & 372 & 310 & 35.9 & 6.2 & 55.8 & 40.8 & 16778 & 9 & 9.1 \\
\hline Nov. 4 & 1430 & 117.8 & 2828 & & 1957 & 151.1 & 182.3 & 733 & 46 & 370 & 311 & 35 & 6.2 & 3.8 & -14 & 1125 & & 31 \\
\hline Nov.4 & 1445 & 1178 & 2832 & & 19 & & & 74 & & 38 & 312 & 34.9 & 7.5 & 22.6 & 0. & & & 4 \\
\hline Nov.4 & 1500 & 118. & 284.1 & & & 48.5 & 182.6 & 75 & 47 & 38 & 315 & 34.6 & 7.1 & 225 & .1 .1 & 11 & 255.7 & 22.0 \\
\hline Nov.4 & 1515 & 1174 & 284.2 & $N / A$ & 197.2 & 149.0 & 181.8 & 746 & 467 & 383 & 311 & 34.4 & 72 & 23.6 & 0.8 & 11264 & 265.8 & 22.6 \\
\hline Nov. 4 & 1530 & 117.2 & 2841 & N/A & 196.8 & 147.0 & & 780 & 46 & 385 & 313 & 35 & 7.2 & 25.8 & 0.7 & 11611 & & $25: 1$ \\
\hline Nov 4 & 154 & $\ldots$ & & & & & & & & 38 & & 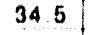 & $?$ & & & & 240.8 & 10 \\
\hline Nov. 4 & 1600 & 117.8 & 281.2 & Nin & & 148.7 & 181.5 & 78 & 46 & 386 & 314 & 34.6 & 6.9 & 2 & 0. & 1142.2 & 280.3 & 24.8 \\
\hline Nov 4 & 1615 & 117.2 & 279.8 & $N / A$ & 186.6 & 147.8 & 182.4 & 773 & 483 & 386 & 311 & 33.0 & 7.3 & 22.8 & 0.4 & 1167.0 & 264.2 & 22.5 \\
\hline Nov. 4 & 1630 & 1178 & 279.9 & 1. & & 146.2 & 180.4 & 80 & 51 & 385 & $\therefore 13$ & 30 & 70 & 21.8 & .0 .2 & 1181.7 & 260.2 & 5.4 \\
\hline 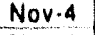 & 164 & & & & & & & & & & & & 7. & 17. & 1 & & 2018 & 2 \\
\hline Nov-4 & 1700 & 117.6 & 279.6 & $N / A$ & 2004 & 147.3 & 179.9 & 788 & 520 & 380 & 308 & 311 & 7.0 & 19.4 & 1.1 & 1175.8 & 228.1 & 22.1 \\
\hline Nov 4 & 1715 & 123.6 & 278.8 & $N / A$ & 2013 & 151.4 & 1850 & 786 & 506 & 386 & 308 & 31.4 & 7.1 & 223 & 2.4 & 1220.3 & 2716 & 25.7 \\
\hline Jov. 4 & 730 & 122.2 & 278.8 & $N / A$ & 1996 & 1484 & 182.8 & 82 & 524 & 383 & 311 & 320 & 74 & 21.7 & 0.1 & 12387 & 269.0 & 23.9 \\
\hline & 745 & 3.3 & & & $198 ?$ & & 4. 1 & B & 300 & 383 & 305 & & $?$ & 22.2 & 04 & 12203 & $270 ?$ & 25.1 \\
\hline & 1800 & 122.3 & 275.5 & $\mathrm{~N} / \mathrm{I}$ & 198.7 & 150 & 185.1 & 78 & 50 & 373 & 306 & 31.6 & 6.9 & 20.0 & -1. & 1187.2 & 238.0 & 23.0 \\
\hline Vov.4 & 1815 & 123.4 & 276.4 & $N / A$ & 198.7 & 148.8 & 1817 & 825 & 533 & 380 & 307 & 31.8 & 67 & 212 & 1.4 & 12446 & 264.3 & 26.2 \\
\hline
\end{tabular}


C2D LIME INJECIION DATA AUQUST 17, TO NOVEMBEA 16. 1992

\begin{tabular}{|c|c|c|c|c|c|c|c|c|c|c|c|c|c|c|c|c|c|c|}
\hline Dato & fimo & $\begin{array}{l}\text { Bollo! } \\
\text { Load } \\
\text { MW }\end{array}$ & $\begin{array}{l}\text { Duct } \\
\text { Inlol } \\
\text { Tomp of }\end{array}$ & $\begin{array}{l}\text { Avg "C", } \\
\text { Beclian" } \\
\text { Tomp op" }\end{array}$ & $\begin{array}{l}\text { Duct } \\
\text { Oullat } \\
\text { Tamp of }\end{array}$ & $\begin{array}{l}\text { Flue dos: } \\
\text { Flow in } \\
\text { KSCFM }\end{array}$ & $\begin{array}{l}\text { Flue Ons } \\
\text { flow Out } \\
\text { kecrim }\end{array}$ & $\begin{array}{l}\text { sor } \\
\text { Iniol } \\
\text { ppm }\end{array}$ & $\begin{array}{c}802 \\
\text { Oullul } \\
\text { ppm }\end{array}$ & $\begin{array}{l}\text { Non } \\
\text { inlel } \\
\text { ppm }\end{array}$ & $\begin{array}{l}\text { NOx } \\
\text { Outle! } \\
\text { ppm }\end{array}$ & $\begin{array}{l}\text { Lime } \\
\text { Flow } \\
\text { opm }\end{array}$ & $\operatorname{Tim}_{\text {Conc }}$ & $\begin{array}{c}\text { SO2 } \\
\text { Anmoval } \\
\%\end{array}$ & $\begin{array}{c}\text { NOK } \\
\text { Homar } \\
4\end{array}$ & $\begin{array}{c}802 \\
\ln \mid 01 \\
\operatorname{lo} \mid \mathrm{Hi}\end{array}$ & $\begin{array}{l}80 \text { ? } \\
\text { Hemoved } \\
\text { Ib/H? }\end{array}$ & Lilizene \\
\hline Nor. 4 & 1830 & 1826 & 2712 & $N / A$ & 1989 & 1500 & 1841 & 810 & 526 & 383 & 312 & 31.8 & 60 & 207 & 01 & 12376 & 2563 & 247 \\
\hline Nov. 4 & 1845 & 1230 & 2774 & $N / A$ & 1985 & 1494 & 1818 & 022 & 516 & 311 & 300 & 31.3 & 1.3 & 237 & 1.3 & 1244.3 & 294.5 & 27.2 \\
\hline Nov. 4 & 1900 & 1232 & 2766 & $N / A$ & 1883 & 148.8 & 1836 & 808 & 522 & 374 & 307 & 318 & 60 & $20 ?$ & 12 & 12174 & $246 ?$ & 241 \\
\hline Nov 4 & 1015 & 1226 & $275 ?$ & $N / A$ & 1083 & 150.3 & 1031 & 706 & 515 & 375 & 313 & 315 & 60 & 214 & 1.7 & 12158 & 2602 & 253 \\
\hline Nov. 4 & 1030 & 122.7 & 2752 & $N: A$ & 1070 & 147.8 & 1833 & 810 & 525 & 360 & 302 & 315 & 70 & 186 & 13 & 12130 & 2378 & 228 \\
\hline Nor. 4 & 1045 & 1241 & 2757 & $N / A$ & 1976 & 1469 & 1811 & 823 & 546 & 371 & 298 & 317 & 7.5 & 182 & 0.4 & 12237 & 2228 & 107 \\
\hline Nov. 4 & 2000 & 122.3 & 2761 & $N / A$ & 1980 & 1403 & 1842 & 025 & 537 & 371 & 315 & 310 & 70 & 108 & 50 & 12484 & 2472 & 234 \\
\hline Nov. 4 & 2015 & 1214 & 2750 & $N / A$ & 2003 & 1493 & 182.8 & 819 & 531 & $38 ?$ & 305 & 28.5 & 16 & 206 & 21 & 12388 & 2640 & 24.7 \\
\hline Nor.d & 2030 & 1230 & 2750 & $N / A$ & 2003 & 148.0 & 1813 & 830 & 560 & 381 & 311 & 30.6 & 73 & 180 & 14 & 12735 & 220.2 & 116 \\
\hline Nov. 4 & 2045 & 1226 & 2152 & $N / A$ & 2000 & 1488 & $100 ?$ & 843 & $56 ?$ & 374 & 304 & 298 & 13 & 103 & 1.6 & 12702 & 2450 & 23.7 \\
\hline Nov. 4 & 2100 & 1210 & 2751 & N/A & 2004 & 1505 & 1828 & 843 & 562 & 374 & 304 & 301 & 60 & 100 & 12 & 12847 & 244.7 & 4.0 \\
\hline Nov. 4 & 2115 & 1220 & 2761 & N/A & 2006 & 1406 & 1021 & 843 & 557 & 374 & 209 & 20.8 & 1.2 & 196 & 28 & 12770 & 250.3 & 4.5 \\
\hline Nor.4 & 2130 & 121.8 & 2756 & $N / A$ & 2004 & 1526 & 1844 & 056 & 546 & 386 & 310 & 30.8 & 7.6 & 23.0 & 3.0 & 13233 & 3042 & 12 \\
\hline Nor. 4 & 2146 & 1234 & 274.0 & $N / A$ & 2006 & 148.8 & 1810 & 853 & 568 & 378 & 303 & 20.4 & 73 & 196 & 3.3 & 12954 & 2637 & 40 \\
\hline Nov 4 & 2200 & 121.6 & 2745 & $N / A$ & 201.2 & 153.0 & 1834 & 825 & 546 & 375 & 303 & 30.2 & 60 & 207 & 30 & 12781 & 2648 & 60 \\
\hline Nov. 4 & 2215 & 118.3 & 275.4 & $N / A$ & 2011 & 1537 & 1832 & 012 & 560 & 376 & 301 & 303 & 71 & 177 & 41 & 12637 & 274.3 & 20 \\
\hline Nov. 4 & 2230 & 120.8 & 2743 & $N / A$ & 2000 & 151.3 & 1827 & A28 & 559 & 374 & 301 & 30.3 & 7.1 & 185 & 31 & 12693 & 235.5 & 3.1 \\
\hline Nov. 4 & 2245 & 110.5 & 2740 & $N / A$ & 2004 & 1517 & 1823 & 832 & 560 & 374 & 305 & 205 & 72 & 191 & 1.0 & 12788 & 2444 & 1.3 \\
\hline Nov. 4 & 2300 & 1208 & 2740 & $N / A$ & 2000 & 1514 & 1830 & 832 & 660 & 374 & 305 & 29.8 & 64 & 106 & 1.3 & 12763 & 2378 & 63 \\
\hline Nov. 4 & 2315 & 118.6 & 2747 & $N / A$ & 2004 & 1531 & 1843 & 832 & 560 & 374 & 305 & 29.9 & 73 & 100 & 17 & 1280.6 & 244.0 & 23.6 \\
\hline Nor. 4 & 2330 & 120.2 & 273.5 & $N / A$ & 2002 & 1521 & 182.7 & 832 & 575 & 379 & 304 & 29.0 & 60 & 168 & 24 & 1282.2 & 217.2 & 22.2 \\
\hline Noy.4 & 2345 & 1189 & 2738 & $N / A$ & 1996 & 1522 & 1832 & 832 & 554 & 374 & 306 & 29.9 & 66 & 109 & $O B$ & 12830 & 2556 & 7.4 \\
\hline$A V$ & AOES & 118.1 & 278.2 & $N / A$ & 1089 & 1504 & 1791 & 7676 & 5245 & 3650 & 313.8 & 312 & 6.5 & 180 & 1.9 & 11690 & 2167 & 22.4 \\
\hline Nov. 5 & 0 & 118.8 & 2737 & $N / A$ & 1094 & 1514 & 1823 & 832 & 547 & 374 & 308 & 290 & 70 & 208 & 0.7 & 12763 & 2650 & 260 \\
\hline Nov. 5 & 16 & 1198 & 2743 & $N / A$ & 2000 & 1532 & 1843 & 832 & 559 & 355 & 318 & 301 & 70 & 102 & .9 .7 & 12014 & 405 & 24.0 \\
\hline Nov. 5 & 30 & 120.2 & 2726 & $N / A$ & 190.2 & 151.4 & 1828 & 823 & 571 & 350 & 318 & 29.3 & 6.7 & 162 & 90 & 12622 & 204.8 & 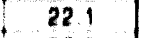 \\
\hline Nov. 5 & 45 & 1124 & 2741 & $N / A$ & 1991 & 150.0 & $180 \mathrm{~B}$ & 8.31 & 546 & 366 & 311 & 29.5 & 68 & 208 & 25 & 12622 & 2622 & 27.6 \\
\hline Nov. 6 & 100 & 1150 & 2736 & $N / A$ & 1985 & 148.2 & $180 ?$ & B31 & 572 & 360 & 200 & 298 & 71 & 16.1 & 11 & 1247.8 & 2005 & 10.9 \\
\hline Nor. 5 & 115 & 116.0 & 274.2 & $N / A$ & 1992 & 1501 & 182.3 & 806 & 550 & 372 & 318 & 295 & 7.2 & 171 & 3.0 & 12258 & & 0 \\
\hline Nor. 5 & 130 & 115.2 & 2756 & $N / A$ & 2002 & 1488 & 1823 & 813 & 567 & 377 & 313 & 29.5 & 71 & 15.1 & .11 & 12330 & 1867 & 108 \\
\hline Nov. 5 & 145 & 1150 & 2751 & N/A & 1988 & 150.7 & 1828 & 818 & 546 & 369 & 315 & 30 & 7.0 & 10.0 & 3.5 & 12482 & 237.2 & 1 \\
\hline Nov. 5 & 200 & 164 & 272.5 & N/A & 2029 & 147.6 & 1787 & 823 & 581 & 373 & 312 & 26.0 & 73 & 130 & .13 & 12305 & 1500 & .1 \\
\hline Nov. 5 & 215 & 1156 & 2716 & $N / A$ & 2043 & 1496 & 1790 & 818 & 587 & 378 & 314 & 256 & 12 & 141 & 0.4 & 12381 & 74.7 & 0 \\
\hline Nov. 5 & 230 & 1140 & 2721 & $N / A$ & 202.4 & 1483 & 181.8 & 861 & 609 & 376 & 308 & 279 & 7.1 & 13.8 & 01 & 13030 & 180.5 & 2 \\
\hline Nov 5 & 245 & 1146 & 270.3 & $N / A$ & 201.4 & 148.6 & 1783 & 850 & 591 & 381 & 306 & 271 & 6.7 & 166 & 3.8 & 12804 & 2120 & 4.7 \\
\hline Nov. 5 & 300 . & 1157 & 2726 & $N / A$ & 2031 & 1504 & 1707 & 853 & 612 & 370 & 306 & 256 & 1.1 & 14.3 & 10 & 12007 & 1856 & 5 \\
\hline Nov. 5 & 315 & 1148 & 2726 & IA & 2014 & 1504 & 179.8 & 872 & 600 & 375 & 300 & 278 & 70 & 164 & 1 & 4 & 66 & \\
\hline Nov 5 & 330 & 1156 & 2726 & $N / A$ & 2002 & 1506 & $181 \mathrm{n}$ & 864 & 600 & 381 & 311 & 284 & 70 & 162 & 1.4 & 13100 & 2130 & 6 \\
\hline Nor 5 & 345 & 1141 & 2730 & $N / A$ & 188.5 & 140. & 180.6 & B74 & 571 & 374 & 308 & 284 & 7.1 & 21.3 & 0.8 & 13273 & 2833 & 6 \\
\hline Nov. 5 & 400 & 1164 & 2732 & $N / A$ & 1883 & 1481 & 1780 & 836 & 580 & 361 & 309 & 601 & 72 & $15 ?$ & 38 & 12537 & 1064 & 5 \\
\hline Nov. 5 & 415 & 1145 & 272.1 & N/A & 1987 & 1491 & 170.3 & 846 & 589 & 377 & 309 & 287 & 69 & 16.3 & 1.6 & 12770 & 2080 & \\
\hline Nor. 5 & 430 & 1148 & 2718 & $N / A$ & 1979 & 1494 & 1804 & 840 & 587 & 383 & 312 & 291 & 68 & 156 & 14 & 12715 & 1988 & 2 \\
\hline Nov. 5 & 445 & 1161 & 2713 & N/A & 1968 & 1480 & 1782 & 854 & 500 & 376 & 306 & 28.2 & 7.2 & 168 & 20 & 1281.2 & 215.2 & 6 \\
\hline Nov. 5 & 500 & 1153 & 2723 & $N / A$ & 1958 & 147.7 & 178 & 855 & 588 & 74 & 0 & & 73 & 17 & & 12601 & 74 & \\
\hline Nov. 5 & 515 & 1160 & 2728 & $N / A$ & 1947 & 1495 & 179.8 & 871 & 567 & 374 & 303 & 311 & 73 & 218 & 2.7 & 13188 & 287.0 & 7 \\
\hline Nov. 5 & 530 & 1143 & 2721 & $N, A$ & 1926 & 1516 & 1804 & 873 & 559 & 378 & 308 & 323 & 13 & 23.8 & 20 & 15 & 0.7 & 17 \\
\hline Nov. 5 & 545 & 1163 & 2730 & $N / A$ & 1927 & 150.1 & 101.1 & 861 & $56 ?$ & 378 & 06 & 322 & 72 & 213 & 2.3 & 13003 & 2790 & 4 \\
\hline Nov 5 & 600 & 1143 & 273.5 & $N / A$ & 1830 & 151.2 & 17 & 851 & 567 & 372 & 09 & 30 & 71 & 1 & 1.5 & 13035 & 275.5 & 5 \\
\hline Nov. 5 & 615 & 1148 & 271.2 & $N / A$ & 1924 & 152.0 & 180.5 & 865 & 558 & 373 & 10 & 31.1 & 73 & 23.4 & 13 & & 15 & $\theta$ \\
\hline Nov -5 & 630 & 1154 & 2718 & A & 192.7 & 1501 & 1808 & 866 & 562 & 376 & 06 & 320 & 72 & 21.8 & 18 & 69 & 66 & 62 \\
\hline Nov. 5 & 645 & 144 & 2735 & A & 192.7 & 152.1 & 1792 & 876 & 564 & 375 & 14 & 31 & 73 & 241 & 16 & 98 & 58 & 01 \\
\hline Nov. 5 & 700 & 155 & 272.2 & $a$ & 1930 & 148.8 & 177.8 & 8 & 558 & 363 & 311 & & 69 & 222 & 2.5 & 12. & 3.0 & 7 \\
\hline Nov 5 & 715 & 1150 & 2716 & $N / A$ & 1937 & 1504 & 180.4 & 856 & 567 & 80 & 309 & 3 & 72 & 207 & 24 & 13050 & 6 & 2 \\
\hline Nov. 5 & 730 & 1154 & 272.7 & A & 1833 & 1486 & 1763 & 878 & 584 & 364 & 301 & 287 & 69 & 212 & 19 & 13233 & 2803 & 6.0 \\
\hline Nov. 5 & 745 & 1164 & 2734 & A & 1837 & 149.9 & 1781 & 864 & 578 & 380 & 308 & 30 & 7.3 & 206 & 37 & 13128 & $270 ?$ & 5.7 \\
\hline Nov. 5 & 800 & 114.3 & 2738 & $A$ & 1937 & 0 & 0 & 851 & 578 & 373 & 312 & 3 & 69 & $?$ & ? & 0.0 & 0 & D \\
\hline Nov 5 & B 15 & 1160 & 2730 & $N / A$ & 194.5 & 1504 & 179.8 & 874 & 556 & 367 & 297 & 300 & 6.9 & 241 & 34 & 4 & 5 & 27 \\
\hline ov. 5 & 830 & 1158 & 2747 & A & 1958 & 1500 & 189.0 & 878 & 583 & 380 & 311 & 29.9 & 72 & 20.5 & 1.8 & 13445 & 2756 & 70 \\
\hline Nov. 5 & 845 & 115.7 & 2750 & & 1859 & 1.3 & 1 & 874 & 600 & 375 & 07 & 29.9 & 7.4 & 20.8 & 5.6 & 11.5 & 2.4 & 0.2 \\
\hline Nov.5 & 900 & 1148 & 274.2 & & 196.8 & 1518 & 1802 & 6 & 6 & 374 & 1 & 29.6 & 69 & 20.2 & 13 & 13173 & & 76 \\
\hline Nov. 5 & 815 & 114.9 & 273.7 & $N / A$ & 1868 & 150.6 & 180 & 877 & 586 & 373 & 287 & 294 & 72 & 198 & 46 & 13380 & 2658 & 65 \\
\hline Nov. 5 & 830 & 114.5 & 2733 & A & 1873 & 1515 & 181.3 & 853 & 568 & 363 & 305 & 292 & 10 & 204 & .0 .7 & 13100 & 6 & 5 \\
\hline Nov. 5 & 945 & 115.1 & 2732 & & 1963 & 150.8 & 1820 & 869 & 572 & 368 & 304 & 293 & 71 & 207 & 0.2 & 13285 & 2746 & 27.8 \\
\hline Nov. 5 & 1000 & 1154 & 274.2 & & 1958 & 151.8 & $180 . !$ & 856 & 569 & 358 & 311 & 30.7 & 7.2 & 21.2 & 2.6 & 13172 & 278.8 & 66 \\
\hline Nov. 5 & 1015 & 114.5 & 2730 & $N / A$ & 196.2 & 151.6 & 1801 & 855 & & 373 & 289 & 295 & 71 & 209 & 5.0 & 13131 & & 7.6 \\
\hline $2 v-5$ & 1030 & 114.8 & 73.3 & N/A & 1056 & 1518 & 1798 & 858 & 569 & 372 & 286 & 30.0 & 7.3 & 21.5 & 58 & 13195 & & 73 \\
\hline$=1$ & 1045 & 14.8 & 2734 & & 196.9 & 152.2 & 181.5 & 860 & 549 & 368 & 297 & 29.8 & 71 & 239 & 41 & 13260 & 317.4 & 316 \\
\hline Nov. 5 & 1100 & 1158 & 2728 & & 200.9 & 150.7 & 178 & 850 & 605 & 360 & 297 & 30.4 & 73 & 159 & 1.5 & 13114 & 208.0 & 19.7 \\
\hline Nov. 5 & 1116 & 1148 & 2733 & $N / A$ & 2004 & 1536 & 180 & 852 & 580 & 367 & 297 & 28.7 & 7.1 & 185 & 51 & 13258 & 5 & 5.4 \\
\hline v. 5 & 1130 & 152 & 271.8 & N/A & 197.9 & 153.6 & 1819 & 866 & 581 & 356 & 296 & $33 . ?$ & 7.6 & 20.5 & 1.5 & 1348.3 & & 2.7 \\
\hline$v \cdot 5$ & 1145 & 115.1 & 271.8 & N & 200.1 & 153.1 & 180.3 & 856 & 595 & 364 & 293 & 274 & 7.8 & 182 & 5.1 & 13284 & 241.7 & 23.4 \\
\hline v. 5 & 1200 & 1198,8 & & & 199.9 & 151.8 & 180. & 848 & 583 & 354 & 298 & 278 & 8.6 & 185 & 0.3 & 13048 & 240.8 & 211 \\
\hline Nov. 5 & 1215 & 1154 & 271.5 & N/A & 1889 & 152.3 & 179 & 862 & 582 & $36 \theta$ & 298 & 28. & 8.1 & 206 & 51 & 13300 & 274.1 & 24.9 \\
\hline Nov 5 & 1230 & 114.3 & 273.6 & $N / A$ & 199.6 & 150.2 & 1779 & 883 & 600 & 359 & 295 & 278 & 82 & 19.6 & 20 & 13444 & 2631 & 24.1 \\
\hline
\end{tabular}


CZDIIME INJCION DAYA AUOUST 17, 10 NOVEMBEA 16, 1992

\begin{tabular}{|c|c|c|c|c|c|c|c|c|c|c|c|c|c|c|c|c|c|c|}
\hline Deto & Timo & $\begin{array}{l}\text { Bolle! } \\
\text { Loed } \\
\text { MN }\end{array}$ & $\begin{array}{l}\text { Duct } \\
\text { Inlol } \\
\text { Tomp of }\end{array}$ & $\begin{array}{l}\text { Avg " } \\
\text { 8uchic } \\
\text { Temp }\end{array}$ & $\begin{array}{l}\text { Duct } \\
\text { Outlat } \\
\text { Tamp of }\end{array}$ & $\begin{array}{l}\text { Flue Gas } \\
\text { Flow In } \\
\text { kBcrm }\end{array}$ & $\begin{array}{l}\text { Fue Oa: } \\
\text { Fiow Ou } \\
\text { Kectim }\end{array}$ & $\begin{array}{l}802 \\
\ln 101 \\
p p m\end{array}$ & $\begin{array}{l}\text { Ooz } \\
\text { Outloi" } \\
\text { ppm }\end{array}$ & $\begin{array}{l}\text { Non } \\
\text { Iniel } \\
\text { ppm }\end{array}$ & $\begin{array}{l}\text { Non } \\
\text { Oullet } \\
\text { ppm }\end{array}$ & $\begin{array}{l}\text { lime } \\
\text { Flow: } \\
\text { opm }\end{array}$ & $\lim _{\infty}$ & $\begin{array}{c}50 ? \\
\text { Homova! }\end{array}$ & $\begin{array}{c}\text { No } \\
\text { Homar } \\
\text { val }\end{array}$ & 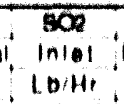 & $\begin{array}{l}802 \\
\text { Aemoved } \\
\text { Lb/H: }\end{array}$ & limo \\
\hline Nor. 5 & 1245 & 1165 & $212 ?$ & $N / A$ & 1091 & 1500 & 171.5 & 814 & 604 & 352 & 281 & 218 & B? & 210 & 21 & 13806 & 3027 & 217 \\
\hline Nor. 5 & 1300 & 1167 & 2114 & $N / A$ & 1990. & 1400 & 1782 & 001 & 500 & 357 & 292 & 284 & 80 & 201 & 26 & $1352 ?$ & 2710 & 251 \\
\hline Nor. 5 & 1315 & 1149 & 2736 & $N / A$ & 1996 & 1510 & 1717 & 1069 & 502 & 360 & 200 & $28 \mathrm{~J}$ & 10 & 180 & 53 & 13301 & 2644 & 24.5 \\
\hline Nur. 5 & 1330 & 1168 & 2716 & $N A$ & 1001 & 1505 & 1765 & 696 & 598 & 356 & 203 & 270 & 81 & 200 & 26 & 13660 & 2037 & $26 \mathrm{~J}$ \\
\hline Nov. 5 & 1345 & 1162 & 2714 & $N / A$ & $198 \mathrm{~B}$ & 1516 & 1015 & 860 & 573 & 362 & $30 ?$ & 283 & 62 & 202 & $0 ?$ & 13208 & 2671 & 241 \\
\hline Nor 5 & 1100 & 1142 & 2715 & $N / A$ & 1070 & 1489 & 1763 & 84? & 571 & 361 & 295 & 296 & 10 & 199 & 20 & 12863 & 2556 & 226 \\
\hline Nor 5 , & 1415 & 1165 & 2604 & $N / A$ & 1973 & 1486 & 1718 & 870 & 545 & 370 & 207 & 273 & 60 & $25 ?$ & 48 & 13193 & 3364 & 92.5 \\
\hline Nov 5 , & 1430 & 1153 & 2713 & $N / A$ & 1900 & 1512 & 1815 & 067 & 573 & 310 & 208 & 206 & 11 & 207 & 20 & 13280 & 2752 & 14 \\
\hline Noy 5 , & 1445 & 1147 & 2711 & $N / A$ & 1972 & 1467. & 1848 & 870 & 568 & 371 & 205 & 266 & 10 & 178 & 4 & 12837 & 2302 & 27 \\
\hline Nor. 5 , & 1500 & 1148 & 2693 & $N / A$ & 1990 . & 1406 & 1804 & 864 & 586 & 371 & 200 & 271 & 62 & 102 & $? 7$ & 13004 & 238 & 23 \\
\hline Nor-5 & 1515 & 1150 & $270 ?$ & $N / A$ & 1937 & 1484 & 183.5 & 878 & 545 & 362 & 290 & 290 & 77 & 234 & 11 & 13222 & 3000 & 291 \\
\hline Nor 5 & 1530 & 1144 & 2708 & $N / A$ & 1922 & 1500 & 1814 & 855 & 878 & 360 & 200 & 31.5 & 6.2 & 186 & 28 & 13062 & 2430 & 107 \\
\hline Nor 5 & 1545 & 1150 & 2711 & $N / A$ & 1810 & 1400 & 1807 & 876 & 575 & 967 & 287 & 326 & 37 & 208 & 30 & 13204 & $278 . ?$ & 232 \\
\hline Nor 3 & 1600 & 1130 & 2732 & $N / A$ & 1817 & 151.7 & 1828 & 045 & 662 & 358 & 293 & & 01 & 100 & 1.4 & 12004 & 2589 & 2 \\
\hline Nor. 6 , & 1615 & 1165 & 2710 & $N / A$ & 1905 & 1493 & 1800 & 894 & 564 & 371 & 289 & 32 & 17 & 239 & 30 & 13520 & 3235 & 276 \\
\hline Nor.5 & 1630 & 1145 & 2718 & $N / A$ & 1900 & 1408 & 1837 & 017 & 563 & 366 & 296 & 340 & 10 & 214 & 10 & 13317 & $284 ?$ & 21.7 \\
\hline Nov 5 & 1045 & 1166 & $270 ?$ & $N / A$ & 190.1 & 1405 & 1807 & 081 & 369 & 312 & 300 & 31.1 & 80 & 200 & 25 & 13405 & 2704 & 228 \\
\hline Nor.5 & 1100 & 1130 & 2718 & $N / A$ & 1807 & 1520 & 1820 & 650 & 542 & 365 & 281 & 33.3 & B 1 & 240 & 30 & 13212 & & 246 \\
\hline Nor. 5 , & 1715 & 1157 & 2608 & NiA & 1898 & 1496 & 1800 & 674 & 540 & 360 & 296 & 313 & 80 & 240 & 20 & 13246 & 3176 & 260 \\
\hline Nor 5 , & 1730 & 1141 & 212.3 & $N / A$ & 1911 & 1514 & 1823 & 861 & 656 & 367 & 290 & 318 & 10 & 22.3 & 1.8 & 13206 & 2046 & 246 \\
\hline Nor 1 , & 1745 & 1157 & 2602 & $N / A$ & 1002 & 1498 & 1800 & 076 & 568 & 375 & 300 & 310 & 01 & 220 & 37 & 13270 & 2020 & 244 \\
\hline Nor 6 , & 1800 & 1153 & 2698 & $1 / A$ & 1913 & 1510 & 1916 & 668 & 570 & 374 & 304 & 313 & 70 & 211 & $2 ?$ & 13278 & 2800 & 230 \\
\hline Nor 5 & 1815 & 1140 & 2703 & $N A$ & 1810. & $1503 \%$ & 1832 & B84 & 567 & 379 & 304 & $318:$ & 62 & 218 & 23 & 13460 & 2045 & 237 \\
\hline Nor. 5 & 1830 & 1150 & 2712 & NiA & 1810 & 1511 & 1823 & 869 & 552 & 371 & 297 & 315 & 19 & 235 & 35 & .13310 & 3123 & 20 \\
\hline Nov 5 & 1845 & 1146 & 11 & $N / A$ & 1805. & 1517 & 1832 & B?2 & 556 & 367 & 302 & 38 & B. 1 & 229 & 00 & 13400 & 3072 & \\
\hline Nor 6 & 1800 & 1137 & 11 & $1 / A$ & $19 ! 1$ & 1511 & 1825 & 066 & 560 & 365 & 301 & 311 & 62 & 220 & 04 & .13264 & 2918 & 240 \\
\hline Nor.s & 1915 & 1151 & 2113 & $N / A$ & 1001 & 1484 & 176.7 & 886 & 589 & 361 & 299 & 310 & 62 & 220 & 14 & 13328 & 2028 & 23 \\
\hline Nor. 3 & 1030 & 1185 & 28 & $N / A$ & 1804 & 1481 & 1704 & 896 & 880 & 360 & 209 & 332 & 82 & 216 & 1.0 & & & 23 \\
\hline Nov. 3 & 1045 & 1148 & 114 & $1 / \mathrm{A}$ & 1886 & 1471 & 1708 & 811 & 574 & 372 & 200 & & 10 & 104 & 21 & 12 & 2523 & 20 \\
\hline Nov. 5 & 2000 & 115.8 & 2107 & $N / A$ & 1677 & 1476 & 1817 & 872 & 576 & 370 & 208 & 341 & 81 & 18.7 & 00 & 13038 & 243.4 & 11 \\
\hline Nov. 5 & 2015 & 1138 & 2. & $N: A$ & 1808 & 1483 & 181.6 & 670 & 575 & 374 & 296 & 320 & 79 & 189 & 31 & 13 & 2634 & $2 !$ \\
\hline Nor 5 & 2030 & 114.8 & 2724 & A & 1874 & 1461 & 1810 & 079 & 568 & 363 & 293 & 327 & 78 & 19.9 & $0 ?$ & 13000 & 2502 & 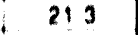 \\
\hline Nav. 5 & 2045 & 1151 & 2726 & $N / A$ & 1880 & 1463 & $180 ?$ & 870 & 572 & 366 & 281 & 332 & B? & 198 & 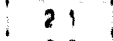 & 19 & 502 & , \\
\hline Nor 5 & 2100 & 1154 & 271.7 & $N / A$ & $180 ?$ & 145.8 & 1707 & 810 & 572 & 366 & 290 & 32 & 81 & 188 & 22 & 12902 & 7 & 206 \\
\hline Nov. 5 & 2115 & 1153 & 2742 & $N A$ & 1804 & 1510 & 1860 & 870 & 500 & 366 & 281 & 3 & 78 & 187 & 50 & 13 & 7 & $J$ \\
\hline Nov 5 & 2130, & $116 ?$ & 2800 & iA & 1913 & 1507 & 1856 & 870 & 532 & 376 & 283 & & 18 & 254 & & 18 & 3 & \\
\hline Nov. 5 & 2145 & 1246 & 2804 & $N / A$ & 1034 & 1528 & 1880 & 896 & 573 & 376 & 294 & 34 & 04 & 214 & 30 & 76 & 2971 & 4 \\
\hline Nov. 5 & 2200 & 1378 & 2119 & $N / A$ & 1958 & 1604 & 1070 & 200 & 508 & 356 & 204 & $34 \mathrm{~d}$ & 84 & 180 & 18 & 14631 & 63.3 & 181 \\
\hline Nov. 5 & 2215 & 1436 & 2783 & /A & 196.8 & 1691 & 2087 & 215 & 587 & 360 & 281 & $36 ?$ & 80 & $20 \mathrm{~B}$ & 0 & 15 & 1 & \\
\hline Nor. 5 & 2230 & 1451 & 2791 & A & 1088 & 1600 & 2037 & 814 & 610 & 360 & 283 & 3 & 81 & 180 & 2 & 15 & 8 & \\
\hline Nov 5 & 2245 & 1444 & 2790 & $N / A$ & 1088 & 1708 & 2016 & 000 & $50 !$ & 360 & 292 & 3 & 64 & 200 & 1 & .16 & 5 & 227 \\
\hline Nor. 5 & 2300 . & 1387 & 2701 & $N / A$ & 1090 & $165 ?$ & 2011 & 000 & 501 & 360 & 292 & 34.7 & 18 & 211 & 15 & 16 & 8 & 0 \\
\hline Nov 5 & 2315, & 1428 & 2180 & $N / A$ & 1908. & 16 & 205 & 800 & 626 & 360 & 305 & & a 0 & 150 & & & & \\
\hline Nor. 5 & 2330 & 1454 & 278.2 & $N A$ & 2007 & 171.3 & 2006 & 200 & 608 & 360 & 280 & 35 & 78 & 182 & 17 & 15773 & 2863 & 216 \\
\hline Nov 8 & 2345 & 1440 & 2797 & $N / A$ & 1998 & $18 B 8$ & $200 \mathrm{~s}$ & 000 & 600 & 360 & 297 & 360 & 79 & 205 & 20 & 15543 & 184 & 136 \\
\hline & IAOES, & 1177 & 2730 & $N / A$ & 1057 & 1485 & 1789 & 8667 & 5761 & 3680 , & 3010 ? & $30 ?$ & 76 & 200 & 1.5 & 13051 & $260 . A$ & 6 \\
\hline Nov.6 & 0 & $139 \mathrm{~d}$ & 2790 & $N / A$ & 1007 & 17 & 2086 & 008 & $58 B$ & 360 & & & 81 & 243 & 60 & (2) & 5 & \\
\hline Nov 6 & 15 & 1467 & 2770 & $N / A$ & 2009 & 1776 & 2137 & 844 & 585 & 340 & 3 & 36 & a & 16.6 & 00 & 9.5 & 1 & 78 \\
\hline Nov. 6 & 30 & 1417 & 2780 & $N / A$ & 2011 & 1768 & 210.6 & 840 & 571 & 356 & 334 & 36.8 & B 1 & 190 & 18 & $1504 \mathrm{a}$ & 364 & $0 !$ \\
\hline Nov 6 & 45 & 1454 & 279.2 & $N / A$ & 2012 & 1775 & 2109 & 856 & 601 & 342 & 315 & 38 & 10 & 166 & 02 & 15303 & 5.2 & 4 \\
\hline Nov. 6 & 100 & $146 ?$ & 2806 & $N / A$ & 2027. & 17 & 2092 & 857 & 585 & 342 & 3 & 35 & 80 & $18 ?$ & 04 & & 4 & \\
\hline Nov. 6 & 115 & 1397 & $279 ?$ & $N / A$ & 2012 & 1721 & 2068 & 882 & 591 & 353 & 326 & 35.2 & 83 & 184 & 110 & 78 & 986 & $A$ \\
\hline ov. 6 & 130 & 1437 & 2770 & A & 2046 & 176.1 & 2086 & 873 & 625 & 364 & 311 & 32 & B 1 & 151 & .13 & 15575 & 57 & 8 \\
\hline 6 & 145 & 1433 & 2718 & & 2030 & 17 & $210 ?$ & 888 & 570 & 365 & 3 & 3 & 81 & 230 & 17 & 40 & 0 & \\
\hline 2. 6 & 215 & $129 ?$ & 2781 & A & 2107 & 16 & 1030 & 834 & 603 & 38 & 3 & 28 & 86 & 165 & 50 & 8 & 3 & 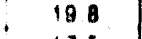 \\
\hline Nov. 6 & 230 & 1377 & 2757 & $N A$ & 2069 & 1606 & 1087 & 876 & 643 & 362 & 3 & 28 & 84 & 140 & 00 & 1 & 0 & 8 \\
\hline or. 6 & 245 & 1437 & 2766 & A & 2086 & 1772 & 2063 & 862 & 644 & 365 & 3 & 30 & 82 & 160 & 0 & 15833 & 8 & 1 \\
\hline 6 & 300 & 143.3 & 2780 & & 2083 & 17 & 2081 & 874 & 622 & 3 & 3 & 3 & $8 ?$ & 171 & 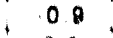 & 3 & & \\
\hline 6 & 315 & 1382 & $277 ?$ & & $205 ?$ & 17 & $108 ?$ & 887 & 622 & 365 & 3 & 32 & 84 & 18.8 & 28 & .15420 & 0 & 2 \\
\hline av. 6 & 390 & 138.1 & 2775 & $N A$ & 2044 & 1738 & 2041 & 884 & 613 & 367 & 304 & 33 & 80 & 186 & 27 & 15665 & 1 & 8 \\
\hline ov. 6 & 146 & 137. & 2770 & A & 2032 & 1706 & 1898 & 802 & 617 & 3 & 30 & 32 & 80 & 189 & 4 & 96 & 8 & 53 \\
\hline 6 & 400 & 142.2 & & & 2033 & & 2043 & 85 & 600 & & 3 & 3 & 81 & 169 & 3 & & 1 & $?$ \\
\hline v. 6 & 416 & 1393 & & & 2027 & 17 & 2045 & 8 & & 3 & 2 & 3 & 80 & 186 & 48 & & & \\
\hline or 6 & 430 & 1380 & 2770 & $N / A$ & 2025 & 1744 & 2037 & 898 & 617 & 374 & 302 & 32 & 81 & 108 & 5 & 73 & 41 & B \\
\hline 6 & 445 & 1373 & 2750 & A & 2014 & 17 & 2013 & 900 & 606 & 3 & $3 c$ & 32 & B. 1 & 21.7 & b & B 1 & & $\theta$ \\
\hline & 500 & 142.8 & & & 2014 & & & $\theta$ & & & & & 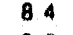 & 20.6 & & & & \\
\hline Nov. 6 & 515 & 1377 & 276.7 & A & 2028 & 1721 & 2014 & Q14 & 617 & 372 & 306 & 32 & B 2 & 208 & 36 & & & \\
\hline ov. 6 & 530 & 1400 & 2764 & A & 2037 & 1722 & 2019 & 810 & 612 & 371 & 30 & 31 & 78 & 212 & 52 & 82 & 56 & 8.3 \\
\hline ov. 6 & 545 & 1462 & 276.8 & & 2052 & 1 & 25 & 8 & 633 & & & 32 & $?$ & 198 & 35 & 10 & & 8 \\
\hline Vov. 6 & 600 & 1427 & & & 2076 & & & 90 & 651 & 373 & r. & 28 & 80 & 17.8 & 46 & 16326 & & \\
\hline Vov. 6 & 615 & 1383 & 275.5 & $N / A$ & 2066 & 1712 & 1873 & 918 & 643 & 356 & 302 & 294 & 80 & 193 & 2.1 & 15028 & 3068 & 274 \\
\hline$v \cdot 6$ & 630 & 1376 & 274.8 & $N / A$ & 2067. & 1718 & 1983 & 810 & 644 & 368 & 300 & 293 & 80 & 18.1 & 5.7 & 15993 & 3058 & 274 \\
\hline 20 & 645 & 1382 & 2744 & & 2048 & 1700 & 1958 & 016 & 632 & 363 & 28 & 30 & 82 & 20.5 & 55 & 15774 & 3230 & 272 \\
\hline Vov. 6 & 700 & 1450 & 2747 & $N / A$ & 2035 & 1780 & 2097 & 020 & 015 & 365 & 296 & 327 & 17 & 212 & 45 & 16588 & 3513 & 293 \\
\hline
\end{tabular}


C2D IIME NJECTION DATA AUQUST 17, 10 NOVEMBEA 16, 1992

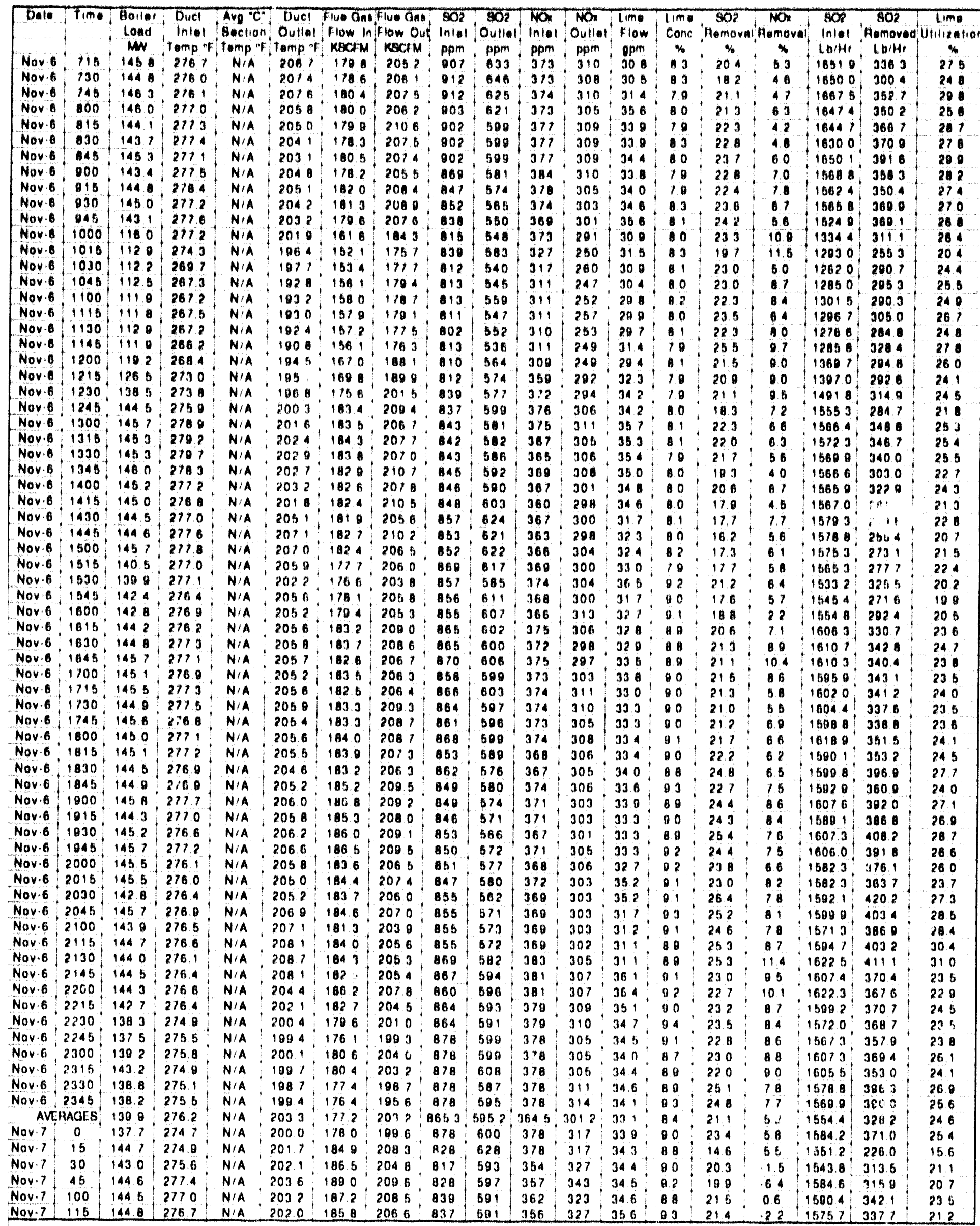


CZD LIMI INJECTION DATA AUQUST 17. TO NOVEMASE 16. 190 ?

\begin{tabular}{|c|c|c|c|c|c|c|c|c|c|c|c|c|c|c|c|c|c|c|}
\hline Dalo & fimo & $\begin{array}{l}\text { Boilo? } \\
\text { Load } \\
\text { MN }\end{array}$ & $\begin{array}{c}\text { Duct } \\
\text { Iniol } \\
\text { Tomp of }\end{array}$ & $\begin{array}{l}\text { Avg "C", } \\
\text { Section," } \\
\text { Tump of }\end{array}$ & $\begin{array}{l}\text { Duct } \\
\text { Outlat } \\
\text { Tampont }\end{array}$ & $\begin{array}{l}\text { Flue Ons } \\
\text { flow in } \\
\text { KECKM }\end{array}$ & $\begin{array}{l}\text { Tlue Ons } \\
\text { Flow Out } \\
\text { KECAM }\end{array}$ & $\begin{array}{l}02 \\
\ln 101 \\
\mathrm{ppm}\end{array}$ & $\begin{array}{c}802 \\
\text { Outloi } \\
\text { ppm }\end{array}$ & $\begin{array}{l}\text { NO } \\
\text { iniol } \\
\text { ppm }\end{array}$ & $\begin{array}{l}\text { NO } \\
\text { Oullel } \\
\text { ppim }\end{array}$ & $\begin{array}{l}\text { lime } \\
\text { low } \\
\text { gpm }\end{array}$ & 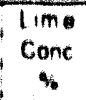 & $\begin{array}{c}80 ? \\
\text { Hemoval } \\
\text { \% }\end{array}$ & Nar & $\begin{array}{l}902 \\
\ln |0| \\
\mathrm{L} / \mathrm{H} \mid\end{array}$ & $\begin{array}{c}802 \\
\text { Romoveo } \\
\text { lo/Hit }\end{array}$ & $\begin{array}{c}\lim \\
\text { Ililizelic } \\
*\end{array}$ \\
\hline ov. 7 & 130 & 1430 & 2712 & $N / A$ & 2023 & 1850 & 2004 & 837 & 584 & उEA & 315 & 351 & 19 & 217 & 4.1 & 16741 & & 20 \\
\hline $28-7$ & 145 & 1394 & 2751 & $N / A$ & 1994 & 1716 & 1976 & 040 & 580 & 363 & 310 & $34 \mathrm{~s}$ & 92 & 23.2 & b) & 15123 & 3513 & 231 \\
\hline Nor. 7 & 200 & 1413 & $273 \mathrm{~s}$ & $N / A$ & 1994 & $102 ?$ & 2055 & 956 & 560 & 385 & 126 & 345 & 90 & 255 & 18 & 15800 & 1035 & 271 \\
\hline Nor. 7 & 215 & 1450 & 2744 & $N / A$ & 2017 & 1879 & 2001 & 856 & 585 & 391 & 330 & 342 & 91 & 243 & 65 & 10205 & 3062 & 265 \\
\hline Nov. 7 & 230 & 1940 & 2753 & $N / A$ & 2026 & 1802 & 2103 & 858 & 576 & 303 & 32 & 345 & 00 & 241 & 55 & 16301 & 4028 & 274 \\
\hline Nor 7 & 245 & 1431 & 2761 & $N / A$ & 2010 & 185.1 & 2016 & 846 & 556 & $30 ?$ & 320 & 350 & 12 & 250 & 57 & 16864 & 11113 & 250 \\
\hline Nov. 7 & 300 & 1422 & 2741 & $N / A$ & 2016 & 1860 & 2052 & 044 & 570 & 365 & 323 & 342 & 68 & 255 & 1.4 & & 1063 & $8 ?$ \\
\hline Nor. 1 & 315 & 1422 & 75.4 & $N / A$ & 2026 & 1854 & 2048 & 838 & 578 & 366 & 323 & 3.10 & Q? & 240 & 76 & 15760 & 3117 & 250 \\
\hline Nor. 7 & 330 & 1426 & 15.3 & $N / A$ & 2044 & 1058 & 2050 & 635 & 579 & 385 & 326 & 120 & 16 & 231 & 60 & 15 & 3631 & 276 \\
\hline Nor. 7 & 345 & 1410 & 0 & $N / A$ & 2033 & 1826 & 2042 & 044 & 574 & 300 & 327 & 330 & 0. & 230 & 6 & 15606 & 3730 & 256 \\
\hline Nov. 7 & 100 & 1400 & 731 & $N / A$ & 2028 & $10 \% ?$ & 2028 & 84? & 570 & 304 & 323 & $31 \%$ & 0.2 & 234 & 65 & & 3641 & 600 \\
\hline Nor 7 & 415 & 1981 & 2120 & $N / A$ & 2016 & 1714 & 1066 & 856 & 564 & 382 & 324 & 3 & 91 & 211 & 50 & 3 & $416 \mathrm{~B}$ & 01 \\
\hline Nov. 7 & 430 & 1300 & 2 & $N / A$ & 2018 & $176 \mathrm{~B}$ & 1959 & 050 & 512 & 381 & 319 & 31.7 & 90 & 262 & 17 & 36 & 4032 & $10 \mathrm{~B}$ \\
\hline Nor. 7 & 445 & 1376 & 142 & $N / A$ & 2026 & 170.0 & 2004 & 058 & B) & 382 & & 31 & & 250 & 64 & & $300 ?$ & 16 \\
\hline Nor. 7 & 500 & 1373 & 2730 & $N / A$ & 2021 & 1780 & 1983 & 851 & B) & 387 & 319 & & 00 & 247 & 02 & 6 & 3701 & 14 \\
\hline Nov 7 & 515 & 1374 & 9 & $N$ & 2016 & 11 & 10 & 858 & 575 & 300 & 322 & 31 & 84 & 263 & 00 & 11 & 0 & 1 \\
\hline Nor. 7 & 530 & 1300 & 116 & $N / A$ & 2001 & 1700 & 2040 & 848 & 583 & 384 & 317 & 32 & 03 & 236 & 50 & & & 3 \\
\hline Nor 7 & 645 & 1396 & 2720 & $N / A$ & 2020 & 1800 & 201 & 850 & 501 & 385 & 311 & & & 236 & 4 & $2 !$ & 0 & 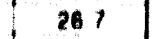 \\
\hline Nov. 7 & 600 & 1404 & 11 & $N / A$ & 2010 & 1804 & 1004 & 856 & 578 & 386 & 32 & 30 & a & 253 & 15 & & 1 & 00 \\
\hline Nor, 1 & 615 & $143 \mathrm{~B}$ & 11 & $N A$ & 2058 & 184 & 2058 & 150 & 588 & $38 ?$ & 323 & 30 & 00 & 231 & 60 & 16 & & 03 \\
\hline Nor. 7 & 630 & 1435 & 2733 & $N / A$ & 2063 & 184.4 & $204 ?$ & 060 & 500 & 385 & 310 & 30 & & 226 & 10 & 16 & 14 & 74 \\
\hline Nor. 7 & 645 & 1441 & & $N / A$ & 2069 & 1859 & $206 \mathrm{~A}$ & 860 & 594 & 363 & 320 & & 9 & 231 & 11 & & 5 & 80 \\
\hline Nor 7 ; & 700 & 1429 & 45 & $N / A$ & 2070 & 1050 & 2061 & 860 & 593 & $3 \times 4$ & 323 & 31 & 10 & 235 & 67 & & 1 & 00 \\
\hline Nor.? & 115 & 1456 & 2146 & $N / A$ & $20 \mathrm{HA}$ & 1856 & 2085 & 863 & 598 & 395 & & 3 & 10 & 221 & 74 & 6 & $389 B$ & 63 \\
\hline Noy. 7 & 130 & 1448 & 2754 & $N A$ & 2053 & $185 ?$ & & 013 & $60 ?$ & 311 & 315 & 3 & 92 & 236 & 76 & 0 & 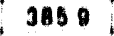 & 64 \\
\hline Nov 7 & 745 & 1440 & 3 & $N / A$ & 0 & 1050 & 20 & 857 & 680 & 364 & & & 0 & 255 & ? & & & D \\
\hline Nor 7 , & 000 & 1045 & 2740 & $N / A$ & 2048 & 1049 & 2075 & 861 & $8 / 3$ & 375 & 319 & & 80 & 254 & 44 & 31 & 0.1 & 1 \\
\hline Nor 7 & 815 & 1455 & & $N / A$ & 2053 & 18 & 2 & 945 & 501 & 311 & 31 & 33 & 00 & 229 & 64 & 6 & 6 & 5 \\
\hline Nov. 7 & 130 & 1452 & ? & $N / A$ & $204 ?$ & 185 & 2073 & 85.3 & 517 & 360 & 316 & & 9? & 251 & 16 & 16 & 13 & 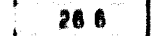 \\
\hline Nor 7 & 845 & 1450 & 2768 & $N / A$ & 2040 & 1813 & 20 & 014 & $5 / 4$ & 376 & 30 & 34 & $9 ?$ & 256 & 100 & $5 !$ & 0 & 2 \\
\hline Nov 7 & 900 & 1443 & & $N / A$ & 2042 & 1891 & 21 & Ba? & 564 & 3 & 3 & 35 & 91 & 257 & 66 & 3 & 10 & 271 \\
\hline Nov? & 815 & 1451 & $B$ & $N / A$ & 2015 & 1月8? & & 834 & 41 & 376 & 306 & & 00 & 284 & 101 & & & 6 \\
\hline Nov 7 & 930 & 1430 & 2753 & $N / A$ & $201 ?$ & 1863 & 20 & ayt & 544 & 360 & 30 & 3 & 92 & 270 & 03 & 15 & 4361 & 61 \\
\hline Nov 7 & 945 & 1421 & & $N A$ & 2016 & 1857 & 20 & 642 & 562 & 366 & $30 \%$ & 3 & 94 & 218 & B & 18 & 60 & 18 \\
\hline Nov 7 & 1000 & 1433 & 2140 & $N / A$ & 2001 & 18 & 2 & 037 & (5) & 36 & 204 & & 0 & 230 & , & & & \\
\hline & 1015 & 1430 & 2744 & $N / A$ & 2008 & 1861 & 20 & Bal & 560 & 366 & ana & 34 & 03 & 261 & 60 & 19 & 3 & 271 \\
\hline Nov $7 !$ & 1030 & $198 ?$ & $274 a$ & $N / A$ & $200 ?$ & 1798 & 1896 & hat & $56 ?$ & $30 ?$ & 303 & 33 & a & 265 & 118 & $154 / 4$ & 110.8 & 202 \\
\hline 7 & 1005 & $197 \mathrm{~B}$ & 2730 & $N / A$ & $189 ?$ & 18 & 3000 & 850 & 503 & 300 & 316 & & 00 & 26.7 & & 3 & 4163 & \\
\hline Nor 1 & 1100 & 1374 & 3 & $N / A$ & 1988 & 11 & 10. & 846 & $50 ?$ & 376 & 307 & 33 & 89 & 26.7 & 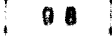 & $1 !$ & $\theta$ & \\
\hline Noy. 7 & 1116 & 1980 & $272 ?$ & Ni & 1984 & 1768 & 1050 & 946 & 502 & 305 & 308 & 33 & 90 & 267 & 6 & 3 & 4041 & 200 \\
\hline 11 & 1130 & 1423 & 10 & $N / A$ & 2013 & 18 & 2 & 851 & 12 & & 300 & & 0 & 7 & & 11 & & \\
\hline Nov. 7 & 1105 & 1417 & 2737 & $N / A$ & 2001 & $181 ?$ & 20 & 64? & 571 & 371 & 303 & 34 & 90 & 252 & 0 & 15 & 6 & 28 \\
\hline Noy. 2 & 1200 & 1301 & 2738 & $N / A$ & 2005 & 1783 & 1065 & $60 ?$ & 658 & $36 ?$ & 307 & 32 & 10 & 280 & 5 & 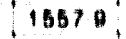 & 4357 & 3 \\
\hline 7 & 1215 & 1378 & 2741 & $N / A$ & 2008 & 17 & & 654 & 600 & 360 & 04 & & 9? & $23 ?$ & 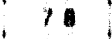 & 0 & 3580 & \\
\hline Nov? & 1230 & 1374 & 274 ? & $N: A$ & 2000 & 1738 & 1020 & 656 & 502 & 364 & 300 & 32 & $9 ?$ & 275 & 00 & 2 & $\theta$ & , \\
\hline ov. 7 ! & 1245 & 1380 & 2749 & $N / A$ & 2006 & 1751 & 1844 & 870 & 510 & 378 & 305 & 32 & 90 & 213 & 103 & $1543 \mathrm{~J}$ & 4216 & 301 \\
\hline ov.? & 1300 & 130.1 & 21 & & 2032 & 11 & 8 & 856 & $5 / 0$ & 364 & 290 & & 01 & 243 & 10 & 1 & $\theta$ & 2 \\
\hline 7 & 1315 & 1464 & $?$ & $N$ & 2039 & 10 & $?$ & OAA & 668 & 367 & 206 & 31 & 00 & 261 & 111 & 18 & & \\
\hline & 1330 & 144.0 & 2 & $N / A$ & 2035 & 1070 & 2013 & B36 & 570 & 362 & 290 & 344 & o a & 234 & a a & 68 & 7 & 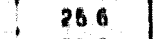 \\
\hline Nor 7 & 1345 & 1401 & 5 & $\mathbf{N}$ & 2040 & 17 & N & 013 & 576 & $36 ?$ & 300 & & $?$ & 60 & 14 & 1 & 9 & 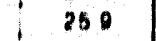 \\
\hline .1 & 1400 & 1388 & & $N / A$ & 2034 & 17 & 18 & 846 & 560 & 386 & 315 & 34 & 10 & 240 & 60 & & & 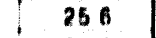 \\
\hline ov. 7 & 1415 & 140.5 & 1.8 & $N / A$ & 2031 & 1804 & 1002 & a5? & 664 & 380 & 312 & 32 & 01 & 260 & 0 & 10 & 7 & 0. \\
\hline 3. & 1430 & 1383 & & & $203 \mathrm{~A}$ & 17 & 98 & 851 & 677 & 367 & 317 & 7 & 00 & 4? & 01 & 12 & 1 & 11 \\
\hline Nor. 7 & 1445 & 1304 & & $N / A$ & 2043 & 178 & 10 & 956 & 5 & 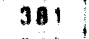 & 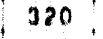 & 3 & 02 & 41 & 6 & & & $\because$ \\
\hline Nov. 7 & 1500 & 1370 & 2) & $N / A$ & 2037 & 17 & 180 & BaA & $5 n 1$ & 385 & 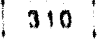 & 30 & 93 & $3 ?$ & 10 & 4 & 3 & . \\
\hline 11 & 1515 & 1370 & & & 2043 & 17 & $196 !$ & 805 & $5 / 8$ & 380 & 100 & 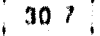 & 60 & 250 & 00 & 14 & & 3 \\
\hline Noy 7 & 1530 & 137.7 & & $N / A$ & 30. & & 10 & A: & & & & & 00 & 26 & & & & 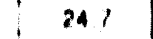 \\
\hline Nov? & 1545 & 1373 & 2734 & $N / A$ & 2020 & 11 & 10 & 062 & b & 1 & 6 & & 00 & $b$ & 7 & 4 & 0 & $20 \mathrm{~J}$ \\
\hline bv. 7 & 1600 & 1373 & 146 & $N: A$ & 2022 & 11 & 1062 & 月5 7 & 0 & 360 & 307 & 32 & 9? & 212 & 65 & 00 . & A & 53 \\
\hline & 161 & 10 & & & 20 & & & & & & & & $8 ?$ & & & & & 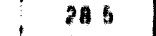 \\
\hline Nor 1 & 1630 & 1424 & 2741 & $N / A$ & 2011 & 1801 & 100 & 866 & 526 & 370 & 1 & & 10 & 3 & 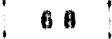 & & & \\
\hline 7 & 1645 & 1382 & 36 & $A$ & $109 ?$ & 1716 & 1 & 866 & 647 & 913 & 300 & 356 & B 8 & 280 & 65 & $1650 \mathrm{C}$ & 1500 & 100 \\
\hline 7 & 1700 & na. & & & 200.8 & 180 & & 870 & 586 & 360 & 302 & 3 & A A & 241 & 82 & 16053 & 17 & 1 \\
\hline Nov. 7 , & 1715 & 1461 & 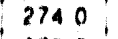 & $N$ & 2036 & 18 & & $B 64$ & & & & & 01 & 246 & 9 & & & \\
\hline ov. 7 & 1730 & 1452 & 159 & $N / A$ & $205 ?$ & 1873 & 2066 & 855 & 601 & 3日7 & 313 & 331 & B日 & 237 & 107 & 16224 & 3844 & 212 \\
\hline & & & & & & & & 850 & 579 & 3 & 69 & & 8 & 240 & 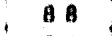 & 66 & 115 & 8 \\
\hline Nov. 7 & 1000 & 1381 & 273 & $N / A$ & 2004 & $17 !$ & 195 & 856 & 56 & & 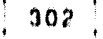 & & 0 & & & & & \\
\hline v. 7 & 1815 & 1403 & B & & $200 \mathrm{~A}$ & 170 & 203 & 065 & 572 & 36 & 300 & 33 & 80 & 251 & 6.5 & 15720 & 394. & 276 \\
\hline & 183 & & & & 20 & & & & 5 & & 302 & 34 & 1 & 255 & 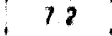 & & 017 & 60 \\
\hline Nov 1 & 1845 & 1424 & 2738 & $N / A$ & $202 ?$ & 1800 & 2020 & 872 & 500 & 367 & 303 & 323 & 02 & 237 & 0 & & & \\
\hline Vor? & 1900 & 1424 & 74.2 & $N / A$ & $20 C 6$ & 1831 & 3070 & 850 & 566 & 36 ! & 904 & 349 & B $\mathrm{A}$ & 255 & 68 & $1593 \mathrm{~A}$ & 1063 & 276 \\
\hline & 年 & 192.2 & & & 2024 & 181,8 & 2037 & 361 & 570 & , & 306 & 323 & A ? & 259 & 6.6 & 6 & 4104 & 5 \\
\hline Nov.? & 1930 & 1380 & 27 & N & 2028 & 171 & 19 & 8 & $5 \mathrm{~B}$ & 37 & 30 & $\pi$ & B & 237 & 75 & & 3640 & $\% a$ \\
\hline oy. 7 & 1845 & $142 ?$ & $c 0$ & NiA & 2028 & 1781 & 1007 & $86 ?$ & 590 & $36 \mathrm{~B}$ & 303 & 316 & 80 & 236 & 81 & 15640 & 3696 & 274 \\
\hline
\end{tabular}

CZDD Demo

Page 11.21

Quarterly Report No. 8 


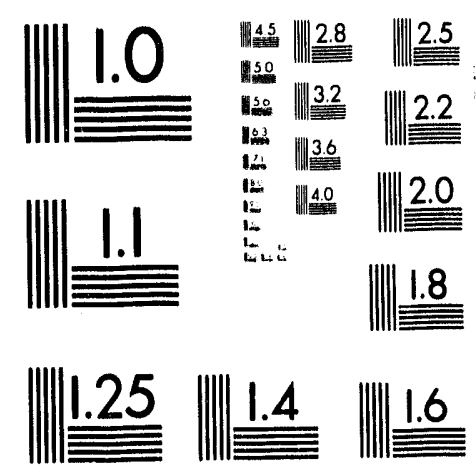



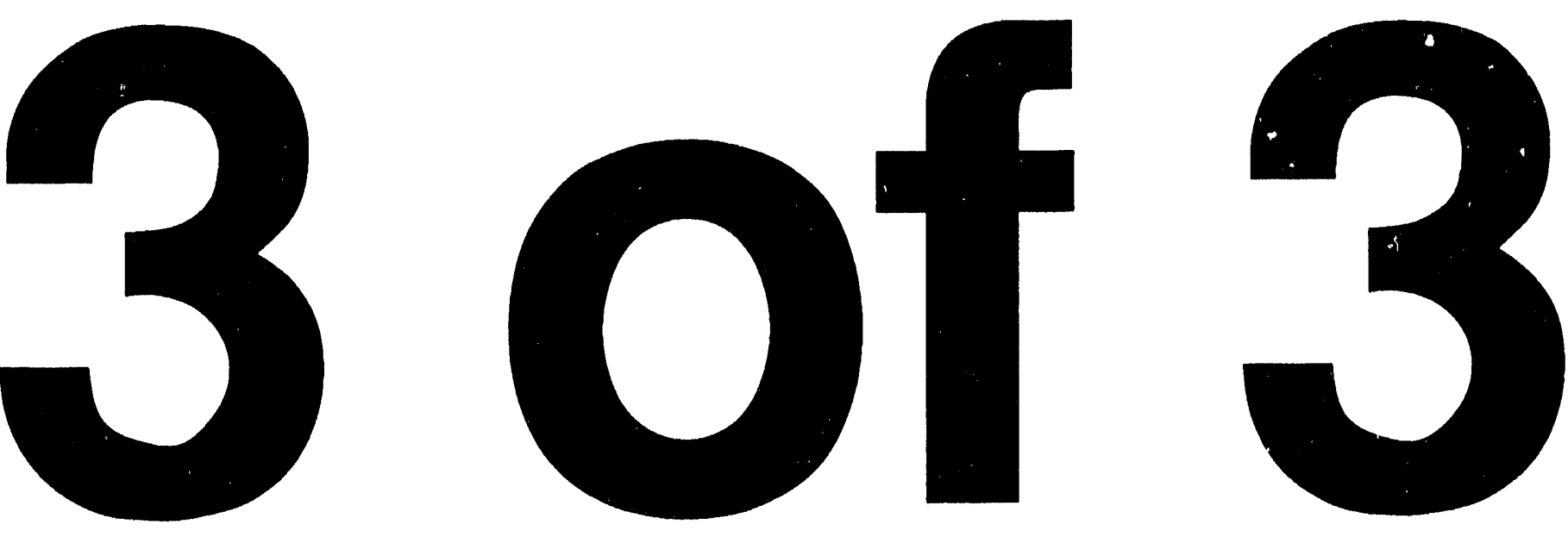


\begin{tabular}{|c|c|c|c|c|c|c|c|c|c|c|c|c|c|c|c|c|c|c|}
\hline Dato & Time & Boiler & Duct & Avg "C" & Duct & Flue Gas & Flue Gas & $\mathrm{SO} 2$ & SO2 & NOx & NOX & $\operatorname{Lim} \theta$ & $\operatorname{Lim} \theta$ & 502 & NOx & SO2 & SO2 & Lime \\
\hline & & Load & $\ln |0|$ & Soction & Outlet & Flow $\mathrm{In}$ & Flow out & In|e| & Outlet & $\operatorname{In} \mid \theta t$ & Outlat & Flow & Conc & Removal & Removal & inlet & Removed & Utilization \\
\hline & & $\mathrm{MN}$ & Temp oF & Temp ${ }^{\circ} \mathrm{F}$ & Temp oF & KSCFM & $\mathrm{KSCFM}$ & $\mathrm{ppm}$ & $\mathrm{ppm}$ & $\mathrm{ppm}$ & $\mathrm{ppm}$ & $\mathrm{gpm}$ & $\%$ & $\%$ & $\%$ & $\mathrm{Lb} / \mathrm{Hr}$ & $\mathrm{Lb} / \mathrm{Hr}$ & $\%$ \\
\hline Nov. 7 & 000 & 142.5 & 272.6 & $N / A$ & 202.6 & 179.8 & 201.3 & 864 & 586 & 372 & 303 & 32.0 & 8.8 & 24.0 & 8.8 & 1573.8 & 377.8 & 28.0 \\
\hline Nov.7 & 2015 & 141.8 & 274.2 & N/A & 203.8 & 180.3 & 206.2 & 849 & 586 & 376 & 309 & 32.3 & 9.1 & 21.1 & $5 . \overline{9}$ & 1551.5 & 327.4 & 23.2 \\
\hline Nov. 7 & 2030 & 139.2 & 273.3 & $N / A$ & 202.1 & 175.7 & 196.9 & 865 & 573 & 370 & 311 & 32.2 & 9.2 & 25.8 & 5.8 & 1539.7 & 397.6 & 28.0 \\
\hline Nov. 7 & 2045 & 143.1 & 273.5 & N/A & 203.8 & 186.4 & 208.7 & 852 & 587 & 373 & 307 & 32.8 & 9.1 & 22.8 & 7.7 & 1609.8 & 367.8 & 25.6 \\
\hline Nov. 7 & 2100 & 145.9 & 274.2 & $N / A$ & 204.0 & 184.4 & 208.1 & 852 & 584 & 373 & 309 & 32.8 & 8.8 & 22.6 & 6.5 & 1592.6 & 360.4 & 26.1 \\
\hline Nov. 7 & 2115 & 146.1 & 274.6 & N/A & 203.8 & 183.6 & 206.0 & 852 & 574 & 373 & 304 & 33.4 & 9.0 & 24.4 & 8.4 & 1585.7 & 387.6 & 26.8 \\
\hline Nov. 7 & 2130 & 143.0 & 274.2 & $N / A$ & 202.8 & 178.7 & 201.1 & 882 & 583 & 380 & 303 & 32.8 & 9.0 & 25.6 & 10.3 & 1597.7 & 409.0 & 28.8 \\
\hline Nov-7 & 2145 & 142.3 & 273.8 & N/A & 203.0 & 179.5 & 204.1 & 873 & 578 & 375 & 304 & 32.6 & 9.0 & 24.8 & 7.8 & 1588.4 & 393.2 & 27.8 \\
\hline Nov. 7 & 2200 & 138.1 & 272.7 & N/A & 201.2 & 175.3 & 196.1 & 859 & 570 & 374 & 304 & 32.3 & 9.4 & 25.8 & 9.0 & 1525.5 & 394.0 & 27.0 \\
\hline Nov. 7 & 2215 & 145.2 & 273.3 & N/A & 203.1 & 183.5 & 207.6 & 860 & 584 & 373 & 309 & 32.6 & 9.0 & 23.1 & 6.4 & 1598.7 & 369.5 & 26.3 \\
\hline Nov. 7 & 2230 & 145.8 & 274.2 & N/A & 202.6 & 185.7 & 210.5 & 854 & 580 & 377 & 309 & 34.8 & 8.8 & 23.1 & 7.1 & 1607.6 & 370.8 & 25.3 \\
\hline Nov-7 & 2245 & 143.8 & 274.7 & N/A & 203.2 & 182.9 & 206.0 & 845 & 584 & 379 & 308 & 33.1 & 9.1 & 22.1 & 8.6 & 1565.7 & 346.0 & 23.9 \\
\hline Nov -7 & 2300 & 144.9 & 274.2 & $N / A$ & 203.6 & 184.3 & 207.6 & 845 & 584 & 379 & 308 & 34.2 & 9.1 & 22.1 & 8.6 & 1577.7 & 348.5 & 23.3 \\
\hline Nov -7 & 2315 & 144.8 & 275.0 & N/A & 203.4 & 185.5 & 206.5 & 845 & 586 & 379 & 308 & 33.9 & 9.0 & 22.8 & 9.6 & 1588.0 & 362.1 & 24.8 \\
\hline Nov-7 & 2330 & 142.4 & 274.1 & N/A & 204.3 & 182.6 & 205.0 & 845 & 581 & 379 & 317 & 31.9 & 9.0 & 22.7 & 6.1 & 1563.1 & 355.5 & 25.8 \\
\hline Nov. 7 & 2345 & 141.3 & 274.3 & N/A & 204.3 & 180.6 & 202.4 & 845 & 583 & 379 & 320 & 32.0 & 9.2 & 22.6 & 5.4 & 1546.0 & 349.6 & 4.7 \\
\hline \multicolumn{2}{|c|}{ AVERAGES } & 141.7 & 274.4 & N/A & 202.6 & 181.8 & 202.7 & 852.3 & 577.3 & 375.2 & 312.2 & 33.1 & 9.0 & 24.4 & 7.2 & 1569.4 & 383.5 & 6.8 \\
\hline Nov.8 & 0 & 140.0 & 274.0 & $N / A$ & 204.6 & 180.2 & 201.5 & 845 & 571 & 379 & 311 & 31.4 & 8.8 & 24.5 & 8.2 & 1542.6 & 377.9 & 28.6 \\
\hline Nov-8 & 15 & 142.2 & 273.7 & $N / A$ & 203.2 & 181.7 & 204.1 & 845 & 583 & 379 & 312 & 33.1 & 8.8 & 22.5 & 7.6 & 1555.4 & 350.0 & 25.1 \\
\hline Nov-8 & 30 & 144.8 & 274.2 & $N / A$ & 203.9 & 184.9 & 207.1 & 845 & 569 & 344 & 314 & 33.0 & 9.1 & 24.6 & -2.2 & 1582.8 & 388.9 & 27.0 \\
\hline Nov-8 & 45 & 144.5 & 275.2 & $N / A$ & 2048 & 186.8 & 207.6 & 845 & 594 & 358 & 316 & 33.5 & 9.0 & 21.8 & 1.8 & 1598.1 & 348.8 & 24.9 \\
\hline Nov-8 & 100 & 144.4 & 275.0 & N/A & 2046 & 186.5 & 2078 & 845 & 578 & 361 & 331 & 32.8 & 9.2 & 23.8 & -2.1 & 1596.5 & 379.6 & 26.2 \\
\hline Nov-8 & 115 & 144.8 & 275.5 & N/A & 205.8 & 187.4 & 207.8 & 845 & 584 & 366 & 324 & 32.6 & 9.1 & 23.3 & 1.9 & 1604.2 & 373.8 & 6.3 \\
\hline Nov. 8 & 130 & 143.4 & 274.3 & N/A & 205.6 & 185.4 & 207.2 & 815 & 590 & 370 & 325 & 31.9 & 8.8 & 19.1 & 1.8 & 1530.9 & 292.5 & 21.8 \\
\hline Nov-8 & 145 & 141.0 & 273.4 & $N / A$ & 204.8 & 181.2 & 202.5 & 816 & 589 & 364 & 336 & 31.1 & 9.2 & 19.3 & -3.1 & 1497.2 & 288.9 & 21.0 \\
\hline Nov-8 & 200 & 140.4 & 270.9 & $N / A$ & 203.5 & 180.0 & 200.1 & 826 & 564 & 362 & 329 & 31.3 & B. 9 & 24.0 & -1.0 & 1505.5 & 362.1 & 27.1 \\
\hline Nov-8 & 215 & 140.5 & 270.6 & N/A & 202.2 & 180.0 & 206.0 & 837 & 602 & 389 & 327 & 33.9 & 9.2 & 17.6 & 3.6 & 1525.6 & 268.2 & 17.9 \\
\hline Nov. 8 & 230 & 140.4 & 273.4 & N/A & 205.2 & 180.9 & 206.6 & 837 & 599 & 379 & 333 & 30.2 & 8.9 & 18.3 & 0.3 & 1534.1 & 280.5 & 21.8 \\
\hline Nov. 8 & 245 & 140.7 & 273.2 & $N / A$ & 205.9 & 178.2 & 2000 & 849 & 604 & 377 & 320 & 29.5 & 8.9 & 20.2 & 4.7 & 1532.7 & 309.0 & 24.6 \\
\hline Nov-8 & 300 & 138.3 & 272.7 & $N / A$ & 205.6 & 178.1 & 200.8 & 844 & 598 & 380 & 324 & 30.1 & 9.2 & 20.2 & 4.0 & 1523.7 & 307.3 & 3.1 \\
\hline Nov. 8 & 315 & 138.1 & 273.1 & $N / A$ & 204.7 & 178.7 & 200.8 & 843 & 592 & 384 & 324 & 31.2 & 9.2 & 21.1 & 5.2 & 1526.3 & 322.1 & 23.4 \\
\hline Nov. 8 & 330 & 136.8 & 273.9 & N/A & 204.8 & 177.5 & 198.5 & 844 & 590 & 386 & 321 & 30.7 & 8.8 & 21.8 & 7.1 & 1518.6 & 331.2 & 25.6 \\
\hline Nov. 8 & 345 & 140.8 & 272.4 & N/A & 204.9 & 189.2 & 202.1 & 849 & 602 & 389 & 329 & 30.9 & 9.3 & 20.9 & 5.6 & 1559.4 & 25.9 & 23.6 \\
\hline Nov-8 & 400 & 140.1 & 272.0 & $N / A$ & 2052 & 179.2 & 202.2 & 861 & 602 & 399 & 330 & 31.0 & 9.3 & 21.0 & 6.6 & 1563.1 & 329.0 & 23.8 \\
\hline Nov-8 & 415 & 140.1 & 272.7 & $N / A$ & 204.1 & 182.3 & 204.2 & 868 & 582 & 395 & 325 & 31.8 & 9. & 24.9 & 7.7 & 1603.2 & 399.9 & 28.8 \\
\hline Nov- 8 & 430 & 142.0 & 273.8 & $N / A$ & 205.0 & 181.6 & 202.6 & 870 & 606 & 390 & 327 & 31.4 & 8.9 & 22.3 & 6.6 & 1600.6 & 356.8 & 26.6 \\
\hline Nov. 8 & 445 & 141.1 & 273.2 & N/A & 202.7 & 180.4 & 203.2 & 873 & 584 & 395 & 321 & 34.2 & 8.8 & 24.6 & 8.4 & 1595.5 & 392.3 & 27.2 \\
\hline Nov-8 & 500 & 143.2 & 273.5 & $N / A$ & 2048 & 185.6 & 207.7 & 871 & 594 & 390 & 327 & 31.7 & 93 & 23.6 & 6.0 & 1637.7 & 386.9 & 27.3 \\
\hline Nov. 8 & 515 & 143.3 & 273.7 & N/A & 203.4 & 184.8 & 205.0 & 880 & 599 & 390 & 324 & 33.3 & 8.9 & 24.4 & 7.7 & 1647.5 & 402.5 & 28.3 \\
\hline Nov-8 & 530 & 142.1 & 273.8 & $N / A$ & 203.1 & 185.6 & 204.4 & 874 & 592 & 389 & 325 & 33.1 & 9.1 & 25.4 & 7.8 & 1643.4 & 417.6 & 28.9 \\
\hline Nov-8 & 545 & 141.4 & 273.5 & N/A & 202.8 & 183.8 & 201.5 & 882 & 599 & 382 & 320 & 32.3 & 8.8 & 25.6 & 8.2 & 16432 & 420.5 & 30.9 \\
\hline Nov-8 & 600 & 141.2 & 272.5 & N/A & 202.5 & 181.5 & 202.0 & 885 & 589 & 390 & 317 & 32.2 & 9.1 & 25.9 & 9.6 & 1628.2 & 421.8 & 30.0 \\
\hline Nov-8 & 615 & 141.8 & 273.5 & $N / A$ & 203.3 & 181.7 & 202.1 & 889 & 601 & 385 & 314 & 32.2 & 9.3 & 24.8 & 9.3 & 1637.4 & 405.9 & 28.2 \\
\hline Nov-8 & 630 & 137.8 & 271.8 & $N / A$ & 200.1 & 179.9 & 199.1 & 901 & 589 & 397 & 324 & 33.2 & 9. & 27.7 & 9.6 & 1642.8 & 1.9 & 31.0 \\
\hline Nov-8 & 645 & 136.8 & 271.0 & N/A & 199.1 & 179.3 & 200.9 & 890 & 580 & 397 & 326 & 35.7 & 9.0 & 26.9 & 7.9 & 1616.6 & 435.2 & 28.3 \\
\hline Nov-8 & 700 & 136.7 & 273.0 & $N / A$ & 199.6 & 176.7 & 194.9 & 895 & 580 & 394 & 327 & 32.1 & 8.8 & 28.5 & 8.5 & 1602.0 & 455.8 & 33.7 \\
\hline Nov-8 & 715 & 140.8 & 272.3 & $N / A$ & 201.8 & 181.6 & 202.4 & 890 & 595 & 389 & 313 & 31.6 & 9.0 & 25.5 & 10.4 & 1638.3 & 417.3 & 30.6 \\
\hline Nov-8 & 730 & 146.5 & 272.9 & N/A & 203.5 & 186.5 & 207.0 & 887 & 601 & 387 & 31 & 32.4 & 9.0 & 24.8 & 8.5 & 1676.8 & 5.6 & 29.8 \\
\hline Nov. 8 & 745 & 145.7 & 274.2 & N/A & 204.4 & 188.8 & 206.9 & 881 & 595 & 391 & 319 & 32.8 & 8.9 & 26.0 & 10.6 & 1686.0 & 439.0 & 31.4 \\
\hline Nov-8 & 800 & 145.7 & 274.7 & $N / A$ & 204.8 & 188.7 & 208.3 & 883 & 596 & 389 & 316 & 32.9 & 9.0 & 25.5 & 10.2 & 1689.0 & 430.3 & 30.3 \\
\hline Nov-8 & 815 & 142.8 & 273.4 & $N / A$ & 204.4 & 184.6 & 205.7 & 872 & 593 & 389 & 31 & 35.5 & 97 & 24.2 & 8.6 & 1630.8 & 40 & 25 \\
\hline Nov-8 & 830 & 143.0 & 273.8 & $N / A$ & 202.3 & 183.3 & 202.7 & 893 & 602 & 391 & 30 & 33.3 & 8.8 & 25.4 & 13.0 & 1659.0 & 421.8 & 30.1 \\
\hline Nov-8 & 845 & 144.8 & 275.2 & $N / A$ & 201.6 & 183.8 & 205.8 & 888 & 598 & 379 & 315 & 34.6 & 9.1 & 24.6 & 6.9 & 1654.4 & 406.7 & 26.9 \\
\hline Nov-8 & 900 & 143.4 & 275.9 & N/A & 200.9 & 184.0 & 202.8 & 888 & 582 & 385 & 31 & 34.7 & 9.1 & 27.8 & 11.2 & 1655.3 & 459.6 & 30.3 \\
\hline Nov-8 & 815 & 139.4 & 276.0 & $N / A$ & 200.8 & 179.8 & 199.2 & 873 & 578 & 371 & 31 & 34.6 & 9.0 & 26.7 & 7.4 & 15911 & 425.5 & 28.5 \\
\hline Nov -8 & 930 & 144.1 & 275.1 & $N / A$ & 200.8 & 181.3 & 203.0 & 887 & 576 & 373 & 300 & 34.0 & 8.9 & 27.3 & 9.8 & 1629.2 & 445.5 & 30.7 \\
\hline Nov-8 & 945 & 145.8 & 274.5 & $N / A$ & 201.2 & 183.5 & 204.0 & 875 & 585 & 378 & 305 & 34.5 & 8.8 & 25.7 & 10.2 & 1626.6 & 417.7 & 28.7 \\
\hline Nov -8 & 1000 & 139.7 & 273.6 & N/A & 199.6 & 176.5 & 195.1 & 883 & 573 & 369 & 300 & 33.1 & 8. & 28.3 & 10.0 & 1578.9 & 447.2 & 31.7 \\
\hline Nov -8 & 1015 & 139.2 & 272.6 & N/A & 199.4 & 178.2 & 195.3 & 868 & 590 & 36 & 3 & 33.4 & 9 & 25.5 & 0 & 1567.9 & 399.6 & 26.8 \\
\hline Nov-8 & 1030 & 139.9 & 272.3 & $N / A$ & 199.1 & 178.5 & 200.5 & 888 & 591 & 369 & 300 & 33.5 & 8.9 & 25.2 & 8.7 & 1605.8 & 405.4 & 28.4 \\
\hline Nov-8 & 1045 & 137.9 & 271.0 & $N / A$ & 196.2 & 175.7 & 191.6 & 893 & 565 & 367 & 296 & 33.2 & 9.0 & 310 & 12.1 & 1590.2 & 493.4 & 34.4 \\
\hline Nov-8 & 1100 & 138.4 & 271.3 & N/A & 198.5 & 175.4 & 192.6 & 892 & 589 & 364 & 300 & 34.5 & 8.9 & 27.5 & 9.6 & 1585.0 & 435.8 & 29.6 \\
\hline $2 v-8$ & 1115 & 137.7 & 271.8 & N/A & 197.3 & 174.0 & 190.8 & 890 & 580 & 3 & 298 & 33.3 & 9.0 & 28.5 & 10.7 & 1569.7 & 447.7 & 31.2 \\
\hline Nov -8 & 1130 & 140.4 & 271.5 & $N / A$ & 199.4 & 173.7 & 192.3 & 895 & 580 & 365 & 300 & 31.8 & 9.1 & 28.3 & 9.0 & 1574.8 & 444.9 & 32.0 \\
\hline vov-8 & 1145 & 146.8 & 273.3 & $N / A$ & 2012 & 185.5 & 2030 & 898 & 588 & 380 & 309 & 33.5 & 9. & 28.3 & 19.1 & 1687.4 & 478.2 & 32.3 \\
\hline Vov - 8 & 1200 & 146.2 & & 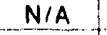 & 2014 & 182.5 & 202.2 & 892 & 589 & 37 & 29 & 32.8 & 9 & $=$ & 12.4 & 1650.1 & 443.7 & 30.6 \\
\hline Jov-8 & 1215 & 146.3 & 274.0 & $N / A$ & 202.7 & 183.7 & 203.7 & 897 & 599 & 368 & 305 & 32.4 & 8.9 & 25.9 & 8.0 & 1669.2 & 432.1 & 31.3 \\
\hline Jov-8 & 1230 & 145.1 & 273.6 & $N / A$ & 202.0 & 181.8 & 203.6 & 893 & 599 & 376 & 307 & 33.0 & 8.7 & 24.8 & 8.4 & 1644.7 & 408.2 & 29.7 \\
\hline Jov-8 & 1245 & 143.4 & 272.6 & $N / A$ & 201.1 & 180.1 & 197.9 & 893 & 594 & 370 & 303 & 32.1 & 8.1 & 26.9 & 10.0 & 1629.3 & 438.5 & 35.4 \\
\hline Jov-8 & 1300 & 143.5 & 271.8 & 11 & 201.3 & 179.7 & 200.8 & 907 & 606 & 368 & 300 & 32.1 & 7.3 & 25.3 & 8.8 & 1651.0 & 418.3 & 37.6 \\
\hline Jov-8 & 1315 & 142.7 & 272.1 & $N / A$ & 2010 & 174.9 & 191.6 & 903 & 597 & 363 & 301 & 31.2 & 6.7 & 27.6 & 9.2 & 1599.8 & 441.1 & 44.6 \\
\hline$\sqrt{o v-8}$ & 1330 & 142.2 & 273.0 & N/A & 200.8 & 176.5 & 194.8 & 896 & 610 & 364 & 302 & 32.4 & 6.0 & 24.9 & 8.5 & 1602.8 & 399.1 & 43.6 \\
\hline tov-8 & 1345 & 142.8 & 274.5 & $N / A$ & 201.8 & 175.7 & 195.9 & 903 & 605 & 366 & 295 & 31.7 & 7.5 & 25.2 & 10.3 & 1607.1 & 405.5 & 35.9 \\
\hline tov-8 & 1400 & 141.1 & 275.9 & $N / A$ & 201.9 & 177.3 & 196.1 & 881 & 585 & 362 & 306 & 32.4 & 8.4 & 26.6 & 6.3 & 1582.4 & 420.3 & 32.3 \\
\hline
\end{tabular}




\begin{tabular}{|c|c|c|c|c|c|c|c|c|c|c|c|c|c|c|c|c|c|c|}
\hline Date & Time & Boller & Duct & $A v g$ "C" & Duct & Flue Gas & Flue Gas & $\mathrm{SO}_{2}$ & SO2 & $\mathrm{NOX}$ & NOX & $\operatorname{Lim} \theta$ & $\operatorname{Lim} \theta$ & $\mathrm{SO} 2$ & $\overline{\mathrm{NO}}$ & 502 & SO2 & Lime \\
\hline & & Load & Inlet & Section & Outlet & Flow in & Flow Ou & inlet & Outlet & $\ln l e \mid$ & Outlet & Flow & Conc & Removal & Removal & Inlet & Ramoved & tilizati \\
\hline & & MN & Temp of & Temp of & Temp of & KSCFM & KSCFM & $\mathrm{ppm}$ & $\mathrm{ppm}$ & $\mathrm{ppm}$ & $\mathrm{ppm}$ & $\mathrm{gpm}$ & $\%$ & $\%$ & $\%$ & $\angle \mathrm{D} / \mathrm{HP}$ & L.b/Hr & $\%$ \\
\hline Nov.8 & 1415 & 146.6 & 275.9 & N/A & 201.8 & 184.6 & 205.9 & 874 & 567 & 335 & 316 & 35.5 & 8.8 & 277 & 107 & 1635.4 & 453.6 & 303 \\
\hline Nov- 8 & 1430 & 144.4 & 276.8 & $\mathrm{~N} / \mathrm{A}$ & 202.3 & 184.1 & 203.8 & 862 & 571 & 377 & 316 & 34.8 & 9.6 & 26.7 & 72 & 1608.6 & 429.6 & 26.7 \\
\hline Nov-8 & 1445 & 144.6 & 276.1 & $\mathrm{~N} / \mathrm{A}$ & 202.4 & 180.5 & 198.1 & 866 & 578 & 366 & 306 & 34.2 & 9.2 & 26.8 & 8.1 & 1584.5 & 425.4 & 28.2 \\
\hline Nov.8 & 1500 & 143.1 & 275.0 & $\mathrm{~N} / \mathrm{A}$ & 201.4 & 176.7 & 195.9 & 876 & 580 & 370 & 29 & 33.2 & 9.2 & 26.6 & 11.8 & 1568.1 & 417.1 & 28.4 \\
\hline Nov-8 & 1515 & 143.2 & 274.4 & $N / A$ & 200.8 & 177.5 & 187.5 & 882 & 576 & 363 & 302 & 33.5 & 9.1 & 27.3 & 7.4 & 1586.0 & 433.4 & 29.6 \\
\hline Nov. 8 & 1530 & 143.3 & 276.0 & N/A & 200.9 & 177.1 & 195.8 & 875 & 581 & 365 & 301 & 33.7 & 87 & 26 & 8.8 & & 417.4 & -60.0 \\
\hline Nov-8 & 1545 & 142.9 & 277.2 & $N / A$ & 201.1 & 178.0 & 197.4 & 889 & 593 & 370 & 296 & 34.4 & 9.3 & 26.0 & 11.3 & 1603.1 & 417.3 & 27.2 \\
\hline Nov-8 & 600 & 141. & 278.7 & N/A & 201.4 & 176.2 & 196.9 & 887 & 587 & 369 & 297 & 34.2 & 8.8 & 26.0 & 10.1 & 1583.3 & 411.5 & 28.5 \\
\hline Nov-8 & 1615 & 137. & 278.8 & $N / A$ & 202.0 & 174.3 & 192.2 & 872 & 593 & 365 & 299 & 34.4 & 8.7 & 25.0 & 9.9 & 1539.8 & 384.2 & 26.8 \\
\hline Nov- 8 & 1630 & 138.8 & 278.4 & N/A & 202.2 & 174.8 & 194.2 & 872 & 565 & 377 & 296 & 33.7 & 9.2 & 28.0 & 12.8 & 1544.2 & 431.7 & 29.0 \\
\hline Nov-8 & 1645 & 141.9 & 276.4 & N/A & 203.3 & 177.0 & 198.1 & 877 & 581 & 362 & 300 & 33.1 & 0 & 25.9 & 73 & 1572.6 & 406.6 & 285 \\
\hline Nov-8 & 1700 & 142.3 & 276.5 & N/A & 203.6 & 179.1 & 198.7 & 872 & 572 & 370 & 296 & 33.2 & 9.1 & 27.3 & 11.4 & 1582.2 & 431.6 & 29.8 \\
\hline Nov-8 & 1715 & 142 & 275.2 & N/A & 203.1 & 177.9 & 199.2 & 868 & 572 & 367 & 29 & 33.0 & 9.1 & 26.3 & 9.0 & 1565.3 & 411.8 & 28 \\
\hline Nov. 8 & 1730 & 141. & 275.1 & N/A & 201.9 & 176.4 & 187.9 & 876 & 578 & 363 & 29 & 33.6 & 9.4 & 26.1 & 8.5 & 1566.4 & 408.4 & 26.9 \\
\hline Nov-8 & 1745 & 142.8 & 274.6 & N/A & 201.0 & 177.8 & 198.1 & 870 & 572 & 371 & 299 & 34.1 & 8.8 & 26.7 & 10.1 & 1567.1 & 419.0 & 29,2 \\
\hline Nov-8 & 1800 & 146.4 & 274.4 & N/A & 202.0 & 183.0 & 202.4 & 87 & 572 & 37 & 30 & \begin{tabular}{|l|}
33.6 \\
\end{tabular} & 80 & 27.7 & 10.0 & 1621.3 & 449.3 & 31.0 \\
\hline Nov-8 & 1815 & 147.1 & 274.8 & N/A & 201.6 & 181.2 & 200.7 & 85 & 564 & 367 & 30 & 34.5 & 8.8 & 26.9 & 9.3 & 1568.6 & 421.7 & \\
\hline Nov-8 & 1830 & 146.2 & 274.8 & $N / A$ & 203.4 & 181. & 203.7 & 85 & 561 & 36 & 29 & 32.8 & 9.0 & 26.3 & 9.3 & 1570.5 & 413.7 & 28 \\
\hline Nov-8 & 1845 & 146. & 274 & N/ & 200.5 & 182. & 204.5 & 84 & 550 & 36 & 30 & 35 & 9.0 & 27.5 & 8.4 & 1570.4 & 431.9 & 2 \\
\hline Nov.8 & 1900 & 145. & 274.5 & N/A & 200.8 & 180.7 & 201.0 & 843 & 566 & 369 & 30 & 33 & 8.7 & 25.4 & 9.6 & 1543.4 & 391.7 & 278 \\
\hline Nov-8 & 1915 & 146.4 & 274.5 & $N / A$ & 202.2 & 181.8 & 201.2 & 844 & 565 & 365 & 30 & 33.5 & 9.1 & 26.0 & 8.5 & 1555.4 & 404.6 & 27.7 \\
\hline Nov-8 & 1930 & 143.8 & 274.3 & $N / A$ & 2014 & 181.1 & 201.5 & 840 & 554 & 36 & 30 & 34.2 & 8.9 & 26.7 & 8.8 & & 411.3 & 28 \\
\hline Nov- 8 & 1945 & 145.2 & 273.8 & $\mathrm{~N} / \mathrm{A}$ & 2016 & 1822 & 2014 & 84 & 552 & 3 & 29 & 33 & 8 & 28.2 & 10 & 1568.0 & 441.6 & \\
\hline No & 2000 & 146. & 273 & $\mathrm{~N}$ & 2016 & 183.7 & 202.7 & 84 & 571 & 38 & 31 & 34 & 8.9 & 25.3 & 12. & 15700 & 397.3 & \\
\hline No & 2015 & 145.8 & 273 & N' & 199.0 & 185.4 & 203.6 & 853 & 564 & 37 & 31 & 37.6 & 8 & 27.5 & 9.6 & 1603.1 & 4407 & \\
\hline Nov-8 & 2030 & 146.1 & 273.2 & $\mathrm{~N} / \mathrm{A}$ & 200.6 & 185.1 & 204.5 & 860 & 554 & 37 & 30 & 34.2 & 8. & 28.9 & 10.1 & 1613.6 & 465.7 & 3 \\
\hline Nov. 8 & 2045 & 142.2 & 272.5 & $N / A$ & 201.1 & 180.8 & 199.4 & 849 & 564 & 37 & 30 & 32.9 & 9 & 26.8 & 10 & & 4165 & \\
\hline Nov-8 & 100 & 143.0 & 271 & $\overline{N / 4}$ & 199.4 & 179. & 196 & 84 & 563 & 37 & 30 & 33 & 9 & 27.1 & 10 & 1541.3 & 418.2 & \\
\hline No & 2115 & 143. & 2.71 & $\mathrm{~N} /$ & 200 & 179. & 198.3 & 84 & 580 & 37 & 30 & 32 & 9 & 24.3 & 10. & 1541.3 & 375.2 & \\
\hline Nov. 8 & 2130 & 142 & 271 & $\mathrm{~N} /$ & 199.0 & 184.4 & 204.5 & $=$ & 577 & 38 & 30 & 354 & 9 & 28.1 & 12. & 1661.7 & 466.2 & 30. \\
\hline Nov-8 & 2145 & 143.7 & 271.0 & N/A & 199.1 & 182.5 & 200.3 & 8 & 581 & 37 & 30 & 34.1 & 5 & 28.0 & 11.7 & & 457.4 & \\
\hline Nov- 8 & 2200 & 146.1 & 271.1 & N/A & 200.0 & 183.8 & 1994 & 87 & 576 & 37 & 30 & 32 & 9 & 28.6 & 13 & & & \\
\hline Nov. 8 & 2215 & 146.1 & 270 & At & 201. & 18 & 203 & & 58. & 37 & & & $a$ & 26.0 & 9. & & 423.7 & \\
\hline Nov-8 & 2230 & 146.4 & 269 & & 200 & & 199 & & 583 & 37 & $3 c$ & 33 & 9 & 27.9 & 11. & & s & \\
\hline Nov. 8 & & 145. & 270 & & 203. & 18 & 199 & 8 & 591 & 37 & 30 & 29.1 & 9 & 27.1 & 11 & 16 & .1 & 34 \\
\hline Nov. 8 & 00 & 145. & 269 & NI & 205.3 & 18 & 199.6 & 8 & 591 & 37 & 30 & 29.8 & 9. & 26.3 & 10 & & 8 & \\
\hline Nov. 8 & 2315 & 134.3 & 268. & Nit & 203.0 & 17 & 188.5 & 8 & 591 & 37 & 30 & 30.1 & 9 & 27.1 & 11 & & & \\
\hline Nov. 8 & 2330 & 196.7 & 271. & $N / A$ & 2004 & 15 & 169. & & 597 & 37 & 3 & 30 & & 27.8 & 11 & & & \\
\hline Nov. 8 & 2345 & 105.9 & & & 198 & & 162.5 & 727 & 533 & 321 & 31 & 28 & 8 & 22.3 & -4 & 8 & 25 & 20 \\
\hline$A V E$ & AGES & 142. & 273. & $\mathrm{~N} /$ & 2020 & 18 & 200.0 & 869.2 & 583.3 & 374.8 & 309.7 & 32 & 8 & 25.5 & 8 & & & \\
\hline Nov. 9 & 0 & 106.9 & 275 & $\mathrm{~N}$ & 198.7 & 15 & 162.7 & 744 & 526 & 328 & 313 & 28 & & 24 & & & & \\
\hline Nov-9 & 15 & 107.3 & 273. & & 198.1 & & 162 & 7 & 524 & 3 & 3 & 28 & 8 & & & & & \\
\hline Nov.9 & 30 & 106.8 & 275 & & 19 & 15 & 16 & 7 & & & 3 & & & 27 & & & & \\
\hline Nov-9 & 45 & 110.2 & 277 & & 19 & & 16 & 88 & 52 & 28 & 2 & 2 & 9 & 36.7 & .4. & & & 38 \\
\hline & 100 & 114 & 269. & & 197. & 15 & 16 & 1 & 5 & 30 & 2 & 2 & 8 & 24.4 & & & & \\
\hline Nov-9 & 115 & 113 & 26 & & 196.6 & & 16 & 8 & & 2 & & & & & & & & \\
\hline Nov.9 & 130 & 111 & 26 & & 19 & & & 8 & 5 & 3 & & 2 & & & & & & \\
\hline No & 145 & 112 & 26 & & 19 & & & & 5 & & & 2 & & 26 & & & & 25 \\
\hline & 200 & 106 & & & 19 & & 16 & 8 & 5 & 30 & 26 & 26 & & 27 & & & & \\
\hline & 21 & 10 & & & 19 & & 16 & & 5 & & & & & 27 & & & & \\
\hline 9 & 2 & 10 & 26 & & 19 & & & & & & & & & & & & & \\
\hline No & 24 & 10 & 26 & & 19 & & 16 & & 5 & & 2 & 2 & & 32 & & & & \\
\hline Nov-9 & 300 & 10 & 26 & $\mathrm{~N}$ & 19 & & 16 & 8 & 57 & & 5 & $\frac{-26}{26}$ & & 30.4 & & & & \\
\hline & 315 & 108 & 26 & & 19 & & 16 & - & 57 & 32 & 27 & 26 & & 27 & & & & \\
\hline & 330 & 10 & 26 & & 19 & & & & & & & & & & & & & \\
\hline 9 & 3 & 10 & 26 & & 19 & & & & & & & & 9. & 28 & & & & \\
\hline & 400 & 10 & 26 & & 19 & & 16 & 8 & 58 & & 27 & 27 & 9. & 28 & & & & \\
\hline & 415 & 108 & 267 & $\mathrm{~N} / \mathrm{A}$ & 196 & & & & 57 & & 27 & 26 & & 30 & & & & \\
\hline & 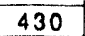 & & 26 & & 19 & & & & & & & & & & & & & \\
\hline & & & & & & & & & & & & & & & & & & \\
\hline & 5 & & 26 & & & & & & 5 & & 2 & & & & 10 & & & \\
\hline & 515 & 10 & 269.6 & $\mathrm{~N}$ & 19 & & 16 & 863 & 57 & 3 & 2 & 26 & & 28 & & & & \\
\hline & 530 & & 268. & $N$ & 19 & & & & & & & & & & & & & \\
\hline & & & & & & & & & & & & & & & & & & \\
\hline & & & & & & & & & & & & & & & & & & \\
\hline & & & & & & & & & & & 2 & & & 25 & 1 & & 37 & \\
\hline & 630 & & 268.7 & $\mathrm{~N} /$ & 19 & & 16 & 87 & 55 & 33 & 27 & 2 & & & & & & \\
\hline & 645 & 110 & 268 & $N$ & & & & & & & & & & & & & & \\
\hline & 70 & & & & & & & & & & & & & & & & & 2 \\
\hline & 71 & & & & & & 16 & & 53 & 33 & 2 & & 8. & 32.5 & 0 & & 42 & 5 \\
\hline & 730 & & 26 & $N$ & 195 & & & $\overline{85}$ & 54 & 33 & 2 & 26 & 9 & & & & & \\
\hline & 745 & 10 & & & & & & & & & & & & & & & & \\
\hline & 80 & & & & & & & & & & & & & 36.9 & 13.8 & & 77.0 & 66.9 \\
\hline bv-9 & 815 & 99.4 & 57.1 & $\mathrm{~N} / \mathrm{A}$ & 190.9 & 152.4 & 161.9 & 837 & 497 & 336 & 272 & 30.3 & 8.9 & 36.9 & 13.8 & 1291.6 & 477.0 & 36.9 \\
\hline
\end{tabular}


CZD LIME INJECTION DATA - AUGUST 17, TO NOVEMBER 16, 1992

\begin{tabular}{|c|c|c|c|c|c|c|c|c|c|c|c|c|c|c|c|c|c|c|}
\hline Dale & Time & Boller & Dual & $A \vee g$ "C $C^{\prime \prime}$ & Duat & Flue Ges & Flue Gas & 502 & $\mathrm{SO} 2$ & Nox & $\mathrm{NO} x$ & $\operatorname{Lim} \theta$ & $\operatorname{Lim} \theta$ & 502 & $\mathrm{NO} x$ & 50 ? & $\mathrm{SO} 2$ & Lime \\
\hline & & Load & $\ln |0|$ & Section & Outlol & Flow in & Flow Out & $t \ln |\theta|$ & Outlet & $\ln |\theta|$ & Oullal & Flow & Conc & Removal & Removal & $\ln |0|$ & Remove & Jilizatic \\
\hline & & $M N$ & Tomp ${ }^{\circ} \mathrm{F}$ & Temp ${ }^{\circ} \mathrm{F}$ & Tomp 'F & KSCFM & KSCFM & $\mathrm{ppm}$ & $\mathrm{ppm}$ & $\mathrm{ppm}$ & $\mathrm{ppm}$ & $\mathrm{gPm}$ & $\%$ & $\%$ & $\%$ & $\therefore \mathrm{b} / \mathrm{Hr}$ & LO/HI & $\%$ \\
\hline Nov-9 & 830 & 109.4 & 267.1 & $N / A$ & $10 n 9$ & 1524 & 161.8 & 837 & 497 & 336 & 272 & 30.3 & 8.9 & 36.9 & 13.8 & 1291.6 & 477.0 & 36.9 \\
\hline Nov. 9 & 845 & 109.4 & 2671 & $N / A$ & 190.9 & 152.4 & 161.9 & 837 & 497 & 336 & 272 & 30.3 & 8.9 & 36.9 & 13.8 & 1291.6 & 477.0 & 36.8 \\
\hline Nov.9 & 900 & 109.4 & 267.1 & N/A & 190.9 & 152.4 & 161.9 & 837 & 497 & 336 & 272 & 30.3 & 8.8 & 36.9 & 13.8 & 12916 & 477.0 & 36.9 \\
\hline \multicolumn{2}{|c|}{ AVERAGES } & 109.6 & 268.5 & $N / A$ & 195.0 & 150.9 & 161.8 & 852.0 & 551.1 & 326.2 & 276.5 & 27.9 & 9.0 & 30.6 & 9.0 & 1301.8 & 398.3 & 32.8 \\
\hline Nov. 12 & 1130 & 116.8 & 286.7 & $N / A$ & 200.2 & 159.7 & 181.9 & 755 & 531 & 330 & 274 & 37.1 & 0.0 & 20.0 & 5.5 & 1221.8 & 244.1 & 0.0 \\
\hline Nov. 12 & 1145 & 110.4 & 286.2 & N/A & 189.0 & 160.1 & 182.8 & 739 & 426 & 323 & 267 & 37.3 & 0.0 & 34.2 & 5.7 & 1198.3 & 409.6 & 0.0 \\
\hline Nov.12 & 1200 & 122.3 & 284.8 & N/A & 198.7 & 165.0 & 187.3 & 728 & 438 & 321 & 268 & 37.5 & 0.0 & 31.7 & 5. & 1216.5 & 385.8 & 0.0 \\
\hline Nov-12 & 1215 & 121.1 & 286.5 & $N / A$ & 198.8 & 161.3 & 183.5 & 762 & 459 & 326 & 271 & 36.8 & 0.0 & 31.5 & 5. & 1244.7 & 391.8 & 0.0 \\
\hline Nov-12 & 1230 & 1221 & 286.5 & N/A & 189.1 & 161.7 & 182.8 & 742 & 447 & 325 & 269 & 36.8 & 0.0 & 31.8 & 6.4 & 1215.2 & 386.9 & 0.0 \\
\hline Nov $\cdot 12$ & 1245 & 121.2 & 287.1 & $N / A$ & 199.5 & 162.0 & 183.4 & 758 & 451 & 324 & 271 & 37.2 & 0.0 & 32.7 & 53 & 1244.3 & 406.8 & 0.0 \\
\hline Nov-12 & 1300 & 121.7 & 286.7 & $N / A$ & 199.4 & 1611 & 181.1 & 742 & 447 & 329 & 273 & 37.0 & 18.3 & 32.3 & 6.7 & 1211.5 & 391.8 & 11.4 \\
\hline Nov-12 & 1315 & 122.4 & 287.5 & N/A & 199.4 & 158.7 & 181.6 & 762 & 445 & 332 & 274 & 37.0 & 18.0 & 33.2 & 5.5 & 1225.4 & 407.2 & 2.1 \\
\hline Nov 12 & 1330 & 120.1 & 287.7 & N/A & 199.8 & 158.4 & 181.9 & 754 & 441 & 328 & 271 & 36.9 & 17.7 & 32.8 & 5.2 & 1209.5 & 396.1 & 12.0 \\
\hline Nov-12 & 1345 & 120.3 & 288.2 & $N / A$ & 200.8 & 160.7 & 183.0 & 753 & 427 & 333 & 277 & 36.8 & 16.5 & 35.4 & 5.3 & 1225.4 & 434.0 & 14.2 \\
\hline Nov-12 & 1400 & 121.6 & 285.9 & $N / A$ & 199.9 & 160.0 & 181.8 & 743 & 424 & 330 & 269 & 36.8 & 14.6 & 35.1 & 7.5 & 1204.0 & 422.4 & 15.9 \\
\hline Nov-12 & 1415 & 120.6 & 287.0 & $N / A$ & 199.5 & 156.2 & 178.3 & 786 & 470 & 325 & 265 & 36.7 & 12.9 & 31.8 & 7.2 & 1243.3 & 394.8 & 7.0 \\
\hline Nov.12 & 1430 & 119.0 & 286.4 & N/A & 199.2 & 158.2 & 179.0 & 719 & 427 & 300 & 263 & 36.5 & 12.1 & 32.7 & 1.1 & 1152.0 & 377.0 & 17.5 \\
\hline Nov 12 & 1445 & 121.8 & 287.0 & $N / A$ & 2008 & 163.6 & 184.5 & 716 & 42 & 31 & 28 & 36.8 & 11.2 & 33.5 & .3 .2 & 1187.2 & 397.7 & 9.9 \\
\hline Nov. 12 & 1500 & 119.8 & 285.7 & N/A & 1984 & 161.8 & 183.4 & 717 & 407 & 303 & 265 & 38.5 & 10.1 & 35.6 & 0.8 & 1175.7 & 418.9 & 2.3 \\
\hline Nov-12 & 1515 & 121.3 & $285 . \overline{1}$ & N/A & 198.7 & 163.1 & 1842 & 703 & 400 & 293 & 270 & 37.5 & 9.7 & 35.7 & .4 .1 & 1162.1 & 415.0 & 3.7 \\
\hline Nov-12 & 1530 & 120.4 & 284.7 & N/A & 197.3 & 164.1 & 185.8 & 703 & 37 & 306 & 267 & 37.7 & 10.0 & 393 & 14 & 1169.2 & 458.8 & 5.3 \\
\hline Nov-12 & 1545 & 122.8 & 289.2 & $N / A$ & 200.3 & 168.2 & 191.9 & 805 & 450 & 31 & 286 & 382 & 10.1 & 36.2 & 4.0 & 13710 & 486.7 & $=$ \\
\hline Nov-12 & 1600 & 137.8 & 291.9 & $\mathrm{~N} / \mathrm{A}$ & 204.2 & 179.3 & 203.0 & 732 & 454 & 342 & 291 & 39.9 & 10.2 & 29.8 & 3.8 & 1330.2 & 397.0 & 0.2 \\
\hline Nov 12 & 1615 & 135.5 & 291.8 & $N / A$ & 204.8 & 179.6 & 2029 & 742 & 448 & 328 & 295 & 39.8 & 9.9 & 31.7 & .1 .4 & 1349.7 & 428.3 & 2.5 \\
\hline Nov-12 & 1630 & 146.6 & 291.9 & N/A & 2050 & 178.4 & 2021 & 785 & 447 & 326 & 299 & 39.5 & 9.7 & 354 & -3.7 & 1418.2 & & 2 \\
\hline Nov. 12 & 1645 & 145.1 & 291.9 & N/A & 2054 & 178.8 & 202.3 & 796 & 487 & 355 & 304 & 39.5 & 10.3 & 30.8 & 2.8 & 1441.1 & 443.7 & 2.6 \\
\hline Nov-12 & 1700 & 143.1 & 291.9 & N/A & 205.2 & 178.1 & 202.0 & 797 & 485 & 356 & 312 & 39.8 & 9.7 & 31.0 & 0.6 & 1437.3 & 445.5 & 24.0 \\
\hline Nov-12 & 1715 & 142.9 & 291.1 & $N / A$ & 205.5 & 178.7 & 202.3 & 768 & 477 & 335 & 298 & 39.1 & 10.1 & 29.6 & 0.9 & 1389.8 & .9 & 6 \\
\hline Nov.12 & 1730 & 141.7 & 290.5 & N/A & 205.0 & 178.9 & 201.5 & 768 & 481 & 331 & 293 & 39.5 & 10.2 & 29.5 & 02 & 1392.3 & 411.1 & 1 \\
\hline Nov-12 & 1745 & 141.8 & 290.5 & $N / A$ & 205.5 & 178.1 & 204.0 & 758 & 482 & 336 & 309 & 38.1 & 10.0 & 27.2 & .5 .1 & 13680 & 2.6 & 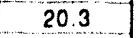 \\
\hline Nov. 12 & 1800 & 141.4 & 290.2 & N/A & 2060 & 178.8 & 202.7 & 765 & 48 & 330 & 293 & 38.1 & 9.8 & 28.1 & .0 .7 & 1385.1 & & \\
\hline Nov-12 & 1815 & 141.7 & 290.1 & NIA & 2064 & 178.6 & 200.6 & 766 & 48 & 320 & 289 & 37.7 & 10.2 & 283 & -1.4 & 54 & 3 & \\
\hline Nov-12 & 1830 & 141.7 & 290.3 & N/A & 2069 & 179.1 & 201.8 & 767 & 498 & 328 & 284 & 37.5 & 9.8 & 26.9 & 2.5 & 13911 & 373.7 & 9 \\
\hline Nov. 12 & 1845 & 141.4 & 290.7 & $N$ & 207.2 & 179.0 & 2021 & 775 & 498 & 328 & 308 & 37.7 & 10.1 & 27.4 & .5 .9 & 14057 & 3858 & 0 \\
\hline Nov. 12 & 1900 & 140.4 & 290.2 & $N / A$ & 207.4 & 177.7 & 199.7 & 774 & 49 & 331 & 294 & & 10. & 27.9 & 0.3 & & & \\
\hline Nov-12 & 1915 & 140.4 & 290.0 & N/A & 207.8 & 178.0 & 199.5 & 766 & 50 & 320 & 291 & 36.9 & 10 & 25.7 & .1 .7 & 13816 & 6 & \\
\hline Nov-12 & 1930 & 140.3 & 2902 & & 2078 & 1786 & 199.3 & 777 & 512 & 331 & 287 & 36.9 & 10.1 & 26.4 & 3.3 & 1405.3 & 1.2 & 6 \\
\hline Nov-12 & 1945 & 140.7 & 290.3 & N/A & 207.9 & 177.2 & 199.2 & 777 & 51 & 331 & 309 & 36.8 & 10 & 25.0 & -4.9 & 1395.2 & 8.5 & 4 \\
\hline Nov-12 & 2000 & 140.4 & 291.3 & $N / A$ & 208.1 & 178.5 & 200.8 & 789 & 52 & 33 & 29 & 37 & 1 & & 0 & 71. & 0 & ? \\
\hline Nov-12 & 2015 & 140.8 & 291.3 & $N / A$ & 208.6 & 178.6 & 2010 & 781 & 52 & 322 & 292 & 370 & 101 & 24.3 & .1 .8 & 3.4 & 1 & 18 \\
\hline Nov-12 & 2030 & 133.7 & 291.4 & N/A & 208.7 & 178.1 & 2007 & 765 & 52 & 330 & 292 & 36.8 & 10.2 & 23.2 & 0.1 & 1379.7 & 0.1 & 177 \\
\hline Nov-12 & 2045 & 130.8 & 290.7 & N/A & 208.2 & 179.4 & 201.8 & 731 & 47 & 33 & 30 & 37 & 9.6 & 27.6 & -4.8 & 1328.2 & 6.8 & 3 \\
\hline Nov-12 & 2100 & 1344 & 290.6 & N/A & 206.6 & 170.0 & 1916 & 731 & 476 & 331 & 296 & 35 & 10.3 & 26.5 & 05 & 12 & 1 & \\
\hline Nov-12 & 2115 & 131.1 & 291.2 & $N / A$ & 206.5 & 170.8 & 196.0 & 73 & 50 & 369 & 289 & 36.3 & 101 & 21.3 & 10.0 & 1264.5 & 94 & 2 \\
\hline Nov. 12 & 2130 & 134.5 & 291.2 & N/A & 206.7 & 17 & 199.0 & 73 & 49 & 357 & 276 & 36.0 & 10.3 & 22.6 & 11.2 & 1281.5 & 9.3 & 16.1 \\
\hline Nov-12 & 2145 & 130.9 & 290.1 & $N / A$ & 206.3 & 170.6 & 194.6 & 788 & 4 & 350 & 29 & 36.0 & 10.1 & 29.2 & 5. & 1.4 & .0 & 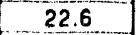 \\
\hline Nov-12 & 2200 & 130.5 & 288.1 & $N / A$ & 206.2 & 171.1 & 195.9 & 786 & 484 & 355 & 291 & 35.9 & 9.7 & 29.5 & 6. & 1362.8 & 9 & \\
\hline Nov-12 & 2215 & 137.8 & 287.7 & N/A & 207.3 & 174.9 & 98.6 & 79 & 49 & 350 & 287 & 36.0 & 9.8 & 29.4 & 6.8 & 1406.3 & 4.1 & 4.4 \\
\hline $\mid v-12$ & 2230 & 136.3 & 288.6 & $N / A$ & 2065 & & 1.1 & 786 & 496 & 352 & 308 & 3 & 10.1 & 28.8 & 1. & 1419.2 & 8.4 & 2.5 \\
\hline$A V E$ & RAGES & 131.5 & 289.0 & $N / A$ & 203.7 & 170.8 & 193.4 & 757.9 & 469.4 & 330.3 & 285.8 & 37.4 & 9.6 & 29.9 & & 22 & & 4 \\
\hline 1.13 & 845 & 145.4 & 277.0 & $N / A$ & 202.6 & 178.4 & 204.0 & 773 & 698 & 366 & 304 & 32.1 & 0.0 & -3.3 & 5 & 5 & 6 & 0 \\
\hline 1.13 & 1500 & 137.3 & 277.4 & 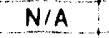 & $: 97.5$ & 168.1 & 194.8 & 6 & 66 & 35 & 30 & 35.2 & 11.4 & 4.4 & 0 & 1371.9 & 8 & 1 \\
\hline 1.13 & 1515 & 135.5 & 281.8 & N/A & 195.3 & 1646 & 192.6 & 819 & 58 & 3 & 295 & 38.3 & 11.0 & 15.9 & 1.9 & 5.0 & 8 & 0.6 \\
\hline ov. 13 & 1530 & 135.8 & 283.2 & $N / A$ & 195.4 & 163.9 & 194.5 & 817 & 574 & 354 & 295 & 38.7 & 11.2 & 16 & 1.1 & & & 0.7 \\
\hline ev-13 & 1545 & 136.0 & 284.1 & N/A & 195.3 & 163.7 & 3.9 & 0 & 574 & 32 & 298 & 38.9 & 10.0 & 16.6 & 0.3 & & & 10 \\
\hline 1.13 & 1600 & 138.5 & 284.1 & $N / A$ & 196.0 & 168.6 & 197.2 & 810 & 566 & 353 & 29 & 39 & 9.1 & 18.2 & 1.8 & 13828 & .9 & 4.7 \\
\hline 1.13 & 615 & 140.7 & 283.5 & $N / A$ & 197.7 & 1764 & 05.9 & 793 & 559 & 360 & 301 & 396 & 8 & 17.7 & 2.4 & 1417.5 & .3 & 6.6 \\
\hline$v-13$ & 30 & 5.0 & 283.2 & & 198.2 & 1766 & 0.0 & 797 & & 359 & 304 & & 7 & 1 & 1.4 & & & .5 \\
\hline$v-13$ & 1645 & 145.2 & 282.6 & N/A & 198.6 & 1812 & 2074 & 700 & 561 & 3 & 3 & 0 & 9 & 100 & 20 & 42 & & 0 \\
\hline-13 & 1700 & 5.3 & 281.7 & & 985 & 1801 & 2089 & 791 & 566 & 357 & 313 & $39 ?$ & 5.8 & 170 & .1. & 1442.7 & 7 & 2.7 \\
\hline$v-13$ & 1715 & 4.6 & 2 & & 99.0 & 180.9 & 210.2 & 789 & 559 & 336 & 309 & 39.1 & 5.4 & 17.7 & .7 .0 & 14454 & 9 & 5.8 \\
\hline ov-13 & 1730 & 144.9 & 282.0 & $N / A$ & 991 & 180.8 & 207.8 & 795 & 5 & 348 & 312 & 39.1 & 6.6 & 18.0 & -3.0 & 1456.3 & & 1.4 \\
\hline v-13 & 1745 & 145.0 & 282.0 & N/A & 1993 & 179.4 & 2089 & 796 & 571 & 357 & 306 & 389 & 8.5 & 164 & 0.0 & 14460 & 2374 & 151 \\
\hline 1.13 & 1800 & 5.2 & & & 199.1 & 179.5 & 210.1 & 798 & 568 & 358 & 305 & 392 & 89 & 16.7 & 0.4 & 14513 & 242.2 & 145 \\
\hline 1.13 & 1815 & & 281.8 & & 1996 & 180.7 & & 796 & 571 & 341 & 307 & 390 & 95 & 16.4 & 4.8 & 1456.4 & 238.1 & 134 \\
\hline v-13 & 1830 & 144.0 & 280.6 & $N / A$ & 202.2 & 1806 & 2100 & 797 & 5 & & 298 & 38.0 & 9.7 & 14.6 & -1.6 & & & 12.0 \\
\hline$v \cdot 13$ & 1845 & 4.8 & 279.7 & $N / A$ & 2014 & 180.0 & 207.1 & 790 & 564 & 352 & 322 & 37.5 & 9.7 & 18.0 & .5 .3 & 1441.1 & & 14.8 \\
\hline & 1900 & & 1.4 & A & 1994 & 179.6 & 206.5 & 795 & 551 & 352 & 299 & 37.7 & 9.1 & 20.3 & 2.6 & 14457 & 292.9 & 17.8 \\
\hline$v-13$ & 1915 & 145.2 & 2 & & 00.2 & & & 784 & 560 & 356 & 312 & .9 & 82 & 17.9 & .0 .8 & 14402 & 258.3 & 17.8 \\
\hline ov-13 & 1930 & 145.1 & 279.7 & N/A & 2008 & 182.8 & 209.2 & 783 & 563 & & 310 & & 9.4 & 17.7 & -2.2 & 1449.5 & 256.1 & 154 \\
\hline $0 v \cdot 13$ & 1945 & 144.8 & 278.6 & $N / A$ & 2003 & 180.8 & 206.1 & 773 & 553 & 349 & 305 & & 98 & 18.5 & 04 & 1416.2 & 262.5 & 15.1 \\
\hline $\begin{array}{r}v .13 \\
\end{array}$ & 2000 & 144.4 & 278.2 & $N / A$ & 201.0 & 1814 & 207.9 & 785 & 558 & 347 & 304 & 35.6 & 10.2 & 18.5 & .0 .3 & 1443.0 & 267.6 & 15.3 \\
\hline$v \cdot 13$ & 2015 & & & & 200.8 & 176.6 & 2018 & 781 & 569 & 341 & 310 & 35.3 & 9.1 & 16.7 & .39 & 1396.7 & 233.3 & 15.1 \\
\hline$v-13$ & 2030 & 136.2 & 278.1 & $N / A$ & 199.3 & 172.8 & 196.6 & 771 & 551 & 356 & 295 & 345 & 8.4 & 18.7 & 5.7 & 1350.0 & 252.5 & 18.2 \\
\hline
\end{tabular}


CZD LIME INJECTION DATA - AUGUST 17, TO NOVEMBER 16, 1992

\begin{tabular}{|c|c|c|c|c|c|c|c|c|c|c|c|c|c|c|c|c|c|c|}
\hline Date & Time & Boiler & Duat & Avg "C" & Duct & Flue Gas & Flue Gas & $\mathrm{SO} 2$ & $\mathrm{SO} 2$ & NOX & NOx & $\operatorname{Lim} \theta$ & $\operatorname{Lim} \theta$ & $\mathrm{SO} 2$ & NOx & $\mathrm{SO} 2$ & $\mathrm{SO2}$ & $\operatorname{Lim} \theta$ \\
\hline & & Load & Inlol & Soction & Oullel & Flow in & Flow out & $\ln |\theta|$ & Outlet & $\ln |0|$ & Outlat & Flow & Conc & Removal & Pemoval & Inle! & Removes & tilizan \\
\hline & & $M N$ & Temp of & Temp of & Temp F $\mathrm{F}$ & KSCFM & KSCFM & $\mathrm{ppm}$ & $\mathrm{ppm}$ & $\mathrm{ppm}$ & $\mathrm{ppm}$ & $\mathrm{gpm}$ & $\%$ & $\%$ & $\%$ & $\mathrm{Lb} / \mathrm{HP}$ & Lb/HI & $\%$ \\
\hline Nov. 13 & 2130 & 144.5 & 278.6 & N/A & 2058 & 1813 & 206.9 & 787 & 574 & 373 & 300 & 33.5 & 7.6 & 16.8 & 8.4 & 1445.8 & 243.6 & 20.1 \\
\hline Nov.13 & 2145 & 142.0 & 277.3 & N/A & 199.4 & 179.4 & 206.2 & 808 & 537 & 380 & 292 & 38.0 & 7.0 & 23.6 & 11.6 & 14678 & 345.9 & 27.4 \\
\hline Nov. 13 & 2200 & 135.7 & 277.3 & $N / A$ & 184.5 & 171.1 & 194.1 & 798 & 537 & 365 & 292 & 37.6 & 7.6 & 23.7 & 9.1 & 1383.4 & 327.3 & 241 \\
\hline Nov.13 & 2215 & 138.8 & 277.6 & N/A & 197.6 & 181.3 & 207.7 & 808 & 548 & 365 & 296 & 36.5 & 9.4 & 22.3 & 7.2 & 1483.3 & 330.1 & 20.0 \\
\hline Nov - 13 & 2230 & 143.5 & 277.5 & $N / A$ & 199.1 & 182.5 & 209.1 & 783 & 549 & 371 & 307 & 36.2 & 10.2 & 19.6 & 5.1 & 14473 & 284.2 & 15.9 \\
\hline Nov. 13 & 2245 & 141.5 & 277.4 & N/A & 198.3 & 177.5 & 204.0 & 798 & 551 & 371 & 296 & 35.5 & 10.6 & 20.6 & 8.1 & 14342 & 295.4 & 16.2 \\
\hline Nov. 13 & 2300 & 136.0 & 277.3 & $N / A$ & 197.0 & 170.2 & 196.0 & 788 & 551 & 371 & 295 & 35.2 & 10.6 & 20.4 & 7.9 & 1375.3 & 281.0 & 15.6 \\
\hline Nov-13 & 2315 & 135.6 & 275.8 & N/A & 196.4 & 170.2 & 187.1 & 788 & 548 & 371 & 296 & 34.6 & 10.5 & 20.4 & 7.4 & 1375.3 & 278.9 & 15.8 \\
\hline Nov-13 & 2330 & 135.8 & 275.4 & $N / A$ & 195.7 & 169.4 & 196.3 & 798 & 534 & 371 & 298 & 34.9 & 102 & 22.5 & 7.0 & 1368.8 & 307.7 & 17.8 \\
\hline Nov. 13 & 2345 & 135.3 & 275.0 & $N / A$ & 186.1 & 171.3 & 198.3 & 798 & 534 & 371 & 306 & 34.4 & 10.2 & 22.6 & 4.6 & 1384.1 & 312.3 & 18.4 \\
\hline \multicolumn{2}{|c|}{ AVERAGES } & 141.4 & 279.7 & N/A & 198.7 & 176.3 & 203.6 & 794.4 & 566.3 & 357.4 & 302.5 & 37.1 & 8.7 & 17.6 & 2.1 & 1418.4 & 250.4 & 16.4 \\
\hline Nov. 14 & 0 & 135.5 & 275.0 & N/A & 195.8 & 170.5 & 195.9 & 727 & 530 & 319 & 305 & 34.6 & 10.4 & 16.3 & -9.5 & 1256.2 & 205.1 & 11.8 \\
\hline Nov. 14 & 15 & 144.8 & 276.3 & N/A & 198.2 & 180.1 & 206.5 & 727 & 528 & 318 & 302 & 35.5 & 10.2 & 16.7 & .9 .0 & 1327.0 & 222.1 & 12.7 \\
\hline Nov-14 & 30 & 142.9 & 277.0 & N/A & 197.2 & 181.5 & 206.3 & 733 & 544 & 332 & 306 & 36.7 & 99 & 15.7 & -4.5 & 1347.4 & 211.4 & 12.1 \\
\hline Nov.14 & 45 & 144.3 & 276.6 & $N / A$ & 200.8 & 181.0 & 209.3 & 735 & 554 & 336 & 316 & 29.2 & 9.2 & 12.8 & .9 .0 & 1348.3 & 174.5 & 13.5 \\
\hline Nov-14 & 100 & 144.7 & 274.7 & $N / A$ & 198.1 & 180.9 & 206.1 & 739 & 553 & 337 & 306 & 36.3 & 8.4 & 14.8 & .3 .3 & 1353.8 & 200.2 & 13.7 \\
\hline Nov-14 & 115 & 145.2 & 274.7 & $N / A$ & 197.8 & 178.0 & 207.0 & 758 & 551 & 335 & 306 & 35.5 & 9.0 & 16.0 & .5 .5 & 1375.8 & 220.3 & 14.4 \\
\hline Nov-14 & 130 & 143.6 & 275.4 & $N / A$ & 198.2 & 178.7 & 204.7 & 758 & 550 & 343 & 307 & 35.4 & 10.4 & 16.9 & .2 .6 & 1371.7 & 232.0 & 13.0 \\
\hline Nov-14 & 145 & 142.4 & 275.3 & N/A & 196.2 & 179.3 & 205.9 & 757 & 538 & 342 & 320 & 37.3 & 10.3 & 18.4 & .7 .4 & 1375.4 & 253.0 & 13.6 \\
\hline Nov-14 & 200 & 138.2 & 277.4 & NIA & 195.7 & 177.4 & 202.5 & 750 & 543 & 343 & 310 & 36.7 & 9.9 & 17.4 & -3.0 & 1348.2 & 234.2 & 13.4 \\
\hline Nov-14 & 215 & 139.4 & 276.4 & $N / A$ & 195.2 & 175.0 & 198.3 & 767 & 533 & 358 & 305 & 36.0 & 10.3 & 21.3 & 3.5 & 1360.1 & 280.3 & 16.2 \\
\hline Nov-14 & 230 & 138.4 & 274.4 & $N / A$ & 196.0 & 172.9 & 200.5 & 775 & 518 & 356 & 312 & 35.4 & 10.5 & 22.6 & -1.8 & 1357.8 & 306.3 & 17.0 \\
\hline Nov-14 & 245 & 142.0 & 273.9 & $N / A$ & 197.5 & 176.5 & 204.4 & 766 & 539 & 352 & 305 & 34.2 & 10.7 & 18.5 & -0.3 & 1369.1 & 253.9 & 14.3 \\
\hline Nov-14 & 300 & 137.2 & 274.3 & $N / A$ & 194.9 & 174.2 & 200.0 & 764 & 517 & 361 & 304 & 36.1 & 10.2 & 223 & 3.4 & 1347.7 & 299.9 & 16.9 \\
\hline Nov-14 & 315 & 136.4 & 276.9 & $N / A$ & 195.4 & 176.2 & 201.9 & 765 & 524 & 361 & 298 & 36.2 & 10.2 & 21.5 & 5.5 & 13650 & 293.8 & 16.5 \\
\hline Nov-14 & 330 & 141.1 & 274.5 & $N / A$ & 194.7 & 174.9 & 203.3 & 771 & 518 & 355 & 295 & 36.6 & 10.1 & 22.0 & 3.5 & 1366.4 & .3 & 16.8 \\
\hline Nov-14 & 345 & 1378 & 273.3 & $N / A$ & 196.2 & 171.9 & 196.6 & 772 & 531 & 357 & 299 & 33.6 & 10.1 & 21.4 & 4.0 & 1343.9 & 287.2 & 17.5 \\
\hline Nov. 14 & 400 & 142.0 & 273.2 & $N / A$ & 198.0 & 177.3 & 2064 & 782 & 539 & 359 & 300 & 36.9 & 10.2 & 19.8 & 3.0 & 1405.0 & 277.6 & 15.3 \\
\hline Nov. 14 & 415 & 144.8 & 274.3 & N/A & 196.6 & 180.0 & 208.9 & 773 & 529 & 339 & 304 & 35.7 & 10.3 & 20.6 & .4 .2 & 1409.0 & 290.2 & 16.3 \\
\hline Nov. 14 & 430 & 141.3 & 275.2 & N/A & 196.1 & 175.4 & 201.9 & 780 & 522 & 351 & 310 & 35.3 & 10.1 & 23.0 & -1.7 & 1385.5 & 318.5 & 18.5 \\
\hline Nov.14 & 445 & 145.0 & 275.0 & NIA & 197.8 & 179.3 & 207.2 & 781 & 541 & 353 & 300 & 35.1 & 9.6 & 20.0 & 1.8 & 1418.1 & 283.4 & .5 \\
\hline Nov-14 & 500 & 141.1 & 272.6 & $N / A$ & 197.9 & 179.9 & 207.3 & 775 & 528 & 356 & 301 & 35.7 & 10.1 & 21.5 & 2.7 & 1411.9 & 303.7 & 17.5 \\
\hline Nov-14 & 515 & 140.9 & 272.6 & $N / A$ & 196.1 & 179.1 & 206.2 & 774 & 536 & 358 & 296 & 37.4 & 9.9 & 20.3 & 4.8 & 1404.7 & 19 & 0 \\
\hline Nov.14 & 530 & 138.7 & 272.7 & $N / A$ & 196.7 & 1714 & 197.5 & 794 & 541 & 354 & 299 & 33.0 & 10.2 & 21.5 & 2.7 & 1378.3 & 296.8 & 18.3 \\
\hline Nov-14 & 545 & 135.9 & 274.3 & $N / A$ & 196.4 & 171.6 & 195.9 & 795 & 532 & 360 & 293 & 33.8 & 9.9 & 23.7 & 7.2 & 1382.2 & 327.3 & 203 \\
\hline Nov.14 & 600 & 140.7 & 275.5 & N/A & 198.3 & 175.3 & 201.9 & 795 & 543 & 348 & 298 & 33.4 & 100 & 21.4 & 1.5 & 1411.1 & 1.4 & 18.7 \\
\hline Nov-14 & 615 & 140.9 & 273.9 & $N / A$ & 198.2 & 175.9 & 201.0 & 796 & 544 & 344 & 305 & 33.8 & 9.8 & 21.9 & .1 .5 & 1418.1 & 311.3 & 19.5 \\
\hline Nov. 14 & 630 & 145.1 & 273.5 & $N / A$ & 199.7 & 181.3 & 209.8 & 789 & 548 & 354 & 304 & 34.9 & 10.4 & 19.6 & 0.5 & 1449.5 & 284.6 & 16.2 \\
\hline ov. 14 & 645 & 139.0 & 272.2 & $N / A$ & 201.1 & 177.3 & 206.0 & 784 & 536 & 365 & 333 & 33.9 & 10.3 & 206 & -5.9 & 1407.8 & 290.1 & 17.2 \\
\hline$v \cdot 14$ & 700 & 138.6 & 271.9 & N/A & 199.4 & 172.2 & 199.8 & 779 & 520 & 358 & 307 & 32 & 9.9 & 226 & 0.7 & 1359.3 & 307.5 & 20.1 \\
\hline Nov.14 & 715 & 138.2 & 271.3 & $N / A$ & 199.1 & 172.6 & 187.1 & 782 & 541 & 366 & 309 & 33.2 & 10.1 & 20 & 3.6 & 13 & 286.5 & 177 \\
\hline ov.14 & 730 & 143.8 & 272.1 & $N / A$ & 201.5 & 177.5 & 205.9 & 783 & 530 & 363 & 305 & 34.6 & 10.2 & 21.6 & 2.4 & 1408.3 & 303.5 & 8 \\
\hline $2 v \cdot 14$ & 745 & 142.4 & 271.6 & $N / A$ & 200.6 & 174.7 & 202.2 & 772 & 530 & 361 & 304 & 33.1 & 10.0 & 20.5 & 2.5 & 1366.7 & 280.7 & 17.6 \\
\hline Nov. 14 & 800 & 145.0 & 272.6 & $N / A$ & 202.0 & 177.8 & 204.3 & 775 & 541 & 363 & 306 & 318 & 9.9 & 19.7 & 3.1 & 1395.4 & 275.6 & 8.2 \\
\hline Nov-14 & 815 & 145.4 & 273.9 & $N / A$ & 203.1 & 178.6 & 202.0 & 759 & 529 & 361 & 308 & 29.7 & 9.9 & 21.2 & 3.3 & 6 & 8 & 00 \\
\hline Nov. 14 & 830 & 144.5 & 273.4 & N/A & 204.1 & 178.5 & 204.8 & 763 & 544 & 359 & 310 & 30.8 & 10.3 & 18.2 & 1.1 & 1379.2 & 251.4 & 16.4 \\
\hline Nov-14 & 845 & 144.7 & 274.4 & $N / A$ & 204.3 & 181.0 & 207.8 & 775 & 544 & 366 & 334 & 31.6 & 10.0 & 19.4 & .4 .6 & 1421.4 & 276.1 & 18.1 \\
\hline Nov-14 & 900 & 144.9 & 274.1 & N/A & 204.4 & 178.1 & 204.2 & 782 & 552 & 364 & 310 & 30.8 & 10.1 & 19. & 2.2 & 14 & 268.3 & 7.9 \\
\hline Nov-14 & 815 & 141.6 & 272.6 & $N / A$ & 202.4 & 177.6 & 205.1 & 772 & 539 & 366 & 306 & 33.3 & 9.9 & 20.6 & 3.5 & & & \\
\hline ov.14 & 830 & 141.1 & 272.9 & $N / A$ & 202.8 & 174.7 & 201.6 & 777 & 543 & 368 & 310 & 30.7 & 10.1 & 19.4 & 2.8 & 1375.5 & 267.4 & 78 \\
\hline Nov-14 & 945 & 141.1 & 273.3 & $N / A$ & 205.1 & 178.1 & 201.7 & 781 & 570 & 37 & 318 & 29.1 & 10.0 & 17.4 & 3.2 & 1409.5 & 244.6 & 17.4 \\
\hline Nov-14 & 1000 & 140.3 & 273.1 & $N / A$ & 204.2 & 175.5 & 202.7 & 785 & 573 & 362 & 307 & 29.9 & 10.0 & 15.7 & 1.9 & 1396.0 & 219.2 & 15.2 \\
\hline Nov.14 & 1300 & 145.9 & 274.5 & NIA & 196.2 & 180.4 & 207.2 & 801 & 579 & 372 & 316 & 36.3 & 9.6 & 16.9 & 2.2 & 14 & 247.6 & 10 \\
\hline$v \cdot 14$ & 1315 & 146.4 & 274.1 & N/A & 195.6 & 180.4 & 209.9 & 807 & 564 & 373 & 31 & 37.9 & 10.5 & 18.6 & 3.3 & 1474.9 & 274.7 & 14.3 \\
\hline v. 14 & 1330 & 146.3 & 274.0 & N/A & 1950 & 180.1 & 210.5 & 800 & 565 & 364 & 312 & 40.0 & 10.0 & 17.5 & .0 .2 & 1459.8 & 255.9 & 13.3 \\
\hline Nov-14 & 1345 & 145.8 & 276.5 & $N / A$ & 194.1 & 180.9 & 209.5 & 814 & 566 & 369 & 308 & 38.3 & 9.9 & 19.5 & 3.3 & 1491.0 & 290.7 & 15.9 \\
\hline Nov-14 & 1400 & 146.3 & 276.8 & N/A & 194.5 & 179.4 & 210.6 & 819 & 565 & 367 & 309 & 37.9 & 9.9 & 19.0 & 1.2 & & 2 & 5.6 \\
\hline$v-14$ & 1415 & 146.1 & 276.5 & $N / A$ & 194.3 & 180.9 & 210.3 & 803 & 532 & 369 & 308 & 39.4 & 9.9 & 23.0 & 3.1 & 1470.8 & 338.4 & 18.0 \\
\hline$v \cdot 14$ & 1430 & 146.2 & 275.8 & $N / A$ & 195.0 & 180.8 & 212.8 & 800 & 545 & 372 & 317 & 38.4 & 10.2 & 19.9 & .0 .2 & 1465.5 & 291.5 & 15.4 \\
\hline Nov. 14 & 1445 & 146.7 & 275.4 & $N / A$ & 193.9 & 181.9 & 207.8 & 797 & 554 & 373 & 317 & 37.9 & 10.0 & 20.7 & 3.1 & 1468.9 & & 16.6 \\
\hline Nov.14 & 1500 & 146.2 & 276.2 & $N / A$ & 194.3 & 180.4 & 207.0 & 796 & 559 & 373 & 318 & 37.7 & 10.3 & 19.5 & 2.3 & 1454.9 & 283.6 & 5.1 \\
\hline ov-14 & 1515 & 146.1 & 276.7 & $N / A$ & 195.4 & 181.2 & 210.1 & 794 & 536 & 374 & 322 & 38.7 & 10.1 & 21.7 & 0.2 & 1457.0 & 316.0 & 16.7 \\
\hline Nov-14 & 1530 & 146.8 & 275.5 & 185.7 & 194.5 & 181.4 & 210.4 & 797 & 535 & 373 & 316 & 41.7 & 10.1 & 22.2 & 1.6 & 1463.9 & 324.5 & 16.0 \\
\hline Nov-14 & 1545 & 146.8 & 275.5 & 185.7 & 194.5 & 181.4 & 210.4 & 797 & 535 & 373 & 316 & 41.7 & 10.1 & 22.2 & 1.6 & 1463.9 & 324.5 & 16.0 \\
\hline Nov-14 & 1600 & 145.7 & 278.6 & 181.2 & 187.3 & 181.2 & 210.5 & 803 & 549 & 365 & 304 & 36.8 & 9.8 & 0 & 3.4 & & & 17.5 \\
\hline Nov-14 & 1615 & 145.7 & 278.6 & 181.2 & 197.3 & 181.2 & 210.5 & 803 & 549 & 365 & 304 & 36.8 & 9.8 & 20.6 & 3.4 & 1474.2 & 303.3 & 17.5 \\
\hline Nov.14 & 1645 & 146.6 & 275.6 & 181.2 & 197.6 & 180.0 & 207.2 & 784 & 545 & 372 & 311 & 36.2 & 9.9 & 20.1 & 3.9 & 1430.0 & 286.9 & 16.6 \\
\hline Nov.14 & 1700 & 146.6 & 276.3 & 182.9 & 198.5 & 179.5 & 206.3 & 783 & 550 & 369 & 311 & 35.5 & 9.9 & 19.4 & 3.3 & 1424.2 & 275.6 & 16.3 \\
\hline Nov-14 & 1715 & 144.5 & 276.5 & 182.8 & 198.5 & 178.1 & 202.6 & 792 & 546 & 374 & 312 & 35.3 & 9.9 & 21.5 & 5.0 & 1428.4 & 307.6 & 18.3 \\
\hline Nov-14 & 1730 & 143.1 & 273.5 & 184.3 & 198.2 & 177.7 & 206.1 & 803 & 525 & 356 & 311 & 35.4 & 10.3 & 24.2 & .1 .4 & 1444.9 & 349.4 & 19.8 \\
\hline Nov-14 & 1745 & 6.2 & 273.4 & 186.8 & 199.2 & 182.7 & 210.7 & 786 & 527 & 372 & 315 & 36.0 & 10.0 & 22.7 & 2.4 & 1455.2 & 331.0 & 19.1 \\
\hline Nov-14 & 1800 & 146.1 & 274.8 & 181.3 & 198.9 & 184.1 & 2111.2 & 773 & 541 & 374 & 321 & 35.8 & 10.0 & 19.7 & 1.4 & 1441.1 & 284.5 & 16.5 \\
\hline Nov.14 & 1815 & 145.3 & 274.8 & 181.7 & 198.3 & 179.6 & 206.6 & 778 & 531 & 371 & 321 & 35.1 & 9.9 & 21.4 & 0.6 & 14150 & 303.5 & 18.1 \\
\hline Nov-14 & 1830 & 145.5 & 273.5 & 181.6 & 198.4 & 181.4 & 209.3 & 784 & 543 & 374 & 313 & 34.6 & 10.0 & 20.1 & 3.2 & 1440.2 & 289.8 & 17.4 \\
\hline
\end{tabular}


CZD LIME INJECTION DATA - AUGIJST 17, TO NOVEMBER 16, 1992

\begin{tabular}{|c|c|c|c|c|c|c|c|c|c|c|c|c|c|c|c|c|c|c|}
\hline Date & Time & Boiler & Dual & Avg "C & Ducl & Fluo Gas & Flue Ges & 502 & S02 & $\sqrt{0 x}$ & NOx & $\operatorname{Lim} \theta$ & $\operatorname{Lim} \theta$ & 502 & NOx & 502 & $\mathrm{SO} 2$ & $\operatorname{Lim} \theta$ \\
\hline & & Load & Inlei & Section & Outlet & Flow in & Flow Out & Inlei & Oullet & Inle| & Outlel & Flow & Conc & Removal & Removal & $\ln |\theta|$ & Removed & tilizatio \\
\hline & & MN & Tomp o $F$ & Temp of & Tomp o & KSCFM & KSCFM & $\mathrm{ppm}$ & $\mathrm{ppm}$ & $\mathrm{ppm}$ & $\mathrm{ppm}$ & $\mathrm{gPm}$ & $\%$ & $\%$ & $\%$ & $\mathrm{Lb} / \mathrm{HI}$ & $L b / H_{1}$ & $\%$ \\
\hline Nov. 14 & 1845 & 1462 & 272.8 & 181.4 & 198.1 & 180.5 & 207.3 & 798 & 535 & 379 & 318 & 34.6 & 9.7 & 23.1 & 3.6 & 1459.4 & 336.8 & 20.8 \\
\hline Nov. 14 & 1900 & 142.2 & 272.5 & 180.3 & 197.0 & 177.2 & 203.3 & 784 & 524 & 378 & 321 & 34.7 & 9.7 & 23.4 & 26 & 1407.8 & 329.2 & 20.3 \\
\hline Nov-14 & 1915 & 142.8 & 271.8 & 180.8 & 197.3 & 177.6 & 204.2 & 780 & 534 & 372 & 315 & 33.8 & 10.2 & 224 & 2.7 & 1421.7 & 3180 & 18.0 \\
\hline Nov-14 & 1930 & 142.8 & 272.7 & 180.8 & 197.2 & 176.8 & 204.0 & 781 & 528 & 371 & 310 & 34.3 & 10.2 & 21.9 & 34 & 1388.3 & 306.8 & 18.2 \\
\hline Nov. 14 & 1845 & 142.3 & 272.7 & 180.1 & 196.6 & 175.2 & 203.2 & 781 & 517 & 372 & 313 & 34.7 & 10.0 & 23.2 & 2.3 & 13857 & 321.0 & 19.2 \\
\hline Nov -14 & 2000 & 142.4 & 273.5 & 179.9 & 196.8 & 174.8 & 202.1 & 187 & 528 & 373 & 315 & 34.3 & 9.8 & 224 & 2.3 & 1393.1 & 311.7 & 19.3 \\
\hline Nov-14 & 2015 & 142.2 & 272.1 & 178.8 & 1977 & 177.0 & 205.4 & 781 & 525 & 376 & 320 & 332 & 10.2 & 22.1 & 1.2 & 1400.8 & 309.0 & 18.9 \\
\hline Nov-14 & 2030 & 142.3 & 272.8 & 1808 & 196.8 & 176.8 & 205.3 & 768 & 510 & 373 & 316 & 34.5 & 10.2 & 22.8 & 1.7 & 1375.0 & 314.0 & 18.5 \\
\hline Nov 14 & 2045 & 142.8 & 2733 & 180.2 & 1962 & 1781 & 206.5 & 775 & 506 & 370 & 313 & 35.5 & 10.0 & 24.3 & 1.9 & 13978 & 339.8 & 19.8 \\
\hline Nov-14 & 2100 & 142.7 & 2735 & 181.4 & 197.7 & 176.0 & 201.7 & 775 & 512 & 370 & 314 & 33.5 & 9.9 & 24.2 & 2.6 & 1381.3 & 334.7 & 20.8 \\
\hline Nov-14 & 2115 & 146.8 & 272.9 & 181.5 & 197.3 & 179.1 & 209.1 & 775 & 520 & 370 & 303 & 35.2 & 10.0 & 21.7 & 4.2 & 1405.6 & 304.8 & 18.0 \\
\hline Nov. 14 & 2130 & 143.0 & 273.5 & 181.3 & 196.8 & 173.6 & 200.8 & 775 & 534 & 385 & 309 & 34.0 & 0.6 & 20.2 & 7.1 & 1362.4 & 275.5 & 17.5 \\
\hline Nov.14 & 2145 & 139.3 & 273.8 & 182.2 & 197.3 & 172.1 & 189.7 & 775 & 544 & 383 & 313 & 33.6 & 9.6 & 18.6 & 5.2 & 1350.7 & 251.0 & 16.2 \\
\hline Nov-14 & 2200 & 137.7 & 273.4 & 181.3 & 196.4 & 170.0 & 186.5 & 775 & 536 & 378 & 313 & 33.5 & 10.0 & 20.0 & 4.2 & 1334.2 & 267.1 & 16.5 \\
\hline Nov.14 & 2215 & 137.3 & 272.6 & 182.0 & 196.6 & 171.4 & 186.6 & 778 & 533 & 380 & 306 & 32.8 & 9.8 & 21.5 & 7.6 & 1351.3 & 280.6 & 18.7 \\
\hline Nov. 14 & 2230 & 138.0 & 272.7 & 182.5 & 197.1 & 170.0 & 195.6 & 773 & 537 & 375 & 307 & 32.3 & 10.1 & 20.1 & 5.9 & 1330.7 & 267.5 & 17.0 \\
\hline Nov-14 & 2245 & 138.0 & 274.3 & 185.4 & 198.3 & 175.0 & 201.6 & 777 & 521 & 375 & 317 & 34.0 & 9.7 & 22.8 & 2.5 & 1378.0 & 313.6 & 19.8 \\
\hline Nov-14 & 2300 & 141.5 & 273.8 & 187.8 & 199.1 & 178.6 & 206.0 & 777 & 521 & 375 & 317 & 34.2 & 9.9 & 22.7 & 2.4 & 14062 & 318.6 & 195 \\
\hline Nov. 14 & 2315 & 144.8 & 274.8 & 182.4 & 199.9 & 184.0 & 210.7 & 777 & 556 & 375 & 317 & 34.2 & 10.1 & 18.9 & 3.1 & 1448.7 & 261.8 & 15.7 \\
\hline Nov.14 & 2330 & 143.1 & 275.2 & 182.4 & 138.7 & 179.9 & 208.9 & 777 & 550 & 375 & 313 & 35.9 & 10.0 & 17.9 & 2.9 & 14164 & 253.4 & 14.6 \\
\hline Nov.14] & 2345 & 143.3 & 274.4 & 179.4 & 196.6 & 175.7 & 201.2 & 748 & 546 & 375 & 314 & 35.7 & 10.2 & 16.4 & 4.1 & 13318 & 218.7 & 12.4 \\
\hline \multicolumn{2}{|c|}{ AVERAGES } & 142.8 & 274.3 & 182.1 & 197.8 & 177.7 & 204.8 & 778.5 & 538.0 & 363.1 & 310.1 & 35.0 & 10.0 & 20.3 & 1.4 & 14016 & 284.8 & 16.8 \\
\hline Nov-15 & 0 & 138.8 & 274.3 & 179.3 & 185.8 & 173.8 & $202 ?$ & 748 & 545 & 375 & 313 & 35.3 & 9.8 & 150 & 2.5 & 13174 & 198.1 & 11.8 \\
\hline Nov- 15 & 15 & 141.6 & 275.0 & 180.0 & 1961 & 1784 & 205.4 & 748 & 544 & 334 & 312 & 36.0 & 8.8 & 16.4 & .7 .4 & 1352.2 & 221.1 & 13.0 \\
\hline Nov 15 & 30 & 139.1 & 276.5 & 180.0 & 1956 & 1769 & 201.7 & 748 & 556 & 344 & 31 & 36.0 & 10.1 & 15.3 & -4.0 & 13408 & 205.6 & 11.7 \\
\hline Nov. 15 & 45 & 140.8 & 2749 & 179.7 & 1954 & 177.7 & 199.8 & 752 & 558 & 345 & 328 & 35.2 & 10.1 & 16.7 & .6 .7 & 1354.1 & 5 & 13.1 \\
\hline Nov. 15 & 100 & 141.3 & 2738 & 176.5 & 195.0 & $177 ?$ & 202.5 & 761 & 553 & 358 & 318 & 34.3 & 9.8 & 17.2 & .1 .1 & 1370.3 & 235.7 & 4.6 \\
\hline Nov-15 & 115 & 141.5 & 2739 & 182.3 & 196.1 & 1804 & 207.2 & 761 & 544 & 361 & 315 & 36.6 & 10.1 & 17.9 & -0.3 & 1391.1 & 249.1 & 14.0 \\
\hline Nov 15 & 130 & 142.1 & 273.2 & 1851 & 196.0 & 179.1 & 207.8 & 775 & 537 & 360 & 306 & 36.7 & 10.2 & 19.7 & 1.3 & 1406.5 & 277.0 & 5.3 \\
\hline Nov-15 & 145 & 142.7 & 275.5 & 177.7 & 185.2 & 180.1 & 204.1 & 770 & 540 & 353 & 329 & 36.3 & 9.8 & 20.5 & .5 .7 & 1404.3 & 287.7 & 6 \\
\hline Nov. 15 & 200 & 143.7 & 277.0 & 178.9 & 1962 & 182.8 & 207.2 & 765 & 537 & 350 & 316 & 36.9 & 9.9 & 20.5 & -2.3 & 14171 & 290.8 & 16.5 \\
\hline $\mathrm{v} \cdot 15$ & 215 & 146.2 & 274.3 & 182.5 & 195.8 & 185.7 & 212.5 & 772 & 516 & 365 & 310 & 38.1 & 9.7 & 23.6 & 28 & 1452.8 & 342.8 & 19.3 \\
\hline Nov-15 & 230 & 145.3 & 274.3 & 182.0 & 186.6 & 182.5 & 210.4 & 778 & 530 & 363 & 311 & 36.6 & 10.2 & 21.5 & 1.2 & 1437.8 & & 1 \\
\hline ov.15 & 245 & 143.3 & 274.6 & 182.8 & 197.1 & 182.3 & 2100 & 782 & 535 & 367 & 300 & 36.8 & 10.9 & 21.2 & 3.1 & 1443.7 & 3.4 & 7.1 \\
\hline Nov-15 & 300 & 1454 & 276.5 & 178.6 & 196.7 & 186.0 & 210.7 & 787 & 527 & 361 & $3 c$ & 37.3 & 9.7 & 24.1 & 32 & 14824 & $\therefore$ & 20.5 \\
\hline$v-15$ & 315 & 146.1 & 277.3 & 180.8 & 198.3 & 187.5 & 211.1 & 775 & 542 & 358 & 3 & 36.6 & 9.6 & 20 & 23 & 1471.5 & 3 & 8.5 \\
\hline Nov-15 & 330 & 146.1 & 274.1 & 183.7 & 197.5 & $184 . ?$ & 210.3 & 786 & 533 & 360 & 5 & 36.5 & 10.0 & 22 & 0 & 1.1 & 5 & 9.1 \\
\hline Nov. 15 & 345 & 146.2 & 273.8 & 183.3 & 197.9 & 182.9 & 206.6 & 785 & 541 & 366 & 304 & 35.5 & 9.8 & 23.2 & 6.0 & 1472.3 & 340.8 & 0.3 \\
\hline$v-15$ & 400 & 143.8 & 273.6 & 1778 & 196.3 & 180.2 & 208.4 & 794 & 522 & 362 & 307 & 35.6 & 10.3 & 24. & 1.7 & 1449.8 & 347.4 & 19.6 \\
\hline Nov-15 & 415 & 144.1 & 275.5 & 180.3 & 197.8 & 182.3 & 2100 & 797 & 546 & 36 & 30 & 35.7 & 10 & & & 1.2 & 4 & 7.4 \\
\hline Nov. 15 & 430 & 145.3 & 275.2 & 180.2 & 197.7 & 182.3 & 207.3 & 800 & 539 & 361 & 304 & $35 \%$ & 10.2 & $=$ & 4.5 & 1477.6 & 6 & 9.6 \\
\hline$v-15$ & 445 & 146.4 & 276.5 & 181.0 & 198.4 & 182.9 & 209.5 & 800 & 537 & 350 & 30 & 35.8 & 100 & 23. & .0 .2 & 14816 & 342.8 & 19.8 \\
\hline$v \cdot 15$ & 500 & 143.8 & 277.2 & 181.1 & 198.5 & 182.0 & 20 & 78 & 534 & 36 & & 35.8 & 10. & 2 & 47 & 3 & 353.6 & 8.8 \\
\hline Nov-15 & 515 & 142.7 & 274.2 & 182.1 & 1982 & 178.6 & 207.1 & 813 & 537 & 36 & 3 & 34.5 & 10.2 & 2 & 2.6 & 2 & 3 & 3 \\
\hline Nov. 15 & 530 & 142.7 & 273.7 & 184.1 & 197.5 & 180.0 & 207.0 & 821 & 538 & 36 & 301 & 358 & 10.3 & 24.6 & 5.5 & 1497.3 & 9 & 67 \\
\hline$v-15$ & 545 & 138.2 & 2738 & 1804 & 197.3 & 174.7 & 200.2 & 824 & 558 & 36 & 300 & 33.5 & 10.4 & 22.4 & 6.4 & $14 !$ & 326.8 & 19.4 \\
\hline$v \cdot 15$ & 600 & 140.6 & 273.5 & 184.2 & 197.5 & 175.5 & 203.4 & $8<7$ & 546 & 36 & 30 & 34.5 & 10 & & 3.3 & 14 & 3 & 20.1 \\
\hline Nov-15 & 615 & 141.7 & 273.3 & 1842 & 197.7 & 1753 & 202.1 & 837 & 544 & 36 & 2 & 34.4 & 10 & 25 & 6 & & & .0 \\
\hline Nav. 15 & 630 & 144.7 & 273.6 & 187.4 & 198.5 & 178.8 & 209.0 & 826 & 550 & 36 & 298 & 36.1 & 10.6 & 22.1 & 4.5 & & 8 & 7.9 \\
\hline $1-15$ & 645 & 142.3 & 272.8 & 1854 & 5 & 1803 & 207.5 & 808 & 540 & 36 & 303 & 33.8 & 10.1 & 23.1 & 5.4 & 14 & 6 & 20.7 \\
\hline$v-15$ & 730 & 144.4 & 274.1 & 1930 & 2026 & 181.6 & 206.1 & 81 & 682 & 3 & 3 & 32.4 & 10.4 & & 6 & 14 & 74.8 & 4.6 \\
\hline Nov. 15 & 745 & 143.4 & 273.7 & 185.1 & 200.6 & 181.3 & 2053 & 803 & 561 & 37 & 312 & 33.2 & 9.8 & 20.8 & 6.5 & 14 & 30 & 19.6 \\
\hline Nov-15 & 800 & 143.8 & 274.8 & 184.3 & 199.6 & 181.2 & 204.1 & 798 & 574 & 379 & 314 & 34.3 & 10.0 & 19.1 & 6.6 & 14651 & 278.1 & 16.9 \\
\hline$v-15$ & 815 & 137.3 & 274.2 & 184.0 & 199.1 & 178.5 & 201.6 & 796 & 571 & 369 & 303 & 33.1 & 10.5 & 18.9 & 7.4 & 14 & 272.5 & 16.2 \\
\hline Nov. 15 & 830 & 146.5 & 274.8 & 185.3 & 200.0 & 184.2 & 208.8 & 813 & 580 & 366 & 303 & 34.4 & 9.9 & & 5.9 & 15 & 291.3 & 17.7 \\
\hline Nov-15 & 845 & 146.3 & 2753 & 185.6 & 200.4 & 184.8 & 211.7 & 791 & 564 & 373 & 310 & 34.4 & 10.2 & $\cdots$ & 4.9 & & & \\
\hline Nov. 15 & 1100 & 145.2 & 274.9 & 185.9 & 199.9 & 181.4 & 206.8 & 813 & 587 & 381 & 316 & 35.1 & 9. & 176 & 5.4 & 14 & 5 & 16.2 \\
\hline-15 & 1115 & 145.4 & 275.0 & 184.2 & 199.1 & 181.5 & 208.6 & 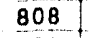 & 580 & 380 & 313 & 350 & 10.0 & 17.5 & 5.3 & 14 & 26 & 154 \\
\hline Vov-15 & 1130 & 143.8 & 272.9 & 183.5 & 198.5 & 180.4 & 204.8 & & 573 & 380 & 31 & 344 & 10.7 & 18.9 & 6.4 & & 276.9 & 15.5 \\
\hline Nov-15 & 1145 & 145.2 & 273.1 & 183.7 & 198.3 & 178.8 & 205.7 & 8 & 601 & 377 & 313 & 34.0 & 10.6 & 16.2 & 4.3 & & & \\
\hline$v \cdot 15$ & 1200 & 146.3 & 2732 & 184.7 & 199.4 & 182.5 & 210.1 & 838 & 595 & 372 & 307 & 340 & 103 & 18.2 & 4.8 & & 2 & 16.7 \\
\hline$v-15$ & 1215 & 146.4 & 273.3 & 187.0 & 199.9 & 181.1 & 210.9 & 838 & 613 & 374 & 304 & 34.3 & 10.1 & 14.8 & 5.1 & 1537.6 & 226.9 & 13.6 \\
\hline Nov-15 & 1230 & 145.2 & 272.2 & 188.6 & 199.7 & 180.5 & 207.2 & 841 & 578 & 369 & 300 & 34.9 & 10.1 & 21.2 & 6.7 & 1538.0 & 325.7 & 19.1 \\
\hline Nov. 15 & 1245 & 146.3 & 274.2 & 181.6 & 1980 & 180.7 & 205.9 & 844 & 595 & 37 & 21 & 350 & 9.9 & 18.7 & 4.7 & & & 18.3 \\
\hline ov-15 & 1300 & 16.2 & 275.3 & 1833 & 198.5 & 181.9 & 210.8 & 839 & 601 & 371 & 307 & 35.4 & 10.0 & 17.0 & 4.3 & & 9 & 15.3 \\
\hline-15 & 1315 & 6.3 & 274.6 & 182.9 & 198.1 & 181.9 & 209.7 & 842 & 592 & 381 & 314 & 34.9 & 10.2 & 18.8 & 4.9 & 1550.8 & 2922 & 17)$. \\
\hline Nov-15 & 1330 & 146.0 & 273.1 & 182.7 & 197.8 & & 08.7 & & 583 & & 314 & 34.4 & 10.1 & 19.2 & 3.8 & 1525.0 & 292.5 & 17.4 \\
\hline Nov. 15 & 1345 & 145.5 & 273.3 & 1832 & 198.2 & 180.1 & 2076 & 837 & 597 & 380 & 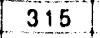 & 34.4 & 10.1 & 17.7 & 4.5 & & & 16.1 \\
\hline$v-15$ & 1400 & 145.0 & 273.3 & 186.1 & 198.4 & 178.5 & 207.7 & 844 & 579 & 382 & 313 & 34.0 & 10.1 & 20.1 & 4.7 & 1525.5 & 307.1 & 18.5 \\
\hline$v \cdot 15$ & 15 & 2.8 & 274.7 & 188.1 & 199.4 & 176.7 & 206.4 & 844 & 601 & 370 & 305 & 34.3 & 10.0 & 16.8 & 3.7 & 1510.8 & 254.2 & 15.4 \\
\hline Nov-15 & 1430 & 144.0 & 274.7 & 180.2 & & 176.0 & 204.8 & 844 & 558 & 381 & 306 & 34.5 & 10.1 & 23.1 & 6.3 & 1504.9 & 348.0 & 207 \\
\hline Nov.15 & 1445 & 142.1 & 274.2 & 1826 & 197.5 & 172.6 & 198.6 & & 582 & & 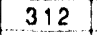 & 33 & 9 & & 57 & 14628 & 291.9 & 18.2 \\
\hline Vov-15 & 1500 & 141.5 & 274.7 & 182.7 & 197.7 & 172.7 & 200.4 & 841 & 581 & 381 & 314 & 33.5 & 10. & 19.8 & 4. & 1471.6 & 292.1 & 17.5 \\
\hline lov-15 & 1515 & 39.1 & 2.73 .6 & 182.3 & 197.5 & 170.9 & 198.8 & 838 & 575 & 383 & 312 & 33.4 & 10.0 & 20.3 & 5.0 & 1451.0 & 293.9 & 18 \\
\hline
\end{tabular}


CZD LIME INJECTION DATA - AUGUST 17, TO NOVEMBER 16. 1992

\begin{tabular}{|c|c|c|c|c|c|c|c|c|c|c|c|c|c|c|c|c|c|c|}
\hline Date & Time & oiler & Duct & Avg "C" & Duct & Flue Gas & Flue Gas & 502 & SO? & NOX & NOx & $\operatorname{Lim} \theta$ & $\operatorname{Lim} \theta$ & $\mathrm{SO} 2$ & NOx & $\mathrm{SO}_{2}$ & 552 & $\operatorname{Lim\theta }$ \\
\hline & & Load & $\ln \mid \theta t$ & Section & Outlol & Flow in & Flow ol & $\ln |\theta|$ & Outlet & $\ln |0|$ & Outlol & Flow & Conc & Removal & Removal & Inlet & Removed & Utilizalion \\
\hline & & $\mathrm{MW}$ & Temp of & Temp of & Temp of & KSCFM & KSCFM & $\mathrm{ppm}$ & $\mathrm{ppm}$ & $\mathrm{ppm}$ & $\mathrm{ppm}$ & $\mathrm{gpm}$ & $\%$ & $\%$ & $\%$ & $\mathrm{LB} / \mathrm{H}$ & $\mathrm{Lb} / \mathrm{Hr}$ & $\%$ \\
\hline Nov. 15 & 1530 & 138.0 & 274.2 & 182.5 & 197.1 & 169.0 & 194.8 & 826 & 574 & 378 & 312 & 33.1 & 10.0 & 18.9 & 4.8 & 14135 & 281.0 & 17.6 \\
\hline Nov. 15 & 1545 & 138.7 & 274.4 & 1834 & 1981 & 171.3 & 1988 & 825 & 563 & 379 & 13 & 32.9 & 10.1 & 20.8 & 4.2 & 1431.0 & 98.0 & 18.6 \\
\hline Nov-15 & 1600 & 138.4 & 275.2 & 183.6 & 197.7 & 169.4 & 185.2 & 838 & 565 & 375 & 05 & 32.9 & 10.3 & 22.3 & 6. & 1438.3 & 20.8 & 19.6 \\
\hline Nov. 15 & 1615 & 143.5 & 274.3 & 185.2 & 199.6 & 177.0 & 207.2 & 82 & 564 & 370 & 306 & 33.4 & 100 & 18.8 & 3. & 1475.8 & 93.0 & 18.2 \\
\hline Nov - 15 & 1630 & 142.2 & 3.6 & 182.8 & 198.9 & 174.3 & 204.5 & 819 & 559 & 377 & 308 & 34.1 & 9.9 & 199 & 4 & 5.5 & 88.3 & 17.7 \\
\hline Nov-15 & 1645 & 145.3 & 273.8 & 1858 & 199.6 & 179.5 & 2098 & 81 & 5 & 37 & 30 & 34 & 10 & 20.5 & 5 & 0.4 & 2.8 & 18.0 \\
\hline Nov. 15 & 1700 & 145.5 & 274.0 & 183.1 & 198.2 & 179.2 & 207.1 & B1 & 53 & 374 & 09 & 36.2 & 10.3 & 24.6 & 4.3 & 9.7 & 4.5 & 0.2 \\
\hline Nov. 15 & 1715 & 145.4 & 275.1 & 181.7 & 198.8 & 178.8 & 206.2 & 809 & 550 & 366 & 308 & 34.7 & 10.1 & 21.7 & 2.8 & $5 . \overline{6}$ & 7.6 & 18.8 \\
\hline Nov-15 & 1730 & 1460 & 274.8 & 1.9 & 198.3 & 1 & 204.4 & 82 & 55 & 3 & & 34.9 & 99 & 22.4 & 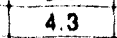 & & 0.3 & \\
\hline Nov. 15 & 1745 & 145.9 & 274.4 & 182.3 & 198.3 & 176.6 & 204.8 & 83 & 555 & 374 & 30 & 34.2 & 10 & 22.6 & 4.8 & 6.8 & 6.3 & 20.0 \\
\hline Nov. 15 & 1800 & 145.8 & 274.6 & 181.8 & 198.1 & 175.4 & 204.7 & 825 & 546 & 370 & 30 & 34.9 & 10.1 & 22.8 & 4.2 & 1465.3 & 33.9 & 19.6 \\
\hline Nov. 15 & 1815 & 145.3 & 275.5 & 182.0 & 198.5 & 180.8 & 206.8 & 800 & 532 & 370 & 304 & 35.2 & 10.4 & 24.0 & 5.8 & 1465.5 & 351.8 & 19.8 \\
\hline Nov-15 & 1830 & 146.2 & 277.1 & 184.1 & 2000 & 183.0 & 211.3 & 803 & 530 & 37 & 30 & 35 & 10.0 & 23.8 & 4.3 & 1487.8 & 4.1 & 20.7 \\
\hline Nov. 15 & 1845 & 142.5 & 275.5 & 182.9 & 198.3 & 176.5 & 203.3 & 796 & 527 & 36 & & 34 & & & 2 & & & - \\
\hline$A V E$ & RAGES & 143.6 & 274.5 & 182.9 & 198.1 & 179.2 & 206.0 & 807.8 & 559.5 & 3683 & 309.0 & 34.9 & 10.1 & 20.4 & 3.5 & 1466.4 & 298.7 & 17.6 \\
\hline
\end{tabular}




\section{PROCEDURES FOR CALCULATING $\mathrm{SO}_{2}$ AND NO $\mathrm{x}$ REMOVAL AND PERCENTAGE OF LIME UTILIZATION FOR CZD CONTINUOUS OPERATION}

Going into the second phase from parametric testing to continuous CZD demonstration, automated and integrated with Boiler \#15 at Seward Station, all the operating data are automatically recorded. The calculations are computerized, and the results recorded.

The lime slurry concentration is set and controlled by the nuclear-type density gauge and transmitter.

The environmental monitoring system (EMS) for monitoring CZD operations includes:

- Duct and stack opacity

- $\mathrm{SO}_{2}$ in and out - by dilution system*

- $\quad \mathrm{NO}_{\mathrm{x}}$ in and out - by dilution system*

- $\mathrm{CO}_{2}$ in and out - by dilution system*

*Flue gas samples are filtered and diluted with air (one volume flue gas with 200 volumes of air) before they are analyzed.

The flow of flue gas in the duct is monitored at three locations using the differential temperature type. Two locations use multipoint probes. These flow measurements, combined with the environmental measurements, form the basis for determining the percentages of $\mathrm{SO}_{2}$ and $\mathrm{NO}_{x}$ reduction by CZD.

During CZD operations, the following data are recorded every 15 minutes:

\begin{tabular}{lll}
\hline Field Name & Field Type & Formula \\
\hline Date & Date & \\
Time & Time & \\
R/C Out Temp ${ }^{\circ} \mathrm{F}$ & Number & \\
I.D. Fan Out Temp ${ }^{\circ} \mathrm{F}$ & Number \\
Flue gas flow in KSCFM & Number \\
Flue gas flow out KSCFM & Number \\
Lime slurry flow gpm & Number \\
Lime slurry header & Number \\
pressure psig & \\
Atomizing air flow & Number \\
SCFM &
\end{tabular}




\begin{tabular}{|c|c|c|}
\hline Fleld Name & Field Type & Formula \\
\hline B-duct opacity \% & Number & \\
\hline Stack opacity \% & Number & \\
\hline Slurry solids \% & Number & \\
\hline Boiler load MW & Number & \\
\hline $\mathrm{SO}_{2}$ inlet $\mathrm{ppm}$ & Number & \\
\hline $\mathrm{SO}_{2}$ outlet ppm & Number & \\
\hline $\mathrm{NO}_{x}$ inlet $\mathrm{ppm}$ & Number & \\
\hline $\mathrm{NO}_{x}$ outlet ppm & Number & \\
\hline $\mathrm{CO}_{2}$ inlet $\%$ & Number & \\
\hline $\mathrm{CO}_{2}$ outlet $\%$ & Number & \\
\hline $\begin{array}{l}\text { Average C-cross section } \\
\text { Temp }{ }^{\circ} \mathrm{F}\end{array}$ & Number & \\
\hline $\begin{array}{l}\text { Lowest C-cross section } \\
\text { Temp }{ }^{\circ} \mathrm{F}\end{array}$ & Number & \\
\hline $\mathrm{SO}_{2}$ in $\mathrm{SCFM}$ & Calculation Number & $\begin{array}{l}\text { (Flue gas [FG] flow in } \mathrm{KSCFM} \times \mathrm{SO}_{2} \text { inlet } \\
\mathrm{ppm} / 1000\end{array}$ \\
\hline $\mathrm{SO}_{2}$ out $\mathrm{SCFM}$ & Calculation Number & (FG flow out $\mathrm{KSCFM} \times \mathrm{SO}_{2}$ outlet $\mathrm{ppm}$ ) $/ 1000$ \\
\hline $\mathrm{NO}_{x}$ in SCFM & Calculation Number & (FG flow in $\mathrm{KSCFM} \times \mathrm{NO}_{\mathrm{x}}$ inlet $\mathrm{ppm}$ ) $/ 1000$ \\
\hline $\mathrm{NO}_{x}$ out SCFM & Calculation Number & $\left(\right.$ FG flow out $\mathrm{KSCFM} \times \mathrm{NO}_{\mathrm{x}}$ outlet $\left.\mathrm{ppm}\right) / 1000$ \\
\hline $\mathrm{SO}_{2}$ removal \% & Calculation Number & $100\left(\mathrm{SO}_{2}\right.$ in $\mathrm{SCFM}-\mathrm{SO}_{2}$ out $\left.\mathrm{SCFM}\right) / \mathrm{SO}_{2}$ in $\mathrm{SCFM}$ \\
\hline $\mathrm{NO}_{x}$ removal \% & Calculation Number & $100\left(\mathrm{NO}_{x}\right.$ in SCFM - NO $\mathrm{N}_{x}$ out SCFM)/NO $\mathrm{NO}_{x}$ in SCFM \\
\hline $\begin{array}{l}\mathrm{SO}_{2} \text { in } \mathrm{lb} / \mathrm{MM} \text { Btu } \\
\mathrm{SO}_{2} \text { out } \mathrm{lb} / \mathrm{MM} \text { Btu }\end{array}$ & $\begin{array}{l}\text { Calculation Number } \\
\text { Calculation Number }\end{array}$ & $\begin{array}{l}0.03 \times \mathrm{SO}_{2} \text { inlet } \mathrm{ppm} / \mathrm{CO}_{2} \text { inlet } \% \\
0.03 \times \mathrm{SO}_{2} \text { outlet } \mathrm{pnm} / \mathrm{CO}_{2} \text { outlet } \%\end{array}$ \\
\hline $\mathrm{NO}_{x}$ in $\mathrm{lb} / \mathrm{MM} \mathrm{Btu}$ & Calculation Number & $0.0218 \times \mathrm{NO}_{x}$ inlet $\mathrm{ppm} / \mathrm{CO}_{2}$ inlet \% \\
\hline $\mathrm{NO}_{x}$ out $\mathrm{lb} / \mathrm{MM}$ Btu & Calculation Number & $0.0218 \times \mathrm{NO}_{x}$ outlet $\mathrm{ppm} / \mathrm{CO}_{2}$ outlet $\%$ \\
\hline $\mathrm{SO}_{2}$ reduction $\%$ & Calculation Number & $\frac{100 \times\left(\mathrm{SO}_{2} \text { in } \mathrm{lb} / \mathrm{MM} \mathrm{Btu}-\mathrm{SO}_{2} \text { out } \mathrm{lb} / \mathrm{MM} \mathrm{Btu}\right)}{\left(\mathrm{SO}_{2} \text { in } \mathrm{lb} / \mathrm{MM} \mathrm{Btu}+0.00001\right)}$ \\
\hline $\mathrm{NO}_{x}$ reduction \% & Calculation Number & $\frac{100 \times\left(\mathrm{NO}_{x} \text { in } \mathrm{lb} / \mathrm{MM} \mathrm{Btu}-\mathrm{NO}_{x} \text { out lb/MM Btu }\right)}{\left(\mathrm{NO}_{x} \text { in lb/MM Btu }+0.00001\right)}$ \\
\hline Dilution $\mathrm{SO}_{2} \%$ & Calcularion Number & $100 \times\left(\mathrm{SO}_{2}\right.$ inlet $\mathrm{ppm} / \mathrm{SO}_{2}$ outlet ppm - 1.0) \\
\hline Dilution $\mathrm{NO}_{\mathbf{x}} \%$ & Calculation Number & $100 \times\left(\mathrm{NO}_{x}\right.$ inlet $\mathrm{ppm} / \mathrm{NO}_{\mathrm{x}}$ outlet $\left.\mathrm{ppm}-1.0\right)$ \\
\hline Dilution $\mathrm{CO}_{2} \%$ & Calculation Number & $100 \times\left(\mathrm{CO}_{2}\right.$ inlet $\% / \mathrm{CO}_{2}$ outlet $\left.\%-1.0\right)$ \\
\hline \multirow{3}{*}{$\begin{array}{l}\text { Dilution flue gas flow } \\
\text { Line useage } \mathrm{lb} \text { mol } \backslash \mathrm{min}\end{array}$} & \multirow{3}{*}{$\begin{array}{l}\text { Calculation Number } \\
\text { Calculation Number }\end{array}$} & $100 \times$ (FG flow out KSCFM/FG flow in KSCFM -1.0) \\
\hline & & $\begin{array}{c}\text { Lime flow gpm } \times 8.3456 \times \text { Slurry SGU } \\
\times \text { (Slurry Solids \%/100) } \\
\end{array}$ \\
\hline & & Lime mol wt. \\
\hline $\mathrm{SO}_{2}$ removed $\mathrm{lb} \mathrm{mol} \backslash \mathrm{min}$ & Calculation Number & $\begin{array}{l}\left(\mathrm{FG} \text { flow in } \mathrm{KSCFM} \times \mathrm{SO}_{2} \text { inlet } \mathrm{ppm} / 1000\right) \times\left(\mathrm{SO}_{2}\right. \\
\text { reduction } \% / 100) / 379\end{array}$ \\
\hline Lime utilization \% & Calculation Number & $\begin{array}{l}100 \times \mathrm{SO}_{2} \text { removed lb-mol } \backslash \mathrm{min} / \text { Lime useage } \\
\mathrm{lb} \mathrm{mol} \backslash \min \end{array}$ \\
\hline
\end{tabular}




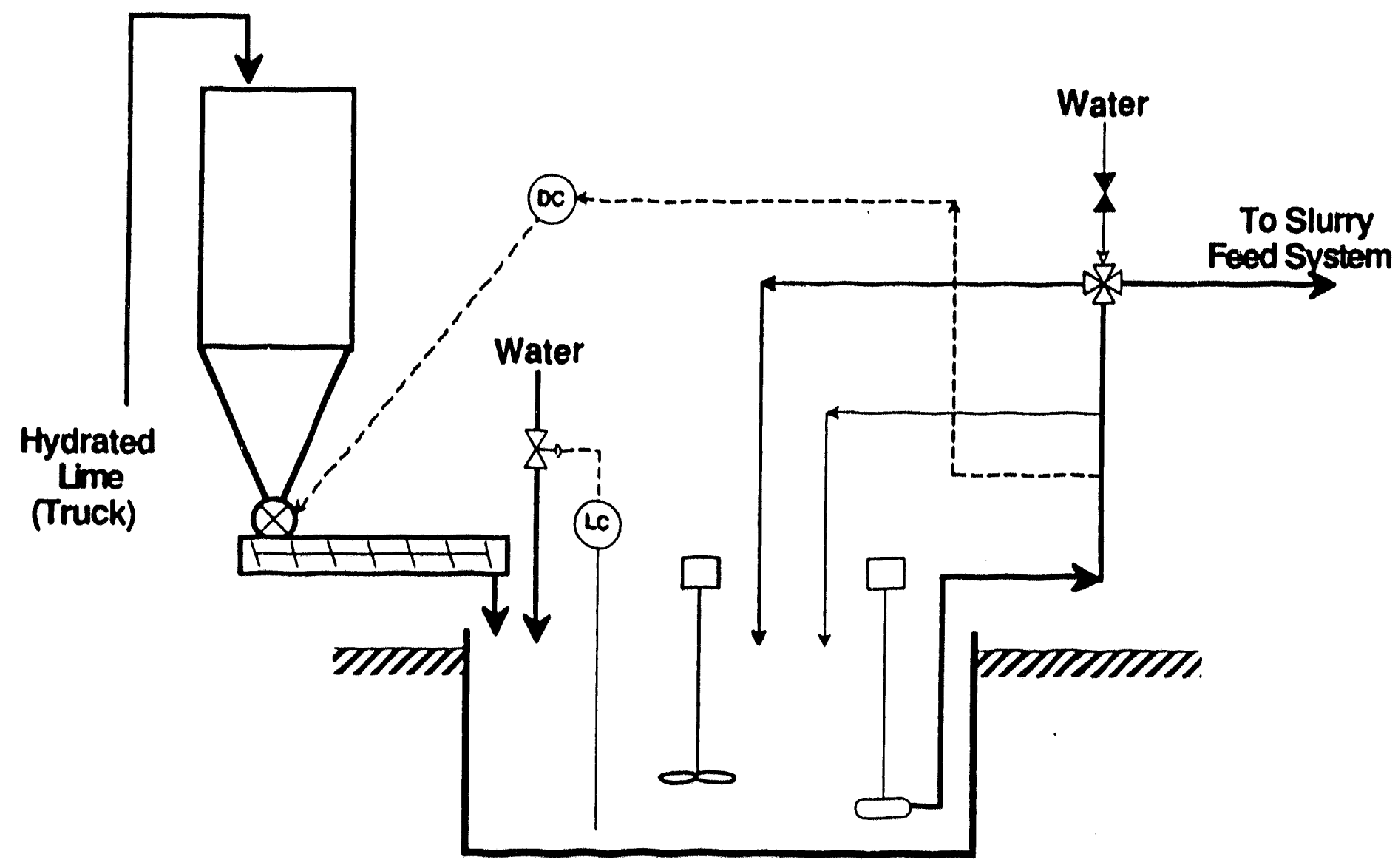

Lime Storage and Slurry Preparation - Continuous Operation 


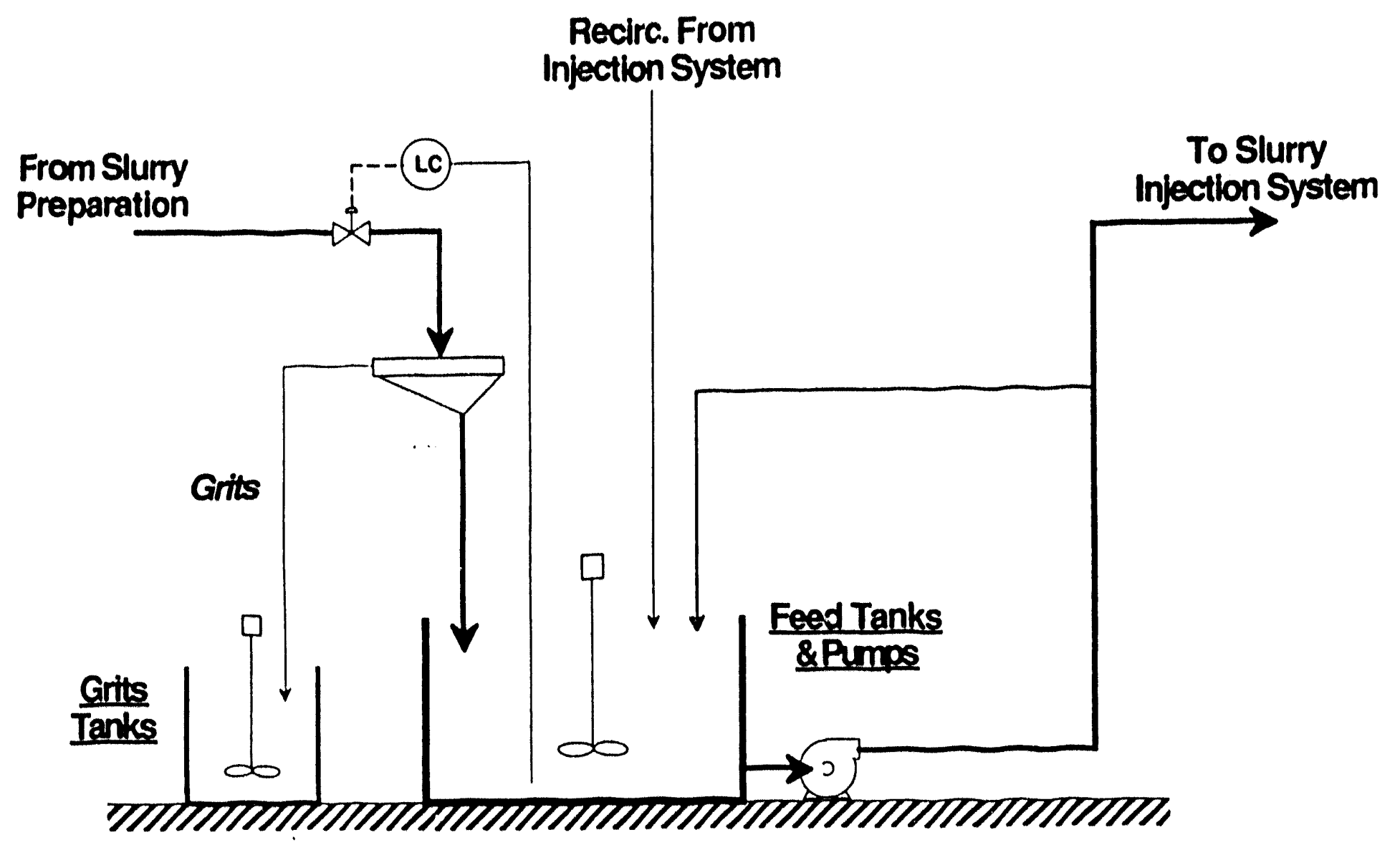

Lime Slurry Feed System - Continuous Operation 


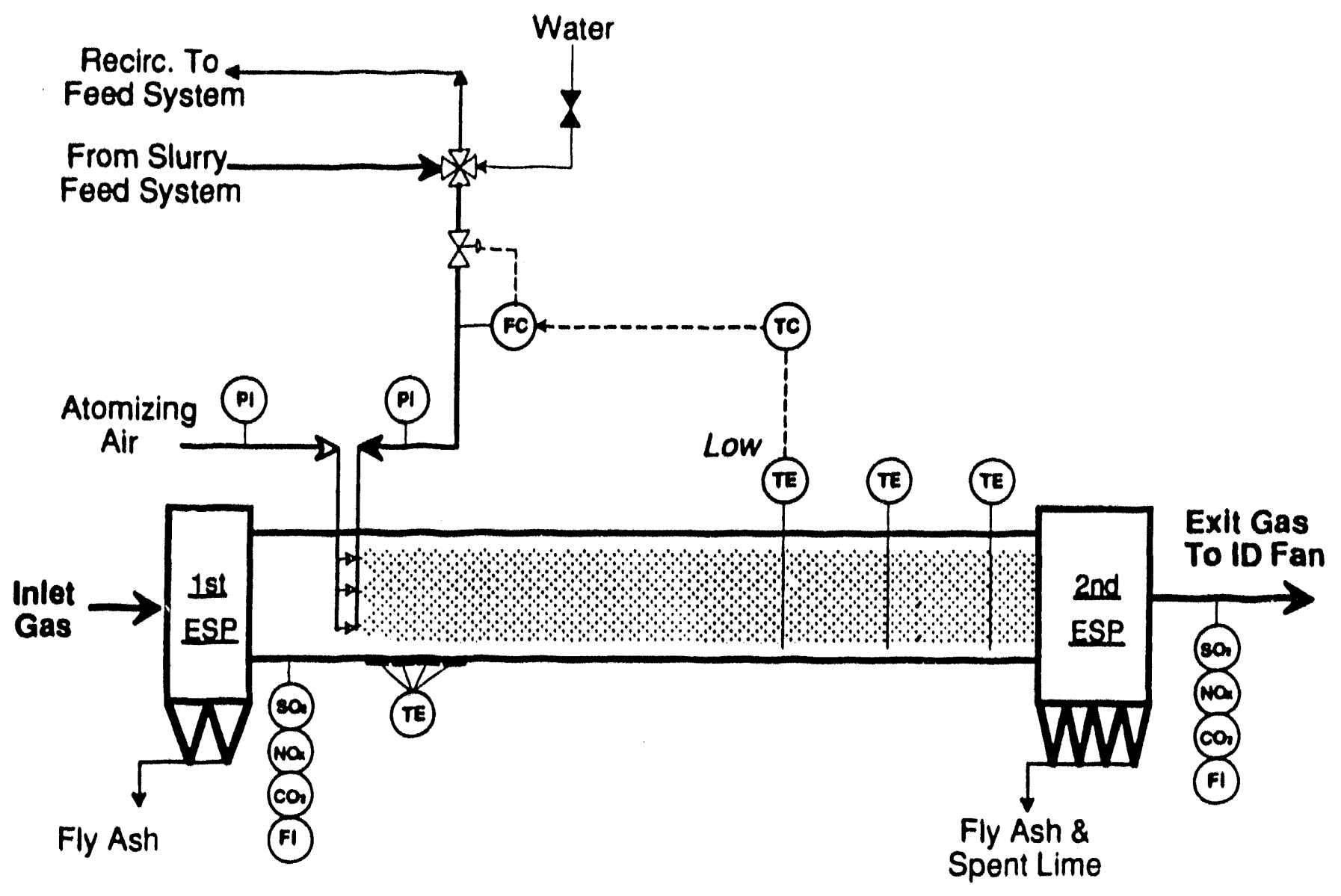

Lime Slurry Injection - Continuous Operation 


\section{Lime Utilization Calculations}

GIVEN:

Lime Sp. Gr. $=2.29=142.965 \mathrm{lb} / \mathrm{cu} \mathrm{ft} \quad(2.29 \times 62.43)$
$1 \mathrm{gal} H 20=8.345677 \mathrm{lb}=0.13368 \mathrm{cu} \mathrm{ft}$
THEN:

$\begin{array}{rrrr}\begin{array}{c}\text { Slurry Conc } \\ \text { WT } \%\end{array} & \begin{array}{c}\text { Slursy Wt } \\ \text { LB/GAL }\end{array} & \begin{array}{c}\text { Slurry Vol } \\ \text { CU FT }\end{array} & \text { Slurry SG } \\ 16 \% & 9.94 & 0.1448 & 1.0991 \\ 15 \% & 9.82 & 0.1440 & 1.0923 \\ 14 \% & 9.70 & 0.1432 & 1.0856 \\ 13 \% & 9.59 & 0.1424 & 1.0790 \\ 12 \% & 9.48 & 0.1416 & 1.0725 \\ 11 \% & 9.38 & 0.1409 & 1.0661 \\ 10 \% & 9.27 & 0.1402 & 1.0597 \\ 9 \% & 9.17 & 0.1395 & 1.0534 \\ 8 \% & 9.07 & 0.1388 & 1.0472 \\ 7 \% & 8.97 & 0.1381 & 1.0411 \\ 6 \% & 8.88 & 0.1374 & 1.0350 \\ 5 \% & 8.78 & 0.1368 & 1.0290 \\ 4 \% & 8.69 & 0.1361 & 1.0231 \\ 3 \% & 8.60 & 0.1355 & 1.0172 \\ 2 \% & 8.52 & 0.1349 & 1.0114 \\ 1 \% & 8.43 & 0.1343 & 1.0057 \\ \% \% & 8.35 & 0.1337 & 1.0000\end{array}$

Slurry Wt calculation $8.345677 /$ (1-slurry conc. wt.\% lime)

Slurry Vol calculation ((slurry wt * slurry lime conc) / 142.965) + 0.13368

Slurry SG calculation (slurry wt / slurry vol) / 62.43

Lime Used, in LB-MOLSMinute:

For Dolomitic Lime

(Slurry flow [gpm] • 8.34568 * Slurry SG • Lime conc. [\%*.01]) / 70.4

For Calcitic Lime

(Slurry flow [gpm] • 8.34568 • Slurry SG • Lime conc. [\%*.01]) / 74

SO2 Removed, in LB-MOLS/Minute:

((( (Flue gas in [KSCFM] ' SO2 in [PPM])/1000) sO2 removed [\%*.01]) / 379)

Lime Utilization:

(LB-MOLS SO2 Removed / LB-MOLS Lime used) 100 


\section{Lime Utilization Calculations}

\section{TONS LIME PER HOUR (Dry Weight)}

Slurry Lime Concentration, Wt. \%

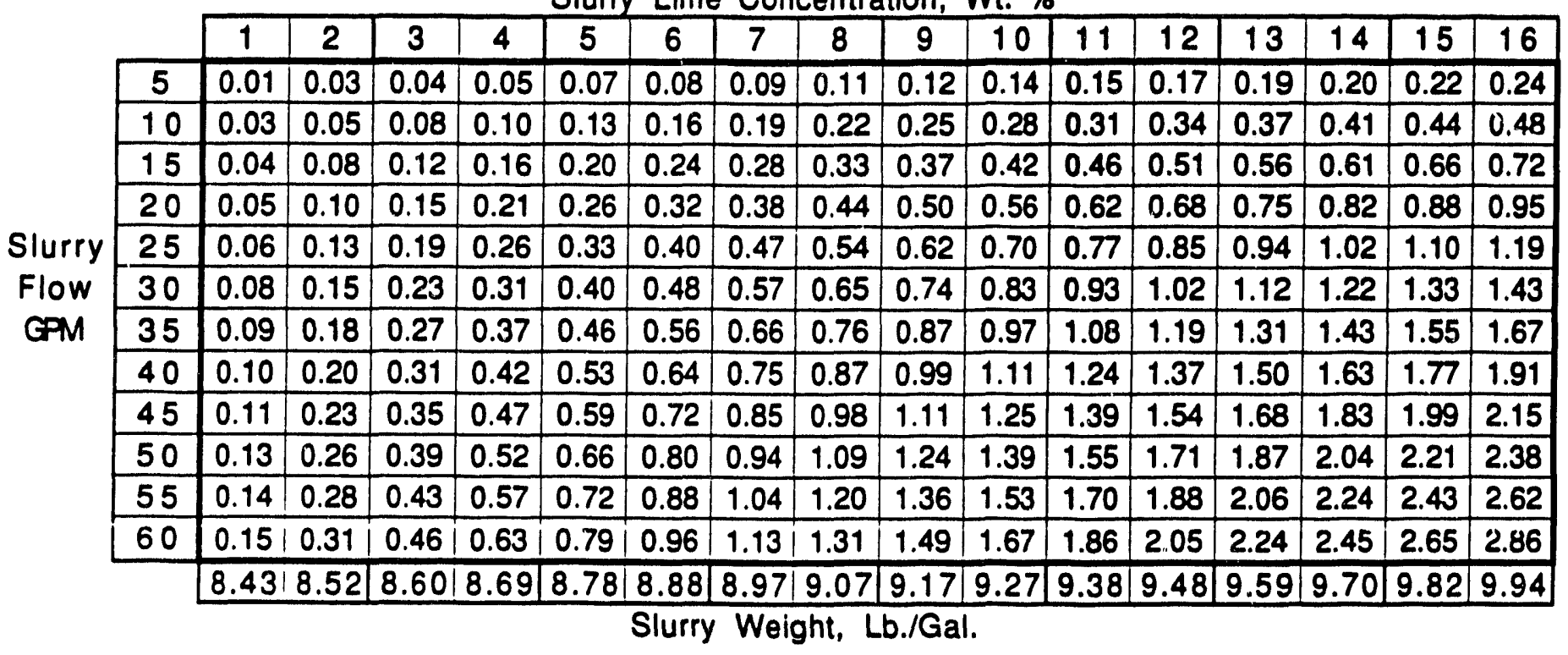


Appendix I

\section{Buell ESP Fields Operating Log}




\begin{tabular}{|c|c|c|c|c|c|c|c|c|c|c|c|c|c|c|c|c|c|c|c|c|c|}
\hline Dat & Time & $\begin{array}{l}\text { Water } \\
\text { Flow } \\
\text { gpm }\end{array}$ & $\begin{array}{l}\text { Lime } \\
\text { Flow } \\
\text { gom }\end{array}$ & $\begin{array}{l}\text { SWK } \\
\text { Q } \\
\% \\
\end{array}$ & $\begin{array}{r}B \\
\% \\
\% \\
\%\end{array}$ & & $\begin{array}{c}1 \mathrm{spk} \\
\mathrm{mn} \\
\end{array}$ & & $\begin{array}{l}2 \\
\text { spow } \\
\text { mn }\end{array}$ & & $\begin{array}{c}3 \\
\text { spok } \\
\mathrm{mn}\end{array}$ & & $\begin{array}{l}\text { spow } \\
\mathrm{mnn}\end{array}$ & & $\mathrm{mn}$ & $k W$ & & KW & $\mathbf{m}$ & & spen \\
\hline 1011492 & 800 & 21.0 & 0.0 & 11.6 & 11.2 & 27.9 & 3.1 & 25.5 & 9.3 & 51.4 & 5.5 & 362 & 8.7 & 200 & 14.9 & 41.7 & 94 & 363 & 9.8 & 515 & 4.9 \\
\hline $10 / 1492$ & 815 & $\mathbf{Z} .0$ & 0.0 & 11.4 & 102 & 282 & 3.0 & 30.3 & 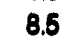 & 56.7 & 20 & 40.3 & - & 288 & 118 & 41.4 & 78 & 360 & 1.1 & $\infty 0$ & 33 \\
\hline $10 / 1492$ & 800 & 25.6 & 0.0 & 129 & 124 & 27.8 & 3.0 & 30.6 & 5.6 & 57.0 & 35 & 40.3 & 6.0 & 30.4 & 168 & $3 a 7$ & 10.5 & 348 & 1.2 & 518 & 3.1 \\
\hline $10 / 1492$ & 845 & 0.0 & 24.9 & 129 & 11.9 & 20.7 & 3.1 & 28.6 & 52 & 572 & 3.0 & 39.7 & 83 & 20.7 & 143 & 4.0 & 10.5 & $\mathbf{3 5 5}$ & 8.9 & 98 & 30 \\
\hline $10 / 1492$ & 900 & 0.0 & 25.0 & 128 & 122 & 29.4 & 3.1 & 28.9 & 5.5 & 56.5 & 3.7 & 39.7 & 7.5 & 286 & 162 & 421 & 10.6 & 352 & 1.1 & 48,4 & 30 \\
\hline $10 / 1492$ & 915 & 0.0 & 25.0 & 120 & 14.4 & $\mathbf{2 9 . 4}$ & 3.1 & 28.9 & 5.5 & 56.5 & 3.7 & 30.7 & 7.5 & 266 & 162 & 421 & 10.6 & 362 & 1.1 & 484 & 30 \\
\hline $10 / 1492$ & 900 & 0.0 & 25.3 & 14.9 & 16.2 & 29.4 & 3.1 & 23.9 & 5.5 & 56.5 & 3.7 & 30.7 & 7.5 & 266 & 16.2 & 421 & 10.6 & 362 & 1.1 & 184 & 30 \\
\hline $10 / 1492$ & 946 & 0.0 & 25.4 & 14.7 & 17.5 & 29.4 & 3.1 & $\mathbf{2 8 . 9}$ & 5.5 & 56.5 & 3.7 & 30.7 & 7.5 & 236 & 162 & 421 & 10.6 & 352 & 1.1 & 18.4 & 30 \\
\hline Q/1492 & 1000 & 0.0 & 25.3 & 15.8 & 20.2 & 29.4 & 3.1 & 28.8 & 5.5 & 56.5 & 3.7 & 30.7 & 7.5 & 28.6 & 162 & 421 & 10.6 & 352 & 1.1 & 184 & 30 \\
\hline 21492 & 1015 & 0.0 & 25.4 & 17.1 & 23.2 & 29.4 & 3.1 & 28.9 & 5.5 & 56.5 & 3.7 & 30.7 & 7.5 & 266 & 162 & 421 & 10.6 & 362 & 1.1 & 124 & 30 \\
\hline $10 / 14 / 92$ & 1000 & 0.0 & 28.9 & 15.9 & 20.8 & 29.4 & 3.1 & $\mathbf{2 8 . 9}$ & 5.5 & 56.5 & 3.7 & 39.7 & 7.5 & 266 & 162 & 421 & 10.6 & 362 & 1.1 & 184 & 30 \\
\hline V14492 & 1045 & 0.0 & 34.6 & 122 & 14.4 & 29.4 & 3.1 & 28.9 & 5.5 & 56.5 & 3.7 & 30.7 & 7.5 & 26.6 & 162 & 421 & 10.6 & 362 & 1.1 & 484 & 30 \\
\hline V14492 & 1100 & 0.0 & 33.0 & 9.4 & 13.9 & 29.4 & 3.1 & 28.9 & 5.5 & 565 & 3.7 & $\mathbf{3 9 . 7}$ & 75 & 26.6 & 162 & 421 & 106 & 362 & 1.1 & 424 & 30 \\
\hline 114/92 & 1115 & 0.0 & 320 & 10.1 & 14.8 & 20.4 & 3.1 & 28.9 & 5.5 & 565 & 3.7 & $\mathbf{3 0 . 7}$ & 75 & 68 & 162 & 121 & 10.6 & 362 & 1.1 & 42.4 & 30 \\
\hline $10 / 1492$ & 1130 & 0.0 & 320 & 128 & 15.4 & 29.4 & 3.1 & 28.9 & 5.5 & 56.5 & 3.7 & 30.7 & 7.5 & 26 & 162 & 121 & 106 & 362 & 1.1 & 424 & 30 \\
\hline $10 / 1492$ & 1145 & 0.0 & 31.8 & 129 & 15.7 & 29.4 & 3.1 & 28.9 & 5.5 & 56.5 & 3.7 & 30.7 & 7.5 & $\mathbf{3 6}$ & 162 & 21 & 10.6 & 362 & 1.1 & 424 & 30 \\
\hline $10 / 14 / 92$ & 1200 & 0.0 & 324 & 14.2 & 17.1 & 20.4 & 3.1 & 28.9 & 5.5 & 56.5 & 3.7 & 30.7 & 75 & 236 & 162 & 121 & 106 & 362 & 1.1 & 48.4 & 30 \\
\hline 14492 & 1215 & 0.0 & 323 & 15.0 & 17.5 & 29.4 & 3.1 & 28.9 & 5.5 & 56.5 & 3.7 & 39.7 & 7.5 & 26.6 & 162 & 121 & 10.6 & 352 & 1.1 & 48.4 & 30 \\
\hline $14 / 92$ & 1230 & 0.0 & 327 & 14.9 & 19.6 & 20.4 & 3.1 & 23.9 & 5.5 & 56.5 & 3.7 & 39.7 & 7.5 & $\mathbf{2 6} 6$ & 162 & 121 & 10.6 & 362 & 1.1 & 48.4 & 30 \\
\hline 10/14/92 & 1245 & 0.0 & 328 & 13.4 & 21.0 & 20.4 & 3.1 & 28.9 & 5.5 & 56.5 & 3.7 & 30.7 & 7.5 & 28.6 & 16.2 & 121 & 10.6 & 352 & 1.1 & 48.4 & 30 \\
\hline 1492 & 1300 & 0.0 & 33.9 & 14.1 & 18.0 & 20.4 & 3.1 & 28.9 & 5.5 & 56.5 & 3.7 & 30.7 & 7.5 & $\mathbf{2 6 6}$ & 162 & 421 & 10.6 & 362 & 1.1 & 484 & 30 \\
\hline $10 / 14 / 92$ & 1315 & 0.0 & 35.3 & 129 & 16.6 & 29.4 & 3.1 & 28.9 & 5.5 & 56.5 & 3.7 & $\mathbf{3 0 . 7}$ & 75 & $\mathbf{2 B 6}$ & 162 & 421 & 10.6 & 362 & 1.1 & 48.4 & 30 \\
\hline $10 / 14 / 92$ & 1330 & 0.0 & 34.2 & 14.9 & 18.7 & 20.4 & 3.1 & 28.9 & 5.5 & 56.5 & 3.7 & 39.7 & 7.5 & 28.6 & 162 & 121 & 10.6 & 332 & 1.1 & 48.4 & 30 \\
\hline $10 / 1492$ & 1345 & 0.0 & 34.0 & 13.0 & 17.1 & 34.5 & 1.2 & 31.2 & 2.0 & 548 & 16 & 43.2 & 1.3 & 64.4 & 1.7 & 484 & 19 & $\$ .0$ & 1.5 & $\infty 0$ & 12 \\
\hline $10 / 1492$ & 1400 & 0 & 36.1 & 16.3 & 19.8 & 0.0 & $?$ & 0.0 & $?$ & 0.0 & $?$ & 00 & $?$ & 0.0 & $?$ & 0.0 & $?$ & 0.0 & 100 & 0.0 & $?$ \\
\hline $10 / 14 / 92$ & 1415 & 0.0 & 34.4 & 16.6 & 21.5 & 20.1 & 3.1 & 21.5 & 15.0 & 47.7 & 88 & 38.9 & 65 & 30.7 & 130 & 46.7 & 10.4 & 330 & 1.0 & 50.0 & 33 \\
\hline $10 / 14 / 92$ & 1430 & 0 & 34.2 & 18.1 & 25.6 & 29.7 & 3.0 & 18.9 & 16.0 & 39.6 & 10.2 & 36.0 & 11.1 & 318 & 132 & 123 & 10.6 & 34.7 & 1.1 & 473 & 30 \\
\hline $10 / 14 / 92$ & 1445 & 0.0 & 35.2 & 16.0 & 223 & 29.4 & 3.1 & 18.1 & 16.8 & 38.6 & 10.6 & 36.5 & 0.8 & 32,4 & 11.6 & 430 & 10.0 & 36.0 & 1.0 & 47.1 & 38 \\
\hline $10 / 1492$ & 1500 & 0 & 35.5 & 15.0 & 224 & 29.4 & 3.1 & 19.1 & 16.8 & 38.6 & 10.6 & 36.5 & 9.8 & $3 a, 4$ & 11.6 & 430 & 10.0 & 360 & 1.0 & 47.1 & 38 \\
\hline $2 / 1492$ & 1515 & 0.0 & 35.0 & 16.3 & 21.9 & 28.5 & 3.0 & 222 & 13.5 & 49.8 & 88 & 38.6 & 3.7 & 30.1 & 220 & 4.4 & 136 & 337 & 1.1 & 43.6 & 3.1 \\
\hline $10 / 14 / 92$ & 1530 & 0.0 & 34.7 & 15.3 & 23.2 & 29.3 & 3.1 & 227 & 14.8 & 50.3 & 74 & 36.7 & 5.7 & 36.0 & 16.7 & 47.4 & 73 & 402 & 8.1 & 4.3 & 30 \\
\hline d/1492 & 1545 & 0.0 & 36.0 & 16.6 & 20.7 & 28.4 & 3.0 & 20.9 & 13.1 & 53.1 & 7.7 & 37.4 & 33 & 32,4 & 202 & 498 & 10.8 & 30.5 & 8.7 & $\infty 8$ & 52 \\
\hline $10 / 1492$ & 1600 & 0.0 & 34.4 & 158 & 26.4 & 29.3 & 3.2 & 227 & 15.1 & 51.0 & 9.6 & 37.5 & 10.1 & 285 & 17.6 & 138 & 123 & 366 & 0.1 & 47.4 & 32 \\
\hline Q/1492 & 1615 & 0.0 & 34.1 & 14.7 & 19.9 & 29.4 & 3.1 & 23.1 & 15.8 & 56.7 & 42 & 38.1 & 3.0 & 30.1 & 160 & 45.4 & 11.4 & 37.0 & 70 & 512 & 3.1 \\
\hline $10 / 1492$ & 1600 & 0.1 & 33.5 & 17.2 & 18.2 & 29.2 & 3.0 & 26.2 & 10.6 & 58.5 & 59 & 38.7 & 3.0 & 30.7 & 16.1 & 450 & 93 & 300 & 1.0 & 47.4 & 36 \\
\hline $10 / 1492$ & 1645 & 0. & 36.1 & 14.4 & 17.0 & 28.7 & 3.2 & $\mathbf{2 8 . 8}$ & 9.8 & 57.5 & 36 & 37.5 & 3.0 & 31.6 & 15.3 & 482 & 11.1 & 302 & 8.3 & 467 & 3.1 \\
\hline $10 / 1492$ & 1700 & 0.1 & 329 & 15.6 & 20.1 & 29.4 & 31 & 25.7 & 10.1 & 56.6 & 6.9 & 388 & 3.2 & 326 & 11.1 & 488 & 84 & 388 & 8.0 & 50.1 & 3.1 \\
\hline $10 / 1492$ & 1715 & 0.1 & 328 & 17.1 & 20.4 & 29.1 & 3.1 & 24.7 & 10.8 & 50.4 & 7.1 & 39.0 & 33 & 33.0 & 127 & $\infty 0.4$ & 92 & 41.1 & 8.6 & 50.1 & 3.1 \\
\hline $10 / 1492$ & 1730 & 0.1 & 324 & 16.8 & 21.0 & 292 & 3.1 & 24.8 & 15.3 & 56.3 & 5.6 & 30.3 & 3.7 & 320 & 18.7 & 518 & 102 & 412 & 8.8 & 97.4 & 33 \\
\hline 10/14/92 & 1745 & 0.1 & 31.9 & 16.1 & 20.8 & 29.1 & 3.2 & $\mathbf{2 0 . 0}$ & 13.6 & 54.7 & 69 & 39.2 & 3.6 & 332 & 10.6 & 50.7 & 80 & 413 & 8.0 & $\infty \mathbf{s}$ & 33 \\
\hline $10 / 1492$ & 1800 & 0.1 & 31.8 & 15.3 & 17.8 & 29.2 & 3.1 & 26.3 & 9.5 & $\$ 9.3$ & 4.1 & 38.0 & 3.0 & 30.3 & 138 & 500 & 86 & 10.7 & 1.0 & 505 & 30 \\
\hline $10 / 1492$ & 1815 & 0.1 & 327 & 14.5 & 17.5 & 20.1 & 3.0 & 27.6 & 7.2 & $\mathbf{5 0 . 4}$ & 32 & 39.5 & 33 & 30.5 & 148 & 484 & 85 & 30.9 & 8.6 & 80 & 30 \\
\hline $10 / 1492$ & 1830 & 0.1 & 34.2 & 16.1 & 17.8 & 28.6 & 3.0 & 27.2 & 10.2 & 60.7 & 3.4 & 38.4 & 3.0 & 232 & 11.4 & 465 & 68 & 37.3 & 92 & 60 & 30 \\
\hline $10 / 1492$ & 1845 & 0.1 & 30.3 & 17.0 & 18.9 & 28.9 & 3.0 & 25.5 & 11.4 & $\mathbf{5 8 . 4}$ & 6.1 & 39.2 & 32 & 340 & 108 & 432 & 79 & 30.1 & 83 & $\$ 2$ & 3.5 \\
\hline 10/14/92 & 1900 & 0.1 & 329 & 16.2 & 18.7 & 28.7 & 3.2 & $\boldsymbol{2 6 . 0}$ & 10.8 & 50.8 & 40 & 39.1 & 3.0 & 337 & 11.1 & 520 & 5.1 & 433 & 8.7 & 5 & 3.7 \\
\hline $10 / 1492$ & 1915 & 0.0 & 325 & 16.4 & 18.8 & $\mathbf{2 8 . 9}$ & 3. & 25.1 & 11.6 & 58.7 & 5.4 & 39.0 & 3. & 320 & 163 & 108 & 78 & 41.6 & 78 & 500 & 30 \\
\hline 10/1492 & 1900 & 0.0 & 33.0 & 16.7 & 19.4 & 28.5 & 32 & 25.8 & 11.3 & 583 & 5.3 & 380 & 38 & 332 & 10.0 & 48 & 79 & $\$ .6$ & 8.7 & sod & 30 \\
\hline $10 / 14 / 92$ & 1945 & 0.0 & 36 & 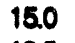 & 14.5 & 27.7 & 3. & 27.3 & 5.1 & 59.0 & 3.0 & 37.2 & 30 & 318 & 11.7 & 484 & 86 & $\$ 0.1$ & 7.4 & $\infty 03$ & 3.0 \\
\hline 10/1492 & 2000 & 0.0 & 28.0 & 19.5 & 18.6 & 20.2 & 4.7 & 21.6 & 14.7 & 57.5 & 4.4 & 38.3 & 33 & 324 & 11,4 & 0.1 & 68 & 108 & 8.2 & & 30 \\
\hline $10 / 14 / 92$ & 2015 & 0.0 & 29.8 & 17.5 & 20.1 & 20.0 & 3.1 & 24.3 & 12.6 & 56.5 & 42 & 30.0 & 3.7 & 322 & 136 & 496 & OA & 0.1 & 1.1 & 40 & 30 \\
\hline $10 / 1492$ & 2000 & 0.0 & 31.5 & 160 & 21.2 & 20.1 & 3. & 20,4 & 11.8 & 56.0 & 6.0 & 30.1 & 3.4 & 320 & 132 & 47.5 & 102 & 303 & 12 & 64 & 30 \\
\hline 10/14/92 & 2045 & 00 & 29.7 & 17.9 & 21.1 & $\mathbf{2 8 . 0}$ & 32 & 20.0 & 15.5 & 5 & 3.5 & 30.8 & 3. & 31.7 & 128 & 40 & $7 A$ & 396 & 86 & $\mathbf{4 a 4}$ & 3.1 \\
\hline $10 / 1492$ & 2100 & 29.0 & 0.0 & 21.0 & 27.1 & 28.1 & 3.0 & 18.6 & 16.4 & 472 & 72 & 33.9 & 8.4 & 28.4 & 166 & 4.0 & 10.4 & $\mathbf{3 6 . 0}$ & 1.0 & 40.1 & 3.6 \\
\hline 10/14/92 & 2115 & 26.3 & 0.0 & 15 & 25.1 & 27.0 & 3.0 & 14.7 & 21.4 & 37.7 & 10.6 & 20.4 & 38 & 20.1 & 16 & 45.6 & 90 & 37.5 & 0.5 & 100 & 39 \\
\hline $10 / 1492$ & 2130 & $\mathbf{3 9 . 6}$ & 0.0 & 13.9 & 14.1 & 312 & 3.0 & 21.3 & 12.7 & 56.7 & 3.7 & 38.5 & 3.0 & 15 & 13 & 452 & 10.5 & 336 & 1.1 & 512 & 32 \\
\hline $10 / 1492$ & 2145 & 20.9 & 0.0 & 129 & 19.3 & 27.9 & 3.1 & 16.1 & 20.0 & 425 & 10.8 & 33.7 & 112 & 253 & 128 & 43.7 & 10.1 & 350 & 92 & 460 & 3.1 \\
\hline /14/92 & 2000 & 25.6 & 0.0 & 14.1 & 21,4 & 27.5 & 3.2 & 14.6 & 18.4 & 44.6 & 70 & 37.5 & 89 & 280 & 142 & 120 & 99 & 348 & 1.0 & 472 & 33 \\
\hline 1600 & 2215 & ఒ. & 0.0 & 15.8 & 20. & 272 & 3.0 & 15.4 & 16.4 & 43.4 & 75 & 358 & 80 & 253 & 17.4 & 412 & 12.7 & 31.3 & 9.9 & 484 & 41 \\
\hline $114 / 92$ & 2230 & 25.8 & 0.0 & 16.6 & 20.7 & 27.2 & 5.5 & 13.5 & 19.6 & 39,6 & 88 & 327 & 10.0 & 26.7 & 184 & 413 & 121 & 330 & 1.1 & 60 & 36 \\
\hline & 2245 & 25.8 & 0.0 & 15.8 & 23.3 & 27.5 & 3.1 & 14.0 & 19.3 & 39.4 & 9.1 & 323 & 11.1 & 27.3 & 186 & 300 & 132 & 328 & 1.1 & 488 & 3.1 \\
\hline 402 & 2300 & 30.2 & 0.0 & 123 & 15.0 & 20.3 & 3.0 & 19.5 & 12.0 & 50.1 & 4.5 & $\$ 0.7$ & 3.7 & & 126 & 5 & 11.7 & 350 & 98 & 488 & 37 \\
\hline & 2315 & s & 0.0 & 11.6 & 15.1 & 20.0 & 3.0 & 202 & 14.4 & 53.8 & 49 & 41.0 & 3.7 & 27.1 & 158 & 21 & 10.6 & 340 & 99 & 521 & 35 \\
\hline & 2300 & 30.6 & 0.0 & 11.2 & 11.3 & 29.3 & 3.0 & 24.1 & 8.8 & 550 & 2 & 40.7 & 3.1 & 30.0 & 14 & 460 & 113 & 372 & 9 & 521 & 30 \\
\hline & 2345 & 30.6 & 0.0 & 9.7 & 11.7 & 29.2 & 3.0 & 26.9 & 9.2 & 55.8 & 3. & 39.8 & 3.0 & 20.1 & 13.1 & 421 & 113 & 346 & 98 & $\$ .6$ & 3.0 \\
\hline & 0 & 30.6 & 0.0 & 8.2 & 11.3 & 29.8 & 3.0 & 282 & 5.3 & 552 & 29 & $\$ 0.3$ & 34 & 9.6 & 142 & 20 & 11.7 & 34.6 & 8.6 & 51.3 & 32 \\
\hline
\end{tabular}




\begin{tabular}{|c|c|c|c|c|c|c|c|c|c|c|c|c|c|c|c|c|c|c|c|c|c|}
\hline Date & Time & $\begin{array}{c}\text { Water } \\
\text { Flow } \\
\text { gpm }\end{array}$ & $\begin{array}{l}\text { Lime } \\
\text { Flow } \\
\text { gpm }\end{array}$ & $\begin{array}{l}\text { sik } \\
\alpha_{p} \\
\%\end{array}$ & $\begin{array}{l}B \\
\alpha_{\%}^{p}\end{array}$ & & $\begin{array}{l}\text { sple } \\
\text { mn }\end{array}$ & & $\begin{array}{l}2 \\
\text { spks } \\
\mathrm{mn}\end{array}$ & & epl & & $\mathrm{mn}$ & & & KW & & & & & mn \\
\hline $10 / 1592$ & 15 & 30.8 & 0.0 & 11.6 & 98 & 293 & 3.0 & 30.8 & 6.2 & 55.2 & 29 & 30.6 & 3.0 & 230 & 120 & 126 & 74 & 332 & 7.9 & 40.1 & 3.0 \\
\hline $10 / 1592$ & 30 & 30.4 & 0.0 & 9.9 & 11.5 & 29.1 & 3.0 & 26.8 & 8.7 & 54.8 & 30 & 39.7 & 3.0 & 27.6 & 108 & 432 & 105 & 349 & 8.3 & 525 & 30 \\
\hline $10 / 15192$ & 45 & 30.4 & 00 & 10.0 & 10.5 & $\mathbf{2 8 . 0}$ & 3.0 & 30.0 & 6.6 & 55.9 & 20 & 384 & 3.0 & 20.7 & 142 & 41.4 & 11.1 & 318 & 8.6 & 502 & 3.0 \\
\hline $10 / 1502$ & 100 & 30.5 & 0.0 & 16.3 & 15.3 & 29.5 & 3.0 & 26.3 & 8.1 & 54.9 & 29 & $\$ .7$ & 3.7 & 280 & 150 & 326 & 11.9 & 33.1 & 9.2 & 43.5 & 3.0 \\
\hline $10 / 1592$ & 115 & 30.5 & 0.0 & 10.4 & 118 & $\mathbf{2 9 . 0}$ & 3.0 & $\mathbf{2 6 . 3}$ & 8.1 & 54.6 & 30 & $\$ 0.6$ & 3.1 & 2.4 & 189 & 182 & 110 & 328 & 8.8 & 49.4 & 32 \\
\hline $10 / 1592$ & 130 & 30.6 & 0.0 & 11.6 & 148 & 28.8 & 3.0 & 25.2 & 8.5 & 543 & 3.0 & 0.9 & 3.1 & 272 & 139 & 4.1 & 10.8 & $2 / 48$ & 0.3 & 53.2 & 3.0 \\
\hline $10 / 1592$ & 145 & 30.6 & 0.0 & 10.9 & 15.0 & $\mathbf{2 8 , 9}$ & 3.0 & 25.6 & 7.8 & 54.6 & 29 & $\$ 0.6$ & 3.9 & 23.7 & 14.4 & 485 & 11.0 & 34.4 & 9.7 & 50.4 & 3.1 \\
\hline $10 / 15 / 92$ & 200 & 30.6 & 0.0 & 17.6 & 18.2 & 29.4 & 3.0 & 23.1 & 8.5 & 528 & 43 & $\$ 0.6$ & 73 & 24.6 & 16.1 & 302 & 121 & 31.1 & 8.9 & 53.3 & 3.1 \\
\hline $10 / 15,92$ & 215 & 30.7 & 0.0 & 127 & 13.9 & 28.4 & 3.0 & 24.6 & 14.7 & 530 & 3.7 & 41.4 & 7.8 & 28.4 & 16.1 & 0.2 & 11.5 & 325 & 1.1 & 520 & 3.0 \\
\hline $10 / 15 / 92$ & 200 & 29.0 & 0.0 & 18.8 & 13.4 & 28.9 & 3.0 & 26.4 & 7.1 & 542 & 29 & 41.7 & 3.1 & 282 & 130 & 425 & 0.8 & 348 & 9.2 & 526 & 32 \\
\hline $10 / 1592$ & 245 & 28.1 & 0.0 & 11.0 & 120 & 28.5 & 3.0 & 31.2 & 5.1 & 56.4 & 20 & $\$ 0.5$ & 3.0 & 2008 & 150 & 0.7 & 121 & 324 & 1.1 & 522 & 3.0 \\
\hline $10 / 15 / 92$ & 300 & 26.8 & 0.0 & 19.5 & $10 . i$ & 29.1 & 3.0 & 30.7 & 9.8 & 56.4 & 20 & 02 & 30 & 20.5 & 132 & 37.5 & 128 & 31.0 & 1.1 & 502 & 30 \\
\hline $10 / 15 / 92$ & 315 & 25.7 & 0.0 & 8.9 & 90 & 28.6 & 3.0 & 33.0 & 3.3 & 558 & 29 & $\$ 0.1$ & 30 & 31.4 & 128 & 163 & 135 & 338 & 7.1 & $\mathbf{4 6 5}$ & 3.0 \\
\hline $10 / 15 / 92$ & 300 & 25.9 & 0.0 & 11.3 & 99 & 28.8 & 3.0 & 33.7 & 3.2 & 560 & 20 & 39.7 & 3.0 & 314 & 128 & 41.1 & 88 & 303 & 8.7 & 51.4 & 30 \\
\hline $10 / 15 / 92$ & 345 & 25.8 & 0.0 & 122 & 9.0 & 28.6 & 3.0 & 33.0 & 3.1 & 56.3 & 20 & 37.4 & 3.0 & 31.5 & 10.4 & 406 & 58 & 300 & 5.8 & 521 & 32 \\
\hline $10 / 1592$ & 400 & 25.6 & 0.0 & 10.7 & 85 & 29.2 & 3.0 & 33.8 & 3.1 & 562 & 20 & 38.1 & 30 & 30.5 & 120 & 321 & 64 & 31.1 & 6.8 & 50.9 & 32 \\
\hline $10 / 1592$ & 415 & 25.6 & 0.0 & 15.6 & $8 B$ & 28.7 & 3.0 & 30.3 & 3.1 & 56.0 & 20 & 350 & 3.0 & 273 & 160 & 552 & 128 & 288 & 1.1 & 47.3 & 6.5 \\
\hline $10 / 1502$ & 450 & 25.8 & 0.0 & 14.1 & 8.5 & 29.0 & 3.0 & 328 & 3.1 & 56.1 & 20 & 33.0 & 3.0 & 27.7 & 21.7 & $\overline{30.1}$ & 172 & 29.4 & 13 & 51.9 & 3.0 \\
\hline $10 / 15 / 92$ & 445 & 24.9 & 0.0 & 13.8 & 83 & 28.8 & 3.0 & 325 & 3.1 & 56.3 & 20 & 36.3 & 3.0 & 321 & 10.1 & 403 & 115 & 335 & 8.5 & 502 & 3.1 \\
\hline $10 / 15 / 92$ & 500 & 24.3 & 0.0 & 11.8 & 8.4 & 28.8 & 3.0 & 33.5 & 4.8 & 56.0 & 20 & 37.0 & 3.0 & 31.0 & 119 & $\$ 0.0$ & 124 & 300 & 12 & 50.9 & 3.0 \\
\hline $10 / 15 / 92$ & 515 & 20.9 & 0.0 & 11.6 & 8.4 & 28.7 & 3.0 & 31.7 & 4.7 & 56.6 & 20 & 38.1 & 3.0 & 280 & 158 & 37.1 & 183 & 202 & 9.7 & 50.9 & 3.0 \\
\hline $10 / 15 / 92$ & 550 & 23.9 & 0.0 & 11.4 & 96 & 28.4 & 3.0 & 323 & 4.7 & 55.8 & 20 & 37.5 & 30 & 230 & 148 & 323 & 106 & 31.0 & 1.0 & 49.7 & 3.0 \\
\hline $10 / 1592$ & 5.45 & 21.7 & 0.0 & 11.5 & 11.6 & 28.4 & 3.0 & 25.0 & 13.8 & 527 & 4.4 & $\$ 0.6$ & 36 & 248 & 16.6 & 332 & 120 & 312 & 1.0 & 51.4 & 3.0 \\
\hline $10 / 1592$ & $\infty \infty$ & 23.7 & 0.0 & 35.0 & 17.1 & 28.1 & 13.4 & 21.0 & 16.0 & 51.0 & 50 & 41.7 & 72 & 24.1 & 248 & $\$ 0.4$ & 13.7 & 31.8 & 12 & 43.7 & 3.0 \\
\hline $10 / 1592$ & 615 & 23.8 & 0.0 & 11.4 & 15.6 & 28.5 & 42 & 19.1 & 14.3 & 48.9 & 56 & $\mathbf{0 . 5}$ & 60 & 285 & 273 & 27 & 163 & 303 & 12 & 49.0 & 3.0 \\
\hline $10 / 15 / 92$ & 60 & 24.2 & 00 & 13.0 & 19.3 & 28.1 & 3.0 & 14.7 & 18.1 & 38.3 & 10.2 & 324 & 10.6 & 272 & 140 & 420 & 9.7 & 305 & 8.8 & 47.3 & 3.0 \\
\hline $10 / 15 / 92$ & 645 & $\mathbf{2 3 . 9}$ & 0.0 & 13.1 & 19.7 & 28.2 & 3.7 & 15.2 & 18.1 & 33.0 & 9.4 & 332 & 9.3 & 274 & 170 & 40 & 108 & 348 & 8.6 & 49.6 & 3.0 \\
\hline $10 / 15.92$ & 700 & $\mathbf{2 0 . 4}$ & 0.0 & 13.5 & 19.9 & 28.3 & 3.7 & 15.2 & 16.6 & 333 & 7.7 & 321 & 83 & 270 & 168 & 462 & 98 & 352 & 7.0 & 41.8 & 30 \\
\hline $10 / 1592$ & 715 & 23.4 & 0.0 & 126 & 17.9 & 28.2 & 4.1 & 16.6 & 15.7 & $\$ 0.6$ & 6.7 & 34.3 & 0.4 & 281 & 138 & 121 & 11.1 & 34.0 & 1.0 & 41.8 & 30 \\
\hline $2 / 15 / 92$ & 730 & 25.3 & 0.0 & 13.8 & 18.6 & 27.7 & 3.0 & 16.4 & 17.0 & 424 & 7.4 & 33.7 & 9.5 & 296 & 13.1 & 420 & 10.0 & 368 & 8.7 & 48.1 & 3.0 \\
\hline $10 / 1592$ & 746 & 27.3 & 0.0 & 13.6 & 20.4 & 27.9 & 3.2 & 18.4 & 13.6 & 45.0 & 60 & 35.4 & 8.6 & 30.0 & 130 & 403 & 11.1 & 34.4 & 8.5 & 48.6 & 3.0 \\
\hline $10 / 15.92$ & 800 & 27.9 & 0.0 & 11.9 & 17.4 & 27.9 & 3.0 & 19.9 & 13.5 & 49.5 & 4.7 & 37.4 & 24 & 238 & 186 & 4.1 & 112 & 336 & 8.9 & 51.6 & 3.7 \\
\hline $10 / 15 / 92$ & 815 & $\mathbf{2 8 . 0}$ & 0.0 & 125 & 15.0 & 27.9 & 3.0 & 20.5 & 118 & 48.1 & 58 & 33.1 & 9.5 & 202 & 125 & 44 & 11.0 & 350 & 8.7 & 49.7 & 3.0 \\
\hline $10 / 15 / 92$ & 800 & 27.8 & 0.0 & 13.1 & 16.2 & 27.9 & 3.0 & 21.7 & 12.3 & 40.4 & 4.7 & 32.3 & 68 & $\overline{20.8}$ & 133 & 46.7 & 6.3 & 368 & 7.6 & $49 B$ & 3.0 \\
\hline $10 / 15 / 92$ & 845 & 27.9 & 00 & 122 & 17.1 & 27.9 & 3.0 & 20.9 & 13.7 & 50.8 & 4.7 & 37.4 & 93 & 28 & 136 & 10.7 & 19.1 & 340 & 9.3 & 4.1 & 3.0 \\
\hline $10 / 15 / 92$ & 900 & 27.8 & 0.0 & 0.1 & 15.7 & 27.8 & 3.0 & 21.4 & 13.3 & 80.1 & 48 & 38.1 & 88 & 203 & 125 & 20 & 113 & 30.1 & 1.1 & 47.7 & 3.0 \\
\hline $0 / 15.92$ & 915 & 27.8 & 0.0 & 125 & 16.4 & 28.0 & 3.0 & 21.2 & 12.5 & 50.5 & 56 & 39.9 & 8.1 & 300 & 11.4 & 408 & 120 & 324 & 9.6 & 521 & 3.6 \\
\hline $0 / 15 / 92$ & 980 & 27.8 & 0.0 & 11.9 & 16.7 & 27.8 & 3.0 & 212 & 11.9 & 51.4 & $4 A$ & 37.0 & 9.1 & 200 & 11.1 & 40 & 103 & 34.7 & 1.0 & 49.1 & 3.0 \\
\hline $10 / 15 / 92$ & 945 & 27.8 & 0.0 & 11.0 & 17.0 & 28.0 & 3.0 & 21.7 & 11.3 & 50.7 & 43 & 37.8 & 88 & 20.7 & 138 & 40.1 & 100 & 350 & 1.1 & 48.5 & 3.0 \\
\hline $0 / 15 / 92$ & 1000 & $\mathbf{2 6 . 0}$ & 0.0 & 9.8 & 14.6 & 28.2 & 3.0 & 23.2 & 112 & 50.4 & 36 & 40.9 & 7.0 & 31.2 & 158 & 468 & 11.3 & 368 & 9.2 & 53.6 & 3.0 \\
\hline $10 / 15 / 92$ & 1015 & 27.1 & 0.0 & 121 & 13.4 & 223 & 3.0 & 220 & 10.1 & 518 & 6.0 & 40.7 & 7.8 & 30.3 & 130 & 468 & 120 & 348 & 0.4 & 53.5 & 3.0 \\
\hline $10 / 15 / 92$ & 1000 & 28.9 & 0.0 & 120 & 14.5 & 28.3 & 3.0 & 25.9 & 7.8 & 53.4 & 4.1 & 410 & 72 & 28.7 & 126 & 44 & 11.4 & 34.3 & 9.1 & 533 & 3.0 \\
\hline $10 / 1592$ & 1045 & 27.6 & 0.0 & 10.1 & 13.7 & 28.3 & 3.0 & 27.5 & 7.3 & 55.2 & 3.1 & 429 & 58 & 208 & 16.1 & 486 & 10.6 & 34.3 & 8.7 & 538 & 3.0 \\
\hline $10 / 15,92$ & 1100 & 290 & 0.0 & 8.6 & 126 & 28.5 & 3.0 & 27.9 & 4.6 & 54.9 & 3.4 & 41.4 & 4.4 & 288 & 133 & 124 & 110 & 338 & 9.0 & 530 & 3.0 \\
\hline $10 / 1592$ & 1115 & 28.8 & 0.0 & 10.8 & 11.7 & 28.2 & 3.0 & 28.7 & 8.1 & 57.0 & 33 & 424 & 3.0 & $\mathbf{2 6 5}$ & 15.1 & 41.1 & 162 & 31.3 & 8.5 & 508 & 3.0 \\
\hline $10 / 1592$ & 1130 & $\mathbf{2 6 . 2}$ & 0.0 & 10.0 & 14.3 & 20.0 & 3.0 & 268 & 10.3 & 57.0 & 3.5 & 41.9 & 8.6 & 255 & 16.6 & 21 & 124 & 31.0 & 1.0 & 504 & 3.0 \\
\hline $1015 / 52$ & 1145 & 26.3 & 0.0 & 10.3 & 15.0 & 20.5 & 3.0 & 21.4 & 12.3 & 51.1 & 60 & 38.7 & 7.6 & 258 & 13.0 & 103 & 122 & 31.0 & 9.3 & 538 & 3.0 \\
\hline$\alpha 15 / 92$ & 1200 & 35.6 & 0.0 & 10.6 & 13.8 & 28.7 & 3.0 & 33.8 & 5.1 & 50.6 & 29 & 40.8 & 3.0 & 270 & 142 & 120 & 144 & 320 & 9.4 & 542 & 3.1 \\
\hline$\alpha 1592$ & 1215 & 34.7 & 0.0 & 135 & 120 & 28.1 & 3.0 & 35.4 & 3.1 & 60.8 & 29 & 30.7 & 3.0 & 272 & 143 & 128 & 120 & 302 & 9.3 & 515 & 3.0 \\
\hline$\alpha 1592$ & 1230 & 34.0 & 0.0 & 9.6 & 126 & 28.2 & 3.0 & 35.3 & 3.2 & 612 & 29 & 30.5 & 3.0 & 279 & 148 & 128 & 112 & 300 & 1.1 & 544 & 3.0 \\
\hline $10 / 15 / 92$ & 1245 & 34.5 & 00 & 148 & 13.4 & 285 & 3.0 & 34.7 & 3.1 & 61.4 & 29 & 388 & 3.0 & 279 & 128 & 120 & 108 & 304 & 9.2 & 543 & 30 \\
\hline $10 / 1592$ & 1300 & 342 & 0.0 & 9.0 & 120 & 28.1 & 3.0 & 34.5 & 3.1 & 61.5 & 30 & 32.0 & 3.0 & 27.1 & 158 & $\infty 00$ & 123 & 30.0 & 1.1 & 46.3 & 3.0 \\
\hline $10 / 15.92$ & 1315 & 33.1 & 0.0 & 8.3 & 123 & 28.3 & 3.0 & 34.7 & 3.3 & 61.7 & 30 & 38.6 & 3.0 & 233 & 187 & 41.1 & 108 & 523 & 1.1 & 54.9 & 3.0 \\
\hline $10 / 15 / 92$ & 1330 & 20.5 & 0.0 & 127 & 126 & 28.7 & 3.0 & 33.5 & 7.9 & 618 & 30 & 38.8 & 3.0 & 24.4 & 164 & 408 & 118 & 30.2 & 8.7 & 53.8 & 3.0 \\
\hline $10 / 15 / 92$ & 1345 & 329 & 0.0 & 14.4 & 13.6 & 28.4 & 3.0 & 34.6 & 3.8 & 61.7 & 30 & $\mathbf{3 a 0}$ & 3.0 & 253 & 163 & $\mathbf{1 0 . 4}$ & 120 & 31.7 & 1.1 & 54.1 & 3.0 \\
\hline $10 / 15 / 92$ & 1400 & 35.5 & 0.0 & 127 & 11,9 & 28.7 & 3.0 & 34.6 & 3.2 & $\infty 2$ & 30 & 37.9 & 30 & 232 & 160 & 334 & 167 & 308 & 1.1 & 53.0 & 3.0 \\
\hline $10 / 15 / 92$ & 1415 & 34.3 & 0.0 & 13.1 & 11.4 & 288 & 3.0 & 36.1 & 3.1 & 61.6 & 30 & 30.3 & 3.3 & 250 & 100 & Da & 133 & 30.3 & 1.1 & 48.2 & 3.0 \\
\hline $10 / 15 / 92$ & 1430 & 33.8 & 0.0 & 11.7 & 11.3 & 28.3 & 3.0 & 36.5 & 3.1 & 61.4 & 30 & 320 & 30 & 248 & 160 & 324 & 138 & 203 & 1.1 & 54.7 & 3.0 \\
\hline $10 / 15 \times 92$ & 1445 & 31.4 & 0.0 & 13.4 & 18.1 & 28.7 & 3.0 & 35.6 & 3.2 & 620 & 3.0 & 39.7 & 3.0 & 240 & 178 & $\Delta .1$ & 140 & 303 & 1.1 & 51.0 & 3.0 \\
\hline $0 / 15 / 92$ & 1500 & 35.3 & 0.0 & 10.0 & 13.5 & 28.6 & 3.0 & 34.6 & 3.3 & 024 & 30 & 390 & 30 & 21,4 & 210 & 332 & 182 & 250 & 1.1 & 54.0 & 3.0 \\
\hline $0 / 1502$ & 1515 & 30.7 & 0.0 & 10.6 & 120 & 28.4 & 3.0 & 34.2 & 5.6 & 201 & 3.0 & $\mathbf{0 . 4}$ & 3.1 & 201 & 186 & 308 & 140 & 250 & 1.4 & 5.5 & 3.6 \\
\hline $10 / 15 / 92$ & 1530 & 30.9 & 0.0 & 11.0 & 13.4 & 282 & 3.0 & 320 & 6.7 & 61.6 & 29 & 41.4 & 3.1 & 208 & 17.8 & 532 & 142 & 273 & 12 & 53.1 & 3.1 \\
\hline$\alpha 15 \times 92$ & 1545 & 0.1 & 30.1 & 10.9 & 123 & 30.3 & 32 & 34.7 & 4.9 & $\infty 2$ & 20 & 40.5 & 3.1 & $\overline{282}$ & 185 & 398 & 143 & 31.0 & 13 & 55.5 & 3.0 \\
\hline$\alpha 1592$ & 1600 & 0.1 & 28.9 & 11.8 & 14.6 & 293 & 3.1 & 323 & 13.1 & 61.8 & 20 & 41.1 & 3.7 & 20.1 & 200 & 385 & 165 & $\mathbf{2 6 5}$ & 12 & 55.2 & 3.0 \\
\hline $10 / 1592$ & 1615 & 0.1 & 27.5 & 126 & 188 & 28.7 & 6.7 & 226 & 12.3 & 50.2 & 45 & 412 & 4.1 & 21.7 & 21.1 & 36.1 & 168 & 230 & 12 & 529 & 3.0 \\
\hline
\end{tabular}




\begin{tabular}{|c|c|c|c|c|c|c|c|c|c|c|c|c|c|c|c|c|c|c|c|c|c|}
\hline Dops & Time & $\begin{array}{l}\text { Water } \\
\text { Flow } \\
\text { gom }\end{array}$ & $\begin{array}{l}\text { Limo } \\
\text { Flow } \\
\text { gpm }\end{array}$ & $\begin{array}{l}\text { Sik } \\
\text { Op } \\
\%\end{array}$ & $\begin{array}{l}B \\
O_{p} \\
\%\end{array}$ & & $\begin{array}{l}1 \text { sple } \\
\mathrm{mn}\end{array}$ & & $\begin{array}{l}2 \\
\text { eple } \\
\mathrm{mn}\end{array}$ & & $\mathrm{mn}$ & KW & $m n$ & & & KW & $m$ & KW & $m n$ & & $m n$ \\
\hline $10 / 1592$ & $\begin{array}{l}1650 \\
1645\end{array}$ & $\begin{array}{l}0.1 \\
0.1\end{array}$ & $\begin{array}{l}27.7 \\
27.7\end{array}$ & $\begin{array}{l}11.5 \\
14.3\end{array}$ & $\begin{array}{l}19.1 \\
232\end{array}$ & 28.6 & $\begin{array}{l}3.1 \\
3.3\end{array}$ & $\begin{array}{l}24.4 \\
21.7\end{array}$ & 129 & $\$ 02$ & 58 & 41.1 & 7.1 & 2016 & 17.0 & 0.1 & 105 & 31.3 & 0.5 & 53.8 & 3.0 \\
\hline $\begin{array}{l}10 / 1502 \\
10 / 1502\end{array}$ & $\begin{array}{l}1646 \\
1700\end{array}$ & $\begin{array}{l}0.1 \\
0.1\end{array}$ & $\begin{array}{l}27.7 \\
26.8\end{array}$ & $\begin{array}{l}14.3 \\
15.1\end{array}$ & $\begin{array}{l}25.2 \\
47.8\end{array}$ & $\begin{array}{l}28.3 \\
28.5\end{array}$ & $\begin{array}{l}3.3 \\
3.1\end{array}$ & $\begin{array}{l}21.7 \\
20.8\end{array}$ & $\begin{array}{l}13.6 \\
14.7\end{array}$ & $\begin{array}{l}54.4 \\
533\end{array}$ & $\begin{array}{l}6.8 \\
64\end{array}$ & $\begin{array}{l}\$ 0.6 \\
33.3\end{array}$ & $\begin{array}{l}7.4 \\
8.6\end{array}$ & $\begin{array}{l}20.7 \\
200\end{array}$ & $\begin{array}{l}10.8 \\
18.3\end{array}$ & $\begin{array}{l}37.7 \\
324\end{array}$ & $\begin{array}{l}161 \\
148\end{array}$ & 20.2 & 1.2 & $\begin{array}{l}51.1 \\
48.8\end{array}$ & 3.0 \\
\hline & $\begin{array}{l}1700 \\
1715\end{array}$ & 0.1 & $\mathbf{2 8 . 6}$ & 13.6 & 20.5 & 28.3 & 3.5 & $\mathbf{2 0 . 4}$ & 16.8 & $\begin{array}{l}50.3 \\
50.8\end{array}$ & 99 & 38.7 & 9.3 & 21.4 & 20.8 & 37.7 & 163 & 27.9 & $\begin{array}{l}1.1 \\
1.2\end{array}$ & $\begin{array}{l}48.8 \\
49.3\end{array}$ & $\begin{array}{l}3.0 \\
3.0\end{array}$ \\
\hline $10 / 15 / 92$ & 1750 & 02 & 30.0 & 14.7 & 222 & 28.4 & 3.0 & 226 & 122 & 57.4 & 62 & 30.9 & 4.9 & $\mathbf{2 0 . 7}$ & 20.4 & 378 & 168 & 29.1 & 1.1 & 49.8 & 30 \\
\hline $10 / 15 / 92$ & 1745 & 02 & 29.7 & 127 & 19.4 & 28.5 & 3.8 & 24.0 & 126 & 58.5 & 5.4 & 40.4 & 38 & 222 & 21.0 & 328 & 150 & 27.5 & 9.9 & 522 & 3.0 \\
\hline $10 / 1502$ & 1800 & 02 & 30.0 & 124 & 18.3 & 28.6 & 3.0 & 25.0 & 11.5 & 57.6 & 75 & $\mathbf{4 0 . 6}$ & 6.0 & 220 & 234 & 378 & 220 & 27.7 & 1.7 & 512 & 3.0 \\
\hline $10 / 45 / 92$ & 1815 & 02 & 29.7 & 126 & 17.4 & 28.5 & 3.1 & 24.4 & 10.6 & 58.0 & 55 & 40.8 & 5.0 & 223 & 24.0 & 382 & 16.6 & 20.0 & 1.1 & 50.3 & 3.0 \\
\hline $10 / 15 / 92$ & 1800 & 0.2 & 29.9 & 128 & 17.1 & 28.4 & 3.0 & 24.8 & 10.3 & 57.1 & 3.9 & 40.8 & 42 & 200 & 20.7 & $3 \mathbf{2}$ & 167 & 27.0 & 12 & 492 & 30 \\
\hline $10 / 15 / 92$ & 1845 & 0.1 & 30.2 & 13.3 & 16.7 & 28.4 & 3.1 & 24.4 & 10.6 & 57.7 & 5.7 & 41.1 & 38 & 23.0 & 20.0 & 335 & 14.5 & 26.7 & 9.9 & 44.5 & 30 \\
\hline $10 / 15 / 92$ & 1800 & 0.1 & 29.6 & 15.1 & 17.7 & 28.4 & 3.1 & 24.1 & 11.0 & 54.8 & 4.1 & 40.8 & 4.4 & 222 & 20.7 & 30.5 & 16.1 & 288 & 1.0 & 51.5 & 30 \\
\hline $10 / 1592$ & 1915 & 0.1 & 29.5 & 16.4 & 17.4 & 28.4 & 3.0 & 242 & 11.4 & 56.3 & 65 & $\mathbf{0 . 5}$ & 4.3 & 280 & 178 & 386 & 143 & 20.6 & 10.0 & 51.8 & 3.1 \\
\hline $10 / 15 / 92$ & 1800 & 0.1 & 20.4 & 17.3 & 18.3 & 28.5 & 3.0 & 23.4 & 14.0 & 548 & 53 & 40.0 & 10.3 & 218 & 325 & 35.7 & 20.4 & 200 & 1.3 & 49.8 & 30 \\
\hline $10 / 15 / 92$ & 1945 & 0.1 & 28.7 & 14.5 & 16.8 & 28.5 & 3.0 & 24.1 & 13.3 & 558 & 4.7 & 40.1 & 3.8 & 238 & 236 & $3 a 3$ & $\mathbf{0 0 0}$ & 282 & 12 & 50.2 & 30 \\
\hline $10 / 1592$ & 2000 & 0.1 & 28.4 & 16.4 & 18.4 & 28.7 & 3.1 & 23.4 & 12.6 & 54.3 & 48 & $\$ .2$ & 4.7 & 208 & 17.4 & 321 & 126 & 27.6 & 12 & 50.5 & 35 \\
\hline $10 / 15 / 92$ & 2015 & 0.1 & 28.7 & 17.7 & 19.5 & 278 & 42 & 220 & 13.5 & 621 & 43 & 39.7 & 4.5 & 20.1 & 20.9 & 30.4 & 143 & 28.7 & 1.0 & 502 & 3.0 \\
\hline $10 / 15 / 92$ & 2000 & 0.1 & $\mathbf{2 8 . 0}$ & 16.8 & 20.3 & $\mathbf{2 8 . 7}$ & 3.0 & 20.8 & 14.3 & 50.6 & 76 & $\mathbf{3 8 . 4}$ & 5.4 & $\mathbf{2 8 6}$ & 14.4 & 332 & 18.6 & 27.0 & 12 & 48.5 & 3.7 \\
\hline $10 / 15 / 92$ & 2045 & 0.1 & 27.7 & 16.2 & 25.4 & 28.2 & 3.1 & 20.7 & 14.1 & 54.0 & 69 & 37.7 & 11.7 & 27.1 & 150 & $\mathbf{3 6 5}$ & 16.3 & 27.7 & 1.1 & 43.7 & 3.0 \\
\hline $10 / 15 / 92$ & 2100 & 0.1 & $\mathbf{2 8 . 0}$ & 15.4 & 24.0 & 28.3 & 3.0 & 20.7 & 14.5 & 51.1 & 79 & $\mathbf{3 8 . 4}$ & 63 & 204 & 178 & 33.1 & 17.0 & 282 & 1.0 & 47.1 & 3.0 \\
\hline $10 / 1592$ & 2115 & 0.1 & 27.2 & 14.4 & 26.5 & 28.7 & 3.1 & 21.6 & 14.6 & 54.1 & 49 & 38.6 & 56 & 234 & 172 & 308 & 142 & 24.4 & 1.1 & 46.5 & 32 \\
\hline $10 / 1502$ & 2130 & 0.1 & 26.9 & 15.6 & 28.5 & 28.6 & 3.2 & $\mathbf{2 0 . 7}$ & 14.7 & 49.0 & 52 & 37.6 & 4.2 & 27.9 & 21.3 & 38.6 & 163 & 2030 & 1.1 & 43.6 & 3.0 \\
\hline $10 / 15 P 2$ & 2145 & 0.1 & $\mathbf{2 6 . 6}$ & 17.0 & 30.1 & 28.8 & 4.6 & $\mathbf{2 0 . 3}$ & 13.6 & 53.2 & 38 & 37.6 & 3.7 & 20.6 & 136 & 324 & 18.1 & 20.0 & 1.0 & 45.8 & 3.0 \\
\hline $10 / 1592$ & 2200 & 0.1 & 26.6 & 16.3 & 31.0 & 28.1 & 3.0 & 21.1 & 13.8 & $\mathbf{5 0} .0$ & 4.4 & 36.9 & 4.1 & 29.1 & 168 & 37.4 & 14.0 & 20.3 & 1.0 & 45.8 & 32 \\
\hline $10 / 1592$ & 2215 & 0.1 & 27.4 & 17.0 & 31.4 & 28.7 & 3.0 & 21.2 & 12.3 & 54.3 & 6.4 & 37.9 & 3.4 & 27.7 & 13.1 & 30.0 & 128 & 31.2 & 1.1 & 478 & 3.0 \\
\hline $10 / 15 / 92$ & 2200 & 0.0 & 26.6 & 18.7 & 34.3 & 0.0 & $?$ & 0.0 & $?$ & 0.0 & $?$ & 0.0 & $?$ & 00 & $?$ & 00 & $?$ & 0.0 & 10.0 & 0.0 & $?$ \\
\hline $10 / 15 / 92$ & 2245 & 0.0 & 25.6 & 21.8 & 41.9 & 27.5 & 12.0 & 19.7 & 15.0 & 51.7 & 75 & 33.9 & 9.5 & 278 & 160 & 300 & 17.7 & 31.0 & 8.6 & 46.4 & 3.0 \\
\hline $10 / 15 / 92$ & $\mathbf{2 0 0}$ & 0.0 & 28.6 & 18.0 & 28.2 & $\mathbf{2 9 . 0}$ & 3.0 & 20.9 & 13.3 & 520 & 7.0 & 38.8 & 56 & $\mathbf{2 3 0}$ & 183 & 40.8 & 135 & 30.4 & 9.4 & 47.7 & 3.1 \\
\hline $10 / 15 / 92$ & 2315 & 0.0 & 29.4 & 15.2 & 24.9 & $\mathbf{2 8 . 6}$ & 3.9 & 19.9 & 12.0 & 54.7 & $5 A$ & 308 & 4.6 & 286 & 150 & 330 & 135 & 285 & 1.1 & 49.3 & 3.0 \\
\hline $10 / 15 / 92$ & 2300 & 0.0 & 29.4 & 13.9 & 20.2 & 28.4 & 3.0 & 21.0 & 13.8 & 54.6 & 42 & 41.1 & 43 & 278 & 122 & 306 & 140 & 283 & 1.0 & 50.6 & 3.0 \\
\hline $2 / 15 / 92$ & 2045 & 0.0 & 28.0 & 13.8 & 24.0 & $\mathbf{2 8 . 4}$ & 3.1 & 21.7 & 112 & 54.6 & 4.1 & 41.1 & 52 & 26.7 & 20.8 & 370 & 18.7 & 272 & 1.4 & 50.4 & 3.6 \\
\hline $10 / 1692$ & 0 & 0.0 & 28.5 & 14.9 & 24.1 & 28.6 & 3.1 & 222 & 127 & 57.7 & 3.0 & 39.8 & 7.7 & 284 & 16.6 & 208 & 11.4 & 30.3 & 1.1 & 40.1 & 3.0 \\
\hline $10 / 1692$ & 15 & 0.0 & 29.8 & 14.5 & 23.2 & 27.8 & 3.1 & 220 & 128 & 57.5 & 3.1 & $\$ 0.8$ & 38 & 312 & 118 & 4.6 & 138 & 30.9 & 8.4 & 474 & 3.0 \\
\hline $10 / 1692$ & 30 & 0.0 & 328 & 10.7 & 15.7 & 28.2 & 3.0 & $\overline{28.6}$ & 11.3 & 521 & 3.1 & 43.3 & 3.0 & 285 & 12.4 & 30.7 & 121 & 282 & 9.7 & 48.1 & 3.0 \\
\hline $0 / 1692$ & 46 & 00 & 27.6 & 126 & 228 & 28.7 & 3.1 & 21.9 & 12.5 & 54.7 & 52 & 41.0 & 9.5 & 238 & 172 & 402 & 11.3 & 30.4 & 8.0 & 51.1 & 3.1 \\
\hline$\alpha / 1692$ & 100 & 0.0 & $\mathbf{2 6 . 8}$ & 16.8 & 30.1 & 28.7 & 3.0 & 25.0 & 11.1 & 54.8 & 29 & $\mathbf{3 8 . 9}$ & 3.0 & $x \times 6$ & 14.1 & 40.7 & 115 & 30.9 & 1.0 & 472 & 3.0 \\
\hline 21692 & 115 & 0.0 & 28.4 & 172 & 27.4 & 29.3 & 6.4 & $\mathbf{2 3 . 7}$ & 10.8 & 66.5 & 30 & 39.7 & 3.0 & 20.4 & 18.4 & 443 & 127 & 33.3 & 1.0 & 48.7 & 3.0 \\
\hline $0 / 1692$ & 130 & 0.0 & $\mathbf{2 8 . 4}$ & 13.8 & 25.7 & 27.9 & 3.0 & 20.1 & 10.4 & 56.0 & 3.6 & 412 & 4.3 & 288 & 162 & 330 & 18.0 & 28.6 & 1.1 & 49.3 & 3.0 \\
\hline $10 / 1692$ & 145 & 0.0 & 26.4 & 10.7 & 21.2 & 27.7 & 3.0 & 228 & 10.5 & 52.4 & 30 & 423 & 62 & 30.7 & 190 & 33.0 & 152 & 28.7 & 1.1 & 48.1 & 3.2 \\
\hline $10 / 1692$ & 200 & 0.0 & 26.5 & 11.0 & 16.8 & 27.7 & 3.0 & 227 & 11.4 & 53.9 & 3.5 & 41.9 & 3.7 & 20.2 & 14.6 & 365 & 12.7 & 27.5 & 9.7 & 46.3 & 3.0 \\
\hline $10 / 1692$ & 215 & 0.0 & 26.5 & 9.3 & 14.3 & 28.5 & 3.0 & 26.1 & 8.5 & 51.6 & 3.7 & 43.3 & 3.4 & 29.6 & 175 & 378 & 11.7 & 30.7 & 1.1 & 527 & 32 \\
\hline $1016 / 92$ & 200 & 0.0 & 25.0 & 9.4 & 17.0 & 27.6 & 3.0 & 21.1 & 13.4 & 55.6 & 30 & 422 & 6.0 & 30.7 & 11.3 & 365 & 136 & 283 & 1.1 & 516 & 3.0 \\
\hline $10 / 16 / 92$ & 245 & 0.0 & 28.6 & 7.6 & 124 & 282 & 3.0 & 27.3 & 7.6 & 53.1 & 20 & 428 & 3.0 & 30.1 & 17.0 & 35.3 & 144 & 27.6 & 1.0 & 531 & 3.0 \\
\hline $10 / 16.92$ & 300 & 0.0 & 25.7 & 7.5 & 15.6 & $\mathbf{2 8 . 1}$ & 3.0 & 23.1 & 15.1 & 526 & 3.6 & 427 & 43 & 322 & 133 & 36.7 & 132 & 290 & 1.1 & 525 & 3.4 \\
\hline 101692 & 315 & 0.0 & 26.6 & 9.0 & 16.2 & 27.8 & 3.0 & 21.8 & 11.8 & 529 & 40 & 420 & 6.0 & $\mathbf{3 3 0}$ & 0.7 & 303 & 109 & 328 & 1.1 & 515 & 3.0 \\
\hline $10 / 1692$ & 300 & 0.0 & 26.2 & 10.4 & 16.7 & 27.6 & 3.0 & 20.8 & 125 & 55.6 & 60 & 41.1 & 52 & $\mathbf{3 a 5}$ & 124 & $\boldsymbol{\omega 0 . 0}$ & 102 & 33.2 & 10.0 & 507 & 4.4 \\
\hline $10 / 1692$ & 345 & 0.0 & 25.0 & 11.0 & 17.3 & 27.6 & 3.0 & 222 & 9.9 & 54.2 & 5.1 & 424 & 4.9 & 313 & 88 & 330 & 143 & 29.6 & 1.1 & 517 & 3.0 \\
\hline$\alpha / 16 / 92$ & 400 & 0.0 & 26.1 & 13.4 & 17.4 & 27.5 & 3.0 & 21.3 & 122 & 562 & 20 & 421 & 5.3 & 31.5 & 148 & 33.4 & 13.7 & 30.3 & 1.1 & 485 & 3.0 \\
\hline$\alpha / 16 / 92$ & 415 & 0.0 & 25.0 & 8.6 & 13.9 & 27.6 & 3.0 & 24.3 & 10.5 & 53.5 & 3.4 & 43.1 & 3.0 & 30.1 & 13.4 & 324 & 11.7 & 320 & 1.1 & 45 & 3.0 \\
\hline $10 / 1692$ & 430 & 0.0 & 25.6 & 120 & 14.1 & $\mathbf{2 8 . 6}$ & 3.0 & 232 & 10.5 & 527 & 20 & 4.0 & 3.6 & 336 & 11.0 & 323 & 123 & 320 & 1.1 & 48.5 & 3.3 \\
\hline $10 / 1692$ & 446 & 0.0 & 25.6 & 10.1 & 13.9 & 28.1 & 3.0 & 21.7 & 12.3 & 49.6 & 50 & 42.1 & 4.7 & 300 & 10.0 & 375 & 110 & 324 & 1.0 & 515 & 3.2 \\
\hline $10 / 1692$ & 500 & 0.0 & 292 & 8.9 & 125 & 29.5 & 3.0 & 25.5 & 10.3 & 53.4 & 29 & 431 & 30 & 332 & 08 & 398 & 112 & 332 & 1.0 & 514 & 3.0 \\
\hline $10 / 1692$ & 515 & 0.0 & 25.0 & 9.0 & 15.6 & 27.8 & 3.0 & 226 & 10.5 & 54.7 & 3.7 & 418 & 3.0 & 341 & 93 & 321 & 10.1 & 327 & 10.0 & 510 & 3.0 \\
\hline $10 / 1692$ & 500 & 0.0 & 25.2 & 10.9 & 178 & 27.7 & 32 & 21.5 & 10.9 & 568 & 29 & $\mathbf{\$ 0 . 6}$ & 3.0 & 34.7 & 10.8 & 300 & 84 & 350 & 1.1 & 50.8 & 3.1 \\
\hline $10 / 1692$ & 546 & 0.0 & 25.1 & 11.4 & 20.4 & 27.7 & 3.0 & 226 & 9.9 & 56.0 & 33 & 39.6 & 4.5 & 34.0 & 10.0 & 41.6 & 7.6 & 35.7 & 6.8 & 44.6 & 3.0 \\
\hline $10 / 1692$ & $\infty \infty$ & 0.0 & 25.2 & 37.3 & 30.8 & $\mathbf{2 8 . 3}$ & 4.0 & 20.7 & 122 & 522 & 33 & 362 & 30 & 30.1 & 104 & 412 & 10.6 & 300 & 9.8 & 45.8 & 3.4 \\
\hline $10 / 1692$ & 615 & 0.0 & 25.3 & 17.8 & 27.6 & 28.6 & 3.1 & 223 & 13.4 & $\boldsymbol{\infty} 1$ & 20 & 330 & 3.8 & 27.8 & 168 & $\$ 27$ & 10.3 & 34.0 & 8.6 & 46.3 & 4.5 \\
\hline $10 / 1692$ & 60 & 0.0 & 25.6 & 16.9 & 27.8 & 28.2 & 3.0 & 223 & 10.4 & 53.6 & 3.1 & 37.1 & 32 & 318 & 104 & 24 & 11.4 & 358 & 8.8 & 472 & 3.6 \\
\hline $0 / 1692$ & 645 & 0.0 & 25.6 & 18.0 & 329 & 28.7 & 3.0 & 221 & 13.3 & 512 & 29 & 38.1 & 3.1 & 344 & 09 & 122 & 92 & 336 & 8.4 & 46.0 & 30 \\
\hline $0 / 1692$ & 700 & 0.0 & 29.6 & 127 & 20.4 & 272 & 3.0 & 23.4 & 112 & 57.0 & 33 & 402 & 3.0 & 330 & 110 & 427 & 95 & 350 & 9.6 & 48.4 & 30 \\
\hline$\alpha 1692$ & 715 & 0.0 & 27.8 & 19.2 & 31.6 & 27.4 & 4.8 & 20.0 & 14.5 & 51.5 & 29 & 358 & 53 & 32.7 & 85 & 48.7 & 102 & 387 & 9.6 & 43.6 & 3.0 \\
\hline $0 / 1692$ & 700 & 0.0 & 28.0 & 14.8 & 262 & 27.5 & 3.0 & 24.0 & 14.6 & 535 & 3.4 & 37.3 & 3.1 & 340 & 119 & 400 & 93 & 37.5 & 82 & 47.9 & 32 \\
\hline $0 / 1692$ & 745 & 22.9 & 0.0 & 126 & 23.9 & 272 & 3.0 & 19.5 & 19.7 & 455 & 120 & 36.7 & 124 & 337 & 108 & 458 & 03 & 30.8 & 9.5 & 48.4 & 38 \\
\hline$\alpha / 1692$ & 800 & 26.7 & 0.0 & 16.3 & 26.1 & 27.2 & 3.4 & 15.8 & 16.2 & 123 & 95 & 35.7 & 88 & sa. & 138 & $\mathbf{4 6 5}$ & 11.3 & 381 & 88 & 48.3 & 3.0 \\
\hline V1692 & 815 & 27.8 & 0.0 & 11.4 & 17.8 & 27.6 & 3.0 & 20.0 & 15.5 & 53.1 & 40 & 41.8 & 3.7 & 288 & 127 & 493 & 11.7 & $\mathbf{3 9 . 0}$ & 9.0 & 522 & 3.3 \\
\hline $0 / 1692$ & 850 & 28.2 & 0.0 & 128 & 17.4 & $\mathbf{2 8 . 0}$ & 3.0 & 19.6 & 11.3 & 47.1 & 40 & 30.8 & 4.3 & 28.1 & 134 & 432 & 124 & 348 & 1.0 & 50.0 & 33 \\
\hline
\end{tabular}




\begin{tabular}{|c|c|c|c|c|c|c|c|c|c|c|c|c|c|c|c|c|c|c|c|c|c|}
\hline Dets & Time & $\begin{array}{l}\text { Water } \\
\text { Flow } \\
\text { gpm }\end{array}$ & $\begin{array}{l}\text { Lime } \\
\text { Flow } \\
\text { gpm }\end{array}$ & $\begin{array}{l}\text { S.k } \\
O_{0} \\
\%\end{array}$ & $\begin{array}{r}8 \\
\alpha_{0}^{\circ} \\
\end{array}$ & & $\mathrm{mn}$ & & $\begin{array}{l}2 \\
\text { sple } \\
\mathrm{mn}\end{array}$ & $K W$ & $\mathrm{mn}$ & & $m$ & & & $\mathrm{KW}$ & & KW & $\mathrm{mn}$ & & $m n$ \\
\hline 101692 & 846 & 28.3 & 0.0 & 14.0 & 18.8 & 28.1 & 3.0 & 18,9 & 15.3 & 45.6 & 8.1 & 40.8 & 10.3 & 200 & 138 & 48.0 & 128 & 35.0 & 8.9 & 48.8 & 3.0 \\
\hline 104692 & 900 & 28.2 & 0.0 & 126 & 18.3 & 28.1 & 3.0 & 17.4 & 16.5 & 43.7 & 76 & 388 & 8.6 & 278 & 14.4 & 427 & 113 & 34.1 & 1.0 & 50.2 & 3.4 \\
\hline $\begin{array}{c}101692 \\
10 / 1692\end{array}$ & $\begin{array}{l}915 \\
900\end{array}$ & $\begin{array}{l}27.9 \\
31.4\end{array}$ & $\begin{array}{l}0.0 \\
0.0\end{array}$ & $\begin{array}{l}14.2 \\
14.4\end{array}$ & $\begin{array}{l}19.4 \\
15.4\end{array}$ & $\begin{array}{l}\mathbf{2 8 . 2} \\
\mathbf{2 0 . 4}\end{array}$ & $\begin{array}{l}3.0 \\
3.0\end{array}$ & $\begin{array}{l}17.4 \\
21.6\end{array}$ & $\begin{array}{l}14.3 \\
11.2\end{array}$ & $\begin{array}{l}43.2 \\
51.8\end{array}$ & $\begin{array}{l}60 \\
32\end{array}$ & $\begin{array}{l}37.7 \\
45.3\end{array}$ & $\begin{array}{l}8.6 \\
6.4\end{array}$ & $\begin{array}{l}30.7 \\
30.1\end{array}$ & $\begin{array}{l}142 \\
120\end{array}$ & $\begin{array}{l}443 \\
433\end{array}$ & $\begin{array}{l}11.6 \\
10.7\end{array}$ & $\begin{array}{l}362 \\
348\end{array}$ & $\begin{array}{l}9.4 \\
8.1\end{array}$ & $\begin{array}{l}49.4 \\
522\end{array}$ & $\begin{array}{l}3.0 \\
3.2\end{array}$ \\
\hline 101692 & 945 & 35.0 & 0.0 & 123 & 128 & 30.2 & 3.0 & 28.6 & 5.3 & 53.5 & 20 & $\mathbf{4 3 . 0}$ & 3.6 & 298 & 10.4 & 43.4 & 108 & 343 & 12 & 51.7 & 3.0 \\
\hline 101692 & 1000 & 35.4 & 0.0 & 10.3 & 10.9 & 29.7 & 3.0 & 31.5 & 3.8 & 56.0 & 29 & 41.0 & 3.0 & 205 & 145 & 30.1 & 0.7 & 328 & 9.2 & 488 & 3.0 \\
\hline 101692 & 1015 & 35.6 & 0.0 & 11.3 & 11.4 & 29.3 & 3.0 & 33.2 & 3.1 & 55.8 & 30 & 39.8 & 3.0 & 30.4 & 142 & 493 & 112 & 358 & 8.9 & 48.6 & 3.3 \\
\hline $10 / 16 / 22$ & 1000 & 36.0 & 0.0 & 123 & 10.3 & 29.0 & 3.0 & 30.3 & 3.6 & 86.8 & 20 & 39.4 & 3.0 & 31.1 & 155 & 430 & 100 & 33.1 & 8.8 & 49.7 & 3.0 \\
\hline 10/16/92 & 1045 & 35.6 & 0.0 & 11.1 & 10.4 & 29.2 & 3.0 & 33.7 & 3.1 & 565 & 30 & 39.4 & 3.0 & 288 & 11.1 & 309 & 8.1 & 34.1 & 1.1 & 51.8 & 3.0 \\
\hline 1016:92 & 1100 & 36.5 & 0.0 & 8.7 & 11.4 & 20.1 & 3.0 & 33.8 & 32 & 57.8 & 20 & 328 & 3.0 & 20.0 & 17.5 & 425 & 123 & 30.7 & 1.0 & 50.4 & 3.1 \\
\hline $10 / 16 / 92$ & 1115 & 36.4 & 0.0 & 15.9 & 10.6 & 28.9 & 3.0 & 33.7 & 3.4 & 56.9 & 3.0 & 30.3 & 3.0 & 27.6 & 108 & 128 & 95 & 34.1 & 9.5 & 53.3 & 3.0 \\
\hline $10 / 1692$ & 1130 & 35.8 & 0.0 & 15.7 & 10.8 & 29.1 & 3.0 & 33.7 & 3.2 & 572 & 29 & 30.0 & 3.0 & 202 & 138 & 420 & 120 & 338 & 8.4 & 51.0 & 3.0 \\
\hline $10 / 1692$ & 1145 & 35.7 & 0.0 & 8.6 & 11.3 & 28.8 & 3.0 & 34.5 & 3.1 & 58.1 & 20 & 303 & 3.0 & 278 & 17. & 408 & 115 & 31.0 & 8.6 & 53.4 & 3.1 \\
\hline 101692 & 1200 & 35.5 & 0.0 & 10.3 & 11.8 & $\mathbf{2 8 . 6}$ & 3.0 & 34.6 & 3.5 & 5.5 & 20 & 302 & 3.0 & 27.6 & 160 & 40.6 & 134 & 324 & 9.9 & 538 & 3.0 \\
\hline 101692 & 1216 & 35.4 & 0.0 & 123 & 11.8 & 28.7 & 3.0 & 34.5 & 3.2 & 623 & 3.1 & 30.1 & 3.0 & 27.7 & 141 & 41.7 & 13.0 & $3 a 7$ & 12 & 523 & 3.0 \\
\hline 1692 & 1200 & 35.4 & 0.0 & 129 & 11.8 & 28.6 & 3.0 & 34.8 & 3.1 & 895 & 29 & 38.9 & 3.0 & 27.4 & 11.7 & 412 & 85 & 30.1 & 9.9 & 53.4 & 3.0 \\
\hline $10 / 1892$ & 1245 & 35.2 & 0.0 & 127 & 11.0 & 28.2 & 3.0 & 34.5 & 3.6 & 58.8 & 30 & 38.5 & 3.0 & 27.9 & 16.7 & 123 & 108 & 33.9 & 8.4 & 526 & 3.0 \\
\hline 101692 & 1300 & 35.4 & 0.0 & 122 & 11.3 & 28.3 & 3.0 & 36.1 & 3.1 & 58.7 & 20 & 40.1 & 30 & 288 & 124 & 422 & 11.4 & 30.4 & 9.7 & 527 & 3.0 \\
\hline 16.92 & 1315 & 35.1 & 0.0 & 12.7 & 14.7 & 28.4 & 3.0 & 35.1 & 3.1 & 58.8 & 3.0 & 39.8 & 3.0 & 20.7 & 18.7 & $\$ .4$ & 120 & 323 & 1.1 & 50.3 & 3.0 \\
\hline $10 / 1692$ & 1350 & 35.6 & 0.0 & 13.2 & 11.6 & 28.2 & 3.0 & 33.4 & 3.1 & 50.6 & 30 & 37.4 & 3.0 & 27.2 & 15.7 & 41.4 & 14.6 & 320 & 1.1 & 510 & 3.0 \\
\hline $2 / 692$ & 1345 & 33.5 & 0.0 & 128 & 10.8 & 28.2 & 3.0 & 34.7 & 3.1 & 58.6 & 30 & 30.7 & 3.0 & 27.0 & 165 & 43.7 & 123 & 330 & 8.5 & 51.8 & 3.0 \\
\hline $0 / 1692$ & 1400 & 324 & 0.0 & 11.0 & 120 & 28.2 & 3.0 & 34.6 & 3.1 & 588 & 3.0 & 384 & 31 & 282 & 14.4 & 420 & 123 & 34.0 & 9.5 & 18.3 & 3.0 \\
\hline 1692 & 1415 & 326 & 0.0 & 10.9 & 13.2 & 28.5 & 3.1 & 34.8 & 3.2 & 582 & 3.0 & 30.8 & 3.0 & 234 & 166 & 41.7 & 119 & 33.8 & 1.0 & 466 & 3.0 \\
\hline 1692 & 1450 & 326 & 0.0 & 11.9 & 120 & 28.1 & 3.1 & 34.7 & 3.7 & 58.0 & 20 & 40.3 & 3.0 & 288 & 17.5 & 42.7 & 122 & 30.7 & 1.0 & 452 & 3.0 \\
\hline Q/1692 & 1445 & 29.6 & 0.0 & 16.1 & 29.0 & 28.9 & 3.2 & 27.3 & 11.1 & 527 & 70 & 38.1 & 83 & 220 & 142 & 438 & 104 & 34.1 & 8.7 & 470 & 30 \\
\hline 1692 & 1500 & 0.0 & 33.0 & 112 & 128 & 28.9 & 3.2 & 30.2 & 12.4 & 57.6 & 20 & 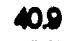 & 3.0 & 27.6 & 16.1 & 436 & 125 & 34.1 & 8.7 & 473 & 3.0 \\
\hline 92 & 1515 & 0.0 & 35.0 & 10.8 & 11.5 & 28.3 & 3.0 & 318 & 6.0 & 57.9 & 29 & $\$ .5$ & 30 & 296 & 173 & 424 & 121 & 34.5 & 1.1 & 521 & 3.0 \\
\hline $10 / 1692$ & 1530 & 0.0 & 35.2 & 10.7 & 11.5 & 28.0 & 3.0 & 34.5 & 3.8 & 58.2 & 29 & $\$ .1$ & 30 & 288 & 165 & 433 & 11.9 & 34.0 & 9.4 & 524 & 30 \\
\hline $10 / 1692$ & 1545 & 0.0 & 35.4 & 10.2 & 10.6 & $\mathbf{2 8 . 3}$ & 3.1 & 34.8 & 3.1 & 58.7 & 29 & 30.5 & 3.0 & 27.8 & 31.0 & 48.6 & 132 & 38.7 & 9.3 & 517 & 3.0 \\
\hline $2 / 1692$ & 1600 & 0.0 & 34.4 & 120 & 126 & 29.4 & 3.2 & 30.7 & 3.1 & 58.7 & 30 & 38.4 & 3.0 & 27.3 & 180 & 4.7 & 128 & 33.4 & 1.1 & 518 & 3.0 \\
\hline $10 / 1692$ & 1615 & 0.0 & 34.3 & 120 & 123 & 29.4 & 3.1 & 30.7 & 3.6 & 58.7 & 30 & 39.4 & 3.0 & 228 & 34.4 & $\mathbf{0 . 4}$ & 21.7 & 29.4 & 1.5 & 512 & 3.0 \\
\hline $10 / 1692$ & 1600 & 0.0 & 33.9 & 122 & 11.5 & 28.7 & 3.0 & 33.5 & 4.9 & 57.7 & 30 & 40.3 & 3.0 & 24.4 & 16.7 & 39.4 & 17.6 & 27.1 & 1.1 & 510 & 3.0 \\
\hline $10 / 1692$ & 1645 & 0.0 & 34.1 & 123 & 11.4 & $\mathbf{2 8 . 7}$ & 3.1 & 325 & 4.6 & 58.3 & 29 & 40.0 & 3.0 & 24.2 & 220 & 30.7 & 17.5 & 2.5 & 10 & 515 & 3.0 \\
\hline $10 / 16 / 92$ & 1700 & 0.0 & 33.3 & 11.9 & 11.3 & $\mathbf{2 9 . 0}$ & 3.1 & 31.8 & 5.8 & 58.0 & 29 & 40.3 & 3.0 & 231 & 184 & 0.7 & 13.3 & 30.3 & 1.1 & 47.7 & 3.0 \\
\hline $10 / 692$ & 1715 & 0.0 & 33.2 & 121 & 120 & $\mathbf{2 8 . 9}$ & 3.1 & 31.2 & 6.2 & 58.1 & 29 & 40.2 & 3.0 & 250 & 20.4 & 40.6 & 123 & $\mathbf{3 0 . 5}$ & 9.5 & 49.1 & 3.0 \\
\hline $10 / 1692$ & 1750 & 0.0 & 31.7 & 11.8 & 122 & 28.9 & 3.0 & 27.9 & 10.6 & 58.3 & 3.0 & 399 & 3.0 & 256 & 16.7 & $\boldsymbol{4 0 . 0}$ & 14.3 & $\mathbf{3 0 . 6}$ & 12 & 50.8 & 3.0 \\
\hline $10 / 1692$ & 1745 & 0.0 & 312 & 3.9 & 13.3 & 28.2 & 3.1 & $\mathbf{2 6 . 6}$ & 8.9 & 58.6 & 30 & 39.8 & 3.0 & 250 & 162 & 40.4 & 13.1 & 30.4 & 8.8 & 60.4 & 3.0 \\
\hline 91692 & 1800 & 0.0 & 31.5 & 3.6 & 13.0 & 28.2 & 3.2 & $\mathbf{2 B . 1}$ & 10.8 & 57.6 & 3.6 & $\$ 0.1$ & 3.1 & 256 & 17.1 & 41.5 & 11.8 & 31.7 & 9.1 & 49.0 & 3.0 \\
\hline V1692 & 1815 & 0.0 & 33.0 & 126 & 123 & 23.2 & 3.0 & 28.5 & 8.5 & 57.1 & 3.6 & 30.7 & 3.0 & 248 & 14.6 & 39.7 & 16.0 & 298 & 10 & 47.9 & 3.0 \\
\hline 692 & 1850 & 0.0 & $\mathbf{3 0 . 8}$ & 13.9 & 15.1 & 27.9 & 3.0 & $\mathbf{2 5 . 0}$ & 128 & 552 & 50 & 30.4 & 3.0 & 248 & 160 & 37.1 & 138 & 29.1 & 1.1 & 48.6 & 3.0 \\
\hline 692 & 1845 & 0.0 & 31.1 & 14.0 & 16.6 & 27.6 & 3.0 & 222 & 129 & 52 & 5.3 & 30.7 & 75 & 208 & 180 & $\operatorname{san}$ & 13.7 & 28.5 & $\boldsymbol{O A}$ & 45.2 & 3.0 \\
\hline $10 / 1692$ & 1800 & 0.0 & 30.5 & 148 & 18.7 & 27.6 & 3.0 & 20.8 & 13.0 & 51.1 & $5 A$ & 30.4 & 63 & 203 & 165 & $\mathbf{3 5 0}$ & 130 & 28.7 & 12 & 48.1 & 6.8 \\
\hline 1692 & 1915 & 0.0 & 30.1 & 16.6 & 19.5 & 27.6 & 3.0 & 19.7 & 14.1 & 50.2 & 6.1 & 38.0 & 50 & 20.7 & 21.8 & 348 & 142 & 27.1 & 12 & 46.5 & 31 \\
\hline $10 / 1692$ & 1900 & 0.0 & 29.6 & 15.7 & 19.8 & 27.5 & 3.0 & 19.1 & 168 & 47.8 & 6.4 & 37,9 & 3.8 & 244 & 103 & 35.1 & 18.1 & 27.9 & 14 & 41.7 & 3.0 \\
\hline $10 / 1692$ & 1945 & 0.0 & $\mathbf{2 8 . 8}$ & 16.3 & 20.3 & 27.4 & 3.0 & 19.0 & 15.2 & 47.7 & 6.5 & 37.7 & 8.1 & 256 & 17.4 & 363 & 162 & 27.6 & 10 & 46.2 & 19 \\
\hline $10 / 1692$ & 2000 & 0.0 & $\mathbf{2 8 . 2}$ & 15.8 & 224 & 27.4 & 3.1 & 17.6 & 142 & 46.4 & 72 & 37.5 & 43 & 288 & 164 & 33.1 & 17.3 & $\mathbf{2 0 . 7}$ & 11 & 45.4 & 3.0 \\
\hline 101692 & 2015 & 0.0 & 27.9 & 18.0 & 25.6 & 27.4 & 3.0 & 16.8 & 17.9 & 45.7 & 92 & 38.1 & 6.9 & 280 & 145 & 350 & 168 & 26.7 & 11 & 44.3 & 3.1 \\
\hline $10 / 1692$ & 2000 & 0.0 & 27.2 & 18.5 & 29.0 & 27.2 & 4.8 & 16.8 & 18.1 & 45.3 & 10.8 & 36.7 & 9.1 & 265 & 138 & 380 & 14.1 & $\mathbf{2 B 3}$ & 13 & 42.0 & 4.4 \\
\hline 692 & 2045 & 0.0 & 26.8 & 18.6 & 320 & 27.6 & 3.0 & 15.9 & 15.8 & 33.6 & 8.4 & 33.6 & 80 & 240 & 15.1 & 348 & 14.0 & 263 & 10 & 40.2 & 30 \\
\hline 1692 & 2100 & 0.0 & 27.7 & 18.6 & 321 & 27.7 & 3.0 & 16.5 & 17.9 & 43.5 & 9.5 & 36.7 & 4.4 & 234 & 17.1 & $\mathbf{3 3 . 0}$ & 142 & 273 & 98 & 41.5 & 3.1 \\
\hline 1692 & 2115 & 0.0 & 27.5 & 17.9 & 34.4 & 27.7 & 3.1 & 16.2 & 18.4 & 37.0 & 95 & 34.3 & 11.1 & 266 & 17.4 & 358 & 138 & 278 & 12 & 36.5 & 3.0 \\
\hline 1692 & 2130 & 18.5 & 0.0 & 21.9 & 38.7 & 27.7 & 3.7 & 16.3 & 223 & 423 & 125 & 34.0 & 128 & 204 & 208 & $3 a 4$ & 14.4 & 285 & 12 & 43.7 & 4.3 \\
\hline $10 / 16 / 92$ & 2145 & 29.3 & 0.0 & 13.1 & 21.7 & 29.8 & 3.1 & 16.0 & 15.3 & 39.1 & 82 & 33.6 & 9.6 & 233 & 14.3 & 358 & 14.7 & 28.1 & 1.1 & 44.2 & 3.1 \\
\hline 101692 & 2200 & 30.2 & 0.0 & 120 & 18.3 & 31.1 & 3.1 & 16.9 & 148 & 41.7 & 6.1 & 38.7 & 80 & 278 & 160 & 24 & 150 & 27.7 & 12 & 44.0 & 30 \\
\hline 1016.92 & 2215 & 27.0 & 0.0 & 126 & 21.1 & $\mathbf{2 9 . 0}$ & 4.1 & 14.3 & 20.5 & 321 & 10.4 & 31.2 & o. & 26.7 & 138 & 360 & 140 & & 12 & 45.8 & 4.0 \\
\hline 101692 & 22000 & 25.9 & 0.0 & 14.0 & 26.8 & 28.6 & 6.2 & 129 & $\mathbf{2 0 . 6}$ & 31.0 & 11.8 & 28.1 & 113 & 233 & 163 & 358 & 146 & 287 & 1.1 & 45.4 & 35 \\
\hline 101692 & 2245 & 27. & 0.0 & 5.3 & 268 & $\mathbf{2 8 . 4}$ & 42 & 15.0 & 18.4 & 32.0 & 79 & 33.0 & 8.0 & 250 & 16.4 & 347 & 146 & 275 & 12 & 47.1 & 30 \\
\hline 10169 & 2000 & 27 & 0.0 & 3.9 & 233 & 28.8 & 5.6 & 15.1 & 16.5 & 38.0 & 74 & 33.6 & 8.0 & $\mathbf{2 8 8}$ & 1388 & 36.7 & 128 & 286 & 8.5 & 40 & 13.0 \\
\hline 104692 & 2215 & 27.9 & 0.0 & 4.6 & 19.4 & 30.2 & 3.0 & 14.7 & 18.0 & 36.0 & 8.5 & 31.0 & 10.5 & 2016 & 19.1 & 303 & 140 & 280 & 1.1 & 47 & 53 \\
\hline 1692 & 2000 & 28.3 & 0.0 & 3.0 & 17.7 & 30.6 & 3.0 & 16.9 & 14.1 & 37.8 & 74 & 33.2 & 88 & 224 & 133 & 35.0 & 127 & 282 & 1.0 & $\infty .0$ & 10.3 \\
\hline 1692 & 2345 & $\mathbf{2 8 . 5}$ & 0.0 & 11.2 & 16.6 & 30.1 & 3.0 & 20.8 & 11.8 & 47.1 & 5.6 & 30.3 & 7.6 & 230 & 130 & 30.1 & 138 & 27.0 & 1.3 & 51.4 & 4.8 \\
\hline v17/2R & 0 & 28.5 & 0.0 & 121 & 14.3 & $\mathbf{3 0 . 9}$ & 3.2 & 18.0 & 14.6 & 427 & 6.0 & 323 & 9.3 & 268 & 160 & 30.3 & 16.7 & 242 & 12 & 48.2 & 8.5 \\
\hline & 15 & 31.6 & 0.0 & 9.6 & 11.1 & 30.9 & 3.2 & 18.0 & 14.6 & 427 & 6.0 & 323 & 9 & 258 & 160 & 303 & 16.7 & 242 & 12 & 48.2 & 8.5 \\
\hline & 30 & 27.8 & 0.0 & 10.7 & 11.6 & 30.9 & 3.2 & 18.0 & 14.6 & 427 & 60 & 323 & 9.3 & 208 & 160 & 300 & 16.7 & 242 & 12 & 48.2 & 8.5 \\
\hline 01792 & 45 & 28.3 & 0.0 & 10.7 & 13.8 & 30.9 & 3.2 & 18.0 & 14.6 & 427 & 6.0 & 323 & 9.3 & 258 & 180 & 303 & 16.7 & 242 & 12 & 48.2 & 8.5 \\
\hline
\end{tabular}




\begin{tabular}{|c|c|c|c|c|c|c|c|c|c|c|c|c|c|c|c|c|c|c|c|c|c|}
\hline Data & Time & $\begin{array}{l}\text { Water } \\
\text { Flow } \\
\text { gpm }\end{array}$ & $\begin{array}{l}\text { Lime } \\
\text { Flow } \\
\text { ggm }\end{array}$ & $\begin{array}{l}S_{*} \\
Q_{0} \\
\% \\
\end{array}$ & $\begin{array}{l}B \\
\rho_{0}^{\circ} \\
\%\end{array}$ & & eple & KW & spew & & & $\mathrm{KW}$ & & & & KW & & & & KW & \\
\hline $10 / 1702$ & 100 & 0.0 & 31.4 & 10.7 & 14.0 & 30.9 & 3.2 & 18.0 & 14.6 & 427 & 60 & $\begin{array}{l}323 \\
303\end{array}$ & 9.3 & 208 & 160 & 303 & 167 & 242 & 12 & 48.2 & 85 \\
\hline $10 / 17 / 92$ & 115 & 0.0 & 34.8 & 0.1 & 10.3 & 30.9 & 3.2 & 18.0 & 14.6 & 427 & 60 & & 93 & 20 & 180 & 303 & 167 & 242 & 12 & 482 & 8.5 \\
\hline $\begin{array}{l}10 / 17 / 92 \\
10 \times 17 / 92\end{array}$ & $\begin{array}{l}130 \\
146\end{array}$ & $\begin{array}{l}0.0 \\
0.0\end{array}$ & $\begin{array}{l}34.6 \\
33.9\end{array}$ & $\begin{array}{l}121 \\
16.6\end{array}$ & $\begin{array}{l}9.5 \\
9.7\end{array}$ & $\begin{array}{l}30.9 \\
30.8\end{array}$ & $\begin{array}{l}3.2 \\
3.2\end{array}$ & $\begin{array}{l}18.0 \\
18.0\end{array}$ & $\begin{array}{l}14.6 \\
14.6\end{array}$ & $\begin{array}{l}427 \\
427\end{array}$ & $\begin{array}{l}60 \\
60\end{array}$ & $\begin{array}{l}323 \\
323\end{array}$ & $\begin{array}{l}93 \\
93\end{array}$ & $\begin{array}{l}258 \\
208\end{array}$ & $\begin{array}{l}160 \\
160\end{array}$ & $\begin{array}{l}303 \\
303\end{array}$ & $\begin{array}{l}16.7 \\
16.7\end{array}$ & $\begin{array}{l}242 \\
242\end{array}$ & $\begin{array}{l}12 \\
12\end{array}$ & $\begin{array}{l}48.2 \\
482\end{array}$ & $\begin{array}{l}8.5 \\
8.5\end{array}$ \\
\hline $10 / 17 / 92$ & 200 & 0.0 & 36.1 & 120 & 0.3 & 30.9 & 3.2 & 18.0 & 14.6 & 427 & 6.0 & 323 & 9.3 & 258 & 160 & 303 & 167 & 242 & 12 & 482 & 8.6 \\
\hline $\begin{array}{l}10 / 1792 \\
1017992\end{array}$ & $\begin{array}{l}215 \\
250\end{array}$ & $\begin{array}{l}0.0 \\
0.0\end{array}$ & $\begin{array}{l}38.5 \\
34.9\end{array}$ & $\begin{array}{l}9.4 \\
9.5\end{array}$ & $\begin{array}{r}11.2 \\
8.6\end{array}$ & $\begin{array}{l}30.9 \\
30.9\end{array}$ & $\begin{array}{l}3.2 \\
3.2\end{array}$ & $\begin{array}{l}18.0 \\
18.0\end{array}$ & $\begin{array}{l}14.6 \\
14.6\end{array}$ & $\begin{array}{l}427 \\
427\end{array}$ & $\begin{array}{l}6.0 \\
6.0\end{array}$ & $\begin{array}{l}323 \\
323\end{array}$ & $\begin{array}{l}9.3 \\
9.3\end{array}$ & $\begin{array}{l}288 \\
288\end{array}$ & $\begin{array}{l}160 \\
160\end{array}$ & $\begin{array}{l}303 \\
303\end{array}$ & $\begin{array}{l}167 \\
167\end{array}$ & $\begin{array}{l}242 \\
242\end{array}$ & $\begin{array}{l}1.2 \\
12\end{array}$ & $\begin{array}{l}48.2 \\
48.2\end{array}$ & $\begin{array}{l}8.5 \\
8.5\end{array}$ \\
\hline $10 / 1702$ & 245 & 0.0 & 35.4 & 7.0 & 8.5 & 30.8 & 3.2 & 18.0 & 14.6 & 127 & 6.0 & 323 & 0.3 & 258 & 160 & 303 & 16.7 & 242 & 12 & 48.2 & 8.5 \\
\hline $10 / 17 / 82$ & 300 & 0.0 & 34.9 & 9.4 & 88 & 30.9 & 3.2 & 18.0 & 14.6 & 427 & 60 & 323 & 93 & 280 & 160 & 30.3 & 16.7 & 242 & 12 & 482 & 86 \\
\hline $10 / 17 / 22$ & 315 & 0.0 & 34.1 & 8.7 & 8.7 & 30.9 & 3.2 & 18.0 & 14.6 & 427 & 6.0 & 323 & 9.3 & 208 & 160 & 30.3 & 167 & 24.2 & 12 & 482 & 8.5 \\
\hline $10 / 7 / 92$ & 350 & 0.0 & 34.0 & 11.0 & 9.1 & 30.9 & 3.2 & 18.0 & 14.8 & 427 & 60 & 323 & 9.3 & 288 & 160 & 303 & 16.7 & 242 & 12 & 482 & 85 \\
\hline $10 / 17 / 92$ & 345 & 0.0 & 30.5 & 8.2 & 92 & 30.9 & 3.2 & 18.0 & 14.6 & 427 & 60 & 323 & 93 & $\mathbf{2 8 B}$ & 160 & 303 & 167 & 242 & 12 & 482 & 8.5 \\
\hline $10 / 7 / 92$ & 400 & 0.0 & 33.6 & 8.1 & 9.4 & 30.9 & 3.2 & 18.0 & 14.6 & 427 & 6.0 & 323 & 0.3 & 208 & 16.0 & 303 & 167 & 242 & 12 & 42.2 & Q.8 \\
\hline $10 / 17 / 82$ & 415 & 0.0 & 38.8 & 9.4 & 11.8 & 30.9 & 3.2 & 18.0 & 14.6 & 427 & 6.0 & 323 & 9.3 & 280 & 160 & 30.3 & 16.7 & 242 & 12 & 48.2 & 85 \\
\hline 1017792 & 450 & 0.0 & 33.5 & 8.7 & 8.8 & 30.0 & 3.2 & 18.0 & 14.6 & 427 & 6.0 & 323 & 0.3 & 208 & 160 & 30.3 & 16.7 & 242 & 12 & 48.2 & as \\
\hline $10 / 17 \theta 2$ & 445 & 0.0 & 327 & 8.6 & 98 & 30.8 & 3.2 & 18.0 & 14.6 & 427 & 6.0 & 323 & 0.3 & 208 & 16.0 & 50.3 & 16.7 & 242 & 12 & 48.2 & 8.6 \\
\hline $10 / 7 / 92$ & 500 & 0.0 & 32.8 & 8.0 & 0.1 & 30.8 & 3.2 & 18.0 & 14.6 & 427 & 60 & 323 & 98 & $\overline{258}$ & 16.0 & 30.3 & 167 & 242 & 12 & 48.2 & 8.5 \\
\hline $10 / 17 / 92$ & 515 & 0.0 & 324 & 6.4 & 9.4 & 30.0 & 3.2 & 18.0 & 14.6 & 427 & 6.0 & 323 & 0.3 & 368 & 160 & $\cos$ & 167 & 242 & 12 & 48.2 & 85 \\
\hline $10 / 17 / 92$ & 550 & 0.0 & 320 & 6.8 & 9.6 & 30.9 & 3.2 & 18.0 & 14.6 & 427 & 60 & 323 & 9.3 & 208 & 160 & $\operatorname{sos}$ & 16.7 & 242 & 1.2 & 48.2 & 85 \\
\hline $10 / 17 / 22$ & 645 & 0.0 & 324 & 7.2 & $9 B$ & 30.8 & 3.2 & 18.0 & 14.6 & 427 & 6.0 & 323 & 9.3 & 200 & 160 & 303 & 167 & 242 & 12 & 482 & 8.5 \\
\hline $10 / 17 / 92$ & $\infty$ & 0.0 & 327 & 6.7 & 92 & 30.9 & 3.2 & 18.0 & 14.6 & 427 & 6.0 & 323 & 9.3 & 208 & 160 & 503 & 167 & 242 & 12 & 48.2 & 8.5 \\
\hline $10 / 17 / 92$ & 615 & 0.0 & 33.5 & 7.2 & 8.9 & 30.8 & 3.2 & 18.0 & 14.6 & 427 & 6.0 & 323 & 93 & 208 & 160 & 303 & 167 & 242 & 1.2 & 48.2 & 8.5 \\
\hline $10 / 17 / 92$ & 650 & 0.0 & 321 & 7.0 & 10.6 & 30.9 & 3.2 & 18.0 & 14.6 & 427 & 60 & 323 & 93 & 208 & 16.0 & 30.3 & 16.7 & 242 & 12 & 48.2 & 8.5 \\
\hline $10 / 1782$ & 645 & 0.0 & 31.7 & 8.0 & 120 & 30.9 & 3.2 & 18.0 & 14.6 & 427 & 6.0 & 323 & 93 & 208 & 160 & 303 & 16.7 & 242 & 12 & 482 & 8.5 \\
\hline $10 / 1792$ & 700 & 0.0 & 323 & 7.9 & 11.4 & 30.8 & 32 & 18.0 & 14.9 & 427 & 60 & 323 & 93 & $\mathbf{2 5 8}$ & 160 & 303 & 16.7 & 242 & 12 & 482 & 8.6 \\
\hline $10 / 17 / 92$ & 715 & 0.0 & 31.5 & 8.7 & 13.3 & 30.9 & 3.2 & 18.0 & 14.6 & 427 & 60 & 323 & 9.3 & 258 & 160 & 303 & 16.7 & 242 & 12 & 48.2 & 8.6 \\
\hline $10 / 47 / 92$ & 730 & 0.0 & 30.7 & 120 & 19.0 & 30.0 & 3.2 & 18.0 & 14.6 & 427 & 6.0 & 323 & 9.3 & 208 & 16.0 & 30.3 & 167 & 242 & 12 & 48.2 & 8.5 \\
\hline $0 / 17 / 92$ & 745 & 0.0 & 34.0 & 8.9 & 13.5 & 30.8 & 3.2 & 18.0 & 14.6 & 427 & 6.0 & 323 & 93 & 208 & 160 & 30.3 & 167 & 242 & 12 & 482 & 8.5 \\
\hline $10 / 17 / 92$ & 800 & 0.0 & 328 & 10.3 & 15.1 & 0.0 & $?$ & 0.0 & $?$ & 0.0 & $?$ & 00 & $?$ & 0.0 & $?$ & 00 & $?$ & 00 & 10.0 & 00 & $?$ \\
\hline $2 / 1792$ & 815 & 0.0 & 329 & 10.5 & 13.5 & 28.1 & 3.1 & 21.0 & 8.0 & 528 & 3.4 & 41.5 & 3.1 & 321 & 98 & 428 & 8.7 & 37.8 & 0.3 & 50.0 & 6.7 \\
\hline $10 / 17 / 92$ & 850 & 0.0 & 34.4 & 10.8 & 16.8 & 27.7 & 3.0 & 18.2 & 15.8 & 53.7 & 30 & 40.4 & 4.0 & 327 & 11.8 & $\mathbf{4 3 5}$ & 79 & $\$ 0.3$ & 9.5 & 50.0 & 5.8 \\
\hline 10/17/92 & 845 & 0.0 & 321 & 11.0 & 17.6 & 27.4 & 3.3 & 19.0 & 14.2 & 529 & 3.1 & 41.1 & 33 & 396 & 10.4 & 51.4 & 38 & 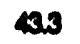 & 8.0 & 48.9 & 5.0 \\
\hline 1017792 & 900 & 0.0 & 31.5 & 13.1 & 22.7 & 26.6 & 5.1 & 16.7 & 16.5 & 54.3 & 35 & 30.2 & 3.0 & 326 & 18.1 & 486 & 10.0 & 37.1 & 9.6 & 48.4 & 10.0 \\
\hline 10/17/92 & 915 & 0.0 & 30.2 & 126 & 51.0 & 27.0 & 3.0 & 17.7 & 17.2 & 53.7 & 3.3 & 303 & 5.4 & 346 & 123 & 468 & 88 & $3 a 0$ & 8.5 & 46.8 & 3.4 \\
\hline $10 / 17 / 92$ & 900 & 0.0 & 31.1 & 125 & 24.2 & 27.3 & 3.0 & 17.4 & 17.5 & 54.1 & 3.7 & 39.6 & 30 & 34.4 & 83 & 465 & 83 & 30.0 & 9.5 & 49.9 & 6.9 \\
\hline 101792 & 945 & 0.0 & 324 & 126 & 18.0 & 27.7 & 3.1 & 19.8 & 11.6 & 520 & 3.4 & 4.9 & 35 & 328 & 0.1 & 40 & 78 & 320 & 92 & 47.1 & 3.1 \\
\hline $0 / 1792$ & 1000 & 0.0 & 33.1 & 10.4 & 17.8 & 27.1 & 3.0 & 20.2 & 10.5 & 53.0 & 32 & 41.8 & 3.6 & 354 & 95 & 182 & 8.8 & 303 & 8.2 & 4.7 & 3.6 \\
\hline $0 / 1792$ & 1015 & $0 . n$ & 30.2 & 34.8 & 227 & 27.2 & 3.6 & 15.6 & 16.5 & 40.6 & 75 & $\mathbf{3 8 . 3}$ & 93 & 343 & 10.8 & 42 & 88 & 37.6 & 9.5 & 40.4 & 7.4 \\
\hline $10 / 17 / 92$ & 1000 & 0.0 & 320 & 13.7 & 19.8 & 27.3 & 3.0 & 17.6 & 13.9 & 48.4 & 6.1 & 424 & 3.7 & 320 & 93 & 4.0 & OS & 32.4 & 8.6 & 50.7 & 7.0 \\
\hline 10/17/92 & 1045 & 0.0 & 28.5 & 15.4 & 27.0 & 27.1 & 3.1 & 16.0 & 16.9 & 46.2 & 73 & 33.8 & 7.4 & 38.7 & 10.1 & 426 & 18.1 & 37.0 & 7.7 & 47.5 & 8.8 \\
\hline $10 / 17 / 92$ & 1100 & 0.0 & 25.2 & 17.8 & 31.4 & 27.1 & 3.3 & 13.3 & 19.5 & 28.1 & 123 & 20.3 & 10.7 & 320 & 150 & 120 & 10.6 & 36.6 & 8.9 & 38.4 & 5.0 \\
\hline 10/17/92 & 1115 & 29.3 & 00 & 15.0 & 27.1 & 28.3 & 4.3 & 14.3 & 18.8 & 38.0 & 0.1 & 34.0 & 0.6 & 396 & 11.4 & 482 & 102 & 372 & 8.5 & 471 & 9.8 \\
\hline $10 / 17 / 92$ & 1130 & 28.0 & 0.0 & 16.2 & 24.4 & $\mathbf{2 8 . 9}$ & 4.1 & 13.5 & 17.8 & 33.6 & 10.8 & 326 & 98 & 358 & 00 & 486 & 05 & 37.6 & 8.5 & 40 & 5.3 \\
\hline 10/17/92 & 1145 & 29.3 & 0.0 & 14.7 & 23.6 & 20.2 & 4.7 & 14.6 & 18.6 & 36.0 & 9.0 & 34.1 & 10.6 & 50 & 9.7 & 182 & 84 & 321 & 8.0 & 46.6 & 6.9 \\
\hline $10 / 17 / 92$ & 1200 & 29.8 & 0.0 & 15.4 & 23.8 & 30.1 & 3.5 & 14.3 & 17.7 & 36.0 & 8.8 & 33.6 & 10.4 & 30.7 & 122 & 48 & 88 & 30.2 & 0.2 & 477 & 42 \\
\hline $10 / 17 / 92$ & 1215 & 28.7 & 0.0 & 17.7 & 28.1 & 28.5 & 4.7 & 15.3 & 16.3 & 36.0 & 99 & 38.1 & 7.9 & $\mathbf{3 0 0}$ & 98 & 46.1 & 10.1 & 30.1 & 0.4 & 46.9 & 7.5 \\
\hline $10 / 17 / 92$ & 12200 & 320 & 0.0 & 13.8 & 19.0 & 23.1 & 5.0 & 123 & 13.8 & $\mathbf{2 8 . 8}$ & 6.9 & $\mathbf{2 6 . 0}$ & 88 & 34.4 & 10.0 & 44 & 95 & 38.6 & 8.0 & 46.9 & 3.9 \\
\hline $10 / 17 / 92$ & 1245 & 34.1 & 0.0 & 13.0 & 10.4 & 30,3 & 3.0 & 20.4 & 10.1 & 48.7 & 48 & 44.7 & 42 & 30 & 94 & 480 & 14.4 & 300 & 8.9 & 517 & 4.2 \\
\hline $10 / 1792$ & 1300 & 35.1 & 00 & 118 & 168 & 30.4 & 3.0 & 20.8 & 12.1 & 47.9 & 5.5 & 41.7 & 6.4 & 362 & 8.7 & 47.7 & 83 & 413 & 7.8 & 526 & 5.0 \\
\hline $10 / 17 / 92$ & 1316 & 34.8 & 0.0 & 11.8 & 15.8 & 30.1 & 3.0 & २3.5 & 10.6 & 48.8 & 53 & 432 & 4.6 & 37.4 & $\boldsymbol{O B}$ & 423 & 08 & 405 & 8.1 & 50.8 & 5.5 \\
\hline$\alpha 17 / 92$ & 1300 & 35.3 & 0.0 & 9.7 & 129 & 30.6 & 3.0 & 25.5 & 7.7 & 512 & 38 & 48.7 & 3.4 & 350 & 120 & 188 & 09 & 32.4 & 0.1 & 51.1 & 5.7 \\
\hline $10 / 7702$ & 1345 & 35.3 & 0.0 & 10.2 & 13.7 & 20.0 & 3.0 & 29.3 & 8.1 & 54.2 & 20 & 422 & 32 & 372 & 120 & 488 & 05 & 323 & 8.7 & 522 & 4.0 \\
\hline $0 / 1792$ & 1400 & 35.1 & 0.0 & 120 & 15.4 & $\mathbf{2 9 . 4}$ & 3.0 & 21.4 & 12.1 & 44.6 & 76 & 39.9 & 8.1 & 360 & 93 & 40 & OA & 393 & 1.0 & 49.8 & 4.9 \\
\hline $0 / 17 / 22$ & 1416 & 36.0 & 0.0 & 10.6 & 17.0 & 20.2 & 3.0 & 221 & 11.5 & 48.5 & 79 & 39.9 & 65 & $\mathbf{3 3 0}$ & 79 & 42 & 104 & 324 & 9.0 & 450 & 5.4 \\
\hline$Q / 17 / 92$ & 1430 & 35.1 & 0.0 & 11.8 & 16.2 & $\mathbf{2 9 . 4}$ & 3.0 & 21.7 & 14.2 & 44.4 & 6.1 & 30.4 & 7.0 & 360 & 103 & 128 & 82 & 303 & 0.0 & 4.6 & 62 \\
\hline $0 / 17 P 2$ & 1445 & 35.1 & 0.0 & 11.6 & 19.5 & 202 & 3.0 & 20.6 & 14.5 & 45.6 & 54 & 30.3 & 83 & 303 & 0.7 & 47 & 118 & 320 & 8.9 & 50.1 & 4.6 \\
\hline $0 / 1792$ & 1500 & 35.3 & 0.0 & 13.1 & 17.4 & 29.2 & 3.0 & 21.6 & 162 & 453 & 69 & 30.8 & 79 & 34.4 & 168 & 124 & 126 & 373 & 0.6 & 47.4 & 3.7 \\
\hline $0 / 17 / 92$ & 1515 & 35,3 & 00 & 10.1 & 18.0 & $\mathbf{2 9 . 4}$ & 3.0 & 20.1 & 132 & 428 & 8.1 & 37.6 & 9.4 & 348 & 94 & 42.7 & 09 & 320 & 0.4 & 473 & 0.4 \\
\hline O/1702 & 1530 & 35.2 & 0.0 & 121 & 18.6 & 20.1 & 3.0 & 221 & 124 & 45.0 & 72 & 32.7 & 9.7 & 350 & 128 & 42.7 & 00 & 37.7 & 1.3 & 49.9 & 6.1 \\
\hline V17/22 & 1545 & 34.6 & 0.0 & 125 & 21.4 & 288 & 3.1 & $\overline{23.0}$ & 8.5 & 47.5 & 48 & 30.1 & 60 & 308 & 103 & 160 & 5.4 & 30.7 & 6.7 & 48.4 & 55 \\
\hline V17N2 & 1600 & 30.7 & 0.0 & 11,1 & 224 & 28.4 & 3.0 & 178 & 16.4 & 330 & 95 & 321 & 113 & 340 & 128 & $\$ 27$ & 0.7 & 37.1 & 1.0 & 47.3 & 42 \\
\hline $0 / 1792$ & 1616 & 30.5 & 0.0 & 112 & 21.8 & 28.1 & 3.0 & 18.2 & 123 & 33.7 & 72 & 33.7 & 86 & 348 & 143 & 403 & 92 & 302 & 8.4 & 47.1 & 4.0 \\
\hline Q/17P2 & 1650 & 30.6 & 0.0 & 13.8 & 25.7 & 28.9 & 3.4 & 16.3 & 172 & 33.5 & 10.8 & 28.6 & 10.7 & 327 & 140 & 442 & 82 & 37.7 & 9.6 & 46.6 & 4.6 \\
\hline & 1645 & 30.5 & 0.0 & 126 & 20.7 & $\mathbf{2 8 . 4}$ & 3.1 & 18.7 & 123 & 38.4 & 7.7 & 326 & 8.7 & 381 & 90 & $\$ 02$ & 48 & 420 & 5.9 & 46.8 & 120 \\
\hline$\alpha / 1782$ & 1700 & 30.5 & 0.0 & 10.7 & 20.4 & 28.1 & 3.0 & 221 & 15.2 & 44.6 & 98 & 372 & 102 & 325 & 120 & 123 & 10.4 & 38.7 & 9.9 & 49.7 & 6.8 \\
\hline
\end{tabular}




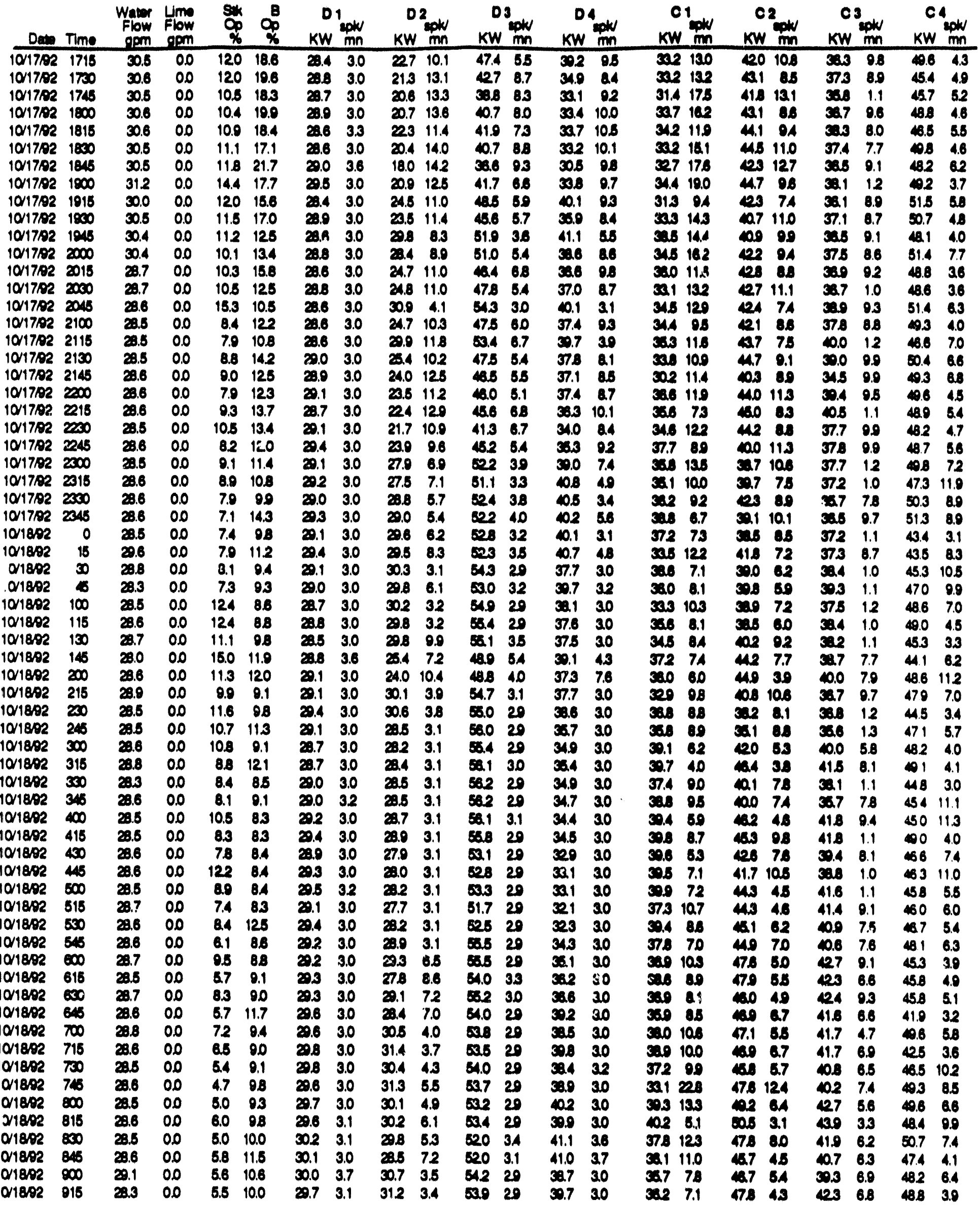




\section{Des Time}

Water Ume Flow Flow open

10/1892 90 1014802 045 28.50 .0

$10 / 18921000$ 29.6 $29.6 \quad 0.0$

28.60 .0

28.60 .0

28.70 .0

28.90 .0

1018021045

1018921100

10/1802 1115

$\begin{array}{lll}0.0 & 6.9 & 10.0\end{array}$

$\begin{array}{llll}27.9 & 0.0 & 8.9 & 12.9\end{array}$

$\begin{array}{llll}28.5 & 0.0 & 7.2 & 10.7\end{array}$

101802 1146

$101802 \quad 1200$

$10 / 1802 \quad 1218$

10/1802 1220

10/1802 1248

$10 / 1802 \quad 1300$

10/1892 1315

1018021500

$10 / 1802 \quad 1346$

$10 / 1802 \quad 1400$

1011892 1415

$10 / 18021450$

10/1892 1446

$1011892 \quad 1500$

1018P2 1515

101802 1580

$10 / 1892 \quad 1545$

1011802 1600

101802 1816

$10 / 18921600$

$0 / 18921646$

$.01802 \quad 1700$

10/1802 1715

$10 / 1892 \quad 1730$

$10 / 1892 \quad 1745$

$10 / 1892 \quad 1800$

$101802 \quad 1815$

$101892 \quad 1860$

10/18P2 1845

$10 / 1802 \quad 1000$

10/1892 1915

$10 / 1802 \quad 1800$

$10 / 1802 \quad 1945$

$10 / 18022000$

$10 / 18922015$

101892 2000

10/1892 2046

10118922100

10189222115

$10 / 18922130$

101892 2145

101802 2200

$10 / 18922215$

10/1892 2200

$101802 \quad 2245$

$10 / 8902200$

101892 2315

10/1892 2500

10/1802 2015

1018920

- orever 16

0/1002 30

$10 / 1002 \quad 16$

10/1092 100

10/1802 115

10/1002 130 $\begin{array}{llll}28.6 & 0.0 & 6.8 & 11.0\end{array}$

$\begin{array}{lllll}28.6 & 0.0 & 8.8 & 10.3\end{array}$

$\begin{array}{lllll}28.6 & 0.0 & 5.5 & 11.1\end{array}$

$\begin{array}{llll}28.4 & 0.0 & 5.4 & 9.8\end{array}$

$\begin{array}{llll}\mathbf{2 8 . 0} & 0.0 & 5.6 & 11.4\end{array}$

$\begin{array}{llll}\mathbf{2 8 . 6} & 0.0 & 6.1 & 10.0 \\ \mathbf{2 8 . 6} & 0.0 & 6.1 & 10.7\end{array}$

28.50 .0

28.70 .0

28.60 .0

$\begin{array}{ll}6.3 & 11.7\end{array}$

$6.0 \quad 9.6$

$\begin{array}{llll}28.8 & 0.0 & 7.8 & 10.3\end{array}$

$\begin{array}{lllll}28.8 & 0.0 & 7.5 & 10.2\end{array}$

$\begin{array}{lllll}28.5 & 0.0 & 6.1 & 10.0\end{array}$

$28.7 \quad 0.0$

$\begin{array}{ll}6.2 & 10.3\end{array}$

28.60 .0

28.60 .0

$8.0 \quad 11.6$

$0.0 \quad 31.0$

6.810 .6

$0.0 \quad 33.8$

$\begin{array}{ll}7.8 .1 \\ 70 & 11.5\end{array}$

$\begin{array}{lllll}0.0 & 34.1 & 18.8 & 14.5\end{array}$

$\begin{array}{llll}0.0 & 34.1 & 129 & 11.3\end{array}$

$\begin{array}{llll}0.0 & 35.1 & 10.3 & 122\end{array}$

$\begin{array}{llll}0.0 & 320 & 8.0 & 121\end{array}$

$0.0 \quad 31.6$

$0.0 \quad 31.2$

$0.0 \quad 31.7$

9.610 .6

0.010 .8

$0.0 \quad 31.8$

0.0320

$\begin{array}{lll}8.8 & 11.1\end{array}$

$8.5 \quad 132$

0.0320

$0.0 \quad 31.7$

$0.0 \quad 31.8$

$0.0 \quad 320$

281148

$11.8 \quad 16.6$

$\begin{array}{llll}0.0 & 20.9 & 13.5 & 210\end{array}$

$\begin{array}{lllll}0.0 & 30.3 & 13.4 & 21.0\end{array}$

$0.0 \quad 328$

$0.0 \quad 28.4$

12317.1

13.018 .8

$\begin{array}{llll}297 & 00 & 128 & 17.1\end{array}$

$\begin{array}{llll}0.0 & 20.0 & 14.1 & 184\end{array}$

$\begin{array}{lllll}0.0 & 20.4 & 13.0 & 12.1\end{array}$

on $30.4 \quad 10.3 \quad 128$

$\begin{array}{lllll}0.0 & 28.7 & 11.8 & 14.1\end{array}$

$\begin{array}{llll}0.0 & 20.2 & 13.4 & 17.6\end{array}$

$\begin{array}{llll}0.0 & 20.9 & 13.3 & 16.3\end{array}$

$\begin{array}{lllll}0.0 & 20.7 & 13.1 & 20.3\end{array}$

$\begin{array}{lllll}0.0 & 29.4 & 12 . & 16.8\end{array}$

$\begin{array}{lllll}0.0 & 31.7 & 12.6 & 16.1\end{array}$

$\begin{array}{lllll}0.0 & 30.7 & 13.6 & 17.2\end{array}$

$\begin{array}{llll}0.0 & 30.6 & 14.4 & 16.8\end{array}$

$\begin{array}{llll}0.0 & 320 & 13.4 & 124\end{array}$

$\begin{array}{lllll}0.0 & 31.4 & 120 & 11.4\end{array}$

$\begin{array}{lllll}0.0 & 34.8 & 13.6 & 98\end{array}$

$\begin{array}{llll}0.0 & 29.7 & 10.8 & 11.1\end{array}$

$\begin{array}{llll}0.0 & 30.5 & 0.1 & 10.2\end{array}$

$0.0 \quad 20.7$

$0.0 \quad 30.2$
$13.5 \quad 15.3$

D1 1 D $\mathrm{KW}$ mn

soly D3 $\begin{array}{llll}30.2 & 6.4 & 525 & 3.1\end{array}$

$\begin{array}{llllll}30.1 & 3.0 & 30.2 & 6.4 & 520 & 3.1 \\ 30.4 & 3.0 & 30.4 & 4.8 & \mathbf{3 0} & 29\end{array}$

$\begin{array}{llllll}30.5 & 3.1 & 30.8 & 5.5 & 50.3 & 29 \\ 20.9 & 30 & 30.2 & 62 & 0.4 & 29\end{array}$

20.930

$\$ 8.420$

20.93 .0

$20.6 \quad 4.3$

8.030

30.63 .1

$29.7 \quad 4.5$

62720

$20.9 \quad 3.0$

30.36 .3

82432

$30.1 \quad 3.2$

$27.8 \quad 8.4$

51.358

$30.0 \quad 3.0$

$28.6 \quad 5.9$

3.1

20.93 .0

28.87 .8

82338

30.13 .0

31.33 .1

C48 29

30.13 .0

$31.4 \quad 3.3$

84.49

$31.3 \quad 3.1$

84.620

$30.2 \quad 3.0$

31.06 .8

64120

$30.4 \quad 3.0$

2807.6

20.5 20

$\$ 0.420$

$20.1 \quad 8.7$

52.730

30.23 .0

$31.6 \quad 4.6$

8420

$30.6 \quad 3.0$

$20.7 \quad 4.9$

8.1 20

$\mathbf{3 0 . 0} 3.0$

$31.7 \quad 4.4$

8.6 30

$30.1 \quad 3.0$

$320 \quad 3.1$

8.820

$321 \quad 3.2$

85.420

30.83 .0

33.23 .1

84.820

30.73 .0

$30.6 \quad 3.0$

33.43.

84.83 .1

20.43 .0

3213.1

85020

83020

29.83 .0

$320 \quad 3.1$

68.529

29.53 .0

31.43 .1

68820

84420

$326 \quad 3.6$

58.720

58.52

$\mathbf{3 0 . 7} 3.0$

$\begin{array}{lll}33.0 & 4.2\end{array}$

88.620

$30.0 \quad 3.0$

$31.7 \quad 5.1$

83220

28.27 .5

85.930

$\begin{array}{llll}\mathbf{3 0 . 4} & 4.0 & \mathbf{2 3 . 1} & 8.7\end{array}$

58.029

$\mathbf{3 0 . 6} 8.1$

23.410 .0

$828 \quad 38$

54.43 .1

30.24 .2
$70.7 \quad 4.8$

23.810 .2

62082

22211.0

$20.8 \quad 48$

49880

43.672

$20.6 \quad 4.3$

21.012.

29.16 .0

10.714 .6

41.07 .1

$\begin{array}{ll}2.0 & 4.7\end{array}$

18.614 .6

41.175

$29.7 \quad 3.2$

29.63 .4

2212

6138.

21.612 .8

43.77 .7

42.66

$47.4 \quad 45$

30.73 .4

29.03 .0

200131

47.448

21.711 .8

$\begin{array}{lll}51.3 & 5.7\end{array}$

2023.0

$227 \quad 0.7$

5.340

$20.6 \quad 123$

51.17 .1

$288 \quad 3.1$

20.713 .1

48.175

$\begin{array}{ll}4.1 & 78 \\ 0.8 & \end{array}$

10.9123

$28.0 \quad 3.0$

20.23 .0

21.814 .4

80.85 .1

$528 \quad 4.4$

23.013 .2

200

208132

64.67 .1 


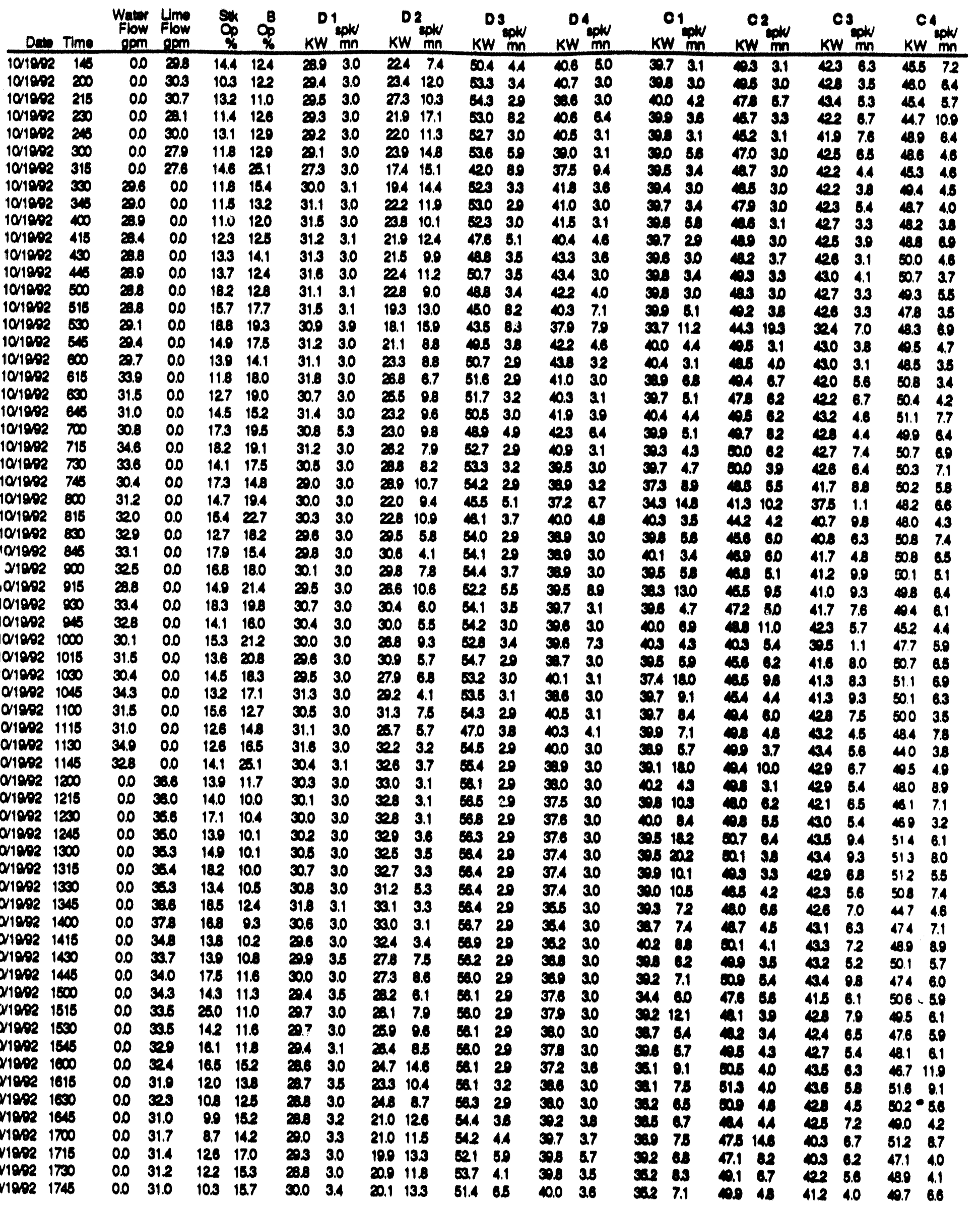


Den Time 10/1902 1600 1018021818 1018021850 10/1002 1846 1018021000 10/1002 1915 $10 / 10021000$ 10/1002 1045 101002 2000 1019022015 101902 2000 1010922046 $10 / 19022100$ 10/1002 2115 10/1002 2130 1018022145 10/1002 2000 101902 2218 $10 / 19022200$ 10/1002 2245 $10 / 19022 \times 00$ $10 / 1002 \quad 2316$ $101002 \times 2 \times 0$ 1010022345 1020020 10 2002 15 10200230 102002 40 \begin{tabular}{cc}
102002 & 100 \\
\hline 20002 & 115
\end{tabular} $102002 \quad 130$ $\begin{array}{ll}102002 & 145 \\ 102002 & 200\end{array}$ 102002 215 102002200 102002245 1020002 300 102009315 102002350 10r2002 345 102002400 $102092 \quad 415$ 10200240 102002448 102002 E00 102002 515 10roper 50 lorapr sis 102002 600 10roper 616 102008 600 102002 6is 102002700 102002715 102002 700 10200027is 102002 600 102002 816 100002 exp croper an - orcorar con 102002 015 102002 c00 10200r ons 102009 1000
Waver Lim Flow Flow op op $\mathrm{kW}$ mn $\begin{array}{llll}0.0 & 33.4 & 112 & 13.7\end{array}$ $0.0 \quad 508 \quad 11.1 \quad 16$. $0.0 \quad 313 \quad 18.1 \quad 162$ $\begin{array}{llll}0.0 & 31.8 & 16.4 & 14.8\end{array}$ $\begin{array}{llll}0.0 & 31.0 & 16.1 & 14.3\end{array}$ $\begin{array}{llll}0.0 & 30.7 & 16.9 & 10.0\end{array}$ $\begin{array}{llll}0.0 & 30.1 & 14.6 & 17.6\end{array}$ $\begin{array}{lllll}0.0 & 30.1 & 16.0 & 17.7\end{array}$ $\begin{array}{llll}0.0 & 30.6 & 15.1 & 18.3\end{array}$ $\begin{array}{llll}0.0 & 327 & 14.5 & 16.0\end{array}$ $\begin{array}{llll}0.0 & 29.9 & 18.3 & 182\end{array}$ $\begin{array}{lllll}0.0 & 29.9 & 128 & 16.8\end{array}$ $\begin{array}{llll}0.0 & 20.8 & 124 & 18.1\end{array}$ $\begin{array}{llll}0.0 & 2.8 & 10.8 & 17.0\end{array}$ $\begin{array}{llll}0.0 & 2.8 & 10.8 & 10.2\end{array}$ $\begin{array}{llll}0.0 & 2.1 & 11.8 & 2.0 .3\end{array}$ $\begin{array}{llll}0.0 & 33.8 & 0.8 & 16.0\end{array}$ $\begin{array}{llll}0.0 & 203 & 0.6 & 14.3\end{array}$ $\begin{array}{lllll}0.0 & 324 & 10.8 & 16.6\end{array}$ $\begin{array}{llll}0.0 & 2.0 & 152 & 20.8\end{array}$ $\begin{array}{llll}0.0 & 28.7 & 123 & 22 .\end{array}$ $\begin{array}{lllll}0.0 & 27.0 & 14.4 & 27.7\end{array}$

$\begin{array}{llll}0.0 & 20.3 & 11.6 & 21.4\end{array}$ $\begin{array}{lllll}0.0 & 27.6 & 15.8 & 29.6\end{array}$ $\begin{array}{lllll}320 & 00 & 120 & 202\end{array}$ $\begin{array}{lllll}302 & 00 & 11.8 & 17.7\end{array}$ $\begin{array}{llll}326 & 0.0 & 13.6 & 10.4\end{array}$ $\begin{array}{llll}321 & 0.0 & 10.0 & 17.1\end{array}$ $\begin{array}{llll}328 & 0.0 & 11.1 & 13.6\end{array}$ $\begin{array}{lllll}32.1 & 0.0 & 28.8 & 16.5\end{array}$ $\begin{array}{llll}31.6 & 0.0 & 11.4 & 152\end{array}$ $\begin{array}{llll}31.6 & 00 & 21.0 & 13.3\end{array}$ $\begin{array}{llll}30.1 & 0.0 & 126 & 13.9\end{array}$ $\begin{array}{llll}31.6 & 0.0 & 102 & 182\end{array}$ $\begin{array}{llll}320 & 0.0 & 16.8 & 14.0\end{array}$ $\begin{array}{llll}323 & 0.0 & 8.3 & 13.1\end{array}$ $\begin{array}{llll}324 & 0.0 & 7.0 & 13.7\end{array}$ $\begin{array}{llll}31.9 & 0.0 & 7.8 & 10.0\end{array}$ $\begin{array}{llll}30.6 & 0.0 & 8.0 & 13.0\end{array}$ $\begin{array}{llll}33.4 & 00 \quad 122 & 11.1\end{array}$ $\begin{array}{llll}28.9 & 0.0 & 7.0 & 10.3 \\ 31 . & 0.0 & 8.5 & 9 .\end{array}$ $\begin{array}{llll}31.0 & 0.0 & 0.0 & 8.0 \\ 31.7 & 0.0 & 8.0 & 8.8\end{array}$ $\begin{array}{llll}31.8 & 00 & 8.8 & 83\end{array}$ 31.20 .0 31.700 31.90 .0 7.80 31.900

$0.036 \quad 0.711 .5$ $\begin{array}{llll}0.0 & 34.1 & 135 & 10.4\end{array}$ $\begin{array}{llll}0.0 & 333 & 120 & 88\end{array}$ $0.0 \quad 31 \quad 148 \quad 10.8$ $\begin{array}{lllll}0.0 & 328 & 14.3 & 93\end{array}$ $\begin{array}{llll}0.0 & 327 & 13.7 & 88\end{array}$ $\begin{array}{llll}00 & 321 & 162 & 03\end{array}$ $\begin{array}{llll}00 & 327 & 167 & 86\end{array}$ $\begin{array}{llll}0.0 & 25 & 16.0 & 8.4\end{array}$ $00 \quad 326 \quad 163 \quad 98$ $\begin{array}{llll}0.0 & 318 & 163 & 04\end{array}$ $\begin{array}{llll}0.0 & 2.1 & 148 & 10.0\end{array}$ $\begin{array}{llll}0.0 & 322 & 18.7 & 10.0\end{array}$ $\begin{array}{llll}0.0 & 36.6 & 17.3 & 172\end{array}$ $\begin{array}{llll}0.0 & 33.0 & 17.1 & 9.8\end{array}$ $\begin{array}{llll}0.0 & 31.1 & 0.0 & 0.8\end{array}$ $\begin{array}{llll}0.0 & 30.4 & 16.7 & 11.0\end{array}$ $\begin{array}{ll}30.0 & 3.0 \\ 28.0 & 32\end{array}$

$2.4 \quad 3.0$

2303.2

232 3.0

2384.1

28.64 .1

2326.6

$2.4 \quad 3.0$

210.0

$28.7 \quad 3.0$

28.16 .0

$2.4 \quad 3.0$

230.8

2.453

2826.0

$2.8 \quad 3.4$

$2.7 \quad 3.0$

$2.8 \quad 3.0$

27.73 .1

27.43 .0

$27.8 \quad 6.8$

$27.8 \quad 3.0$

$272 \quad 3.7$

$312 \quad 3.0$

5123.0

50.83 .0

$30.9 \quad 3.0$

$30.4 \quad 3.0$

$503 \quad 3.0$

5023.0

30.3 .0

5023.0

$30.8 \quad 3.0$

$30.8 \quad 3.0$

$50.0 \quad 3.0$

$\mathbf{0 . 8} 3.0$

$2.1 \quad 3.0$

$\mathbf{5 0 . 6} 3.0$

30.83 .0

$30.7 \quad 3.0$

$30.3 \quad 3.0$

2003.0

$209 \quad 3.0$

2.63 .0

$2.0 \quad 3.0$

$290 \quad 3.0$

$208 \quad 3.0$

2.13 .0

$50.3 \quad 3.0$

20.9 .0

29.80

29.93 .0

$29.7 \quad 3.4$

29.53 .0

$20.0 \quad 3.0$

2.09 .0

$290 \quad 3.0$

$297 \quad 3.0$

29.13 .0

$203 \quad 3.0$

30.13 .0

$29.8 \quad 3.0$

$2.4 \quad 3.0$

23.89 .0

$D 2$

cow 03 04 Kw inn

61 nw in $\begin{array}{llllll}24.6 & 8.0 & 843 & 30 & 320 & 30\end{array}$ 21.710 .5 608 30. 10.8120 62746 40.520 $\begin{array}{llllll}217 & 02 & 5 \times 4 & 30 & 30.7 & 3.6\end{array}$ 24.611 .6 $19.2 \quad 12.2$ 19.416 .4 $18.8 \quad 13.0$ ans 3.1 3263 $\begin{array}{llll}03 & 44 \quad 396 & 73\end{array}$ $\begin{array}{llll}40.4 & 5.5 & 30.6 & 3.2\end{array}$ 80.34 .9 21.113 .8

62430 $24.8 \quad 0.1$ 52820 $30.8 \quad 43$ $21.0 \quad 124$ 19.910 .7 19811.6 21.2112 18.013 .0 10.718 .4 $228 \quad 03$ 22210.6 21.913 .0 $17.9 \quad 14.5$ 18.217. 16.918 .3 20.116 .6 16.916 .0 19.410 .6 2278.6 $288 \quad 6.3$ 28.162 51.039 $0.1 \quad 48$ $60.3 \quad 4.4$ 80.989 00.230 $60.4 \quad 42$
0.9 80833

a.1 30

50.032 63242 $821 \mathrm{BA}$ 4636.8 46.948 32.8 3058 $308 \quad 43$ $308 \quad 42$ $30.9 \quad 7.4$ $30.4 \quad 43$ 30.56 3.120 30.733 30.730 32338 37.96 .8 $\begin{array}{llll}51.1 & 7.1 & 30.9 & 48\end{array}$ 427.4 4738 37.149 4.030 41.927 0032 49.14 .6 29.68 $\begin{array}{llll}40.4 & 4.1 & 423 & 46\end{array}$ $\begin{array}{llll}512 & 29 & 30.4 & 30\end{array}$ 7.0

51.120 $39.0 \quad 31$ $28.6 \quad 4.0$

c0.4 3.0 0.335 3.6 


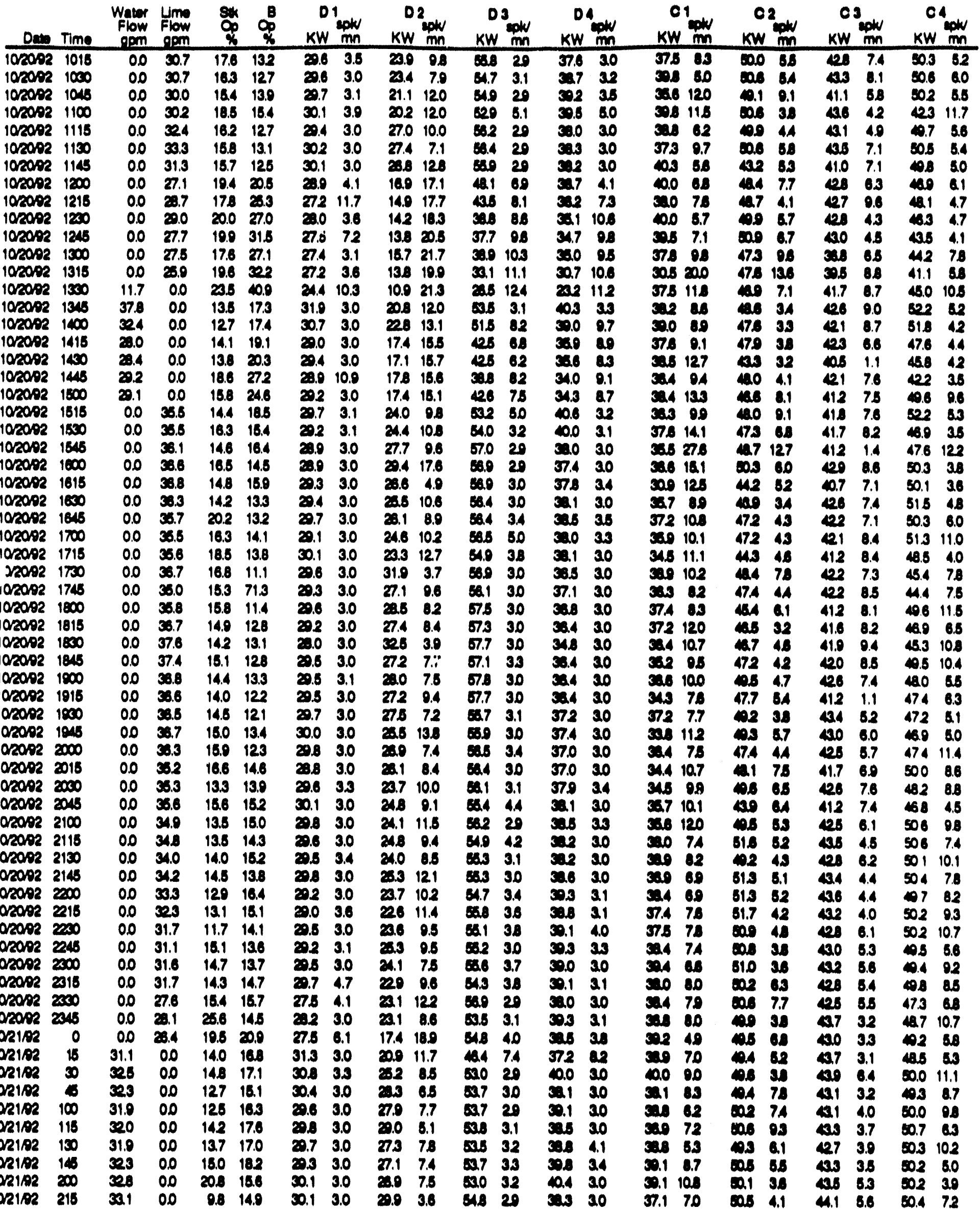




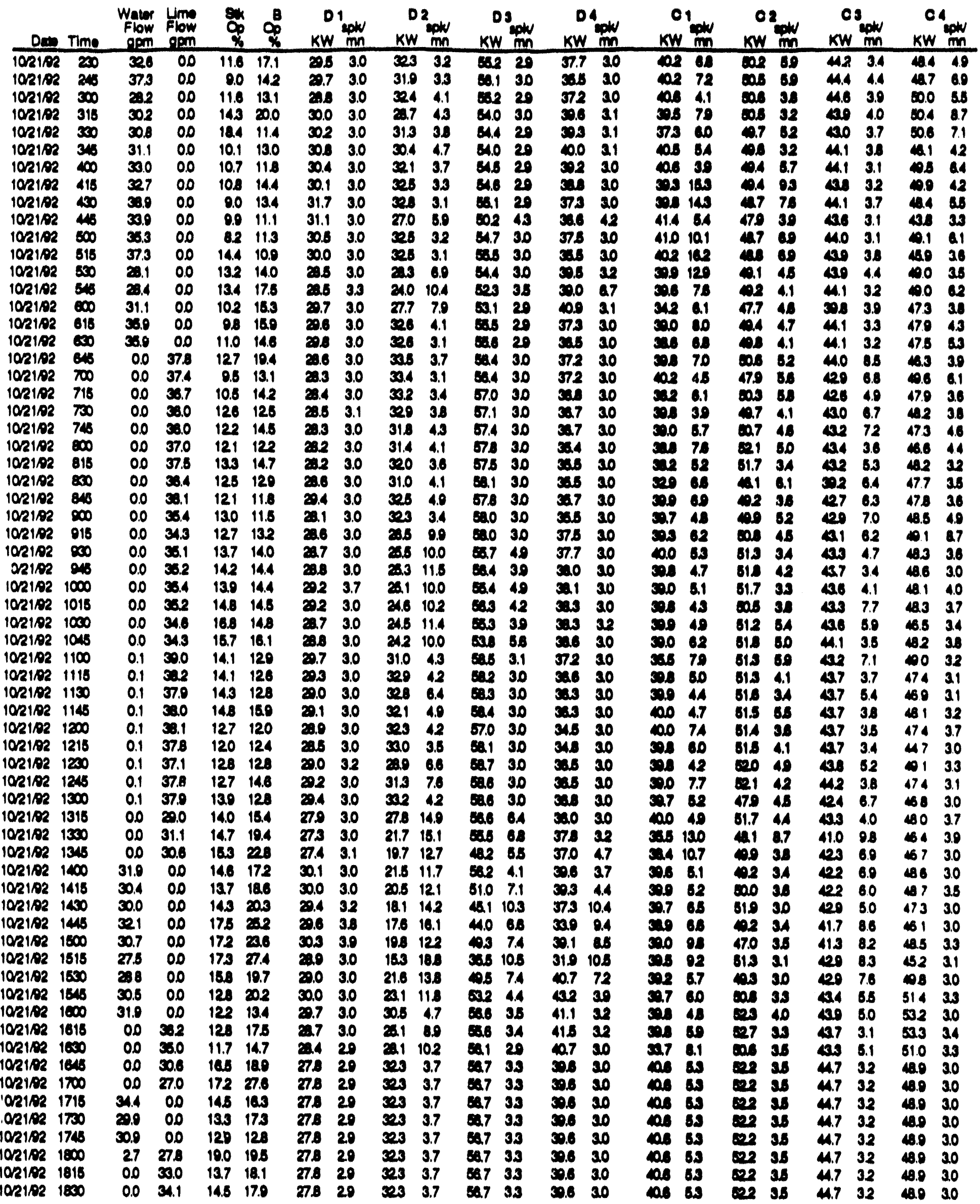




\begin{tabular}{|c|c|c|c|c|c|c|c|c|c|c|c|c|c|c|c|c|c|c|c|c|c|}
\hline Date & Time & $\begin{array}{l}\text { Water } \\
\text { Flow } \\
\text { gpm }\end{array}$ & $\begin{array}{l}\text { Lime } \\
\text { Flow } \\
\text { gpm }\end{array}$ & $\begin{array}{l}S k \\
O_{p} \\
\%\end{array}$ & $\begin{array}{l}B \\
\% \\
\%\end{array}$ & & spiks & & $\begin{array}{l}2 \\
\text { sple } \\
\mathrm{mn}\end{array}$ & & $\begin{array}{l}\text { spol } \\
\mathrm{mn}\end{array}$ & & $\mathrm{mn}$ & & $\mathrm{m}$ & KW & $m$ & & & KW & \\
\hline 2192 & 1845 & 0.0 & 33.4 & 15.9 & 16.8 & 278 & 29 & 323 & 3.7 & 56.7 & 33 & 30.6 & 30 & 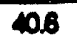 & 53 & 522 & 35 & 4.7 & 32 & 48.9 & 3.0 \\
\hline $21 / 92$ & 1900 & 0.0 & 33.1 & 14.7 & 18.9 & 278 & 29 & 323 & 3.7 & 66.7 & 33 & 39.6 & 3.0 & 40.8 & 53 & 522 & 35 & 4.7 & 32 & 48.9 & 3.0 \\
\hline $21 / 92$ & 1915 & 0.0 & 34.1 & 15.4 & 19.4 & 278 & 2.9 & 323 & 3.7 & 56.7 & 33 & 39.6 & 3.0 & $\$ 0.6$ & 53 & 022 & 35 & 4.7 & 3.2 & 48.9 & 30 \\
\hline $21 / 92$ & 1900 & 0.0 & 34.0 & 162 & 18.5 & 27.8 & 29 & 323 & 3.7 & 56.7 & 33 & 39.6 & 30 & $\$ .6$ & 5.3 & 22 & 35 & 4.7 & 32 & 48.9 & 3.0 \\
\hline $21 / 92$ & 1845 & 0.0 & 30.9 & 13.9 & 21.0 & 27.8 & 29 & 323 & 3.7 & 56.7 & 33 & 39.6 & 3.0 & 40.6 & 83 & 522 & 35 & 4.7 & 32 & 48.9 & 3.0 \\
\hline $1 / 92$ & 2000 & 0.0 & 34.1 & 13.5 & 18.4 & 278 & 2.9 & 323 & 3.7 & 56.7 & 33 & 39.6 & 3.0 & 40.6 & 53 & 52 & 35 & 4.7 & 32 & 489 & 3.0 \\
\hline 1192 & 2015 & 0.0 & 34.0 & 13.9 & 16.8 & 278 & 29 & 323 & 3.7 & 56.7 & 33 & 39.6 & 3.0 & $\boldsymbol{\infty} .8$ & 53 & $\$ 22$ & 38 & 4.7 & 32 & 489 & 3.0 \\
\hline $1 / 92$ & $\mathbf{2 0 0 0}$ & 0.0 & 34.0 & 13.9 & 18.2 & 278 & 20 & 323 & 3.7 & 56.7 & 33 & 30.6 & 3.0 & 10.6 & 53 & $\$ 2$ & 38 & 47 & 32 & 489 & 3.0 \\
\hline $1 / 92$ & 2045 & 0.0 & 34.7 & 15.8 & 158 & 278 & 29 & 323 & 3.7 & 56.7 & 33 & 39.6 & 3.0 & 4.8 & 53 & 52 & 35 & 4.7 & 32 & 48.9 & 3.0 \\
\hline $1 / 92$ & 2100 & 0.0 & 35.2 & 13.8 & 16.2 & 27.8 & 2.9 & 323 & 3.7 & 56.7 & 33 & 30.6 & 3.0 & $\mathbf{4 0 . 6}$ & 83 & 822 & 35 & 4.7 & 32 & 48.9 & 3.0 \\
\hline 2192 & 2115 & 0.0 & 35.8 & 13.1 & 15.4 & 27.8 & 29 & 323 & 3.7 & 56.7 & 33 & 39.6 & 3.0 & 40.6 & 5.3 & 522 & 36 & 4.7 & 32 & 489 & 30 \\
\hline $21 / 92$ & 2130 & 0.0 & 36.1 & 15.2 & 15.3 & 27.8 & 29 & 323 & 3.7 & 56.7 & 33 & 39.6 & 3.0 & 406 & 53 & 522 & 35 & 4.7 & 32 & 48.9 & 30 \\
\hline $11 / 92$ & 2145 & 0.0 & 36.8 & 13.2 & 13.3 & 27.8 & 29 & 323 & 3.7 & 56.7 & 33 & 39.6 & 30 & 406 & 53 & 522 & 35 & 4.7 & 3.2 & 48.9 & 30 \\
\hline $1 / 92$ & 200 & 0.0 & 34.3 & 15.6 & 13.5 & 27.8 & 29 & 323 & 3.7 & 567 & 33 & 39.6 & 3.0 & 106 & 53 & 52 & 38 & 4.7 & 32 & 48.9 & 3.0 \\
\hline $1 / 92$ & 2215 & 0.0 & 31.5 & 13.4 & 19.4 & 27.8 & 29 & 323 & 3.7 & 56.7 & 33 & 39.6 & 3.0 & $\boldsymbol{\phi} .6$ & 53 & 522 & 38 & 4.7 & 3.2 & 48.9 & 3.0 \\
\hline $1 / 92$ & 2200 & 0.0 & 31.9 & 14.6 & 16.6 & 278 & 2.9 & 323 & 3.7 & 56.7 & 33 & 30.6 & 3.0 & $\$ 8$ & 83 & 52 & 38 & 4.7 & 32 & 48.9 & 3.0 \\
\hline $1 / 92$ & 2245 & 0.0 & 328 & 13.5 & 14.8 & 27.8 & 2.9 & 323 & 3.7 & 56.7 & 33 & 39.6 & 30 & $\boldsymbol{\omega 0 . 6}$ & 53 & 52 & 35 & 4.7 & 32 & 489 & 3.0 \\
\hline $1 / 92$ & 2300 & 0.0 & 327 & 20.8 & 14.5 & 27.8 & 2.9 & 323 & 3.7 & 56.7 & 33 & 39.6 & 3.0 & 40.8 & 53 & 22 & 35 & 4.7 & 3.2 & 48.9 & 3.0 \\
\hline $1 / 92$ & 2315 & 0.0 & 33.5 & 127 & 15.0 & 278 & 29 & 323 & 3.7 & 56.7 & 33 & 39.6 & 30 & $\$ 0.6$ & 53 & 82 & 35 & 4.7 & 32 & 48.9 & 3.0 \\
\hline $1 / 92$ & 2330 & 0.0 & 33.6 & 123 & 127 & 28.0 & 2.9 & 31.9 & 7.1 & 56.6 & 30 & 39.7 & 30 & $\boldsymbol{\phi 0 . 6}$ & 58 & 02 & 48 & 44.7 & 5.0 & 40.5 & 37 \\
\hline $1 / 92$ & 2345 & 0.0 & 34.4 & 17.2 & 16.6 & 28.0 & 2.9 & 31.9 & 7.1 & 56.6 & 30 & 39.7 & 30 & $\infty 6$ & 58 & 522 & 48 & 4.7 & 5.0 & 4.5 & 3.7 \\
\hline 292 & 0 & 0.0 & 34.6 & 128 & $1 i .6$ & 28.0 & 2.9 & 31.8 & 7.1 & 56.6 & 30 & 39.7 & 30 & $\$ 0.8$ & 58 & 82 & 48 & 447 & 5.0 & 49.5 & 3.7 \\
\hline 292 & 15 & 0.0 & 35.4 & 14.1 & 142 & $\mathbf{2 8 . 0}$ & 29 & 31.9 & 7.1 & 56.6 & 30 & 39.7 & 30 & 10.6 & 58 & 022 & 48 & 4.7 & 5.0 & 49.5 & 3.7 \\
\hline 2992 & 30 & 0.0 & 35.8 & 14.1 & 20.7 & $\mathbf{2 8 . 0}$ & 29 & 31.9 & 7.1 & 56.6 & 30 & 39.7 & 30 & $\$ 06$ & $\mathbf{5 8}$ & 822 & 48 & 4.7 & 5.0 & 49.5 & 3.7 \\
\hline 292 & 46 & 0.0 & 37.4 & 14.7 & 15.2 & $\mathbf{2 8 . 0}$ & 2.9 & 31.9 & 7.1 & 56.6 & 30 & 30.7 & 3.0 & 106 & $\mathbf{5 8}$ & $\infty 2$ & 48 & 4.7 & 5.0 & 40.5 & 3.7 \\
\hline 29 & 100 & 0.0 & 38.1 & 15.5 & 16.6 & $\mathbf{2 8 . 0}$ & 2.8 & 31.8 & 7.1 & 56.6 & 30 & 39.7 & 3.0 & 106 & 58 & $\$ 22$ & 48 & 4.7 & 5.0 & 49.5 & 3.7 \\
\hline 292 & 115 & 0.0 & 35.2 & 122 & 11.8 & $\mathbf{2 8 . 0}$ & 29 & 31.9 & 7.1 & 56.6 & 30 & 30.7 & 3.0 & 106 & 58 & 822 & 46 & 4.7 & 5.0 & 40.5 & 3.7 \\
\hline 192 & 130 & 00 & 29.1 & 10.8 & 129 & 280 & 29 & 31.9 & 7.1 & 56.6 & 30 & 39.7 & 3.0 & 106 & $\mathbf{S B}$ & 22 & 48 & 4.7 & 5.0 & 49.5 & 3.7 \\
\hline 29 & 145 & 0.0 & 27.6 & 122 & 14.9 & $\mathbf{2 B . 0}$ & 29 & 31.8 & 7.1 & 56.6 & 30 & 39.7 & 3.0 & $\$ 0.6$ & 58 & 522 & 46 & 44.7 & 5.0 & 49.5 & 3.7 \\
\hline 9 & 200 & 0.0 & $\mathbf{2 8 . 1}$ & 0.1 & 13.6 & 28.0 & 2.9 & 31.8 & 7.1 & 56.6 & 30 & 39.7 & 30 & $\$ 06$ & 58 & 502 & 46 & 44.7 & 0 & 49.5 & 3.7 \\
\hline 292 & 215 & 0.0 & $27 . y$ & 120 & 18.9 & 28.0 & 2.9 & 31,8 & 7.1 & 56.6 & 3.0 & 39.7 & 3.0 & $\$ 0.8$ & 68 & 52 & 48 & 4.7 & 5.0 & 49.5 & 3.7 \\
\hline 0,92 & 200 & 0.0 & 19.0 & 31.7 & 37.0 & 28.0 & 29 & 31.9 & 7.1 & 56.6 & 30 & 39.7 & 30 & $\boldsymbol{4 0 . 6}$ & 88 & 502 & 48 & 44.7 & 5.0 & 48.5 & 3.7 \\
\hline 209 & 245 & 0.0 & 28.0 & 9.6 & 16.4 & 28.0 & 2.9 & 31.9 & 7.1 & 56.6 & 30 & 39.7 & 30 & $\$ .8$ & 58 & 52 & 48 & 4.7 & 5.0 & 49.5 & 3.7 \\
\hline 100 & 300 & 0.0 & $\mathbf{2 8 . 1}$ & 10.1 & 11.1 & 8.0 & 29 & 31.9 & 7.1 & 56.6 & 30 & 30.7 & 3.0 & 108 & 88 & $\sqrt{22}$ & 48 & 4.7 & 5.0 & 49.5 & 3.7 \\
\hline 08 & 315 & 0.0 & 34.0 & 30.8 & 16.5 & 28.0 & 2.9 & 31.9 & 7.1 & 56.6 & 3.0 & 30.7 & 3.0 & $\$ 0.8$ & 58 & 22 & 46 & 4.7 & 5.0 & 49.5 & 3.7 \\
\hline 29 & 330 & 0.0 & 27.7 & 120 & 19.6 & 28.0 & 29 & 31.9 & 7.1 & 56.6 & 30 & 30.7 & 30 & $\$ .6$ & 58 & $\overline{22}$ & 48 & 4.7 & 5.0 & 49.5 & 3.7 \\
\hline 9 & 345 & 0.0 & 31.0 & 14.4 & 20.8 & 28.0 & 29 & 31.9 & 7.1 & 56.6 & 30 & 39.7 & 30 & $\$ 0.6$ & 58 & 52 & 46 & 4.7 & 5.0 & 4.5 & 3.7 \\
\hline 9 & 400 & 0.0 & 31.2 & 17.6 & 16.4 & 28.0 & 29 & 31.0 & 7.1 & 56.6 & 3.0 & 39.7 & 3.0 & $\$ 0.6$ & 68 & 522 & 46 & 4.7 & 5.0 & 49.5 & 3.7 \\
\hline 9 & 415 & 00 & 34.3 & 14.4 & 14.7 & 28.0 & 29 & 31.9 & 7.1 & 56.6 & 30 & 39.7 & 30 & 408 & 68 & $\sqrt{2}$ & 48 & 4.7 & 5.0 & 495 & 3.7 \\
\hline 9 & 430 & 0.0 & 34.6 & 13.1 & 13.0 & $\mathbf{2 8 . 0}$ & 29 & 31.9 & 7.1 & 56.6 & 30 & 39.7 & 3.0 & $\$ .6$ & 58 & $\$ 2$ & 48 & 4.7 & 5.0 & 49.5 & 3.7 \\
\hline 9 & 445 & 0.0 & 35.3 & 31.2 & 14.8 & 28.0 & 29 & 31.9 & 7.1 & 56.6 & 30 & 39.7 & 30 & $\$ 0.6$ & 58 & $\overline{22}$ & 46 & 4.7 & 5.0 & 4.5 & 3.7 \\
\hline 9 & 500 & 0.0 & 35.5 & 13.1 & 14.4 & 8.0 & 29 & 1.9 & 7.1 & 56.6 & 38 & 39.7 & 3. & $\$ .6$ & 58 & 522 & 46 & 4.7 & 0 & 9.5 & 3.7 \\
\hline 9 & 515 & 0.0 & 36.4 & 13.0 & 14.6 & 80 & 29 & 31.9 & 7.1 & 56.6 & 30 & 30.7 & 3.0 & $\$ 0.6$ & 5 & 102 & 46 & 4.7 & 6.0 & 95 & 3.7 \\
\hline & 530 & 0.0 & 36.0 & 11.8 & 129 & 8.0 & 29 & 31.8 & 7.1 & 56.6 & 30 & 30.7 & 3.0 & 10.6 & 58 & 122 & 48 & 4.7 & 5.0 & 4.5 & 3.7 \\
\hline 92 & 545 & 0.0 & 35.2 & 13.0 & 13.6 & 3.0 & 29 & 31.9 & 7.1 & 56.6 & 3.0 & 39.7 & 3.0 & $\$ 06$ & 58 & $\sqrt[52]{22}$ & 46 & 4.7 & 5.0 & 45 & 3.7 \\
\hline & 600 & 0.0 & 36.9 & 13.3 & 15.3 & 3.0 & 29 & 31.9 & 7.1 & 56.6 & 30 & 39.7 & 3.0 & $\$ 0.6$ & 58 & 52 & 46 & 4.7 & 5.0 & 45 & 3.7 \\
\hline 22 & 615 & 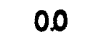 & 37.1 & 14.3 & 13.2 & 8.0 & 29 & 31.9 & 7.1 & 56.6 & 30 & 39.7 & 30 & 40.6 & 58 & 522 & 46 & 4.7 & 5.0 & 4.5 & 3.7 \\
\hline 229 & $\varpi$ & 0.0 & 35.9 & 15.7 & 15.5 & 28.0 & 2.9 & 31.9 & 7.1 & 56.6 & 30 & 39.7 & 30 & 10.6 & 58 & 22 & 48 & 4.7 & 5.0 & 4.5 & 3.7 \\
\hline 2292 & 645 & 0.0 & 29.1 & 165 & 19.4 & $\mathbf{2 8 . 0}$ & 29 & 31.9 & 7.1 & 56.6 & 30 & 39.7 & 30 & $\Delta .6$ & 58 & $\$ 22$ & 46 & 47 & 15.0 & 49.5 & 3.7 \\
\hline 2292 & 700 & 0.0 & 30.0 & 11.0 & 160 & 28.0 & 29 & 31.9 & 7.1 & 56.6 & 30 & 39.7 & 30 & 106 & 58 & 522 & 48 & 4.7 & Si. & 40.5 & 3.7 \\
\hline 2292 & 715 & 0.0 & 30.2 & 15.9 & 198 & 220 & 20 & 31.9 & 7.1 & 56.6 & 30 & 30.7 & 30 & 10 & $8 B$ & $\$ 22$ & 48 & 4.7 & 5.0 & 49.5 & 3.7 \\
\hline & 730 & 0.0 & 282 & 15. & 14.6 & 28.0 & 29 & 31.9 & 7.1 & 56.6 & 30 & 39.7 & 3.0 & 108 & 5 & $\$ 22$ & 48 & 47 & 5.0 & 49.5 & 3.7 \\
\hline & 745 & 00 & 28.9 & 15.0 & 18.5 & 28.0 & 29 & 31.9 & 7.1 & 56.6 & 30 & 39.7 & 30 & $\$ 0.6$ & 5. & 522 & 48 & 44.7 & 5.0 & 49.5 & 3.7 \\
\hline $28 c$ & 100 & 0.0 & 28.5 & 16.4 & 18.4 & 28.6 & 3.3 & 20.1 & 14.6 & 50.8 & 60 & 37.0 & 26 & 303 & s. & 505 & 58 & 40.3 & 8.5 & 523 & 8.7 \\
\hline & 815 & 0.0 & $\mathbf{2 9 . 1}$ & 6.6 & 19.5 & 288 & 3.0 & 19.1 & 13.2 & 45.9 & 7.4 & 37.6 & 86 & 305 & 3 & 800 & 35 & 420 & 4.1 & .9 .9 & 7.5 \\
\hline & 800 & 0.0 & 321 & 13.5 & 17.1 & 29.5 & 3.0 & 23.7 & 10.8 & 55.1 & 3.1 & 38.4 & 33 & 300 & 43 & 80.7 & 38 & 420 & 8.3 & 50.5 & 52 \\
\hline 2292 & 845 & 0.0 & 30.3 & 13.7 & 15.4 & 28.4 & 3.0 & 21.4 & 128 & 54.4 & 4.1 & $\$ .1$ & 3.7 & 380 & 6.7 & $\Delta .1$ & 48 & 421 & 43 & 51.5 & 7.4 \\
\hline & 900 & 00 & 29.0 & 127 & 15.1 & 28.1 & 3.2 & 212 & 120 & 50 & 5.1 & 4.0 & 3.7 & 303 & 58 & 28 & $4 A$ & 122 & 6.3 & 48.7 & 7.6 \\
\hline 20 & 915 & 0.0 & $\mathbf{2 9 . 0}$ & 127 & 15.1 & 28.1 & 3.2 & 212 & 120 & 50 & 5.1 & 40.0 & 3.7 & 303 & 58 & $\mathbf{4 5}$ & 44 & $\$ 22$ & 6.3 & 48.7 & 7.6 \\
\hline$\infty 0$ & 900 & 0.0 & 29.0 & 127 & 15.1 & $\mathbf{2 8 . 1}$ & 32 & 21.2 & 12.0 & 53.0 & 5.1 & 40.0 & 3.7 & 303 & 5.8 & $\omega \mathbf{B}$ & 44 & 422 & 6.3 & 48.7 & 7.6 \\
\hline & 945 & 0.0 & 30.3 & 13.7 & 18.8 & 29.3 & 3.0 & $\mathbf{2 0 . 3}$ & 123 & 50.6 & 63 & 30.3 & 86 & 308 & 58 & $\$ 24$ & 43 & 124 & 9.1 & 520 & 7.4 \\
\hline & 1000 & 00 & 31.7 & 15.9 & 38.1 & 29.3 & 3.0 & 23.1 & 11.0 & 525 & 48 & 39.6 & 75 & 396 & 4.7 & 500 & 34 & 120 & 4.9 & 524 & 5.0 \\
\hline & 1015 & 00 & 31.7 & 10.9 & 18.6 & $\mathbf{2 9 . 1}$ & 4.1 & 226 & 11.8 & 529 & 5.4 & 39.5 & 5.1 & 30.7 & 7 & 51.4 & 42 & $\mathbf{4 3 . 0}$ & 5.4 & 522 & 5.7 \\
\hline & $1 \alpha$ & 30.7 & 0.0 & 7.9 & 18.3 & 30.1 & 3.0 & 220 & 8.8 & 528 & 3. & 39.7 & 55 & 39.0 & & $\infty 08$ & 43 & 120 & 4.5 & 50.4 & 5.1 \\
\hline 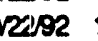 & 1045 & 00 & .7 & 10.5 & 19.7 & 9.3 & 3.0 & 23.9 & 10.0 & 542 & 3 & 39.7 & 7. & 3.5 & & 5 & 40 & 135 & 3.1 & 532 & 85 \\
\hline
\end{tabular}




\section{Das Timo} 1022921100 102292 1115 1022821130 $102202 \quad 1145$ 1022021200 1022921215 10228921250 1022921246 1022921300 1022921316 1022921350 1022921345 1022021400 $1020 p 21415$ 1022921450 1020921445 10222921500 $102292 \quad 1515$ $102292 \quad 1550$ 102202 1545 $102292 \quad 1600$ 1022921615 1022921600 1022921645 1022921700 1022921715 1022921730 1022921745 1022921800 उ2092 1815 10222921830 1022921845 1022921900 1022921915 1022921960 10222921945 1022922000 1022922015 1022922000 10222922045 1022822100 1022922115 1022922130 $10 / 22922145$ 10222922200 $102292 \quad 2215$ 1022922200 $102292 \quad 2245$ 1022922000 $102292 \quad 2315$ 1022922350 1022922045 1023920 10239215 10239253 102392 1023592100 1023P2 115 $1023192 \quad 130$ OR2395 145 Orzsis 200 $102392 \quad 215$ 1023592200 102392245 1023192300

Water Lim

$\begin{array}{llll}\text { Water Limo } & \text { Sk } & \mathbf{3} \\ \text { Flow Flow } & \text { Op } & \text { Op } \\ \text { gpm gom } & \% & \%\end{array}$

D1 spew

D 2

D3

D4

C1

62

opin

${ }_{3} 3$

soly

$c 4$ KW mn KW mn KW mn KW m

$\begin{array}{llll}0.0 & 322 & 9.9 & 15.5 \\ 0.0 & 327 & 7.1 & 16.6\end{array}$

$\begin{array}{llll}0.0 & 27.0 & 7.9 & \mathbf{2 0 . 0}\end{array}$

$20.5 \quad 3.0$

28.472

29.83 .0

28.25 .5

$0.0 \quad 27.1$

$11.1 \quad 19.3$

28.13 .0

21.317 .1

$\begin{array}{lll}27.8 & 4.7\end{array}$

$0.0 \quad 26.6$

$0.0 \quad 28.7$

0.124 .0

$27.7 \quad 3.1$

18.1129

$0.0 \quad 31.1$

0.123 .5

27.63 .0

3230.0

19.327 .7

28.93 .0

$\begin{array}{ll}17.0 & 15.3\end{array}$

30.24 .1

18.215 .4

$\begin{array}{lllll}28.7 & 0.0 & 16.0 & 20.6\end{array}$

$\begin{array}{llll}28.8 & 0.0 & 142 & 226\end{array}$

$\begin{array}{lllll}28.7 & 0.0 & 13.4 & 20.6\end{array}$

20.63 .0

18.114 .4

30.23 .0

20.4132

$\begin{array}{llll}28.0 & 0.0 & 17.9 & 24.1\end{array}$

20.93 .0

20112

$29.7 \quad 3.0$

23.311 .7

$\begin{array}{lllll}28.3 & 0.0 & 14.5 & 21.0\end{array}$

29.43 .0

20.512 .3

21.911 .9

$29.8 \quad 3.0$

20.93 .0

24.58 .1

$\begin{array}{llll}0.0 & 35.4 & 15.2 & 18.1\end{array}$

$\begin{array}{llll}0.0 & 35.0 & 120 & 16.0\end{array}$

$\begin{array}{lllll}0.0 & 34.2 & 13.7 & 18.2\end{array}$

$\begin{array}{lllll}0.1 & 34.4 & 13.4 & 21.6\end{array}$

$30.1 \quad 3.2$

27.98 .0

$29.7 \quad 3.0$

2817.3

$\begin{array}{lllll}0.1 & 31.5 & 14.0 & 21.3\end{array}$

$\begin{array}{lllll}0.1 & 36.2 & 13.6 & 15.9\end{array}$

$\begin{array}{lllll}30.5 & 4.1 & 24.7 & 7.1\end{array}$

$29.9 \quad 3.0$

$\begin{array}{llll}20.1 & 3.0 & \mathbf{3 0} .5 & 6.5\end{array}$

$\begin{array}{llll}0.1 & 35.9 & 124 & 14.6\end{array}$

$\begin{array}{lllll}0.1 & 36.3 & 15.3 & 14.2\end{array}$

$\begin{array}{llll}0.1 & 37.0 & 14.2 & 15.1\end{array}$

$\begin{array}{llll}0.1 & 37.7 & 11.2 & 16.2\end{array}$

$\begin{array}{llll}0.1 & 35.5 & 11.1 & 15.7\end{array}$

$\begin{array}{llll}0.1 & 36.9 & 126 & 14.3\end{array}$

$\begin{array}{llll}0.1 & 36.7 & 15.2 & 15.3\end{array}$

$\begin{array}{llll}0.1 & 37.2 & 14.3 & 15.4\end{array}$

$\begin{array}{lllll}0.1 & 34.4 & 14.9 & 14.3\end{array}$

$\begin{array}{llll}0.1 & 35.4 & 11.2 & 13.1\end{array}$

$\begin{array}{lllll}0.1 & 35.8 & 11.8 & 15.3\end{array}$

$\begin{array}{llll}0.1 & 35.9 & 123 & 10.9\end{array}$

$\begin{array}{lllll}0.1 & 34.7 & 15.8 & 18.2\end{array}$

$\begin{array}{llll}0.1 & 36.6 & 11.9 & 14.8\end{array}$

$\begin{array}{llll}0.1 & 35.8 & 14.1 & 16.3\end{array}$

$\begin{array}{lllll}0.1 & 35.2 & 127 & 15.3\end{array}$

$\begin{array}{lllll}0.1 & 35.1 & 14.4 & 16.3\end{array}$

$\begin{array}{lllll}0.0 & 35.1 & 19.3 & 15.8\end{array}$

$\begin{array}{llll}0.0 & 35.2 & 13.8 & 16.1\end{array}$

$\begin{array}{llll}0.0 & 38.2 & 14.7 & 16.1\end{array}$

$\begin{array}{llll}0.0 & 35.6 & 13.4 & 121\end{array}$

$\begin{array}{llll}00 & 35.2 & 123 & 123\end{array}$

$\begin{array}{llll}0.0 & 30.9 & 17.5 & 19.1\end{array}$

$\begin{array}{llll}0.0 & 328 & 11.6 & 126\end{array}$

$\begin{array}{llll}0.0 & 33.6 & 21.7 & 15.1\end{array}$

$\begin{array}{llll}0.0 & 33.2 & 13.2 & 14.9\end{array}$

$\begin{array}{llll}0.0 & 33.9 & 11.9 & 15.2\end{array}$

$\begin{array}{llll}0.0 & 327 & 10.6 & 14.8\end{array}$

$\begin{array}{llll}0.0 & 32.7 & 13.2 & 16.3\end{array}$

$\begin{array}{llll}0.0 & 36.7 & 13.8 & 16.6\end{array}$

$\begin{array}{llll}0.0 & 31.3 & 11.0 & 129\end{array}$

$\begin{array}{lllll}0.0 & 327 & 14.4 & 14.5\end{array}$

$\begin{array}{llll}0.0 & 31.6 & 125 & 125\end{array}$

$\begin{array}{llll}0.0 & 29.6 & 11.8 & 18.4\end{array}$

$\begin{array}{llll}0.0 & 30.3 & 20.0 & 221\end{array}$

$0.0 \quad 30.2$

$0.0 \quad 30.4$

12314.1

\begin{tabular}{llll}
0.0 & 29.1 & 11.7 & 132 \\
\hline & & 18.6
\end{tabular}

$\begin{array}{llll}27.9 & 0.0 & 15.1 & 18.7\end{array}$

$\begin{array}{llll}292 & 0.0 & 16.6 & 16.4\end{array}$

$\begin{array}{llll}27.9 & 0.0 & 155 & 21.1\end{array}$

$\begin{array}{lllll}28.6 & 0.0 & 14.5 & 18.2\end{array}$

$29.1 \quad 0.0 \quad 15.7 \quad 220$

$\begin{array}{lllll}29.1 & 0.0 & 17.9 & 24.1\end{array}$

\subsection{3 .0}

20.93 .0

29.13 .0

29.53 .0

$20.7 \quad 3.0$

30.13 .0

$30.1 \quad 3.0$

$20.8 \quad 3.0$

$28.5 \quad 3.0$

$20.4 \quad 3.0$

$30.0 \quad 3.0$

3023.0

30.23 .0

$30.6 \quad 3.0$

$30.0 \quad 3.0$

$298 \quad 3.0$

$29.6 \quad 3.0$

$30.0 \quad 3.0$

$30.5 \quad 3.0$

$30.8 \quad 3.0$

29.93 .0

3023.0

$30.4 \quad 3.0$

$30.1 \quad 3.0$

30.23 .0

$30.3 \quad 3.0$

30.23 .0

30.43 .0

30.23 .0

$30.8 \quad 3.0$

20.53 .0

20.73 .0

$30.5 \quad 3.0$

20.43 .0

30.83 .2

29.63 .1

29.93 .0

27.03 .0

$30.3 \quad 3.0$

$30.0 \quad 3.0$

$30.4 \quad 3.0$

30.13 .0

29.23 .0

$29.6 \quad 3.0$

$30.3 \quad 3.2$
$29.6 \quad 6.0$

28.65 .5

$34.4 \quad 3.3$

$30.5 \quad 5.5$

$\begin{array}{ll}30.3 & 5.7\end{array}$

$\begin{array}{ll}31.0 & 6.7\end{array}$

31.56 .6

30.25 .4

3245.4

28.86 .4

$\begin{array}{llll}55.9 & 3.1 & 30.3 & 3.6\end{array}$

5653.1

54.162

52375

45.065

35.80 .0

$\begin{array}{llll}47.5 \quad 69 \quad 341 & 86\end{array}$

$\begin{array}{llll}523 & 5.7 \quad 382 & 45\end{array}$

$\begin{array}{llll}40.2 & 6.1 & 35.6 & 9.3\end{array}$

$\begin{array}{llll}51.8 & 62 & 37.6 & 82\end{array}$

$548 \quad 44 \quad 333 \quad 85$

$\begin{array}{llll}50.4 & 6.7 & 352 & 10.4\end{array}$

$\begin{array}{llll}4.5 & 66 & 356 & 9.1\end{array}$

68.443

50.43 .4

68.23 .1

58948

$\begin{array}{ll}57.5 & 3.7\end{array}$

60.140

62720

60.53 .0

60.2 3.5

63.429

63029

62220

$\begin{array}{lll}428 & 4.7\end{array}$

$428 \quad 37$

$41.7 \quad 36$

$41.7 \quad 3.6$

$41.5 \quad 32$

$40.6 \quad 3.1$

$40.1 \quad 3.0$

$40.8 \quad 2.1$

$308 \quad 3.0$

38.93 .0

39030

30.93 .0

61.73 .1

39.93 .0

0220

30.73 .0

2620 


\begin{tabular}{|c|c|c|c|c|c|c|c|c|c|c|c|c|c|c|c|c|c|c|c|c|c|}
\hline Date & Time & $\begin{array}{l}\text { Water } \\
\text { Flow } \\
\text { gpm }\end{array}$ & $\begin{array}{l}\text { Lime } \\
\text { Flow } \\
\text { ogm }\end{array}$ & $\begin{array}{l}\text { Sik } \\
O_{p} \\
x \\
\end{array}$ & $\stackrel{8}{\%}$ & $\begin{array}{r}\text { D1 } \\
\text { KW } \\
\end{array}$ & $\begin{array}{l}\text { splw } \\
\mathrm{mn}\end{array}$ & & $\begin{array}{l}2 \\
\text { spk } \\
\mathrm{mn} \\
\end{array}$ & & $\begin{array}{l}3 \\
\text { sple } \\
\text { mn }\end{array}$ & & $\mathrm{mn}$ & & $m$ & & $\mathrm{~m}$ & $\mathrm{KW}$ & $\mathrm{mn}$ & & $\mathrm{mn}$ \\
\hline $\begin{array}{l}102392 \\
102302\end{array}$ & $\begin{array}{l}315 \\
300\end{array}$ & $\begin{array}{l}320 \\
27.5\end{array}$ & $\begin{array}{l}0.0 \\
0.0\end{array}$ & 14.8 & 18.0 & 30.9 & 3.0 & 28.8 & 5.2 & 50.3 & 29 & 427 & 3.5 & 402 & 40 & 483 & 4.1 & 426 & 3.0 & 46.3 & 10 \\
\hline $\begin{array}{l}1023192 \\
10233192\end{array}$ & $\begin{array}{l}350 \\
345\end{array}$ & $\begin{array}{l}27.5 \\
28.4\end{array}$ & $\begin{array}{l}O D \\
0.0\end{array}$ & $\begin{array}{l}13.6 \\
12.4\end{array}$ & 23.6 & 20.9 & $\begin{array}{l}3.0 \\
3.0\end{array}$ & $\begin{array}{l}\mathbf{2 0 . 8} \\
\mathbf{2 0 . 9}\end{array}$ & 8.9 & 472 & 38 & 372 & 73 & 41.4 & 36 & 0.1 & 72 & 130 & 3.2 & 51.6 & 7.7 \\
\hline $\begin{array}{l}102392 \\
102392\end{array}$ & 400 & 31.1 & 0.0 & $\begin{array}{l}124 \\
13.2\end{array}$ & $\begin{array}{l}18.4 \\
7.5\end{array}$ & 29.3 & $\begin{array}{l}3.0 \\
3.2\end{array}$ & $\begin{array}{l}23.9 \\
27.9\end{array}$ & $\begin{array}{r}10.0 \\
3.5\end{array}$ & 49.4 & 6.1 & 39.9 & 9.9 & $\prod_{303}^{02}$ & $\begin{array}{l}80 \\
38\end{array}$ & 44 & 34 & 436 & 3.0 & 53.8 & 48 \\
\hline 1023192 & 415 & 26.9 & 0.0 & $\begin{array}{l}13.2 \\
13.6\end{array}$ & $\begin{array}{l}20.5 \\
17.7\end{array}$ & $\begin{array}{l}\mathbf{3 0 . 6} \\
\mathbf{2 9 . 0}\end{array}$ & $\begin{array}{l}3.2 \\
3.0\end{array}$ & 20.3 & $\begin{array}{l}3.5 \\
6.7\end{array}$ & $\begin{array}{l}50.4 \\
50.7\end{array}$ & 20 & $\begin{array}{r}420 \\
106\end{array}$ & $\begin{array}{l}4.7 \\
4.9\end{array}$ & 108 & $\begin{array}{l}38 \\
48\end{array}$ & 485 & $\begin{array}{l}6.5 \\
88\end{array}$ & $\begin{array}{l}409 \\
128\end{array}$ & $\begin{array}{l}3.0 \\
30\end{array}$ & $\begin{array}{l}46.3 \\
521\end{array}$ & $\begin{array}{l}3.1 \\
7.4\end{array}$ \\
\hline 102302 & 400 & 20.8 & 0.0 & 15.5 & $\mathbf{2 5 . 6}$ & $\mathbf{2 8 3}$ & 48 & 17.2 & 14.6 & 38.6 & 89 & 278 & 11.1 & 412 & 6.1 & 486 & 80 & $\$ 20$ & 3.0 & 473 & 5.0 \\
\hline 1023192 & 445 & 20.8 & 0.0 & 16.3 & 28.3 & $\mathbf{2 8 . 1}$ & 3.0 & 15.6 & 15.6 & 325 & 10.0 & 25.0 & 10.8 & $\infty 09$ & 36 & 4.1 & 33 & 44.1 & 3.1 & 48.8 & 7.4 \\
\hline 1023192 & 500 & 20.8 & 0.0 & 15.3 & २8.1 & 28.3 & 3.0 & 15.3 & 15.1 & 320 & 0.4 & 25.7 & 10.1 & 40.9 & 30 & 488 & 80 & 435 & 3.0 & 48.9 & 58 \\
\hline 102392 & 515 & 20.5 & 0.0 & 14.6 & 27.0 & 28.2 & 3.0 & 18.3 & 9.7 & 38.4 & 62 & 312 & 92 & 412 & 38 & 0.4 & 4.7 & 44.6 & 3.4 & 48.1 & 4.0 \\
\hline 1023992 & 600 & 24.6 & 0.0 & 15.6 & 220 & 28.3 & 3.0 & 212 & 12.6 & 40.8 & 79 & 36.4 & 98 & 412 & $3 A$ & $\$ 2$ & 85 & 430 & 3.0 & 522 & 8.1 \\
\hline 102392 & 545 & 26.5 & 0.0 & 13.4 & 23,6 & 28.5 & 3.0 & $\mathbf{2 0 . 4}$ & 7.9 & 46.3 & 45 & 37.6 & 7.4 & 412 & 40 & 0.1 & $4 A$ & 4.1 & 3.2 & 527 & 5.7 \\
\hline 1023192 & $6 \infty$ & 0.0 & 28.4 & 14.6 & 19.6 & $\mathbf{2 8 . 4}$ & 3.0 & 23.6 & 7.5 & 46.8 & 5.4 & 34.6 & 9.3 & 41.0 & 3.1 & 40 & $8 A$ & 4.0 & 3.0 & 527 & 55 \\
\hline 1023592 & 615 & 0.0 & 27.9 & 16.6 & 24.7 & $\mathbf{2 8 . 4}$ & 3.0 & 224 & 11.4 & 48.0 & 40 & 34.7 & 7.8 & 0.7 & 93 & & 64 & 43.3 & 3.1 & 521 & 63 \\
\hline $10 / 2392$ & 60 & 0.0 & 30.3 & 13.8 & 18.8 & 292 & 3.6 & 23.2 & 10.0 & 48.8 & 43 & 33.6 & 6.6 & 37.9 & 48 & 4.3 & 40 & 48.1 & 3.3 & 51.0 & 6.1 \\
\hline $10 / 2392$ & 645 & 0.0 & $\mathbf{3 0 . 5}$ & 13.5 & 16.8 & 20.1 & 3.7 & 20.1 & 7.6 & 47.0 & 39 & 38.8 & 78 & $\mathbf{0 B}$ & 78 & 4.5 & 60 & 436 & 3.0 & 512 & 7.0 \\
\hline 102392 & 700 & 0.0 & $\mathbf{3 0 . 5}$ & 142 & 19.3 & $\mathbf{2 8 . 0}$ & 3.7 & 225 & 129 & 50.5 & 63 & $\mathbf{4 0 . 6}$ & 86 & $\$ .7$ & 74 & 90 & 88 & 426 & 3.5 & 48.4 & 53 \\
\hline 1023902 & 715 & 0.0 & 30.6 & 14.6 & 16.7 & 28.7 & 3.0 & 23.0 & 7.9 & 80.0 & 40 & 30.9 & 4.0 & 398 & 38 & $\infty 08$ & SA & 182 & 3.1 & 50.9 & 6.6 \\
\hline $10 / 2392$ & 730 & 0.0 & 31.1 & 16.5 & 16.5 & $\mathbf{2 9 . 4}$ & 3.4 & 20.0 & 120 & $\mathbf{5 0 . 4}$ & 39 & 39.5 & 4.7 & 0.1 & 43 & 51.1 & $\mathbf{5 B}$ & 432 & 4.8 & 50.4 & 7.8 \\
\hline 102392 & 745 & 0.0 & 31.6 & 128 & 16.2 & $\mathbf{2 9 . 0}$ & 3.0 & 24.3 & 8.7 & 542 & 20 & 39.6 & 38 & 309 & 69 & 818 & OS & 48.1 & 3.1 & 513 & 7.1 \\
\hline $10 / 2392$ & 800 & 0.0 & 31.4 & 14.9 & 19.2 & 29.1 & 4.0 & 21.0 & 124 & 81.0 & 5.9 & $\mathbf{3 a . 7}$ & 7.9 & 0.1 & 54 & 821 & 48 & $\mathbf{4 3 . 0}$ & 3.4 & 50.4 & 6.1 \\
\hline 102392 & 815 & 0.0 & 31.4 & 14.9 & 19.2 & $\mathbf{2 9 . 1}$ & 4.0 & 21.0 & 12.4 & 51.0 & 58 & $\mathbf{3 8 . 7}$ & 7.9 & $\$ 2$ & SA & 821 & 48 & 420 & 3.4 & 50.5 & 6.1 \\
\hline 1023/92 & 800 & 0.0 & 35.2 & 124 & 13.4 & $\mathbf{2 9 . 3}$ & 3.0 & 28.6 & 4.0 & 56.6 & 29 & 37.6 & 3.0 & 40.1 & 40 & 81.8 & 4.1 & 40.7 & 39 & 507 & 4.3 \\
\hline 102392 & 845 & 0.0 & 34.7 & 13.1 & 11.4 & 29.8 & 3.0 & 28.4 & 6.7 & 5.3 & 29 & 37.9 & 3.1 & 39.6 & 69 & 80.6 & 6.1 & 42.1 & $11 . e$ & 48.8 & 5.4 \\
\hline 1023192 & 900 & 0.0 & 34.5 & 17.3 & 16.6 & 29.7 & 3.0 & 20.6 & 5.9 & 568 & 20 & 37.5 & 30 & 372 & 80 & 812 & 8.1 & 43.1 & 42 & 466 & 5.0 \\
\hline 1023392 & 915 & 0.0 & 34.1 & 121 & 13.3 & 29.5 & 3.0 & 20.7 & 5.1 & 86.2 & 20 & 37.4 & 30 & 30.8 & 8.1 & 81.9 & 38 & 48.4 & 3.4 & 473 & 8.8 \\
\hline 1023192 & 900 & 0.0 & 34.1 & 16.4 & 15.7 & 29.8 & 3.0 & 30.2 & 6.6 & 658 & 20 & 37.1 & 3.0 & 40.3 & 32 & 81.6 & $7 A$ & 434 & 3.6 & 512 & 6.6 \\
\hline 1023392 & 245 & 0.0 & 34.4 & 14.3 & 125 & $\mathbf{2 9 . 9}$ & 3.0 & 30.8 & 3.8 & 562 & 20 & 368 & 3.0 & 30.5 & 53 & 820 & 8.1 & 406 & 32 & 515 & 8.2 \\
\hline 102392 & 1000 & 0.0 & 34.5 & 13.3 & 128 & 20.9 & 3.0 & 31.4 & 4.0 & 5.7 & 3.0 & 33.7 & 30 & 30.1 & 48 & 81.8 & 80 & 433 & 3.9 & 504 & 5.1 \\
\hline $10 / 2392$ & 1015 & 0.0 & 34.6 & 14.2 & 13.8 & 292 & 3.0 & 321 & 3.2 & 57.9 & 29 & 34.7 & 3.0 & 30.6 & 59 & 81.7 & 63 & 435 & 3.4 & 431 & 3.3 \\
\hline 0,2392 & 1000 & 0.0 & 33.8 & 123 & 10.8 & 29.0 & 3.0 & 325 & 4.5 & 57.8 & 20 & 35.4 & 30 & 100 & 4.1 & $\mathbf{2 0}$ & 49 & 420 & 5.3 & 502 & 5.6 \\
\hline $10 / 2392$ & 1045 & 0.0 & 33.6 & 13.5 & 14.8 & 29.2 & 3.0 & 312 & 3.8 & 57.6 & 20 & 332 & 30 & 402 & 48 & 520 & 48 & 405 & 3.1 & 40 & 3.2 \\
\hline 102392 & 1100 & 0.0 & 35.8 & 14.1 & 123 & 29.9 & 3.0 & 31.8 & 3.5 & 57.9 & 20 & 38.6 & 3.0 & 402 & 34 & 20 & 89 & 485 & 4.1 & 512 & 6.3 \\
\hline 102392 & 1115 & 0.0 & 35.9 & 16.1 & 14.3 & 29.8 & 3.0 & 326 & 32 & 80.1 & 20 & 36.5 & 30 & $\$ 0.4$ & $3 \mathbf{B}$ & 523 & 66 & 426 & 5.3 & 520 & 6.5 \\
\hline $10 / 23 / 92$ & 1130 & 0.0 & 36.2 & 14.2 & 14.2 & 30.3 & 3.0 & 324 & 4.3 & 80.4 & 29 & $\mathbf{3 a 7}$ & 30 & 37.5 & 58 & 822 & 7.1 & 400 & 4.6 & 522 & 7.0 \\
\hline 102392 & 1145 & 0.0 & 36.3 & 17.8 & 125 & 30.3 & 3.0 & 327 & 3.5 & 58.4 & 20 & 37.1 & 30 & 40.2 & 40 & 824 & 58 & 430 & 4.6 & 538 & 50 \\
\hline $10 / 2392$ & 1200 & 0.0 & 36.2 & 13.1 & 18.1 & 30.0 & 3.0 & 329 & 3.3 & $\mathbf{5 8 . 7}$ & 29 & 37.4 & 30 & $\mathbf{0 . 6}$ & 34 & 81.6 & 40 & 43.7 & 5.5 & 51.1 & 52 \\
\hline V23.92 & 1215 & 0.0 & 34.9 & 14.5 & 14.6 & 292 & 3.0 & 34.4 & 3.1 & 60.4 & 20 & 362 & 3.0 & 40.2 & 38 & 51.7 & 65 & 43.5 & 5.6 & 50.6 & 4.1 \\
\hline 1023992 & 1230 & 0.0 & 33.6 & 14.1 & 13.3 & 28.7 & 3.0 & 31.9 & 6.7 & 60.6 & 20 & 365 & 3.0 & 402 & 5.5 & 520 & 48 & 43.7 & 8.0 & 47.5 & 4.3 \\
\hline 123192 & 1245 & 0.0 & 35.6 & 125 & 123 & 29.1 & 3.0 & 33.4 & 6.6 & $\boldsymbol{\infty}, 3$ & 29 & 372 & 3.0 & 30.7 & 5.7 & $\mathbf{\infty 0 . 1}$ & 4.7 & 48.0 & 8.8 & 56.4 & 4.9 \\
\hline 392 & 1300 & 0.1 & 36.4 & 14.0 & 13.3 & 30.0 & 3.0 & 323 & 4.6 & 59.8 & 20 & 382 & 3.0 & 40.5 & 56 & 521 & 45 & 4.0 & 45 & 56.4 & 4.5 \\
\hline 1023192 & 1315 & 0.1 & 38.1 & 14.6 & 13.9 & $\mathbf{3 0 . 3}$ & 3.0 & 329 & 4.4 & 60.7 & 20 & 32.1 & 30 & $\$ 0.5$ & 59 & 51.1 & 3.1 & 42.6 & 78 & 52.1 & 3.0 \\
\hline 102392 & 1350 & 0.1 & 38.6 & 16.8 & 132 & 29.9 & 3.0 & 34.8 & 3.1 & 61.6 & 29 & 37.3 & 3.0 & 40.4 & 5.7 & $\mathbf{\infty 0 2}$ & 43 & 43.4 & 64 & 49.7 & 3.0 \\
\hline 102392 & 1345 & 0.1 & 38.5 & 13.0 & 14.0 & $\mathbf{2 0 . 7}$ & 3.0 & 34.0 & 42 & 620 & 20 & 373 & 3.0 & 306 & 80 & 42 & 44 & 43.0 & 73 & 52.0 & 3.0 \\
\hline $10 / 2392$ & 1400 & 0.1 & $\mathbf{3 8 . 6}$ & 13.0 & 14.9 & 20.7 & 3.0 & 34.0 & 4.2 & 620 & 20 & 37.3 & 3.0 & 30.6 & 80 & 42 & 44 & 43.0 & 73 & 52.0 & 30 \\
\hline 102392 & 1415 & 0.1 & 38.5 & 13.0 & 14.8 & 29.7 & 3.0 & 34.0 & 4.2 & 620 & 20 & 37.3 & 3.0 & 30.6 & 50 & 42 & 44 & 43.0 & 73 & 52.0 & 3.0 \\
\hline $10 / 23 / 92$ & 1430 & 0.1 & 38.5 & 13.0 & 14.9 & 99.7 & 3.0 & 34.0 & 42 & 620 & 20 & 37.3 & 3.0 & 398 & 50 & 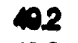 & 44 & 43.0 & 73 & 52.0 & 30 \\
\hline 1023192 & 1445 & 0.1 & 40.8 & 13.8 & 126 & 20.6 & 3.0 & 36.6 & 3.3 & 623 & 29 & 38.6 & 3.0 & 330 & 64 & 493 & 32 & 43.4 & 80 & 56.2 & 3.0 \\
\hline 102392 & 1500 & 0 & 38.5 & 130 & 14.9 & 9.7 & 3.0 & 34.0 & 42 & 620 & 20 & 57.3 & 30 & 398 & 50 & 42 & 44 & 43.0 & 73 & 52.0 & 3.0 \\
\hline 2392 & 1515 & 0.1 & 38.5 & 3.0 & 14.9 & 20.7 & 3.0 & 34.0 & 4.2 & 620 & 20 & 37.3 & 3.0 & 396 & 80 & 02 & 44 & 430 & 73 & 52.0 & 3.0 \\
\hline 392 & 1530 & 0.1 & 38.5 & 13.0 & 14.9 & 29.7 & 3.0 & 34.0 & 4.2 & 620 & 20 & 37.3 & 3.0 & 30.6 & 5.0 & $\$ 2$ & 44 & 42.0 & 73 & 52.0 & 30 \\
\hline 392 & 1545 & 02 & 37.8 & 15.5 & 13.4 & 29.2 & 3.0 & 33.3 & 4.4 & 63.8 & 20 & 37.3 & 30 & 34.1 & 127 & 482 & 35 & 43.4 & 126 & 56.2 & 3.0 \\
\hline 3992 & 1600 & 02 & 33.6 & 16.5 & 126 & 28.2 & 3.0 & 328 & 4.9 & 626 & 20 & 326 & 30 & 35.6 & 0.1 & 802 & 6.1 & 42 & 73 & 54.7 & 30 \\
\hline 023392 & 1615 & 02 & 34.1 & 13.0 & 11.8 & 28.4 & 3.0 & $\mathbf{2 0 . 4}$ & 5.7 & 622 & 29 & 39.0 & 3.0 & 35.7 & 88 & 60.1 & 5.3 & 45 & 6.1 & 56.8 & 3.0 \\
\hline 102392 & 1000 & 02 & 36.2 & 11.8 & 13.7 & 29.4 & 3.0 & 30.0 & 7.3 & 61.9 & 29 & 40.0 & 3.0 & $\mathbf{2 8 6}$ & 13.0 & 47.4 & 78 & 37.0 & 78 & 57.5 & 3.0 \\
\hline 102392 & 1645 & 02 & 36.8 & 121 & 13.5 & 29.7 & 3.0 & 320 & 5. & 201 & 20 & $\$ 0.0$ & 32 & 300 & 136 & 40 & 43 & 4.0 & 68 & 56.7 & 3.1 \\
\hline 102392 & 1700 & 02 & 36.9 & 13.0 & 14.3 & 298 & 3.0 & 31.6 & 0.0 & 61.6 & 20 & 10.3 & 30 & 31.3 & 114 & $\mathbf{\infty 2 2}$ & 54 & 4.0 & 7.1 & 57.8 & 3.0 \\
\hline $10 / 23192$ & 1715 & 02 & $\mathbf{3 8 . 9}$ & 133 & 14.6 & 202 & 3.0 & 35.3 & 3.1 & 605 & 20 & 37.5 & 30 & 200 & 134 & 487 & 65 & 40.9 & 7.6 & 56.2 & 3.0 \\
\hline 102392 & 1750 & 0. & 34.6 & 15.1 & 14.8 & 28.1 & 3.0 & 24.9 & 108 & 61.2 & 30 & 0.7 & 31 & 30.1 & 94 & $\mathbf{4 2 7}$ & 42 & 120 & 8.1 & 51.2 & 3.0 \\
\hline 100 & 1745 & 02 & $\mathbf{3 3 . 0}$ & 13.6 & 17.8 & 28.8 & 3.0 & 25.8 & 8.6 & 61.1 & 35 & 40.8 & 30 & 312 & 128 & 473 & 58 & 425 & 121 & 98 & 3.0 \\
\hline 192 & 1800 & 02 & 34.8 & 126 & 16.5 & 28.6 & 3.5 & 29.8 & 6.1 & 61.2 & 20 & 40.3 & 30 & 31.5 & 127 & $\infty 2$ & 3.7 & $\mathbf{4 8 0}$ & 8.6 & 575 & 3.0 \\
\hline$\sqrt{23492}$ & 1815 & 02 & 30.5 & 0.1 & 19.0 & 278 & 3.0 & 242 & 9.2 & $\boldsymbol{6 0 . 5}$ & 35 & $\$ 0.0$ & 21 & 300 & 127 & $\mathbf{\infty 0 . 7}$ & 4.7 & 48 & 76 & 5.7 & 3.0 \\
\hline Q23192 & 18200 & 02 & 30.8 & 172 & 21.2 & 23.4 & 3.0 & 21.8 & 118 & 52.1 & 36 & 39.4 & 58 & 307 & 182 & 827 & 122 & 4.0 & 58 & 55.3 & 3.0 \\
\hline & 1845 & 02 & 30.3 & 13.5 & 14.3 & 28.1 & 3.0 & 25.3 & 9.8 & 50.2 & 30 & $\$ .0$ & 31 & 31.7 & 127 & 512 & 34 & 4.7 & 6.4 & 549 & 3.0 \\
\hline & 1800 & 02 & 31.7 & 15.1 & 20.7 & 28.1 & 3.0 & 225 & 11.5 & 80.4 & 32 & $\$ .8$ & 3.4 & 30.2 & 236 & 524 & 13.1. & 4.4 & 6.5 & 56.5 & 30 \\
\hline 102392 & 1915 & 02 & 30.9 & 15.2 & 17.8 & 28.1 & 3.0 & 24.1 & 9.5 & 58.6 & 4.1 & 41.0 & 3.7 & 330 & 162 & 51.4 & 48 & 48 & 6.8 & 54.4 & 3.0 \\
\hline
\end{tabular}




\begin{tabular}{|c|c|c|c|c|c|c|c|c|c|c|c|c|c|c|c|c|c|c|c|c|c|}
\hline Date & Time & $\begin{array}{l}\text { Water } \\
\text { Flow } \\
\text { opm }\end{array}$ & $\begin{array}{l}\text { Ume } \\
\text { Flow } \\
\text { gom }\end{array}$ & $\begin{array}{l}\text { s.k } \\
\text { क } \\
\%\end{array}$ & $\begin{array}{r}\mathbf{B} \\
\rho^{\circ} \\
\end{array}$ & $\begin{array}{r}0 \\
K W \\
\end{array}$ & & & $\begin{array}{l}\text { sple } \\
\mathrm{mn}\end{array}$ & & $\mathrm{mn}$ & & & $\mathbf{K W}$ & & $K W$ & $m$ & $\mathrm{KW}$ & $m n$ & $\mathrm{~kW}$ & $m n$ \\
\hline 02392 & 1800 & 02 & 30.4 & 16.6 & $\begin{array}{l}20.2 \\
170\end{array}$ & 28.2 & 3.0 & 23.2 & 7.4 & 55.0 & 40 & 39.8 & $\begin{array}{l}5.9 \\
15\end{array}$ & $\begin{array}{l}34.1 \\
36.2\end{array}$ & $\begin{array}{r}168 \\
71\end{array}$ & $\begin{array}{l}511 \\
620\end{array}$ & $\begin{array}{l}65 \\
38\end{array}$ & $\begin{array}{r}4.6 \\
4.0\end{array}$ & $\begin{array}{l}3.7 \\
6.0\end{array}$ & $\begin{array}{l}51.6 \\
563\end{array}$ & 30 \\
\hline $\begin{array}{l}102392 \\
102392\end{array}$ & $\begin{array}{l}1945 \\
2000\end{array}$ & 02 & 27.7 & 13.0 & 17.9 & $\begin{array}{l}27.9 \\
27.9\end{array}$ & $\begin{array}{l}3.0 \\
3.0\end{array}$ & 24.4 & 14.4 & 57.1 & 5.7 & $\begin{array}{l}402 \\
0.1\end{array}$ & $\begin{array}{l}4.5 \\
3.7\end{array}$ & $\begin{array}{l}302 \\
326\end{array}$ & $\begin{array}{l}7.1 \\
98\end{array}$ & 60 & $\begin{array}{l}316 \\
80\end{array}$ & $\begin{array}{l}18.0 \\
46.4\end{array}$ & $\begin{array}{l}6.0 \\
6.3\end{array}$ & $\begin{array}{l}8.3 \\
4.5\end{array}$ & $\begin{array}{l}3.0 \\
30\end{array}$ \\
\hline $\begin{array}{l}1023922 \\
102392\end{array}$ & $\begin{array}{l}2000 \\
2015\end{array}$ & $\begin{array}{l}02 \\
02\end{array}$ & $\begin{array}{l}27.9 \\
29.0\end{array}$ & $\begin{array}{l}15.2 \\
16.8\end{array}$ & $\begin{array}{l}20.2 \\
23.6\end{array}$ & $\begin{array}{l}27.9 \\
27.7\end{array}$ & $\begin{array}{l}3.0 \\
3.0\end{array}$ & $\begin{array}{l}19.8 \\
21.7\end{array}$ & $\begin{array}{l}13.4 \\
11.3\end{array}$ & $\begin{array}{l}56.0 \\
55.3\end{array}$ & $\begin{array}{l}36 \\
49\end{array}$ & $\begin{array}{l}40.1 \\
38.9\end{array}$ & 7.4 & 326 & 1603 & 828 & 33 & 46.1 & 4.7 & 81.0 & 3.0 \\
\hline 102302 & 2000 & 02 & 28.1 & 14.7 & 20.0 & 27.8 & 3.0 & 232 & 10.5 & 50.4 & 8.7 & 30.4 & 5.9 & 33.0 & 79 & 828 & 60 & 452 & 3.9 & 46.7 & 3.0 \\
\hline 1023992 & 2045 & 0.1 & 30.5 & 15.4 & 19.3 & 282 & 3.0 & $\mathbf{2 0 . 2}$ & 13.1 & 525 & 4.6 & $\mathbf{\omega . 6}$ & 79 & 335 & 136 & 818 & 4.7 & 42 & 4.0 & 51.5 & 3.0 \\
\hline 102392 & 2100 & 0.1 & 32.5 & 14.4 & 16.3 & 28.8 & 3.0 & 28.1 & 9.7 & 57.6 & 43 & 398 & 3.5 & 32.6 & 88 & 50.4 & 34 & $\mathbf{6 0 3}$ & 6.3 & 34.9 & 3.0 \\
\hline 102392 & 2115 & 0.1 & 34.5 & 12.8 & 13.6 & 29.2 & 3.0 & 27.9 & 5.5 & 57.3 & 3.0 & 30.1 & 3.0 & 349 & 10.4 & 51.5 & 79 & 43.0 & 5.5 & 53.4 & 3.0 \\
\hline $10 / 2392$ & 2130 & 0.1 & 328 & 14.2 & 14.5 & 20.1 & 3.0 & 23.9 & 9.6 & 56.2 & 4.1 & 30.0 & 3.7 & 30.7 & 68 & 80 & 4.1 & 4.7 & 3.7 & 54.3 & 30 \\
\hline 102392 & 2145 & 0.1 & 33.8 & 14.8 & 15.2 & 288 & 3.0 & 20.0 & 7.3 & 56.6 & 3.7 & 30.1 & 3.0 & 303 & 88 & 526 & 3.1 & 4.4 & 5.4 & 54.1 & 3.0 \\
\hline $10 / 23192$ & 2000 & 0.1 & 324 & 14.6 & 13.3 & $\mathbf{2 0 . 4}$ & 3.0 & 20.0 & 8.0 & 563 & 38 & 39.6 & 3.7 & 30.1 & 95 & 500 & 48 & 4.4 & B.5 & 80.7 & 3.0 \\
\hline 023992 & 2216 & 0.1 & 35.2 & 11.7 & 13.4 & $\mathbf{2 9 . 0}$ & 3.1 & 25.5 & 5.4 & 56.0 & 3.1 & 37.9 & 36 & 300 & 9.1 & $\omega .6$ & 38 & 43.0 & 8.6 & 625 & 3.0 \\
\hline 22392 & 2200 & 0.1 & 32.6 & 13.9 & 13.8 & 28.6 & 3.0 & 20.3 & 9.1 & 57.7 & 34 & 395 & 30 & 33.4 & 100 & 51.1 & 30 & 405 & 9.1 & 342 & 3.0 \\
\hline Q239192 & 2245 & 0.1 & 325 & 15.1 & 15.6 & $\mathbf{2 0 . 0}$ & 3.0 & 220 & 102 & 58.8 & 3.7 & 39.0 & 3.4 & 362 & 08 & 480 & 38 & 426 & 6.3 & 51.0 & 32 \\
\hline 10/23192 & 2000 & 0.1 & 324 & 15.8 & 15.8 & $\mathbf{2 8 . 7}$ & 3.0 & 24.9 & 78 & 57.1 & 3.1 & 38.6 & 3.4 & 318 & 80 & 62 & 30 & 430 & 3.8 & 518 & 3.0 \\
\hline 102392 & 2315 & 27.8 & 0.0 & 14.6 & 20.6 & 29.3 & 3.0 & 20.0 & 10.3 & 48.5 & 60 & $\mathbf{3 5 . 8}$ & 9.1 & 346 & 60 & 51.1 & 38 & 4.4 & 3.4 & 48.1 & 3.0 \\
\hline $102 \times 192$ & 2350 & 31.9 & 0.0 & 15.6 & 18.5 & 30.8 & 3.0 & 25.3 & 7.8 & 53.3 & 3.1 & $\$ 08$ & 33 & 378 & $8 A$ & $\mathbf{0 . 7}$ & 30 & 488 & 3.1 & 53.7 & 3.1 \\
\hline $102 \times 192$ & 2345 & 31.9 & 0.0 & 16.5 & 18.5 & 30.8 & 3.0 & 25.3 & 7.6 & 53.3 & 3.1 & 40.8 & 33 & 378 & 6A & $\boldsymbol{\infty} .7$ & 30 & 48 & 3.1 & 53.7 & 3.1 \\
\hline $10 / 2492$ & 0 & 31.1 & 0.0 & 13.9 & 13.4 & 20.9 & 3.0 & 27.8 & 7.0 & 54.0 & 20 & 39.6 & 30 & 324 & 6.4 & 61.1 & 33 & 4.5 & 3.0 & 526 & 40 \\
\hline 02492 & 15 & 31.1 & 0.0 & 13.9 & 13.4 & 29.0 & 3.0 & 27.8 & 7.0 & 84.0 & 20 & 39.6 & 3.0 & 324 & 64 & 51.1 & 33 & 4.6 & 3.0 & 526 & 4.0 \\
\hline $1 / 2492$ & 30 & 31.1 & 0.0 & 13.9 & 13.4 & $\mathbf{2 0 . 9}$ & 3.0 & 27.8 & 7.0 & 54.0 & 20 & 39.6 & 3.0 & 38.4 & $6 A$ & 51.1 & 33 & 45 & 3.0 & 526 & 40 \\
\hline 102492 & 45 & 31.1 & 0.0 & 13.9 & 13.4 & 29.9 & 3.0 & 27.8 & 7.0 & 54.0 & 20 & $\mathbf{3 9 . 6}$ & 3.0 & 324 & 64 & 61.1 & 33 & 44.5 & 3.0 & 526 & 4.0 \\
\hline 102402 & 100 & 33.9 & 0.0 & 14.8 & 122 & $\mathbf{3 0 . 8}$ & 3.0 & 29.6 & 3.3 & 53.1 & 3.0 & $\$ .0$ & 3.0 & 37.6 & 74 & $\mathbf{\infty 0 . 6}$ & 32 & 4.3 & 3.1 & 532 & 3.8 \\
\hline 102492 & 115 & 33.9 & 0.0 & 14.0 & 122 & $\mathbf{3 0 . 8}$ & 3.0 & 20.6 & 3.3 & 53.1 & 30 & $\$ 0.0$ & 3.0 & 37.6 & 74 & $\infty 26$ & 32 & 443 & 3.1 & 532 & 3.8 \\
\hline 102492 & 130 & 33.6 & 00 & 20.4 & 129 & 28.7 & 3.0 & 30.2 & 3.1 & 56.1 & 20 & 334 & 30 & 344 & 63 & 808 & SA & 4.6 & 3.0 & 49.0 & 4.3 \\
\hline 102492 & 145 & 33.6 & 0.0 & 20.4 & 129 & 28.7 & 3.0 & 30.2 & 3.1 & 56.1 & 29 & 33.4 & 30 & 34.4 & 63 & $\infty 08$ & 54 & 4.6 & 3.9 & 49.9 & 4.3 \\
\hline $10 / 24 / 92$ & 200 & 29.6 & 0.0 & 10.7 & 78 & 28.8 & 3.0 & 31.7 & 3.1 & 57.4 & 20 & 34.3 & 3.0 & 320 & 82 & .04 & 42 & 4.1 & 33 & 502 & 4.6 \\
\hline $10 / 2492$ & 215 & 30.2 & 0.0 & 11.5 & 37.6 & 20.2 & 3.0 & 31.8 & 3.1 & 57.3 & 20 & 34.0 & 3.0 & 30.6 & 9.1 & 81.4 & 48 & 430 & 43 & 51.3 & 32 \\
\hline 102492 & 200 & 29.8 & 0.0 & 21.5 & 8.6 & 29.0 & 3.0 & 320 & 3.1 & 573 & 20 & 33.0 & 30 & 326 & 10.1 & 203 & 88 & 435 & 3.5 & 49.3 & 4.1 \\
\hline $0 / 2492$ & 245 & 29.9 & 00 & 19.6 & 86 & $\mathbf{2 9 . 0}$ & 3.0 & 31.5 & 3.1 & 565 & 32 & 328 & 30 & 33.4 & $\boldsymbol{Q S}$ & 93 & 58 & 120 & 3.1 & 50.5 & 4.9 \\
\hline $10 / 2492$ & 300 & 29.9 & 0.0 & 17.5 & 17.6 & $\mathbf{2 9 . 7}$ & 3.1 & $\mathbf{2 8 . 7}$ & 3.1 & 529 & 20 & 323 & 3.0 & 33.4 & 10.5 & $\mathbf{4 6 5}$ & 8.7 & 122 & 6.1 & 4.0 & 42 \\
\hline 102492 & 315 & 30.3 & 0.0 & 14.6 & 8.4 & 29.0 & 3.0 & 29.6 & 3.1 & 54.0 & 29 & 326 & 30 & 330 & 88 & 805 & 88 & 49.1 & 6.6 & 49.2 & 3.6 \\
\hline 102492 & 350 & 29.8 & 0.0 & 10.6 & 10.7 & 20.2 & 3.0 & 29.5 & 3.1 & 53.8 & 20 & 325 & 3.0 & 366 & 70 & 810 & 3.7 & 443 & 43 & 4.1 & 30 \\
\hline 102402 & 345 & 30.6 & 0.0 & 11.7 & 92 & 29.1 & 3.0 & $\mathbf{2 9 . 0}$ & 3.1 & 63.0 & 20 & 320 & 3.0 & 363 & 72 & 002 & 48 & 438 & 3.6 & 426 & 3.1 \\
\hline $10 / 2492$ & 400 & 30.5 & 0.0 & 11.5 & 8.4 & 29.3 & 3.0 & $\mathbf{2 9 . 0}$ & 3.1 & 526 & 20 & 31.7 & 30 & 338 & 03 & 512 & 48 & 4.1 & 3.4 & 49.8 & 4.4 \\
\hline $10 / 2492$ & 415 & 28.8 & 0.0 & 11.8 & 8.4 & 29.0 & 3.0 & $\mathbf{2 8 . 3}$ & 3.1 & 51.5 & 20 & 31.1 & 3.0 & 362 & 102 & 800 & 30 & 43 & 3.5 & 422 & 3.3 \\
\hline 02492 & 430 & 30.1 & 0.0 & 17.7 & 8.8 & 29.0 & 3.0 & 28.2 & 3.1 & 512 & 29 & 30.9 & 3.0 & 36.4 & 92 & $\infty 0.7$ & 44 & 4.7 & 4.2 & 48.4 & 5.2 \\
\hline $0 / 2492$ & 445 & 30.1 & 0.0 & 13.8 & 8.5 & 28.7 & 3.0 & 28.0 & 3.1 & 50.0 & 29 & 30.7 & 3.0 & 323 & 74 & 516 & 3.8 & 4.0 & 3.5 & 480 & 3.5 \\
\hline 02492 & 500 & 30.3 & 0.0 & 13.0 & 83 & 28.7 & 3.0 & 27.9 & 3.1 & $\mathbf{8 0 . 6}$ & 20 & 30.6 & 3.0 & 300 & 76 & $\mathbf{\infty 0 3}$ & 44 & 4.5 & 3.1 & 470 & 3.6 \\
\hline 02492 & 515 & 29.6 & 0.0 & 13.6 & 16.6 & ฉ8.6 & 3.0 & 27.8 & 3.0 & 80.5 & 20 & 30.6 & 30 & 37.3 & 8.1 & 806 & 43 & 43.7 & 3.7 & 450 & 42 \\
\hline 02492 & 500 & 29.9 & 0.0 & 127 & 8.1 & $\mathbf{2 8 . 9}$ & 3.0 & 28.0 & 3.0 & $\infty .7$ & 20 & 30.6 & 3.0 & 306 & 103 & 51.1 & 38 & 42 & 4.3 & 43.4 & 42 \\
\hline 02492 & 546 & 20.7 & 0.0 & 11.9 & 82 & 28.8 & 3.0 & 28.0 & 3.1 & 80.8 & 20 & 30.8 & 30 & 348 & 8.1 & 803 & 4.1 & 485 & 3.4 & 28 & 4.3 \\
\hline $10 / 2492$ & $6 \infty$ & 30.4 & 0.0 & 14.1 & 8.4 & 20.0 & 3.0 & 27.9 & 3.1 & $\mathbf{5 0 . 4}$ & 29 & $\mathbf{5 0 . 9}$ & 30 & 343 & 92 & 800 & 43 & 438 & 4.7 & 430 & 5.7 \\
\hline $10 / 2492$ & 615 & 30.2 & 0.0 & 11.8 & 8.0 & 29.4 & 3.0 & 28.6 & 3.0 & 520 & 29 & 31.0 & 30 & 363 & 85 & 000 & 39 & 42 & 3.3 & 48.3 & 5.4 \\
\hline 02492 & 600 & 30.1 & 0.0 & 11.6 & 8.1 & 29.4 & 3.0 & 28.6 & 3.1 & 522 & 29 & 31.1 & 30 & 347 & 04 & 61.3 & 48 & 43.0 & 4.3 & 438 & 3.8 \\
\hline $10 / 2492$ & 645 & 30.1 & 0.0 & 13.7 & 83 & 29.6 & 3.0 & 28.8 & 3.1 & 527 & 29 & 31.4 & 3.0 & 328 & 8.1 & 485 & 69 & 412 & 4.6 & 456 & 4.5 \\
\hline 02492 & 700 & 27.4 & 0.0 & 11.0 & 82 & 28.1 & 3.0 & 30.2 & 3.1 & 56.5 & 29 & 329 & 3.0 & 30 & 92 & 208 & 38 & 433 & 6.0 & 480 & 3.4 \\
\hline $0 / 2492$ & 715 & 27.9 & 0.0 & 13.8 & 90 & 288 & 3.0 & 31.6 & 3.1 & 57.0 & 20 & 33.9 & 3.0 & 342 & 133 & 51.7 & $3 A$ & 4.6 & 4.0 & 500 & 3.3 \\
\hline $0 / 2492$ & 730 & 27.9 & 0.0 & 163 & 120 & 28.8 & 3.0 & 31.8 & 4.0 & 56.3 & 20 & 36.1 & 3.0 & 343 & 103 & 51.1 & 4.7 & 4.6 & 3.2 & 405 & 3.3 \\
\hline $0 / 2492$ & 745 & 28.0 & 0.0 & 16.7 & 14.1 & 28.5 & 3.0 & 28.6 & 6.9 & 54.9 & 20 & 39.1 & 3.1 & 34.4 & 133 & 51.6 & 38 & 4.6 & 3.1 & 53.3 & 4.4 \\
\hline O2492 & 800 & 27.9 & 0.0 & 16.3 & 122 & 29.0 & 3.0 & 29.6 & 4.1 & 55.1 & 20 & 302 & 30 & 34.7 & 141 & 824 & 68 & 42 & 3.6 & 53.0 & 3.6 \\
\hline OR492 & 815 & 27.1 & 0.0 & 13.5 & 14.3 & 20.1 & 3.4 & 24.9 & 112 & 524 & 40 & 30.9 & 32 & 384 & 54 & 58 & 4.1 & 42 & 3.1 & 432 & 3.0 \\
\hline 0,2492 & 830 & 27.9 & 0.0 & 15.6 & 14.6 & 28.6 & 3.0 & 24.7 & 8.0 & 51.8 & 35 & 41.1 & 62 & 37.3 & 126 & 51.7 & 5.1 & 430 & 6.0 & 508 & 3.0 \\
\hline 02492 & 845 & 27.4 & 0.0 & 15.5 & 23.3 & 28.9 & 3.0 & 23.0 & 13.6 & 51.7 & 5.7 & $\$ 1.0$ & 7.6 & 332 & 02 & $\infty 8$ & 48 & 46.1 & 3.0 & 531 & 4.6 \\
\hline $0 / 2402$ & $\infty \infty$ & 25.7 & 0.0 & 13.9 & 20.1 & 28.9 & 3.0 & 25.4 & 9.2 & 50.8 & 38 & 41.1 & 5.1 & 362 & 122 & $\infty 0$ & 36 & 448 & 5.1 & 54.1 & 4.3 \\
\hline OR492 & 915 & $\mathbf{2 8 . 0}$ & 0.0 & 14.4 & 21.9 & 30.6 & 3.1 & 25.8 & 7.4 & 50.4 & 36 & 42.1 & 7.0 & 325 & 8.8 & 478 & 4.7 & 308 & 4.8 & 544 & 4.4 \\
\hline 102492 & 900 & 27.1 & 0.0 & 16.4 & 15.8 & 28,4 & 3.0 & 220 & 8.7 & 44.8 & 75 & 36.7 & 7.7 & 388 & 128 & $\infty$ & 50 & 48 & 5.8 & 54.1 & 5.3 \\
\hline 02492 & 945 & 26.6 & 0.0 & 15.0 & 14.4 & 29.8 & 3.0 & 24.1 & 10.9 & 48.7 & 7.1 & 383 & 88 & 37.6 & 126 & 204 & 3.7 & 4.9 & 3.1 & 54.7 & 32 \\
\hline OR492 & 1000 & 27.0 & 0.0 & 14.7 & 16.7 & 29.7 & 3.0 & 20.6 & 9.6 & 458 & 40 & 32.4 & 65 & 37.1 & 128 & 500 & 58 & 427 & 38 & 53.5 & 3.5 \\
\hline 102492 & 1015 & 27.0 & 0.0 & 202 & 20.9 & 29.6 & 3.0 & 252 & 7.5 & $\mathbf{5 0 . 7}$ & 6.1 & 41.6 & 25 & 37.1 & 88 & 527 & 3.7 & 45.0 & 3.3 & 53.6 & 3.1 \\
\hline $0 / 2492$ & 1000 & 26.8 & 0.0 & 15.0 & 14.7 & 29.1 & 3.0 & 23.7 & 8.8 & 46.7 & 62 & 350 & 88 & 35 & 95 & 824 & 4.1 & 42 & 3.1 & 53.0 & 3.6 \\
\hline$\sqrt{2492}$ & 1045 & 27.1 & 0.0 & 128 & 14.1 & 29.0 & 3.4 & 220 & 8.3 & 45.4 & 42 & 37.3 & 7.2 & 30 & 88 & 01 & 3.7 & 4.9 & 32 & 49.8 & 3.0 \\
\hline 192 & 1100 & 27.0 & 0.0 & 13.8 & 14.4 & 29.5 & 3.0 & 20.1 & 9.5 & 47.4 & 5.5 & 36.4 & 7.5 & 35.4 & 02 & 512 & 4.1 & 443 & 6.5 & 54.9 & 3.0 \\
\hline NeW & 1115 & 27.2 & 0.0 & 17.0 & 17.0 & 29.8 & 3.0 & 202 & 9.0 & 46.9 & 58 & 36.9 & 98 & 365 & 112 & exo & 33 & 45.1 & 3.5 & 54.7 & 3.0 \\
\hline ORAS & 1130 & 27.0 & 0.0 & 14.4 & 14.9 & 29.8 & 3.0 & 222 & 10.1 & 44.6 & 6.1 & 355 & 92 & 34.1 & 8.5 & 50 & 5.7 & 43.1 & 4.3 & 54.7 & 3.3 \\
\hline
\end{tabular}




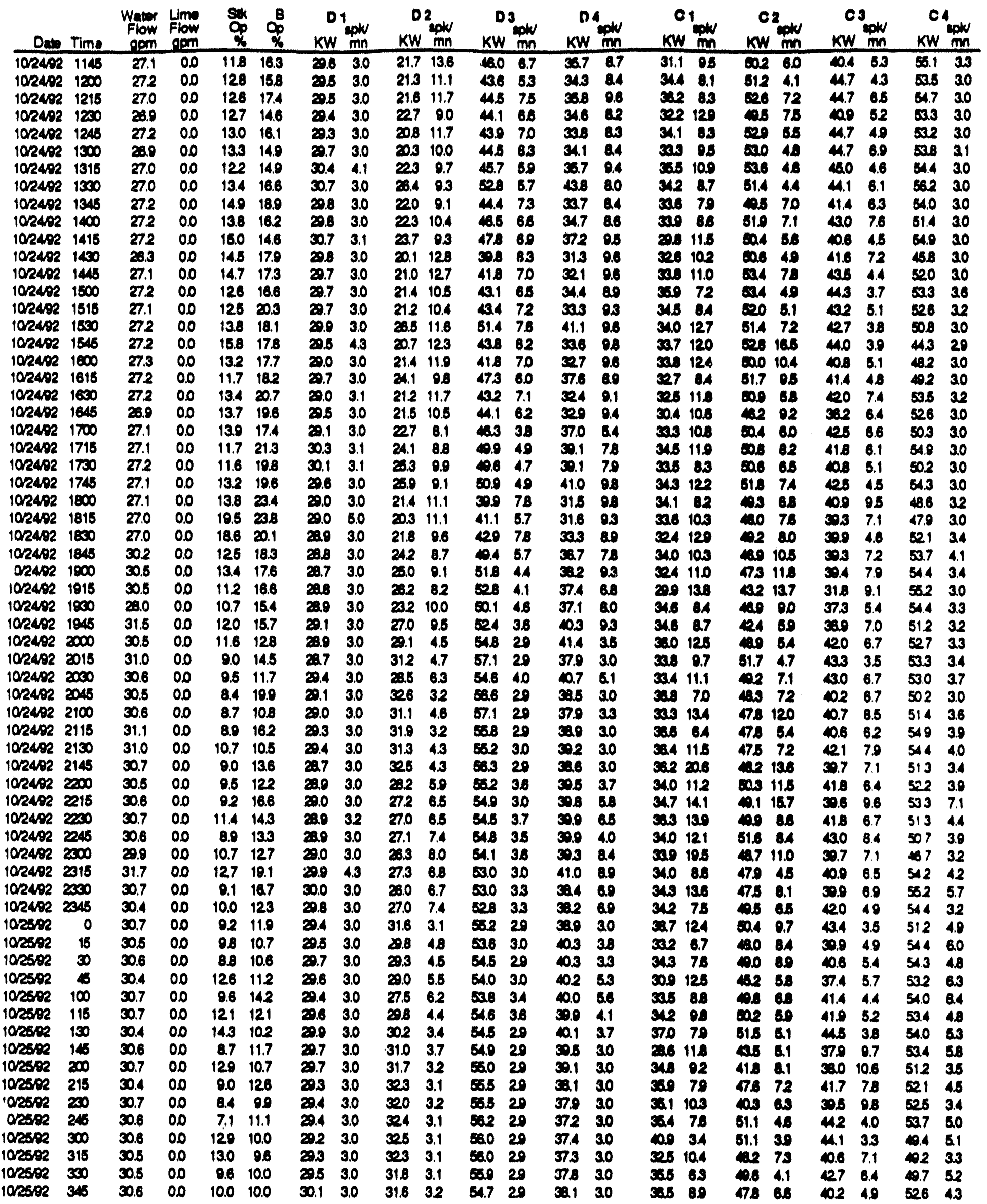




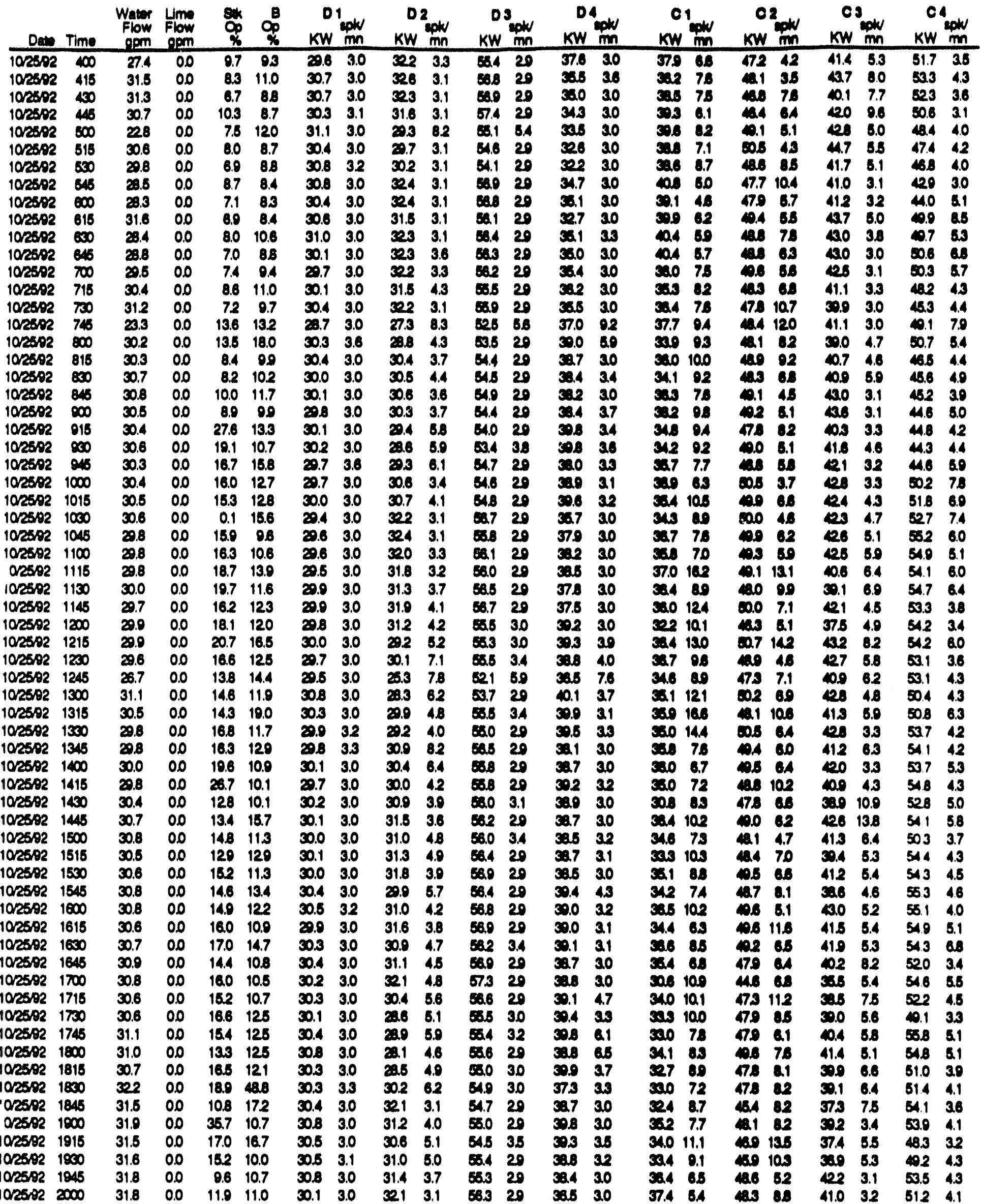




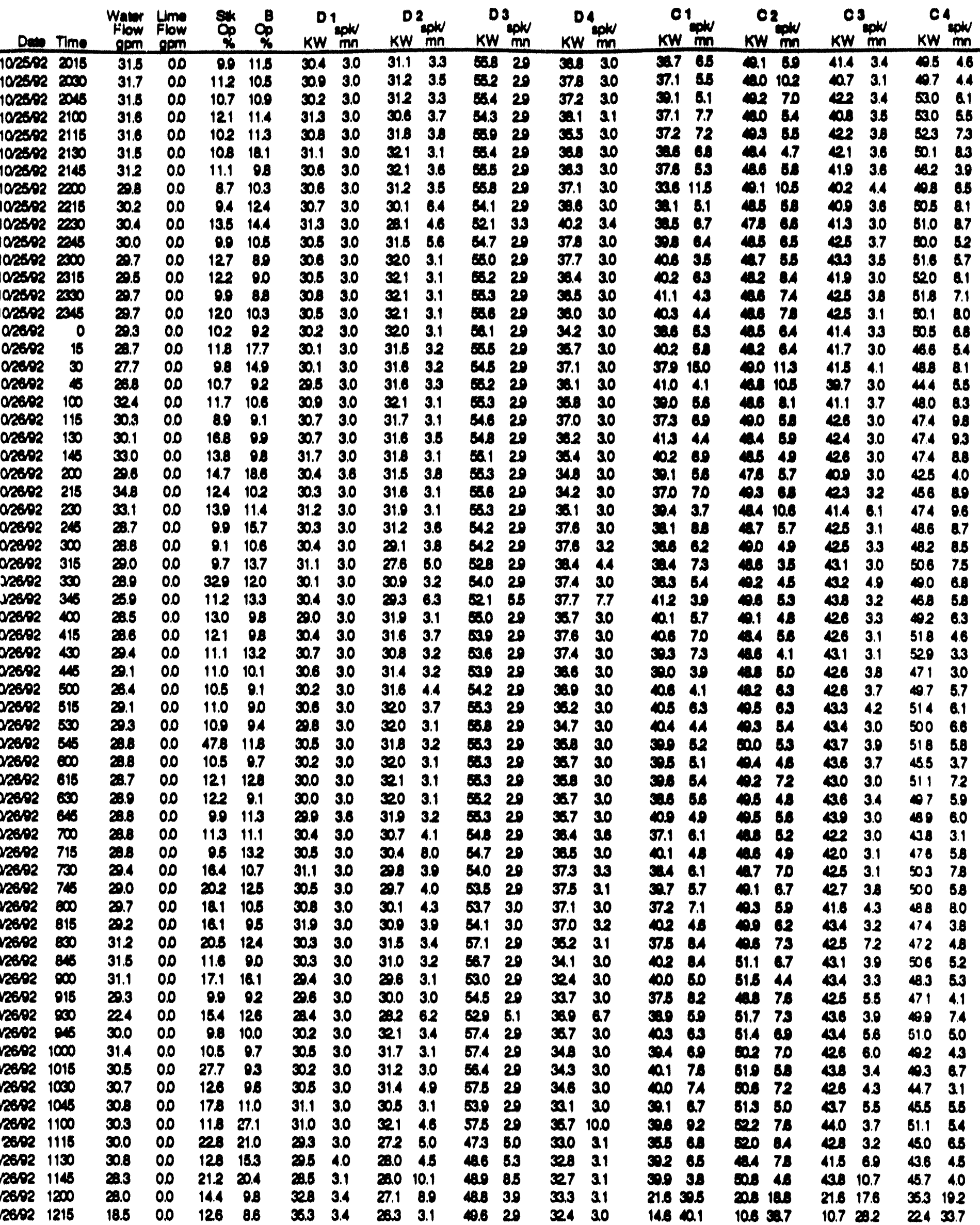




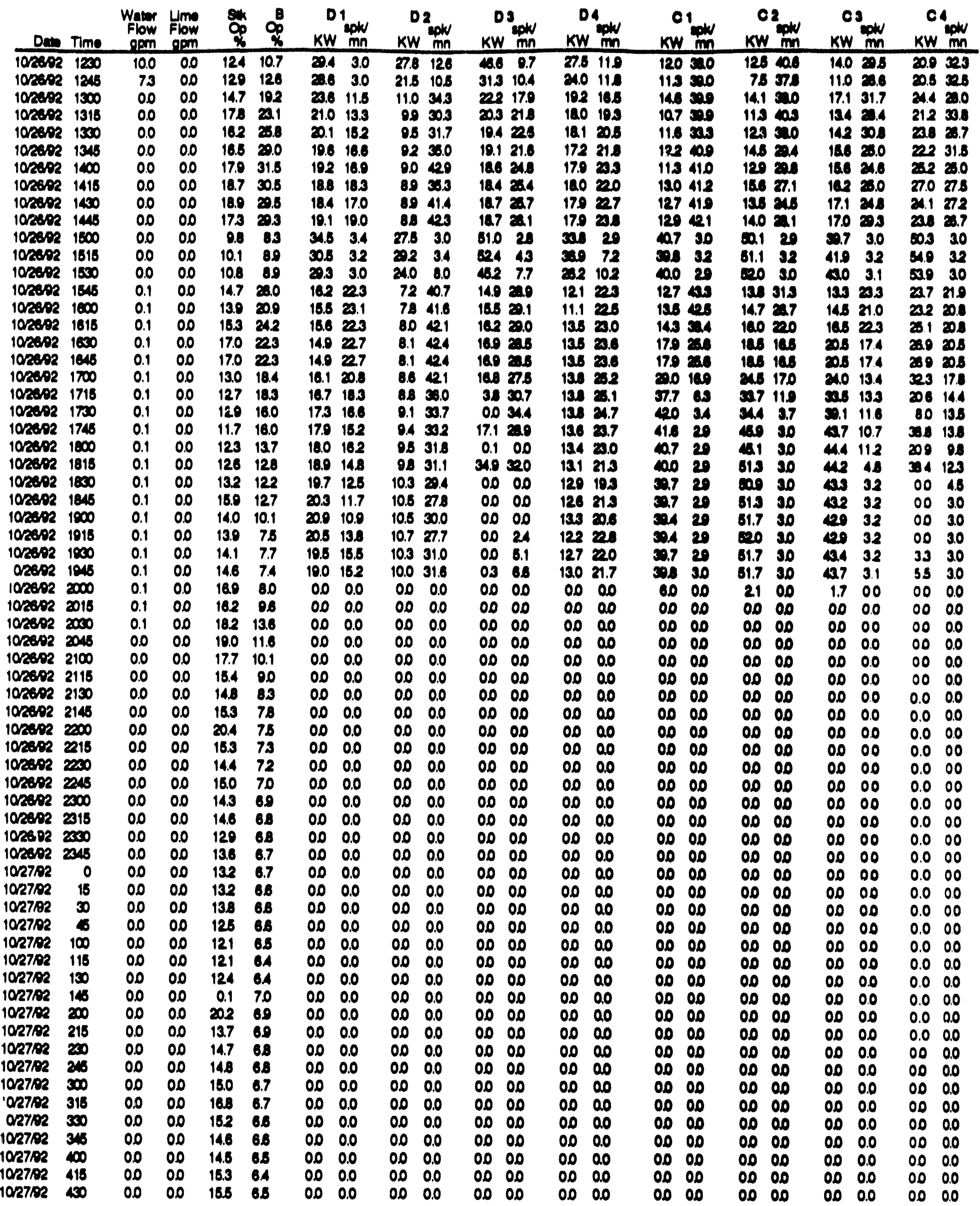




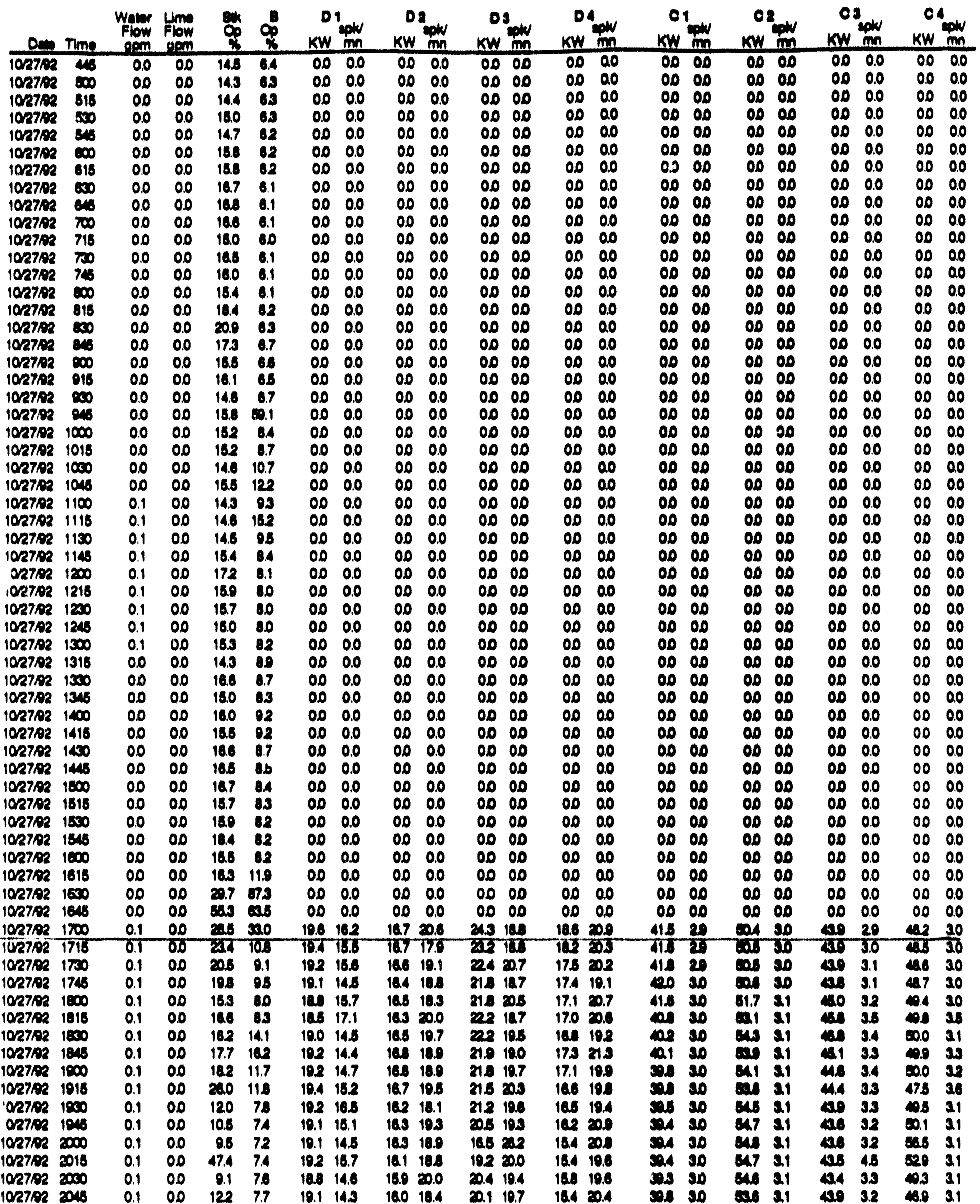




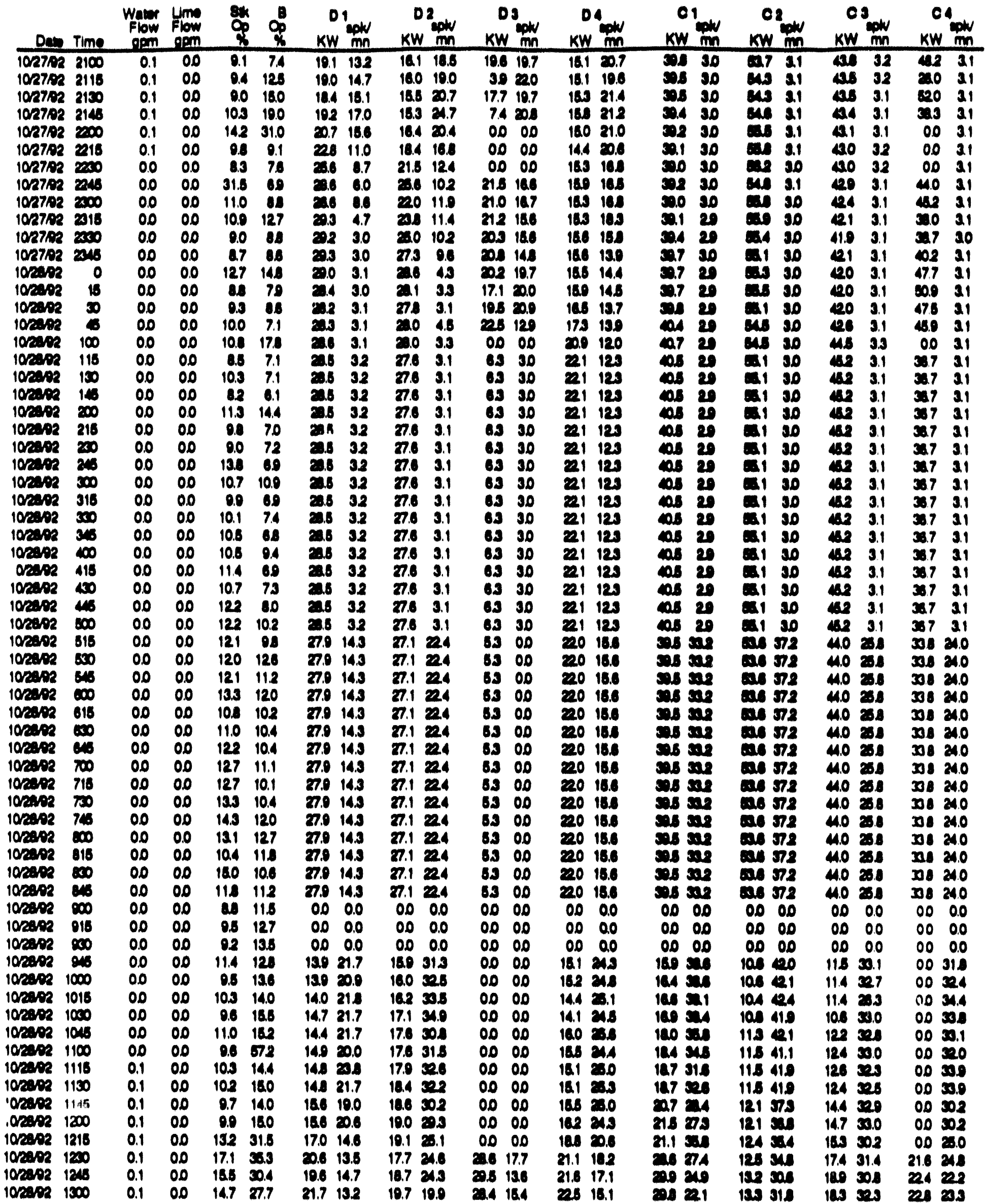




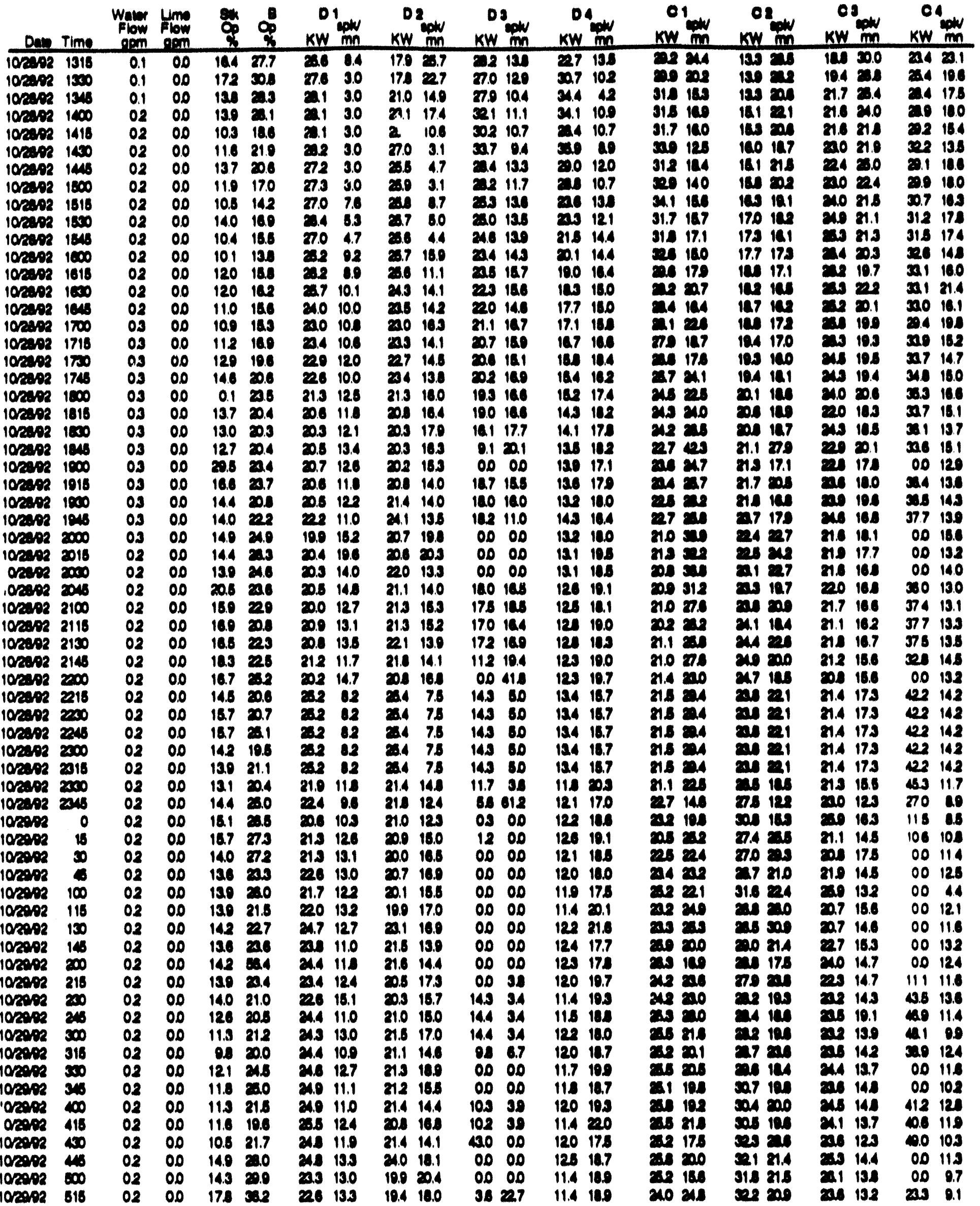




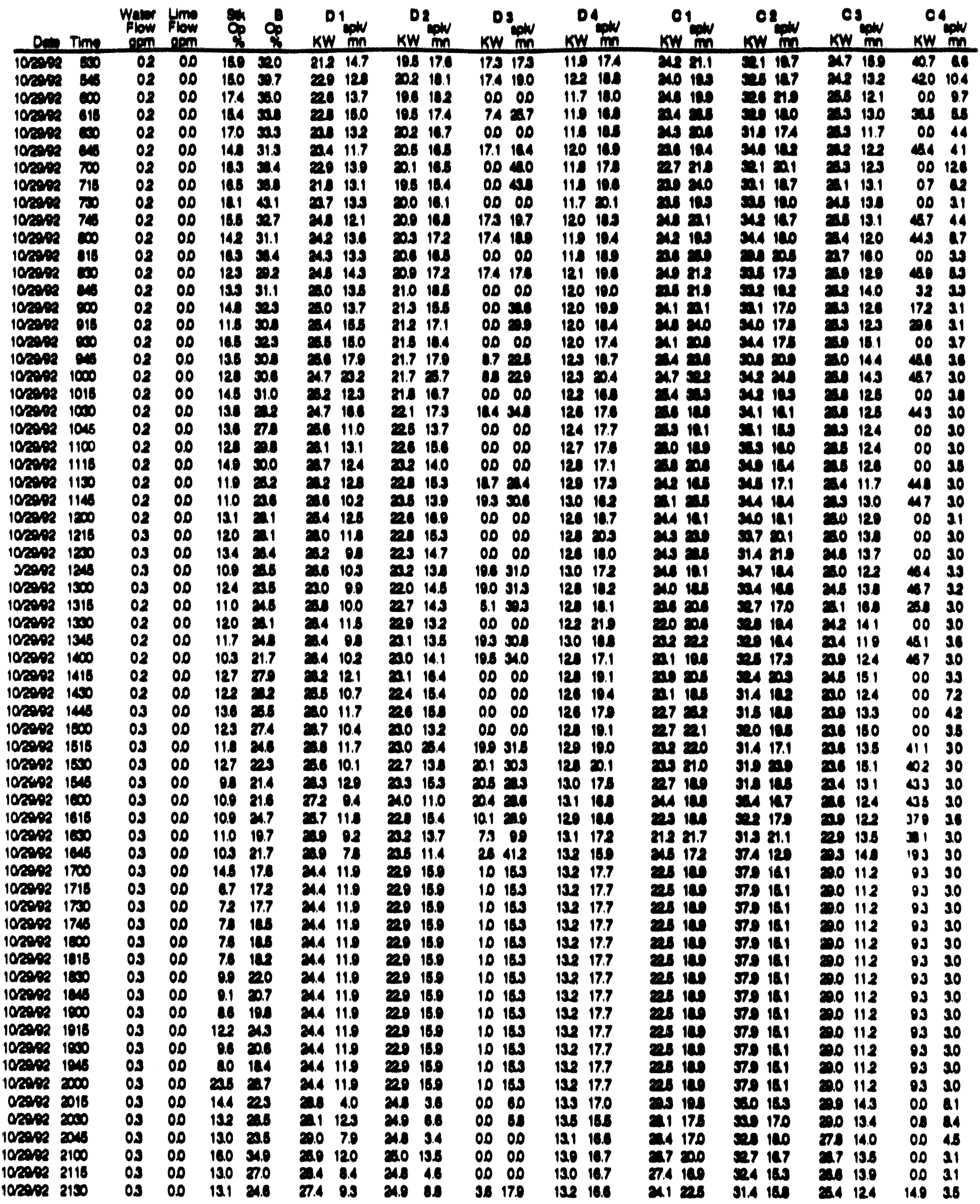




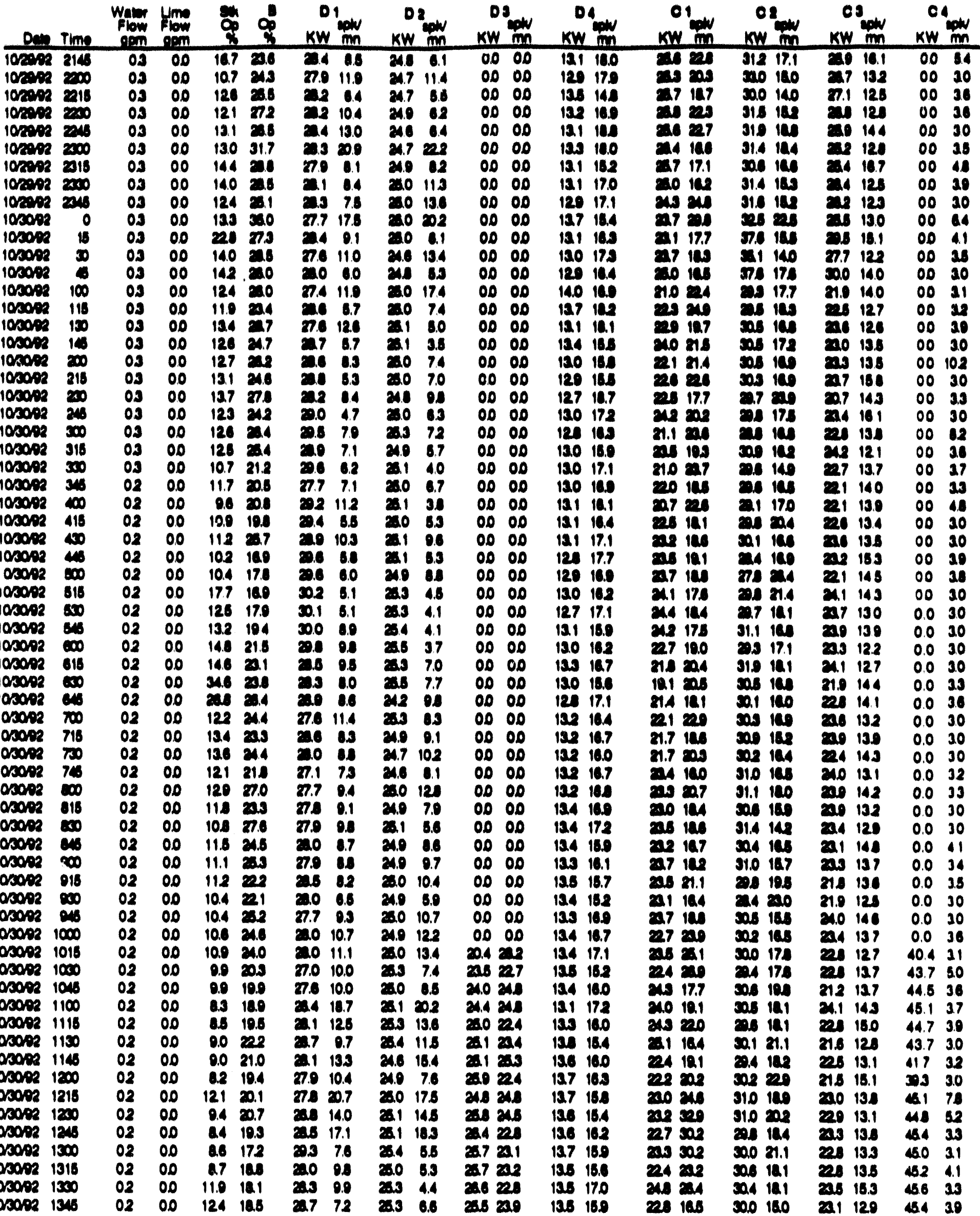




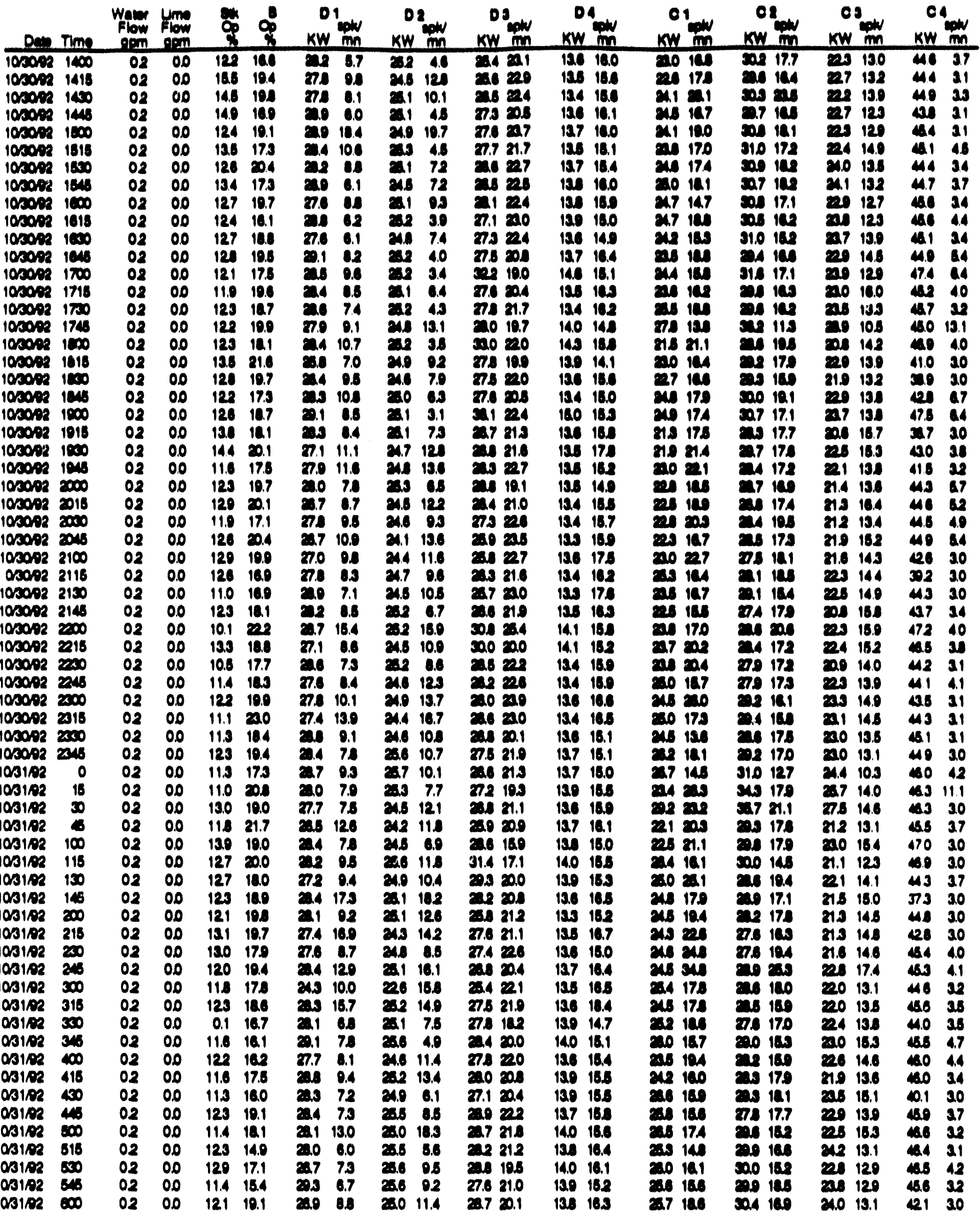




\begin{tabular}{|c|c|c|c|c|c|c|c|c|c|c|c|c|c|c|c|c|c|c|c|c|c|}
\hline Dens & Time & $\begin{array}{l}\text { Water } \\
\text { Flow } \\
\text { gpm }\end{array}$ & $\begin{array}{l}\operatorname{Limo}_{\text {flow }} \\
\text { gpm }\end{array}$ & $\begin{array}{l}\text { s.k } \\
\text { op } \\
\%\end{array}$ & $\begin{array}{l}B \\
\infty \\
\%\end{array}$ & & own & & $\begin{array}{l}2 \\
\text { sple } \\
\mathrm{mn}\end{array}$ & & spov & $\mathrm{KW}$ & $m$ & $\mathrm{KW}$ & & KW & & $\mathrm{KW}$ & $m n$ & & $m$ \\
\hline $1031 / 92$ & 615 & 02 & 00 & 11.7 & 152 & 28.7 & 6.6 & 25.5 & 7.0 & 282 & 18.6 & 14.0 & 14.3 & 203 & 14.7 & 288 & 180 & 227 & 132 & 43.9 & 3.0 \\
\hline $1031 / 92$ & $\infty 0$ & 02 & 00 & 121 & 16.0 & 28.1 & 6.4 & 25.5 & 62 & $\overline{26.7}$ & 21.7 & 14.0 & 15.5 & 246 & 143 & 208 & 16.7 & 222 & 13.6 & 43.5 & 30 \\
\hline $10031 / 92$ & 645 & 02 & 0.0 & 121 & 16.0 & 28.7 & 6.7 & 25.1 & 9.3 & 27.1 & 19.9 & 13.7 & 15.7 & 8.7 & 180 & 303 & 184 & 208 & 13.3 & 426 & 4.1 \\
\hline $1031 / 92$ & 700 & 02 & 0.0 & 13.1 & 17.0 & 88.2 & 8.6 & 25.2 & 6.2 & 27.8 & 21.1 & 14.0 & 15.0 & 280 & 143 & 305 & 130 & 24.4 & 13.7 & 39.8 & 11.8 \\
\hline $10 / 31 / 92$ & 715 & 02 & 00 & 11.0 & 17.0 & $\mathbf{2 8 . 0}$ & 8.4 & 25.4 & 8.2 & 27.7 & 21.5 & 138 & 15.1 & 258 & 162 & 203 & 136 & 24.7 & 128 & 463 & 32 \\
\hline $10 / 31 / 92$ & 730 & 02 & 0.0 & 13.4 & 18.0 & 28.0 & 9.4 & 24.6 & 12.9 & 27.6 & 20.7 & 13.7 & 149 & 264 & 189 & 208 & 182 & 228 & 14.7 & 458 & 4.1 \\
\hline $1031 / 92$ & 745 & 02 & 0.0 & 129 & 17.9 & 28.5 & 7.8 & 24.8 & 9.8 & 26.8 & 21.1 & 136 & 15.6 & 245 & 163 & 302 & 166 & 208 & 13.0 & 124 & 3.0 \\
\hline $10 / 31 / 92$ & 800 & 02 & 0.0 & 124 & 16.6 & 28.5 & 6.8 & 25.1 & 9.1 & 268 & 21.7 & 13.7 & 15.3 & 258 & 17.9 & 302 & 152 & 24.6 & 13.4 & 452 & 4.0 \\
\hline $1031 / 92$ & 815 & 02 & 00 & 124 & 16.4 & $27 B$ & 6.7 & 25.1 & 4.1 & 29.6 & 19.0 & 143 & 14.6 & 234 & 15.4 & 340 & 14.7 & 280 & 121 & 46.0 & 4.4 \\
\hline $1031 / 92$ & 800 & 02 & 0.0 & 13.5 & 16.4 & 26.5 & 9.9 & 24.9 & 6.8 & 28.7 & 20.3 & 13.9 & 163 & 248 & 165 & 237 & 180 & 20 & 13.7 & 45.9 & 30 \\
\hline 103192 & 845 & 02 & 0.0 & 13.7 & 16.5 & 27.5 & 7.0 & 24.6 & 8.2 & 27.9 & 221 & 139 & 17.0 & $\mathbf{2 0 7}$ & 184 & 200 & 17.1 & 20.1 & 15.1 & 43.1 & 3.0 \\
\hline $1031 / 92$ & 800 & 02 & 0.0 & 126 & 16.9 & 27.6 & 72 & 24.6 & 15.6 & 279 & 222 & 14.1 & 15.6 & $\mathbf{2 0 4}$ & 156 & 202 & 16.7 & 224 & 13.8 & 452 & 3.1 \\
\hline $10 / 31 / 92$ & 915 & 02 & 00 & 125 & 15.6 & 288 & 6.8 & 25.2 & 9.7 & 283 & $\mathbf{2 0 . 9}$ & 14.0 & 15.9 & 27.4 & 158 & 30.7 & 182 & 242 & 13.1 & 45.4 & 3.4 \\
\hline 1031,92 & 900 & 02 & 0.0 & 13.6 & 17.1 & 27.1 & 76 & 24.8 & 11.1 & 27.3 & 21.3 & 13.8 & 16.1 & 24.7 & 212 & 220 & 180 & 218 & 16.0 & 43.9 & 4.4 \\
\hline 1031,92 & 845 & 02 & 0.0 & 13.3 & 16.4 & 27.8 & 9.8 & 25.4 & 4.3 & 27.3 & 212 & 139 & 15.3 & 245 & 16.1 & 200 & 143 & 205 & 13.0 & 45.6 & 30 \\
\hline $1031 / 92$ & 1000 & 0.2 & 0.0 & 126 & 18.0 & 27.1 & 7.6 & 25.3 & 8.8 & 28.7 & 227 & 14.1 & 14.7 & 220 & 173 & 220 & 17.6 & 20.6 & 14.1 & 44.6 & 3.0 \\
\hline $10 / 31 / 92$ & 1015 & 02 & 0.0 & 129 & 16.2 & 28.5 & 11.4 & $\mathbf{2 5 . 3}$ & 72 & 282 & 21.3 & 14.1 & 16.5 & 240 & 180 & 200 & 183 & 232 & 14.4 & 45.4 & 30 \\
\hline $1031 / 92$ & 1000 & 02 & 0.0 & 126 & 18.4 & 28.7 & 7.5 & 25.1 & 9.4 & 28.9 & 20.8 & 138 & 162 & 27.1 & 172 & 20.1 & 163 & 223 & 162 & 45.5 & 30 \\
\hline $1031 / 92$ & 1045 & 02 & 0.0 & 10.0 & 15.2 & 29.4 & 8.7 & 25.4 & 5.9 & 20.1 & 19.0 & 13.8 & 16.4 & 27.1 & 139 & 208 & 150 & 235 & 14.5 & 45.0 & 33 \\
\hline $10 / 3192$ & 1100 & 02 & 0.0 & 11.2 & 16.1 & 28.2 & 13.9 & 25.2 & 14.4 & 29.2 & 220 & 14.0 & 162 & 237 & 16.1 & 20.1 & 172 & 201 & 13.8 & 43.7 & 3.0 \\
\hline $10 / 31 / 92$ & 1115 & 02 & 0.0 & 11.0 & 15.8 & 29.5 & 5.9 & 25.6 & 4.3 & 202 & 19.0 & 14.0 & 15.9 & 250 & 163 & 205 & 166 & 20.4 & 14.4 & 46.5 & 3.0 \\
\hline $1031 / 92$ & 1130 & 02 & 0.0 & 10.4 & 9.9 & $\mathbf{2 8 . 3}$ & 9.7 & 25.1 & 121 & 28.6 & 19.5 & 14.0 & 15.0 & 270 & 156 & 205 & 16.1 & 23.7 & 14.4 & 46.0 & 30 \\
\hline $1031 / 92$ & 1145 & 02 & 00 & 10.0 & 16.6 & 29.0 & 5.8 & 25.6 & 3.5 & 28.3 & 21.3 & 14.1 & 14.9 & 27.4 & 13.1 & 30.4 & 158 & 24.0 & 13.6 & 45.3 & 32 \\
\hline $1031 / 92$ & 1200 & & 00 & 10.8 & 178 & 27.3 & 8.8 & 24.8 & 11.0 & 29.0 & 208 & 142 & 14.4 & 286 & 216 & 208 & 16.7 & 208 & 14.4 & 43.9 & 3.9 \\
\hline $10 / 31 / 92$ & 1215 & 0 & 0.0 & 10.8 & 16.1 & 29.3 & 7.3 & 25.6 & 7.0 & 30.0 & 20.0 & 142 & 140 & 266 & 140 & 300 & 150 & 245 & 14.1 & 46.8 & 4.1 \\
\hline $10 / 31 / 92$ & 1230 & 0 & 0.0 & 10.2 & 17.0 & 28.3 & 6.9 & 25.5 & 4.4 & 28.7 & 20.6 & 142 & 163 & 280 & 18.5 & 303 & 17.8 & 24.1 & 15.0 & 46.5 & 8.2 \\
\hline $10 / 31 / 92$ & 1245 & 0. & 0.0 & 10.9 & 15.7 & 29.2 & 9.3 & 25.6 & 6.2 & 28.7 & 18.6 & 14.1 & 17.7 & 2014 & 168 & 30.1 & 175 & 24.1 & 13.6 & 46.6 & 3.0 \\
\hline $1031 / 92$ & 1300 & 0 & 0.0 & 11.3 & 18.6 & 27.8 & 9.3 & 25.4 & 122 & 29.2 & 19.9 & 14.3 & 148 & 287 & 180 & 208 & 10.1 & 24.0 & 13.8 & 46.7 & 3.4 \\
\hline $1031 / 92$ & 1315 & 02 & 0.0 & 10.6 & 15.0 & 29.2 & 10.1 & 25.6 & 5.2 & 28.5 & 20.3 & 143 & 15.9 & 2088 & 183 & $\mathbf{5 0 . 1}$ & 15.1 & 24.6 & 17.1 & 46.7 & 3.0 \\
\hline$Q_{3} \| 92$ & 1330 & 02 & 0.0 & 9.8 & 19.6 & 27.9 & 10.2 & 25.2 & 16.0 & 30.0 & 19.0 & 142 & 15.2 & $\mathbf{2 5 0}$ & 143 & 30.1 & 15.4 & 24.2 & 15.8 & 16.9 & 3.0 \\
\hline $10 / 31 / 92$ & 1345 & 02 & 0.0 & 10.3 & 16.2 & 29.4 & 7.0 & 25.6 & 6.7 & 29.0 & 212 & 14.3 & 15.1 & 27.3 & 142 & 30.5 & 168 & 20.9 & 13.7 & 46.9 & 3.0 \\
\hline $10 / 31 / 92$ & 1400 & 02 & 0.0 & 10.2 & 16.6 & 28.8 & 8.5 & ఒ.5 & 42 & 28.7 & 18.9 & 14.3 & 148 & 250 & 152 & 302 & 17.6 & 242 & 13.9 & 41.9 & 3.0 \\
\hline $10 \Omega 1 / 92$ & 1415 & 02 & 0.0 & 10.2 & 16.7 & 28.2 & 10.1 & 25.6 & 4.4 & 28.1 & 21.5 & 14.3 & 15.6 & 280 & 14.7 & 30.5 & 148 & 24.4 & 14.6 & 46.2 & 3.0 \\
\hline $10 / 31 / 92$ & 1430 & 02 & 0.0 & 10.1 & 16.7 & $\mathbf{2 8 . 6}$ & 8.3 & 25.6 & 6.0 & 30.1 & 21.0 & 14.4 & 15.6 & 27.1 & 160 & 31.1 & 15.1 & 25.1 & 14.4 & 46.2 & 3.0 \\
\hline $10 / 31 / 92$ & 1445 & 02 & 0.0 & 10.9 & 18.1 & 28.1 & 13.4 & 25.5 & 14.7 & 29.5 & 20.8 & 14.4 & 14.9 & 2055 & 17.5 & 283 & 163 & 228 & 14.1 & 45.8 & 3.0 \\
\hline $10 / 31 / 92$ & 1500 & 02 & 00 & 9.7 & 17.1 & ฉ.9 & 10.9 & 25.5 & 5.7 & 28.9 & 20.4 & 14.5 & 15.1 & 28.7 & 17.9 & 31.4 & 165 & 255 & 142 & 43.0 & 30 \\
\hline $10 / 31 / 92$ & 1515 & 02 & 0.0 & 10.0 & 18.6 & 29.2 & 10.0 & 25.5 & 72 & 29.6 & 228 & 14.1 & 16.7 & 25.7 & 180 & 285 & 164 & 23.6 & 14.5 & 43.4 & 3.0 \\
\hline $1031 / 92$ & 1530 & 02 & 0.0 & 11.5 & 17.8 & 27.8 & 16.3 & 25.0 & 15.4 & 29.7 & 21.6 & 14.5 & 172 & 285 & 18.6 & 31.1 & 15.7 & 250 & 13.8 & 40.8 & 3.7 \\
\hline $10 / 31 / 92$ & 1545 & 02 & 0.0 & 11.1 & 17.5 & 20.5 & 10.2 & 25.6 & 3.4 & 30.1 & 172 & 14.2 & 15.9 & 270 & 19.6 & 30.5 & 220 & 25.1 & 13.4 & 477 & 3.6 \\
\hline $10 / 31 / 92$ & 1600 & 02 & 0.0 & 10.3 & 17.1 & $\mathbf{2 8 . 7}$ & 10.4 & 25.4 & 4.5 & 364 & 162 & 15.3 & 13.6 & 286 & 160 & 335 & 186 & 233 & 13.3 & 480 & 3.0 \\
\hline $1031 / 92$ & 1615 & 0 & 0 & 124 & 20.8 & 278 & 8.8 & 25.6 & 3.4 & 323 & 16.1 & 152 & 14.2 & 288 & 19.1 & 306 & 180 & 24.2 & 13.7 & 458 & 3.0 \\
\hline $10 / 31 / 92$ & 1600 & 02 & 00 & 13.0 & 17.5 & 25.9 & 6.2 & 24.7 & 5.8 & 28.9 & 208 & 14.2 & 15.1 & 27.1 & 182 & 318 & 158 & 258 & 14.0 & 437 & 3.0 \\
\hline $10 / 31 / 92$ & 1645 & 02 & 0.0 & 121 & 15.0 & 27.9 & 125 & 25.6 & 3.1 & 332 & 19.1 & 15.0 & 142 & 285 & 37.7 & 303 & 192 & 230 & 15.4 & 466 & 3.0 \\
\hline $1031 / 92$ & 1700 & 0.2 & 00 & 11.8 & 162 & $\mathbf{2 8 . 0}$ & 7.0 & 25.6 & 3.4 & 298 & 188 & 14.3 & 14.6 & 218 & 210 & 308 & 15.0 & 24.1 & 13.5 & 469 & 3.2 \\
\hline $1031 / 92$ & 1715 & 02 & 0.0 & 121 & 16.4 & 28.4 & 11.3 & 25.5 & 6.6 & 308 & 20.1 & 142 & 145 & 27.1 & 16.7 & 31.5 & 16.1 & 25.4 & 127 & 475 & 3.2 \\
\hline $10 / 31 / 92$ & 1730 & 02 & 0.0 & 128 & 172 & 27.7 & 78 & 25.3 & 5.3 & 292 & 18.5 & 14.2 & 14.0 & 284 & 13.1 & 30.6 & 166 & 24.7 & 13.7 & $\$ 6.6$ & 3.0 \\
\hline $10 / 31 / 92$ & 1745 & 02 & 0.0 & 127 & 182 & $\mathbf{2 8 . 0}$ & 7.8 & 25.4 & 72 & 20.1 & 18.4 & 14.1 & 14.6 & 288 & 14.0 & 30.1 & 167 & 25.1 & 14.5 & 466 & 3.0 \\
\hline $10 / 31 / 92$ & 1800 & 02 & 00 & 128 & 16.3 & 28.5 & 5.2 & 25.2 & 5.7 & 308 & 18.5 & 14.2 & 15.1 & 258 & 150 & 313 & 14.1 & 25.0 & 12.6 & 464 & 3.0 \\
\hline $10 / 31 / 92$ & 1815 & 02 & 0.0 & 25 & 17.7 & 27.4 & 108 & 1 & 5.3 & 29.4 & 24.6 & 142 & 18.3 & 20.0 & 206 & $\mathbf{2 8 5}$ & 160 & 232 & 15.3 & 470 & 3.0 \\
\hline 10/31/92 & 1830 & 02 & 00 & 127 & 162 & 12 & 7. & 0 & 5.8 & 298 & 202 & 14.4 & 159 & 288 & 140 & $\overline{805}$ & 156 & 22.6 & 14.9 & 16.7 & 33 \\
\hline 10/31/92 & 1845 & 0 & 0 & 22 & 158 & 8 & 8 & 252 & 6.7 & 28.6 & 21.1 & 14.1 & 15.5 & 278 & 136 & 300 & 162 & 230 & 13.0 & 43.8 & 3.0 \\
\hline 10/31/92 & 1900 & 0 & & & 6 & 28.5 & 6.2 & $\overline{252}$ & 4.7 & $\mathbf{2 0 . 4}$ & 20.2 & 14.4 & 14.5 & 27.7 & 210 & 208 & 181 & 243 & 14.0 & 457 & 30 \\
\hline $10 / 31 / 92$ & 1915 & 0 & & & & 28.9 & 5.5 & $\overline{25.4}$ & 3.7 & 30.3 & 17.4 & 142 & 15.1 & 284 & 13.4 & 208 & 103 & 21.8 & 133 & 46.0 & 3.0 \\
\hline $1031 / 9$ & 1950 & & 0. & & 15.5 & 28.7 & 5.8 & 25.4 & 4.8 & 28.8 & 19.1 & 14.3 & 16.0 & 204 & 122 & $\overline{20.5}$ & 165 & 227 & 13.6 & 46.9 & 8.0 \\
\hline $1031 / 92$ & 1845 & 02 & 00 & & 15.3 & 29.6 & 7.7 & 25.4 & 32 & 34.0 & 135 & 15.5 & 132 & 288 & 158 & 324 & 17.1 & 25.7 & 13.6 & 48.4 & 32 \\
\hline $1031 / 92$ & 2000 & 02 & 00 & 11.4 & 17.4 & 26.9 & 8.8 & 255 & 5.1 & 29.8 & 18.1 & 14.3 & 14.1 & 282 & 15.7 & 30.6 & 17.1 & 24.4 & 14.4 & 47.0 & 42 \\
\hline $1031 / 92$ & 2015 & 02 & 0.0 & 14.4 & 15.8 & 223 & 4.4 & 25.3 & 4.5 & 29.6 & 19.2 & 142 & 16.1 & 273 & 146 & 286 & 16.7 & 200 & 13.6 & 47.4 & 4.0 \\
\hline 103192 & 2000 & 02 & 0.0 & 126 & 16.5 & 28.1 & 6.4 & 252 & 5.3 & 29.0 & 19.7 & 142 & 15.1 & 286 & 16.1 & 227 & 156 & 23.7 & 132 & $\infty 0.9$ & 30 \\
\hline Q $131 / 92$ & 2045 & 02 & 00 & 127 & 16.0 & 28.5 & 6.2 & 25.2 & 3.7 & 34.1 & 17.8 & 152 & 143 & 225 & 164 & $30 \mathrm{~s}$ & 13.7 & 253 & 15.3 & 421 & 30 \\
\hline $0,31 / 92$ & 2100 & 02 & 00 & 13.8 & 152 & 28.7 & 6.2 & 24.9 & 6.4 & 322 & 20.0 & 15.0 & 15.3 & 286 & 146 & 203 & 160 & 250 & 13.9 & 473 & 32 \\
\hline $031 / 92$ & 2115 & 0 & 0.0 & 125 & 172 & 29.1 & 9.3 & 25.1 & 48 & $\overline{20.7}$ & 20.0 & 143 & 140 & 27.7 & 130 & 205 & 17.1 & 228 & 15.1 & 46.3 & 3.0 \\
\hline 03192 & 2130 & 1 & 0.0 & 122 & 16.7 & 28.3 & 6.9 & z5.1 & 3.6 & 34.1 & 20.5 & 15.0 & 16.7 & 273 & 18.7 & 225 & 187 & $\overline{235}$ & 13.1 & 47.6 & 3.0 \\
\hline $031 / 92$ & 2145 & 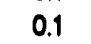 & 0.0 & 14.1 & 15.7 & $\mathbf{2 8 . 6}$ & 7.0 & 250 & 4.1 & 28.9 & 21.5 & 14.4 & 16.3 & 262 & 14.1 & 206 & 13.7 & 239 & 14.2 & 427 & 30 \\
\hline 03192 & 2000 & 0.1 & 0.0 & 13.1 & 17.2 & 29.6 & 6.4 & 25.0 & 4.1 & 29.1 & 19.2 & 142 & 15.9 & 232 & 17.7 & 20.1 & 16.1 & 221 & 13.7 & 45.5 & 3.6 \\
\hline $031 / 92$ & 2215 & 0.1 & 00 & 13.3 & 16.9 & 278 & 8.7 & 24.8 & 8.0 & 28.6 & 220 & 14.4 & 15.5 & $\overline{26.1}$ & 14.4 & 298 & 163 & 23.4 & 138 & 443 & 3.0 \\
\hline
\end{tabular}




\begin{tabular}{|c|c|c|c|c|c|c|c|c|c|c|c|c|c|c|c|c|c|c|c|c|c|}
\hline Date & Time & $\begin{array}{l}\text { Water } \\
\text { Flow } \\
\text { gpm }\end{array}$ & $\begin{array}{l}\text { Limo } \\
\text { Flow } \\
\text { opm }\end{array}$ & $\begin{array}{l}\text { Suk } \\
\text { क } \\
\%\end{array}$ & $\begin{array}{l}\mathrm{B} \\
\stackrel{p}{\%}^{\circ}\end{array}$ & & $\begin{array}{l}1 \\
\text { sow } \\
\text { mn }\end{array}$ & & $\begin{array}{l}2 \\
\mathrm{spow} \\
\mathrm{mn}\end{array}$ & & $\begin{array}{l}\mathrm{pk} \\
\mathrm{mn}\end{array}$ & $\mathrm{KW}$ & $m n$ & $\mathrm{KW}$ & & $K W$ & & KW & $m n$ & & $m n$ \\
\hline $1031 / 92$ & 2000 & 0.1 & 0.0 & 124 & 16.6 & 29.2 & 10.3 & $\mathbf{2 6 . 1}$ & 4.1 & 20.7 & 20.3 & 14.4 & 16.2 & 28.1 & 188 & 20.0 & 14.1 & 24.4 & 16.0 & 45.8 & 3.0 \\
\hline $1031 / 9 ?$ & 2245 & 0.1 & 0.0 & 12.8 & 16.3 & 20.2 & 7.7 & 20.1 & 4.7 & 20.6 & 19.6 & 14.6 & 18.0 & 23.6 & 134 & 30.4 & 13.1 & 24.7 & 13.9 & 45.2 & 3.0 \\
\hline $10 / 31 / 92$ & 2000 & 0.1 & 0.0 & 14.4 & 17.7 & 25.7 & 8.2 & 23.9 & 8.7 & 20.1 & 20.0 & 14.3 & 14.7 & 275 & 17.6 & 20.6 & 173 & 24.7 & 13.3 & 45.1 & 3.0 \\
\hline $10 / 31 / 92$ & 2015 & 0.1 & 0.0 & 125 & 16.5 & 28.9 & 8.0 & 25.2 & 3.4 & 20.1 & 20.9 & 14.6 & 16.1 & 221 & 123 & 312 & 16.1 & 258 & 120 & 46.7 & 5.6 \\
\hline $10 / 31 / 92$ & 2300 & 0.1 & 0.0 & 15.3 & 17.5 & 23.6 & 7.6 & 23.7 & 9.8 & 27.7 & 20.7 & 14.5 & 163 & 293 & 138 & 313 & 166 & 202 & 121 & 426 & 3.0 \\
\hline $10 / 31 / 92$ & 2345 & 0.1 & 0.0 & 13.8 & 15.7 & 28.8 & 5.9 & 20.5 & 3.5 & 31.8 & 19.0 & 15.4 & 14.3 & 305 & 11.6 & 32.1 & 160 & 28.4 & 138 & 44.3 & 3.6 \\
\hline $11 / 1 / 92$ & 0 & 0.1 & 0.0 & 13.2 & 16.0 & 28.8 & 8.6 & 25.4 & 3.7 & 35.1 & 20.5 & 16.0 & 148 & 284 & 13.0 & 323 & 15.1 & 208 & 13.5 & 45.2 & 3.0 \\
\hline $11 / 1 / 92$ & 15 & 0.1 & 0.0 & 14.8 & 16.7 & 28.4 & 98 & 28.4 & 5.2 & $28 B$ & 10.6 & 14.6 & 15.5 & 234 & 138 & 30.1 & 110 & 24.2 & 126 & 420 & 30 \\
\hline $11 / 1 / 92$ & 30 & 0.1 & 0.0 & 13.3 & 17.0 & 26.8 & 6.7 & 24.7 & 7.6 & 29.8 & 15.6 & 14.7 & 13.6 & 202 & 128 & 32.6 & 13.6 & 25.9 & 122 & 44.1 & 4.6 \\
\hline $11 / 1 / 02$ & 45 & 0.1 & 0.0 & 10.4 & 17.6 & 28.9 & 12.6 & 25.3 & 14.6 & 28.9 & 21.6 & 14.7 & 16.8 & 28.1 & 160 & 343 & 14.9 & 27.8 & 12.1 & 47.4 & 4.0 \\
\hline $11 / 1 / 92$ & 100 & 0.1 & 0.0 & 10.1 & 19.1 & 28.4 & 7.7 & 25.2 & 8.5 & 30.6 & 19.2 & 140 & 14.1 & 30.5 & 102 & 307 & 122 & 272 & 121 & 47.6 & 31 \\
\hline $11 / 1 / 92$ & 115 & 0.1 & 0.0 & 9.9 & 16.1 & 20.3 & 8.3 & 25.3 & 3.4 & 330 & 14.6 & 15.4 & 13.7 & 238 & 16.6 & 200 & 163 & 202 & 13.0 & 48.3 & 3.3 \\
\hline $11 / 1 / 92$ & 130 & 0.1 & 0.0 & 8.8 & 15.0 & 20.6 & 6.0 & $\mathbf{2 0 . 4}$ & 3.1 & 33.9 & 15.6 & 16.7 & 13.0 & 203 & 13.7 & 30.7 & 136 & $\overline{230}$ & 13.7 & 48.4 & 30 \\
\hline $11 / 1 / \theta 2$ & 145 & 0.1 & 0.0 & 9.5 & 15.8 & 28.6 & 7.7 & 2.6 & 62 & 298 & 18.6 & 148 & 15.8 & 200 & 138 & 203 & 14.7 & 24.1 & 148 & 4.7 & 3.0 \\
\hline $11 / 1 / \theta 2$ & 200 & 0.1 & 0.0 & 16.3 & 17.7 & $\mathbf{2 8 . 3}$ & 0.7 & $\mathbf{2 6 . 2}$ & 11.6 & 28.4 & 19.1 & 14.7 & 14.8 & 27.6 & 226 & 30.1 & 182 & 24.5 & 14.5 & 46.0 & 3.0 \\
\hline $11 / 1 / \theta 2$ & 215 & 0.1 & 0.0 & 10.3 & 15.8 & 28.8 & 6.7 & 25.3 & 38 & 31.4 & 18.0 & 14.5 & 14.6 & 20.1 & 128 & 30.8 & 14.4 & 248 & 13.5 & 46.5 & 37 \\
\hline $11 / 1 / 92$ & 200 & 0.1 & 0.0 & 8.4 & 15.8 & $\overline{20.4}$ & 8.0 & $\mathbf{2 0 . 5}$ & 3.2 & 31.2 & $\$ 7.6$ & 148 & 148 & 28.7 & 182 & 30.8 & 14.2 & 252 & 13.0 & 43.1 & 3.0 \\
\hline $11 / 1 / 92$ & 245 & 02 & 0.0 & 10.4 & 15.0 & 28.0 & 8.0 & 20.2 & 14.0 & 20.0 & 17.7 & 14.7 & 15.2 & 278 & 180 & 23.7 & 160 & $\overline{200}$ & 13.0 & 472 & 3.7 \\
\hline 11/1/92 & 300 & 02 & 0.0 & 10.9 & 16.5 & 29.0 & 7.6 & $\overline{\mathbf{2 5 . 6}}$ & 4.7 & 20.8 & 18.7 & 15.0 & 14.5 & 23 & 17.4 & 20.6 & 15.5 & $\mathbf{2 3 . 6}$ & 142 & 47.4 & 3.0 \\
\hline 11/1/92 & 315 & 02 & 0.0 & 9.9 & 15.0 & 29.7 & 3.6 & $\mathbf{2 5 . 4}$ & 3.7 & 31.1 & 18.7 & 14.9 & 13.8 & 28.5 & 138 & 503 & 148 & 24.4 & 12.6 & 44.6 & 3.5 \\
\hline $11 / 1 / 92$ & 300 & 0.2 & 0.0 & 10.2 & 16.8 & 29.0 & 8.3 & 25.6 & 3.1 & 30.8 & 17.5 & 14.7 & 148 & 2.1 & 162 & 30.3 & 152 & 25.2 & 13.1 & 47.5 & 5.7 \\
\hline $11 / 1 / 92$ & 345 & 02 & 0.0 & 10.4 & 16.9 & 29.1 & 5.9 & 25.4 & 4.0 & 30.8 & 18.1 & 14.6 & 14.5 & 28.0 & 15.0 & 30.1 & 163 & 250 & 12.6 & 45.9 & 3.6 \\
\hline $11 / 1 / 92$ & 400 & 02 & 0.0 & 10.1 & 16.5 & 29.5 & 7.8 & $\mathbf{2 5 . 4}$ & 3.7 & 31.5 & 18.6 & 14.6 & 14.1 & 286 & 142 & 30.0 & 12.1 & 20.0 & 14.8 & 47.6 & 3.0 \\
\hline $11 / 1 / 92$ & 415 & 02 & 0.0 & 10.0 & 15.4 & 29.6 & 10.5 & 20.5 & 4.0 & 30.6 & 17.9 & 14.6 & 14.1 & 288 & 189 & 200 & 15.1 & 24.7 & 138 & 46.6 & 3.9 \\
\hline $11 / 1 / 92$ & 430 & 02 & 0.0 & 10.5 & 17.0 & 29.0 & 13.1 & 20.5 & 13.4 & 29.7 & 18.3 & 14.7 & 15.4 & 27.3 & 130 & 208 & 162 & 200 & 14.0 & 47.1 & 3.1 \\
\hline $11 / 1 / 92$ & 445 & 02 & 0.0 & 10.4 & 14.8 & 29.8 & 8.1 & 25.6 & 4.2 & 30.8 & 18.4 & 15.2 & 13.5 & 286 & 16.4 & 31.0 & 143 & 2055 & 13.1 & 472 & 3.0 \\
\hline $11 / 1 / 92$ & 500 & 02 & 0.0 & 10.2 & 16.5 & 28.8 & 14.5 & 25.6 & 14.9 & 20.8 & 19.4 & 14.9 & 148 & 280 & 142 & 313 & 130 & 202 & 129 & 47.7 & 30 \\
\hline $11 / 1 / 92$ & 515 & 02 & 0.0 & 10.1 & 15.4 & 29.5 & 8.4 & 25.6 & 3.1 & 30.8 & 188 & 15.1 & 14.2 & 272 & 163 & 30.4 & 16.4 & 245 & 14.0 & 47.3 & 3.0 \\
\hline $11 / 1 / 92$ & 530 & 02 & 0.0 & 10.9 & 15.7 & 29.0 & 9.9 & $\mathbf{2 5 . 6}$ & 4.2 & 31.8 & 18.8 & 15.0 & 14.5 & 22 & 266 & 31.8 & 185 & 20.6 & 13.3 & 478 & 3.0 \\
\hline $11 / 1 / 92$ & 545 & 02 & 0.0 & 11.6 & 15.4 & 29.2 & 8.0 & 25.5 & 4.6 & 30.8 & 19.4 & 15.0 & 14.0 & 280 & $\overline{184}$ & 312 & 16.6 & 232 & 14.9 & 47.7 & 3.0 \\
\hline $11 / 1 / 92$ & 600 & 02 & 0.0 & 10.3 & 19.4 & 28.2 & 16.0 & 25.0 & 13.9 & 31.2 & 18.8 & 14.9 & 15.2 & 23.6 & 17.6 & 31.0 & 152 & 27.0 & 13.7 & 47.8 & 5.1 \\
\hline $11 / 1 / 92$ & 615 & 0.2 & 0.0 & 10.9 & 15.5 & 28.3 & 8.1 & 25.7 & 3.8 & 20.1 & 18.0 & 15.0 & 16.0 & 27.7 & 16.4 & 300 & 136 & 20.3 & 13.7 & 46.7 & 3.0 \\
\hline $11 / 192$ & 60 & 02 & 0.0 & 10.0 & 15.2 & 29.3 & 10.0 & $\mathbf{8 0 . 7}$ & 3.9 & $\mathbf{3 0 . 0}$ & 17.4 & 14.7 & 15.7 & 24.9 & 16.6 & 20.4 & 14.7 & 242 & 13.1 & 47.7 & 3.3 \\
\hline $11 / 1 / 92$ & 645 & 02 & 0.0 & 10.1 & 14.7 & 29.8 & 4.5 & 25.6 & 3.7 & $\mathbf{2 9 . 6}$ & 18.0 & 15.3 & 13.4 & 23.1 & 14.0 & 312 & 166 & $\mathbf{2 3 . 4}$ & 12.3 & 47.3 & 3.1 \\
\hline $11 / 192$ & 700 & 02 & 0.0 & 9.5 & 14.5 & $\mathbf{2 0 . 0}$ & 7.9 & 25.6 & 3.4 & 31.9 & 16.0 & 14.6 & 15.0 & 28.1 & 108 & 322 & 13.6 & 280 & 13.4 & 48.7 & 3.0 \\
\hline $11 / 1 / 92$ & 715 & 02 & 0.0 & 10.2 & 14.4 & 29.5 & 7.6 & 26.7 & 3.2 & 320 & 16.7 & 15.1 & 15.9 & 288 & 182 & 286 & 15.1 & 25.3 & 13.9 & 47.9 & 3.0 \\
\hline 11/1/92 & 730 & 02 & 0.0 & 9.4 & 15.0 & 29.4 & 7.8 & 25.7 & 4.4 & 326 & 15.3 & 15.1 & 14.4 & 282 & 10.9 & 318 & 16.4 & 26.6 & 12.5 & 47.5 & 3.0 \\
\hline $11 / 1 / 92$ & 745 & 02 & 0.0 & 10.2 & 14.3 & 29.6 & 4.0 & 26.7 & 3.4 & 33.6 & 18.3 & 15.3 & 15.0 & 273 & 150 & 325 & 15.7 & $\mathbf{2 6 . 7}$ & 13.1 & 48.4 & 3.0 \\
\hline $11 / 1 / 92$ & 800 & 02 & 0.0 & 9.5 & 13.3 & 20.2 & 5.1 & 258 & 3.6 & 321 & 18.3 & 14.4 & 148 & 270 & 132 & 313 & 15.1 & 20.3 & 13.2 & 48.3 & 3.0 \\
\hline $11 / 1 / 92$ & 815 & 02 & 0.0 & 11.1 & 16.3 & 28.9 & 7.4 & $\overline{25.8}$ & 4.0 & 31.4 & 16.1 & 15.3 & 13.0 & 302 & 10.4 & 340 & 13.7 & $\overline{288}$ & 11.7 & 45.0 & 3.0 \\
\hline $11 / 1 / 92$ & 830 & 02 & 0.0 & 10.1 & 15.7 & 28.8 & 8.9 & 25.7 & 7.1 & 20.5 & 18.3 & 14.6 & 14.6 & 255 & 172 & 30.5 & 154 & 24.5 & 14.3 & 445 & 3.0 \\
\hline 11/1/92 & 845 & 02 & 0.0 & 11.5 & 15.6 & 26.1 & 5.1 & 24.3 & 5.8 & 20.8 & 16.2 & 14.6 & 14.5 & 27.1 & 132 & 20.6 & 130 & 24.4 & 126 & 46.7 & 3.2 \\
\hline $11 / 1 / 92$ & 900 & 02 & 0.0 & 10.7 & 14.7 & 29.8 & 4.1 & 25.7 & 3.1 & 35.8 & 15.8 & 15.7 & 15.6 & 28.6 & 13.6 & 332 & 11.7 & 27.5 & 120 & 459 & 3.0 \\
\hline $11 / 1 / 92$ & 915 & 02 & 0.0 & 8.8 & 15.5 & 28.5 & 7.1 & 25.8 & 6.6 & 30.8 & 18.3 & 14.9 & 13.9 & $\mathbf{2 8 0}$ & 135 & 20.7 & 148 & 25.0 & 125 & 48.5 & 3.0 \\
\hline $11 / 1 / 92$ & $\infty 00$ & 02 & 0.0 & 9.2 & 16.6 & 29.3 & 9.5 & 25.8 & 6.1 & 30.5 & 20.0 & 15.3 & 15.0 & 23.1 & 16.4 & 300 & 14.1 & 248 & 13.5 & 473 & 3.0 \\
\hline $11 / 1 / 92$ & 946 & 02 & 0.0 & 10.9 & 16.0 & 27.9 & 17.1 & 25.4 & 11.0 & 321 & 20.5 & 15.0 & 13.7 & 258 & 118 & 20.7 & 133 & 202 & 120 & 486 & 3.6 \\
\hline $11 / 1 / 92$ & 1000 & 02 & 0.0 & 10.4 & 15.4 & 28.7 & 7.8 & 25.8 & 3.8 & 30.6 & 18.6 & 15.0 & 14.2 & 25.6 & 127 & 31.4 & 15.4 & $\overline{20.1}$ & 13.1 & 48.2 & 3.8 \\
\hline $11 / 1 / 92$ & 1015 & 02 & 00 & 9.7 & 14.9 & 20.4 & 7.6 & 20.8 & 4.4 & 31.9 & 16.3 & 15.2 & 158 & 27.5 & 15.1 & 0.2 & 160 & 24.5 & 129 & 477 & 3.0 \\
\hline $11 / 1 / 82$ & 1000 & 02 & 0.0 & 8.7 & 14.8 & $\mathbf{2 0 . 6}$ & 8.3 & 25.8 & 3.9 & 320 & 16.8 & 15.0 & 148 & 27.0 & 146 & 508 & 14.0 & 20.4 & 13.2 & 48.3 & 4.8 \\
\hline $11 / 1 / 92$ & 1045 & 02 & 00 & 8.2 & 15.2 & 27.8 & 8.2 & 25.6 & 5.7 & 30.8 & 162 & 15.0 & 13.3 & 284 & 180 & 308 & 160 & 24.4 & 14.2 & 47.6 & 3.0 \\
\hline $11 / 1 / 92$ & 1100 & 02 & 00 & 10.2 & 13.6 & 20.1 & 5.0 & 25.5 & 5.4 & 31.6 & 17.7 & 14.8 & 15.1 & 248 & 16.1 & 30.0 & 16.7 & $\mathbf{2 3 . 9}$ & 122 & 477 & 3.0 \\
\hline $11 / 1 / 92$ & 1115 & 02 & 0.0 & 9.5 & 14.7 & $\mathbf{2 8 . 9}$ & 10.2 & 28.0 & 4.6 & 31.8 & 16.4 & 15.2 & 13.6 & 28.7 & 127 & 308 & 158 & 24.7 & 13.7 & 46.3 & 3.3 \\
\hline $11 / 1 / 92$ & 1130 & 02 & 0.0 & 9.9 & 14.1 & 20.9 & 4.6 & 26.0 & 3.2 & 31.1 & 18.1 & 15.4 & 15.1 & 28.7 & 112 & 31.4 & 122 & 24.9 & 125 & 426 & 3.1 \\
\hline $11 / 1 / 92$ & 1145 & 02 & 0.0 & 10.2 & 15.5 & 29.0 & 9.4 & 258 & 7.6 & 323 & 16.4 & 14.8 & 14.5 & 256 & 127 & 203 & 128 & 24.7 & 14.0 & 47.1 & 3.0 \\
\hline $11 / 1 / 92$ & 1200 & 02 & 0.0 & 9.8 & 15.4 & 27.8 & 7.1 & 25.6 & 8.6 & 30.0 & 18.6 & 15.1 & 15.2 & 255 & 16.0 & 30.0 & 156 & 248 & 13.0 & 48.2 & 3.0 \\
\hline $11 / 1 / 92$ & 1215 & 02 & 0.0 & 9.1 & 15.3 & 28.5 & 6.1 & 25.9 & 3.7 & 31.0 & 172 & 14.9 & 143 & 249 & 173 & 302 & 148 & 240 & 133 & 47.6 & 3.0 \\
\hline $11 / 1 / 92$ & 1220 & 02 & 00 & 10.4 & 16.7 & 28.9 & 72 & 25.5 & 9.7 & 31.8 & 16.1 & 15.1 & 13.4 & 250 & 18.5 & 50.5 & 152 & 245 & 127 & 442 & 3.0 \\
\hline $11 / 1 / 92$ & 1245 & 02 & 0.0 & 9.2 & 14.6 & 28.6 & 5.5 & 25.8 & 5.0 & 31.4 & 18.5 & 14.9 & 130 & 242 & 134 & 20.6 & 146 & 200 & 14.1 & 46.1 & 3.0 \\
\hline $11 / 1 / 92$ & 1300 & 02 & 0.0 & 9.7 & 14.9 & 20.1 & 7.2 & 25.9 & 5.4 & 323 & 18.5 & 15.3 & 14.2 & $\mathbf{2 0 5}$ & 19.1 & 30.7 & 16.1 & 24.4 & 13.6 & 469 & 3.0 \\
\hline $11 / 1 / 92$ & 1315 & 02 & 0.0 & 9.6 & 138 & 28.5 & 6.1 & 25.9 & 6.5 & 31.5 & 16.6 & 14.9 & 132 & 20.1 & 14.4 & 303 & 130 & 240 & 128 & 48.4 & 3.1 \\
\hline $11 / 1 / 92$ & 1330 & 02 & 0.0 & 9.3 & 13.8 & 29.5 & 5.1 & 25.8 & 3.5 & 28.7 & 17.0 & 15.0 & 14.7 & 212 & 18.6 & 30.1 & 127 & 245 & 14.6 & 48.2 & 30 \\
\hline $11 / 1 / 92$ & 1345 & 02 & 0.0 & 9.5 & 15.3 & $\mathbf{2 9 . 0}$ & 8.2 & $\mathbf{2 6 . 0}$ & 5.9 & 30.0 & 180 & 15.0 & 142 & 250 & 153 & 300 & 152 & 240 & 127 & 46.6 & 3.4 \\
\hline $11 / 1 / 92$ & 1400 & 02 & 0.0 & 8.6 & 15.8 & 29.3 & 13.6 & 25.8 & 14.2 & 20.9 & 21.6 & 15.2 & 14.1 & 242 & 17.0 & 20.4 & 152 & 23.6 & 13.4 & 48.1 & 3.7 \\
\hline $1 / 1 / 92$ & 1415 & 02 & 0.0 & 9.2 & 15.4 & 20.0 & 7.9 & 25.9 & 6.5 & 31.4 & 20.0 & 15.3 & 13.8 & 20.1 & 26.7 & 20.3 & 212 & 220 & 14.2 & 48.9 & 3.1 \\
\hline $11 / 1 / 92$ & 1430 & 02 & 0.0 & 9.3 & 15.6 & 29.6 & 6.5 & 25.9 & 5.2 & 30.6 & 17.3 & 14.9 & 14.4 & 253 & 152 & 28.1 & 154 & 227 & 14.8 & 429 & 3.0 \\
\hline
\end{tabular}




\begin{tabular}{|c|c|c|c|c|c|c|c|c|c|c|c|c|c|c|c|c|c|c|c|c|c|}
\hline Dats & Time & $\begin{array}{l}\text { Wator } \\
\text { Flow } \\
\text { gpm }\end{array}$ & $\begin{array}{l}\text { Limo } \\
\text { Flow } \\
\text { gpm }\end{array}$ & $\begin{array}{l}S * k \\
\phi_{0} \\
\end{array}$ & $\begin{array}{r}B \\
O_{0}^{0} \\
\% \\
\end{array}$ & $\begin{array}{r}01 \\
K W \\
\end{array}$ & $\begin{array}{l}1 \\
\text { splw } \\
\mathrm{mn}\end{array}$ & & $\begin{array}{l}\text { sple } \\
\text { mn }\end{array}$ & & $\begin{array}{l}3 \\
\mathrm{mn}\end{array}$ & $\begin{array}{r}D \\
K W \\
\end{array}$ & iples & & & $\mathrm{KW}$ & $m n$ & $K W$ & $\mathrm{mn}$ & $\mathrm{KW}$ & $\mathrm{mn}$ \\
\hline $11 / 1 / 22$ & 1446 & 02 & 0.0 & 8.6 & 17.7 & $\mathbf{2 9 . 5}$ & 8.3 & 25.8 & 3.6 & $\mathbf{2 0 . 9}$ & 182 & 15.3 & 13.6 & 286 & 162 & 20.7 & 146 & 20.7 & 17.0 & $\$ 2$ & 3.0 \\
\hline $11 / 1 / 92$ & 1500 & 02 & 00 & 8.6 & 15.3 & 29.3 & 6.3 & 25.8 & 4.8 & 20.7 & 17.2 & 14.6 & 13.7 & 252 & 16.7 & 20.4 & 149 & 20.6 & 14.0 & 47.4 & 3.2 \\
\hline $11 / 1 / 92$ & 1615 & 02 & 0.0 & 8.8 & 17.2 & 29.4 & 7.6 & 25.7 & 7.5 & 312 & 19.7 & 14.7 & 14.6 & 24.4 & 14.6 & 2023 & 167 & 21.6 & 16.5 & 46.7 & 3.1 \\
\hline 11/1/92 & 1550 & 02 & 0.0 & 8.7 & 15.1 & 29.5 & 8.1 & 25.8 & 4.6 & 29.7 & 19.3 & 14.7 & 15.8 & 24.0 & 17.1 & 286 & 185 & 200 & 15.5 & 47.8 & 3.4 \\
\hline $11 / 1 / 92$ & 1545 & 02 & 0.0 & 8.2 & 15.0 & 28.8 & 7.5 & 25.8 & 3.5 & 31.1 & 16.6 & 14.8 & 15.8 & 28.6 & 15.3 & 29.6 & 135 & 24.0 & 14.5 & 47.6 & 4.7 \\
\hline $11 / 1 / 92$ & 1600 & 02 & 0.0 & 9.1 & 14.3 & 29.4 & 5.6 & 25.8 & 4.1 & 30.9 & 16.0 & 15.1 & 14.2 & 24.0 & 16.1 & 30.1 & 130 & 23.8 & 12.7 & 44.8 & 11.0 \\
\hline $11 / 1 / 92$ & 1615 & 02 & 0.0 & 9.0 & 18.3 & 29.8 & 5.3 & 25.8 & 8.1 & 324 & 162 & 15.1 & 14.1 & 28.1 & 152 & 20.6 & 163 & 24.0 & 13.7 & 46.8 & 3.0 \\
\hline $11 / 1 / 92$ & 1600 & 02 & 0.0 & 10.3 & 16.3 & 28.4 & 7.0 & 26.8 & 4.8 & 36.7 & 14.1 & 15.9 & 13.3 & $2 \mathbf{2 6}$ & 19.5 & 30.4 & 17.0 & 24.1 & 12.6 & 492 & 15.3 \\
\hline $11 / 1 / 92$ & 1645 & 02 & 0.0 & 10.3 & 16.0 & 26.9 & 8.7 & 26.0 & 9.3 & 20.6 & 17.7 & 15.1 & 138 & 243 & 163 & 202 & 149 & 23.1 & 13.3 & 48.2 & 4.3 \\
\hline 11/1/92 & 1700 & 02 & 0.0 & 10.0 & 15.2 & 20.4 & 8.2 & 258 & 4.3 & 31.0 & 18.4 & 18.0 & 15.0 & 24.0 & 17.1 & 20.4 & 14.6 & $2 \mathbf{8}$ & 126 & 48.9 & 3.1 \\
\hline $11 / 1 / \theta 2$ & 1715 & 02 & 0.0 & 9.0 & 14.8 & 29.4 & 5.5 & 26.8 & 4.6 & 268 & 228 & 16.1 & 13.8 & 22.6 & 178 & 20.0 & 16.4 & 221 & 128 & 46.7 & 4.0 \\
\hline $11 / 1 / 92$ & 1730 & 02 & 0.0 & 8.8 & 17.3 & 28.3 & 9.2 & 25.7 & 9.5 & 28.1 & 203 & 16.0 & 14.7 & 226 & 206 & 200 & 18.1 & 21.5 & 18.2 & 47.0 & 3.8 \\
\hline $11 / 1 / 82$ & 1745 & 02 & 0.0 & 9.9 & 15.2 & $\mathbf{2 0 . 9}$ & 0.4 & 25.9 & 8.2 & 204 & 23.4 & 15.0 & 148 & 24.0 & 102 & 22.6 & 16.7 & 223 & 14.6 & 47.0 & 42 \\
\hline $11 / 1 / 92$ & 1800 & 02 & 0.0 & 8.7 & 16.2 & 28.8 & 112 & 23.6 & 11.6 & 282 & 17.5 & 15.0 & 15.5 & 208 & 180 & 23.1 & 163 & 227 & 14.0 & 46.8 & 3.1 \\
\hline 11/1/92 & 1815 & 02 & 0.0 & 9.1 & 17.3 & 27.5 & 12.8 & 25.2 & 16.3 & 28.4 & 192 & 14.0 & 15.4 & 221 & 20.5 & $\mathbf{2 8 . 0}$ & 18.0 & 21.8 & 13.8 & 45.8 & 3.1 \\
\hline $11 / 1 / 92$ & 1800 & 02 & 0.0 & 8.7 & 14.1 & 282 & 8.7 & 25.6 & 7.6 & 30.4 & 19.2 & 148 & 13.6 & $\mathbf{2 1 6}$ & 17.1 & 20.0 & 160 & 20.1 & 12.6 & 47.2 & 3.1 \\
\hline 11/1/92 & 1845 & 02 & 0.0 & 9.7 & 16.1 & 28.1 & 14.2 & 25.6 & 13.9 & $\mathbf{2 8 . 4}$ & 19.8 & 15.0 & 13.5 & 20.0 & 19.8 & 27.5 & 16.1 & 220 & 13.1 & 45.7 & 3.1 \\
\hline 11/1/92 & 1900 & 02 & 0.0 & 8.5 & 15.4 & 29.2 & 9.4 & 25.6 & 4.5 & 30.7 & 198 & 15.3 & 14.1 & 23.7 & 160 & 29 & 163 & 24.5 & 13.6 & 46.8 & 3.4 \\
\hline $11 / 1 / 92$ & 1915 & 0.1 & 0.0 & 10.0 & 16.5 & 29.3 & 11.8 & 25.4 & 12.3 & 29.8 & 18.0 & 14.8 & 15.3 & 24.0 & 120 & 22.7 & 184 & 227 & 14.2 & 45.4 & 3.1 \\
\hline 11/1/92 & 1900 & 0.1 & 0.0 & 10.1 & 14.6 & $\mathbf{2 9 . 7}$ & 8.0 & 25.6 & 4.8 & 28.6 & 19.1 & 14.9 & 142 & 20.7 & 330 & 270 & 21.3 & 20.5 & 16.6 & 44.9 & 5.7 \\
\hline $11 / 1 / 92$ & 1945 & 0.1 & 0.0 & 0.1 & 17.0 & 27.5 & 11.3 & 25.6 & 5.8 & 31.4 & 17.4 & 15.0 & 13.5 & 227 & 19.4 & 27.7 & 173 & 212 & 14.9 & 45.3 & 3.1 \\
\hline $11 / 1 / 92$ & 2000 & 0.1 & 0.0 & 9.7 & 14.8 & 28.8 & 7.8 & 25.4 & 5.1 & 20.3 & 17.6 & 152 & 13.1 & 202 & 185 & 225 & 18.0 & 228 & 13.6 & 45.7 & 3.0 \\
\hline $11 / 1 / 92$ & 2015 & 0.1 & 0.0 & 10.1 & 14.4 & $\mathbf{2 9 . 4}$ & 13.8 & 20.3 & 8.6 & 30.0 & 18.1 & 14.6 & 143 & 250 & 21.9 & $\overline{283}$ & 16.9 & $\overline{23.0}$ & 13.7 & 43.7 & 3.0 \\
\hline $11 / 1 / 92$ & 2000 & 0.1 & 0.0 & 9.8 & 14.9 & 29.6 & 6.3 & 25.3 & 5.0 & 31.1 & 13.3 & 15.0 & 13.9 & 27.0 & 126 & 30.5 & 13.9 & 24.6 & 126 & 45.4 & 3.0 \\
\hline 11/1/92 & 2045 & 0.1 & 0.0 & 9.1 & 13.7 & $\mathbf{2 9 . 1}$ & 4.7 & $\mathbf{2 5 . 4}$ & 3.5 & 31.0 & 18.5 & 15.3 & 14.3 & 236 & 158 & 288 & 14.4 & 23.0 & 15.8 & 45.9 & 3.6 \\
\hline 11/1/92 & 2100 & 0.1 & 0.0 & 8.7 & 14.7 & 30.0 & 4.9 & 25.3 & 3.8 & 30.6 & 18.8 & 15.2 & 13.5 & 248 & 16.0 & 28.1 & 15.1 & 230 & 121 & 448 & 3.0 \\
\hline 11/1/92 & 2115 & 0.1 & 0.0 & 13.8 & 14.4 & $\mathbf{2 9 . 0}$ & 6.7 & 25.0 & 4.6 & 28.8 & 19.0 & 150 & 142 & 282 & 19.5 & 20.3 & 20.0 & 220 & 13.4 & 429 & 3.0 \\
\hline $11 / 1 / 92$ & 2130 & 0.1 & 0.0 & 16.4 & 14.4 & 30.1 & 62 & 25.3 & 3.6 & $\mathbf{2 9 . 0}$ & 18.0 & 15.0 & 143 & $\mathbf{2 8 . 1}$ & 120 & 20.0 & 130 & 24.6 & 13.1 & 427 & 3.2 \\
\hline $11 / 1 / 92$ & 2145 & 0.1 & 0.0 & 15.0 & 15.7 & 29.2 & 13.6 & 25.2 & 8.6 & 20.8 & 19.5 & 15.0 & 14.0 & 27.6 & 16.0 & 20.6 & 15.6 & 24.1 & 14.0 & 43.9 & 3.0 \\
\hline 11/1/92 & 2200 & 0.1 & 0.0 & 13.4 & 14.1 & 29.4 & 5.3 & 25.3 & 48 & 29.0 & 19.5 & 15.2 & 14.1 & 243 & 18.1 & 282 & 14.7 & 226 & 127 & 462 & 73 \\
\hline 11/1/92 & 2215 & 0.0 & 0.0 & 13.8 & 14.5 & 28.9 & 4.2 & 25.2 & 32 & 20.4 & 20.3 & 148 & 15.0 & 232 & 140 & 28.4 & 16.4 & 225 & 14.6 & 46.3 & 6.2 \\
\hline 11/1/92 & 2220 & 0.0 & 0.0 & 15.9 & 13.3 & 30.3 & 3.6 & 20.1 & 3.9 & 20.4 & 18.6 & 15.1 & 138 & 20.7 & 122 & 285 & 163 & 23.4 & 127 & 46.0 & 3.5 \\
\hline $11 / 1 / 92$ & 2245 & 0.1 & 0.0 & 14.6 & 14.0 & 29.7 & 5.5 & 25.1 & 42 & 29.7 & 17.0 & 14.9 & 15.0 & 262 & 133 & 283 & 16.5 & 23.1 & 14.1 & 44.5 & 3.2 \\
\hline 11/1/92 & 2000 & 0.0 & 0.0 & 14.6 & 14.3 & $\mathbf{3 0 . 0}$ & 5.5 & 25.1 & 4.3 & 31.9 & 17.3 & 15.3 & 14.3 & $\mathbf{2 6 8}$ & 13.7 & 286 & 156 & 220 & 13.8 & 47.0 & 3.7 \\
\hline $11 / 1 / 92$ & 2315 & 0.0 & 0.0 & 15.4 & 14.8 & 30.1 & 4.9 & 25.2 & 3.2 & 30.2 & 17.8 & 15.1 & 14.5 & 27.7 & 11.0 & 200 & 138 & 24.4 & 12.8 & 448 & 3.8 \\
\hline $11 / 1 / 92$ & 2330 & 0.0 & 0.0 & 14.9 & 14.8 & $\mathbf{2 9 . 9}$ & 6.7 & 25.1 & 3.1 & 30.9 & 17.0 & 15.4 & 13.8 & 258 & 148 & 20.6 & 17.1 & 23.9 & 14.3 & 46.6 & 9.7 \\
\hline $11 / 1 / 92$ & 2345 & 0.0 & 0.0 & 14.8 & 14.4 & 29.6 & 6.5 & 25.1 & 5.8 & 29.9 & 16.3 & 15.3 & 13.5 & 282 & 96 & 30.6 & 10.1 & 24.6 & 11.5 & 454 & 5.2 \\
\hline 11/292 & 0 & 0.0 & 0.0 & 15.2 & 15.3 & 29.9 & 6.1 & 25.1 & 4.4 & 30.1 & 21.1 & 15.4 & 13.6 & 28.7 & 78 & 339 & 129 & 27.0 & 12.6 & 454 & 4.3 \\
\hline $11 / 292$ & 15 & 0.0 & 0.0 & 16.4 & 128 & 30.3 & 3.2 & 25.1 & 3.3 & 31.0 & 18.3 & 15.0 & 13.6 & 285 & 13.1 & 285 & 153 & 242 & 13.7 & 46.2 & 4.0 \\
\hline $11 / 292$ & 30 & 0.0 & 0.0 & 16.8 & 142 & 30.3 & 3.2 & 25.1 & 3.1 & 30.5 & 9.7 & 178 & 12.4 & $\overline{23.4}$ & 150 & 280 & 18.1 & 23.7 & 14.3 & 483 & 7.1 \\
\hline $11 / 292$ & 45 & 0.1 & 0.0 & 8.4 & 13.9 & $\mathbf{5 0 . 9}$ & 3.3 & 25.1 & 3.2 & 37.6 & 18.8 & 17.5 & 15.2 & 28.1 & 13.1 & 281 & 16.1 & 24.0 & 15.2 & 456 & 3.2 \\
\hline $11 / 292$ & 100 & 0.1 & 0.0 & 7.6 & 13.2 & 30.0 & 7.3 & 252 & 3.1 & 30.1 & 19.5 & 14.6 & 13.6 & 29.6 & 202 & 31.4 & 17.3 & 26.5 & 172 & 462 & 7.2 \\
\hline $11 / 292$ & 115 & 0.1 & 0.0 & 8.0 & 126 & 30.4 & 3.2 & 25.1 & 3.5 & 302 & 18.0 & 15.0 & 16.6 & 33.6 & 153 & 342 & 18.7 & 28.5 & 15.4 & 418 & 3.0 \\
\hline $11 / 292$ & 130 & 0.1 & 0.0 & 7.5 & 124 & 30.6 & 3.2 & 25.2 & 3.1 & 34.9 & 13.9 & 16.5 & 13.1 & 327 & 120 & 29.6 & 160 & 24.6 & 13.2 & 05 & 6.8 \\
\hline $11 / 292$ & 145 & 0.1 & 0.0 & 7.0 & 126 & 30.9 & 3.1 & 25.2 & 3.1 & 326 & 17.5 & 16.5 & 125 & 34.4 & 8.7 & 29.7 & 18.7 & 26.4 & 12.9 & $1: 6$ & 9.5 \\
\hline $11 / 292$ & 200 & 0.1 & 0.0 & 10.1 & 127 & 30.6 & 3.1 & 25.2 & 3.0 & 30.8 & 17.3 & 15.6 & 139 & 312 & 15.0 & 203 & 135 & 25.6 & 142 & $\Delta B$ & 6.7 \\
\hline $11 / 292$ & 215 & 0.1 & 0.0 & 8.9 & 13.0 & $\mathbf{3 0 . 7}$ & 5.1 & 25.1 & 3.0 & 31.6 & 16.3 & 15.3 & 142 & 35.1 & 120 & 31.0 & 127 & 28.3 & 14.0 & 458 & 6.8 \\
\hline $11 / 292$ & 200 & 0.1 & 0.0 & 7.3 & 125 & 31.0 & 3.2 & 25.1 & 3.1 & 329 & 17.9 & 15.8 & 126 & 33.6 & 10.1 & 31.3 & 14.0 & 281 & 13.0 & $48 B$ & 3.3 \\
\hline $11 / 2 / 92$ & 245 & 0.1 & 0.0 & 7.3 & 127 & 31.1 & 3.1 & 25.3 & 3.1 & 34.6 & 15.1 & 15.6 & 13.5 & 36.1 & 93 & 33.4 & 10.2 & 30.1 & 12.5 & 41 & 4.2 \\
\hline 11/292 & 300 & 0.1 & 0.0 & 8.5 & 11.8 & 30.9 & 3.1 & 25.3 & 3.1 & $\mathbf{2 9 . 0}$ & 17.2 & 16.1 & 126 & 35.6 & 11.1 & 33.0 & 142 & 29.8 & 13.5 & 472 & 5.8 \\
\hline $11 / 292$ & 315 & 0.1 & 0.0 & 8.1 & 124 & 31.0 & 3.1 & 252 & 3.1 & 31.3 & 17.1 & 15.7 & 13.6 & 35.6 & 10.0 & 320 & 13.7 & 28.7 & 13.5 & 474 & 3.6 \\
\hline $11 / 292$ & 350 & 0.1 & 0.0 & 7.7 & 125 & 31.2 & 3.1 & 25.3 & 3.3 & 300 & 19.3 & 16.4 & 142 & 360 & 8.7 & 328 & 16.0 & 29.9 & 13.8 & 48.3 & 7.3 \\
\hline $11 / 292$ & 345 & 0.1 & 0.0 & 5.4 & 42 & 31.1 & 3.2 & 25.2 & 3.1 & 34.8 & 15.5 & 158 & 14.0 & 38.7 & 84 & 342 & 10.7 & 324 & 14.5 & 47.8 & 6.9 \\
\hline $11 / 292$ & 400 & 0.1 & 0.0 & 7.0 & 122 & 312 & 3.2 & 25.1 & 3.2 & 30.5 & 13.5 & 15.7 & 13.9 & 37.1 & 7.7 & 349 & 102 & 31.6 & 11.8 & 48.0 & 8.8 \\
\hline $11 / 292$ & 415 & 0.1 & 0.0 & 7.5 & 120 & 31.1 & 3.1 & 25.1 & 3.7 & 329 & 15.5 & 15.8 & 13.7 & $\mathbf{3 6 3}$ & 98 & 353 & 108 & 327 & 127 & 39.0 & 3.0 \\
\hline $11 / 292$ & 430 & 0.1 & 0.0 & 6.9 & 11.5 & 31.3 & 3.2 & 25.2 & 3.0 & 34.4 & 15.8 & 15.5 & 13.7 & 37.0 & 80 & 346 & 10.1 & 31.8 & 132 & 44.5 & 9.4 \\
\hline $11 / 292$ & 445 & 0.1 & 0.0 & 7.4 & 121 & 30.9 & 5.6 & 25.2 & 3.0 & 34.4 & 15.5 & 16.1 & 14.1 & 385 & 85 & 340 & 10.9 & 323 & 11.7 & 45.5 & 6.0 \\
\hline $11 / 292$ & 500 & 0.1 & 0.0 & 8.2 & 123 & 31.1 & 3.3 & 25.3 & 3.1 & 322 & 16.7 & 15.4 & 13.6 & 37.1 & 70 & 34.5 & 10.1 & 320 & 127 & 45.8 & 10.6 \\
\hline $11 / 292$ & 515 & 0.1 & 0.0 & 6.8 & 11.6 & 31.3 & 3.2 & 25.2 & 3.0 & 33.3 & 222 & 15.9 & 13.4 & $\$ 0.0$ & 50 & 35.7 & 98 & 330 & 11.9 & 47.1 & 7.4 \\
\hline 11/292 & 530 & 0.1 & 0.0 & 7.4 & 120 & 312 & 3.2 & 25.3 & 3.1 & 329 & 16.5 & 16.0 & 13.8 & 327 & 58 & 37.1 & 8.1 & 34.5 & 11.7 & 47.4 & 8.9 \\
\hline $11 / 292$ & 545 & 0.1 & 0.0 & 7.7 & 126 & 312 & 3.6 & ఒ. & 3.1 & 324 & 16.0 & 16.1 & 13.4 & 37.9 & 112 & 352 & 9.7 & 30.8 & 128 & 44.6 & 3.7 \\
\hline $11 / 292$ & $6 \infty$ & 0.1 & 0.0 & 7.5 & 11.5 & 31,3 & 3.2 & 25.2 & 3.5 & 34.6 & 15.1 & 16.0 & 13.9 & 39.9 & 64 & 36.1 & 8.5 & 33.6 & 118 & 46.4 & 14.5 \\
\hline $11 / 292$ & 615 & 0.1 & 0.0 & 7.9 & 11.6 & 31.4 & 3.1 & 25.2 & 3.1 & 328 & 15.2 & 15.9 & 13.6 & 37.9 & 9.1 & 35.1 & 96 & 328 & 15.9 & 44.6 & 72 \\
\hline $11 /$ & $\infty 0$ & 0.1 & 0.0 & 7.5 & 11.6 & 31.4 & 3.2 & 252 & 3.0 & 35.0 & 15.3 & 16.5 & 13.1 & $\infty .1$ & 62 & 36.1 & 93 & 33.4 & 14.1 & 47.6 & 9.5 \\
\hline $1 / 292$ & 64 & 0.1 & - & 7.3 & 11.4 & 31.2 & 3.2 & 5.2 & - & 33.6 & 16 & 6 & . & 39.6 & 6 & 36.7 & 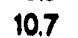 & 31.8 & 11.8 & 45.9 & 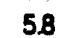 \\
\hline
\end{tabular}




\begin{tabular}{|c|c|c|c|c|c|c|c|c|c|c|c|c|c|c|c|c|c|c|c|c|c|}
\hline Dats & Time & $\begin{array}{l}\text { Water } \\
\text { Flow } \\
\text { gpm }\end{array}$ & $\begin{array}{l}\text { Lime } \\
\text { Flow } \\
\text { ogm }\end{array}$ & $\begin{array}{l}\text { Sux } \\
\% \\
\%\end{array}$ & $\begin{array}{l}8 \\
0^{8} \\
\%\end{array}$ & $\begin{array}{r}01 \\
\mathrm{KW} \\
\end{array}$ & $\begin{array}{l}\text { speks } \\
\text { mn }\end{array}$ & & $\begin{array}{l}\text { spok } \\
\mathrm{mn}\end{array}$ & & $\begin{array}{l}3 \\
\text { mn }\end{array}$ & & & & & & & KW & $\mathrm{mn}$ & $\mathrm{kW}$ & \\
\hline $11 / 292$ & 700 & 0.1 & 00 & 8.7 & 11.8 & 31.0 & 3.5 & $\mathbf{2 0 . 1}$ & 3.0 & 328 & 16.5 & 18.9 & 13.6 & 30.4 & 11.0 & 36 & 103 & sas & 12.1 & 45.2 & 84 \\
\hline $11 / 292$ & 715 & 0.1 & 0.0 & 8.4 & 126 & 31.1 & 8.2 & 80.0 & 3.0 & 320 & 17.0 & 16.0 & 13.4 & 320 & 88 & 34.4 & 108 & 321 & 11.4 & 452 & 3.8 \\
\hline $11 / 292$ & 730 & 0.1 & 0.0 & 202 & 124 & 31.0 & 3.1 & $\mathbf{2 0 . 0}$ & 32 & 327 & 17.4 & 16.0 & 14.1 & 30.4 & 7.7 & 34.4 & 13.1 & 326 & 123 & 45.1 & 4.8 \\
\hline $11 / 2 / 92$ & 745 & 0.1 & 0.0 & 11.8 & 11.8 & 31.1 & 3.2 & 20.1 & 3.0 & 30.0 & 18.6 & 15.6 & 14.3 & 308 & 80 & 38.6 & 11.0 & 30 & 128 & 45.5 & 9.1 \\
\hline $11 / 292$ & 800 & 0.1 & 0.0 & 10.3 & 122 & 31.3 & 3.9 & 20.1 & 3.0 & 34.3 & 16.7 & $15 B$ & 14.4 & 300 & 113 & 300 & 102 & 320 & 11.6 & 47.0 & 72 \\
\hline $11 / 2 / 2$ & 815 & 0.1 & 0.0 & 10.3 & 126 & 30.8 & 3.1 & $\mathbf{2 5 0} 0$ & 3.0 & 34.0 & 17.3 & 16.5 & 13.9 & 33.4 & 213 & 38.1 & 11.5 & 30.9 & 125 & 47.1 & 7.7 \\
\hline $11 / 292$ & 800 & 0.1 & 0.0 & 10.1 & 13.0 & 31.1 & 3.8 & 35.1 & 3.0 & 35.0 & 16.8 & 16.4 & 14.4 & 30.7 & 76 & 34.6 & 11.8 & 342 & 11.4 & 475 & 5.7 \\
\hline $11 / 292$ & 846 & 0.1 & 0.0 & 9.0 & 125 & 31,0 & 3.2 & 25.2 & 3.0 & 34.1 & 17.7 & 16.5 & 14.8 & 320 & 78 & 30.0 & 102 & 34.4 & 162 & 47.1 & 6.1 \\
\hline $11 / 292$ & $\infty 00$ & 0.1 & 0.0 & 9.9 & 122 & 30.7 & 32 & 25.0 & 32 & 33.7 & 172 & 16.7 & 13.8 & 398 & 112 & 330 & 16.1 & 320 & 118 & 429 & 4.8 \\
\hline $11 / 292$ & 915 & 0.1 & 0.0 & 10.1 & 13.2 & 30.6 & 4.0 & 20.0 & 3.0 & 38.7 & 14.7 & 16.7 & 14.1 & 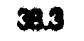 & 4.4 & $4 a 7$ & 32 & 41.0 & 6.3 & 421 & 12.0 \\
\hline 11/2/92 & $\infty 0$ & 0.1 & 0.0 & 10.7 & 11.1 & 30.7 & 3.6 & 20.1 & 3.0 & 34.6 & 12.8 & 16.5 & 14.6 & 503 & $O A$ & 32.1 & 10.3 & 320 & 126 & 42 & 6.7 \\
\hline $11 / 292$ & 945 & 0.1 & 0.0 & 9.9 & 13.0 & 30.5 & 3.5 & 200 & 3.0 & 300 & 168 & 16.5 & 13.1 & 343 & 89 & 304 & 136 & 326 & 123 & 464 & 102 \\
\hline $11 / 292$ & 1000 & 0.1 & 0.0 & 102 & 125 & 30.6 & 3.1 & $\mathbf{2 5 . 0}$ & 3.0 & 34.4 & 19.0 & 16.7 & $14 B$ & & 122 & 302 & 13.1 & 328 & 13.4 & 476 & 80 \\
\hline 11/292 & 1015 & 0.1 & 0.0 & 10.2 & 120 & $\mathbf{3 0 . 3}$ & 3.3 & 20.0 & 3.0 & 33.9 & 18.6 & 16.0 & 132 & 300 & 118 & 32,4 & 11.7 & 302 & 13.8 & 42 & 42 \\
\hline 11/292 & 1000 & 0.1 & 0.0 & 10.4 & 124 & 30.7 & 3.3 & 20.0 & 3.0 & 35.6 & 14.6 & 16.7 & $13 B$ & 34.1 & 123 & 338 & OA & 326 & 118 & 46.3 & 72 \\
\hline $11 / 2 / 92$ & 1045 & 0.1 & 0.0 & 10.6 & 120 & 30.6 & 3.2 & 20.0 & 3.1 & 30.7 & 16.4 & 16.1 & 13.4 & 343 & 123 & 37.7 & 10.4 & 300 & 13.3 & 46.1 & 8.0 \\
\hline $11 / 202$ & 1100 & 0.1 & 0.0 & 11.6 & 125 & 30.0 & 3.2 & 25.1 & 3.1 & 38.1 & 140 & 16.6 & 14.4 & 300 & 8.7 & 3.1 & 130 & 34.7 & 10.2 & 478 & 7.0 \\
\hline $11 / 292$ & 1115 & 0.1 & 0.0 & 11.7 & 124 & 30.9 & 3.3 & 25.1 & 3.4 & 30.3 & 16.4 & 16.3 & 142 & 302 & 84 & 372 & 128 & 308 & 13.8 & 68 & 9.6 \\
\hline 11/292 & 1130 & 0.1 & 0.0 & 15.0 & 13.8 & 30.7 & 3.7 & 25.2 & 3.2 & 34.0 & 16.6 & 163 & 13.0 & 35.6 & 83 & 358 & 98 & 328 & 11.7 & 473 & 10.1 \\
\hline $11 / 292$ & 1145 & 0.1 & 0.0 & 10.8 & 11.8 & 30.9 & 4.0 & 25.2 & 3.3 & 34.0 & 19.5 & 16.3 & 142 & 353 & 11.9 & 33.7 & 10.7 & 30.1 & 128 & 471 & 6.4 \\
\hline $11 / 292$ & 1200 & 0.1 & 0.0 & 10.7 & 125 & 30.8 & 5.8 & 25.2 & 3.2 & 36.0 & 153 & 16.7 & 14.6 & 37.6 & 74 & 303 & 121 & 320 & 127 & 437 & 6.4 \\
\hline $11 / 2 / 92$ & 1215 & 0.1 & 0.0 & 10.8 & 125 & 30.9 & 4.3 & 25.2 & 3.3 & 378 & 125 & 172 & 124 & 33.6 & 10.4 & 303 & 98 & 38.4 & 110 & 408 & 10.3 \\
\hline $11 / 292$ & 1200 & 0.1 & 0.0 & 9.4 & 11.7 & 30.6 & 4.6 & 25.2 & 3.1 & 35.4 & 16.6 & 16.3 & 13.7 & 38.1 & 88 & 38.9 & 10.4 & 338 & 118 & 49 & 10.7 \\
\hline $11 / 292$ & 1245 & 0.1 & 0.0 & 11.0 & 122 & 31.1 & 5.2 & 20.3 & 3.1 & 30.2 & 14.8 & 162 & 13.5 & 372 & 60 & 373 & 127 & 308 & 10.9 & 465 & 0.7 \\
\hline 11/2/92 & 1300 & 0.1 & 0.0 & 11.4 & 11.8 & 31.0 & 3.3 & 20.2 & 3.6 & 33.9 & 16.0 & 16.7 & 138 & 338 & 11.0 & 30.3 & 102 & 324 & 113 & 40 & 43 \\
\hline $11 / 2 / \theta 2$ & 1315 & 0.1 & 0.0 & 11.1 & 124 & 30.6 & 3.2 & 252 & 3.1 & 33.3 & 19.3 & 162 & 148 & 303 & 102 & 32.0 & 120 & 328 & 128 & 40 & 6.1 \\
\hline 11/292 & 1300 & 0.1 & 0.0 & 122 & 121 & 30.7 & 3.4 & 25.2 & 3.1 & 35.4 & 17.4 & 168 & 143 & $\mathbf{3 a 6}$ & 82 & 37.4 & 11.6 & 320 & 148 & 67 & 8.1 \\
\hline $11 / 292$ & 1345 & 0.1 & 0.0 & 11.4 & 121 & 30.9 & 3.2 & 25.2 & 3.5 & 30.1 & 14.6 & 17.1 & 13.7 & 300 & 64 & $\boldsymbol{\varphi 0 . 1}$ & 123 & 330 & 10.7 & $\mathbf{6 3}$ & 9.7 \\
\hline $11 / 292$ & 1400 & 0.1 & 0.0 & 13.5 & 124 & 30.2 & 4.0 & 25.0 & 3.3 & 36.1 & 127 & 16.9 & 13.4 & 345 & 64 & $\boldsymbol{0 . 7}$ & 88 & 37.4 & 168 & 41 & 6.7 \\
\hline $11 / 292$ & 1415 & 0.0 & 0.0 & 17.9 & 122 & 31.0 & 3.0 & 25.5 & 3.1 & $\mathbf{3 9 . 6}$ & 13.4 & 18.5 & 127 & 30.4 & 68 & 37.6 & 95 & 382 & 118 & 474 & 3.5 \\
\hline 11/292 & 1430 & 0.1 & 0.0 & 11.4 & 121 & 30.8 & 3.1 & $\mathbf{2 5 . 3}$ & 3.4 & 38.4 & 143 & 18.0 & 120 & 302 & 93 & 30.4 & 10.6 & 37.9 & 124 & 42 & 3.5 \\
\hline $11 / 292$ & 1445 & 0.1 & 0.0 & 11.7 & 11.7 & 30.8 & 3.1 & 20.3 & 3.2 & 37.0 & 17.0 & 17.3 & 13.0 & 37.7 & 75 & 39.4 & 7.1 & 37.9 & 110 & 135 & 6.0 \\
\hline $11 / 292$ & 1500 & 19.5 & 0.0 & 120 & 13.5 & 324 & 3.3 & $\mathbf{2 0 . 8}$ & 32 & 03.8 & 3.7 & 28.1 & 10.3 & 303 & 60 & 108 & 8.7 & 38.8 & 108 & 467 & 82 \\
\hline $11 / 2 / 92$ & 1515 & 22.2 & 0.0 & 13.4 & 121 & 320 & 3.3 & 27.7 & 3.3 & 520 & 3.1 & 33.6 & 7.7 & 30.6 & 70 & $\$ .5$ & 83 & 30.1 & 10.7 & 446 & 4.7 \\
\hline $11 / 292$ & 1530 & 25.5 & 0.0 & 128 & 129 & 320 & 3.2 & 27.0 & 3.3 & 48.3 & 3.1 & 452 & 4.1 & $\infty .0$ & 42 & 412 & 78 & 388 & 95 & 42.2 & 6.1 \\
\hline $11 / 292$ & 1545 & 28.5 & 0.0 & 11.3 & 10.7 & 30.5 & 3.3 & $\mathbf{2 8 . 3}$ & 3.3 & 46.4 & 33 & 43.3 & 30 & 30.7 & 8.0 & 41.6 & 50 & 30.7 & 96 & 46.7 & 7.0 \\
\hline $1 / 2 / 92$ & $16 \infty$ & 28.6 & 0.0 & 128 & 10.0 & 322 & 3.2 & $\mathbf{2 8 . 9}$ & 3.3 & 45.9 & 3.1 & 41.8 & 3.0 & 30.7 & 42 & 4.0 & 8.8 & 40.3 & 9.3 & 42.4 & 3.4 \\
\hline $11 / 292$ & 1615 & 0.1 & $\mathbf{2 6 . 9}$ & 123 & 10.1 & 30.8 & 3.4 & 29.3 & 3.4 & 46.0 & 3.1 & 423 & 3.0 & 398 & 32 & 432 & 75 & $\mathbf{0 . 3}$ & 135 & 47.1 & 64 \\
\hline $1 / 2 / 92$ & 1600 & 0.1 & 31.3 & 11.8 & 10.6 & 320 & 3.2 & 20.4 & 3.4 & 45.1 & 3.1 & 30.0 & 3.0 & 30.7 & 32 & 120 & 68 & $\boldsymbol{\infty} 0.0$ & 10.8 & 44.8 & 110 \\
\hline $11 / 292$ & 1645 & 0.1 & 31.7 & 11.7 & 99 & 318 & 3.0 & 29.4 & 32 & 448 & 3.1 & 30.0 & 3.0 & 308 & 4.1 & 42.1 & 4.1 & $\$ 0.2$ & 110 & 45.6 & 5.3 \\
\hline $11 / 292$ & 1700 & 0.1 & 30.8 & 129 & 8.8 & 325 & 3.3 & 29.4 & 3.3 & 4.7 & 3.1 & 382 & 3.0 & 39.8 & 32 & 420 & 10.8 & 39.4 & 135 & 46.8 & 6.8 \\
\hline $11 / 2 / 2$ & 1715 & 0.1 & 29.7 & 126 & 121 & 30.4 & 3.1 & 28.6 & 3.4 & 452 & 3.1 & 38.7 & 3.0 & 300 & 35 & 43.7 & 60 & $\mathbf{\infty 0 . 4}$ & 120 & 40.8 & 36 \\
\hline $11 / 2 / 92$ & 1750 & 0.0 & 30.7 & 11.7 & 95 & 312 & 3.3 & $\mathbf{2 0 . 1}$ & 33 & 448 & 3.1 & 372 & 30 & 309 & 35 & 4.6 & 35 & 08 & 93 & 42.7 & 32 \\
\hline 11/2/92 & 1745 & 0.0 & 33.3 & 11.7 & 9.5 & 31.7 & 32 & 28.1 & 3.4 & 4.7 & 3.1 & 38.5 & 30 & 398 & 42 & 40.4 & 3.7 & $\boldsymbol{\infty} .4$ & 90 & 43.5 & 34 \\
\hline $11 / 2 / 92$ & 1800 & 0.0 & 34.6 & 120 & 10.2 & 30.0 & 3.0 & $\mathbf{2 8 . 9}$ & 3.3 & 4.6 & 30 & 358 & 30 & $\$ .1$ & 65 & 460 & 70 & 41.0 & 108 & 43.8 & 112 \\
\hline $11 / 292$ & 1815 & 0.1 & 34.2 & 14.6 & 10.0 & 30.0 & 3.0 & $\mathbf{2 8 , 9}$ & 3.2 & 44.3 & 30 & 34.9 & 30 & 395 & OA & 42 & 5.7 & 30.2 & 100 & 46.0 & 5.6 \\
\hline $11 / 2 / 92$ & 1830 & 0.1 & 30.0 & 13.2 & 9.4 & 30.8 & 3.0 & $\mathbf{2 8 . 9}$ & 3.4 & 44.7 & 3.0 & 38.6 & 3.0 & 30.7 & 4.1 & 435 & 68 & 30.8 & $10 S$ & 43.9 & 46 \\
\hline $11 / 2 / 92$ & 1845 & 0.1 & 35.2 & 13.1 & 95 & 292 & 3.0 & 28.6 & 3.3 & 4.7 & 3.1 & 35.6 & 3.0 & 32.7 & 72 & 4.8 & 58 & $\$ 0.8$ & 99 & 39.6 & 3.0 \\
\hline $11 / 292$ & 1900 & 0.1 & 33.2 & 13.3 & 9.0 & 302 & 3.0 & $\mathbf{2 8 . 3}$ & 3.3 & 45.1 & 30 & 36.4 & 30 & 30.5 & 40 & 438 & 38 & 40.2 & 113 & 41.0 & 87 \\
\hline $11 / 2 / 92$ & 1916 & 0.1 & 33.9 & 124 & 9.1 & 29.8 & 3.0 & $\mathbf{2 8 . 4}$ & 3.3 & 45.3 & 30 & 36.6 & 3.0 & 30.4 & 34 & 423 & 6.7 & 30.9 & 108 & 46.8 & 70 \\
\hline $11 / 2 / 92$ & 1900 & 0.1 & 33.3 & 17.6 & 9.9 & 30.6 & 3.1 & $\mathbf{2 8 . 8}$ & 3.3 & 45.2 & 3.0 & 36.5 & 30 & 30.6 & 30 & 44.5 & 35 & $\$ 0.4$ & 98 & 47.1 & 6.0 \\
\hline 11/292 & 1945 & 0.1 & 33.3 & 14.8 & 8.9 & 30.1 & 32 & 29.0 & 3.3 & 452 & 30 & 33.7 & 3.0 & 308 & 30 & 4.7 & 33 & 0.4 & 9.4 & 44.6 & 4.5 \\
\hline $11 / 2 / 92$ & 2000 & 0.1 & 34.4 & 129 & 9.6 & 29.6 & 3.0 & 28.1 & 32 & 45.8 & 30 & 37.4 & 30 & 303 & 62 & 42 & 82 & 30.0 & 88 & 42.8 & 3.5 \\
\hline $11 / 292$ & 2015 & 0.1 & 34.1 & 14.0 & 92 & 30.1 & 3.0 & $\mathbf{2 8 . 0}$ & 3.3 & 45.0 & 3.0 & 37.1 & 3.0 & 30.7 & 9.7 & 43.7 & 68 & 40.2 & 76 & 44.7 & 5.1 \\
\hline $11 / 292$ & 2000 & 0.1 & 33.2 & 13.8 & BB & 30.1 & 3.1 & $\mathbf{2 9 . 0}$ & 3.3 & 45.4 & 3.0 & 38.8 & 3.0 & 30.8 & 33 & 4.0 & 34 & $\$ .5$ & 0.7 & 44.3 & 52 \\
\hline $11 / 292$ & 2045 & 0.1 & 34.1 & 14.5 & 13.0 & 20.5 & 3.0 & $\mathbf{2 9 . 3}$ & 3.3 & 44.7 & 30 & 34.8 & 3.0 & 30.7 & 88 & 405 & 5A & $\boldsymbol{\infty} .4$ & 8.4 & 43.9 & 7.0 \\
\hline $11 / 282$ & 2100 & 0.1 & 320 & 13.2 & 85 & 28.8 & 3.0 & $\mathbf{2 8 . 3}$ & 3.3 & 458 & 30 & 36.4 & 30 & 300 & 6.1 & 468 & 52 & 41.1 & 8.3 & 44.1 & 4.7 \\
\hline $11 / 292$ & 2115 & 0.1 & 328 & 14.8 & 9.1 & 28.3 & 3.0 & 28.2 & 3.3 & 45.0 & 30 & 35.7 & 30 & 395 & 38 & 4.7 & 48 & $\mathbf{1 0 . 4}$ & 0.5 & $\$ 6$ & 8.1 \\
\hline $11 / 292$ & 2130 & 0.1 & 326 & 15.8 & 8.5 & 28.6 & 3.0 & $\mathbf{8 . 5}$ & 3.4 & 45.0 & 3.0 & 35.0 & 30 & 30.7 & 32 & 138 & 7.1 & $\$ 0.4$ & 83 & 16.6 & 9.4 \\
\hline $11 / 292$ & 2145 & 0.1 & 326 & 14.0 & 8.6 & 28.3 & 3.0 & 28.2 & 3.3 & 44.9 & 3.0 & 36.7 & 3.0 & 30.4 & 33 & 442 & 55 & 402 & 10.1 & 107 & 3.0 \\
\hline $11 / 2 / 92$ & 2200 & 0.1 & 31.8 & 14.1 & 88 & $\mathbf{2 8 . 7}$ & 3.0 & $\overline{28.3}$ & 3.3 & 44.8 & 30 & 35.6 & 30 & 396 & 30 & 40 & $7 A$ & 41.1 & 8.0 & 40 & 5.0 \\
\hline $11 / 292$ & 2215 & 0.1 & 31.7 & 142 & 89 & 28.4 & 3.0 & 28.2 & 3.3 & 4.7 & 30 & 36.4 & 30 & 395 & 20 & 472 & 58 & 41.3 & 8.1 & 456 & 5.1 \\
\hline $11 / 292$ & 2200 & 0.1 & 321 & 13.0 & BB & $\mathbf{2 8 . 4}$ & 3.2 & 28.0 & 3.4 & 4.9 & 3.0 & 36.7 & 3.0 & 36.8 & 48 & 45.6 & 4.7 & $\$ .4$ & 8.1 & 44.8 & 6.1 \\
\hline $11 / 292$ & 2245 & 0.1 & 31.8 & 16.4 & 89 & 28.6 & 3.0 & 28.0 & 3.3 & 45.2 & 3.1 & 35.8 & 3.0 & 39.9 & 40 & 46.1 & 34 & 41.0 & 93 & 46.2 & 5.3 \\
\hline $11 / 292$ & $\mathbf{2 0 0}$ & 0.1 & 30.4 & 15.7 & 10.0 & $\mathbf{2 9 . 0}$ & 3.0 & 28.1 & 3.3 & 45.4 & 3.6 & 36.2 & 3.0 & 30.5 & 20 & $\mathbf{6 . 7}$ & 70 & $\mathbf{0 . 4}$ & 7.7 & 46.1 & 42 \\
\hline
\end{tabular}




\begin{tabular}{|c|c|c|c|c|c|c|c|c|c|c|c|c|c|c|c|c|c|c|c|c|c|}
\hline Das & Time & $\begin{array}{l}\text { Water } \\
\text { Flow } \\
\text { gpm }\end{array}$ & $\begin{array}{l}\operatorname{Lim}_{\text {Flow }} \\
\text { spm }\end{array}$ & $\begin{array}{l}\text { six } \\
0 \\
\%\end{array}$ & $\begin{array}{l}8 \\
\% \\
\%\end{array}$ & & nn & $\mathrm{KW}$ & $\mathrm{mn}$ & & $m n$ & $K W$ & m & $\mathrm{KW}$ & $m$ & KW & $m$ & $K W$ & $\mathrm{mn}$ & kW & $\mathrm{mn}$ \\
\hline $\begin{array}{l}11 / 202 \\
11 / 200\end{array}$ & 2016 & 0.1 & 30.6 & $\begin{array}{l}13.4 \\
130\end{array}$ & $8 B$ & 20.6 & $\begin{array}{l}3.0 \\
30\end{array}$ & 28.1 & 3.3 & 4.3 & 30 & 38.7 & $\begin{array}{l}3.0 \\
30\end{array}$ & $\begin{array}{l}39.6 \\
09\end{array}$ & 3.1 & 486 & 36 & 102 & $\begin{array}{l}9.8 \\
80\end{array}$ & 467 & 4.5 \\
\hline $\begin{array}{l}11 / 202 \\
11 / 202\end{array}$ & $\begin{array}{l}2350 \\
2345\end{array}$ & 02 & $\begin{array}{l}30.4 \\
20.4\end{array}$ & $\begin{array}{l}13.0 \\
14.8\end{array}$ & 8.9 & $\begin{array}{l}202 \\
324\end{array}$ & $\begin{array}{l}3.0 \\
3.3\end{array}$ & $\begin{array}{l}28.2 \\
20.8\end{array}$ & $\begin{array}{l}3.2 \\
3.4\end{array}$ & 488 & 3.4 & $\begin{array}{l}39.0 \\
37.0\end{array}$ & $\begin{array}{l}3.0 \\
3.0\end{array}$ & $\begin{array}{r}02 \\
\mathbf{0 . 0}\end{array}$ & $\begin{array}{l}3.9 \\
3.1\end{array}$ & $\begin{array}{r}44 \\
42\end{array}$ & $\begin{array}{l}49 \\
48\end{array}$ & $\begin{array}{r}21 \\
412\end{array}$ & $\begin{array}{l}8.0 \\
5.7\end{array}$ & $\begin{array}{l}488 \\
49\end{array}$ & $\begin{array}{r}8.8 \\
104\end{array}$ \\
\hline $11 / 3192$ & $\begin{array}{r}240 \\
0\end{array}$ & $\begin{array}{l}02 \\
02\end{array}$ & $\begin{array}{l}29.4 \\
20.6\end{array}$ & $\begin{array}{l}14.8 \\
126\end{array}$ & $\begin{array}{l}8.8 \\
8.7\end{array}$ & $\begin{array}{l}324 \\
31.7\end{array}$ & 3.2 & 20.1 & $\begin{array}{l}3.4 \\
3.4\end{array}$ & $\begin{array}{l}\mathbf{6 . 8} \\
\mathbf{4 6 . 0}\end{array}$ & $\begin{array}{l}30 \\
30\end{array}$ & 37.7 & 3.0 & $\infty .0$ & $\begin{array}{l}3.1 \\
32\end{array}$ & $\begin{array}{r}242 \\
487\end{array}$ & 38 & 426 & 6.5 & 47.7 & 9.0 \\
\hline $11 / 3 / 22$ & 15 & 02 & 30.0 & 10.5 & 92 & 30.4 & 3.0 & 28.8 & 3.3 & 47.0 & 30 & 30.1 & 3.0 & 0.3 & 48 & $\mathbf{0 . 8}$ & 49 & 436 & 73 & 124 & 8.0 \\
\hline $11 / 302$ & 30 & 02 & 20.7 & 10.0 & 89 & 33.4 & 3.3 & 29.6 & 3.4 & $\mathbf{8 . 1}$ & 30 & 38.1 & 3.0 & 300 & 8.7 & 470 & 89 & $\$ 12$ & 6.9 & 424 & 94 \\
\hline $11 / 302$ & 40 & 02 & 20.5 & 10.1 & 88 & 328 & 3.3 & 20.5 & 3.4 & $\mathbf{4 0 . 3}$ & 3.0 & 33.7 & 3.0 & 308 & 30 & $\$ 0$ & $3 A$ & 24 & 8.8 & 45 & 144 \\
\hline $11 / 302$ & 100 & 02 & 20.4 & 0.4 & 68 & 320 & 32 & 20.6 & 3.3 & $\mathbf{4 8 8}$ & 30 & 378 & 20 & 30.4 & 83 & $\mathbf{\infty 0 . 4}$ & 32 & 403 & 8.4 & 46.6 & 0.3 \\
\hline $11 / 302$ & 116 & 02 & 20.6 & 10.1 & 9.6 & 30.0 & 3.0 & 20.8 & 3.3 & 421 & 30 & 38.3 & 30 & 0.1 & 4.7 & 47.1 & 88 & 41.3 & 8.4 & 47.6 & 3.3 \\
\hline $11 / 302$ & 130 & 02 & 28.7 & 10.9 & $0 \$$ & 31.2 & 3.3 & 20.6 & 3.4 & 48 & 30 & 30.7 & 20 & 102 & 60 & 60.0 & 90 & 427 & 11.1 & 4.6 & 3.5 \\
\hline $11 / 302$ & 145 & 02 & 20.4 & 11.7 & 93 & 320 & 3.3 & 204 & 3.4 & 43.7 & 35 & 30.7 & 2.0 & 0.1 & 29 & 80 & 30 & 4.3 & 4.6 & 4.0 & 52 \\
\hline $11 / 302$ & 200 & 02 & 23.8 & 10.1 & 9.0 & 31.3 & 3.3 & 28.7 & 3.4 & 47.1 & 33 & $\mathbf{0 . 3}$ & 30 & 309 & 35 & 4.6 & 48 & 41.1 & 78 & 45.8 & 3.4 \\
\hline $11 / 302$ & 218 & 02 & 20.3 & 10.4 & 90 & 30.6 & 3.0 & 28.5 & 3.3 & $\mathbf{6 . 7}$ & 34 & 30.7 & 3.0 & 0.1 & 34 & 47.6 & 80 & 123 & 7.6 & 47.4 & 82 \\
\hline 11/302 & 200 & 02 & $\mathbf{2 0 . 0}$ & 11.0 & 9.7 & 30.0 & 3.0 & 28.1 & 3.3 & 47.8 & $3 B$ & $\boldsymbol{\omega 0 . 0}$ & 3.0 & 300 & 73 & 486 & 68 & 41.0 & 8.5 & 46.0 & 78 \\
\hline $11 / 302$ & 246 & 02 & 28.7 & 10.2 & 9.0 & 30.4 & 3.1 & 28.2 & 3.3 & 47.1 & 3.1 & 40.3 & 3.0 & 30.7 & 38 & 81.3 & 32 & 428 & 7.0 & 46.7 & 5.1 \\
\hline $11 / 302$ & 300 & 02 & 20.3 & 11.6 & 92 & 20.6 & 3.0 & 28.1 & 3.3 & $\mathbf{4 0 . 9}$ & 3.1 & 38.9 & 30 & 105 & 4.4 & 4.7 & 48 & 41.8 & 0.5 & 49.8 & 7.0 \\
\hline $11 / 302$ & 315 & 02 & 20.4 & 14.0 & 88 & 20.3 & 3.0 & $\mathbf{2 8 . 6}$ & 3.3 & 46.0 & 3.1 & 33.0 & 3.0 & $\omega 02$ & 62 & 4.7 & 8.1 & 41.3 & 02 & $\$ 6.1$ & 4.2 \\
\hline $11 / 392$ & 300 & 02 & $\mathbf{2 0 . 0}$ & 13.2 & 88 & 30.1 & 3.0 & 28.2 & 3.3 & 46.0 & 3.1 & 30.0 & 3.0 & $\mathbf{3 2 . 6}$ & 75 & 48.6 & 6.7 & 412 & 7.7 & 47.5 & 5.5 \\
\hline $11 / 392$ & 346 & 02 & $\mathbf{2 0 . 1}$ & 11.8 & 9.1 & 29.9 & 3.0 & 28.3 & 3.3 & $\mathbf{4 8 8}$ & 3.1 & $\boldsymbol{\omega 0 . 0}$ & 3.0 & 402 & 32 & 460 & 30 & 41.8 & 7.5 & 45.7 & 4.1 \\
\hline $11 / 392$ & 400 & 02 & 20.4 & 121 & 0.1 & 20.5 & 3.1 & 28.0 & 3.3 & 488 & 3.1 & $\$ 0.1$ & 3.0 & 0.1 & 45 & 4.7 & 30 & 41.1 & 0.8 & 46.7 & 58 \\
\hline $11 / 392$ & 415 & 02 & $\mathbf{2 0 . 0}$ & 24.1 & 02 & 20.4 & 3.0 & 284 & 3.3 & 4.4 & 30 & 37.6 & 3.0 & 30.7 & 63 & 40 & 4.1 & $\boldsymbol{0 . 0}$ & 92 & 45.9 & 7.4 \\
\hline $11 / 392$ & 480 & 02 & 20.0 & 11.4 & 9.0 & 20.7 & 3.0 & 28.3 & 3.3 & 45.2 & 3.1 & 37.7 & 3.0 & 20.6 & 33 & 424 & 8.7 & 30.7 & 10.7 & 38.5 & 3.0 \\
\hline $11 / 392$ & 448 & 0.1 & 31.3 & 0.4 & 10.3 & $\mathbf{2 8 . 4}$ & 3.0 & 28.1 & 3.3 & 448 & 30 & 30.7 & 3.0 & 59 & 3.1 & 483 & 48 & 41.1 & 8.7 & 45.4 & 74 \\
\hline $11 / 302$ & 500 & 0.1 & 30.0 & 9.0 & 88 & 20.6 & 3.0 & 27.9 & 3.2 & $\mathbf{4 8 . 3}$ & 3.1 & 30.4 & 3.0 & 308 & $B B$ & 46.7 & 4.1 & 41.8 & 0.2 & 46.7 & 4.5 \\
\hline $11 / 392$ & B15 & 02 & 20.0 & 0.4 & 88 & 20.8 & 3.1 & 28.2 & 3.3 & 458 & 30 & 327 & 30 & 30 & 4.7 & 41.6 & 38 & $\$ .0$ & 0.9 & 46.6 & 5.1 \\
\hline $11 / 302$ & 530 & 0.1 & 29.0 & 9.2 & 6.7 & 30.3 & 3.0 & 28.4 & 3.3 & 46.9 & 3.1 & 38.7 & 3.0 & 20 & 30 & 4.1 & 68 & $\boldsymbol{\phi . 0}$ & 10.1 & 464 & 6.8 \\
\hline $11 / 302$ & 548 & 0.1 & 20.3 & 9.9 & 8.7 & $\mathbf{2 0 . 3}$ & 3.0 & 28.0 & 3.3 & 46.1 & 30 & 302 & 3.0 & $\boldsymbol{\omega} .0$ & 6.7 & 122 & 63 & 41.6 & 8.4 & 45.6 & 4.4 \\
\hline $11 / 392$ & 600 & 0.1 & 28.5 & 8.8 & 88 & 30.1 & 3.1 & 28.2 & 32 & 48.1 & 30 & 30.3 & 30 & 37.0 & 89 & 4.0 & 83 & $\omega .7$ & 8.3 & 46.2 & 52 \\
\hline $11 / 302$ & 615 & 0.1 & 20.2 & 9.8 & 8.7 & 20.9 & 3.1 & $\mathbf{2 8 . 1}$ & 3.3 & 45.8 & 30 & 33.7 & 30 & 0.1 & 3.1 & 40.4 & 44 & 41.7 & 8.3 & 459 & 40 \\
\hline $11 / 392$ & 600 & 0.1 & 29.8 & 10.7 & 8.7 & 20.2 & 3.0 & 28.0 & 3.3 & 46.0 & 3.0 & 38.9 & 3.0 & 0.1 & 3.7 & 420 & 80 & $\mathbf{0 . 3}$ & 6.9 & 45.9 & 7.6 \\
\hline $11 / 392$ & 645 & 0.1 & 29.0 & 120 & 8.7 & 30.1 & 3.1 & 28.3 & 3.3 & $\mathbf{4 5 . 6}$ & 30 & 38.6 & 30 & 300 & 34 & $\$ 2.1$ & 50 & 408 & 10.7 & $\mathbf{4 6 . 3}$ & 4.3 \\
\hline $11 / 392$ & 700 & 0.1 & 20.4 & 126 & 8.0 & 20.6 & 3.1 & 27.0 & 3.3 & 46.1 & 30 & 38.0 & 30 & 30.6 & 62 & 4.0 & 38 & $\mathbf{\omega 0 . 3}$ & 8.4 & 49 & 6.8 \\
\hline $11 / 392$ & 715 & 0.1 & 20.7 & 14.0 & 85 & 20.3 & 3.0 & 28.0 & 3.3 & 4.3 & 30 & 37.0 & 30 & 303 & 7.1 & 422 & 32 & 30.9 & 8.7 & 43.4 & 3.3 \\
\hline $11 / 302$ & 730 & 0.1 & 30.0 & 13.7 & 8.7 & 20.5 & 3.0 & 28.3 & 3.3 & 4.1 & 3.0 & $\mathbf{3 8 . 6}$ & 30 & 30.8 & 34 & $\$ 27$ & 39 & $\boldsymbol{\infty} .0$ & 0.4 & $\$ 14$ & 6.5 \\
\hline 11/3D2. & 745 & 0.1 & 29.8 & 11.3 & 85 & 20.0 & 3.1 & $\mathbf{2 8 . 0}$ & 3.3 & 4.5 & 30 & 38.0 & 3.0 & 98 & 30 & 125 & 5.1 & $\boldsymbol{\omega} .0$ & 7.6 & 372 & 3.0 \\
\hline $11 / 302$ & 800 & 0.1 & 29.8 & 11.0 & 8.6 & 20.2 & 3.0 & 27.8 & 3.3 & 4.1 & 30 & 382 & 3.0 & 308 & 43 & 48 & 44 & 303 & 08 & 384 & 6.1 \\
\hline $11 / 392$ & 815 & 0.1 & 20.2 & 59.0 & 9.0 & 20.1 & 3.1 & 20.0 & 3.3 & 44.6 & 30 & 33.6 & 3.0 & 30.7 & 62 & 487 & $4 A$ & 412 & 9.0 & 43.9 & 9.7 \\
\hline $11 / 392$ & 850 & 0.1 & 30.4 & 122 & 85 & $\mathbf{2 8 . 5}$ & 3.0 & 27.6 & 3.3 & 4.5 & 30 & 38.6 & 3.0 & 39.2 & 120 & 40 & $B A$ & 41.0 & 7.0 & 45.3 & 4.0 \\
\hline $11 / 302$ & 846 & 0.1 & $\mathbf{3 0 . 5}$ & 120 & 9.7 & 28.0 & 3.0 & 28.6 & 3.3 & $\mathbf{1 3 . 6}$ & 30 & 34.7 & 30 & 308 & 8.7 & 462 & 48 & 41.0 & 82 & 434 & 5.0 \\
\hline $11 / 392$ & $\infty 00$ & 0.1 & 20.9 & 120 & 88 & 20.8 & 3.0 & $\mathbf{2 8 . 3}$ & 3.3 & 4.0 & 30 & 30.4 & 3.0 & 30.6 & 48 & 40 & 32 & $\$ .8$ & 8.8 & 46 & 3.7 \\
\hline $11 / 302$ & 916 & 0.1 & 28.7 & 11.6 & 8.7 & 20.3 & 3.1 & 27.0 & 3.3 & 44.9 & 30 & 37.1 & 3.0 & 302 & 64 & 47.4 & 38 & 412 & 8.7 & 450 & 9.1 \\
\hline $11 / 3 / 92$ & 200 & 0.1 & 203 & 10.3 & 93 & 28.0 & 3.0 & 27.6 & 32 & 45.7 & 30 & 382 & 3.0 & 30.4 & 8.4 & 472 & 48 & 41.4 & 7.5 & 458 & 48 \\
\hline $11 / 302$ & 945 & 0.1 & 28.6 & 11.0 & 9.1 & 20.5 & 30 & $\mathbf{2 8 . 0}$ & 3.2 & 48.1 & 30 & 38.7 & 3.0 & 320 & 83 & 473 & 3.1 & 41.6 & 6.1 & 43 & 46 \\
\hline $11 / 3 / 92$ & 1000 & 0.1 & 30.4 & 10.4 & 9.1 & 29.1 & 3.0 & 28.2 & 3.3 & 45.9 & 30 & 39.0 & 30 & 308 & 6.4 & 443 & 32 & $\mathbf{4 0 . 6}$ & 9.0 & 451 & 71 \\
\hline $11 / 302$ & 1015 & 0.1 & 28.7 & 11.8 & 83 & 20.3 & 3.0 & 28.6 & 3.3 & 45.8 & 30 & 38.0 & 3.0 & 303 & 3.7 & 47.4 & 58 & 41.4 & 10.8 & 461 & 8.0 \\
\hline $11 / 3102$ & 1000 & 0.1 & 29.8 & 11.7 & 10.0 & $\mathbf{2 0 . 3}$ & 3.0 & 28.3 & 3.2 & 48.3 & 3.0 & 38.6 & 3.0 & 30.4 & 32 & $\mathbf{4 0 . 4}$ & 49 & 40.0 & 8.8 & 464 & 5.7 \\
\hline $11 / 392$ & 1045 & 0.1 & 30.4 & 11.4 & 95 & 28.9 & 3.0 & 28.5 & 32 & 46.2 & 3.0 & 38.1 & 3.0 & 303 & 3.1 & 468 & 4.7 & $\$ 0.8$ & 8.8 & 450 & 46 \\
\hline $11 / 3192$ & 1100 & 0.1 & 30.4 & 10.4 & 0.4 & 20.1 & 3.0 & 29.0 & 3.3 & 45.9 & 30 & 37.3 & 3.0 & 39.7 & 18.7 & 4.1 & 148 & 0.1 & 11.3 & 466 & 44 \\
\hline $11 / 3 / 92$ & 1116 & 0.1 & 36.5 & 124 & 83 & 20.5 & 3.0 & 29.5 & 3.3 & 4.8 & 30 & 34.6 & 3.0 & 303 & 20 & 430 & 68 & 30.7 & 8.7 & 45.5 & 4.3 \\
\hline $11 / 392$ & 1130 & 0.1 & 36.4 & 10.5 & 93 & 29.0 & 3.0 & 20.9 & 3.3 & 44.4 & 30 & 300 & 3.0 & 39.4 & 3.1 & 46.7 & 4.1 & 41.0 & 9.6 & 430 & 6.1 \\
\hline $11 / 392$ & 1145 & 0.1 & 34.7 & 11.5 & 93 & 29.1 & 3.0 & 30.0 & 3.4 & 4.7 & 30 & 34.3 & 3.0 & 30.8 & 3.1 & 42 & 40 & $\$ 0.6$ & 10.7 & 450 & 47 \\
\hline $11 / 302$ & 1200 & 0.1 & 34.8 & 124 & 8.7 & 20.0 & 3.0 & $\mathbf{2 9}$. & 3.3 & 4.7 & 30 & 34.0 & 30 & 30.3 & 20 & 4.3 & 88 & 0.9 & 8.9 & $4 B$ & 6.7 \\
\hline $11 / 392$ & 1215 & 0.1 & 34.7 & 11.1 & 8.7 & 20.4 & 3.0 & 28.8 & 3.3 & 45.4 & 30 & 34.6 & 30 & 30.6 & 60 & 27 & 80 & $5 B$ & 8.3 & 44.3 & 8.1 \\
\hline $1 / 3902$ & 1220 & 0.1 & 34.9 & 10.6 & 88 & 20.4 & 3.0 & 298 & 3.3 & 45.6 & 30 & 34.4 & 3.0 & 30.4 & 30 & 41 & 33 & 41.1 & 11.0 & 46.8 & 7.2 \\
\hline $1 / 392$ & 1246 & 0.1 & 34.4 & 10.8 & 9.1 & 20.3 & 3.0 & 20.8 & 3.3 & 45.8 & 30 & 34.7 & 3.0 & 398 & 40 & 40 & 70 & 412 & 9.6 & $\infty .1$ & 7.0 \\
\hline $1 / 302$ & 1300 & 0.0 & 36.0 & 121 & 8.6 & 29.5 & 3.0 & 29.8 & 32 & 46.1 & 30 & 348 & 30 & 39.6 & 20 & 46 & 3.7 & 41.4 & 10.4 & 46.5 & 8.4 \\
\hline 1392 & 1316 & 0.0 & 34.6 & 11.0 & 8.7 & $\mathbf{2 0 . 5}$ & 3.0 & 29.0 & 3.3 & 46.1 & 30 & 35.0 & 30 & 30.7 & 3.1 & 123 & 4.1 & $\boldsymbol{\omega 0 . 7}$ & 9.6 & 40 & 93 \\
\hline $1 / 3 \times 2$ & 1300 & 0.0 & 33.9 & 13.0 & 85 & 28.1 & 3.0 & 30.1 & 3.3 & 46.0 & 3.0 & 34.7 & 3.0 & 30.7 & 20 & 122 & 48 & 412 & 9.0 & 45.0 & 7.1 \\
\hline $1 / 3 / 22$ & 1345 & 0.1 & 35.1 & 11.0 & 85 & 20.7 & 3.0 & 29.6 & 3.3 & 46.3 & 30 & 34.0 & 3.0 & 30.8 & 39 & 128 & 88 & 308 & 10.1 & 450 & 9.3 \\
\hline $1 / 3 / 22$ & 1400 & 0.1 & 34.5 & 123 & 8.7 & $\mathbf{2 0 . 4}$ & 3.0 & 29.8 & 3.2 & 46.3 & 30 & 350 & 3.0 & 30.7 & 3.1 & 42 & 38 & 0.0 & 10.8 & 48.4 & 3.0 \\
\hline $1 / 3 P 2$ & 1415 & 0.1 & 30.8 & 11.3 & 8.5 & 20.3 & 3.0 & 20.9 & 32 & 463 & 3.0 & 349 & 30 & 39.8 & 20 & 48 & 8 & 41.0 & 11.9 & $\$ 0.4$ & 3.0 \\
\hline 11/392 & 1430 & 0.1 & 34.4 & 120 & 8.9 & 20.7 & 3.0 & 20.5 & 32 & 46.6 & 30 & 36.5 & 3.0 & 30.7 & 3.1 & 127 & 7.1 & $\mathbf{0 . 1}$ & 10.7 & 43.0 & 3.2 \\
\hline 1/BP2 & 1445 & 0.1 & 34.1 & 13.2 & 8.6 & 29.5 & 3.0 & $\mathbf{2 9 . 7}$ & 32 & 46.4 & 3.0 & 35.2 & 3.0 & 30.6 & 4.7 & 123 & SA & 0.1 & 102 & 412 & 6.7 \\
\hline $\sin 2$ & 1500 & 0.1 & 34.1 & 11.3 & 8.6 & 29.3 & 3.0 & 20.7 & 3.2 & 46.5 & 30 & 36.3 & 3.0 & 39.6 & 20 & 42 & 38 & 40.8 & 12.1 & 47.5 & 4.0 \\
\hline $1 / 392$ & 516 & 0.1 & 3.6 & 25 & 8.7 & 3 & 3.0 & 2.0 & 3.3 & 45.0 & 30 & 41 & 30 & 398 & 30 & 197 & 31 & 10,3 & 11.4 & 48.1 & \\
\hline
\end{tabular}




\begin{tabular}{|c|c|c|c|c|c|c|c|c|c|c|c|c|c|c|c|c|c|c|c|c|c|}
\hline Dens & Time & $\begin{array}{l}\text { Water } \\
\text { Flow } \\
\text { opm }\end{array}$ & $\begin{array}{l}\text { Umo } \\
\text { Flow } \\
\text { gpm }\end{array}$ & $\begin{array}{l}8 * \\
\$ \\
\%\end{array}$ & $\begin{array}{r}\mathrm{B} \\
\% \\
\end{array}$ & $K W$ & $\mathrm{mn}$ & $K W$ & $m n$ & KW & $m n$ & $k W$ & $\mathrm{mn}$ & $\mathrm{KW}$ & $\mathrm{m}$ & $\mathrm{KW}$ & $\mathrm{in}$ & KW & $\mathrm{mn}$ & & $\mathrm{mn}$ \\
\hline $11 / 302$ & 1650 & 0.1 & 324 & 128 & $8 B$ & $\mathbf{2 8 . 9}$ & 3.0 & 29.6 & 3.3 & 48.0 & 30 & 30.9 & 30 & 308 & 30 & 49 & 36 & $\infty .0$ & 90 & 414 & 31 \\
\hline $11 / 302$ & 1846 & 0.1 & 31.4 & 126 & 9.3 & 28.0 & 3.0 & 20.4 & 32 & 47.4 & 30 & $3 a 0$ & 30 & 30.6 & 20 & 41 & 3.1 & $\$ 1.1$ & 6.7 & $\mathbf{2 . 7}$ & 48 \\
\hline $\begin{array}{l}11 / 302 \\
11 / 302\end{array}$ & $\begin{array}{l}1000 \\
1618\end{array}$ & $\begin{array}{l}0.1 \\
0.1\end{array}$ & $\begin{array}{l}312 \\
31.6\end{array}$ & $\begin{array}{l}124 \\
13.8\end{array}$ & $\begin{array}{l}8.8 \\
93\end{array}$ & $\begin{array}{l}20.4 \\
20.1\end{array}$ & $\begin{array}{l}3.0 \\
3.0\end{array}$ & $\begin{array}{l}29.7 \\
20.3\end{array}$ & $\begin{array}{l}3.3 \\
32\end{array}$ & $\begin{array}{r}48.9 \\
47.5\end{array}$ & $\begin{array}{l}30 \\
30\end{array}$ & $\begin{array}{l}38.1 \\
372\end{array}$ & $\begin{array}{l}3.0 \\
3.0\end{array}$ & $\begin{array}{l}30.8 \\
30.9\end{array}$ & $\begin{array}{l}3.1 \\
3.0\end{array}$ & $\begin{array}{l}4.1 \\
47.0\end{array}$ & $\begin{array}{l}30 \\
30\end{array}$ & $\begin{array}{l}41.9 \\
41.7\end{array}$ & $\begin{array}{l}78 \\
78\end{array}$ & $\begin{array}{l}124 \\
150\end{array}$ & $\begin{array}{r}6.2 \\
11.3\end{array}$ \\
\hline $11 / 392$ & 1600 & 0.1 & 31.6 & 128 & 93 & 28.8 & 3.1 & 200 & 3.3 & 47.8 & 3.0 & 37.0 & 30 & 300 & 38 & 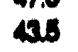 & 4.1 & $\$ 3$ & 8.7 & $\infty 0$ & 80 \\
\hline $11 / 302$ & 1615 & 0.1 & 321 & 128 & 95 & 20.1 & 3.0 & $\mathbf{2 8 . 8}$ & 32 & 18.0 & 30 & 30.8 & 3.0 & 30.3 & 7.7 & 424 & 6.1 & 308 & 10.0 & 0.2 & 20 \\
\hline 11/392 & 1700 & 0.1 & 30.6 & 11.8 & 95 & 20.4 & 3.1 & 20.1 & 3.3 & 47.3 & 3.0 & 37.9 & 3.0 & 30.6 & 34 & 41.4 & 30 & 30.8 & 9.8 & $\mathbf{4 8 . 3}$ & 78 \\
\hline $11 / 3102$ & 1715 & 02 & 30.8 & 121 & 10.1 & 20.1 & 3.0 & 29.7 & 3.3 & 47.6 & 3.0 & 328 & 3.0 & $\omega .0$ & 3.1 & $4 \mathbf{4}$ & 38 & 413 & 7.6 & 40.8 & 30 \\
\hline $11 / 392$ & 1730 & 02 & 30.1 & 128 & 9.7 & 28.8 & 3.0 & 28.3 & 32 & 48.1 & 30 & 326 & 3.0 & 30.7 & 34 & 48 & 4.1 & $\$ 1.0$ & 10.8 & 4.0 & 30 \\
\hline $11 / 302$ & 1746 & 02 & 20.9 & 11.7 & 10.2 & 28.8 & 3.0 & 29.3 & 3.2 & 48.1 & 30 & 32.8 & 3.0 & 30.7 & 34 & 48 & 4.1 & 41.0 & 108 & 40 & 30 \\
\hline $11 / 302$ & 1800 & 02 & 20.2 & 11.0 & 10.4 & $28 \boldsymbol{8}$ & 3.0 & 20.3 & 32 & 48.1 & 30 & 39.6 & 30 & 30.7 & 34 & 40 & 4.1 & 41.0 & 108 & 4.0 & 3.0 \\
\hline $11 / 302$ & 1818 & 02 & 29.6 & 123 & 10.3 & 288 & 3.0 & 28.3 & 3.2 & 48.1 & 30 & 33.5 & 30 & 30.7 & 34 & 448 & 4.1 & 41.0 & 108 & 4.0 & 30 \\
\hline $11 / 302$ & 1800 & 02 & 20.7 & 121 & 10.4 & 28.8 & 3.0 & 20.3 & 3.2 & 48.1 & 30 & 39.6 & 3.0 & 30.7 & 34 & 48 & 4.1 & 41.0 & $10 B$ & 4.0 & 3.0 \\
\hline $11 / 302$ & 1848 & 02 & 28.1 & 13.6 & 10.6 & 23.8 & 3.0 & 28.3 & 3.2 & 48.1 & 30 & 38.6 & 3.0 & 30.7 & 34 & 48 & 4.1 & $\$ 1.0$ & 108 & 4.0 & 3.0 \\
\hline $11 / 302$ & 1000 & 02 & 28.4 & 127 & 11.0 & 288 & 3.0 & 283 & 3.2 & 48.1 & 30 & 30.8 & 3.0 & 30.7 & $3 \mathbf{A}$ & 48 & 4.1 & 41.0 & 108 & 40 & 3.0 \\
\hline $11 / 302$ & 1015 & 02 & 282 & 122 & 10.6 & 29.8 & 3.0 & 28.3 & 32 & 48.1 & 3.0 & 32.6 & 3.0 & 30.7 & 34 & 48 & 4.1 & 41.0 & 10.8 & 40 & 30 \\
\hline $11 / 302$ & 1000 & 02 & $\mathbf{2 8 . 3}$ & 128 & 10.8 & 30.3 & 3.0 & 29.1 & 32 & 47.1 & 30 & 300 & 30 & 308 & 4.1 & 484 & 39 & 41.7 & 8.5 & 45.0 & 8.3 \\
\hline $11 / 302$ & 1946 & 0.2 & 28.2 & 120 & 112 & 30.3 & 3.2 & $\mathbf{2 3 . 2}$ & 3.3 & 47.6 & 30 & $\$ 0.6$ & 3.0 & 30.7 & 68 & 42 & 38 & 41.7 & 8.5 & 45.2 & 7.3 \\
\hline $11 / 392$ & 2000 & 02 & $\mathbf{2 8 . 0}$ & 0.1 & 18 & 0.2 & 3.1 & 28.0 & 32 & 47.3 & 3.0 & $\$ 02$ & 3.0 & 300 & 80 & 18.1 & 3.1 & $\phi .6$ & 118 & 45.0 & 7.5 \\
\hline $11 / 392$ & 2018 & 02 & 28.6 & 13.1 & 11.7 & 30.1 & 3.2 & $\mathbf{2 8 . 1}$ & 3.2 & 47.6 & 30 & 40.8 & 30 & $\mathbf{\phi 0 . 0}$ & 3.1 & 100 & 3.7 & 0.6 & 10.6 & 436 & 6.4 \\
\hline $11 / 302$ & 2000 & 02 & 28.9 & 14.1 & 11.8 & 30.7 & 3.2 & 28.3 & 3.3 & $\mathbf{4 5 . 0}$ & 30 & 332 & 30 & 308 & 50 & 478 & 40 & $\$ 0.8$ & 108 & 427 & 4.6 \\
\hline $11 / 302$ & 2046 & 02 & $\mathbf{2 8 . 2}$ & 15.0 & 11.4 & 20.9 & 3.2 & 27.7 & 3.3 & $\mathbf{4 8 0}$ & 30 & 39.6 & 30 & 30.4 & 3.1 & 498 & 30 & 41.8 & 8.1 & 470 & 0.1 \\
\hline $11 / 302$ & 2100 & 02 & 28.8 & 14.0 & 11.8 & 30.5 & 3.1 & 28.1 & 3.2 & 48.2 & 30 & 326 & 30 & 30.7 & 20 & 98 & 32 & 41.8 & 6.9 & 454 & 40 \\
\hline $11 / 302$ & 2115 & 02 & 20.0 & 120 & 11.7 & 23.1 & 3.0 & 28.8 & 32 & 47.6 & 30 & $\$ .4$ & 30 & 30.3 & 6.7 & 62 & 44 & 41.9 & 6.6 & 47.6 & 88 \\
\hline $11 / 302$ & 2130 & 02 & 28.0 & 123 & 11.6 & 28.3 & 3.0 & 28.8 & 3.1 & 47.6 & 30 & 40.4 & 3.0 & 30.3 & 20 & 478 & 32 & 41.0 & 9.6 & $\Delta 6.2$ & 6.4 \\
\hline $11 / 302$ & 2146 & 02 & $\mathbf{2 8 . 6}$ & 14.3 & 11.8 & 28.4 & 3.0 & $\mathbf{2 6 . 7}$ & 3.2 & 47.4 & 3.0 & $\boldsymbol{\omega 0 . 3}$ & 30 & 30.3 & 38 & 47.4 & $3 \overline{3}$ & $\mathbf{\phi . 0}$ & 7.7 & 46.8 & 4.3 \\
\hline $11 / 392$ & 2200 & 0.1 & 28.8 & 127 & 124 & 28.0 & 3.0 & 28.8 & 3.1 & 47.8 & 30 & $\mathbf{0 . 7}$ & 3.0 & 3.7 & 60 & 40 & 55 & 41.0 & 78 & 453 & 38 \\
\hline $11 / 392$ & 2215 & 0.1 & 28.0 & 13.1 & 11.7 & 288 & 3.0 & 28.7 & 3.2 & 47.4 & 30 & 40.0 & 30 & 30.4 & 34 & 423 & 30 & 413 & $10 . A$ & 475 & 6.7 \\
\hline $11 / 302$ & 2020 & 0.1 & $\mathbf{2 0 . 0}$ & 14.3 & 13.8 & 29.4 & 4.1 & 28.7 & 3.1 & 42.1 & 30 & 412 & 30 & 30.4 & 6.7 & 42 & 83 & 308 & 8.0 & 472 & 47 \\
\hline $11 / 392$ & 2246 & 0.1 & 28.6 & 13.2 & 122 & 29.4 & 3.0 & 28.7 & 32 & 47.9 & 30 & $\$ 0.6$ & 30 & 302 & 68 & 41 & 68 & 103 & 8.7 & 473 & 3.7 \\
\hline $11 / 302$ & 2000 & 0.1 & 28.6 & 126 & 13.8 & 29.7 & 32 & 28.7 & 32 & 48.0 & 30 & 413 & 30 & 30.4 & 3.1 & 460 & 3.1 & $\mathbf{P S}$ & 7.1 & 420 & 37 \\
\hline $1 / 302$ & 2316 & 0.1 & 28.2 & 15.5 & 13.3 & 30.6 & 3.2 & 27.1 & 3.2 & 48.7 & 30 & 121 & 3.0 & 308 & 20 & $\mathbf{4 8}$ & 0.7 & $\$ 1.0$ & 8.1 & $\mathbf{6 . 7}$ & 8.3 \\
\hline $11 / 392$ & 2000 & 0.1 & 28.2 & 122 & 121 & 302 & 3.1 & 27.0 & 3.1 & 4.3 & 30 & 422 & 3.0 & 300 & 3.1 & 476 & 74 & 41.0 & 5.0 & 488 & 6.3 \\
\hline 11/392 & 2345 & 0.1 & 30.2 & 123 & 11.4 & $\mathbf{2 8 . 5}$ & 3.0 & 28.6 & 3.1 & 48.9 & 30 & 41.0 & 3.0 & 39.6 & 20 & 0.1 & 3.1 & 422 & 88 & 477 & 9.7 \\
\hline $11 / 402$ & 0 & 0.1 & 30.8 & 13.8 & 11.7 & $\mathbf{2 8 . 3}$ & 3.0 & $\mathbf{2 3 . 7}$ & 3.1 & 48.1 & 30 & $\boldsymbol{\infty} .3$ & 3.0 & 39.8 & 3.1 & 82 & 30 & 428 & 42 & 472 & 6.0 \\
\hline $11 / 492$ & 15 & 0.1 & 30.1 & 15.0 & 11.8 & $\mathbf{2 3 . 3}$ & 3.0 & 28.8 & 3.1 & 47.7 & 30 & $\omega .0$ & 3.0 & 308 & 40 & 48 & 38 & 41.0 & 7.7 & 471 & 3.6 \\
\hline 11/492 & 30 & 0.1 & 30.6 & 11.8 & 11.8 & $\mathbf{2 0 . 5}$ & 3.0 & 27.4 & 3.1 & 46.6 & 30 & 38.0 & 30 & $\mathbf{1 0 0}$ & 38 & 490 & 62 & 427 & 0.0 & 472 & 5.8 \\
\hline $11 / 492$ & 4 & 0.1 & 31.4 & 11.6 & 11.6 & 28.0 & 3.0 & $\mathbf{2 8 . 7}$ & 3.1 & 472 & 30 & 38.9 & 30 & $\mathbf{3 0 . 8}$ & $B B$ & 80 & 05 & 41.7 & 8.8 & 466 & 76 \\
\hline $11 / 402$ & 100 & 0.1 & 33.1 & 10.7 & 11.0 & 282 & 3.0 & 23.6 & 3.1 & $\mathbf{4 6 . 8}$ & 30 & 382 & 20 & 308 & 10.4 & 403 & 63 & 412 & 78 & $\Delta 1$ & 4.8 \\
\hline $11 / 402$ & 115 & 0.1 & 320 & 10.0 & 10.0 & 29.6 & 32 & 27.7 & 3.2 & 44.8 & 30 & 38.1 & 3.0 & 30.8 & 4.7 & 40 & 6.7 & 412 & 8.0 & 456 & 3.5 \\
\hline $11 / 492$ & 130 & 0.1 & 33.4 & 10.8 & 10.3 & 30.0 & 3.1 & 28.5 & 3.3 & 43.7 & 30 & 34.6 & 3.0 & 398 & 48 & 407 & 80 & 41.1 & 6.8 & 45 & 40 \\
\hline $11 / 492$ & 146 & 0.1 & 33.9 & 10.6 & 9.6 & 288 & 3.0 & 28.0 & 3.3 & 43.7 & 30 & 34.6 & 3.0 & 30.7 & 3.1 & 412 & 3.7 & 40.4 & 8.8 & 45 & 46 \\
\hline $11 / 492$ & 200 & 0.1 & 33.1 & 10.8 & 98 & $\mathbf{2 8 . 8}$ & 3.0 & 28.1 & 3.2 & 43.5 & 30 & 33.7 & 30 & 39.8 & 49 & 47.3 & 33 & 41.3 & 9.2 & 42 & 81 \\
\hline $11 / 492$ & 216 & 0.1 & 34.6 & 128 & 9.7 & $\mathbf{3 0 . 0}$ & 3.0 & 27.0 & 3.2 & 4.1 & 30 & 34.6 & 30 & 39.6 & 40 & 49 & 42 & $\boldsymbol{\infty} .7$ & 9.1 & 45 & 53 \\
\hline $11 / 402$ & 2200 & 0.1 & 30.4 & 11.4 & 9.4 & 202 & 3.0 & 27.3 & 3.2 & 12.1 & 30 & 30.6 & 3.0 & 302 & 30 & 400 & 30 & $\boldsymbol{4 0 . 7}$ & 8.5 & $\$ 1$ & 60 \\
\hline $11 / 402$ & 246 & 0.1 & 34.0 & 11.0 & 92 & 20.4 & 3.0 & 27.2 & 3.2 & 43.3 & 30 & 33.5 & 3.0 & 302 & 30 & 824 & 30 & 127 & 42 & 44 & 76 \\
\hline $11 / 402$ & 300 & 0.1 & 33.6 & 10.0 & 9.5 & 2.7 & 3.0 & 27.3 & 3.2 & 420 & 30 & 332 & 3.0 & 39.3 & 42 & 400 & 32 & 41.4 & 66 & 41 & 6.5 \\
\hline $11 / 492$ & 315 & 0.1 & 326 & 10.7 & 93 & 22.5 & 3.0 & 27.3 & 3.2 & 128 & 30 & 33.1 & 30 & 303 & 20 & 466 & 3.1 & $\boldsymbol{\omega} 0.7$ & 0.4 & 128 & 6.4 \\
\hline $11 / 492$ & 330 & 0.1 & 328 & 13.6 & 89 & 28.7 & 3.0 & 27.6 & 32 & 124 & 30 & 31.7 & 30 & 302 & 32 & 198 & 49 & 40.0 & 76 & 124 & 57 \\
\hline $11 / 402$ & 346 & 0.1 & 325 & 123 & 102 & $\mathbf{2 8 . 7}$ & 3.0 & 27.6 & 3.2 & 423 & 30 & 31.7 & 3.0 & 32.7 & 62 & 47.0 & 38 & 41.1 & 0.0 & $\$ 4$ & 7.7 \\
\hline $11 / 492$ & 400 & 0.1 & 321 & 122 & 9.0 & 28.6 & 3.0 & 27.0 & 3.2 & 43.1 & 30 & 330 & 30 & 303 & 48 & 120 & 30 & 413 & 8.6 & 132 & 6.1 \\
\hline $11 / 402$. & 415 & 0.1 & 31.4 & 14.1 & 0.7 & $\mathbf{2 8 . 3}$ & 3.0 & 27.4 & 3.2 & 428 & 30 & 327 & 3.0 & 303 & 32 & 42 & 33 & 413 & 7.4 & 115 & 3.8 \\
\hline $11 / 492$ & 430 & 0.1 & 31.9 & 11.8 & 96 & 28.8 & 3.0 & 27.1 & 3.2 & 433 & 30 & 30.0 & 3.0 & 30.8 & 50 & $\$ 17$ & 34 & 41.5 & 78 & 421 & 4.0 \\
\hline $11 / 492$ & 445 & 0.1 & 31.6 & 10.9 & 95 & 28.4 & 3.0 & 27.2 & 3.2 & 43.1 & 30 & 330 & 3.0 & 30.4 & 20 & 47.7 & 30 & 41.1 & 7.0 & 410 & 4.8 \\
\hline $11 / 402$ & 500 & 0.1 & 31.6 & 9.7 & 9.6 & 28.6 & 3.0 & 272 & 3.2 & 43.8 & 30 & 332 & 30 & 302 & 20 & 424 & 42 & 41.3 & 0.1 & 43.6 & 52 \\
\hline $11 / 492$ & 515 & 0.1 & 31.3 & 10.0 & 10.2 & 28.8 & 3.0 & 27.0 & 3.2 & 43.7 & 30 & $3 a 7$ & 3.0 & 308 & 30 & 48 & 30 & 41.9 & 62 & $\infty .0$ & 6.0 \\
\hline $11 / 402$ & 500 & 0.1 & 31.3 & 9.6 & 99 & 28.5 & 3.0 & 27.4 & 3.2 & 43.4 & 30 & 303 & 30 & 39.4 & 30 & 480 & 30 & 41.4 & 0.4 & 41.0 & 40 \\
\hline $11 / 402$ & 546 & 0.1 & 27.5 & 2.3 & 10.3 & 20.5 & 3.1 & 27.0 & 3.3 & 126 & 30 & 31.6 & 30 & 30.6 & 3. & 48 & 3 & 102 & 62 & 41.0 & 7.6 \\
\hline $1 / 4902$ & 600 & 0.1 & 26.5 & 9.3 & 122 & 302 & 3.2 & 27.3 & 3.2 & $\mathbf{4 5 . 0}$ & 30 & 35.6 & 30 & 308 & 38 & 002 & 30 & 428 & 4.7 & 40 & 6.6 \\
\hline $1 / 402$ & 615 & 0.1 & 26.1 & 122 & 120 & 30.6 & 3.0 & 27.1 & 32 & 46.0 & 3.0 & 330 & 30 & $3 a 8$ & 69 & 47.7 & 82 & 412 & 6.6 & 45.6 & 4.7 \\
\hline $1 / 402$ & 60 & 0.1 & 25.0 & 13.0 & 13.0 & 30.3 & 3.1 & 27.0 & 32 & $\mathbf{4 7 . 0}$ & 3.0 & 38.3 & 30 & 30.6 & 42 & 48 & 30 & 124 & 88 & 120 & 8.4 \\
\hline $1 / 492$ & 645 & 0.1 & 25.9 & 129 & 11.8 & 30.0 & 3.2 & 27.3 & 32 & 46.7 & 30 & 37.0 & 3.0 & 40.0 & 3.1 & 81.6 & 30 & 126 & 3.6 & 448 & 98 \\
\hline $1 / 402$ & 700 & 0.1 & 27.4 & 132 & 112 & $\mathbf{2 0 . 4}$ & 3.1 & 26.9 & 3.2 & 46.0 & 30 & 38.8 & 30 & 30.4 & 3.8 & $47 \mathrm{~s}$ & 3.1 & 412 & 7.0 & 421 & 3.1 \\
\hline $1 / 4 / 92$ & 715 & 0.1 & 26.4 & 15.4 & 11,3 & $\mathbf{3 0 . 4}$ & 3.1 & 27.3 & 3.2 & 44.6 & 30 & 34.8 & 3.0 & 30.3 & 30 & 486 & 3.1 & $\omega .6$ & 8.1 & 43.1 & 5.4 \\
\hline $1 / 492$ & 730 & 0.1 & 25.7 & 14.4 & 13.1 & $\mathbf{2 0 . 5}$ & 3.0 & 25.8 & 3.2 & 48.3 & 30 & 32.1 & 3.0 & 303 & 10 & $\Delta 7$ & 38 & 413 & 7.0 & 442 & 42 \\
\hline
\end{tabular}




\begin{tabular}{|c|c|c|c|c|c|c|c|c|c|c|c|c|c|c|c|c|c|c|c|c|c|}
\hline Den & Time & $\begin{array}{l}\text { Water } \\
\text { Flow } \\
\text { opm }\end{array}$ & $\begin{array}{l}\text { Lims } \\
\text { Flow } \\
\text { ogen }\end{array}$ & 8 & $\begin{array}{r}8 \\
\alpha \\
\alpha\end{array}$ & & $\mathrm{mn}$ & & $m n$ & & $\mathrm{mn}$ & $K W$ & $\mathrm{mn}$ & $\mathrm{KW}$ & $\min$ & & $\operatorname{mn}$ & $K W$ & $m$ & $k w$ & $m n$ \\
\hline $\begin{array}{l}11 / 402 \\
11 / 402\end{array}$ & $\begin{array}{l}746 \\
800\end{array}$ & $\begin{array}{l}0.1 \\
0.1\end{array}$ & $\begin{array}{l}28.7 \\
23.8\end{array}$ & $\begin{array}{l}14.8 \\
14.7\end{array}$ & $\begin{array}{l}13.2 \\
138\end{array}$ & $\begin{array}{l}30.0 \\
20.6\end{array}$ & $\begin{array}{l}3.1 \\
3.0\end{array}$ & $\begin{array}{l}28.8 \\
28.6\end{array}$ & $\begin{array}{l}3.2 \\
32\end{array}$ & 4.6 & 30 & 324 & 30 & 322 & 38 & 472 & 12 & $\mathbf{D B}$ & 7.9 & 452 & 10.1 \\
\hline $11 / 402$ & 815 & 0.1 & 28.6 & 14.0 & 132 & 200 & 3.1 & 28.7 & 3.2 & 47.0 & $\begin{array}{l}30 \\
30\end{array}$ & 40.8 & 30 & 32 & $\begin{array}{l}36 \\
30\end{array}$ & 478 & $\begin{array}{l}3.1 \\
30\end{array}$ & $\begin{array}{l}40.4 \\
40.0\end{array}$ & $\begin{array}{l}7.6 \\
7.9\end{array}$ & $\begin{array}{l}47.0 \\
47.0\end{array}$ & $\begin{array}{l}6.4 \\
3.7\end{array}$ \\
\hline $11 / 402$ & 800 & 0.1 & 27.0 & 14.0 & 14.0 & 20.7 & 30 & $\mathbf{8 . 7}$ & 32 & 47.4 & 30 & 0.8 & 3.0 & 35 & 82 & 40 & 80 & $\$ 0.3$ & 73 & 474 & 44 \\
\hline 11/402 & 816 & 0.1 & 27.6 & 14.0 & 13.8 & 20.4 & 3.0 & 28.7 & 32 & 472 & 30 & 102 & 3.0 & 38 & 68 & 418 & 49 & $\boldsymbol{0 . 4}$ & 82 & 4.6 & 8.6 \\
\hline $\begin{array}{l}11 / 402 \\
11 / 402\end{array}$ & $\begin{array}{l}900 \\
916\end{array}$ & $\begin{array}{l}0.1 \\
0.1\end{array}$ & $\begin{array}{l}27.7 \\
30.1\end{array}$ & $\begin{array}{l}13.9 \\
128\end{array}$ & $\begin{array}{l}120 \\
122\end{array}$ & $\begin{array}{r}20.2 \\
20.0\end{array}$ & $\begin{array}{l}3.1 \\
3.0\end{array}$ & $\begin{array}{l}20.7 \\
20.9\end{array}$ & $\begin{array}{l}32 \\
32\end{array}$ & $\begin{array}{r}472 \\
46.0\end{array}$ & $\begin{array}{l}30 \\
30\end{array}$ & $\begin{array}{l}30.6 \\
37.1\end{array}$ & $\begin{array}{l}3.0 \\
1.0\end{array}$ & $\begin{array}{l}303 \\
303\end{array}$ & 44 & 40 & $\begin{array}{l}38 \\
34\end{array}$ & $\begin{array}{l}0.8 \\
0.0\end{array}$ & $\begin{array}{l}8.0 \\
8.6\end{array}$ & $\begin{array}{l}4.6 \\
4.4\end{array}$ & $\begin{array}{l}48 \\
18\end{array}$ \\
\hline $11 / 402$ & $\infty \infty$ & 0.1 & 302 & 125 & 123 & 20 & 3.0 & 272 & 32 & $\mathbf{4 8 . 8}$ & 30 & 37.0 & 30 & 303 & 30 & 478 & 3.7 & $\begin{array}{l}0.0 \\
41.1\end{array}$ & $\begin{array}{l}8.0 \\
7.7\end{array}$ & $\begin{array}{l}4.4 \\
4.7\end{array}$ & 40 \\
\hline $\begin{array}{l}11 / 402 \\
11 / 402\end{array}$ & 1000 & $\begin{array}{l}0.1 \\
0.1\end{array}$ & $\begin{array}{l}29.6 \\
27.3\end{array}$ & $\begin{array}{l}14.6 \\
14.1\end{array}$ & $\begin{array}{l}128 \\
14.2\end{array}$ & $\begin{array}{l}20.3 \\
2.1\end{array}$ & $\begin{array}{l}3.1 \\
3.1\end{array}$ & $\begin{array}{l}27.1 \\
27.4\end{array}$ & $\begin{array}{l}3.2 \\
3.2\end{array}$ & $\begin{array}{l}\mathbf{4 . 0} \\
\mathbf{4 6 . 6}\end{array}$ & $\begin{array}{l}30 \\
30\end{array}$ & $\begin{array}{l}37.7 \\
37.3\end{array}$ & $\begin{array}{l}3.0 \\
3.0\end{array}$ & 302 & $\begin{array}{l}3.1 \\
40\end{array}$ & 46 & $\begin{array}{l}6.1 \\
32\end{array}$ & 0.0 & 7.9 & 408 & $\begin{array}{l}78 \\
80\end{array}$ \\
\hline $11 / 402$ & 1015 & 0.1 & 20.6 & 14.8 & 128 & 200 & 32 & 27.1 & 32 & $\mathbf{4 . 6}$ & 30 & 30 & 3.0 & 30 & 38 & 472 & 34 & $\begin{array}{l}41.1 \\
412\end{array}$ & $\begin{array}{l}8.6 \\
7.6\end{array}$ & 40.1 & $\begin{array}{l}6.0 \\
6.4\end{array}$ \\
\hline $11 / 402$ & 1000 & 0.1 & 23.2 & 14.2 & 13.2 & 30.8 & 32 & 27.7 & 32 & 46.7 & 30 & 37.0 & 30 & & 3.7 & & 34 & $\$ 0.6$ & 11.1 & 412 & 82 \\
\hline $11 / 492$ & 1046 & 0.1 & 28.0 & 13.8 & 127 & 30.6 & 3.1 & 27.9 & 32 & 48.4 & 30 & 37.1 & 30 & & 30 & 47.0 & 30 & $\infty .8$ & 8.3 & 4.3 & 30 \\
\hline $11 / 402$ & 1100 & 0.1 & 29.6 & 14.4 & 121 & 204 & 3.1 & 27.6 & 32 & 48.3 & 30 & 37.0 & 10 & 24 & 30 & 42 & 3.1 & 41.0 & 6.1 & 40 & 53 \\
\hline $11 / 402$ & 1118 & 0.1 & $\mathbf{3 0 . 3}$ & 130 & 10.9 & 29.8 & 3.0 & 27.8 & 3.2 & 44.0 & 30 & 30.6 & 20 & 200 & 80 & 473 & 30 & 412 & 9.3 & $\mathbf{4 6 . 7}$ & 7.3 \\
\hline $11 / 402$ & 1130 & 0.1 & 34.1 & 126 & 10.4 & 20.6 & 3.0 & 27.7 & 32 & 448 & 30 & 308 & 30 & 20 & 20 & 120 & 38 & 41.7 & 72 & 4.7 & 02 \\
\hline $11 / 402$ & 1146 & 0.1 & 35.3 & 16.7 & 11.0 & 20.3 & 3.0 & 282 & 3.1 & 49.6 & 30 & 326 & 30 & 29.6 & 32 & & 3.1 & 41.9 & 84 & 424 & 34 \\
\hline 11/402 & 1200 & 0.1 & 35.1 & 148 & 10.2 & 204 & 3.0 & 27.6 & 3.2 & 4.7 & 30 & 34.0 & 30 & 39.8 & 29 & $\infty$ & 32 & 124 & 0.3 & 4.1 & 10.4 \\
\hline 11/4P2 & 1218 & 0.1 & 35.0 & 13.8 & 96 & 20.6 & 3.0 & 27.6 & 32 & 4.6 & 30 & 30 & 30 & 3.4 & 30 & 00 & 30 & 124 & 62 & 4.6 & 47 \\
\hline $11 / 402$ & 1200 & 0.1 & 34.8 & 140 & 93 & $\mathbf{2 0 . 5}$ & 3.0 & 27.7 & 3.1 & 48 & 30 & 320 & 30 & & 20 & 0.4 & $3 \mathbf{A}$ & 428 & 7.0 & 48.3 & 72 \\
\hline $11 / 402$ & 1246 & 0.1 & 38.0 & 14.0 & 90 & 29.3 & 30 & 23.1 & 3.1 & 40.4 & 30 & 322 & 3.0 & 30.5 & 3.1 & 478 & 30 & 41.1 & 7.6 & 43.0 & 82 \\
\hline $11 / 402$ & 1300 & 0.1 & 34.0 & 16.6 & 08 & 20.0 & 30 & 2.3 .3 & 32 & 40.4 & 30 & 21 & 3.0 & 38 & 38 & $\infty$ & 30 & 421 & 6.1 & 4.3 & 98 \\
\hline $11 / 402$ & 1316 & 0.1 & 346 & 39.3 & 92 & 20.4 & 3.0 & 27.9 & 32 & 48.6 & 30 & 323 & 3.0 & 393 & 20 & 0.4 & 83 & 418 & 8.6 & 428 & 0.3 \\
\hline $11 / 492$ & 1300 & 0.1 & 38.2 & 8.7 & 9.5 & 20.2 & 3.0 & $\mathbf{2 8 . 0}$ & 3.1 & 4.7 & 30 & 323 & 30 & 39.4 & 20 & 48 & 38 & 41,4 & 8.0 & 482 & 68 \\
\hline $11 / 402$ & 1348 & 0.1 & 35.6 & 64.5 & 90 & 20.0 & 3.0 & 20 & 3.2 & 43.6 & 30 & 322 & 30 & 30.4 & 118 & 98 & 30 & 41.8 & 8.6 & 46.4 & 6.3 \\
\hline 11/402 & 1400 & 02 & 34.9 & 0.1 & 0.1 & 20.0 & 3.0 & 23.1 & 32 & 497 & 30 & 322 & 3.0 & 3.4 & 30 & 4.1 & 32 & 41.0 & 8.6 & 46.2 & 62 \\
\hline $11 / 402$ & 1416 & 02 & 36.9 & 18.8 & 0.1 & 288 & 3.0 & $\mathbf{2 0 . 3}$ & 3.2 & $\mathbf{4 . 5}$ & 30 & 321 & 30 & 39 & 20 & 40 & 32 & 41.4 & 0.7 & 4.2 & 4.3 \\
\hline $11 / 402$ & 1450 & 02 & 30.0 & 13.0 & 0.4 & 28.8 & 3.0 & 28.1 & 32 & 43.4 & 30 & 31.8 & 3.0 & 30 & 4.1 & 492 & 32 & 41.3 & 8.0 & 306 & 6.7 \\
\hline $11 / 402$ & 1448 & 02 & 340 & 13.6 & 9.0 & 29.8 & 3.0 & 28.0 & 3.1 & 40.4 & 30 & 31.9 & 3.0 & 30.3 & 20 & $\mathbf{2 2}$ & 30 & 41.6 & 72 & 37.6 & 3.1 \\
\hline $11 / 402$ & 1600 & 02 & 34.6 & 124 & 92 & 292 & 3.0 & 27.0 & 32 & 43.7 & 30 & 320 & 30 & 39.4 & 3.1 & 0.1 & 3.1 & 41.9 & 7.6 & 45.3 & 8.3 \\
\hline $11 / 402$ & 1515 & 02 & 34.4 & 14.8 & 0.7 & 20.0 & 3.0 & 27.8 & 3.1 & 420 & 30 & 323 & 30 & 3.4 & 20 & 81.7 & 30 & 102 & 7.1 & 47 & 10.0 \\
\hline $11 / 402$ & 1500 & 02 & 350 & 127 & 92 & 20.1 & 3.0 & 230 & 3.2 & $\mathbf{4 0 . 8}$ & 30 & 318 & 30 & 3.4 & 30 & 0.0 & 30 & 41.7 & 7.0 & 45.4 & 20 \\
\hline $11 / 402$ & 1845 & 0.2 & 34.5 & 144 & 8.9 & 200 & 3.0 & 27.8 & 3.1 & 40.8 & 30 & 320 & 30 & 30.4 & 29 & ane & 3.1 & 426 & 3.7 & 48 & 7.0 \\
\hline $11 / 492$ & 1600 & 02 & 34.6 & 13.7 & 0.1 & 292 & 3.0 & 27.8 & 32 & 40.7 & 30 & 318 & 30 & 30 & 33 & $\infty .0$ & 32 & 119 & 8.4 & 4.8 & 4.9 \\
\hline 11/402 & 1616 & 02 & 33.9 & 120 & $8 B$ & 20.4 & 3.0 & 27. & 3.1 & 43.6 & 30 & 31.6 & 30 & 3.7 & 30 & $\infty \mathbf{1}$ & 30 & 422 & 5.0 & 4.7 & 33 \\
\hline $11 / 402$ & $16 \times 0$ & 02 & 30.9 & 13.8 & 0.4 & 28.8 & 3.0 & 27.4 & 3.1 & 4.6 & 30 & 302 & 30 & 3.4 & 20 & 613 & 30 & 423 & 5.6 & 454 & 53 \\
\hline $11 / 402$ & 16.45 & 02 & 30.1 & 13.9 & 0.7 & 28.7 & 3.0 & 27.1 & 3.1 & 462 & 30 & 338 & 30 & & 20 & a. & 30 & 420 & 4.4 & 43.1 & 44 \\
\hline 11/492 & 1700 & 02 & 31.1 & 128 & 0.6 & 20.0 & 3.0 & 27.1 & 3.1 & $\mathbf{8 . 2}$ & 30 & 330 & 20 & 308 & 20 & 26 & 34 & 480 & 4.0 & 460 & 2.3 \\
\hline 11/492 & 1716 & 02 & 31.4 & 17.2 & 09 & 28.8 & 3.0 & 272 & 3.1 & $\mathbf{4 5 . 0}$ & 30 & 30.7 & 30 & 30.7 & 38 & 800 & 34 & 120 & 3.6 & 134 & 3.1 \\
\hline 1/402 & 1730 & 02 & 320 & 18.4 & 9.7 & $\mathbf{2 0} .0$ & 3.0 & 23.0 & 32 & 48 & 30 & 30 & 30 & 39.6 & 40 & 81.0 & 3.1 & 126 & 4.6 & 462 & 38 \\
\hline $1 / 4 P 2$ & 1745 & 0.1 & 320 & 18.4 & 93 & 28.7 & 3.0 & 28.0 & 3.1 & 4.6 & 30 & 30.6 & 3.0 & 30.4 & 3.1 & 87 & 30 & 408 & 4.8 & 46 & 3.4 \\
\hline $1 / 402$ & 1800 & 0.1 & 31.6 & 18.7 & 0.4 & 28.3 & 3.0 & 27.4 & 3.2 & 498 & 30 & 38.7 & 3.0 & 30 & 4.1 & 26 & 32 & 427 & 5.3 & $\$ 8$ & 4.7 \\
\hline $1 / 402$ & 1816 & 0.1 & 31.8 & 14.6 & 92 & 28.4 & 3.0 & 27.0 & 3.1 & 490 & 30 & 30.4 & 30 & 303 & 20 & 0.4 & 38 & 41.7 & 6.5 & 44 & 5.0 \\
\hline 11/402 & 1800 & 0.1 & 31.8 & 14.0 & 93 & 29.0 & 3.0 & 28.5 & 3.1 & 42 & 30 & 307 & 30 & 30.4 & 38 & 82 & 30 & 426 & 7.0 & 42 & 6.1 \\
\hline $11 / 402$ & 1845 & 0.1 & 31.3 & 18.3 & 98 & 29.7 & 3.0 & 23.6 & 3.1 & 40 & 30 & $\mathbf{3 . 8}$ & 20 & 30.4 & 10 & 0.7 & 30 & 41.7 & 6.6 & 445 & 44 \\
\hline $1 / 402$ & 1000 & 0.1 & 31.8 & 14.1 & OS & 286 & 3.0 & 28.6 & 3.1 & 40.0 & 30 & 30.3 & 30 & & 38 & 81.1 & 3.1 & 420 & 88 & 426 & 3.7 \\
\hline 11/AD2 & 1915 & 0.1 & 31.5 & 13.8 & 98 & 28.4 & 3.0 & 29.6 & 3.1 & 40.7 & 30 & 303 & 30 & 0.0 & 20 & 0.8 & 30 & 418 & 5.3 & 40 & 32 \\
\hline $11 / 402$ & 1000 & 0.1 & 31.6 & 14.3 & 92 & 28.7 & 3.0 & 28.4 & 3.1 & 4.7 & 30 & 33.3 & 30 & 12 & 30 & 08 & 48 & 41.6 & 6.4 & 422 & 52 \\
\hline $11 / 402$ & 1045 & 0.1 & 31.7 & 18.7 & 0.0 & 20.3 & 3.0 & 28.6 & 3.1 & 49.3 & 30 & 320 & 30 & 803 & 20 & 803 & 30 & 126 & 3.7 & 112 & 3.6 \\
\hline $11 / 492$ & 2000 & 0.1 & 31.8 & 14.0 & 02 & 28.6 & 3.0 & 28.6 & 3.1 & 43.7 & 30 & 30.4 & 30 & 30.6 & 20 & 0.4 & 34 & 418 & 4.4 & 40 & 13.4 \\
\hline $1 / 402$ & 2015 & 0.1 & 28.6 & 16.1 & 11.3 & 28.7 & 3.0 & 23.8 & 32 & 43.5 & 30 & 303 & 30 & 308 & 44 & 427 & 3.1 & 203 & 0.1 & 428 & 33 \\
\hline $1 / 402$ & 2000 & 0.1 & 30.6 & 18.4 & 10.5 & 29.6 & 3.0 & 232 & 3.1 & $\mathbf{M B}$ & 3.0 & 34.4 & 30 & 30.6 & 3.1 & 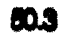 & 84 & 419 & 0.4 & 438 & 7.9 \\
\hline $1 / 402$ & 2045 & 0.1 & 29.8 & 13.8 & 10.7 & 28.4 & 3.0 & 282 & 3.1 & 42 & 30 & 34.4 & 30 & $\mathbf{S O S}$ & 29 & 43 & 30 & 41.0 & 6.0 & 430 & 4.0 \\
\hline $1 / 402$ & 2100 & 0.1 & 30.1 & 16.5 & 11.1 & $\mathbf{2 8 . 3}$ & 3.0 & 28.1 & 3.1 & 4.3 & 3.0 & 34.6 & 30 & 322 & 30 & 478 & 36 & $\omega .7$ & 8.7 & 4.1 & 5.1 \\
\hline $11 / 402$ & 2116 & 0.1 & 29.0 & 15.6 & 11.0 & 223 & 3.0 & 25.0 & 3.1 & 43 & 30 & 34.6 & 30 & & 0.1 & 100 & 30 & 0.1 & 8.5 & 427 & 10.0 \\
\hline $11 / 402$ & 2130 & 0.1 & 30.0 & 14.9 & 10.8 & 283 & 3.0 & 28.0 & 3.1 & 4.3 & 30 & 34.6 & 30 & 303 & 30 & 24 & 33 & $\infty 03$ & 6.7 & 43.0 & 3.5 \\
\hline 11/402 & 2145 & 0.1 & 20.4 & 18.3 & 11.2 & 282 & 3.0 & 23.1 & 3.1 & 4,3 & 30 & 34.6 & 30 & $\boldsymbol{N}$ & 34 & 41 & 3.1 & 4) 9 & 8.0 & 4.4 & 36 \\
\hline 11/402 & 2000 & 0.1 & 30.2 & 16.9 & 11.0 & 21 & 3.0 & 20.1 & 3.1 & 443 & 3.0 & 34.1 & 30 & & 20 & 420 & 32 & 4.5 & 8.0 & 422 & 4.3 \\
\hline 11/402 & 2216 & 0.1 & 30.3 & 18.0 & 121 & 20.2 & 3.0 & 26.7 & 3.1 & 4.4 & 30 & 340 & 30 & 30.1 & 20 & 97. & 3.1 & $\$ 0.4$ & 7.0 & 15.1 & 67 \\
\hline 11/402 & 2000 & 0.1 & 30.3 & 10.8 & 10.8 & 282 & 3.0 & 20.8 & 3.1 & 4.1 & 30 & 342 & 30 & 30.1 & 3.1 & 46 & 3.1 & $\$ 1.0$ & 8.0 & 438 & 3.6 \\
\hline $11 / 402$ & 2216 & 0.1 & 29.6 & 10.8 & 11.6 & $\mathbf{2 8 . 1}$ & 3.0 & 25.7 & 3.1 & 443 & 30 & 340 & 3.0 & 52 & 3.1 & and & 68 & 302 & 11.1 & 482 & 48 \\
\hline 11/402 & 2000 & 0.1 & 20.0 & 11.4 & 11.2 & 28.1 & 3.0 & 26.7 & 3.1 & 4.3 & 30 & 34.6 & 3 & . & 5.1 & 47.8 & 80 & $\omega .5$ & 8.4 & 4.5 & 28 \\
\hline 11/402 & 2015 & 0.1 & $\mathbf{2 0 . 0}$ & 11.4 & 125 & 282 & 3.0 & 20.7 & 3.1 & $\mathbf{4 5 . 0}$ & 30 & 33.7 & 30 & 30.4 & 20 & $\$ 7.1$ & 3.1 & $\mathbf{D B}$ & 8.4 & 40 & 54 \\
\hline $1 / 4902$ & 2350 & 0.1 & 200 & 11.8 & 11.7 & 28.5 & 3.0 & 26.6 & 3.1 & 45.1 & 30 & 35.3 & 30 & 5.9 .4 & 20 & 400 & 80 & $\$ 2$ & 8.2 & 490 & 4.3 \\
\hline $1 / 402$ & 2346 & 0.1 & 29.9 & 128 & 11.9 & 28.2 & 3.0 & 20.5 & 3.1 & 44.0 & 30 & 303 & 30 & 50.4 & 78 & 47.1 & 38 & 40.4 & 6.0 & 45.3 & 6.4 \\
\hline
\end{tabular}




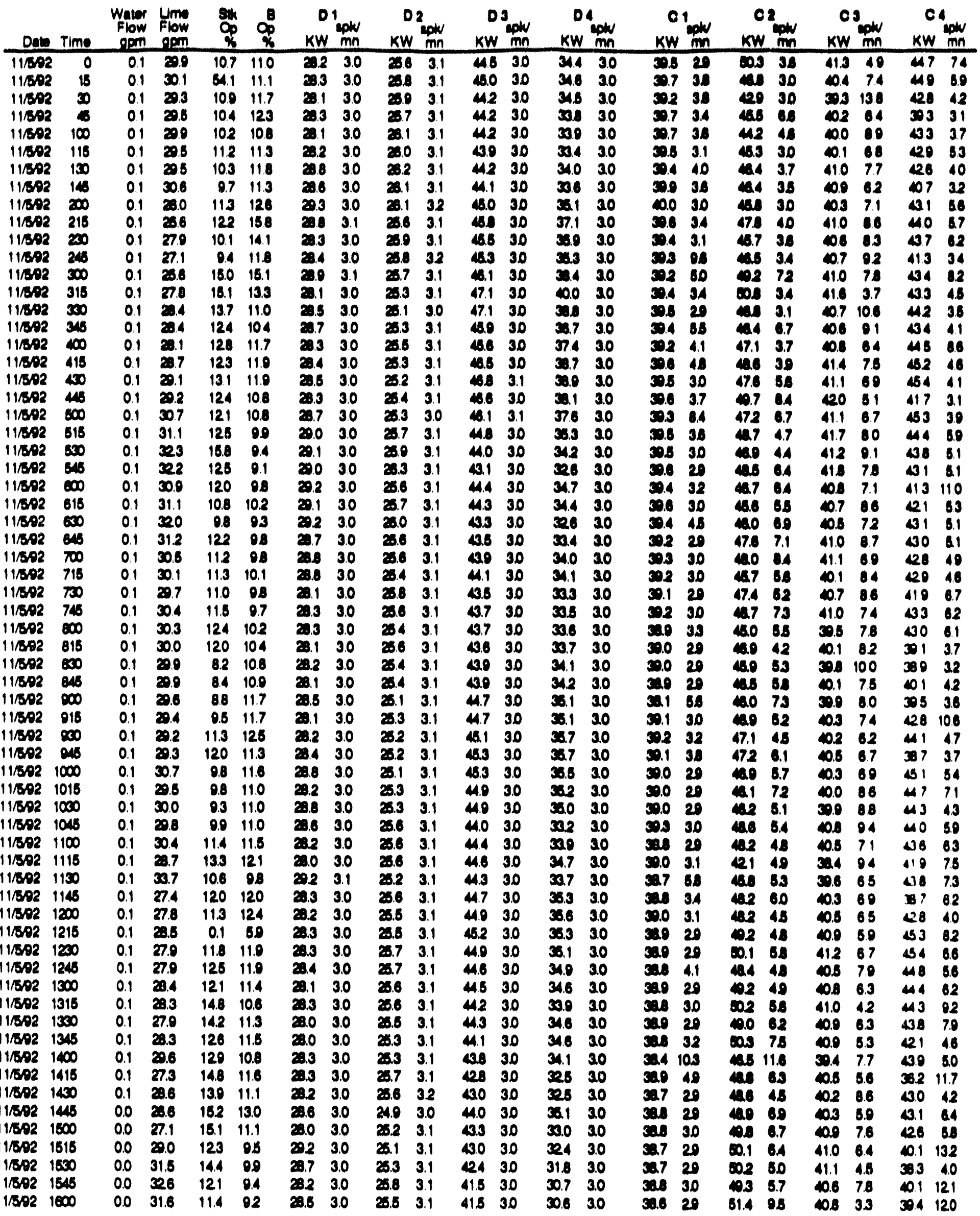




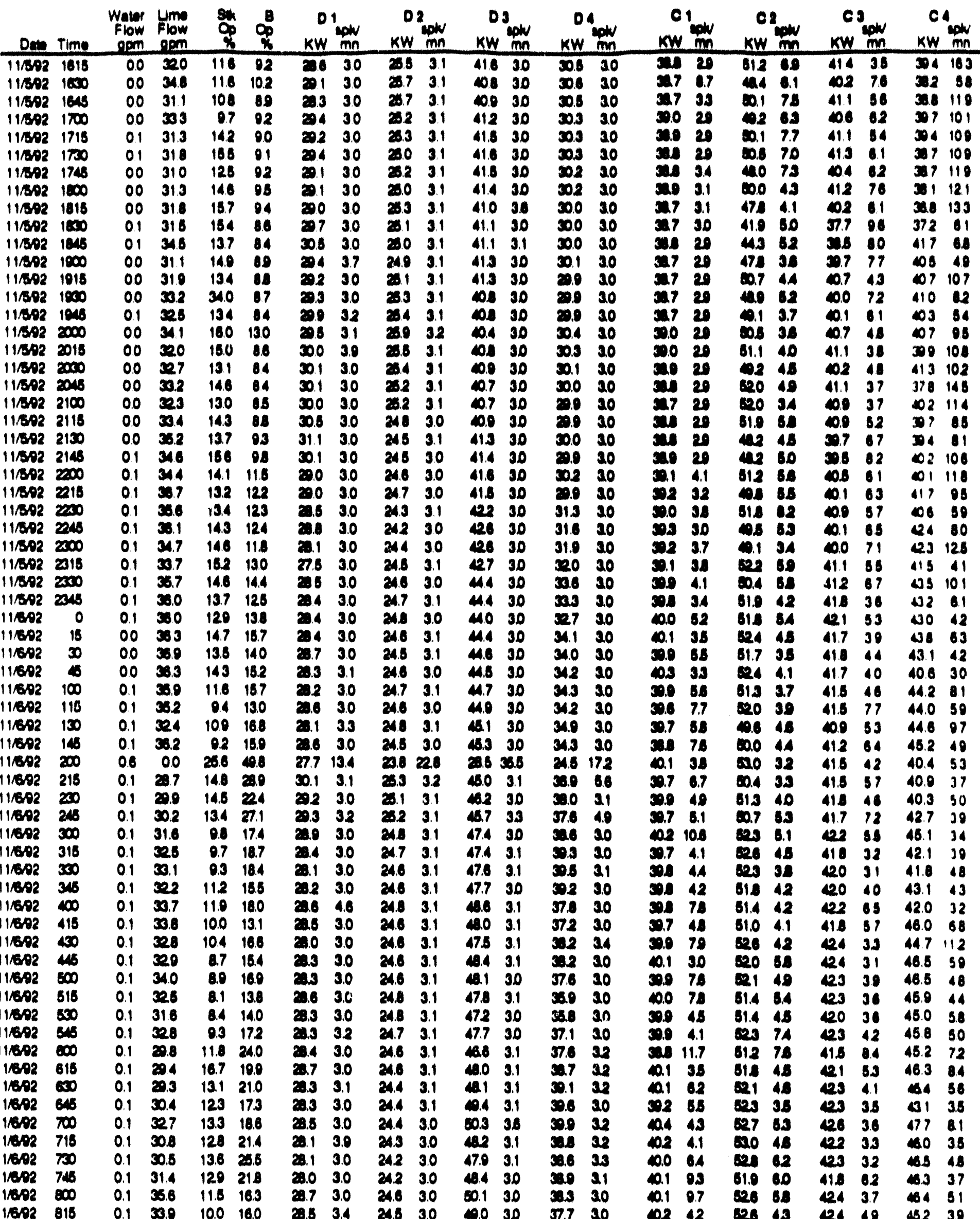




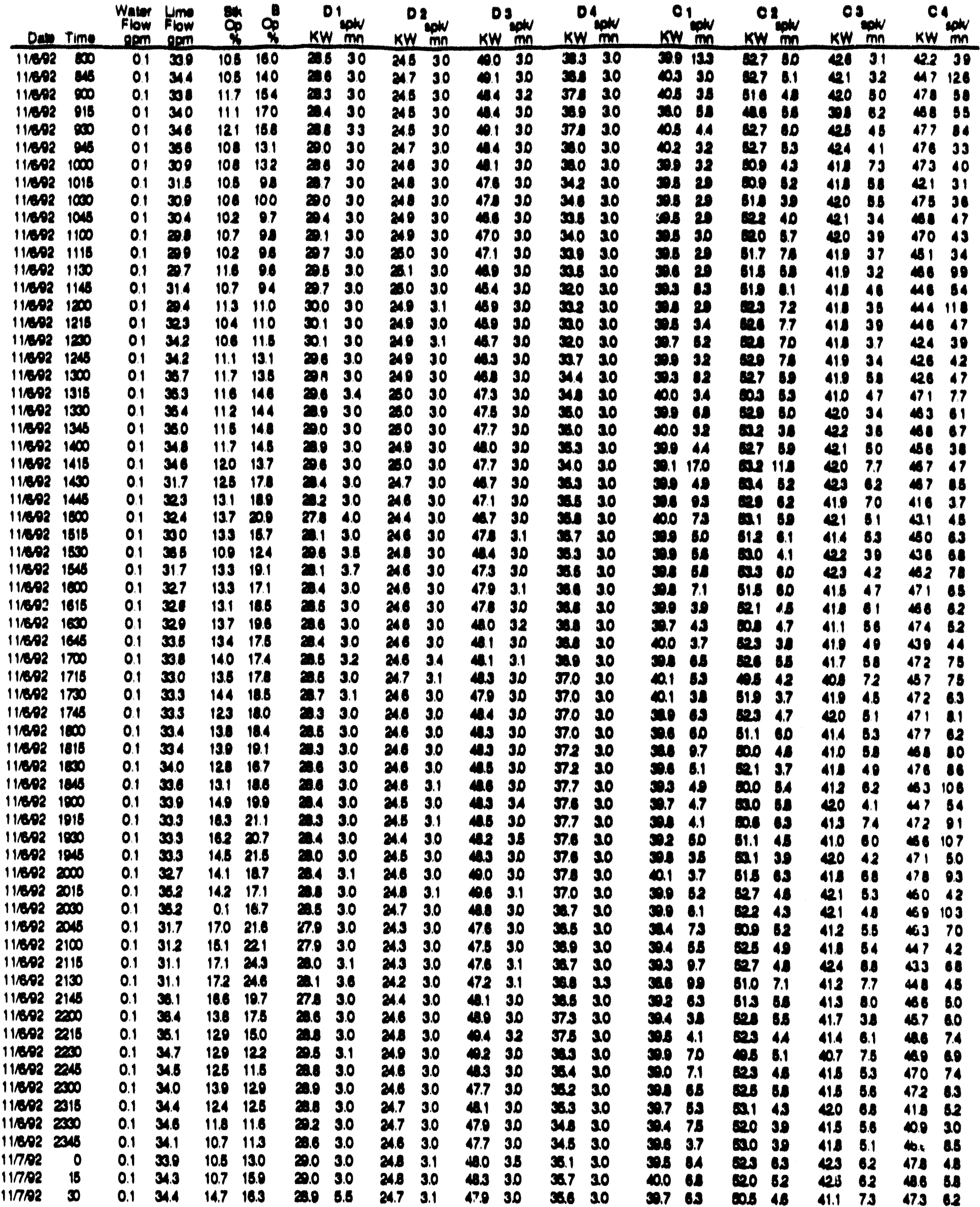




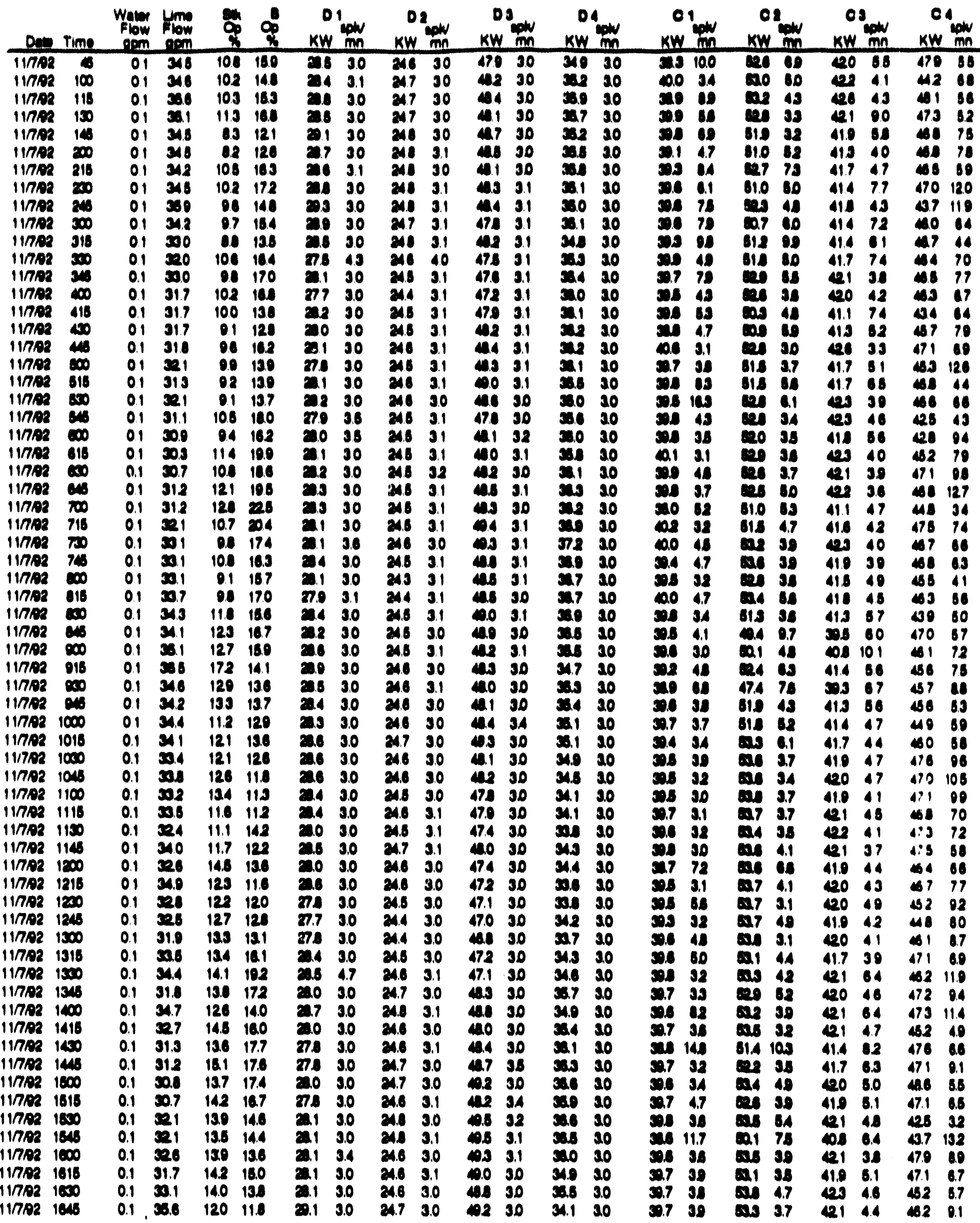




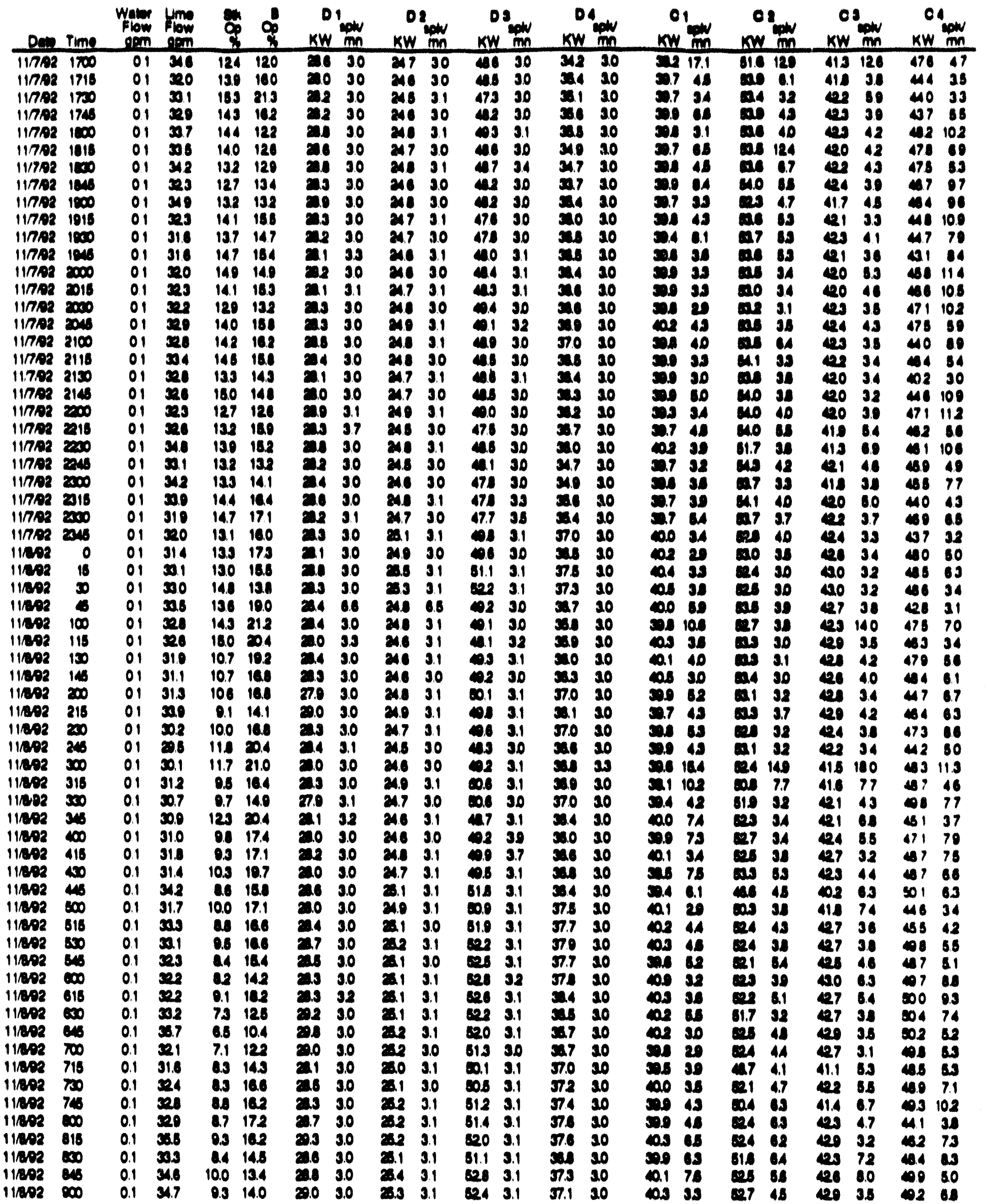




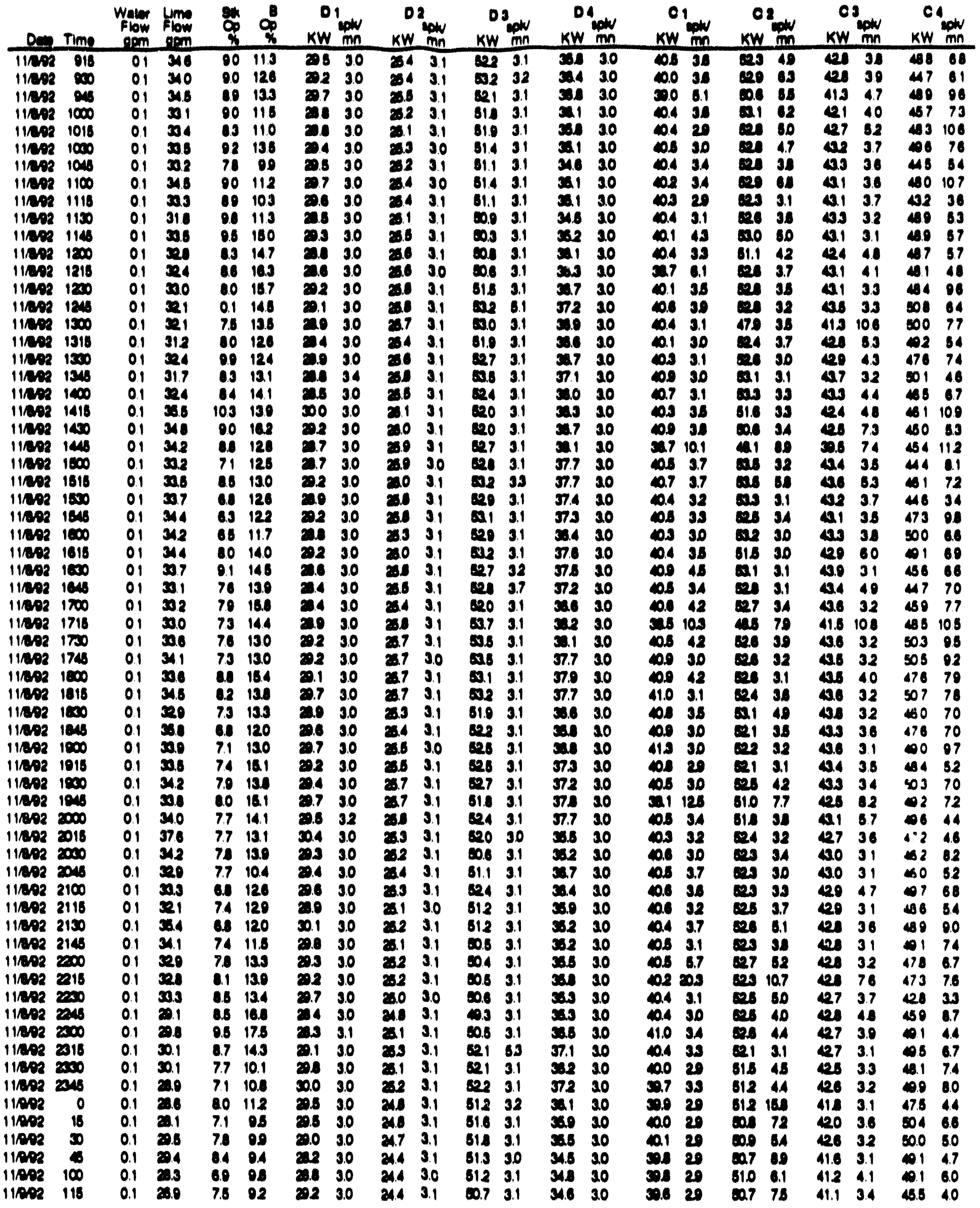




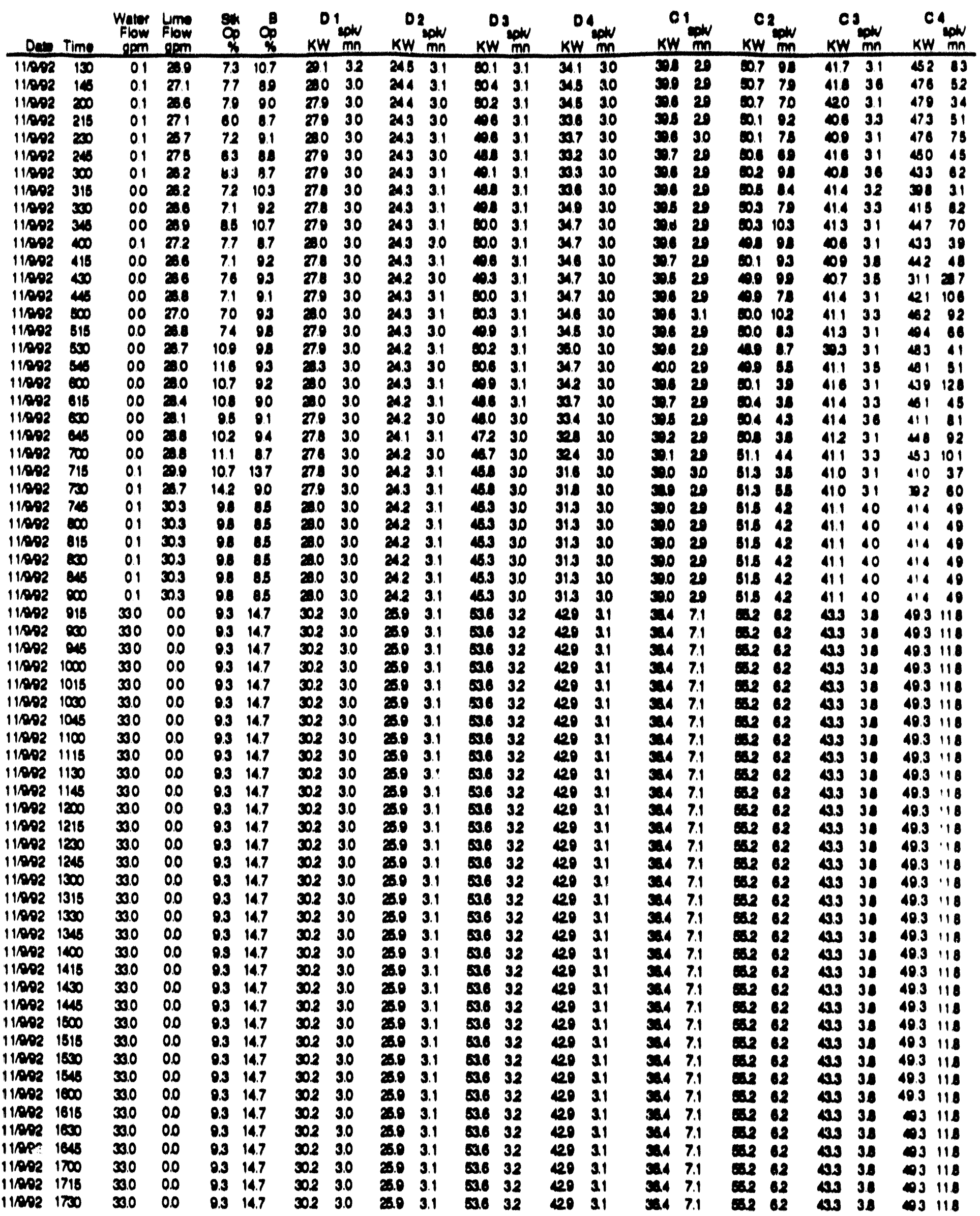




\begin{tabular}{|c|c|c|c|c|c|c|c|c|c|c|c|c|c|c|c|c|c|c|c|c|c|}
\hline Date & Time & $\begin{array}{c}\text { Water } \\
\text { Flow } \\
\text { gpm }\end{array}$ & $\begin{array}{l}\text { Lime } \\
\text { Flow } \\
\text { gpm }\end{array}$ & $\begin{array}{l}\text { Suk } \\
O_{0} \\
\%\end{array}$ & $\begin{array}{l}B \\
q_{0} \\
\%\end{array}$ & & $\begin{array}{l}\text { splw } \\
\text { mn }\end{array}$ & & $\mathrm{mn}$ & & $\begin{array}{l}\mathrm{W} / \\
\mathrm{nn}\end{array}$ & & $\begin{array}{l}\text { spok } \\
\text { mn }\end{array}$ & & $m n$ & $k W$ & mn & & $\begin{array}{l}\text { spk } \\
\text { mnn }\end{array}$ & $\mathrm{KW}$ & $\begin{array}{l}\text { solv } \\
\mathrm{mn}\end{array}$ \\
\hline 11,992 & 1745 & 33.0 & 0.0 & 9.3 & 14.7 & 30.2 & 3.0 & 25.9 & 3.1 & 53.6 & 32 & 429 & 3.1 & 36.4 & 7.1 & 552 & 62 & 43.3 & 38 & 403 & 11.8 \\
\hline $11 / 992$ & 1800 & 33.0 & 0.0 & 9.3 & 14.7 & 30.2 & 3.0 & 25.9 & 3.1 & 50.6 & 32 & 429 & 3.1 & 36.4 & 7.1 & 5.2 & 62 & 43.3 & 3.8 & 49.3 & 11.8 \\
\hline $11 / 3992$ & 1815 & 33.0 & 0.0 & 9.3 & 14.7 & 30.2 & 3.0 & 25.9 & 3.1 & 53.6 & 32 & 429 & 3.1 & 36.4 & 7.1 & 852 & 62 & 43.3 & 3.8 & 49.3 & 11.8 \\
\hline $11 / 392$ & 1830 & 33.0 & 0.0 & 9.3 & 14.7 & 30.2 & 3.0 & 25.9 & 3.1 & 53.6 & 32 & 429 & 3.1 & 36.4 & 7.1 & 552 & 62 & 43.3 & 3.8 & 49.3 & 11.8 \\
\hline $11 / 192$ & 1845 & 33.0 & 0.0 & 9.3 & 14.7 & 30.2 & 3.0 & 25.9 & 3.1 & 53.6 & 32 & 429 & 3.1 & 36.4 & 7.1 & 552 & 62 & 43.3 & 3.8 & 4.3 & 11.8 \\
\hline $11 / 9192$ & 1900 & 33.0 & 0.0 & 9.3 & 14.7 & 30.2 & 3.0 & 25.9 & 3.1 & 53.6 & 32 & 429 & 3.1 & 36.4 & 7.1 & 55.2 & 62 & 43.3 & 3.8 & 4.3 & 11.8 \\
\hline $11 / 9192$ & 1915 & 33.0 & 0.0 & 9.3 & 14.7 & 30.2 & 3.0 & $\mathbf{2 5 . 9}$ & 3.1 & 53.6 & 32 & 429 & 3.1 & 36.4 & 7.1 & 552 & 62 & 43.3 & 3.8 & 49.3 & 11.8 \\
\hline $11 / 9192$ & 1900 & 33.0 & 0.0 & 9.3 & 14.7 & 30.2 & 3.0 & 25.9 & 3.1 & 53.6 & 32 & 429 & 3.1 & 36.4 & 7.1 & 5.2 & 62 & 43.3 & 3.8 & 49.3 & 118 \\
\hline $11 / 9,92$ & 1945 & 33.0 & 0.0 & 9.3 & 14.7 & 30.2 & 3.0 & 25.9 & 3.1 & 53.6 & 32 & 429 & 3.1 & 36.4 & 7.1 & 55.2 & 62 & 43.3 & 3.8 & 49.3 & 11.8 \\
\hline $11 / 9192$ & 2000 & 33.0 & 0.0 & 9.3 & 14.7 & 30.2 & 3.0 & 25.9 & 3.1 & 53.6 & 32 & 429 & 3.1 & 36.4 & 7.1 & 55.2 & 62 & 43.3 & 3.8 & 49.3 & 11.8 \\
\hline $11 / 9 / 92$ & 2015 & 33.0 & 0.0 & 9.3 & 14.7 & 30.2 & 3.0 & 25.9 & 3.1 & 53.6 & 32 & 429 & 3.1 & 36.4 & 7.1 & 552 & 62 & 43.3 & 3.8 & 49.3 & 11.8 \\
\hline $11 / 9 / 92$ & 2000 & 33.0 & 0.0 & 9.3 & 14.7 & 30.2 & 3.0 & 25.9 & 3.1 & 53.6 & 32 & 429 & 3.1 & 36.4 & 7.1 & 552 & 62 & 43.3 & 3.8 & 49.3 & 118 \\
\hline $11 / 9 / 92$ & 2045 & 33.0 & 0.0 & 9.3 & 14.7 & 30.2 & 3.0 & 25.9 & 3.1 & 53.6 & 32 & 429 & 3.1 & 36.4 & 7.1 & 552 & 62 & 43.3 & 3.8 & 49.3 & 11.8 \\
\hline $11 / 9192$ & 2100 & 33.0 & 0.0 & 9.3 & 14.7 & 30.2 & 3.0 & 25.9 & 3.1 & 53.6 & 32 & 429 & 3.1 & 36.4 & 7.1 & 552 & 62 & 43.3 & 3.8 & 49.3 & 11.8 \\
\hline $11 / 992$ & 2115 & 33.0 & 0.0 & $9^{2}$ & 14.7 & 302 & 3.0 & 25.9 & 3.1 & 53.6 & 32 & 429 & 3.1 & 36.4 & 7.1 & 55.2 & 62 & 43.3 & 3.8 & 49.3 & 118 \\
\hline $11 / 9 / 92$ & 2130 & 33.0 & 0.0 & 9.3 & 14.7 & 30.2 & 3.0 & 25.9 & 3.1 & 53.6 & 32 & 429 & 3.1 & 36.4 & 7.1 & 502 & 62 & 43.3 & 3.8 & 49.3 & 11.8 \\
\hline $11 / 9 / 92$ & 2145 & 33.0 & 0.0 & 9.3 & 14.7 & 30.2 & 3.0 & 25.9 & 3.1 & 53.6 & 32 & 429 & 3.1 & 36.4 & 7.1 & 552 & 62 & 43.3 & 3.8 & 49.3 & 11.8 \\
\hline $11 / 992$ & 2200 & 33.0 & 0.0 & 9.3 & 14.7 & 30.2 & 3.0 & 25.9 & 3.1 & 53.6 & 32 & 429 & 3.1 & 36.4 & 7.1 & 552 & 62 & 43.3 & 3.8 & 49.3 & 11.8 \\
\hline $11 / 992$ & 2215 & 33.0 & 0.0 & 9.3 & 14.7 & 30.2 & 3.0 & 25.9 & 3.1 & 53.6 & 32 & 429 & 3.1 & 36.4 & 7.1 & 55.2 & 62 & 43.3 & 3.8 & 49.3 & 11.8 \\
\hline $11 / 992$ & 2200 & 33.0 & 0.0 & 9.3 & 14.7 & 30.2 & 3.0 & 25.9 & 3.1 & 53.6 & 32 & 429 & 3.1 & 36.4 & 7.1 & 552 & 62 & 43.3 & 3.8 & 49.3 & 11.8 \\
\hline $11 / 992$ & 2245 & 33.0 & 0.0 & 9.3 & 14.7 & 30.2 & 3.0 & 25.9 & 3.1 & 53.6 & 32 & 429 & 3.1 & 36.4 & 7.1 & 552 & 62 & 43.3 & 3.8 & 49.3 & 11.8 \\
\hline $11 \times 392$ & 2300 & 33.0 & 0.0 & 9.3 & 14.7 & 30.2 & 3.0 & 25.9 & 3.1 & 53.6 & 32 & 429 & 3.1 & 36.4 & 7.1 & 552 & 62 & 43.3 & 3.8 & 49.3 & 11.8 \\
\hline $11 / 992$ & 2315 & 33.0 & 0.0 & 9.3 & 14.7 & 30.2 & 3.0 & 25.9 & 3.1 & 53.6 & 32 & 429 & 3.1 & 36.4 & 7.1 & 55.2 & 62 & 43.3 & 3.8 & 4.3 & 11.8 \\
\hline $11 / 992$ & 2330 & 33.0 & 0.0 & 9.3 & 14.7 & 30.2 & 3.0 & ఒ5.9 & 3.1 & 53.6 & 32 & 429 & 3.1 & 36.4 & 7.1 & 5.2 & 62 & 43.3 & 3.8 & 49.3 & 11.8 \\
\hline $11 / 992$ & 2345 & 33.0 & 0.0 & 9.3 & 14.7 & 30.2 & 3.0 & 25.9 & 3.1 & 53.6 & 32 & 42.9 & 3.1 & 36.4 & 7.1 & $\overline{50.2}$ & 62 & 43.3 & 3.8 & 49.3 & 11.8 \\
\hline $1 / 1092$ & 0 & 33.0 & 0.0 & 9.3 & 14.7 & 30.2 & 3.0 & 25.9 & 3.1 & 53.6 & 32 & 429 & 3.1 & 36.4 & 7.1 & 552 & 62 & 43.3 & 38 & 4.3 & 11.8 \\
\hline $1 / 1092$ & 15 & 33.0 & 0.0 & 9.3 & 14.7 & 30.2 & 3.0 & 25.9 & 3.1 & 53.6 & 32 & 429 & 3.1 & 36.4 & 7.1 & 552 & 62 & 43.3 & 3.8 & 49.3 & 11.8 \\
\hline $11 / 1092$ & 30 & 33.0 & 0.0 & 9.3 & 14.7 & 30.2 & 3.0 & 25.9 & 3.1 & 53.6 & 32 & 429 & 3.1 & 36.4 & 7.1 & 552 & 62 & 43.3 & 3.8 & 49.3 & 11.8 \\
\hline $1 / 1092$ & 45 & 33.0 & 0.0 & 9.3 & 14.7 & 30.2 & 3.0 & 25.9 & 3.1 & 53.6 & 32 & 429 & 3.1 & 36.4 & 7.1 & 552 & 62 & 43.3 & 3.8 & 49.3 & 11.8 \\
\hline $1 / 1092$ & 100 & 33.0 & 0.0 & 9.3 & 14.7 & 30.2 & 3.0 & 25.9 & 3.1 & 53.6 & 32 & 429 & 3.1 & 36.4 & 7.1 & 552 & 62 & 43.3 & 3.8 & 49.3 & 11.8 \\
\hline $1 / 1092$ & 145 & 33.0 & 0.0 & 9.3 & 14.7 & 30.2 & 3.0 & 25.9 & 3.1 & 53.6 & 32 & 429 & 3.1 & 36.4 & 7.1 & 562 & 62 & 43.3 & 3.8 & 49.3 & 11.8 \\
\hline $1 / 1092$ & $4 x$ & 33.0 & 0.0 & 9.3 & 14.7 & 30.2 & 3.0 & 25.9 & 3.1 & 53.6 & 32 & 429 & 3.1 & 36.4 & 7.1 & 852 & 62 & 43.3 & 3.8 & 49.3 & 11.8 \\
\hline $1 / 1092$ & 145 & 33.0 & 0.0 & 9.3 & 14.7 & 30.2 & 3.0 & 25.9 & 3.1 & 53.6 & 32 & 429 & 3.1 & 36.4 & 7.1 & 52 & 62 & 43.3 & 3.8 & 49.3 & 11.8 \\
\hline $1 / 1092$ & 200 & 33.0 & 0.0 & 9.3 & 14.7 & 30.2 & 3.0 & 25.9 & 3.1 & 53.6 & 32 & 429 & 3.1 & 36.4 & 7.1 & 562 & 62 & 43.3 & 3.8 & 49.3 & 11.8 \\
\hline $1 / 1092$ & 215 & 33.0 & 0.0 & 9.3 & 14.7 & 30.2 & 3.0 & 25.9 & 3.1 & 53.6 & 32 & 429 & 3.1 & 36.4 & 7.1 & 502 & 62 & 43.3 & 3.8 & 49.3 & 11.8 \\
\hline $1 / 1092$ & 200 & 33.0 & 0.0 & 9.3 & 14.7 & 30.2 & 3.0 & ఒ. & 3.1 & 53.6 & 32 & 429 & 3.1 & 36.4 & 7.1 & 55.2 & 62 & 43.3 & 3.8 & 493 & 11.8 \\
\hline $1 / 1092$ & 245 & 33.0 & 0.0 & 9.3 & 14.7 & 30.2 & 3.0 & 25.9 & 3.1 & 53.6 & 32 & 429 & 3.1 & 36.4 & 7.1 & 552 & 62 & 43.3 & 3.8 & 493 & 11.8 \\
\hline $1 / 1092$ & 300 & 33.0 & 0.0 & 9.3 & 14.7 & 30.2 & 3.0 & 25.9 & 3.1 & 53.6 & 32 & 429 & 3.1 & 36.4 & 7.1 & 50.2 & 62 & 43.3 & 3.8 & 49.3 & 11.8 \\
\hline $1 / 1092$ & 315 & 33.0 & 0.0 & 9.3 & 14.7 & 30.2 & 3.0 & 25.9 & 3.1 & 53.6 & 32 & 429 & 3.1 & 36.4 & 7.1 & 552 & 62 & 43.3 & 3.8 & 43 & 118 \\
\hline $1 / 1092$ & 330 & 33.0 & 0.0 & 9.3 & 14.7 & 30.2 & 3.0 & 25.9 & 3.1 & 53.6 & 32 & 429 & 3.1 & 36.4 & 7.1 & 552 & 62 & 43.3 & 3.8 & 43 & 11.8 \\
\hline $1 / 1092$ & 345 & 33.0 & 0.0 & 9.3 & 14.7 & 30.2 & 3.0 & 25.9 & 3.1 & 53.6 & 32 & 429 & 3.1 & 36.4 & 7.1 & 552 & 62 & 433 & 3.8 & 43 & 11.8 \\
\hline $1 / 1092$ & 400 & 33.0 & 0.0 & 9.3 & 14.7 & 30.2 & 3.0 & 25.9 & 3.1 & 53.6 & 32 & 429 & 3.1 & 36.4 & 7.1 & 552 & 62 & 43.3 & 3.8 & 43 & 11.8 \\
\hline $1 / 1092$ & 415 & 33.0 & 0.0 & 9.3 & 14.7 & 30.2 & 3.0 & 25.9 & 3.1 & 53.6 & 32 & 429 & 3.1 & 33.4 & 7.1 & 552 & 62 & 43.3 & 3.8 & 43 & 11.8 \\
\hline $1 / 1092$ & 430 & 33.0 & 0.0 & 9.3 & 14.7 & 30.2 & 3.0 & 25.9 & 3.1 & 53.6 & 32 & 429 & 3.1 & 36.4 & 7.1 & 552 & 62 & 43.3 & 38 & 43 & 11.8 \\
\hline 1/1092 & 445 & 33.0 & 0.0 & 9.3 & 14.7 & 302 & 3.0 & 25.9 & 3.1 & 53.6 & 32 & 429 & 3.1 & 36.4 & 7.1 & 552 & 62 & 43.3 & 3.8 & 43 & 11.8 \\
\hline $1 / 1092$ & 500 & 33.0 & 0.0 & 9.3 & 14.7 & 30.2 & 3.0 & 25.9 & 3.1 & 53.6 & 32 & 429 & 3.1 & 36.4 & 7.1 & $\overline{52}$ & 62 & 43.3 & 38 & $\infty 3$ & 11.8 \\
\hline $1 / 1092$ & 515 & 33.0 & 0.0 & 9.3 & 14.7 & 30.2 & 3.0 & 25.9 & 3.1 & 53.6 & 32 & 429 & 3.1 & 36.4 & 7.1 & 552 & 62 & 43.3 & 3.8 & $\$ 3$ & 11.8 \\
\hline $1 / 1092$ & 530 & 33.0 & 0.0 & 9.3 & 14.7 & 30.2 & 3.0 & 25.9 & 3.1 & 53.6 & 32 & 429 & 3.1 & 36.4 & 7.1 & 55.2 & 62 & 43.3 & 3.8 & 493 & 11.8 \\
\hline $1 / 1092$ & 545 & 30.0 & 0.0 & 9.3 & 14.7 & 30.2 & 3.0 & 25.9 & 3.1 & 53.6 & 32 & 429 & 3.1 & 36.4 & 7.1 & 552 & 6.2 & 43.3 & 38 & 43 & 11.8 \\
\hline $1 / 1092$ & 600 & 33.0 & 0.0 & 9.3 & 14.7 & 30.2 & 3.0 & 25.9 & 3.1 & 50.6 & 32 & 429 & 3.1 & 33.4 & 7.1 & 55.2 & 62 & 433 & 3.8 & 4.3 & 11.8 \\
\hline $1 / 1092$ & 615 & 33.0 & 0.0 & 9.3 & 14.7 & 302 & 3.0 & 25.9 & 3.1 & 53.6 & 32 & 429 & 3.1 & 36.4 & 7.1 & 552 & 62 & 43.3 & 3.8 & 49.3 & 11.8 \\
\hline $1 / 1092$ & 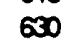 & 33.0 & 0.0 & 9.3 & 14.7 & 30.2 & 3.0 & 25.9 & 3.1 & 53.6 & 32 & 429 & 3.1 & 36.4 & 7.1 & 552 & 62 & 43.3 & 3.8 & 4.3 & 11.8 \\
\hline $1 / 1092$ & 645 & 33.0 & 0.0 & 9.3 & 14.7 & 30.2 & 3.0 & 25.9 & 3.1 & 53.6 & 32 & 429 & 3.1 & 36.4 & 7.1 & 552 & 62 & 43.3 & 3.8 & 493 & 11.8 \\
\hline $1 / 1092$ & 700 & 33.0 & 0.0 & 9.3 & 14.7 & 30.2 & 3.0 & 25.9 & 3.1 & 53.6 & 32 & 429 & 3.1 & 36.4 & 7.1 & 552 & 62 & 433 & 38 & 493 & 118 \\
\hline $1 / 1092$ & 715 & 33.0 & 0.0 & 9.3 & 14.7 & 302 & 3.0 & 25.9 & 3.1 & 53.6 & 32 & 429 & 3.1 & 36.4 & 7.1 & 552 & 62 & 43.3 & 38 & 49.3 & 11.8 \\
\hline $1 / 1092$ & 730 & 33.0 & 0.0 & 9.3 & 14.7 & 30.2 & 3.0 & 25.9 & 3.1 & 53.6 & 32 & 429 & 3.1 & 36.4 & 7.1 & 552 & 62 & 433 & 38 & 49.3 & 118 \\
\hline $1 / 1092$ & 745 & 33.0 & 0.0 & 9.3 & 14.7 & 30.2 & 3.0 & 25.9 & 3.1 & 53.6 & 32 & 429 & 3.1 & 36.4 & 7.1 & 552 & 62 & 43.3 & 38 & 49.3 & 118 \\
\hline $1 / 1092$ & 800 & 33.0 & 0.0 & 9.3 & 14.7 & 30.2 & 3.0 & 25.9 & 3.1 & 53.6 & 32 & 429 & 3.1 & 36.4 & 7.1 & 552 & 62 & 43.3 & 3.8 & 49.3 & 118 \\
\hline $1 / 1092$ & 815 & 30.0 & 0.0 & 9.3 & 14.7 & 30.2 & 3.0 & 25.9 & 3.1 & 53.6 & 32 & 420 & 3.1 & 36.4 & 7.1 & 552 & 62 & 433 & 38 & 49.3 & 11.8 \\
\hline $1 / 1092$ & 800 & 33.0 & 0.0 & 9.3 & 14.7 & 30.2 & 3.0 & 25.9 & 3.1 & 53.6 & 32 & 429 & 3.1 & 36.4 & 7.1 & 50.2 & 62 & 433 & 3.8 & 49.3 & 118 \\
\hline $1 / 1092$ & 845 & 33.0 & 0.0 & 9.3 & 14.7 & 30.2 & 3.0 & 25.9 & 3.1 & 53.6 & 32 & 429 & 3.1 & 36.4 & 7.1 & 52 & 62 & 433 & 38 & 49.3 & 11.8 \\
\hline $1 / 1092$ & 900 & 33.0 & 00 & 9.3 & 14.7 & 30.2 & 3.0 & 25.9 & 3.1 & 53.6 & 32 & 429 & 3.1 & 35.4 & 7.1 & 552 & 62 & 433 & 3.8 & 49.3 & 11.8 \\
\hline $1 / 1092$ & $n 15$ & 33.0 & 0.0 & 9.3 & 14.7 & 30.2 & 3.0 & 25.9 & 3.1 & 53.6 & 32 & 429 & 3.1 & 36.4 & 7.1 & 552 & 62 & 43.3 & 3.8 & 49.3 & 11.8 \\
\hline $1 / 1092$ & 900 & 33.0 & 0.0 & 9.3 & 14.7 & 30.2 & 3.0 & 25.9 & 3.1 & 53.6 & 32 & 429 & 3.1 & 35.4 & 7.1 & 552 & 62 & 43.3 & 3.8 & 4.3 & 11.8 \\
\hline $1 / 1092$ & 945 & 33.0 & 0.0 & 9.3 & 14.7 & 30.2 & 3.0 & 25.9 & 3.1 & 53.6 & 32 & 429 & 3.1 & 36.4 & 7.1 & 552 & 62 & 43.3 & 3.8 & 49.3 & 11.8 \\
\hline
\end{tabular}




\begin{tabular}{|c|c|c|c|c|c|c|c|c|c|c|c|c|c|c|c|c|c|c|c|c|c|}
\hline Dato & Time & $\begin{array}{l}\text { Water } \\
\text { Flow } \\
\text { gpm }\end{array}$ & $\begin{array}{l}\text { Lime } \\
\text { Flow } \\
\text { gpm }\end{array}$ & $\begin{array}{l}\text { Suk } \\
\text { Op } \\
\% \\
\end{array}$ & $\begin{array}{l}B \\
\text { Op } \\
\%\end{array}$ & $\begin{array}{r}01 \\
K W\end{array}$ & $\begin{array}{l}\mathrm{splw} \\
\mathrm{mn}\end{array}$ & & $\begin{array}{l}\text { spow } \\
\mathrm{mn}\end{array}$ & $\mathrm{KW}$ & $\begin{array}{l}\text { spk } \\
\mathrm{mn}\end{array}$ & & $\begin{array}{c}\mathrm{spl} \\
\mathrm{mn}\end{array}$ & & $\begin{array}{l}\mathrm{spl} \\
\mathrm{mn}\end{array}$ & $\mathrm{KW}$ & $\begin{array}{l}\text { splu } \\
\mathrm{mn}\end{array}$ & & & $\mathrm{KW}$ & \\
\hline $11 / 1092$ & 1000 & 33.0 & 0.0 & 9.3 & 14.7 & 30.2 & 3.0 & 25.9 & 3.1 & 53.6 & 32 & 429 & 3.1 & 36.4 & 7.1 & 552 & 62 & 43.3 & 3.8 & 49.3 & 118 \\
\hline $11 / 1092$ & 1015 & 33.0 & 0.0 & 9.3 & 14.7 & 30.2 & 3.0 & 25.9 & 3.1 & 53.6 & 32 & 429 & 3.1 & 38.4 & 7.1 & 552 & 62 & 43.3 & 3.8 & 49.3 & 11.8 \\
\hline $11 / 1092$ & 1000 & 33.0 & 0.0 & 9.3 & 14.7 & 30.2 & 3.0 & 25.9 & 3.1 & 53.6 & 32 & 429 & 3.1 & 36.4 & 7.1 & 552 & 62 & 43.3 & 3.8 & 49.3 & 11.8 \\
\hline $11 / 1092$ & 1045 & 330 & 0.0 & 9.3 & 14.7 & 30.2 & 3.0 & 25.9 & 3.1 & 53.6 & 32 & 429 & 3.1 & 36.4 & 7.1 & 552 & 62 & 43.3 & 3.8 & 49.3 & 11.8 \\
\hline $11 / 1092$ & 1100 & 33.0 & 0.0 & 9.3 & 14.7 & 30.2 & 3.0 & 25.9 & 3.1 & 53.6 & 32 & 429 & 3.1 & 36.4 & 7.1 & 552 & 62 & 43.3 & 3.8 & 49.3 & 11.8 \\
\hline $11 / 1092$ & 1115 & 33.0 & 0.0 & 9.3 & 14.7 & 30.2 & 3.0 & 25.9 & 3.1 & 53.6 & 32 & 429 & 3.1 & 36.4 & 7.1 & 552 & 62 & 433 & 3.8 & 4.3 & 11.8 \\
\hline $11 / 1092$ & 1130 & 33.0 & 0.0 & 9.3 & 14.7 & 30.2 & 3.0 & 25.9 & 3.1 & 53.6 & 32 & 429 & 3.1 & 36.4 & 7.1 & 55.2 & 62 & 43.3 & 3.8 & 49.3 & 11.8 \\
\hline $11 / 1092$ & 1145 & 33.0 & 0.0 & 9.3 & 14.7 & 30.2 & 3.0 & 25.9 & 3.1 & 53.6 & 32 & 429 & 3.1 & 36.4 & 7.1 & 55.2 & 62 & 43.3 & 3.8 & 4.3 & 11.8 \\
\hline $11 / 1092$ & 1200 & 33.0 & 0.0 & 9.3 & 14.7 & 30.2 & 3.0 & 25.9 & 3.1 & 53.6 & 32 & 429 & 3.1 & 36.4 & 7.1 & 552 & 62 & 43.3 & 38 & 4.3 & 11.8 \\
\hline $11 / 10 / 92$ & 1215 & 33.0 & 0.0 & 9.3 & 14.7 & 30.2 & 3.0 & 25.9 & 3.1 & 53.6 & 32 & 429 & 3.1 & 36.4 & 7.1 & 55.2 & 62 & 43.3 & 38 & 4.3 & 11.8 \\
\hline $11 / 1092$ & 1230 & 33.0 & 0.0 & 9.3 & 14.7 & 30.2 & 3.0 & 25.9 & 3.1 & 53.6 & 32 & 429 & 3.1 & 33.4 & 7.1 & 552 & 62 & 43.3 & 3.8 & 49.3 & 118 \\
\hline $11 / 1092$ & 1245 & 33.0 & 0.0 & 9.3 & 14.7 & 30.2 & 3.0 & 25.9 & 3.1 & 53.6 & 32 & 429 & 3.1 & 36.4 & 7.1 & 552 & 62 & 43.3 & 3.8 & 49.3 & 118 \\
\hline $11 / 1092$ & 1300 & 33.0 & 0.0 & 9.3 & 14.7 & 30.2 & 3.0 & 25.9 & 3.1 & 53.6 & 32 & 429 & 3.1 & 36.4 & 7.1 & 552 & 62 & 43.3 & 3.8 & 49.3 & 11.8 \\
\hline $11 / 1092$ & 1315 & 33.0 & 0.0 & 9.3 & 14.7 & 30.2 & 3.0 & 25.9 & 3.1 & 53.6 & 32 & 428 & 3.1 & 36.4 & 7.1 & 552 & 62 & 43.3 & 3.8 & 49.3 & 11.8 \\
\hline $11 / 1092$ & 1300 & 33.0 & 0.0 & 9.3 & 14.7 & 30.2 & 3.0 & 25.9 & 3.1 & 53.6 & 32 & 429 & 3.1 & 36.4 & 7.1 & 55.2 & 62 & 43.3 & 3.8 & 49.3 & 11.8 \\
\hline $11 / 1092$ & 1345 & 30.0 & 0.0 & 9.3 & 14.7 & 30.2 & 3.0 & 25.9 & 3.1 & 53.6 & 32 & 429 & 3.1 & 36.4 & 7.1 & 552 & 62 & 43.3 & 3.8 & 49.3 & 11.8 \\
\hline $11 / 1092$ & 1400 & 33.0 & 0.0 & 9.3 & 14.7 & 30.2 & 3.0 & 25.9 & 3.1 & 53.6 & 32 & 429 & 3.1 & 36.4 & 7.1 & 552 & 62 & $\mathbf{4 3 . 3}$ & 3.8 & 4.3 & 11.8 \\
\hline $11 / 1092$ & 1415 & 33.0 & 0.0 & 9.3 & 14.7 & 30.2 & 3.0 & 25.9 & 3.1 & 53.6 & 32 & 429 & 3.1 & 36.4 & 7.1 & 562 & 62 & 43.3 & 3.8 & 4.3 & 11.8 \\
\hline $11 / 1092$ & 1430 & 33.0 & 0.0 & 9.3 & 14.7 & 30.2 & 3.0 & 25.9 & 3.1 & 53.6 & 32 & 429 & 3.1 & 36.4 & 7.1 & 55.2 & 62 & 43.3 & 3.8 & 49.3 & 11.8 \\
\hline $11 / 1092$ & 1445 & 33.0 & 0.0 & 9.3 & 14.7 & 30.2 & 3.0 & 25.9 & 3.1 & 53.6 & 32 & 429 & 3.1 & 36.4 & 7.1 & 552 & 62 & 43.3 & 3.8 & 4.3 & 11.8 \\
\hline $11 / 1092$ & 1500 & 33.0 & 0.0 & 9.3 & 14.7 & 30.2 & 3.0 & 25.9 & 3.1 & 53.6 & 32 & 429 & 3.1 & 36.4 & 7.1 & 55.2 & 62 & 43.3 & 3.8 & 49.3 & 11.8 \\
\hline $11 / 1092$ & 1515 & 33.0 & 0.0 & 9.3 & 14.7 & 30.2 & 3.0 & 25.9 & 3.1 & 53.6 & 32 & 429 & 3.1 & 36.4 & 7.1 & 552 & 62 & 43.3 & 3.8 & 49.3 & 11.8 \\
\hline $11 / 1092$ & 1530 & 33.0 & 0.0 & 9.3 & 14.7 & 30.2 & 3.0 & 25.9 & 3.1 & 53.6 & 32 & 429 & 3.1 & 36.4 & 7.1 & 55.2 & 62 & 43.3 & 3.8 & 49.3 & 11.8 \\
\hline $11 / 1092$ & 1545 & 33.0 & 0.0 & 9.3 & 14.7 & 30.2 & 3.0 & 25.9 & 3.1 & 53.6 & 32 & 429 & 3.1 & 36.4 & 7.1 & 552 & 62 & 43.3 & 3.8 & 49.3 & 11.8 \\
\hline $11 / 1092$ & 1600 & 33.0 & 0.0 & 9.3 & 14.7 & 30.2 & 3.0 & 25.9 & 3.1 & 50.6 & 32 & 429 & 3.1 & 36.4 & 7.1 & 552 & 62 & 43.3 & 3.8 & 49.3 & 11.8 \\
\hline $11 / 1092$ & 1615 & 33.0 & 0.0 & 9.3 & 14.7 & 30.2 & 3.0 & 25.9 & 3.1 & 53.6 & 32 & 429 & 3.1 & 36.4 & 7.1 & 55.2 & 62 & 43.3 & 3.8 & 49.3 & 11.8 \\
\hline $1 / 1092$ & 1600 & 33.0 & 0.0 & 9.3 & 14.7 & 30.2 & 3.0 & 25.9 & 3.1 & 53.6 & 32 & 429 & 3.1 & 36.4 & 7.1 & 55.2 & 62 & 43.3 & 38 & 49.3 & 11.8 \\
\hline $1 / 1092$ & 1645 & 33.0 & 0.0 & 9.3 & 14.7 & 30.2 & 3.0 & 25.9 & 3.1 & 53.6 & 32 & 429 & 3.1 & 36.4 & 7.1 & 552 & 62 & 43.3 & 3.8 & 49.3 & 118 \\
\hline $1 / 1092$ & 1700 & 33.0 & 0.0 & 9.3 & 14.7 & 30.2 & 3.0 & 25.9 & 3.1 & 53.6 & 32 & 429 & 3.1 & 36.4 & 7.1 & 552 & 62 & 43.3 & 3.8 & 4.3 & 11.8 \\
\hline $1 / 1092$ & 1715 & 33.0 & 0.0 & 9.3 & 14.7 & 30.2 & 3.0 & 25.9 & 3.1 & 53.6 & 32 & 429 & 3.1 & 36.4 & 7.1 & 552 & 62 & 43.3 & 38 & 49.3 & 11.8 \\
\hline $11 / 1092$ & 1730 & 33.0 & 0.0 & 9.3 & 14.7 & 30.2 & 3.0 & 25.9 & 3.1 & 53.6 & 32 & 429 & 3.1 & 36.4 & 7.1 & 56.2 & 62 & 43.3 & 3.8 & 49.3 & 11.8 \\
\hline $11 / 1092$ & 1745 & 33.0 & 0.0 & 9.3 & 14.7 & 30.2 & 3.0 & $\mathbf{2 5 . 9}$ & 3.1 & 53.6 & 32 & 429 & 3.1 & 36.4 & 7.1 & 552 & 62 & 43.3 & 3.8 & 49.3 & 11.8 \\
\hline $11 / 1092$ & 1800 & 33.0 & 0.0 & 9.3 & 14.7 & 30.2 & 3.0 & 25.9 & 3.1 & 53.6 & 32 & 429 & 3.1 & 36.4 & 7.1 & 552 & 62 & 43.3 & 3.8 & 49.3 & 11.8 \\
\hline $11 / 1092$ & 1815 & 33.0 & 0.0 & 9.3 & 14.7 & 30.2 & 3.0 & 25.9 & 3.1 & 53.6 & 32 & 429 & 3.1 & 36.4 & 7.1 & 552 & 62 & 43.3 & 3.8 & 49.3 & 11.8 \\
\hline 11/1092 & 1850 & 33.0 & 0.0 & 9.3 & 14.7 & 30.2 & 3.0 & 25.9 & 3.1 & 53.6 & 32 & 429 & 3.1 & 36.4 & 7.1 & 55.2 & 62 & 43.3 & 3.8 & 49.3 & 11.8 \\
\hline $11 / 1092$ & 1845 & 33.0 & 0.0 & 9.3 & 14.7 & 30.2 & 3.0 & 25.9 & 3.1 & 53.6 & 32 & 429 & 3.1 & 36.4 & 7.1 & 55.2 & 62 & 43.3 & $3 B$ & 49.3 & 11.8 \\
\hline $11 / 1092$ & 1900 & 33.0 & 0.0 & 9.3 & 14.7 & 30.2 & 3.0 & 25.9 & 3.1 & 53.6 & 32 & 429 & 3.1 & 36.4 & 7.1 & 55.2 & 62 & 43.3 & 3.8 & 493 & 11.8 \\
\hline $11 / 1092$ & 1915 & 33.0 & 0.0 & 9.3 & 14.7 & 30.2 & 3.0 & 25.9 & 3.1 & 53.6 & 32 & 429 & 3.1 & 36.4 & 7.1 & 552 & 62 & 43.3 & 3.8 & 49.3 & 11.8 \\
\hline $11 / 1092$ & 1900 & 33.0 & 0.0 & 9.3 & 14.7 & 30.2 & 3.0 & 25.9 & 3.1 & 53.6 & 32 & 429 & 3.1 & 36.4 & 7.1 & 552 & 62 & 43.3 & 3.8 & 493 & 11.8 \\
\hline $11 / 1092$ & 1945 & 33.0 & 0.0 & 9.3 & 14.7 & 30.2 & 3.0 & 25.9 & 3.1 & 53.6 & 32 & 429 & 3.1 & 36.4 & 7.1 & 55.2 & 62 & 43.3 & 3.8 & 43 & 11.8 \\
\hline $11 / 1092$ & 2000 & 30.0 & 0.0 & 9.3 & 14.7 & 30.2 & 3.0 & 25.9 & 3.1 & 53.6 & 3.2 & 429 & 3.1 & 36.4 & 7.1 & 552 & 62 & 43.3 & 3.8 & 43 & 11.8 \\
\hline $11 / 1092$ & 2015 & 33.0 & 0.0 & 9.3 & 14.7 & 30.2 & 3.0 & 25.9 & 3.1 & 53.6 & 32 & 428 & 3.1 & 36.4 & 7.1 & 562 & 62 & 43.3 & 3.8 & 43 & 11.8 \\
\hline $11 / 1092$ & 2000 & 30.0 & 0.0 & 9.3 & 14.7 & 30.2 & 3.0 & 25.9 & 3.1 & 53.6 & 3.2 & 429 & 3.1 & 36.4 & 7.1 & 55.2 & 62 & 43.3 & 3.8 & 43 & 11.8 \\
\hline $11 / 1092$ & 2045 & 30.0 & 0.0 & 9.3 & 14.7 & 30.2 & 3.0 & 25.9 & 3.1 & 53.6 & 32 & 429 & 3.1 & 36.4 & 7.1 & 552 & 62 & 43.3 & 3.8 & $\$ 3$ & 11.8 \\
\hline 11/1092 & 2100 & 33.0 & 0.0 & 9.3 & 14.7 & 30.2 & 3.0 & 25.9 & 3.1 & 53.6 & 32 & 429 & 3.1 & 36.4 & 7.1 & 55.2 & 62 & 43.3 & 38 & 43 & 11.8 \\
\hline 11/1092 & 2115 & 33.0 & 0.0 & 9.3 & 14.7 & 30.2 & 3.0 & 25.9 & 3.1 & 53.6 & 32 & 429 & 3.1 & 36.4 & 7.1 & 55.2 & 62 & 43.3 & 38 & 43 & 11.8 \\
\hline $11 / 1092$ & 2130 & 33.0 & 0.0 & 9.3 & 14.7 & 30.2 & 3.0 & 25.9 & 3.1 & 53.6 & 32 & 429 & 3.1 & 36.4 & 7.1 & 552 & 62 & 43.3 & 3.8 & $\otimes 3$ & 11.8 \\
\hline $11 / 1092$ & 2145 & 33.0 & 0.0 & 9.3 & 14.7 & 302 & 3.0 & 25.9 & 3.1 & 53.6 & 32 & 429 & 3.1 & 36.4 & 7.1 & 55.2 & 62 & 43.3 & 3.8 & 43 & 118 \\
\hline $1 / 1 / 1092$ & 2200 & 33.0 & 0.0 & 9.3 & 14.7 & 30.2 & 3.0 & 25.9 & 3.1 & 53.6 & 32 & 429 & 3.1 & 36.4 & 7.1 & 552 & 62 & 43.3 & 3.8 & 43 & 11.8 \\
\hline $1 / 1092$ & 2215 & 30.0 & 0.0 & 9.3 & 14.7 & 30.2 & 3.0 & 25.9 & 3.1 & 53.6 & 32 & 429 & 3.1 & 36.4 & 7.1 & $\overline{552}$ & 62 & 43.3 & 3.8 & 43 & 11.8 \\
\hline $1 / 1092$ & 2200 & 33.0 & 0.0 & 9.3 & 14.7 & 30.2 & 3.0 & $\mathbf{2 5 . 9}$ & 3.1 & 53.6 & 32 & 429 & 3.1 & 36.4 & 7.1 & 552 & 62 & 43.3 & 3.8 & 40.3 & 11.8 \\
\hline $1 / 1092$ & 2245 & 33.0 & 0.0 & 9.3 & 14.7 & 30.2 & 3.0 & 25.9 & 3.1 & 53.6 & 32 & 429 & 3.1 & 36.4 & 7.1 & 55.2 & 62 & 43.3 & 3.8 & 4.3 & 11.8 \\
\hline $1 / 1092$ & 2300 & 33.0 & 0.0 & 9.3 & 14.7 & 30.2 & 3.0 & 25.9 & 3.1 & 53.6 & 32 & 429 & 3.1 & 36.4 & 7.1 & 552 & 62 & 43.3 & 3.8 & 4.3 & 11.8 \\
\hline $1 / 1092$ & 2315 & 33.0 & 0.0 & 9.3 & 14.7 & 30.2 & 3.0 & 25.9 & 3.1 & 53.6 & 32 & 429 & 3.1 & 36.4 & 7.1 & 55.2 & 62 & 43.3 & 3.8 & 49.3 & 118 \\
\hline $1 / 1092$ & 2030 & 33.0 & 0.0 & 9.3 & 14.7 & 30.2 & 3.0 & 25.9 & 3.1 & 53.6 & 32 & 429 & 3.1 & 36.4 & 7.1 & 552 & 62 & 43.3 & 3.8 & 4.3 & 11.8 \\
\hline $1 / 1092$ & 2345 & 33.0 & 0.0 & 9.3 & 14.7 & 30.2 & 3.0 & ఒ.. & 3.1 & 53.6 & 32 & 429 & 3.1 & 36.4 & 7.1 & 552 & 62 & 43.3 & 3.8 & 49.3 & 11.8 \\
\hline $1 / 11 / 92$ & 0 & 33.0 & 0.0 & 9.3 & 14.7 & 30.2 & 3.0 & 25.9 & 3.1 & 53.6 & 32 & 429 & 3.1 & 36.4 & 7.1 & 552 & 62 & 43.3 & 3.8 & 49.3 & 118 \\
\hline $1 / 11 / 92$ & 15 & 33.0 & 0.0 & 9.3 & 14.7 & 302 & 3.0 & 25.9 & 3.1 & 53.6 & 32 & 429 & 3.1 & 36.4 & 7.1 & 552 & 62 & 43.3 & 3.8 & 49.3 & 11.8 \\
\hline $1 / 11 / 92$ & 30 & 33.0 & 0.0 & 9.3 & 14.7 & 302 & 3.0 & 25.9 & 3.1 & 53.6 & 32 & 429 & 3.1 & 36.4 & 7.1 & 552 & 62 & 43.3 & 3.8 & 49.3 & 118 \\
\hline $1 / 11 / 92$ & 45 & 33.0 & 0.0 & 9.3 & 14.7 & 30.2 & 3.0 & 25.9 & 3.1 & 53.6 & 32 & 429 & 3.1 & 36.4 & 7.1 & 55.2 & 62 & 43.3 & 3.8 & 49.3 & 11.8 \\
\hline $1 / 11 / 92$ & 100 & 30.0 & 0.0 & 9.3 & 14.7 & 30.2 & 3.0 & 25.9 & 3.1 & 53.6 & 32 & 429 & 3.1 & 36.4 & 7.1 & 552 & 62 & 43.3 & 3.8 & 4.3 & 11.8 \\
\hline $1 / 11 / 92$ & 115 & 33.0 & 0.0 & 9.3 & 14.7 & 30.2 & 3.0 & 25.9 & 3.1 & 53.6 & 32 & 429 & 3.1 & 36.4 & 7.1 & 552 & 62 & 433 & 3.8 & 49.3 & 11.8 \\
\hline $1 / 11 / 92$ & 130 & 33.0 & 0.0 & 9.3 & 14.7 & 30.2 & 3.0 & 25.9 & 3.1 & 53.6 & 32 & 429 & 3.1 & 36.4 & 7.1 & 552 & 62 & 43.3 & 3.8 & 49.3 & 11.8 \\
\hline $11 / 92$ & 145 & 33.0 & 0.0 & 9.3 & 14.7 & 30.2 & 3.0 & 25.9 & 3.1 & 53.6 & 32 & 429 & 3.1 & 36.4 & 7.1 & 552 & 62 & 43.3 & 3.8 & 49.3 & 11.8 \\
\hline $1 / 11 / 92$ & 200 & 33.0 & 0.0 & 9.3 & 14.7 & 30.2 & 3.0 & ఒ. & 3.1 & 53.6 & 32 & 429 & 3.1 & 36.4 & 7.1 & 552 & 62 & 43.3 & 3.8 & 49.3 & 11.8 \\
\hline
\end{tabular}


D 2

$\begin{array}{ll}\% & 14.7\end{array}$

$\mathrm{KW} \mathrm{mn}$
$\mathrm{KW} \mathrm{mn}$

D 3 sow

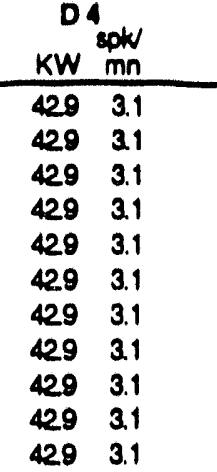

C1 C2 C 3 c4 Date Time $33.0 \quad 0.0$

$11 / 1192220$

$11 / 11 / 92 \quad 245$

$11 / 11 / 92 \quad 300$

$33.0 \quad 0.0$

$11 / 11 / 92 \quad 315$

$11 / 11 / 92 \quad 330$

$11 / 11 / 92 \quad 345$

$11 / 11 / 92 \quad 400$

$11 / 11 / 92 \quad 415$

$11 / 11 / 92 \quad 430$

$11 / 11 / 92 \quad 445$

$11 / 11 / 92 \quad 500$

$11 / 11 / 92 \quad 515$

$11 / 11 / 92 \quad 530$

$11 / 11 / 92 \quad 545$

$11 / 11 / 92 \quad 600$

$11 / 11 / 92 \quad 615$

$11 / 11 / 92 \quad 630$

$11 / 11 / 92 \quad 645$

$11 / 11 / 92 \quad 700$

$11 / 11 / 92 \quad 715$

$11 / 11 / 92 \quad 730$

$11 / 11 / 92 \quad 745$

$11 / 11 / 92 \quad 800$

$11 / 11 / 92 \quad 815$

$11 / 11 / 92 \quad 800$

$11 / 11 / 92 \quad 845$

$11 / 11 / 92 \quad 900$

$11 / 11 / 92 \quad 915$

$1 / 11 / 92 \quad 900$

$11 / 11 / 92 \quad 945$

$11 / 11 / 92 \quad 1000$

$11 / 11 / 92 \quad 1015$

$11 / 11 / 92 \quad 1000$

$11 / 11 / 92 \quad 1045$

$11 / 11 / 92 \quad 1100$

$11 / 11 / 92 \quad 1115$

$11 / 11 / 92 \quad 1130$

$11 / 11 / 92 \quad 1145$

$11 / 11 / 92 \quad 1200$

$11 / 11 / 92 \quad 1215$

$11 / 1192 \quad 1230$

$11 / 11 / 92 \quad 1245$

$11 / 1192 \quad 1300$

$11 / 11 / 92 \quad 1315$

$11 / 11 / 92 \quad 1330$

$11 / 11 / 92 \quad 1345$

$11 / 11 / 92 \quad 1400$

$11 / 11 / 92 \quad 1415$

$11 / 11 / 92 \quad 1430$

$11 / 11 / 92 \quad 1445$

$11 / 11 / 92 \quad 1500$

$11 / 11 / 92 \quad 1515$

$11 / 11 / 92 \quad 1530$

$11 / 11 / 92 \quad 1545$

$11 / 11 / 92 \quad 1600$

$11 / 11 / 92 \quad 1615$

$11 / 11 / 92 \quad 1630$

$11 / 11 / 92 \quad 1645$

$11 / 11 / 92 \quad 1700$

$1 / 11 / 92 \quad 1715$

$11 / 11 / 92 \quad 1730$

$11 / 11 / 92 \quad 1745$

$11 / 11 / 92 \quad 1800$

$11 / 11 / 92 \quad 1815$

$33.0 \quad 0.0$

$33.0 \quad 0.0$

$33.0 \quad 0.0$

$33.0 \quad 0.0$

$33.0 \quad 0.0$

$33.0 \quad 0.0$

$33.0 \quad 0.0$

$\begin{array}{llll}33.0 & 0.0 & 9.3 & 14.7\end{array}$

$33.0 \quad 0.0$

$\begin{array}{llll}33.0 & 0.0 & 9.3 & 14.7\end{array}$

$\begin{array}{llll}33.0 & 0.0 & 9.3 & 14.7\end{array}$

$\begin{array}{llll}33.0 & 0.0 & 9.3 & 14.7\end{array}$

$\begin{array}{llll}33.0 & 0.0 & 9.3 & 14.7\end{array}$

$\begin{array}{llll}33.0 & 0.0 & 9.3 & 14.7\end{array}$

$\begin{array}{llll}33.0 & 0.0 & 9.3 & 14.7\end{array}$

$\begin{array}{llll}33.0 & 0.0 & 9.3 & 14.7\end{array}$

$\begin{array}{llll}33.0 & 0.0 & 9.3 & 14.7\end{array}$

$\begin{array}{llll}33.0 & 0.0 & 9.3 & 14.7\end{array}$

$33.0 \quad 0.0$

$33.0 \quad 0.0$

$\begin{array}{ll}9.3 & 14.7 \\ 9.3 & 14.7\end{array}$

$\begin{array}{llll}33.0 & 0.0 & 9.3 & 14.7\end{array}$

$\begin{array}{llll}33.0 & 0.0 & 9.3 & 14.7\end{array}$

$\begin{array}{llll}33.0 & 0.0 & 9.3 & 14.7\end{array}$

$\begin{array}{llll}33.4 & 0.0 & 9.8 & 15.7\end{array}$

$\begin{array}{llll}30.5 & 0.0 & 19.8 & 27.4\end{array}$

$\begin{array}{llll}25.6 & 0.0 & 126 & 20.5\end{array}$

$\begin{array}{llll}25.6 & 0.0 & 126 & 20.5\end{array}$

$\begin{array}{llll}25.6 & 0.0 & 126 & 20.5\end{array}$

$\begin{array}{llll}321 & 0.0 & 11.3 & 17.2\end{array}$

$\begin{array}{llll}31.2 & 0.0 & 11.9 & 19.0\end{array}$

$\begin{array}{llll}30.1 & 0.0 & 129 & 16.1\end{array}$

$\begin{array}{llll}31.3 & 0.0 & 127 & 17.5\end{array}$

$\begin{array}{llll}30.4 & 0.0 & 120 & 17.6\end{array}$

$\begin{array}{llll}31.2 & 0.0 & 13.1 & 15.6\end{array}$

$\begin{array}{llll}31.2 & 0.0 & 13.1 & 15.6\end{array}$

$\begin{array}{llll}31.3 & 0.0 & 126 & 18.1\end{array}$

$\begin{array}{llll}324 & 0.0 & 10.8 & 13.7\end{array}$

$\begin{array}{llll}328 & 0.0 & 120 & 16.6\end{array}$

$\begin{array}{llll}31.5 & 0.0 & 128 & 15.3\end{array}$

$\begin{array}{llll}32.4 & 0.0 & 12.2 & 16.9\end{array}$

$\begin{array}{llll}33.0 & 0.0 & 10.2 & 122\end{array}$

$\begin{array}{llll}34.0 & 0.0 & 11.7 & 14.6\end{array}$

$\begin{array}{llll}34.2 & 0.0 & 13.1 & 17.6\end{array}$

$\begin{array}{llll}31.7 & 0.0 & 125 & 11.9\end{array}$

$\begin{array}{llll}33.0 & 0.0 & 10.3 & 13.5\end{array}$

$\begin{array}{llll}33.0 & 0.0 & 33.2 & 13.1\end{array}$

$\begin{array}{llll}320 & 0.0 & 9.9 & 13.4\end{array}$

$\begin{array}{llll}320 & 0.0 & 9.9 & 13.4\end{array}$

$\begin{array}{llll}320 & 0.0 & 9.9 & 13.4\end{array}$

$\begin{array}{llll}35.9 & 0.0 & 122 & 15.5\end{array}$

$\begin{array}{llll}34.8 & 0.0 & 10.4 & 10.8\end{array}$

$\begin{array}{llll}35.5 & 0.0 & 11.5 & 14.5\end{array}$

$\begin{array}{llll}35.5 & 0.0 & 124 & 15.4\end{array}$

$\begin{array}{llll}35.8 & 0.0 & 12.6 & 13.8\end{array}$

$\begin{array}{llll}36.7 & 0.0 & 11.1 & 125\end{array}$

$\begin{array}{llll}34.9 & 0.0 & 25.7 & 15.8\end{array}$

$\begin{array}{llll}34.5 & 0.0 & 15.7 & 14.6\end{array}$

$\begin{array}{llll}34.8 & 0.0 & 17.4 & 13.5\end{array}$

$\begin{array}{llll}35.4 & 0.0 & 15.0 & 15.1\end{array}$

$\begin{array}{llll}35.2 & 0.0 & 16.2 & 15.5\end{array}$

$\begin{array}{llll}36.8 & 0.0 & 15.1 & 13.0\end{array}$

$\begin{array}{llll}34.7 & 0.0 & 14.3 & 121\end{array}$

$\begin{array}{llllll}30.2 & 3.0 & 25.9 & 3.1 & 53.6 & 3.2\end{array}$

$\begin{array}{llllll}30.2 & 3.0 & 25.9 & 3.1 & 53.6 & 32\end{array}$

30.23 .0

30.23 .0

25.93 .1

$53.6 \quad 32$

$25.9 \quad 3.1$

$53.6 \quad 32$

30.23 .0

30.23 .0

25.83 .1

$53.6 \quad 32$

30.23 .0

30.23 .0

$30.2 \quad 3.0$

30.23 .0

$30.2 \quad 3.0$

30.23 .0

30.23 .0

$30.2 \quad 3.0$

30.23 .0

30.23 .0

30.23 .0

30.23 .0

30.23 .0

30.23 .0

30.23 .0

$30.2 \quad 3.0$

30.23 .0

30.23 .0

$30.2 \quad 3.0$

30.23 .0

$30.3 \quad 3.0$

$30.8 \quad 3.0$

$29.3 \quad 3.0$

$29.3 \quad 3.0$

$29.3 \quad 3.0$

29.73 .0

29.53 .0

$29.8 \quad 3.0$

30.03 .0

$29.2 \quad 3.0$

29.43 .0

20.43 .0

28.93 .0

$29.5 \quad 3.0$

$30.3 \quad 3.0$

$29.5 \quad 3.2$

20.43 .0

29.53 .0

$20.2 \quad 3.0$

30.13 .0

$29.7 \quad 3.0$

20.53 .0

$30.1 \quad 3.0$

$29.8 \quad 3.0$

$29.8 \quad 3.0$

$29.8 \quad 3.0$

$30.3 \quad 3.0$

25.93 .1

$53.6 \quad 32$

35.93 .1

$53.6 \quad 32$

25.93 .1

$53.6 \quad 32$

25.93 .1

$53.6 \quad 32$

3.1

$536 \quad 32$

$53.6 \quad 3.2$

$53.6 \quad 32$

$429 \quad 3.1$

42.93 .1

$429 \quad 3.1$

3.1

25.93 .1

$53.6 \quad 32$

25.93 .1

$\begin{array}{ll}53.6 & 32 \\ 53.6 & 32\end{array}$

3.1

$53.6 \quad 32$

4293.1

$420 \quad 3.1$

4293.1

$429 \quad 3.1$

$429 \quad 3.1$

25.93 .1

$53.6 \quad 32$

$53.6 \quad 32$

25.93 .1

$53.6 \quad 32$

25.93 .1

$53.6 \quad 32$

$\begin{array}{llll} & 3.1 & 53.6 & 32\end{array}$

25.93 .1

$53.6 \quad 3.2$

5.93 .1

$53.6 \quad 32$

5.83 .1

$53.6 \quad 32$

53.13 .1

26.23 .1

$49.9 \quad 8.8$

25.63 .1

$53.1 \quad 9.7$

25.63 .1

$53.1 \quad 9.7$

25.63 .1

$\begin{array}{ll}53.1 & 9.7\end{array}$

$25.6 \quad 3.1$

$528 \quad 3.1$

$\begin{array}{lllll}25.5 & 3.1 & 53.0 & 3.1\end{array}$

25.53 .1

$53.0 \quad 53$

25.73 .1

$520 \quad 3.1$

$\begin{array}{llll}25.8 & 3.1 & 523 & 32\end{array}$

25.53 .1

51.93 .1

25.53 .1

51.93 .1

$25.3 \quad 3.1$

53.23 .1

25.43 .1

$53,8 \quad 30$

$25.3 \quad 3.0$

53.83 .1

25.43 .1

54.13 .1

25.53 .1

54.43 .1

25.53 .0

54.53 .1

25.53 .1

55.53 .1

25.53 .0

$54.5 \quad 3.1$

25.43 .0

54.73 .1

25.53 .0

55.43 .1

$25.6 \quad 3.0$

$54.0 \quad 3.0$

$25.6 \quad 3.0$

$54.0 \quad 3.1$

$25.6 \quad 3.0$ 


\begin{tabular}{|c|c|c|c|c|c|c|c|c|c|c|c|c|c|c|c|c|c|c|c|c|c|}
\hline Date & Time & $\begin{array}{l}\text { Water } \\
\text { Flow } \\
\text { gpm }\end{array}$ & $\begin{array}{l}\text { Lime } \\
\text { Flow } \\
\text { opm }\end{array}$ & $\begin{array}{l}\text { suk } \\
\phi_{0} \\
\%\end{array}$ & $\begin{array}{r}8 \\
\% \\
\% \\
\end{array}$ & & $\begin{array}{l}1 \\
\text { spl } \\
\mathrm{mn}\end{array}$ & & $\begin{array}{l}2 \\
\text { spow } \\
\mathrm{mn}\end{array}$ & & $\mathrm{mn}$ & & & & $\mathrm{m}$ & $K W$ & & KW & & KW & $m n$ \\
\hline $11 / 11 / 92$ & 1850 & 36.4 & 0.0 & 16.0 & 14.6 & 30.9 & 3.0 & 25.9 & 3.1 & 53.0 & 3.1 & 37.4 & 3.0 & 40.5 & 43 & 55.0 & 3.1 & 43.4 & 32 & 49.7 & 110 \\
\hline $11 / 11 / 92$ & 1845 & 35.5 & 0.0 & 16.4 & 15.5 & 30.5 & $\$ 3.3$ & 26.1 & 3.1 & 542 & 3.1 & 38.1 & 3.0 & $\mathbf{0 . 5}$ & 40 & 84.7 & 30 & 43.7 & 3.1 & 491 & 103 \\
\hline $11 / 11 / 92$ & 1900 & 35.4 & 0.0 & 19.2 & 13.8 & 30.2 & 3.0 & $\mathbf{2 5 . 9}$ & 3.0 & 53.8 & 3.1 & 38.0 & 3.0 & 39.5 & 62 & 50 & 3.1 & 43.6 & 3.6 & 49.8 & 10.9 \\
\hline $11 / 11 / 92$ & 1916 & 34.9 & 0.0 & 14.5 & 14.0 & 30.3 & 3.0 & $\mathbf{2 6 . 1}$ & 3.1 & 54.7 & 3.1 & 38.6 & 3.0 & 38.1 & 122 & 8.1 & 30 & 43.9 & 3.1 & 49.8 & 100 \\
\hline $11 / 1102$ & 1900 & 34.9 & 0.0 & 15.5 & 14.3 & 30.7 & 3.0 & 25.8 & 3.1 & 53.5 & 3.1 & 38.2 & 3.0 & 378 & 63 & 5.7 & 4.1 & 40.9 & 3.4 & 48.2 & 121 \\
\hline $11 / 11 / 92$ & 1945 & 34.8 & 0.0 & 17.7 & 16.6 & 30.4 & 3.0 & $\mathbf{2 6 . 0}$ & 3.0 & 53.0 & 3.1 & 38.0 & 3.0 & 35.3 & 95 & 556 & 50 & 43.7 & 4.4 & 41 & 95 \\
\hline $11 / 11 / 82$ & 2000 & 35.2 & 0.0 & 14.2 & 13.7 & 30.7 & 3.0 & 25.8 & 3.1 & 528 & 3.1 & 37.7 & 3.0 & 37.4 & 7.4 & 586 & 34 & 43.8 & 3.5 & 50.8 & 106 \\
\hline $11 / 11 / 92$ & 2015 & 35.7 & 0.0 & 16.1 & 16.7 & 30.6 & 3.0 & 26.2 & 3.1 & 51.3 & 30 & 36.6 & 3.0 & 35.4 & 8.7 & 5.7 & $3 B$ & 438 & 32 & 4.3 & 5.4 \\
\hline $11 / 11 / 92$ & 2000 & 34.7 & 0.0 & 17.1 & 14.8 & 30.3 & 3.0 & 28.0 & 3.1 & 522 & 3.1 & 37.5 & 3.0 & 326 & 79 & 8.1 & 56 & 43.1 & 4.4 & 47.5 & 74 \\
\hline $11 / 11 / 92$ & 2045 & 34.9 & 0.0 & 14.8 & 122 & 30.2 & 3.0 & 26.1 & 3.1 & 51.0 & 3.1 & 37.1 & 3.0 & 343 & 10.8 & 5.6 & 40 & 438 & 3.3 & 474 & 7.6 \\
\hline $11 / 11 / 92$ & 2100 & 34.7 & 0.0 & 15.5 & 13.4 & 30.2 & 3.0 & $\mathbf{2 6 . 1}$ & 3.2 & 51.7 & 3.0 & 373 & 3.0 & 34.1 & 98 & 860 & 38 & 442 & 3.3 & 48.9 & 6.0 \\
\hline $11 / 11 / 92$ & 2115 & 34.6 & 0.0 & 0.1 & 21.1 & 30.4 & 3.0 & 26.1 & 3.1 & 51.6 & 3.1 & 37.3 & 3.0 & 35.7 & 6.7 & 8.7 & 40 & 438 & 32 & 45.6 & 7.4 \\
\hline $11 / 11 / 92$ & 2130 & 34.5 & 0.0 & 18.1 & 14.9 & 30.4 & 3.0 & 28.0 & 3.1 & 51.8 & 3.1 & 37.4 & 3.0 & 382 & 89 & 540 & 3.1 & 43.5 & 7.6 & 46.6 & 11.7 \\
\hline $11 / 11 / 92$ & 2145 & 34.5 & 0.0 & 16.5 & 14.5 & 30.6 & 3.0 & 28.0 & 3.1 & 527 & 3.1 & 378 & 3.0 & 33.5 & 68 & $\operatorname{sis} 3$ & 40 & 438 & 8.6 & 51.0 & 8.8 \\
\hline $11 / 11 / 92$ & 2200 & 35.9 & 0.0 & 19.4 & 19.5 & 31.0 & 3.0 & $\mathbf{2 8 . 3}$ & 3.1 & 51.0 & 3.1 & 38.1 & 3.0 & 35.9 & 11.1 & 80.6 & 30 & 4.1 & 3.2 & 40.3 & 70 \\
\hline $11 / 11 / 92$ & 2215 & 34.7 & 0.0 & 18.2 & 15.8 & 31.2 & 3.0 & $\mathbf{2 0 . 0}$ & 3.0 & 51.4 & 30 & 36.6 & 3.0 & 34.1 & 8.7 & 8.6 & 53 & 42 & 3.1 & 48.7 & 3.4 \\
\hline $11 / 11 / 92$ & 2200 & 35.3 & 0.0 & 16.6 & 13.3 & 31.0 & 3.0 & $\mathbf{2 0 . 0}$ & 3.1 & 51.4 & 3.1 & 36.8 & 3.0 & 300 & 70 & 50 & 32 & 4.1 & 3.1 & 50.2 & 5.5 \\
\hline $11 / 11 / 92$ & 2245 & 34.6 & 0.0 & 17.0 & 14.2 & 31.0 & 3.0 & 25.9 & 3.1 & 51.3 & 3.1 & 37.1 & 3.0 & 33.1 & 128 & 55.7 & 46 & 4.0 & 3.1 & 49.9 & 4.2 \\
\hline $11 / 11 / 92$ & $2 \varkappa 0$ & 34.7 & 0.0 & 18.6 & 14.6 & 30.7 & 3.0 & 25.9 & 3.1 & 51.3 & 3.0 & 36.0 & 3.0 & 31.7 & 11.7 & 582 & 8.5 & 426 & 3.7 & 50.0 & 40 \\
\hline $11 / 11 / 92$ & 2315 & 34.8 & 0.0 & 16.7 & 17.4 & 30.7 & 3.0 & 26.1 & 3.1 & 526 & 3.1 & 37.7 & 3.0 & 34.4 & 120 & 52 & 3.7 & 4.8 & 3.1 & 50.8 & 6.9 \\
\hline $11 / 11 / 92$ & $23<0$ & 34.5 & 0.0 & 18.1 & 11.7 & 323 & 3.0 & 25.8 & 3.0 & 53.7 & 3.1 & 38.6 & 3.0 & 31.0 & 8.4 & 525 & 43 & 41.4 & 3.5 & 50.7 & 7.2 \\
\hline $11 / 11 / 92$ & 2345 & 326 & 0.0 & 11.4 & 11.1 & 31.8 & 3.0 & 28.4 & 3.1 & 524 & 3.1 & 37.5 & 3.0 & 33.0 & 11.1 & 54.1 & $3 A$ & 4.8 & 3.3 & 50.6 & 4.4 \\
\hline $11 / 1292$ & 0 & 324 & 0.0 & 19.4 & 9.5 & 122 & 3.0 & 26.3 & 3.1 & 51.9 & 3.1 & 37.1 & 3.0 & 30.2 & 5.9 & $\infty$ & 3.1 & 4.7 & 3.1 & 50.5 & 4.0 \\
\hline $11 / 1292$ & 15 & 34.3 & 00 & 8.8 & 10.4 & 324 & 3.0 & 26.6 & 3.1 & 40.7 & 30 & 348 & 30 & 37.0 & 78 & 60.7 & 3.1 & 42 & 3.1 & 49.0 & 3.9 \\
\hline $11 / 1292$ & 30 & 33.3 & 0.0 & 11.4 & 11.2 & 326 & 3.0 & 26.7 & 3.1 & 49.7 & 3.1 & 35.0 & 3.0 & 40.7 & 84 & 203 & 40 & 43.9 & 3.1 & 48.4 & 6.8 \\
\hline $11 / 12 / 92$ & 46 & 34.8 & 0.0 & 127 & 11.3 & 323 & 3.0 & $\mathbf{2 8 . 6}$ & 3.1 & 49.9 & 30 & 348 & 3.0 & $\omega .3$ & 48 & 50.6 & 36 & 433 & 3.3 & 48.8 & 5.1 \\
\hline $11 / 1292$ & 100 & 33.6 & 0.0 & 10.4 & 8.9 & 326 & 3.0 & 28.4 & 3.1 & 49.8 & 3.1 & 35.1 & 3.0 & 40.4 & 36 & 505 & 36 & 427 & 3.1 & 45.8 & 4.9 \\
\hline $11 / 1292$ & 115 & 31.9 & 0.0 & 9.0 & 9.9 & 31.1 & 3.0 & 27.2 & 3.2 & 49.0 & 3.1 & 34.5 & 3.0 & $\$ 0.6$ & 39 & 53.4 & 3.1 & 427 & 3.1 & 45.5 & 3.9 \\
\hline $11 / 12 / 92$ & 130 & 30.3 & 0.0 & 31.8 & 9.1 & 31.3 & 3.0 & 27.1 & 3.2 & 48.9 & 3.1 & 34.7 & 3.0 & 308 & 10.4 & 58.1 & 30 & 43.3 & 3.1 & 46.8 & 3.5 \\
\hline $1 / 1292$ & 145 & 30.7 & 0.0 & 10.8 & 99 & 31.0 & 3.0 & 27.2 & 3.2 & 47.8 & 3.1 & $34 . n$ & 3.0 & $\$ .1$ & 38 & 6032 & 3.9 & 123 & 3.1 & 476 & 3.7 \\
\hline $11 / 1292$ & 200 & 30.8 & 0.0 & 9.0 & 8.5 & 31.5 & 3.0 & 27.3 & 3.2 & 47.1 & 30 & 33.2 & 3.0 & $\$ .2$ & 40 & 50.4 & 30 & 430 & 3.1 & 46.2 & 5.6 \\
\hline $11 / 1292$ & 215 & 30.3 & 0.0 & 11.0 & 93 & 320 & 3.0 & 27.4 & 3.2 & 47.0 & 3.1 & 33.0 & 3.0 & $\$ .5$ & 3.7 & 53.2 & 43 & 43.0 & 3.1 & 467 & 4.1 \\
\hline $11 / 1292$ & 200 & 31.8 & 0.0 & 13.2 & 11.3 & 320 & 3.0 & 27.2 & 3.1 & 472 & 3.0 & 329 & 3.0 & 402 & 3.4 & 0.1 & 46 & 429 & 3.1 & 476 & 5.5 \\
\hline $11 / 1292$ & 245 & 30.6 & 0.0 & 10.4 & 8.7 & 29.6 & 3.0 & 28.2 & 3.3 & 46.2 & 3.0 & 323 & 3.0 & $\$ .1$ & 8.7 & 532 & 30 & 426 & 3.1 & 444 & 3.5 \\
\hline $11 / 1292$ & 300 & 30.6 & 0.0 & 10.4 & 9.3 & 29.8 & 3.0 & 28.2 & 3.2 & 46.1 & 30 & 321 & 3.0 & $\$ .0$ & 33 & 53.4 & 40 & 428 & 3.1 & 46.3 & 5.1 \\
\hline $11 / 1292$ & 315 & 30.6 & 0.0 & 11.2 & 8.7 & 320 & 3.0 & 27.5 & 3.2 & 46.8 & 3.0 & 324 & 3.0 & 30.0 & 6.1 & 52 & 45 & 427 & 3.1 & 429 & 5.4 \\
\hline $11 / 1292$ & 330 & 31.0 & 0.0 & 11.4 & 8.4 & 323 & 3.0 & 27.3 & 3.2 & 46.8 & 3.0 & 325 & 3.0 & $\mathbf{0 . 0}$ & 3.1 & $5 x 0$ & 5.1 & 426 & 3.5 & 417 & 44 \\
\hline $11 / 1292$ & 345 & 30.0 & 0.0 & 10.6 & 8.1 & 32.1 & 3.0 & 27.2 & 3.2 & 47.1 & 3.0 & 33.1 & 35 & 30.8 & 50 & 526 & 63 & $\$ 1.7$ & 3.1 & 455 & 9.9 \\
\hline $11 / 1292$ & 400 & 30.2 & 0.0 & 10.8 & 9.7 & 324 & 3.0 & 27.2 & 32 & 47.3 & 3.1 & 33.4 & 3.0 & $\mathbf{4 0 . 0}$ & 40 & 500 & 4.7 & 422 & 3.1 & 455 & 5.2 \\
\hline $11 / 1292$ & 415 & 30.4 & 0.0 & 128 & 14.1 & 326 & 3.0 & 27.4 & 3.2 & 46.3 & 3.0 & 321 & 3.0 & 40.2 & 32 & 500 & 43 & 427 & 3.1 & 470 & 6.2 \\
\hline $11 / 1292$ & 430 & 30.5 & 0.0 & 121 & 8.4 & 325 & 3.0 & 27.6 & 3.2 & 46.3 & 30 & 321 & 3.0 & 40.1 & 32 & 526 & 5.5 & 427 & 3.1 & 452 & 6.6 \\
\hline $11 / 1292$ & 445 & 31.1 & 0.0 & 10.2 & 8.5 & 327 & 3.0 & 27.7 & 3.2 & 46.2 & 3.1 & 321 & 3.0 & $\$ 0.1$ & 32 & 527 & 52 & 427 & 3.1 & 467 & 8.4 \\
\hline $11 / 1292$ & 500 & 321 & 0.0 & 10.1 & 8.3 & 328 & 3.0 & 27.5 & 32 & 46.2 & 3.0 & 320 & 3.0 & 40.0 & 63 & 50.0 & 63 & 425 & 3.1 & 450 & 44 \\
\hline $11 / 1292$ & 515 & 30,3 & 0.0 & 9.7 & 92 & 324 & 3.0 & 27.4 & 3.2 & 46.4 & 3.1 & 327 & 3.0 & 40.2 & 40 & 53.4 & 5.3 & 426 & 3.1 & 49 & 44 \\
\hline $11 / 1292$ & 530 & 0.7 & 0.0 & 8.8 & 8.5 & 326 & 3.0 & 27.4 & 3.2 & 46.7 & 3.0 & 329 & 3.0 & 40.0 & 34 & 53.1 & 5.4 & 426 & 3.1 & 463 & 6.2 \\
\hline $1 / 1292$ & 545 & 31.0 & 0.0 & 9.5 & 8.7 & 33.1 & 3.0 & 27.5 & 3.2 & 46.5 & 3.0 & 326 & 3.0 & $\infty .1$ & 3.7 & 53.0 & 50 & 426 & 3.1 & 466 & 4.6 \\
\hline $11 / 1292$ & $\infty$ & 31.5 & 0.0 & 9.1 & 92 & 324 & 3.0 & 27.6 & 3.2 & 45.7 & 30 & 31.7 & 3.0 & 39.2 & 40 & 533 & 5.5 & 428 & 3.2 & 459 & 5.3 \\
\hline $11 / 12 / 92$ & 615 & 30.4 & 0.0 & 7.3 & 8.3 & 320 & 3.0 & 27.5 & 3.2 & 45.8 & 3.0 & 321 & 3.0 & $\$ 0.0$ & 38 & 60.2 & 6.3 & 425 & 31 & 438 & 48 \\
\hline $11 / 1292$ & $\infty 0$ & 31.4 & 0.0 & 9.6 & 9.1 & 33.0 & 3.0 & 27.4 & 3.2 & 46.1 & 3.1 & 31.9 & 3.1 & 39.9 & 43 & 527 & 50 & 426 & 3.1 & 127 & 5.0 \\
\hline $11 / 12 / 92$ & 645 & 30.6 & 0.0 & 8.4 & 8.6 & 327 & 3.0 & 27.7 & 3.2 & 45.7 & 30 & 31.8 & 3.0 & $\$ .5$ & 3.7 & 502 & 6.1 & 428 & 3.7 & 454 & 6.2 \\
\hline $11 / 12 / 92$ & 700 & $\mathbf{2 9 . 2}$ & 0.0 & 8.1 & 10.0 & 324 & 3.0 & 27.3 & 3.2 & 45.6 & 30 & 320 & 3.0 & 38.4 & 48 & 53.4 & 4.1 & 422 & 3.1 & 47 & 4.3 \\
\hline $1 / 1292$ & 715 & 31.5 & 0.0 & 11.3 & 8.4 & 325 & 3.0 & 27.5 & 3.2 & 45.5 & 30 & 31.7 & 3.0 & 308 & 33 & $0 \times .5$ & 43 & 422 & 3.1 & 43.6 & 4.1 \\
\hline $1 / 1292$ & 730 & 30.8 & 0.0 & 14.2 & 10.5 & 328 & 3.0 & 27.2 & 3.2 & 45.1 & 3.4 & 31.4 & 3.0 & 39.5 & 64 & 523 & 84 & 421 & 3.1 & 43.8 & 4.7 \\
\hline $1 / 12 / 92$ & 745 & 30.7 & 0.0 & 120 & 8.9 & 322 & 3.0 & 27.8 & 3.3 & 45.2 & 3.0 & 31.4 & 3.0 & 398 & 29 & 53.5 & 96 & 41.7 & 3.1 & 41.8 & 10.0 \\
\hline $1 / 1292$ & 800 & 30.5 & 0.0 & 121 & 8.7 & 327 & 3.0 & 27.3 & 3.2 & 45.5 & 30 & 31.0 & 3.0 & 39.7 & 3.7 & 506 & 6.6 & 420 & 3.3 & 43.4 & 3.8 \\
\hline $1 / 1292$ & 815 & 31.1 & 0.0 & 126 & 8.4 & 329 & 3.0 & 27.3 & 3.2 & 45.5 & 3.1 & 320 & 3.0 & 39.6 & 34 & 525 & 50 & 421 & 3.1 & 425 & 4.5 \\
\hline 1129 & 800 & 31.1 & 0.0 & 126 & 8.4 & 329 & 3.0 & 27.3 & 3.2 & 45.5 & 3.1 & 320 & 3.0 & 39.6 & 34 & 505 & 59 & 21 & 3.1 & 425 & 4.5 \\
\hline $1 / 12 \theta$ & 845 & 31.1 & 0.0 & 126 & 8.4 & 329 & 3.0 & 27.3 & 3.2 & 45.5 & 3.1 & 320 & 3.0 & 39.6 & 3.4 & 505 & 59 & 421 & 3.1 & 425 & 4.5 \\
\hline $1 / 1292$ & 900 & 31.6 & 0.0 & 125 & 8.7 & 31.7 & 3.0 & 28.0 & 32 & 44.8 & 30 & 31.7 & 31 & 39.6 & 3.1 & 50.7 & 64 & 418 & 3.1 & 429 & 5.9 \\
\hline $1 / 1292$ & 915 & 30.9 & 0.0 & 15.3 & 9.1 & 321 & 3.0 & 27.8 & 3.2 & 45.2 & 30 & 320 & 3.0 & 398 & 32 & 54.0 & 5.3 & 422 & 3.1 & 424 & 4.6 \\
\hline $1 / 1292$ & 900 & 30.9 & 0.0 & 15.3 & 9.1 & 32.1 & 3.0 & 27.8 & 32 & 45.2 & 30 & 320 & 3.0 & 39.8 & 32 & 54.0 & 53 & 422 & 3.1 & 424 & 4.6 \\
\hline $1 / 1292$ & 945 & 31.3 & 0.0 & 14.2 & 9.4 & 31.8 & 3.0 & 28.0 & 3.2 & 45.3 & 30 & 323 & 3.0 & 39.7 & 32 & 542 & 75 & 423 & 3.1 & 41.6 & 5.8 \\
\hline $1 / 1292$ & 1000 & 31.6 & 0.0 & 13.4 & 10.5 & 320 & 3.0 & 28.2 & 3.2 & 45.6 & 30 & 324 & 3.0 & 30.3 & 5.0 & 538 & 62 & 420 & 3.6 & 41.1 & 3.2 \\
\hline & 1015 & 31.6 & 0.0 & 13.4 & 10.5 & 320 & 3.0 & 28.2 & 3.2 & 45.6 & 30 & 324 & 3.0 & 39.3 & 50 & 538 & 62 & 420 & 3.6 & 41.1 & 32 \\
\hline $11 / 1292$ & 1000 & 31.6 & 0.0 & 13.4 & 10.5 & 320 & 3.0 & 28.2 & 3.2 & 45.6 & 3.0 & 324 & 3.0 & 39.3 & 59 & 508 & 62 & 420 & 3.6 & 41.1 & 3.2 \\
\hline
\end{tabular}


Date Time

Water Lime

Flow Flow

$11 / 12921045$

$11 / 1292 \quad 1100$

31.80 .0

$11 / 1292 \quad 1115$

$11 / 1292 \quad 1130$

$11 / 1292 \quad 1145$

$11 / 1292 \quad 1200$

$11 / 1282 \quad 1215$

$11 / 1292 \quad 1230$

$11 / 1202 \quad 1245$

$11 / 1292 \quad 1300$

$11 / 1292 \quad 1315$

$11 / 1292 \quad 1350$

$11 / 1292 \quad 1345$

$11 / 1292 \quad 1400$

$11 / 1292 \quad 1415$

$11 / 1202 \quad 1450$

$11 / 1202 \quad 1445$

$11 / 1292 \quad 1500$

$11 / 1292 \quad 1515$

$11 / 12921530$

$11 / 1292 \quad 1545$

$11 / 1292 \quad 1600$

$11 / 1292 \quad 1615$

$11 / 1292 \quad 1600$

$11 / 12921645$

$11 / 1292 \quad 1700$

$11 / 1292 \quad 1715$

$11 / 1292 \quad 1730$

$11 / 1292 \quad 1745$

$1 / 1292 \quad 1600$

, $1 / 1292 \quad 1816$

$11 / 12821850$

$11 / 1292 \quad 1845$

$11 / 1292 \quad 1900$

$11 / 1292 \quad 1915$

$11 / 1292 \quad 1900$

$11 / 1292 \quad 1945$

$11 / 12922000$

$11 / 1292 \quad 2015$

$11 / 12922000$

$11 / 1292 \quad 2045$

$11 / 1292 \quad 2100$

$11 / 12922115$

$11 / 12922130$

$11 / 1292 \quad 2145$

$11 / 12922200$

11/1202 2215

$11 / 1292 \quad 2200$

$11 / 1292 \quad 2245$

$11 / 1292200$

$11 / 1292 \quad 2315$

$11 / 12922300$

$11 / 1292 \quad 2345$

$11 / 1392$

$11 / 13 / 92$

15

$11 / 1392$
$11 / 1392$

$11 / 1392 \quad 100$

$11 / 1302 \quad 115$

$1 / 1392 \quad 130$

$1 / 1392 \quad 145$

$11 / 1392200$

$11 / 1392 \quad 215$

$11 / 1392220$

$11 / 1392 \quad 245$

$0.0 \quad 37.2$

$\begin{array}{llll}31.8 & 0.0 & 14.6 & 9.5 \\ 33.5 & 0.0 & 15.4 & 9.6\end{array}$

31.30

0.137 .1

$0.1 \quad 37.3$

0.137 .5

$\begin{array}{llll}0.1 & 36.8 & 16.8 & 10.4\end{array}$

$\begin{array}{llll}0.1 & 36.9 & 17.2 & 98\end{array}$

$0.1 \quad 37.2$

$0.0 \quad 37.0$

$0.0 \quad 37.0$

$0.0 \quad 36.8$

$0.0 \quad 38.8$

O.D $\quad 36.8$

$0.0 \quad 36.7$

$0.0 \quad 38.6$

$0.0 \quad 33.8$

$\begin{array}{llll}0.0 & 38.5 & 14.3 & 11.5\end{array}$

$\begin{array}{llll}0.0 & 37.5 & 15.4 & 121\end{array}$

$\begin{array}{llll}0.0 & 37.7 & 14.2 & 120\end{array}$

$\begin{array}{llll}0.0 & 38.2 & 17.9 & 14.6\end{array}$

$\begin{array}{llll}0.0 & 30.9 & 15.8 & 17.4\end{array}$

$\begin{array}{llll}0.1 & 39.8 & 17.3 & 18.2\end{array}$

$\begin{array}{llll}0.1 & 39.5 & 14.2 & 16.3\end{array}$

$\begin{array}{llll}0.1 & 30.5 & 122 & 16.2\end{array}$

$\begin{array}{llll}0.0 & 39.8 & 124 & 17.6\end{array}$

$\begin{array}{lllll}0.0 & 30.1 & 128 & 18.3\end{array}$

$\begin{array}{llll}0.1 & 39.5 & 129 & 17.1\end{array}$

$\begin{array}{lllll}0.0 & 38.1 & 13.5 & 18.4\end{array}$

$0.0 \quad 38.1$

$0.0 \quad 37.7$

$13.7 \quad 19.3$

$\begin{array}{llll}0.0 & 37.5 & 14.0 & 19.7\end{array}$

$\begin{array}{lllll}0.0 & 37.7 & 14.7 & 21.3\end{array}$

$\begin{array}{llll}0.0 & 37.1 & 15.6 & 20.1\end{array}$

$\begin{array}{llll}0.0 & 36.9 & 14.8 & 21.0\end{array}$

$0.0 \quad 36.9$

$0.0 \quad 36.8$

$0.0 \quad 37.5$

15.3228

$14.8 \quad 221$

$0.0 \quad 37.0$

ก. 36.8

$16.0 \quad 22.2$

$15.8 \quad 225$

$0.0 \quad 35.9$

$\begin{array}{lll}17.0 & 29.3\end{array}$

$18.1 \quad 29.2$

$14.0 \quad 19.9$

$\begin{array}{llll}0.0 & 36.3 & 16.7 & 24.4\end{array}$

$\begin{array}{llll}0.0 & 36.0 & 15.0 & 221\end{array}$

$\begin{array}{llll}0.0 & 36.0 & 15.1 & 222\end{array}$

$0.0 \quad 35.9$

$0.0 \quad 38.0$

15.520 .0

$0.0 \quad 37.3$

16.423 .5

$34.3 \quad 0.0$

$19.6 \quad 30.6$

$\begin{array}{llll}30.9 & 0.0 & 18.0 & 18.2\end{array}$

$\begin{array}{llll}31.1 & 0.0 & 14.1 & 17.8\end{array}$

$\begin{array}{llll}31.7 & 0.0 & 13.9 & 13.8\end{array}$

$\begin{array}{llll}322 & 0.0 & 11.9 & 124\end{array}$

$\begin{array}{llll}31.7 & 0.0 & 120 & 11.5\end{array}$

$\begin{array}{llll}31.5 & 0.0 & 126 & 13.9\end{array}$

$\begin{array}{llll}31.6 & 0.0 & 123 & 12.5\end{array}$

$\begin{array}{lllll}31.7 & 0.0 & 125 & 11.8\end{array}$

$\begin{array}{llll}31.7 & 0.0 & 10.1 & 11.1\end{array}$

$\begin{array}{llll}31.8 & 0.0 & 11.8 & 11.9\end{array}$

$\begin{array}{lllll}31.7 & 0.0 & 10.8 & 120\end{array}$

$\begin{array}{llll}31.7 & 0.0 & 9.2 & 11.3\end{array}$

$\begin{array}{llll}31.7 & 0.0 & 10.5 & 13.2\end{array}$

$\begin{array}{llll}31.7 & 0.0 & 120 & 13.2\end{array}$

$\begin{array}{llll}323 & 0.0 & 9.7 & 11.6\end{array}$

$\begin{array}{llll}31.5 & 0.0 & 120 & 10.9\end{array}$

D1

KW $\mathrm{mn} \quad \mathrm{KW}$ mn

03

spok

04 mn

$C 1_{\text {ency }}$

$C_{2}$ $\mathrm{mn} \quad \mathrm{kW}$ mn $\mathrm{kW}$ mn KW mn $\mathrm{KW} \mathrm{mm}$

$\begin{array}{llll}126 & 31 & 467 & 76 \\ 42 & 31 & 469 & 50\end{array}$

$\begin{array}{llll}30.2 & 3.0 & \mathbf{8 3 . 5} & 3.2\end{array}$

$\begin{array}{llll}328 & 3.0 & 28.5 & 32\end{array}$

$\begin{array}{llll}472 & 30 & 342 & 30\end{array}$

$\begin{array}{llll}47.6 & 3.1 & 34.8 & 3.0\end{array}$

31.13 .0

20.43 .2

$\begin{array}{llll}47.1 & 3.0 & 34.4 & 3.0\end{array}$

$322 \quad 3.0$

$20.1 \quad 32$

47.43 .0

34.43 .0

$\begin{array}{llll}20 & 3.0 & 28.8 & 3.2\end{array}$

31.13 .0

$20.7 \quad 3.2$

46.030

$30.8 \quad 3.0$

$28.6 \quad 3.2$

46.630

$33.0 \quad 3.0$

33.53 .0

46.530

33.43 .0

$\begin{array}{llll}30.3 & 3.0 & 28.3 & 3.2\end{array}$

30.03 .0

20.43 .0

28.73 .0

28.23 .2

46.43 .0

$30.3 \quad 3.0$

$\begin{array}{llll}4.3 & 30 & 33.2 \quad 3.0\end{array}$

48.130

$30.0 \quad 3.0$

28.132

$4.0 \quad 30$

$30.0 \quad 3.0$

$\begin{array}{cccc}46.0 & 30 & 320 & 3.0\end{array}$

$\begin{array}{llll}28.9 & 5.9 & 27.6 & 3.1\end{array}$

27.26 .6

27.73 .1

48.030

$328 \quad 3.0$

$\begin{array}{llllllll}27.2 & 6.6 & 27.7 & 3.1 & 46.7 & 3.0 & 32.7 & 3.0 \\ 23.6 & 9.6 & 27.2 & 3.1 & 46.0 & 3.0 & 32.8 & 3.0\end{array}$

25.57 .7

27.53 .1

$\begin{array}{cc}28.6 & 0.4\end{array}$

$27.5 \quad 3.1$

46.53 .0

3243.0

19.310 .3

26.78 .0

$27.2 \quad 3.1$

$26.6 \quad 5.7$

$\mathbf{2 8 . 6} 8.0$

27.03 .1

45.130

$320 \quad 3.0$

46230

$320 \quad 3.0$

$28.8 \quad 3.1$

45.53 .0

3213.0

24.310 .0

$28.6 \quad 3.2$

$46.4 \quad 3.0$

3213.0

26.37 .3

$28.3 \quad 3.1$

48.430

3243.0

27.95 .0

28.83 .1

$46.0 \quad 3.0$

3293.0

27.45 .0

$27.0 \quad 3.2$

46.53 .1

3263.0

$26.0 \quad 3.2$

46.33 .0

32.90

$\begin{array}{llll}2.0 & 10.4 & 28.5 & 3.2\end{array}$

29.05 .2

$28.6 \quad 3.2$

46.13 .0

$33.6 \quad 30$

$24.0 \quad 9.2$

$28.4 \quad 3.2$

46.23 .0

$33.3 \quad 3.0$

28.98 .8

$28.3 \quad 3.2$

$458 \quad 3.0$

$303 \quad 30$

$\begin{array}{lll}27.8 & 8.5\end{array}$

46.930

$33.0 \quad 3.0$

$28.0 \quad 13.7$

28.13 .1

45.83 .0

33330

20.43 .4

$48.8 \quad 3.0$

3323.0

30.13 .0

29.43 .4

$25.8 \quad 3.1$

$\begin{array}{llll}3.8 & 25.0 & 3.2\end{array}$

$\begin{array}{lllll}29.1 & 3.0 & 25.7 & 3.1\end{array}$

$\begin{array}{lllll}27.3 & 4.2 & 25.5 & 3.1\end{array}$

$\begin{array}{lllll}20.3 & 3.2 & 25.5 & 3.1\end{array}$

$\begin{array}{lllll}29.0 & 4.1 & 25.5 & 3.2\end{array}$

$\begin{array}{llll}27.1 & 11.4 & 25.5 & 3.2\end{array}$

$\begin{array}{lllll}23.8 & 4.1 & 25.4 & 3.1\end{array}$

$\begin{array}{lllll}22.7 & 3.1 & 25.0 & 3.1\end{array}$

$29.2 \quad 3.9$

25.13 .1

$45.8 \quad 30$

33.33 .0

48.030

$33.3 \quad 3.0$

4.13 .1

33.43 .0

$45.9 \quad 3.0$

$33.3 \quad 3.0$

46.13 .1

33.43 .0

$45.8 \quad 3.0$ 


\begin{tabular}{|c|c|c|c|c|c|c|c|c|c|c|c|c|c|c|c|c|c|c|c|c|c|}
\hline Das & Time & $\begin{array}{l}\text { Water } \\
\text { Flow } \\
\text { Opm }\end{array}$ & $\begin{array}{l}\text { Lime } \\
\text { Flow } \\
\text { ogm }\end{array}$ & $\begin{array}{l}\text { Suk } \\
O_{0} \\
\%\end{array}$ & $\begin{array}{l}8 \\
\phi_{0}^{\circ} \\
\%\end{array}$ & & $\begin{array}{l}1 \mathrm{spw} \\
\mathrm{mn}\end{array}$ & & $\begin{array}{l}\text { solv } \\
\mathrm{mn}\end{array}$ & & $\begin{array}{l}\text { solv } \\
m n\end{array}$ & $K W$ & $m n$ & KW & $m n$ & KW & $m$ & $K W$ & $m n$ & & \\
\hline $11 / 1392$ & 300 & 31.7 & 0.0 & 15.6 & 10.6 & 306 & 30 & 254 & 31 & 4.8 & 3.1 & 36.2 & 3.0 & 34.8 & 102 & 810 & 62 & 128 & 46 & 48 & 51 \\
\hline $11 / 1392$ & 315 & 325 & 0.0 & 121 & 15.0 & 30.3 & 3.0 & 25.8 & 3.1 & $\mathbf{4 6 . 5}$ & 30 & 342 & 3.0 & 38.0 & 10.3 & 523 & 43 & 422 & 63 & 415 & 36 \\
\hline $11 / 1392$ & 300 & 326 & 0.0 & 10.1 & 10.8 & 28.6 & 3.0 & 26.8 & 3.1 & 48 & 30 & 33.8 & 3.0 & 30. & 11.0 & 0.7 & 88 & 41.5 & 6.3 & 123 & 5.5 \\
\hline $11 / 1392$ & 345 & 327 & 0.0 & 11.4 & 120 & 200 & 3.0 & 26.7 & 3.2 & 450 & 3.0 & 30.0 & 3.0 & 338 & 112 & 62 & 42 & 128 & 5.2 & 43.1 & 75 \\
\hline $11 / 1392$ & 400 & 327 & 0.0 & 0.7 & 10.9 & 30.5 & 3.0 & 26.0 & 3.1 & 46.7 & 3.0 & 346 & 3.0 & 38.7 & 09 & 643 & 4.1 & 427 & 3.2 & 43.8 & 52 \\
\hline $11 / 1392$ & 415 & 327 & 0.0 & 10.2 & 11.1 & 30.6 & 3.0 & 260 & 3.1 & 45.4 & 30 & 342 & 3.0 & 348 & 18.0 & 043 & 7.1 & 127 & 36 & 4.1 & 65 \\
\hline $11 / 13992$ & 430 & 33.1 & 0.0 & 11.8 & 10.1 & 30.8 & 3.0 & 26.5 & 3.2 & 48.3 & 3.0 & 33.6 & 3.0 & 30.3 & 11.0 & $\mathbf{\omega . 6}$ & 68 & 41.1 & 5.1 & 43.3 & 41 \\
\hline $11 / 1302$ & 445 & 324 & 00 & 95 & 10.4 & 30.3 & 3.0 & $\mathbf{2 8 . 5}$ & 3.1 & 4.3 & 3.0 & 30.0 & 3.0 & 34.9 & 10.2 & $\mathbf{6 5}$ & 98 & 420 & 62 & 410 & 5.9 \\
\hline $11 / 1302$ & 500 & 326 & 00 & 9.9 & 11.3 & 30.7 & 3.0 & 25.6 & 3.2 & 46.7 & 30 & 34.3 & 3.0 & 3.6 & 124 & 82 & 6.1 & 48.0 & 40 & 122 & 63 \\
\hline $11 / 1392$ & 515 & 327 & 0.0 & 8.0 & 11.7 & 30.8 & 3.0 & 28.1 & 3.2 & 45.6 & 3.0 & 34.3 & 3.0 & 30.4 & 82 & 325 & 80 & 422 & 6.3 & 43.1 & 6.5 \\
\hline $11 / 1392$ & 500 & 326 & 0.0 & 10.8 & 13.2 & 30.8 & 30 & 2.8 .1 & 3.2 & 45.5 & 3.1 & 342 & 3.0 & 37.7 & 120 & 0.1 & 8.7 & 120 & 3.7 & 10.3 & 3.3 \\
\hline $11 / 1302$ & 545 & 326 & 0.0 & 110 & 15.1 & 30.9 & 3.0 & $\mathbf{2 8 . 3}$ & 3.2 & 45.2 & 30 & 340 & 3.0 & 30 & 62 & 020 & 36 & 43.6 & 33 & 41.2 & 44 \\
\hline $11 / 1392$ & $\infty \infty$ & 326 & 0.0 & 8.2 & 123 & 31.0 & 3.0 & 282 & 3.1 & 462 & 3.0 & 33.8 & 3.0 & 35.6 & 14.8 & $6 x 7$ & 65 & 128 & 3.1 & 398 & 3.2 \\
\hline $11 / 1392$ & 615 & 326 & 0.0 & 0.0 & 13.7 & 31.3 & 3.0 & $\mathbf{2 0 . 7}$ & 3.1 & 46.1 & 30 & 348 & 3.0 & 302 & 90 & 643 & 30 & 43.4 & 4.3 & 418 & 41 \\
\hline $11 / 1392$ & 600 & 30.0 & 0.0 & 11.8 & 11.7 & 31.7 & 3.0 & 20.0 & 3.2 & 48.1 & 30 & 332 & 3.1 & 39.6 & 11.7 & $8 \times 6$ & 80 & 43.6 & 40 & 43.1 & 7.7 \\
\hline $11 / 1302$ & 645 & 325 & 0.0 & 13.8 & 15.2 & 31.0 & 3.0 & 25.0 & 3.2 & 40 & 30 & 34.6 & 3.0 & 308 & 203 & 840 & 133 & 432 & 3.6 & 432 & 74 \\
\hline $11 / 1392$ & 700 & 327 & 0.0 & 10.8 & 126 & 30.0 & 3.0 & 28.1 & 3.2 & 45.4 & 30 & 35.1 & 3.0 & 32.6 & 118 & $\infty 27$ & 74 & 43.6 & 10 & 428 & 10 \\
\hline $11 / 13992$ & 715 & 325 & 0.0 & 16.8 & 15.6 & 31.0 & 3.0 & 25.8 & 3.1 & 46.7 & 30 & 38.3 & 3.0 & 30.4 & 0.7 & 842 & 78 & 43.1 & 4.7 & 40.5 & 110 \\
\hline $11 / 1392$ & 730 & 327 & 0.0 & 13.7 & 16.6 & 30.0 & 3.0 & 25.5 & 3.1 & 48.0 & 30 & 346 & 3.0 & 37.0 & 7.7 & 62.6 & 38 & 128 & 50 & 430 & 8.7 \\
\hline $11 / 1392$ & 745 & 327 & 0.0 & 11.7 & 15.3 & 30.8 & 3.0 & 20.4 & 3.1 & $\mathbf{6 . 7}$ & 30 & 35.3 & 3.0 & 38.1 & 10.8 & 84.4 & 38 & 43.1 & 5.0 & 421 & 78 \\
\hline $11 / 1392$ & 800 & 33.2 & 0.0 & 125 & 15.5 & 31.3 & 3.1 & 25.0 & 32 & 47.5 & 33 & 38.1 & 3.1 & 37.7 & 68 & 840 & 108 & 126 & 3.8 & 427 & 72 \\
\hline $11 / 1392$ & 815 & 328 & 0.0 & 129 & 16.4 & 30.0 & 3.0 & 20.6 & 3.1 & 47.6 & 3.0 & 37.6 & 3.0 & 34.4 & 86 & 610 & 16 & 125 & 3.8 & 427 & 6.5 \\
\hline $11 / 1392$ & 800 & 320 & 0.0 & 8.7 & 9.9 & 20.5 & 3.0 & 26.3 & 32 & $\mathbf{4 6 . 1}$ & 3.1 & 36.0 & 3.0 & 34.2 & 10.0 & 84.9 & 49 & 430 & 3.5 & 420 & 6.7 \\
\hline $11 / 1392$ & 845 & 0.0 & 321 & 124 & 112 & 0.0 & 0.0 & 0.0 & 0.0 & 0.0 & 0.0 & 00 & 0.0 & 0.0 & 00 & 0.0 & 00 & 00 & 0.0 & 0.0 & 0.0 \\
\hline $11 / 1302$ & $\infty 00$ & 0.0 & 324 & 8.0 & 11.6 & 0.0 & 0.0 & 0.0 & 0.0 & 0.0 & 0.0 & 0.0 & 0.0 & 0.0 & 00 & 0.0 & 0.0 & 0.0 & 0.0 & 00 & 0.0 \\
\hline $1 / 1392$ & 915 & 0.0 & 321 & 8.8 & 121 & 0.0 & 0.0 & 0.0 & 0.0 & 0.0 & 0.0 & 0.0 & 0.0 & 0.0 & 0.0 & 00 & 0.0 & 0.0 & 0.0 & 0.0 & 0.0 \\
\hline $1 / 1392$ & 900 & 0.0 & 320 & 9.1 & 11.3 & 0.0 & 0.0 & 0.0 & 0.0 & 00 & 0.0 & 0.0 & 0.0 & 00 & 0.0 & 0.0 & 00 & 0.0 & 0.0 & 0.0 & 0.0 \\
\hline $11 / 1392$ & 945 & 327 & 0.0 & 8.8 & 10.8 & 0.0 & 0.0 & 0.0 & 0.0 & 0.0 & 00 & 0.0 & 0.0 & 00 & 00 & 00 & 00 & 00 & 0.0 & 00 & 0.0 \\
\hline $11 / 13992$ & 1000 & $\mathbf{2 8 . 0}$ & 0.0 & 9.9 & 13.5 & 0.0 & 0.0 & 0.0 & 0.0 & 0.0 & 0.0 & 0.0 & 0.0 & 0.0 & 0.0 & 00 & 00 & $O D$ & 0.0 & 0.0 & 0.0 \\
\hline $1 / 1392$ & 1018 & 27.6 & 0.0 & 140 & 13.5 & 0.0 & 0.0 & 0.0 & 0.0 & 00 & 0.0 & 0.0 & 0.0 & 0.0 & 00 & 0.0 & 0.0 & 0.0 & 0.0 & 00 & 0.0 \\
\hline $11 / 13992$ & 1000 & 20.4 & 0.0 & 13.7 & 14.1 & 0.0 & 0.0 & 0.0 & 0.0 & 0.0 & 0.0 & 0.0 & 0.0 & 0.0 & 0.0 & 0.0 & 0.0 & 0.0 & 0.0 & 00 & 0.0 \\
\hline $11 / 13992$ & 1046 & 29.6 & 0.0 & 128 & 14.3 & $\mathbf{2 0 . 7}$ & 3.0 & 25.0 & 3.1 & 49.9 & 3.0 & 30.5 & 3.1 & 34.0 & 88 & 84.6 & 88 & 40.0 & 4.1 & 461 & 32 \\
\hline $11 / 1392$ & 1100 & 30.3 & 0.0 & 127 & 14.2 & 31.2 & 3.0 & 25.6 & 3.1 & 48.7 & 3.1 & 30.3 & 30 & 352 & 7.0 & 54.4 & 4,4 & 43.1 & 5.0 & 463 & 4.8 \\
\hline $11 / 1392$ & 1115 & 325 & 0.0 & 11.0 & 125 & 30.6 & 3.0 & 26.8 & 3.2 & 48.1 & 3.0 & 30.0 & 3.0 & 35.2 & 82 & 6.1 & 70 & 428 & 63 & 46.7 & 86 \\
\hline $11 / 1392$ & 1130 & 326 & 0.0 & 11.5 & 13.9 & 31.2 & 3.0 & 27.0 & 3.2 & $\varphi 0$ & 3.1 & 30.6 & 3.0 & 38.1 & 63 & 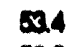 & 6.1 & 41.0 & 4.5 & 458 & 3.2 \\
\hline $11 / 13982$ & 1145 & 323 & 0.0 & 121 & 15.4 & 321 & 3.0 & 26.9 & 3.1 & 48.7 & 30 & 39.5 & 3.0 & 34.7 & 92 & 826 & 4.7 & 120 & 5.1 & 473 & 4.5 \\
\hline $11 / 13,92$ & 1200 & 327 & 0.0 & 11.4 & 11.5 & 31.7 & 3.0 & 27.3 & 3.2 & 48.0 & 3.1 & 38.8 & 3.0 & 30.1 & 10.1 & 51 & 8.6 & 412 & 5.6 & 458 & 5.3 \\
\hline $11 / 1392$ & 1215 & 326 & 0.0 & 10.5 & 120 & 30.0 & 3.0 & 28.3 & 3.3 & $\mathbf{4 6 . 6}$ & 30 & 372 & 3.0 & 31.7 & 10.6 & $\infty .7$ & $B B$ & 30.3 & 8.0 & 448 & 3.4 \\
\hline $11 / 1392$ & 1200 & 327 & 0.0 & 10.1 & 10.5 & 31.2 & 3.0 & 28.2 & 3.2 & 45.0 & 30 & 36.4 & 3.0 & 31.4 & 122 & 43 & 7.4 & 38.8 & 7.5 & 49 & 40 \\
\hline $11 / 1392$ & 1245 & 326 & 0.0 & 23.3 & 144 & 31.0 & 3.0 & 28.5 & 32 & 46.0 & 3.0 & 38.0 & 3.0 & 28.4 & 108 & 49.7 & 7.7 & 36.3 & 6.8 & 448 & 5.2 \\
\hline $11 / 1392$ & 1300 & 325 & 0.0 & 10.3 & 10.1 & 31.2 & 3.0 & $\mathbf{2 8 . 4}$ & 3.3 & 45.6 & 30 & 36.7 & 30 & 30.7 & 122 & 4.7 & 7.7 & 38.6 & 9.2 & 450 & 4.3 \\
\hline $11 / 1392$ & 1315 & 325 & 0.0 & 123 & 13.2 & 31.3 & 3.0 & 28.5 & 3.3 & 45.4 & 3.0 & 35.0 & 3.0 & 342 & 9.7 & 4.0 & 75 & $\omega .1$ & 11.0 & 45 & 6.3 \\
\hline $1 / 13992$ & 1330 & 326 & 0.0 & 0.1 & 18.8 & 30.8 & 3.0 & $\mathbf{2 B . 7}$ & 3.3 & 45.1 & 3.0 & 34.3 & 3.0 & 31.0 & 112 & 475 & 76 & 30.7 & 5.0 & 438 & 3.5 \\
\hline $1 / 1392$ & 1345 & 325 & 0.0 & 126 & 9.5 & 30.8 & 3.0 & 28.7 & 3.3 & $\mathbf{4 5 . 0}$ & 3.0 & 34.0 & 3.0 & 20.0 & 126 & 51.4 & 5.1 & $\$ 1.0$ & 6.1 & 42 & 9.7 \\
\hline $1 / 1392$ & 1400 & 326 & 0.0 & 9.4 & 83 & 30.9 & 3.0 & 28.6 & 3.3 & 44.7 & 30 & 33.7 & 3.0 & 204 & 14.6 & 46.6 & 11.6 & 33.7 & 8.8 & 47 & 5.1 \\
\hline $1 / 13902$ & 1415 & 326 & 0.0 & 6.9 & 90 & 30.6 & 3.0 & 28.9 & 3.3 & 44.4 & 3.0 & 33.4 & 3.0 & 20 & 250 & 51.0 & 13.6 & 39.9 & 72 & 437 & 5.9 \\
\hline $1 / 1392$ & 1430 & 327 & 0.0 & 10.1 & 9.5 & 31.0 & 3.0 & 28.9 & 3.3 & 43.8 & 3.0 & 324 & 3.0 & $\mathbf{3 0 . 0}$ & 10.8 & 51.4 & 78 & 40.0 & 78 & 439 & 3.6 \\
\hline $1 / 1392$ & 1445 & 326 & 0.0 & 8.6 & 11.1 & 30.6 & 3.0 & 28.9 & 3.3 & 44.0 & 3.0 & 327 & 3.0 & 27.5 & 160 & 512 & 70 & $\omega .1$ & 66 & 435 & 4.2 \\
\hline $1 / 1392$ & 1500 & 0.0 & 35.2 & 9.7 & 10.4 & $\mathbf{2 0 . 3}$ & 3.0 & 28.9 & 3.3 & 43.8 & 3.0 & 33.2 & 3.0 & 31.6 & 118 & 588 & 8.5 & $\$ 1.1$ & 5.0 & 433 & 4.6 \\
\hline $1 / 1392$ & 1515 & 0.0 & 38.3 & 10.3 & 90 & 29.1 & 3.0 & 28.7 & 3.3 & 43.8 & 30 & 33.0 & 3.0 & 31.4 & 127 & 50.0 & 39 & 4.5 & 46 & 434 & 5.6 \\
\hline $1 / 13992$ & 1530 & 0.0 & 38.7 & 9.2 & 9.5 & 30.3 & 3.0 & 27.9 & 3.3 & 43.7 & 30 & 33.0 & 30 & 324 & 121 & 58.4 & 3.0 & 45.0 & 3.6 & 43.3 & 3.5 \\
\hline $1 / 1392$ & 1545 & 0.0 & 38.8 & 9.0 & 88 & $\mathbf{2 9 . 7}$ & 3.0 & 27.6 & 32 & 43.8 & 30 & 33.3 & 3.0 & 348 & 85 & 882 & 32 & 4.6 & 3.5 & 428 & 112 \\
\hline $1 / 1392$ & $16 \infty 0$ & 0.1 & 39.3 & 10.6 & 10.4 & 28.9 & 3.0 & $\mathbf{2 8 . 0}$ & 3.3 & 43.4 & 30 & 327 & 3.0 & 34.7 & 7.7 & 58.1 & 4.1 & 4.3 & 4.6 & 424 & 3.3 \\
\hline $1 / 1392$ & 1615 & 0.1 & 39.6 & 10.1 & 10.7 & $\mathbf{2 0 . 2}$ & 3.0 & 27.5 & 3.2 & 44.1 & 3.0 & 33.4 & 3.0 & 257 & 11.5 & 627 & 82 & 37.8 & 3.5 & 421 & 10.5 \\
\hline $1 / 1392$ & 100 & 0.1 & 39.8 & 10.3 & 11.4 & 202 & 3.0 & $\mathbf{2 8 . 6}$ & 3.1 & 448 & 30 & 338 & 3.0 & 30.7 & 125 & $\mathbf{5 6}$ & 39 & 44.0 & 3.6 & .13 .3 & 5.0 \\
\hline $1 / 1392$ & 1646 & 0.1 & 40.0 & 10.4 & 11.9 & 20.3 & 3.0 & 25.0 & 3.1 & 45.5 & 3.0 & 342 & 3.0 & 31.7 & 11.7 & 50 & 6.1 & 44.5 & 4.6 & 4.1 & 6.1 \\
\hline $1 / 1392$ & 1700 & 0.1 & 39.7 & 10.8 & 13.7 & $\mathbf{2 0 . 5}$ & 3.0 & 25.8 & 3.2 & 46.8 & 30 & 34.6 & 3.0 & 325 & 88 & 503 & 44 & 44.0 & 3.6 & 45.1 & 5.5 \\
\hline $1 / 13992$ & 1716 & 0.1 & 39.1 & 11.6 & 121 & 29.1 & 3.0 & 25.6 & 3.1 & 46.0 & 3.0 & 35.0 & 3.0 & 313 & 99 & 60 & 4.7 & 43.6 & 3.3 & 45.0 & 3.4 \\
\hline $1 / 1392$ & 1730 & 0.1 & 39.1 & 129 & 127 & 29.2 & 3.0 & 25.5 & 3.1 & 46.0 & 3.1 & 35.2 & 3.0 & 320 & 98 & 84.0 & 62 & $\$ 0.0$ & 4.4 & 425 & 4.3 \\
\hline $1 / 13992$ & 1745 & 0.1 & 39.8 & 13.4 & 127 & $\mathbf{2 0 . 0}$ & 3.0 & 26.4 & 3.1 & 460 & 30 & 36.3 & 3.0 & 30.4 & 10.1 & 648 & 48 & 43.1 & 3.1 & 41.6 & 5.1 \\
\hline $1 / 1392$ & 1800 & 0.1 & 39.2 & 129 & 13.0 & 29.1 & 3.0 & 25.2 & 3.1 & 45.8 & 30 & 363 & 3.0 & 31.0 & 98 & $\mathbf{E 2}$ & 30 & 4.1 & 3.4 & 41.8 & 4.7 \\
\hline$/ 1392$ & 1816 & 0.1 & 39.0 & 121 & 13.0 & 29.2 & 3.0 & 25.8 & 3.1 & 45.3 & 30 & 348 & 3.0 & 320 & 60 & 50 & 6.7 & 430 & 42 & 45.0 & 11.0 \\
\hline /1392 & 1850 & 0.1 & 38.0 & 127 & 14.2 & 29.3 & 3.0 & 25.5 & 3.1 & 45.5 & 30 & 35.0 & 3.0 & 300 & 0.5 & 64.6 & 53 & 43.1 & 3.4 & 41.6 & 13.3 \\
\hline & 1845 & 0.1 & 37.5 & 128 & 13.3 & $\mathbf{2 9 . 0}$ & 3.0 & 25.2 & 3.1 & 45.8 & 30 & 35.2 & 3.0 & 328 & 10.7 & 56 & 44 & 4.6 & 5.3 & 40.0 & 4.3 \\
\hline 113 & 1900 & 0.1 & 37.7 & 14.5 & 129 & 29.6 & 3.0 & 25.3 & 3.1 & 46.1 & 30 & 34.9 & 3.0 & 37.9 & 7.1 & 62 & 42 & 43.6 & 3.4 & $\mathbf{4 0 . 1}$ & 5.6 \\
\hline
\end{tabular}


Das Time $\begin{gathered}\text { Water Lim } \\ \text { Flow Flow } \\ \text { som opm } \\ \text { opm } \\ \%\end{gathered}$ D 1

Das Time 0.13

13.4150
$\mathrm{KW} \mathrm{mn}$ D2 D 3 04 $C$

$11 / 13021000$ 0.138 .0

$11 / 1392 \quad 1046$ $0.1 \quad 33.7$

$\begin{array}{ll}13.4 & 16.0 \\ 13.1 & 14.1\end{array}$ 13.314 .6

0.135 .6

$144 \quad 16.7$

$\begin{array}{llll}0.1 & 36.3 & 128 & 14.4\end{array}$

$\begin{array}{llll}0.1 & 34.5 & 14.0 & 15.0\end{array}$

$11 / 1302$ 2000

$11 / 1302 \quad 2045$

$\begin{array}{llll}28.6 & 00 & 13.8 & 140\end{array}$

$\begin{array}{llll}321 & 0.0 & 13.1 & 120\end{array}$

31.800

$134 \quad 13.8$

$11 / 1302 \quad 2116$

$11 / 1302 \quad 2130$

$11 / 1302 \quad 2145$

$11 / 13022200$

$11 / 1392 \quad 2216$

$11 / 1302 \quad 200$

$11 / 1302 \quad 2245$

$11 / 13022000$

$11 / 1302 \quad 2016$

$11 / 1302 \quad 200$

$11 / 1302 \quad 2345$

$11 / 1492 \quad 0$

$\begin{array}{lllll}0.1 & 30.6 & 14.6 & 15.3\end{array}$

$\begin{array}{llll}0.0 & 33.0 & 123 & 11.6\end{array}$

$\begin{array}{llll}0.0 & 37.6 & 11.6 & 10.8\end{array}$

$\begin{array}{llll}0.0 & 38.6 & 13.6 & 120\end{array}$

$\begin{array}{llll}0.0 & 36.2 & 13.0 & 14.4\end{array}$

$\begin{array}{llll}0.0 & 35.6 & 120 & 140\end{array}$

$\begin{array}{llll}0.0 & 35.2 & 126 & 11.0\end{array}$

$\begin{array}{llll}0.1 & 34.6 & 128 & 11.6\end{array}$

$\begin{array}{lllll}0.0 & 34.0 & 11.3 & 11.2\end{array}$

$\begin{array}{lllll}0.0 & 34.4 & 123 & 124\end{array}$

$\begin{array}{lllll}0.0 & 34.6 & 11.4 & 11.6\end{array}$

$11 / 1402 \quad 15$

$11 / 140230$

$11 / 1402 \quad 45$

$11 / 1492 \quad 100$

$11 / 1492 \quad 116$

$11 / 1402 \quad 130$

$11 / 1492 \quad 145$

$11 / 1402200$

$11 / 1492 \quad 215$

$1 / 1492230$

$11 / 1492 \quad 245$

$11 / 1492300$

$\begin{array}{llll}0.0 & 35.6 & 120 & 13.6\end{array}$

$\begin{array}{llll}0.0 & 36.7 & 11.2 & 13.6\end{array}$

$\begin{array}{llll}0.0 & 29.2 & 11.6 & 17.6\end{array}$

$\begin{array}{llll}0.0 & 38.3 & 10.0 & 13.2\end{array}$

$\begin{array}{lllll}0.0 & 35.5 & 9.9 & 13.3\end{array}$

$\begin{array}{lllll}0.0 & 35.4 & 9.7 & 15.2\end{array}$

$\begin{array}{llll}0.0 & 37.3 & 16.4 & 127\end{array}$

$\begin{array}{llll}0.0 & 38.7 & 11.8 & 121\end{array}$

$\begin{array}{lllll}0.0 & 33.0 & 9.7 & 128\end{array}$

$0.0 \quad 35.4$

$\begin{array}{rr}9.7 & 128 \\ 11.6 & 11.7\end{array}$

$0.0 \quad 34.2$

$0.7 \quad 13.4$

$8.8 \quad 124$

$\begin{array}{ll}0.0 & 38.1 \\ 0.0 & 36.2\end{array}$

8.511 .6

$11 / 1492350$

$11 / 1492 \quad 345$

$11 / 1492 \quad 400$

$11 / 1492 \quad 415$

$11 / 1492 \quad 400$

$11 / 1492 \quad 446$

$11 / 1492 \quad 500$

$11 / 1492 \quad 515$

$11 / 1492 \quad 500$

$11 / 1402 \quad 645$

$11 / 1492 \quad 600$

$11 / 1492 \quad 615$

$11 / 1492600$

$11 / 1492 \quad 646$

$11 / 1492700$

$11 / 1402716$

$11 / 1402750$

$11 / 1402 \quad 746$

$11 / 1402 \quad 800$

$11 / 1402 \quad 815$

$11 / 1492 \quad 800$

$11 / 1492 \quad 845$

$11 / 1492 \quad 200$

$11 / 1492 \quad 915$

$11 / 1402 \quad 000$

$11 / 1492$ 245

$+1 / 1492 \quad 1000$

$1 / 1492 \quad 1015$

$11 / 14921060$

$11 / 1492 \quad 1045$

$11 / 1492 \quad 1100$

$11 / 1492 \quad 1115$ $\begin{array}{llll}0.0 & 33.6 & 0.0 & 10.6\end{array}$

$\begin{array}{llll}0.0 & 33.6 & 0.4 & 11.1\end{array}$

$\begin{array}{llll}0.0 & 38.9 & 8.7 & 121\end{array}$

$0.0 \quad 35.7$

$0.0 \quad 35.3$

9.013 .6

$\begin{array}{lllll}0.0 & 36.1 & 8.7 & 13.4\end{array}$

$\begin{array}{lllll}0.0 & 36.7 & 10.4 & 14.0\end{array}$

$\begin{array}{llll}0.0 & 37.4 & 8.2 & 13.3\end{array}$

$\begin{array}{lllll}0.0 & 33.0 & 8.8 & 127\end{array}$

$\begin{array}{llll}0.0 & 33.8 & 8.1 & 11.1\end{array}$

$\begin{array}{lllll}0.0 & 33.4 & 8.6 & 12.7\end{array}$

$\begin{array}{llll}0.0 & 33.8 & 10.2 & 15.6\end{array}$

$\begin{array}{llll}0.0 & 34.0 & 11.0 & 15.6\end{array}$

$\begin{array}{llll}0.0 & 30.9 & 11.3 & 17.4\end{array}$

$\begin{array}{lllll}0.0 & 321 & 0.7 & 14.1\end{array}$

$\begin{array}{llll}0.0 & 30.2 & 0.9 & 14.7\end{array}$

$\begin{array}{llll}0.0 & 34.6 & 10.3 & 15.5\end{array}$

$\begin{array}{llll}0.0 & 30.1 & 0.9 & 19.2\end{array}$

$\begin{array}{llll}0.0 & 31.8 & 10.4 & 19.0\end{array}$

$\begin{array}{llll}0.0 & 29.7 & 15.2 & 34.3\end{array}$

$\begin{array}{llll}0.0 & 30.8 & 17.4 & 31.3\end{array}$

$\begin{array}{lllll}0.0 & 31.6 & 17.8 & 33.3\end{array}$

$0.0 \quad 30.8$

$0.0 \quad 33.3$

$\begin{array}{ll}16.6 & 31.7\end{array}$

$0.0 \quad 30.7$

13.023 .1

$\begin{array}{llll}0.0 & 29.1 & 17.8 & 26.1\end{array}$

$\begin{array}{llll}30.6 & 0.0 & 14.3 & 20.9\end{array}$

$\begin{array}{llll}34.6 & 0.0 & 12.2 & 13.4\end{array}$

$\begin{array}{llll}34.3 & 0.0 & 122 & 11.7\end{array}$

$\begin{array}{llll}35.7 & 0.0 & 17.0 & 11.1\end{array}$

$\begin{array}{llll}35.4 & 0.0 & 15.9 & 11.2\end{array}$ $\begin{array}{llll}0.0 & 29.8 & 20.0 & 34.4\end{array}$ $\begin{array}{llll}28.8 & 3.0 & 25.0 & 3.1 \\ 20.0 & 30 & 20.2 & 3.1\end{array}$

$\begin{array}{llll}290 & 3.0 & 24.7 & 30\end{array}$

28.83 .0

$28.8 \quad 3.0$

$29.3 \quad 30$

28.03 .0

31.430

31.24 .1

20.240

3263.0

3243.0

31.13 .0

$31.8 \quad 32$

$30.8 \quad 3.0$

$31.3 \quad 3.0$

$31.0 \quad 3.0$

$320 \quad 3.1$

$31.7 \quad 3.1$

$320 \quad 3.0$

31.63 .0

2213.1

30.23 .6

3243.2

31.53 .0

$31.6 \quad 3.0$

3263.0

3233.0

3263.1

$31.8 \quad 3.0$

$31.8 \quad 3.0$

$33.3 \quad 3.0$

$31.8 \quad 3.0$

3273.0

$323 \quad 3.0$

$320 \quad 3.8$

3253.1

$325 \quad 3.3$

$31.9 \quad 3.0$

$32.8 \quad 3.0$

$33.4 \quad 4.0$

$320 \quad 3.0$

$33.1 \quad 3.0$

32.13 .0

$31.8 \quad 3.0$

$31.3 \quad 3.1$

30.93 .0

$31.3 \quad 3.0$

31.23 .0

$31.0 \quad 3.0$

30.63 .1

$29.5 \quad 3.0$

$28.0 \quad 3.2$

26.04 .1

$27.1 \quad 3.0$

28.43 .0

$28.8 \quad 3.2$

28.35 .3

$27.8 \quad 4.4$

$28.6 \quad 3.2$

$20.0 \quad 3.0$

3245.1

$30.4 \quad 3.2$

$33.7 \quad 3.0$

34.13 .0
24.83 .0

$246 \quad 31$

2473.1

2483.0

24.23 .0

24330

2033.1

$\begin{array}{rr}\mathbf{2 0} 2 & 3.1 \\ \mathbf{2 0} 3 & 3.1\end{array}$

28.23 .1

24.63 .0

24.03 .1

$24.8 \quad 3.1$

20.13 .1

20.23 .1

25.03 .1

25.03 .1

20.03 .1

24.93 .1

25.33 .1

$\begin{array}{ll}24.9 & 3.1\end{array}$

$24.8 \quad 3.1$

24.63 .1

24.83 .1

24.93 .1

24.73 .1

20.63 .1

24.93 .1

24.43 .1

24.93 .1

20.33 .1

20.23 .1

$24.8 \quad 3.1$

24.43 .0

$24.4 \quad 3.1$

24.33 .0

24.43 .1

24.23 .0

24.23 .1

$24.6 \quad 3.1$

24.53 .1

24.23 .1

24.13 .1

$24.0 \quad 3.0$

$24.3 \quad 3.1$

$24.0 \quad 3.1$

20.93 .0

$23.7 \quad 3.1$

$23.6 \quad 3.0$

23.63 .0

20.63 .0

20.53 .1

23.73 .1

$23.6 \quad 3.0$

\subsection{3 .0}

23.83 .1

20.73 .1

20.93 .1

24.93 .1

$\begin{array}{ll}24.7 & 3.1 \\ 24.5 & 3.0\end{array}$

24.73 .1
$24.5 \quad 3.0$

KW mn

$\begin{array}{llll}46.7 & 30 & 302 & 3.0\end{array}$

$\begin{array}{llll}46.6 & 30 & 30.1 & 3.0\end{array}$

$\begin{array}{llll}48.8 & 3.0 & 36.0 & 3.0\end{array}$

$\begin{array}{llll}464 & 30 & 35.6 & 3.0\end{array}$

$47.3 \quad 3.1$

$38.3 \quad 3.0$

$40.630 \quad 35.9 \quad 3.0$

$\begin{array}{lllll}46.8 & 3.1 & 35.8 & 3.0\end{array}$

$\begin{array}{llll}47.4 & 3.0 & 32.5 & 3.0\end{array}$

$\begin{array}{llll}49.3 & 3.1 & 37.7 & 3.0\end{array}$

$\begin{array}{llll}48.6 & 3.0 & 37.3 & 3.0\end{array}$

46.63 .1

4.730 


\begin{tabular}{|c|c|c|c|c|c|c|c|c|c|c|c|c|c|c|c|c|c|c|c|c|c|}
\hline Dat & Time & $\begin{array}{l}\text { Water } \\
\text { Flow } \\
\text { ofem }\end{array}$ & $\begin{array}{l}\operatorname{Lim}_{\text {Flow }} \\
\text { ogm }\end{array}$ & $\begin{array}{l}S \\
\% \\
\%\end{array}$ & $\begin{array}{r}8 \\
9 \\
\times\end{array}$ & & $m n$ & & & $k W$ & & KW & $m n$ & $\mathbf{n W}$ & $\mathrm{mn}$ & KW & $\mathrm{mn}$ & $K W$ & & $k w$ & $\mathrm{mn}$ \\
\hline 11402 & 1130 & 360 & 00 & 142 & 108 & 300 & 3.0 & 247 & 3.1 & 4.5 & 30 & 30.0 & 3.0 & 3.18 & 6.1 & 890 & 8.0 & 431 & 43 & 407 & 43 \\
\hline $\begin{array}{l}11 / 1402 \\
11 / 1402\end{array}$ & $\begin{array}{l}1146 \\
1200\end{array}$ & $\begin{array}{l}336 \\
340\end{array}$ & $\begin{array}{l}0.0 \\
0.0\end{array}$ & $\begin{array}{l}13.8 \\
15.0\end{array}$ & $\begin{array}{l}10.3 \\
120\end{array}$ & $\begin{array}{l}302 \\
321\end{array}$ & $\begin{array}{l}3.6 \\
3.0\end{array}$ & $\begin{array}{l}24.6 \\
24.8\end{array}$ & $\begin{array}{l}3.1 \\
3.1\end{array}$ & $\begin{array}{l}48.7 \\
43\end{array}$ & $\begin{array}{l}3.1 \\
30\end{array}$ & $\begin{array}{l}\mathbf{3 . 3} \\
\mathbf{3 0 . 3}\end{array}$ & $\begin{array}{l}30 \\
30\end{array}$ & $\begin{array}{l}37.3 \\
31.7\end{array}$ & $\begin{array}{l}11.8 \\
10.6\end{array}$ & $\begin{array}{l}2 \times 6 \\
6.4\end{array}$ & $\begin{array}{l}62 \\
0.1\end{array}$ & $\begin{array}{r}423 \\
400\end{array}$ & $\begin{array}{l}14 \\
6.3\end{array}$ & $\begin{array}{l}48 C \\
426\end{array}$ & $\begin{array}{l}60 \\
37\end{array}$ \\
\hline $\begin{array}{l}11 / 14 \theta 2 \\
11 / 14 \theta 2\end{array}$ & $\begin{array}{l}1200 \\
1216\end{array}$ & $\begin{array}{l}340 \\
305\end{array}$ & $\begin{array}{l}0.0 \\
0.0\end{array}$ & 18.2 & 10.8 & 322 & 3.0 & 240 & 3.1 & 40.0 & 30 & $\mathbf{3 0 . 0}$ & 30 & $\mathbf{3 0}$ & 11.3 & $\mathbf{0 0 s}$ & 78 & 308 & 8.8 & 385 & 33 \\
\hline $11 / 1492$ & 1200 & 324 & 00 & 146 & 106 & 310 & 3.0 & 24.6 & 3.0 & 46 & 30 & 30.8 & 3.0 & 32 & 03 & as & 8.7 & $\mathbf{0 . 7}$ & 10 & 118 & 87 \\
\hline $11 / 1402$ & 1246 & 410 & 00 & 120 & 123 & 31.6 & 30 & 24.8 & 3.1 & 44 & 30 & $34 !$ & 30 & 34.1 & 90 & 4.3 & 7.1 & 41.8 & 30 & 430 & 80 \\
\hline $11 / 1402$ & 1300 & 0.0 & 36.3 & 140 & 121 & 314 & 30 & 247 & 3.1 & 46.3 & 30 & 348 & 30 & 378 & as & 82 & 50 & 4.1 & 44 & 427 & 51 \\
\hline $11 / 1402$ & 1316 & 0.0 & 37.0 & 15.4 & 10.5 & 310 & 30 & 34 & 3.1 & 4.1 & 30 & 30.1 & 3.0 & $3 \mathbf{3 0}$ & 0.7 & 84.6 & 59 & 422 & 3.8 & 110 & 66 \\
\hline $11 / 1402$ & 1300 & 00 & 100 & 9.3 & 10.5 & 31.7 & 3.0 & 284 & 3.1 & 40.8 & 30 & 28 & 3.0 & 32.7 & 03 & $\mathbf{2 0 2}$ & 6.1 & $\mathbf{4 3 3}$ & 38 & 118 & 38 \\
\hline $11 / 1402$ & 1345 & 00 & 39.3 & 80 & 128 & 310 & 30 & 248 & 3.1 & 4.6 & 30 & 32 & 3.0 & $\mathbf{3 3 . 3}$ & 88 & $\mathbf{8 . 8}$ & 82 & 4.1 & 4.1 & 124 & 47 \\
\hline $11 / 1402$ & 1400 & 00 & 37.0 & 72 & 10.7 & 31.0 & 3.0 & 216 & 3.1 & 48 & 30 & 302 & 30 & $\mathbf{3 7 . 0}$ & 78 & 63 & $\mathbf{4 8}$ & 40 & 33 & 427 & 44 \\
\hline $11 / 1402$ & 1416 & 0.0 & 304 & 7.3 & 10.4 & 321 & 30 & 204 & 3.2 & 48.6 & 30 & 320 & 30 & 228 & 0.1 & 80 & 82 & 40 & 41 & 110 & 133 \\
\hline $11 / 1402$ & 1430 & 0.0 & 38.4 & 8.8 & 10.8 & 324 & 3.0 & 248 & 3.1 & $\mathbf{4 4}$ & 30 & 20 & 3.0 & 344 & 10.4 & $8 \times 8$ & 127 & 412 & 90 & $\mathbf{4 3}$ & 94 \\
\hline $11 / 1402$ & 1446 & 0.0 & 37.0 & 82 & 11.2 & 30.1 & 3.0 & 244 & 30 & 40 & 30 & $\mathbf{3 0 . 1}$ & 3.0 & 370 & 10.1 & $\mathbf{2}$ & 78 & 493 & 31 & 4.8 & 44 \\
\hline $11 / 1402$ & 1600 & 0.0 & 37.7 & 7.3 & 11.2 & 30.2 & 30 & 24.4 & 3.0 & 46.4 & 30 & 33.6 & 3.0 & 328 & 74 & 20 & 6.7 & 408 & 34 & 48 & 47 \\
\hline $11 / 1402$ & 1818 & 0.0 & 38.7 & 7.0 & 100 & 320 & 30 & 200 & 31 & 4.3 & 30 & 326 & 30 & 37.1 & 10.8 & 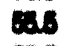 & 7.1 & 405 & 3.1 & 408 & 38 \\
\hline $11 / 1402$ & 1500 & 0.0 & 41.7 & 74 & 10.0 & 33.3 & 30 & 24.7 & 3.1 & 4.3 & 30 & 323 & 3.0 & 57.3 & 78 & a.8 & 72 & 40.8 & 33 & 422 & 82 \\
\hline $11 / 1402$ & 1546 & 00 & 41.7 & 7.4 & 10.9 & 33.3 & 3.0 & 247 & 31 & 4.3 & 30 & 323 & 3.0 & 373 & 78 & 48 & 72 & 436 & 3.3 & 422 & 5.2 \\
\hline $11 / 1402$ & 1600 & 0.0 & 38.8 & 01 & 13.7 & 31.3 & 3.6 & 24.8 & 3.0 & 46.7 & 3.0 & 332 & 30 & $\$ 0.4$ & 4.7 & 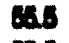 & 62 & 43.5 & 36 & 41 & 74 \\
\hline $1 / 1402$ & 1618 & 00 & 38.8 & 9.1 & 13.7 & 31.3 & 3.6 & 24.6 & 3.0 & $\mathbf{8 . 7}$ & 30 & 332 & 30 & 91 & 4.7 & 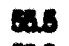 & 62 & 436 & 36 & 41 & 74 \\
\hline $1 / 1 / 492$ & 1600 & 00 & 0.0 & 0.0 & 14.9 & 306 & 30 & 243 & 3.1 & 47.6 & 3.1 & 34.8 & 30 & $3 / 0$ & 80 & 00 & 7.1 & 423 & 43 & 481 & 54 \\
\hline $1 / 1402$ & 1645 & 0.0 & 38.2 & 8.0 & 14.2 & 30.5 & 3.0 & 24.3 & 3.0 & 47.1 & 3.1 & 34.7 & 3.0 & 302 & 09 & 200 & 6.7 & 41.3 & 10 & $\Delta B 1$ & 6.2 \\
\hline $1 / 1402$ & 1700 & 00 & 36.6 & 10.3 & 15.2 & 300 & 30 & 24.2 & 3.1 & 47.6 & 3.1 & 381 & 3.0 & 358 & 20.0 & 8.1 & 78 & 42 & 4.7 & 40 & 31 \\
\hline $1 / 1402$ & 1715 & 0.0 & 35.3 & 13.8 & 16.0 & 20.8 & 32 & 24.2 & 3.0 & 47.7 & 3.1 & 38.1 & 30 & 328 & 78 & 812 & 68 & 428 & 8.0 & 41 & 42 \\
\hline $1 / 1402$ & 1750 & 0.0 & 36.4 & 15.3 & 14.1 & 310 & 3.0 & 24.4 & 3.1 & 46.6 & 3.1 & 320 & 20 & 330 & 7.7 & $\mathbf{8 . 0}$ & 48 & 4.1 & 48 & 456 & 48 \\
\hline $1 / 1492$ & 1746 & 0.0 & 38.0 & 120 & 13.8 & 31.0 & 3.1 & 24.3 & 3.0 & 41 & 30 & 33.6 & 20 & 221 & 73 & $\mathbf{8 3}$ & 88 & 44 & 34 & 463 & 76 \\
\hline $1 / 1402$ & 1800 & 0.0 & 36.8 & 16.3 & 164 & 30.5 & 3.0 & 241 & 3.1 & 473 & 3.1 & 348 & 30 & 328 & 0.7 & 842 & 08 & 438 & 83 & 46.4 & 8.8 \\
\hline $1 / 1492$ & 1816 & 0.0 & 35.1 & 13.7 & 16.2 & 30.7 & 3.0 & 240 & 3.0 & 47.8 & 30 & 34.0 & 20 & 3.7 & 88 & 80 & 82 & 480 & 3.7 & $\mathbf{4 8 . 0}$ & 6.8 \\
\hline $1 / 1492$ & 1800 & 0.0 & 34.6 & 15.2 & 14.0 & 30.6 & 30 & 241 & 3.0 & 47.4 & 30 & 34.0 & 30 & 3.1 & 88 & 818 & 4.7 & 43.6 & 38 & $\mathbf{4 8 4}$ & 70 \\
\hline $1 / 1402$ & 1845 & 0.0 & 346 & 14.7 & 16.8 & 30.7 & 3.0 & 240 & 3.0 & 47.6 & 30 & 32.1 & 10 & 31 & 7.1 & as & 72 & 424 & 44 & 40 & 46 \\
\hline $1 / 1402$ & 1900 & 0.1 & 347 & 148 & 14.3 & 312 & 30 & 240 & 3.0 & 47.0 & 3.1 & 353 & 30 & 5.6 & 64 & $2 \times 5$ & 4.4 & 40.8 & 86 & 409 & 40 \\
\hline $1 / 1492$ & 1816 & 0.0 & 30.8 & 14.8 & 142 & 31.1 & 3.2 & 24.2 & 3.0 & 48.0 & 3.1 & 38.5 & 34 & 30.6 & 8.1 & 84.7 & 4.7 & 4.4 & 3.1 & 438 & 90 \\
\hline $1 / 1492$ & 1000 & 0.1 & 34.3 & 14.8 & 14.6 & 30.0 & 3.0 & 240 & 3.0 & 47.8 & 30 & 35.3 & 30 & 33.0 & 72 & 842 & 70 & 403 & 3.0 & 41 & 71 \\
\hline $1 / 1402$ & 1846 & 00 & 347 & 14.2 & 13.2 & 31.3 & 3.4 & 24.0 & 3.1 & 47.8 & 3.1 & 3.1 & 30 & 372 & 136 & 84 & 10.0 & 438 & 4.8 & 128 & 70 \\
\hline 11492 & 2000 & 0.0 & 34.3 & 14.2 & 13.0 & 31.1 & 3.6 & 210 & 3.0 & 472 & 30 & 34.7 & 3.0 & 38 & 68 & 21 & 18 & 40 & 3.4 & 45.6 & 00 \\
\hline $1 / 1402$ & 2015 & 0.0 & 30.2 & 14.9 & 13.2 & 30.3 & 3.7 & 24.3 & 3.1 & 40.2 & 3.1 & 340 & 3.0 & 30 & 62 & 21 & 68 & 488 & 34 & 430 & 42 \\
\hline $1 / 1492$ & 2000 & 0.0 & 34.5 & 13.4 & 122 & 31.2 & 3.0 & 24.3 & 30 & 488 & 30 & 30.8 & 3.0 & $\mathbf{3 0 3}$ & 83 & 48 & 68 & 4.1 & 3.3 & 48 & 8.3 \\
\hline $1 / 1492$ & 2045 & 0.1 & 35.5 & 14.8 & 15.6 & 31.7 & 3.0 & 240 & 3.1 & 46.2 & 30 & 30.0 & 3.0 & 3.7 & 7.7 & 648 & 68 & 4.1 & 3.0 & 450 & 5.8 \\
\hline $1 / 1492$ & 2100 & 0.1 & 30.5 & 16.9 & 15.9 & 30.8 & 3.7 & 20.9 & 3.0 & 46.9 & 30 & 34.8 & 30 & 380 & 68 & 84.0 & 48 & 486 & 32 & 450 & 4.8 \\
\hline $1 / 492$ & 2115 & 0.1 & 35.2 & 14.8 & 15.0 & 31.8 & 3.0 & 24.0 & 3.1 & 47.7 & 3.1 & 35.1 & 3.0 & 37.9 & 82 & 84.8 & 73 & 42.6 & 37 & $\omega 0$ & 42 \\
\hline $1 / 492$ & 2130 & 0.0 & 340 & 14.6 & 14.3 & 31.1 & 3.0 & 240 & 3.0 & 47.8 & 3.0 & 34.0 & 30 & 373 & 60 & 8.7 & 88 & 4.1 & 34 & 466 & 6.3 \\
\hline 11492 & 2145 & 0.0 & 33.6 & 14.6 & 15.6 & 31.1 & 3.1 & 20.0 & 3.1 & 47.6 & 3.1 & 35.2 & 3.0 & 402 & 88 & 84.7 & 52 & 4.0 & 37 & 458 & 73 \\
\hline 11492 & 2000 & 0.0 & 30.5 & 16.0 & 13.0 & 31.3 & 3.4 & 20.0 & 3.0 & 47.3 & 3.0 & 320 & 3.0 & 3.0 & 7.1 & 8.1 & 60 & 4.1 & 4.3 & 44 & 40 \\
\hline 11402 & 2215 & 0.0 & 320 & 14.3 & 144 & 31.1 & 3.8 & 20.0 & 3.0 & 472 & 3.1 & 34.0 & 30 & 403 & 72 & 8.8 & 68 & 40.0 & 3.4 & 42 & 5.1 \\
\hline 11492 & 2000 & 0.1 & 323 & 14.8 & 16.3 & 30.8 & 6.4 & $\mathbf{2 0 . 0}$ & 3.1 & $\$ 7.6$ & 3.1 & 3.1 & 30 & 37.0 & 62 & 820 & 8. & 420 & 10 & 44 & 4.1 \\
\hline 1402 & 246 & 0.1 & 34.0 & 13.0 & 14.6 & 313 & 3.1 & 24.2 & 3.0 & 48.6 & 30 & 33.1 & 30 & 33.0 & 60 & 848 & 80 & 40.4 & 32 & 45 & 8.0 \\
\hline 1492 & 2000 & 0.1 & 34.2 & 16.6 & 14.4 & 30.0 & 3.1 & 24.2 & 3.1 & 48.4 & 30 & 30.1 & 30 & 528 & 98 & 64.0 & 7.1 & 426 & 34 & 40 & 8.7 \\
\hline 11402 & 2015 & 0.1 & 342 & 13.4 & 21.7 & 31.6 & 3.7 & 24.2 & 3.1 & 49.7 & 3.1 & 35.8 & 3.0 & 30.3 & 89 & 823 & $\mathbf{B . 7}$ & 24 & 3.3 & 457 & 92 \\
\hline 1402 & 2000 & 0.1 & 35.0 & 123 & 19.1 & 31.8 & 8.3 & 24.1 & 3.1 & 9.6 & 3.1 & 38.3 & 3.0 & $\mathbf{5 8 . 0}$ & 60 & 628 & 8.4 & 426 & 41 & 460 & 6.1 \\
\hline 11402 & 2345 & 0.0 & 35.7 & 9.6 & 14.7 & 33.0 & 3.0 & 24.3 & 3.1 & 51.0 & 3.1 & 36.4 & 3.0 & 39.4 & 69 & 513 & 83 & 418 & 40 & 454 & 4.6 \\
\hline 11502 & 0 & 0.0 & 35.3 & 0.3 & 14.8 & 328 & 3.3 & 24.3 & 3.1 & 498 & 3.1 & 30.7 & 3.0 & 396 & 64 & 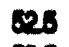 & 83 & 426 & 3.4 & 46 & 52 \\
\hline 11502 & 16 & 0.0 & 36.0 & 9.2 & 14.0 & 326 & 4.7 & 242 & 3.0 & 48.8 & 3.1 & 34.0 & 3.0 & 300 & 68 & 006 & 48 & 43.4 & 3.1 & 40 & 5.6 \\
\hline 11502 & 30 & 0.0 & 36.0 & 7.6 & 13.1 & 328 & 3.5 & 24.3 & 3.1 & 49.1 & 3.1 & 34.3 & 3.0 & 302 & 40 & 80 & 54 & 428 & 32 & 452 & 6.0 \\
\hline on & 4 & 0.0 & 36.2 & 7.8 & 13.4 & 328 & 3.0 & 24.2 & 3.0 & 48.0 & 3.1 & 343 & 3.0 & 33. & 78 & 026 & 5.1 & 433 & 3.2 & 420 & 3.1 \\
\hline 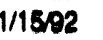 & 100 & 0.0 & 34.3 & 72 & 126 & 30.3 & 3.0 & 243 & 3.1 & 48.5 & 3.1 & 34.5 & 3.0 & 308 & 73 & 805 & 4.1 & 420 & 3.2 & 470 & 5.4 \\
\hline The & 116 & 0.0 & 38.6 & 8.4 & 14.0 & 326 & 43 & 24.4 & 3.1 & 47.4 & 3.1 & $3 a 3$ & 3.0 & 0.6 & 68 & 82.4 & 40 & 4.7 & 3.1 & 462 & 3.0 \\
\hline 11508 & 130 & 0.0 & 38.7 & 0.6 & 125 & 33.6 & 3.0 & 24.4 & 3.0 & 168 & 3.1 & 308 & 20 & 0.1 & 58 & 822 & 88 & 420 & 3.1 & 40 & 3.6 \\
\hline 11502 & 145 & 0.0 & 38.3 & 8.6 & 13.7 & 33.2 & 3.0 & 24.3 & 3.1 & 47.1 & 3.1 & 33.5 & 30 & $\boldsymbol{\omega 0 . 4}$ & 88 & 208 & 4.7 & 424 & 3.1 & 45.1 & 4.6 \\
\hline $1 / 1602$ & 200 & 0.0 & 33.0 & 7.3 & 128 & 321 & 4.3 & 24.8 & 3.0 & $\mathbf{4 6 . 6}$ & 30 & 30.0 & 30 & 372 & 88 & 8.1 & 40 & 406 & 4.0 & 46.1 & 3.7 \\
\hline $1 / 1502$ & 215 & 0.0 & 38.1 & 8.2 & 13.0 & 34.3 & 3.0 & 24.3 & 3.1 & 46.14 & 3.1 & 324 & 3.0 & 324 & 6.7 & 81.5 & 6.1 & 41.1 & 4.0 & 40 & 4.4 \\
\hline $1 / 1502$ & 200 & 0.0 & 38.6 & 7.6 & 127 & 321 & 3.8 & 24.1 & 3.0 & 46.7 & 3.1 & 32.1 & 30 & 50.0 & 73 & $\mathbf{6 x} 7$ & 63 & 4.7 & 3.4 & 41.1 & 3.6 \\
\hline $1 / 15 / 92$ & 246 & 0.0 & 36.8 & 8.5 & 13.6 & 33.8 & 3.1 & 24.2 & 3.1 & 48.6 & 3.1 & 328 & 3.0 & 39.6 & 03 & 628 & 59 & 48.1 & 3.6 & 40.4 & 6.0 \\
\hline $1 / 1592$ & 300 & 0.0 & 37.3 & 7.1 & 128 & 320 & 3.0 & 24.3 & 3.0 & 48.4 & 3.1 & 326 & 30 & 321 & 90 & 827 & 83 & 424 & 32 & 426 & 4.0 \\
\hline 1150 & 315 & 0.0 & 38.6 & 8.2 & 14.5 & 31.8 & 3.0 & 242 & 3.1 & 48.8 & 3.1 & 308 & 3.0 & 373 & 164 & $\mathbf{\infty 0 8}$ & 11.0 & $\$ 0.6$ & 0.1 & 427 & 8.7 \\
\hline$/ 1502$ & 300 & 0.0 & 36.5 & 8.0 & 14.9 & 34.0 & 3.0 & 24.3 & 3.0 & 468 & 30 & 328 & 3.0 & 4.6 & 68 & 826 & 74 & 423 & 3.3 & 43.2 & 68 \\
\hline
\end{tabular}




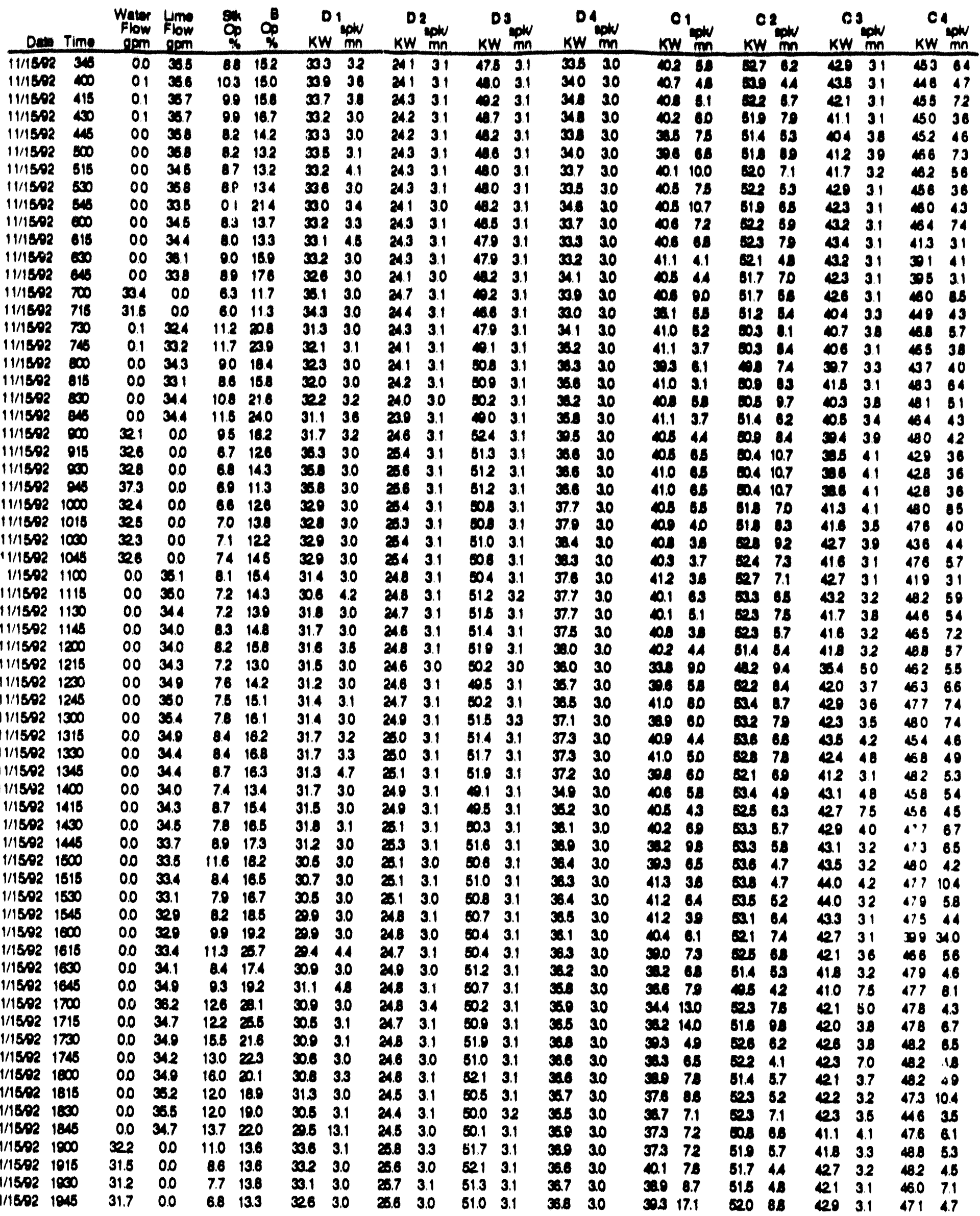




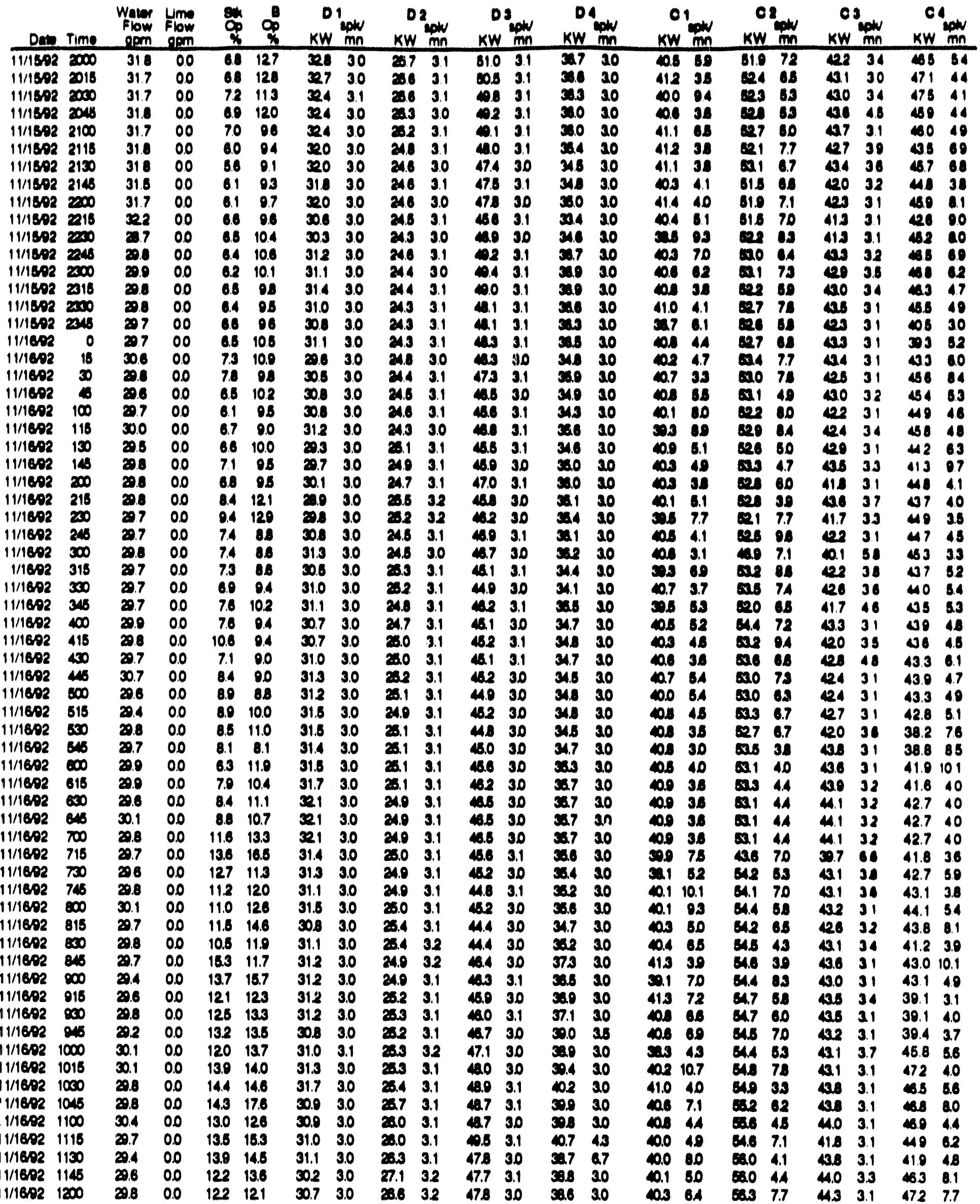


leane 20:4000

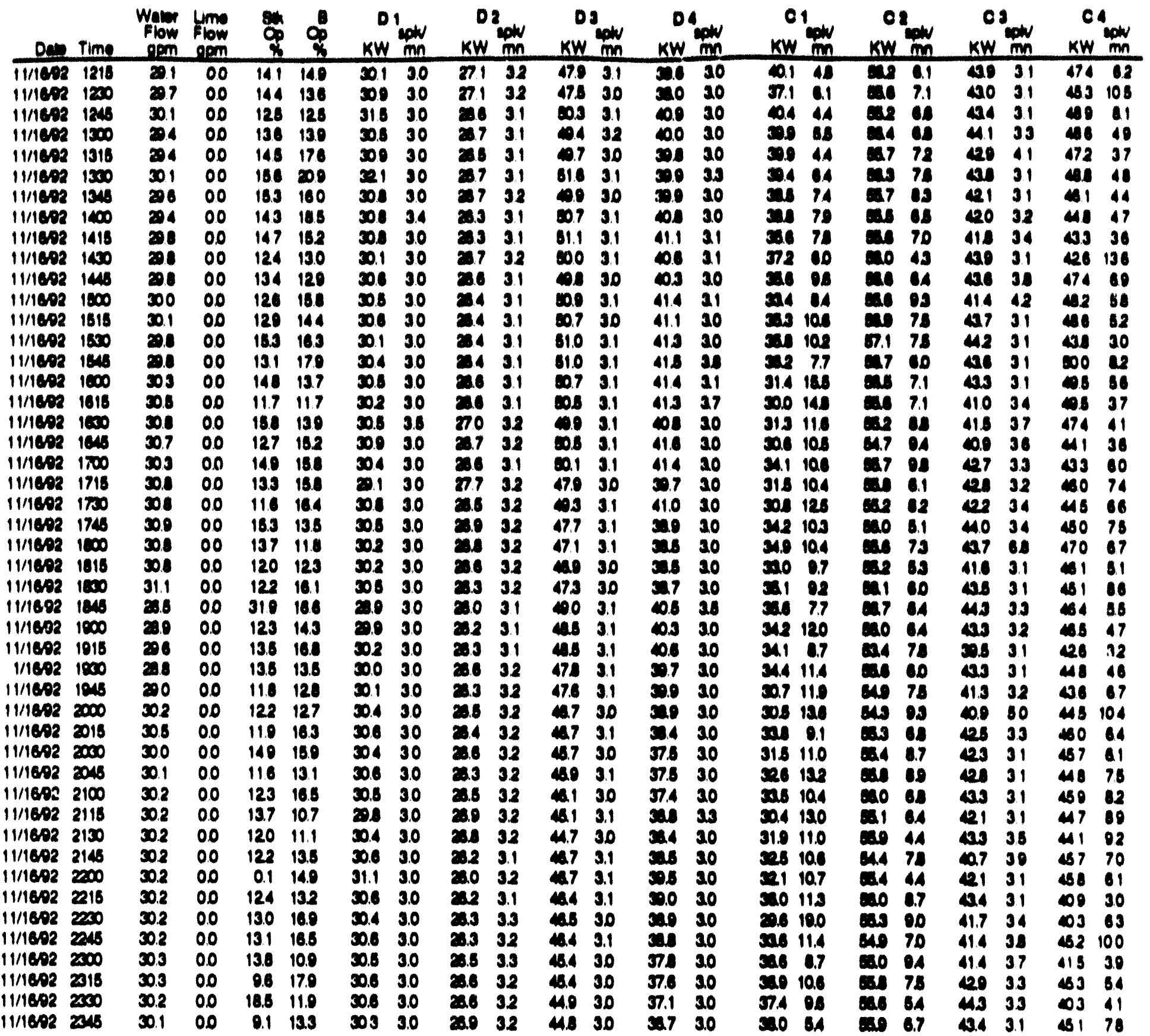



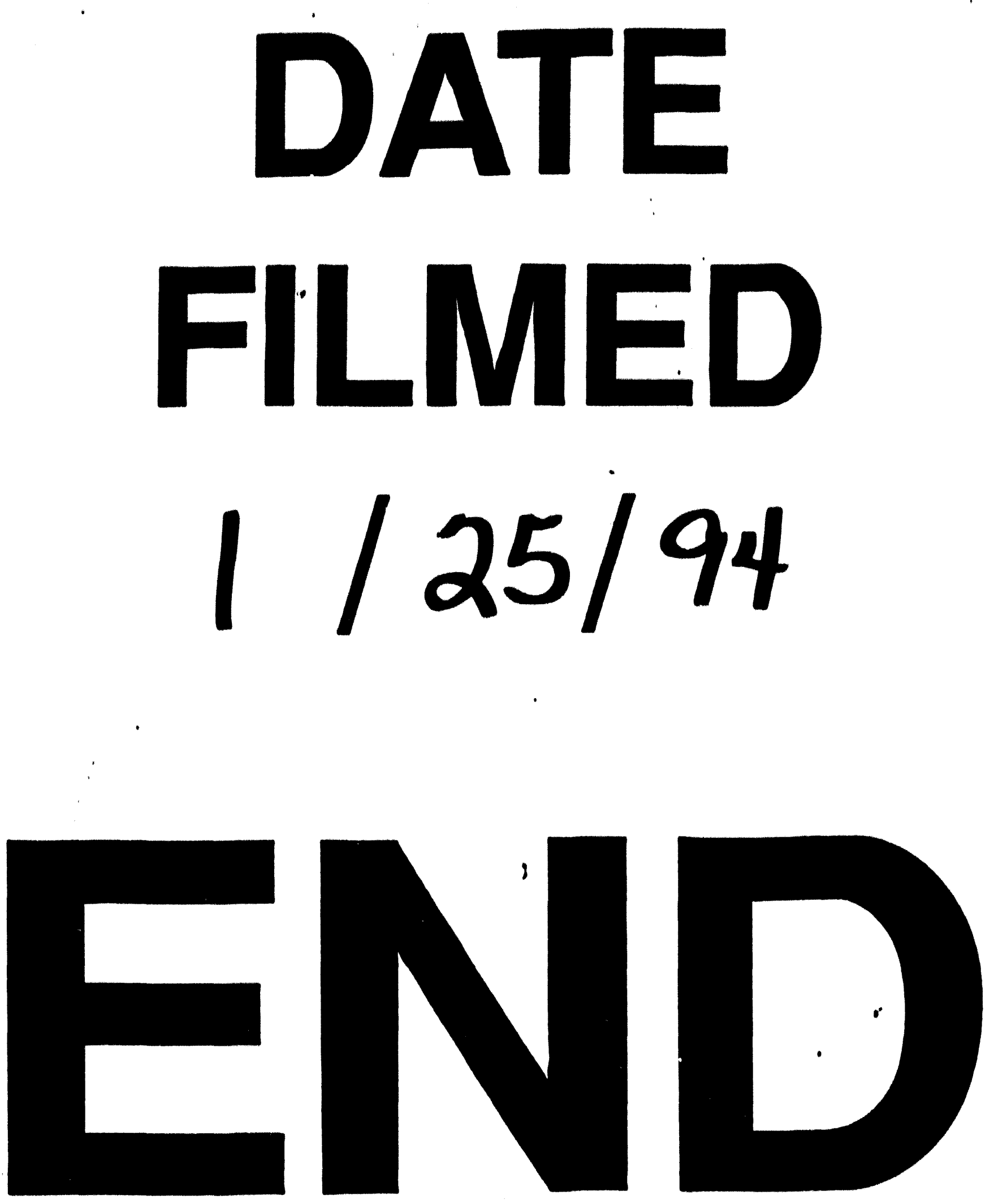
